Universidade Estadual Paulista "Júlio de Mesquita Filho" - UNESP Instituto de Biociências, Letras e Ciências Exatas de São José do Rio Preto

\title{
Análise Filogenética das Espécies de Hemigrammus Gill, 1858 (Characiformes, Characidae)
}

\author{
Jane Piton Serra
}

Orientador: Prof. Dr. Francisco Langeani Neto

Co-orientador: Prof. Dr. Heraldo Antônio Britski

Tese apresentada para obtenção do título de Doutor em Biologia Animal, junto ao Programa de Pós-Graduação em Biologia Animal do Instituto de Biociências, Letras e Ciências Exatas da Universidade Estadual Paulista "Júlio de Mesquita Filho”, Campus de São José do Rio Preto. 
Serra, Jane Piton.

Análise filogenética das espécies de Hemigrammus Gill, 1858 / Jane

Piton Serra. - São José do Rio Preto : [s.n.], 2010.

335 f. : il.; $30 \mathrm{~cm}$.

Orientador: Francisco Langeani

Co-orientador: Heraldo Antônio Britski

Tese (doutorado) - Universidade Estadual Paulista, Instituto de

Biociências, Letras e Ciências Exatas

1. Ictiologia. 2. Filogenia. 3. Peixes - Filogenia. I. Langeani, Francisco. II. Britski, Heraldo Antônio. III. Universidade Estadual Paulista, Instituto de Biociências, Letras e Ciências Exatas. IV. Título.

CDU - 597

Ficha catalográfica elaborada pela Biblioteca do IBILCE Campus de São José do Rio Preto - UNESP

Dedico este trabalho ao meu marido, Wesler e aos meus pais, Laurindo e Janesta 


\section{Jane Piton Serra}

\section{Análise Filogenética das Espécies de Hemigrammus Gill, 1858}

(Characiformes, Characidae)

\section{BANCA EXAMINADORA}

\section{TITULARES}

Prof. Dr.Francisco Langeani Neto

UNESP - São José do Rio Preto

Orientador

Prof. Dr. Luiz Roberto Malabarba

Universidade Federal do Rio Grande do Sul

Prof $^{\mathrm{a}}$. Dr ${ }^{\mathrm{a}}$. Ângela Maria Zanata

Universidade Federal da Bahia

Prof $^{\mathrm{a}}$. Dr ${ }^{\mathrm{a}}$. Katiane Mara Ferreira

Universidade de São Paulo

Prof. Dr. Vinicius de Araújo Bertaco

Universidade Federal do Rio Grande do Sul

\section{SUPLENTES}

Prof. Dr. Ricardo Cardoso Benine

UNESP - Botucatu

Prof. Dr. Reinaldo José Fazzio Feres

UNESP - São José do Rio Preto

Prof. Dr. Valdener Garutti

UFMT - Alto Araguaia

São José do Rio Preto, 08 de Abril de 2010 
Sem taxonomia para dar forma aos tijolos e a sistemática para dizer-nos como colocá-los juntos, os pilares das ciências naturais se transformam num quebra-cabeças sem significado.

Robert M. May.

O valor das coisas não está no tempo em que elas duram, mas na intensidade com que acontecem. Por isso existem momentos inesquecíveis, coisas inexplicáveis e pessoas incomparáveis.

Fernando Pessoa 


\section{AGRADECIMENTOS}

Durante o desenvolvimento deste doutorado, várias pessoas foram de fundamental importância, tanto para os conhecimentos teóricos quanto no apoio sentimental, sendo assim, espero aqui conseguir expressar minha gratidão a cada uma delas (e não me esquecer de ninguém!!). Poderia escrever centenas de páginas para agradecer a cada um, mas como o tempo é curto e o espaço também, vai aí um agradecimento resumido, mas verdadeiro e de coração.

Agradeço em primeiro lugar ao meu orientador (professor, amigo e padrinho de casório) Francisco Langeani, por todo o conhecimento, ictiológico e de vida, durante esses muitos anos (mais de dez!!!!) em que faço parte do laboratório de ictiologia da UNESP de São José do Rio Preto. Agradeço por ter me aturado (reconheço que não é fácil!!), por ter me dado o privilégio de sua convivência (dentro e fora do laboratório) e de ser sua orientada, e agradeço ainda também pelas broncas, discussões e puxões de orelha que me ajudaram a crescer e amadurecer.

Gostaria também de agradecer meu co-orientador Prof. Heraldo A. Britski por me permitir absorver uma ínfima parte de seu incrível conhecimento de ictiofauna, e pelos momentos tão preciosos que passei em sua companhia.

Não poderia deixar de fazer um agradecimento mais do que especial ao amigo Flávio C. T. Lima por compartilhar comigo seu (imenso) conhecimento dos pequenos characídeos, em especial os Hemigrammus da vida. Essa contribuição foi essencial para o desenvolvimento desse trabalho!!

Agradeço imensamente aos professores e aos meus colegas (especialmente Lia e Rodrigo Zieri) de curso de Pós-Graduação em Biologia Animal da UNESP de São José do Rio Preto, por todo o apoio, estimulo e amizade durante esses anos.

Minha muito obrigada aos colegas de laboratório de ictiologia (aos de baixo: Manoela, Fernandinha, Márcio e Rose e aos de cima: Cris, Mônica, Fabrício, Renato e Lílian), por toda a ajuda nas horas de necessidade.

Agradeço de coração as minhas queridas amigas Manoela M. F. Marinho (Manú), Fernanda O. Martins (Fernandinha) e Daiane Simiele (Daia), amigas de laboratório (apertado!), de alegria, de tristeza (e broncas!!), de kombis quebradas e coletas divertidas, de dias ensolarados e chuvosos, que 
chegaram como simples colegas de trabalho e se tornaram amigas incríveis, inseparáveis, indiscutíveis, (às vezes insuportáveis) e totalmente indispensáveis e insubstituíveis na minha vida. A Fernandinha merece um agradecimento especial por ter me aturado na agonia final da tese!!

Minha eterna gratidão à minha família: meus pais Laurindo M. Serra e Janesta L. Piton Serra, minha irmã Kátia Piton Serra, e minha "irmã/mãe" Lucia H. Vieira, pelo amor incondicional, por serem à base de minha formação, por sempre terem me apoiado e me ajudado em todos os momentos da minha vida, por nunca me deixarem desistir de meus sonhos e por sempre fazerem 0 impossível para que eles se realizem. Agradeço também por sempre terem me apoiado na minha empreitada ictiológica, mesmo que nem sempre entendam que peixe serve pra outras coisas além de comida.

Agradeço do fundo do meu coração ao amor da minha vida, meu marido Wesler C. Sanches por todo carinho, dedicação, cuidado e amor que tem comigo. Também por ter cometido a quase insanidade de se casar comigo no meio no doutorado (a melhor coisa que podia ter me acontecido!), e por dividir comigo todos os momentos de mais pura alegria (muitos e incontáveis) e de tristeza (que felizmente são poucos), e ainda me ajudar a superar as dificuldades (nunca é fácil ser atropelado...). Me orgulho muito de você, sempre!

Agradeço também aos meus amigos e parentes por estarem sempre presentes, tornando meus dias mais felizes por compartilharem comigo pizzas, churrascos, pipocas, bolinhos de chuva, jogos de baralho, noites em barzinhos e muitas risadas e conversa fiada (em especial: Wesler, Kátia, Fábio, Liliane, Fernandinha, Manú, Hania, Carina, Alex, Carol, Daia, Guilherme e Paulinho).

Gostaria de agradecer também às seguintes pessoas e coleções pelo empréstimo de material e/ou apoio e atenção durante as visitas às coleções: Mark Sabaj Pérez e John Lundberg (ANSP), Barry Chernoff e Mary Anne Rogers (FMNH), Lucia H. Rapp PyDaniel (INPA), Donald Taphorn e Otto Castillo (MCNG), Zilda Margarete S. Lucena (MCP), Ivan Sazima (MHN), Sonia Fisch-Muller (MHNG), Paulo A. Buckup (MNRJ), Osvaldo T. Oyakawa, José Lima de Figueiredo, Heraldo A. Britski e Flávio C. T. Lima (MZUSP), Carla Pavanelli e Rafaela Ota (NUP), Ângela M Zanata (UFBA), Luiz R. Malabarba, Vinicius Bertaco e Fernando R. Carvalho (UFRGS). 
Agradeço por fim ao Conselho Nacional de Desenvolvimento Científico e Tecnológico (CNPq - Processo no 140465/2006-5) pelo financiamento do presente projeto. 


\section{Aviso}

Este trabalho é parte integrante dos requisitos necessários à obtenção do título de Doutor em Biologia Animal e não deve ser considerado uma publicação zoológica formal. Em consequência, todos os resultados aqui apresentados não podem ser considerados como disponíveis em literatura zoológica. Cópias ou referências públicas a este documento, na sua forma atual, deverão ser feitos após consulta e aprovação prévia da autora. 


\section{CONTEÚDO}

RESUMO

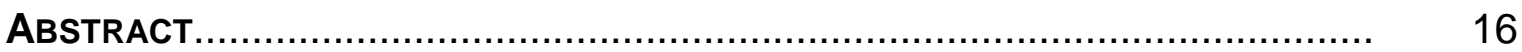

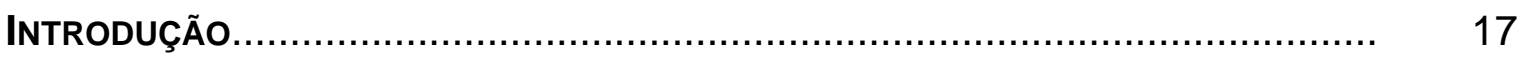

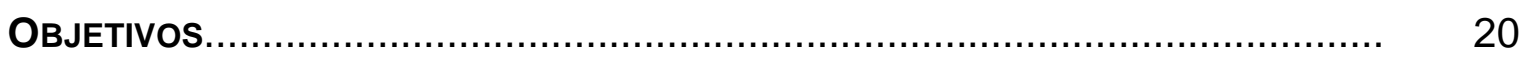

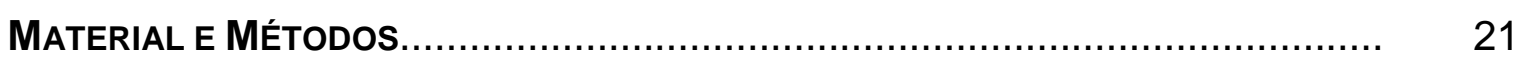

Taxonomia......................................................................... 22

Análise Filogenética.................................................................... 23

MATERIAL ANALISADO................................................................ 24

1- Espécies de Hemigrammus...................................................... 24

2- Espécies fora de Hemigrammus................................................ $\quad 29$

2.1- Gêneros "incertae sedis" em characidae.................................. 29

2.2- Representates do "clado A" .................................................. 33

2.3- Aphyocharacinae................................................................ 33

2.4- Characinae ................................................................ 33

2.5- Iguanodectinae ................................................................ 33

2.6- Cheirodontinae ............................................................. 33

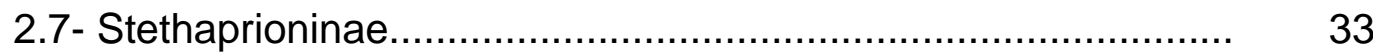

2.8- Grupos basais em Characidae.............................................. 34

RESULTADOS E DISCUSSÃO..................................................................

1- REDESCRIÇÃo E DESCRIÇÃO OSTEOLÓGICA DE HEMIGRAMMUS UNILINEATUS 35

1.1- REDESCRIÇÃO............................................................................. 35

Comentários......................................................................... 39

1.2- DESCRIÇÃO OSTEOLÓGICA.......................................................... 40

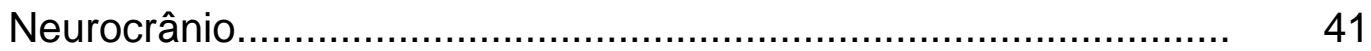

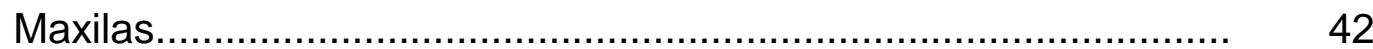

Infra-orbitais, Nasal e Antorbital................................................. 42

Arco palatino, Suspensório e Ossos Operculares.......................... 42

Arco Hióideo e Arco Branquial.................................................... 43

Aparelho de Weber e Supraneurais............................................. 44

Cintura e Nadadeira Peitoral.......................................................... 44

Cintura e Nadadeira Pélvica.......................................................... 45

Nadadeira Dorsal, Adiposa, Anal e Caudal .................................. 45

2- ANÁLISE FILOGENÉTICA................................................................ 47

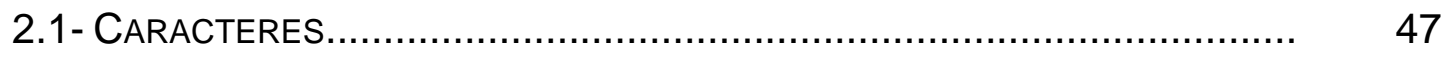

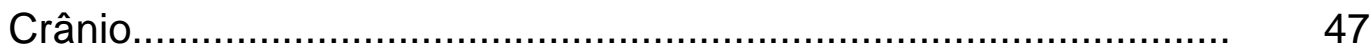

Maxilas.............................................................................. 70

Antorbital, Nasal, Supra-orbital e Infra-orbital................................ 96

Arco Palatino, Suspensório, Ossos Operculares............................ 114

Arco Hióideo....................................................................... 127 
Arcos Branquiais........................................................... 132

Cintura Peitoral .................................................................. 139

Nadadeira Dorsal........................................................... 146

Nadadeira Anal.................................................................. 149

Nadadeira Adiposa............................................................ 153

Nadadeira Caudal............................................................. 154

Vértebras e Supraneurais...................................................... 156

Escamas..................................................................... 160

Colorido......................................................................... 166

Miscelânea...................................................................... 184

2.2- RECONSTRUÇÃo FILOGENÉTICA................................................. 189

Considerações Gerais............................................................. 189

Clados discutidos................................................................ 190

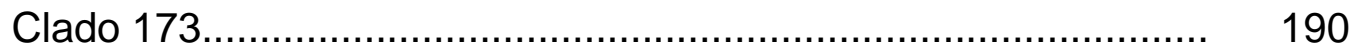

Hemigrammus levis....................................................... 192

Hemigrammus stictus........................................................ 193

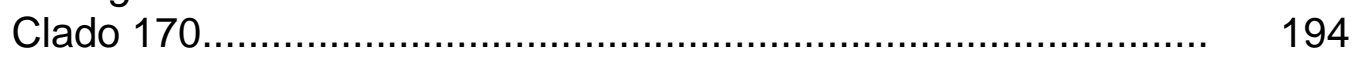

Hemigrammus barrigonae............................................. 195

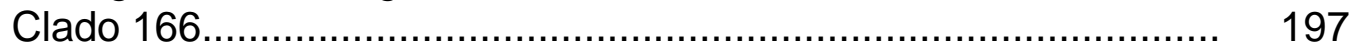

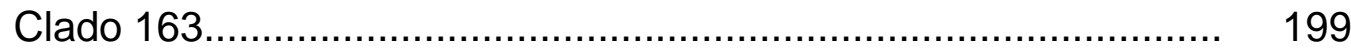

Clado 161 "Gênero Hemigrammus" ............................................ 200

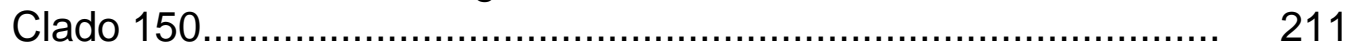

Clado 148...................................................................... 212

Clado 143.......................................................................... 215

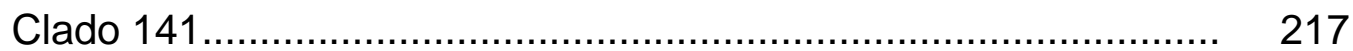

Hemigrammus brevis................................................... 223

Hemigrammus hyanuary.............................................. 224

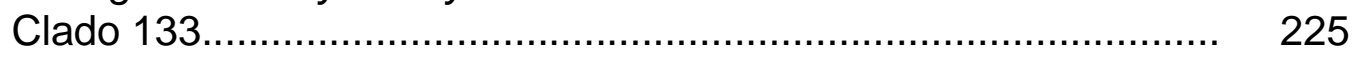

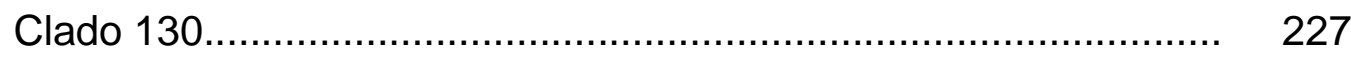

Hemigrammus yinyang................................................... 232

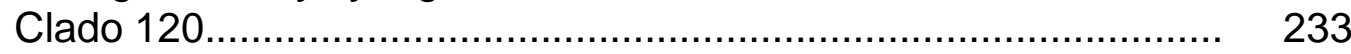

Hemigrammus taphorni...................................................... 236

Hemigrammus pretoensis.................................................... 237

CONSIDERAÇÕES FINAIS.................................................................. 240

REFERÊNCIAS BIBLIOGRÁFICAS.......................................................... 242

TABELAS E FIGURAS............................................................. 254

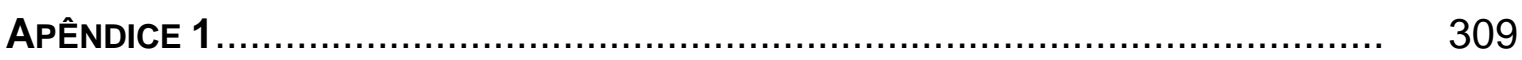

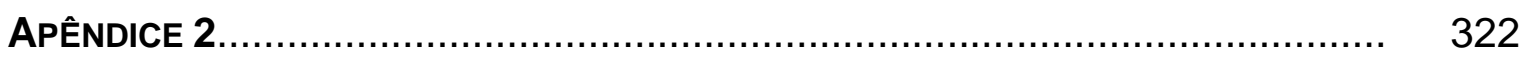




\section{LISTA DE TABELAS, FIGURAS E APÊNDICES}

Tabela 1- Espécies válidas de Hemigrammus, seguidas de sua respectiva localidade-tipo e distribuição geográfica. - Pg. 254.

Tabela 2- Dados morfométricos e merísticos de Hemigrammus unilineatus. Pg. 259.

Tabela 3- Matriz de dados de 165 caracteres e 95 táxons terminais utilizados na análise filogenética. - Pg. 260.

Figura 1- Hemigrammus unilineatus ANSP 147082, 27,7 mm CP, West Indies, Trinidad and Tobago, La Selva river. - Pg. 278.

Figura 2- Hemigrammus unilineatus MZUSP 65409, 25,8 mm CP. Crânio, vista dorsal. - Pg. 279.

Figura 3- Hemigrammus bleheri MCP 14921, 28,4 mm CP. Região anterior do crânio, vista dorsal. - Pg. 279.

Figura 4- Hasemania crenuchoides DZSJRP 11039, 50,7 mm CP. Região anterior do crânio, vista dorsal. - Pg. 280.

Figura 5- Hemigrammus lunatus MZUSP 90274, 27,8 mm CP. Região antertior do crânio, vista dorrsal. - Pg. 280.

Figura 6- Bryconamericus exodon MZUSP 28026, 43,1 mm CP. Crânio, vista dorsal. - Pg. 281.

Figura 7- Hemigrammus unilineatus MZUSP 65409, 25,8 mm CP. Crânio, vista lateral. - Pg. 281.

Figura 8- Bryconamericus exodon MZUSP 28026, 43,1 mm CP. Crânio, vista lateral. - Pg. 282.

Figura 9- Coptobrycon bilineatus DZSJRP 6890, 36,3 mm CP. Crânio, vista lateral. - Pg. 282.

Figura 10- Gymnocorymbus ternetzi DZSJRP 2808, 30,4 mm CP. Crânio, vista lateral. - Pg. 283.

Figura 11- Hemigrammus unilineatus, MZUSP 65409, 25,8 mm CP. Maxilas, vista lateral do lado esquerdo. - Pg. 283.

Figura 12- Coptobrycon bilineatus DZSJRP 6890, 36,3 mm CP. Maxilas, vista lateral do lado esquerdo. - Pg. 284.

Figura 13- Coptobrycon bilineatus DZSJRP 6890, 36,3 mm CP. Maxilas, vista medial do lado esquerdo. - Pg. 284. 
Figura 14- Hemigrammus mimus MZUSP 28018, 22,2 mm CP. Dentário, vista medial do lado esquerdo. - Pg. 285.

Figura 15- Antorbital. A) Hemigrammus unilineatus MZUSP 65409, 28,5 mm $\mathrm{CP}$; B) Hemigrammus hyanuary MZUSP 42348, 22,8 $\mathrm{mm}$ CP; C) Hemigrammus mimus MZUSP 28018, 22,2 mm CP; D) Coptobrycon bilineatus DZSJRP 6889, 36,3 mm CP e E) Lignobrycon myersi MCP 39737, 51,7 mm CP. - Pg. 285.

Figura 16- Hemigrammus unilineatus, MZUSP 65409, 25,8 mm CP. Infraorbitais, antorbital e nasal, vista lateral do lado direito (refletido). - Pg. 286.

Figura 17- Coptobrycon bilineatus DZSJRP 6890, 36,3 mm CP. Infra-orbitais, vista lateral do lado esquerdo. - Pg. 286.

Figura 18- Astyanax altiparanae DZSJRP 3297, 48,8 mm CP. Infra-orbitais, vista lateral do lado esquerdo. - Pg. 287.

Figura 19- Palatino. A) Hemigrammus unilineatus MZUSP 65409, 28,5 mm CP; B) Aphyocharax anisitsi DZSJRP 7573, $29 \mathrm{~mm}$ CP e C) Hasemania crenuchoides DZSJRP 11039, 50,7 mm CP. - Pg. 287.

Figura 20- Hemigrammus unilineatus MZUSP 65409, 25,8 mm CP. Arco palatino, suspensório e ossos operculares, vista lateral do lado esquerdo. - Pg. 288.

Figura 21- Parte do suspensório esquerdo em vista medial, detalhe da fenestra entre metapterigóide e quadrado. A) Hemigrammus eilyos DZSJRP 3092, 22,9 mm CP; B) Hemigrammus taphorni MCNG 55843, 41,3 mm CP e C) Salminus hilarii DZSJRP 3833, 74,8 mm CP. - Pg. 288.

Figura 22- Hemigrammus unilineatus MZUSP 65409, 25,8 mm CP. Parte do arco hióideo, vista ventral do lado esquerdo. - Pg. 289.

Figura 23- Uro-hial, vista ventral (superior) e vista lateral (inferior). A) Hyphessobrycon bentosi MZUSP 77528, 23,6 mm CP; B) Hemigrammus unilineatus MZUSP 65409, 25,8 mm CP; C) Hemigrammus newboldi MZUSP 103066, 33,6 mm CP e D) Brycon cf pesu DZSJRP 3803, 88,8 mm CP. - Pg. 289.

Figura 24- Hemigrammus unilineatus MZUSP 65409, 25,8 mm CP. Parte do arco branquial, vista dorsal. - Pg. 290.

Figura 25- Ceratobranquial 4. A) Hemigrammus marginatus MZUSP 17088, 36,3 mm CP e B) Hemigrammus analis MZUSP 85667, 33,8 mm CP. - Pg. 290.

Figura 26- Hemigrammus unilineatus MZUSP 65409, 25,8 mm CP. Aparelho de Weber. - Pg. 290. 
Figura 27- Hemigrammus unilineatus MZUSP 65409, 25,8 mm CP. Cintura e nadadeira peitoral, vista medial do lado esquerdo. - Pg. 291.

Figura 28- Hemigrammus mimus MZUSP 28018, 22,2 mm CP. Parte da cintura peitoral, vista lateral do lado esquerdo. - Pg. 291.

Figura 29- Coptobrycon bilineatus DZSJRP 6890, 36,3 mm CP. Parte da cintura peitoral, vista medial do lado esquerdo. - Pg. 292.

Figura 30- Bryconamericus exodon MZUSP 28026, 43,1 mm CP. Parte da cintura e nadadeira peitoral, respectivamente vista lateral e vista medial do lado esquerdo. - Pg. 292.

Figura 31- Hemigrammus unilineatus MZUSP 65409, 25,8 mm CP. Cintura e nadadeira pélvica, vista lateral do lado esquerdo. - Pg. 293.

Figura 32- Hemigrammus unilineatus MZUSP 65409, 25,8 mm CP. Nadadeira dorsal. - Pg. 293.

Figura 33- Supraneurais e parte da nadadeira dorsal. A) Hemigrammus unilineatus MZUSP 65409, 25,8 mm CP; B) Gymnocorymbus ternetzi DZSJRP 2808, 37,4 mm CP; C) Serrasalmus maculatus DZSJRP 1903, 47,4 mm CP; D) Poptella paraguayensis DZSJRP 627, 43,2 mm CP e E) Hemigrammus brevis MZUSP 17068, 20,7 mm CP. - Pg. 294.

Figura 34- Hemigrammus unilineatus MZUSP 65409, 25,8 mm CP. Nadadeira anal. - Pg. 295.

Figura 35- Hemigrammus unilineatus MZUSP 65409, 25,8 mm CP. Nadadeira caudal. - Pg. 295.

Figura 36- Colorido em vida. A) Hemigrammus rhodostomus; B) Hemigrammus bleheri e C- Petitella georgiae. Figuras extraídas de www.fishbase.org.br. - Pg. 296.

Figura 37- Nadadeira anal: presença de mancha negra. A) Hemigrammus unilineatus MZUSP 65409, 29 mm CP; B) Pristella maxillaris MZUSP 66678, 29,8 mm CP; C) Hyphessobrycon eques DZSJRP 10863, 19,3 mm CP; D) Moenkhausia hemigrammoides MZUSP 92036, 37 mm CP e E) Hemigrammus ulreyi MZUSP 59538, 30 mm CP. - Pg. 297.

Figura 38- Nadadeira anal: presença de mancha negra. A) Hyphessobrycon eques DZSJRP 10863, 26 mm CP; B) Pristella maxillaris MZUSP 66678, 29,8 $\mathrm{mm}$ CP; C) Hemigrammus unilineatus MZUSP 65409, $29 \mathrm{~mm} \mathrm{CP}$; D) Moenkhausia hemigrammoides MZUSP 92936, $37 \mathrm{~mm}$ CP; E) Hemigrammus rhodostomus MZUSP 17997, 27,1 mm CP e F) Gymnocorymbus ternetzi DZSJRP 10858, 36,5 mm CP. - Pg. 298.

Figura 39- Nadadeira anal: presença de mancha negra na base ou paralela à base da nadadeira. A) Hemigrammus bellottii MZUSP 85729, 28 mm CP; B) 
Hemigrammus boesemani MZUSP 65445, 26,9 mm CP e C) Hemigrammus barrigonae MZUSP 85006, 35 mm CP. - Pg. 299.

Figura 40- Pedúnculo caudal: presença de mancha negra. A) Hemigrammus ocellifer DZSJRP 11208, 20,9 mm CP e B) Hemigrammus newboldi MZUSP 77793, 31,6 mm CP. - Pg. 300.

Figura 41- Nadadeira caudal: presença de manhcas negras. A) Bryconamericus exodon MZUSP 54010, 27,5 mm CP; B) Moenkhausia bonita DZSJRP 11372, 24,8 mm CP; C) Hemigrammus parana DZSJRP 10796, 24,3 mm CP; D) Hemigrammus rhodostomus MZUSP 17997, 27,1 mm CP; E) Bryconops melanurus DZSJRP 6285, 46 mm CP e F) Thayeria obliqua MZUSP 29391, 32,8 mm CP. - Pg. 301.

Figura 42- Narinas. A) Hemigrammus unilineatus ANSP 147082, 27,7 mm CP e B) Coptobrycon bilineatus DZSJRP 29,1 mm CP. - Pg. 302.

Figura 43- Cladograma de consenso estrito das seis árvores mais parcimoniosas geradas pela análise. IC - 0,13; IR -0,44; Passos - 1849. - Pg. 303.

Figura 44- Única árvore mais parcimoniosa gerada após repesagens. IC 0,35; IR - 0,61. - Pg. 304.

Figura 45- Detalhe do clado 173 da única árvore mais parcimoniosa gerada após repesagens. - Pg. 305.

Figura 46- Detalhe do clado 161 da única árvore mais parcimoniosa gerada após repesagens. - Pg. 305.

Figura 47- Detalhe do clado 151 da única árvore mais parcimoniosa gerada após repesagens. - Pg. 306.

Figura 48- Detalhe do clado 136 da única árvore mais parcimoniosa gerada após repesagens. - Pg. 307.

Figura 49- Detalhe do clado 121 da única árvore mais parcimoniosa gerada após repesagens. - Pg. 307.

Apêndice 1- Lista das sinapomorfias para cada clado e táxon. - Pg. 308.

Apêndice 2- Lista da mudança dos estados dos caracteres. Número do caráter, seguido pelo índice de consistência, número de passos e mudança de estado. - Pg. 321. 


\section{RESUMO}

Hemigrammus Gill, (1858) é um dos gêneros mais especiosos dentro da família Characidae, com 51 espécies distribuídas nas Américas do Sul e Central; porém, pouco se sabia sobre suas relações com outros táxons de Characidae, sendo considerado por muitos autores como um grupo não natural (Ellis in Eigenmann,1918; Böhlke, 1955; Weitzman \& Palmer, 1997a; Marinho et al., 2008; Britski \& Lima, 2008; Lima \& Souza, 2009; Lima et al., 2009). As poucas propostas disponíveis de relacionamento para o gênero, apresentavam Hemigrammus como mais proximamente relacionado à Hyphessobrycon, Parapristella, Bryconella, Thayeria e Hasemania (Géry, 1977; Serra, 2003; Benine, 2004; Mirande, 2009), porém, a maioria desses trabalhos analisaram poucas (1-3) espécies do gênero e nenhum deles teve como objetivo principal testar o monofiletismo de Hemigrammus. Sendo assim, os objetivos do presente trabalho foram: redescrever e descrever osteologicamente $H$. unilineatus, espécie-tipo do gênero, avaliar o monofiletismo de Hemigrammus com a utilização do maior número possível de suas espécies válidas e avaliar o relacionamento de suas espécies entre si e com outras de Characidae. Foram analisados 95 táxons e 165 caracteres osteológicos, de morfologia externa e de colorido; a matriz de caracteres foi analisada pelo programa PAUP (4.0b 10 2001), com busca heurística, algoritmo tree-bisection-reconnection para o branch-swapping, random taxon addition sequence, 1000 réplicas e otimização acctran, com repesagens sucessivas. A análise filogenética resultou em seis árvores igualmente parcimoniosas; após repesagens uma única árvore mais parcimoniosa foi gerada, com IC. 0,35 e IR. 0,61. Hemigrammus em seu sensu atual não aparece formando um grupo monofilético. De acordo com os resultados obtidos o gênero fica restrito a sua espécie-tipo e espécies de outros gêneros de Characidae (Hyphessobrycon, Moenkhausia e Pristella). As demais espécies de Hemigrammus analisadas aparecem mais relacionadas entre si ou com outras de Characidae do que com a espécie-tipo do gênero. A nova concepção de Hemigrammus é discutida, bem como todos os clados onde aparecem alocadas as espécies do gênero. 


\section{ABStRact}

Hemigrammus Gill, (1858) is one of the most specious genera within the family Characidae, with 51 valid species distributed in South and Central America. The phylogenetic relationships of the genus with other Characidae are poorly known, and many authors consider that Hemigrammus does not constitute a monophyletic assemblage (Ellis in Eigenmann, 1918, Böhlke, 1955; Weitzman \& Palmer, 1997a; Marinho et al., 2008; Britski \& Lima, 2008, Souza \& Lima, 2009, Lima et al., 2009). The few hypotheses available concerning relationships of Hemigrammus, presented the genus as closely related to Hyphessobrycon, Parapristella, Bryconella, Thayeria, and Hasemania (Géry, 1977; Serra, 2003, Benine, 2004; Mirande, 2009), however, all these studies analyzed only few species (1-3) of the genus, and none aimed to test the monophyly of Hemigrammus. Therefore, the objectives of this study were: redefining and describing osteologically $H$. unilineatus, type species of the genus; evaluate the monophyly of Hemigrammus using the largest possible number of its valid species; and assess the relationships of its species among themselves and with other Characidae. Were analyzed 95 taxa and 165 characters concerning osteology, external morphology and color pattern. Phylogenetic analysis was performed using PAUP (4.0b 10 2001) with heuristic search algorithm, tree-bisection-reconnection for branch-swapping, random taxon addition sequence, 1000 replicates and optimization acctran, with successive reweighting. The phylogenetic analysis resulted in six most parsimonious trees; after reweighting, resulted in a single most parsimonious tree, with IC. 0.35 and IR. 0.61. Hemigrammus in its current sense does not form a monophyletic group. According to the results, the genus Hemigrammus is restricted to its type species and species of other genera of Characidae (Hyphessobrycon, Moenkhausia and Pristella). Other analyzed species of Hemigrammus are more related to each other or with other Characidae species than with the type species of the genus. The new composition of Hemigrammus is discussed, as well as all the clades which include species of the genus 


\section{INTRODUÇ̃̃O}

Hemigrammus Gill (1858) pertence à Characidae, uma das mais numerosas e heterogêneas famílias de Characiformes (Reis et al. 2003), alocando, atualmente, mais de 92 gêneros e 1.100 espécies, muitas anteriormente classificadas em Tetragonopterinae sensu Géry (1977) e hoje relacionadas como "incertae sedis" em vista do desconhecimento a respeito de seu monofiletismo e de suas relações filogenéticas (Reis et al. 2003; Lima et al. 2003; Lima et al., 2007; Mirande, 2009; Javonillo et al., 2010).

O monofiletismo de Characidae vem sendo amplamente discutido na literatura (dentre outros, Weitzman \& Malabarba, 1998; Malabarba \& Weitzman, 2003, Calcagnotto et al., 2005; Mirande 2009; Javonillo et al., 2010), resultando em várias propostas de relacionamento com pontos concordantes e outros divergentes. A realidade é que Characidae abrange muitos grupos (Reis et al, 2003), a maioria deles sem avaliação de monofiletismo, o que torna difícil a realização de análises adequadas da família. Frente a isso, Weitzman \& Malabarba (1998), colocam que para um melhor entendimento dos relacionamentos dentro de Characidae, são necessários estudos filogenéticos que avaliem o possível monofiletismo de seus gêneros e de outros grupos taxonômicos mais abrangentes.

Dentro de Characidae, a subfamília Tetragonopterinae (sensu Géry, 1977) é conhecida, tradicionalmente, pela presença de duas séries de dentes no pré-maxilar, porém não existem evidências de seu monofiletismo, sendo hoje a subfamília reconhecida apenas para Tetragonopterus Cuvier, seu gênero-tipo (Reis et. al, 2003; Lima et. al, 2003; Lima et al., 2007). Segundo Weitzman \& Malabarba (1998), além de não existirem evidências do monofiletismo de Tetragonopterinae (sensu Géry, 1977), muitos de seus gêneros mais especiosos também têm sua natureza monofilética incerta, como é o caso de Astyanax Baird \& Girard, Hyphessobrycon Durbin e Hemigrammus Gill.

Hemigrammus compreende hoje 51 espécies válidas, distribuídas em 10 países da América do Sul (Argentina, Bolívia, Brasil, Colômbia, Guiana, Guiana Francesa, Paraguai, Peru, Suriname e Venezuela) e um país da América Central (Trinidad e Tobago), sendo que no Brasil ocorrem 34 espécies, 17 delas endêmicas (Lima et al., 2003; Lima et al., 2005; Bertaco \& Carvalho, 
2005; Zarske et al., 2006; Lima et al., 2007; Benine \& Lopes, 2007; Zarske \& Géry, 2007; Marinho et al., 2008; Britski \& Lima, 2008; Lima \& Souza, 2009, Lima et al., 2009) (Tabela 1).

Apesar de Hemigrammus ser um dos gêneros mais especiosos de Characidae, pouco se sabe sobre suas relações filogenéticas, seja entre suas espécies, ou entre elas e outros gêneros de Characidae, porém, diversos autores sugerem que Hemigrammus não seja um grupo natural (Ellis in Eigenmann, 1918; Böhlke, 1955; Weitzman \& Palmer, 1997a; Marinho et al., 2008; Britski \& Lima, 2008; Lima \& Souza, 2009; Lima et al., 2009; Javonillo et al., 2010), o que indica que provavelmente muitas espécies hoje alocadas em Hemigrammus sejam mais relacionadas filogeneticamente a outros gêneros de Characidae.

Uma das questões que levanta discussões sobre o monofiletismo de Hemigrammus é o fato dos caracteres que definem o gênero não serem únicos dentro da família, pois como colocado por Lima et al. (2009), a distinção de Hemigrammus de cada um de seus gêneros considerados mais próximos é feita apenas por apenas um único caráter, como: nadadeira caudal com escamas (vs. nua em Hyphessobrycon), linha lateral incompleta (vs. completa em Moenkhausia), pouco dentes no maxilar (vs. muitos em Parapristella), duas séries de dentes no pré-maxilar (vs. uma única em Petitella), presença de nadadeira adiposa (vs. ausência em Hasemania) e lobos da nadadeira caudal de mesmo tamanho (vs. lobo inferior mais alongado em Thayeria).

As discussões sobre o monofiletismo de Hemigrammus envolvem, especialmente, Hyphessobrycon (também considerado não monofilético - Ellis in Eigenmann, 1918; Böhlke, 1955; Weitzman \& Palmer, 1997a; Lima \& Moreira, 2003; Bertaco \& Malabarba, 2005), pois o único caráter que separa os dois gêneros é a presença de escamas sobre a nadadeira caudal em Hemigrammus, e a ausência de tais escamas em Hyphessobrycon (Eigenmann, 1917), não existindo outros caracteres adicionais que os diferenciem satisfatoriamente. Böhlke (1955), discutindo as relações de algumas espécies de Hemigrammus e Hyphessobrycon, afirma que a análise das relações desses dois gêneros é difícil e ambígua e Ellis (in Eigenmann, 1918) destaca também que Hemigrammus e Hyphessobrycon são mais "conveniências" que "entidades". Weitzman \& Palmer (1997a e b) discutem a 
hipótese de Hemigrammus unilineatus, espécie-tipo do gênero, estar relacionada com o clado dos "rosy-tetra" e, dentro desse grupo estar proximamente relacionada com Hyphessobrycon compressus, espécie-tipo de Hyphessobrycon. Segundo os autores, caso essa proximidade filogenética ocorra, os dois gêneros se tornariam sinônimos, o que mudaria o nome genérico usado correntemente para mais de 150 espécies de pequenos tetragonopteríneos.

Os principais trabalhos tratando extensivamente de Hemigrammus são aqueles de Eigenmann (1918) e Géry (1977). Trabalhos publicados após Géry (1977) objetivaram sempre a descrição de novas espécies para o gênero: $H$. bleheri Géry \& Mahnert, 1986; H. mahnerti Uj \& Géry, 1989; H. neptunu Zarske \& Géry, 2002; H. skolioplatus Bertaco \& Carvalho, 2005; H. ora Zarske et al., 2006; H. geisleri Zarske \& Géry, 2007; H. taphorni Benine \& Lopes, 2007; H. parana Marinho et al., 2008; H. silimoni Britski \& Lima, 2008; H. arua Lima et al., 2009 e H. yinyang Lima \& Souza, 2009. Nenhum trabalho, entretanto trata de aspectos filogenéticos das espécies de Hemigrammus, sendo que atualmente o gênero está alocado como "incertae sedis" dentro de Characidae (Lima et al., 2003; Lima et al., 2007) e estaria mais relacionado aos Tetragonopterinae (sensu Géry, 1977) sem quatro dentes na série interna do pré-maxilar e ii, 8 raios na nadadeira dorsal.

Géry (1977) cria um agrupamento denominado de "Hemigrammus and allied genera", que engloba Hemigrammus, Hyphessobrycon, Parapristella, Ramirezella (= Hemigrammus) Bryconella, Thayeria e Hasemania, baseado na ocorrência de linha lateral incompleta e no pequeno tamanho dos exemplares, destacando ainda Hyphessobrycon e Hasemania como os mais semelhantes a Hemigrammus; o autor, porém, não apresenta uma análise filogenética para sustentar esse agrupamento. Serra (2003) em uma análise filogenética sugere que Hemigrammus, Hasemania, Thayeria, Hyphessobrycon e Parapristella sejam mais proximamente relacionados entre si do que a outros grupos de Characidae, baseada, principalmente na presença de linha lateral incompleta e de poucos dentes no maxilar, corroborando a proposta de Géry (1977). Outros trabalhos recentes incluem apenas uma ou poucas espécies de Hemigrammus e Hyphessobrycon (alguns também espécies de Hasemania e Thayeria) e via 
de regra, esses gêneros aparecem proximamente relacionados (Benine, 2004; Moreira, 2007; Mirande, 2009).

É consenso que a definição atual de Hemigrammus, baseada especialmente na presença de linha lateral incompleta e escamas sobre os lobos da nadadeira caudal é pobre, dá margem a muitas dúvidas e possivelmente defina um agrupamento poli ou parafilético. Entretanto, existem poucas análises envolvendo espécies de Hemigrammus e outros táxons de Characidae (Serra, 2003; Benine, 2004; Moreira, 2007 e Mirande, 2009), e essas análises, apesar de sugerirem o relacionamento do gênero com outros Characidae, não avaliam com mais profundidade a questão de seu monofiletismo e as relações entre suas espécies.

\section{OBJETIVOS}

De acordo com o exposto acima, os objetivos do presente trabalho foram:

- Redescrever H. unilineatus, espécie-tipo do gênero e descrever sua osteologia, de modo a fornecer informações mais recentes e abrangentes em relação à descrição original da espécie de Gill (1858)

- Testar o monofiletismo de Hemigrammus

- Avaliar o relacionamento filogenético de suas espécies entre sí

- Avaliar o relacionamento filogenético de Hemigrammus com outros táxons de Characidae. 


\section{MATERIAL e MÉtodos}

O material analisado pertence às seguintes coleções: ANSP (The Academy of Natural Sciences of Philadelphia, USA), DZSJRP (Coleção de Peixes do Departamento de Zoologia e Botânica de São José do Rio Preto), FMNH (Field Museum of Natural History, USA), INPA (Instituto Nacional de Pesquisas da Amazônia), MCNG (Museo de Ciencias Naturales de Guanare, Venezuela), MCP (Museu de Ciência e Tecnologia da Pontifícia Universidade Católica do Rio Grande do Sul), MCZ (Museum of Comparative Zoology, Harvard University, USA), MHN (Museu de História Natural "Prof. Dr. Adão José Cardoso", UNICAMP), MHNG (Museum d'Histoire Naturelle, Genebra, Suíça), MNRJ (Museu Nacional do Rio de Janeiro, Universidade Federal do Rio de Janeiro), MZUSP (Museu de Zoologia da Universidade de São Paulo), NMW (Naturhistorisches Museum, Viena, Áustria), NUP (Núcleo de Pesquisas em Limnologia, Ictiologia e Aqüicultura da Universidade Estadual de Maringá), UFBA (Museu de Zoologia da Universidade Federal da Bahia) e UFRGS (Universidade Federal do Rio Grande do Sul).

$\mathrm{Na}$ lista de material examinado, são fornecidos: número de registro da coleção, dados da localidade de coleta, número total de exemplares em álcool e diafanizados (listados como d\&c) quando disponíveis, e para esses últimos o tamanho dos exemplares.

Exemplares representativos de cada espécie foram diafanizados e corados segundo Taylor \& Van Dyke (1985) e dissecados segundo Weitzman (1974). A nomenclatura osteológica segue Weitzman (1962), com modificações de Monod (1967, 1968), Fink \& Fink (1981) e Vari (1983), segundo a tradução para a língua portuguesa proposta por Castro \& Castro (1987). As abreviações osteológicas utilizadas nas figuras são: aar - ângulo-articular; an - arco neural; ant - antorbital; bb - basibranquial; bh - basi-hial; boc - basioccipital; cb ceratobranquial; cha - cerato-hial anterior; chp - cerato-hial posterior; cla claustro; cle - cleitro; cn - complexo neural; co - coracóide; cp - costela pleural; cv - centro vertebral; d - dentário; eb - epibranquial; ect - ectopterigóide; ef escáfio; eh - espinho hemal; en - espinho neural; ep - epural; epo - epoccipital; esc - escápula; esf - esfenótico; esp - esquírola pélvica; etl - etmóide lateral; exe - extra-escapular; exo - exoccipital; fb - faringo-branquial; fc - fontanela craniana; fpt - fossa pós-temporal; fr - frontal; hb - hipobranquial; hi - 
hiomandibular; hip - hipural; hp - hipial; i - infra-orbital; ic - intercalário; ih inter-hial; iop - interopéculo; mes - mesopterigóide; mese - mesetmóide; meso - mesocoracóide; met - metapterigóide; mx - maxilar; na - nasal; ocm - osso corono-meckeliano; oe - órbito-esfenóide; op - opérculo; ope - osso pélvico; pa - parietal; pal - palatino; pc - pós-cleitro, pd - placa dentígera; pe paresfenóide; pi - processo isquiático; plc - processo lateral do centro vertebral dois; pm - pré-maxilar; pne - processo neural especializado; po - pró-ótico; pop - pré-opérculo; pp - paripural; prt - processo transverso da terceira vértebra; pt - pós-temporal; pte - pteroesfenóide; ptm - peça terminal; ptr - pterótico; qua - quadrado; rar - retroarticular; rb - rastro branquial; rbr - raio branquiostégio; rd - radial distal; re - rinoesfenóide; rm - radial mediano; rp radial proximal; rpc - raios procorrentes; scl - supracleitro; sim - simplético; sn - supraneural; soc - supra-occipital; sop - subopérculo; te - trabécula epifiseana; tr - trípode; uh - uro-hial; un - uroneural; ur - uróstilo; vo - vômer.

\section{Taxonomia}

Para a redescrição de Hemigrammus unilineatus foi feita uma série de 19 medidas e 15 contagens seguindo Fink \& Weitzman (1974) com as seguintes inclusões: 1- contagem de escamas cobrindo a base da nadadeira anal, representadas pelas escamas que se pronunciam sobre os raios da nadadeira; 2- contagem de raios branquiostégios. O comprimento padrão (CP) é expresso em milímetros e as demais medidas expressas como porcentagens do CP, exceto subunidades da cabeça, que são expressas como porcentagens do comprimento da cabeça (CC). Na tabela 2, "N" representa o número de espécimes examinados para cada medida e contagem. Todas as medidas foram feitas de ponto a ponto com paquímetro digital, os dados são aproximados em décimos de milímetro; contagens e medidas foram feitas do lado esquerdo dos exemplares sempre que possível.

Contagens de vértebras, rastros branquiais, raios branquiostégios e número total de dentes do dentário foram feitas somente em exemplares "d\&c". Vértebras do Aparelho de Weber foram contadas como quatro elementos, e as fusionadas (PU1+U1) da região caudal como um elemento único. $\mathrm{Na}$ redescrição de $H$. unilineatus, as contagens são seguidas por sua freqüência entre parênteses. 


\section{Análise Filogenética}

Para a análise filogenética foram utilizados caracteres osteológicos de exemplares "d\&c", caracteres de morfologia externa de exemplares em álcool e caracteres de padrão de colorido de exemplares em álcool e exemplares vivos; entretanto a maior parte das informações sobre colorido em vida foi retirada de literatura, especialmente trabalhos de descrição original ou redescrição das espécies.

A análise filogenética seguiu a metodologia de Hennig (1966, 1968), desenvolvida e elaborada por vários autores (Nelson \& Platinick, 1981; Wiley, 1981; Wiley et al., 1991; Amorim, 1997; Kitching et al., 2000), que reconhece e caracteriza os táxons pela posse e compartilhamento, entre seus membros, de novidades evolutivas únicas e recentes, as sinapomorfias. Para a polarização dos estados dos caracteres foi utilizado o método do grupo externo (Nixon \& Carpenter, 1993).

Frente ao pequeno conhecimento que se tem das relações filogenéticas de Hemigrammus, Tetragonopterinae (sensu Géry, 1977) e Characidae, a hipótese inicial para orientar a busca por características exclusivas e diagnósticas assumiu o gênero Hemigrammus como monofilético, tendo como referência $H$. unilineatus, sua espécie-tipo. Os demais gêneros escolhidos para análise foram separados em quatro níveis:

$1^{\circ}$ - espécies dos gêneros incertae sedis de Characidae: Hyphessobrycon, Parapristella, Bryconella, Thayeria e Hasemania, segundo proposta de relacionamento de Géry (1977) e Serra (2003), que colocam os referidos gêneros como mais proximamente relacionados a Hemigrammus;

$2^{\circ}$ - espécies dos demais gêneros incertae sedis de Characidae (Lima et al., 2003; Lima et al., 2007) sugeridos como pertencentes aos Characidae sem supra-orbital, sem ii, 8 raios na nadadeira dorsal e sem quatro dentes na série interna do pré-maxilar, segundo proposta de Malabarba \& Weitzman (2003);

3- outras espécies de Characidae, envolvendo o cla do A (sensu Malabarba \& Weitzman, 2003) e das subfamílias Cheirodontinae, Characinae, Stethaprioninae, Iguanodectinae e Aphyocharacinae, segundo proposta de relacionamento de Malabarba \& Weitzman (2003);

4- Brycon, Bryconops, Salminus, Serrasalmus, Triportheus, Lignobrycon, Chalceus e Brycinus que segundo diversas hipóteses recentes formam os 
grupos mais basais ou grupos irmãos de Characidae (Buckup, 1998; Weitzman \& Malabarba, 1998; Zanata, 2000; Malabarba e Weitzman, 2003; Zanata \& Vari, 2005; Moreira, 2007; Mirande, 2009)

Devido ao grande número de espécies na família Characidae e a falta de hipóteses de monofiletismo para a maioria de seus gêneros, sempre que possível, foi analisada a espécie-tipo dos gêneros, especialmente os incluídos nos níveis 1 e 2.

A matriz de caracteres foi analisada pelo programa PAUP (4.0b 10 2001, Swofford, não publicado), utilizando busca heurística, algoritmo tree-bisectionreconnection para o branch-swapping, opção random taxon addition sequence, 1000 réplicas e otimização acctran. Os caracteres foram codificados numericamente, não ordenados e tiveram o mesmo peso. Ao resultado obtido aplicou-se repesagem sucessiva dos caracteres com as mesmas definições iniciais.

Os caracteres apresentados englobam tanto novos quanto outros já descritos na literatura, que nesse caso, são seguidos de suas respectivas referências entre parênteses (autor, ano e número do caráter na referida obra).

A maioria das espécies teve seus exemplares (em álcool e "d\&c"), diretamente analisados, porém, para algumas espécies com pouco material disponível, a análise dos caracteres foi complementada com dados de literatura (especialmente descrição original e redescrições) quando possível.

A matriz de dados analisada é apresentada na tabela 3, as listas com as sinapomorfias para cada clado e com as mudanças dos estados dos caracteres são apresentadas nos apêndice 1 e 2 respectivamente.

$\mathrm{Na}$ reconstrução filogenética é apresentado cada um dos clados onde aparecem espécies de Hemigrammus e para esses clados são fornecidos os táxons integrantes e os caracteres de sustentação, seguidos pelo estado sinapomórfico entre parênteses e alguma discussão quando pertinente.

\section{Material analisado:}

1- Espécies de Hemigrammus

Das 51 espécies válidas de Hemigrammus, exemplares de 37 delas (aproximadamente $73 \%$ das espécies descritas) foram analisados. As 14 espécies restantes $(H$. aereus, $H$. cupreus, $H$. elegans, $H$. erythrozonus, $H$. 
geisleri, $H$. guyanensis, $H$. iota, $H$. matei, $H$. maxillaris, $H$. megaceps, $H$. melanochrous, $H$. micropterus, $H$. microstomus e $H$. ora), não foram analisadas pois a obtenção de exemplares de algumas dessas espécies é bastante difícil, visto que existem poucos exemplares, predominantemente tipos, depositados em coleções, entretanto, quando possível, comentários sobre o relacionamento dessas espécies são fornecidos na discussão. Para $H$. neptunus, $H$. luelingi, e H. mahnerti foram analisados somente exemplares em álcool, e essas espécies não foram incluídas na análise filogenética.

Hemigrammus analis Durbin, 1909. MZUSP 74761, Brasil, Amazonas, Negro, igarapé Arraia, margem direita do rio Cuieiras, aprx. $25 \mathrm{Km}$ da boca, 15 de 135 ex., 2 d\&c, 34,4 - 39 mm CP.; MZUSP 85667, Brasil, Amazonas, Rio Preto da Eva no Recanto do Buriti, logo ao lado esquerdo da praia do balneário, 30 de 139 ex., 2 d\&c, 33,8 e 37 mm CP.

Hemigrammus arua Lima, Wosiacki \& Ramos, 2009. Parátipos: MZUSP 100379, Brasil, Pará, Jutiti, Amazonas, São Francisco do Alto Curuá, 6 de 29 ex., 3 d\&c, 17,2 - 19,5 mm CP.

Hemigrammus barrigonae Eigenmann \& Henn, 1914. MZUSP 85006, Brasil, Amazonas, Negro, rio Tiquié, praia imediatamente abaixo da Cachoeira Comprida, 33 ex., 2 d\&c, 32,4 e 34,7 mm CP.; MZUSP 89232, Colômbia, Meta, Hacienda Mozambique, laguna W of N.L. Mozambique (2nd one to the NW), with outlet to the Metica, 15 de 50 ex., 2 d\&c, 22,9-30,5 mm CP.

Hemigrammus bellottii (Steindachner, 1882). Síntipos: NMW 57254, Tabatinga, alto Solimões, expedição Thayer, 4 ex. Material não tipo: MZUSP 85729, Brasil, Amazonas, Rio Preto da Eva, Urubu, primeiro igarapé no ramal ZF10, que sai do Km 113 da estrada para Itacoatiara, 30 de 303 ex., 3 d\&c, $22,9-27,9 \mathrm{~mm} \mathrm{CP}$.

Hemigrammus bleheri Géry \& Mahnert, 1986. MCP 14921, Brasil, Amazonas, Alto Negro, confluência do rio Negro com o rio Urubaxi, 17 ex., 2 d\&c, 28,4 e 29,6 mm CP.

Hemigrammus boesemani Géry, 1959. MZUSP 65445, Suriname, Distrito de Parmaribo, Coropina Creek, afl. do rio Suriname no balneário Republiek, 67 ex., 3 d\&c, 24,1 - 25,9 mm CP.

Hemigrammus brevis Ellis, 1911. MZUSP 47343, Brasil, Minas Gerais, Missões, São Francisco, riacho na fazenda próximo da cidade de Missões, 10 ex., 1 d\&c, 21,6 mm CP.; MCP 17068, Brasil, Bahia, São Francisco, rio da Olaria, $30 \mathrm{~km}$ e de Malhada na estrada para Guanambi, 30 de 150 ex., 2 d\&c, 20,7 e $22 \mathrm{~mm}$ CP.

Hemigrammus coeruleus Durbin, 1908. Síntipos: MCZ 20801, Brasil, Amazonas, Lago Manacapuru (Lago Grande de Manacapuru), 5 de 92 ex. Material não tipo: MZUSP 85685, Brasil, Amazonas, Rio Preto da Eva no Recanto do Buriti, logo ao lado esquerdo da praia do balneário, 30 de 99 ex., 3 d\&c; 27,6 $-33,5 \mathrm{~mm}$ CP. 
Hemigrammus cylindricus Durbin, 1909. ANSP 175656, Guyana, Siparuni, Essequibo river, extensive sanbar near Essequibo campsite, 50 de 102 ex., 3 d\&c, 40,2 e 34,9 mm CP.; MZUSP 35032, Brasil, Amazonas, Negro, rio Urubaxi, perto da boca, alagadiço, 12 de 13 ex., 2 d\&c, 33,1 e 32,8 mm CP.

Hemigrammus gracilis (Lütken, 1875). MZUSP 83795, Brasil, Bahia, Formosa do Rio Preto, São Francisco, rio Preto, no povoado de Cacimbinhas, pela estrada da garganta (Dianópolis - Formosa do Rio Preto), 23 ex., 2 d\&c, 24,6 e 25,9 mm CP.; MNRJ 16397, Brasil, Minas Gerais, Manga, rio São Francisco em Mocambinho (canal artificial de captação na margem direita do rio, 1 ex.; MNRJ 24476, Brasil, Mato Grosso, Nova Canaã do Norte, rio 17 de Agosto (tributário margem esquerda do rio Caarapá, bacia do rio Parado, Teles Pires) estrada MT 320, 4 ex. MZUSP 51373, Brasil, Bahia, Campo Formoso, São Francisco, rio Salitre na estrada para Lage, ca. $40 \mathrm{Km}$ a leste de Lage, Poços de Doce, 10 de 96 ex.

Hemigrammus haraldi Géry, 1961. MZUSP 27761, Brasil, Amazonas, Tefé, Japurá, Costa Japão, baixo rio Japurá, 34 ex., 2 d\&c, 17 e 18 mm CP. (obs. misturado com H. pulcher); MZUSP 18734, Brasil, Amazonas, Manaquiri, Solimões, Lago Janauacá, margem direita do rio Solimões, 1 d\&c, 23,3 mm CP.

Hemigrammus hyanuary Durbin, 1918. MZUSP 42348, Brasil, Amazonas, Solimões, lago Amaná, rio Solimões, 9 ex., 1d\&c, 22,8 mm CP.; MNRJ 9390, Colômbia, próximo a Letícia, alto Amazonas, Solimões, 1 ex.; MNRJ 9391, Colômbia, próximo a Letícia, alto Amazonas, Solimões, 1 ex.; MNRJ 9392, Colômbia, próximo a Letícia, alto Amazonas, Solimões, 1 ex.

Hemigrammus levis Durbin, 1908. Síntipos: MCZ 20738, Brasil, Amazonas, Lagoa do Maximo (Lago Maximo small lake near Parintins), 5 de 189 ex. Material não tipo: DZSJRP11195, Brasil, Pará, Baião, rio Jacundá, drenagem do rio Pará, fazenda Jutaituba; MZUSP 76129, Brasil, Amazonas, Solimões, lago Murumuru, curral de gado, Janauacá, 44 ex., 3 d\&c, 27 37,8 mm CP.; MCP 37129, Brasil, Amazonas, Solimões, lago Murumuru, perto do curral do INPA, Janauacá, 7 ex.

Hemigrammus luelingi Géry, 1964a. MZUSP 85587, Peru, Loreto, Requena, Ucayali, pequena quebrada, afl. Queb. Parnayari, ca. $2 \mathrm{Km}$ da estrada Jenaro Herrera/Colônia Angamos, 2 ex.

Hemigrammus lunatus Durbin, 1918. MZUSP 90274, Brasil, Mato Grosso, Cáceres, Paraguai, rio Sepotuba (trecho inferior), 25 de 41 ex., 2 d\&c, 27,8 e 29 mm CP.; MZUSP 54028, Paraguai, Concepcion, Paraguay, riacho Blandengue, perto da boca com o rio Apa, 1 d\&c, 30,8 mm CP.

Hemigrammus mahnerti Uj \& Géry, 1989. MZUSP 36314, Brasil, Mato Grosso do Sul, Corumbá, Paraguai, vazante do riozinho, fazenda Nhumirim, Nhecolândia, 8 ex.; MZUSP 35922, Brasil, Mato Grosso, Itiquira, Lagoas entre os rios Piquiri e Itiquira, faz. Santo Antônio do Paraíso, 18 ex (obs: misturados com H. tridens); MZUSP 37651, Brasil, Mato Grosso, Vila Bela de Santíssima Trindade, rio Guaporé, Cais da Balsa, 72 ex.

Hemigrammus marginatus Ellis, 1911. Parátipos: FMNH 54431, Brasil, Bahia, Queimadas, rio Itapicuru, 1 de 22 ex.; FMNH 54442, Brasil, Ribera Azul Lagoa, 12 miles from Tiete, 1 de 9 ex. Material não tipo: DZSJRP 12218, 
Brasil, Bahia, Queimadas, rio Itapicuru, logo abaixo da barragem Grande, 30 ex.; DZSJRP 12219, Brasil, Bahia, Barra, Brejo da Cachoeira, rio São Francisco, 10 ex.; MZUSP 57523, Brasil, Bahia, Jussiape, Leste, rio de Contas, ponte na estrada entre Marcolino Moura e Jussiape, 20 de 1073 ex., 2 d\&c, 28 e 29 mm CP.; MZUSP 17088, Brasil, Minas Gerais, Três Marias, rio São Francisco, 30 de 77 ex., 3 d\&c, 35,7 - 39,1 mm CP.; MZUSP 47346, Brasil, Minas Gerais, Missões, São Francisco, riacho na fazenda próximo da cidade de Missões, 5 ex.; MZUSP 38039, Brasil, Minas Gerais, Morada Nova de Minas, São Francisco, margem da represa de Três Marias na volta de Morada Nova de Minas, 15 de 552 ex., 2 d\&c, 28,4 e 30 mm CP.

Hemigrammus mimus Böhlke, 1955. MZUSP 28018, Brasil, Roraima, Caracari, Branco, rio Jufari, Baia Grande, São Bento, 14 ex., 1 d\&c, 22,2 mm CP.

Hemigrammus neptunus Zarske \& Géry, 2002. MZUSP 95352, rio Guaporé, 3 ex.; MZUSP 4444, Brasil, Mato Grosso, Santo Antônio do Leverger, Paraguai, 1 ex.; MZUSP 96686, Brasil, Mato Grosso, Barão de Melgaço, Paraguai, rio Mutum entre Vila do Mimoso e Joselândia, 2 ex.; MZUSP 90439, Brasil, Mato Grosso, Cáceres, Paraguai, rio Sepotuba (trecho inferior), 1 ex.

Hemigrammus newboldi (Fernández-Yépez, 1949). MZUSP 77793, Venezuela, Bolívar, Caura, canõ pequeño margen derecha, frente islã sur raudal 5000, 5 ex.; MZUSP 103066, Venezuela, Barinas, Orinoco, rio Suripa em el camino que pasa por la parcela de Capitanejo, 8 de 15 ex.,2 d\&c, 31,7 e 33,6 mm CP.

Hemigrammus ocellifer (Steindachner, 1882). MZUSP 7332, Brasil, Amazonas, Maués, igarapé Limãozinho, 30 de 105 ex., 3 d\&c 23,9 - 29,6 mm CP; MZUSP 17409, Brasil, Amazonas, Fonte Boa, Solimões, igarapé em Jacaré, próximo de Fonte Boa, 2 d\&c, 29,4 - 29,7 mm CP.; DZSJRP 11152, Brasil, Pará, Baião, igarapé Areia, drenagem do rio laçú, fazenda Jutaituba, 2 ex.; DZSJRP 11190, Brasil, Pará, Baião, rio Jacundá, drenagem do rio Pará, fazenda Jutaituba, 22 ex.; DZSJRP 11208, Brasil, Pará, Portel, rio Arapari, drenagem do rio Pacajá, fazenda Jutaituba, 2 ex.; DZSJRP 11122, Brasil, Pará, Bagre, igarapé Santo Antônio, drenagem do rio Jacundá, fazenda Jutaituba, 2 ex.

Hemigrammus orthus Durbin, 1909. MZUSP 25395, Brasil Pará, Tapajós, boca do igarapé Pimental, margem direita do rio Tapajós, povoado Pimental (PARNA), 30 de 55 ex., 3 d\&c, 16,7-18,7 mm CP.

Hemigrammus parana Marinho, Carvalho, Langeani \& Tatsumi, 2008. Holótipo: DZSJRP 6217, Brasil, São Paulo, Santa Clara D'Oeste, antigo porto de areia (conhecido como velho Adão); Parátipos: DZSJRP 10796, Brasil, São Paulo, Santa Clara D'Oeste, pequeno braço do rio próximo ao antigo porto de areia (conhecido como velho Adão), 354 ex., 3 d\&c, 22,6 - 33,4 mm CP.; DZSJRP 8907, Brasil, Mato Grosso do Sul, Aparecida do Taboado, córrego do Pântano, reservatório de Ilha Solteira, 197 ex., 4 d\&c, 22,2 - 29,9mm CP.

Hemigrammus pretoensis Géry, 1965. INPA 13228, Brasil, Amazonas, Manaus, 8 de 14 ex., 2 d\&c, 34,7 e 39,6 mm CP.; MZUSP 73980, Brasil, Amazonas, Manaus, igarapé Marianil, rio Negro, 1 ex. 
Hemigrammus pulcher Ladiges, 1938. MCP 28177, Peru, Loreto, Amazonas, Quebrada Pintuyacu a $43 \mathrm{Km}$ SW de lquitos na estrada para Nauta, 2 ex.; MCP 28203, Peru, Loreto, Amazonas, rio Itaya a $57 \mathrm{~km}$ SW de lquitos na estrada para Nauta, 13 ex, 2 de 3 ex d\&c, 18, 5 e 27,1.

Hemigrammus rhodostomus Ahl, 1924. MCP 39780, Brasil, Acre, Purus, igarapé sem nome (afluente do rio Taquari, ca. De $50 \mathrm{~km}$ a SE de Sena Madureira na BR-364, 40 de 99 ex., 2 d\&c, 26,8 e 28,7 mm CP.; MCP 27252, Brasil, Pará, Amazonas, rio Capim e lagoa marginal junto a balsa entre Paragominas e Tomé Açu, ca. $56 \mathrm{Km}$ a O. de Paragominas, 2 ex.; MZUSP 17997, Brasil, Pará, Badajpos, Capim, lago Bernardino, perto de Badajós, rio Capim, 30 de 153 ex., 3 d\&c, 28,4 - 30,5 mm CP.; MZUSP 29435, Brasil, Amazonas, Negro, rio Negro, confluência com o rio Urubaxi, 25 de 6681, 3 d\&c, 27,4-31,3 mm CP.

Hemigrammus rodwayi Durbin, 1909. MZUSP 65436, Suriname, Distrito de Parmaribo, Coropina Creek, afl. do rio Suriname, no balneário Republiek, 25 ex., 2 d\&c, 19,8 e 26,3 mm CP.

Hemigrammus schmardae (Steindachner, 1882). Síntipos: NMW 57979, Tabatinga, alto rio Solimões, expedição Thayer, 3 ex. Material não tipo: MZUSP 58621, Brasil, Amazonas, Santa Isabel do rio Negro, canal entre lagos em São João, perto de Tapurucuara (rio Negro), 30 de 126 ex., 3d\&c, 24,1-24,9 mm CP.

Hemigrammus silimoni Britski \& Lima, 2008. Parátipos: MZUSP 93562, Brasil, Mato Grosso, Sapezal, Tapajós, rio Juruena, jusante PCH Telegráfica, 2 de 8 ex., 2 d\&c., 17,1 e 23, 5 mm CP.; MZUSP 93561, Brasil, Mato Grosso, Sapezal, Tapajós, rio Juruena, jusante PCH Telegráfica, 1 ex.; MZUSP 93562, Brasil, Mato Grosso, Sapezal, Tapajós, rio Juruena, jusante PCH Telegráfica, 1 de 6 ex.

Hemigrammus skolioplatus Bertaco \& Carvalho 2005. Parátipos: MCP 37825, Brasil, Mato Grosso, Tapajós, rio Doze de Outubro, entre Comodoro e Vilhena (RO) na BR 364, 3 de 5 ex, 1 d\&c, 27,7 mm CP.

Hemigrammus stictus (Durbin, 1909). MZUSP 75471, Brasil, Amazonas, Manaus, Negro, igarapé Jaradá, afluente da margem direita do rio Cuieiras, cerca de $40 \mathrm{~km}$ da boca, 20 de 42 ex., 2 d\&c, 33,1 e 36,1 mm CP..

Hemigrammus taphorni Benine \& Lopes, 2007. Parátipos: MCNG 55843, Venezuela, Estado de Bolívar, afluente do rio Caura NE (CVG - Tecmin Grp 1-heliport), 22 ex., 2 d\&c, 40,2 e 41, 3 mm CP.

Hemigrammus tridens Eigenmann, 1907. UFRGS 9682, Paraguay, San Pedro, rio Aguaray (and associated run-off ditch) at bridge on dirt hwy (Rite 3), 2,1 $\mathrm{Km} \mathrm{N}$ of jct w/road (E) to Capitan Bado, 10 de 50 ex., 2 d\&c, 20,1 e 20, 4 MM CP; MZUSP 35922, Brasil, Mato Grosso, Itiquira, Lagoas entre os rios Piquiri e Itiquira, faz. Santo Antônio do Paraíso, 17 ex (obs: misturados com $H$. mahnerti).

Hemigrammus ulreyi (Boulenger, 1895). MZUSP 59538, Brasil, Mato Grosso do Sul, rio Verde de M.G., rio Negro, Anhuma, abaixo da estrada, menor conexão com o canal principal, 30 de 206 ex., 3 d\&c, 25,2-32 mm CP. 
Hemigrammus unilineatus (Gill, 1858). ANSP 70182, West Indies, Trinidad/Tobago, sent from Port-of-Spain, 1 ex.; ANSP 147082, West Indies, Trinidad/Tobago, La Selva river, 2 ex.; UFPB 3958, Brasil, Paraíba, rio Caiana, afluente da margem direita do rio Camarutuba, Reserva Biológica Guaribas, 8 ex.; MZUSP 65409, Suriname, Distrito Brokopondo, Maykaboeka Creek, Gros Rosebel Area, Area de concessão Golden Star, 2 d\&c, 25,7 e 28,5 mm CP; MZUSP 63759, Brasil, Pernambuco, Igarassu, igarapé Jacoca (também chamado Tabatinga), Refúgio Ecológico Charles Darwin, 11 ex.; MZUSP 78011, Brasil, Rondônia, Calama, igarapé de água preta, 20 ex.; MZUSP 6278, Brasil, Amazonas, Lago Castro, boca do rio Purus, 6 ex.; MZUSP 17877, Brasil, Pará, igarapé Açu, perto de São Domingos do Capim, 1 ex.; MZUSP 16534, Brasil, Pará, Utinga, Belém, 2 ex.; MZUSP 16897, Brasil, Pará, Belém, 1 ex.; MZUSP 16543, Brasil, Pará, Castanhal, drenagem do rio Capim, rio Apeu, Boa Vista, 39 ex.; MZUSP 103617, Equador, drenagem do rio Napo, rio Tambococha, quebrada a $3.000 \mathrm{~m}$ de la confluencia em el rio Yasuní, 5 ex.; MZUSP 17417, Brasil, Amazonas, Fonte Boa, drenagem do rio Solimões, Igarapé em Jacaré, próximo a Fonte Boa, 64 ex.

Hemigrammus vorderwinkleri Géry, 1963. MZUSP 88327, Brasil, Amazonas, Negro, igarapé Ira (primeiro tributário da margem direita do rio Tiquié), acima de Taracuá, 2 ex.; MZUSP 18684, Brasil, Mato Grosso, Vila Bela da Santíssima Trindade, Madeira, Lagoa do rio Guaporé em Mato Grosso, 6 de 60 ex., 1 d\&c, 23,1 mm CP.; MZUSP 85007, Brasil, Amazonas, Negro, rio Tiquié, praia imediatamente abaixo da Cachoeira Comprida, 30 de 61 ex., 2 d\&c, 25,2 e 27,3 mm CP.

Hemigrammus yinyang Lima \& Souza, 2009. Parátipos: MZUSP 92534, Brasil, Amazonas, Negro, igarapé Castanha (afl, rio Tiquié), próximo a foz, 6 de 27 ex.; MZUSP 93250, Brasil, Amazonas, Negro, igarapé Cunuri (ou Macucu), margem oposta do porto da comunidade de São José II, 3 d\&c, 16,6 - 23,3 $\mathrm{mm}$ CP.

Hemigrammus sp. n. - DZSJRP 6811, Brasil, São Paulo, Junqueirópolis, córrego Sucuri, porção represada (ponto 2), fazenda Alvorada, 40 ex., 2 d\&c, 27,3 e 27,7 mm CP.; DZSJRP 4762, Brasil, São Paulo, Rosana, Usina Hidrelétrica de Rosana, rio Paranapanema (limpeza de turbinas), 592 ex.; 2 d\&c, 25,5 e 29,7 mm CP.

\section{2- Espécies analisadas fora de Hemigrammus}

2.1- Gêneros Incertae Sedis em Characidae, sem ii, 8 e quatro dentes na série interna do pré-maxilar

Astyanax jacobinae - Parátipo: UFBA 02793, Brasil, Bahia, Jacobina, rio da Jaqueira, abaixo da Cachoeira Araponga, afl. do rio Itapicuru-mirim, bacia do Itapicuru, $1 \mathrm{~d} \& \mathrm{c}, 32,2 \mathrm{~mm}$ CP.

Astyanax mexicanus - UFRGS 9681, Mexico, San Luis Potosi, rio Ojo Frio, bridge on $\mathrm{N}$ edge of Rascon, 5 de 50 ex., 1 d\&c, 47,5 mm CP. 
Astyanax altiparanae - DZSJRP 10139, Brasil, São Paulo, Cândido Rodrigues, riacho afluente do rio dos Porcos, margem direita, drenagem Tietê/Batalha, 33 ex., 1 d\&c, 50,6 mm CP.; DZSJRP 3297, Brasil, São Paulo, Palestina, rio Turvo, Boturuna, 2 d\&c, 48,8 e 51,4 mm CP.

Bryconella pallidifrons - MZUSP 17486, Brasil, Amazonas, Fonte Boa, Solimões, Lago Mari-Mari, Ati-Paraná, NW de Fonte Boa, 30ex., 2 d\&c, 19 e 19,3 mm CP.

Bryconexodon juruenae - DZSJRP 6727, Brasil, Mato Grosso, Carlinda, riacho Corgão (ponto médio), Teles Pires, Tapajós, 1 ex.; DZSJRP 6731, Brasil, Mato Grosso, Carlinda, riacho Corgão (ponto médio), Teles Pires, Tapajós, 5 ex., $1 \mathrm{~d} \& \mathrm{c}, 83 \mathrm{~mm} \mathrm{CP}$.

Coptobrycon bilineatus - DZSJRP 6610; Brasil, São Paulo, Bertioga, rio Itatinga, sob a ponte de concreto, $2 \mathrm{Km}$ do centro de visitntes do Parque das Neblinas, 150 ex.; DZSJRP 6890, Brasil, São Paulo, Bertioga, Parque das Neblinas, desembocadura de riacho afluente da margem direita do rio Itatinga, $5^{\mathbf{a}}$ ponte de madeira na estrada de terra saindo do centro de visitantes do parque, 2 d\&c, 28,1 e 36,3 $\mathrm{mm}$ CP.

Deuterodon iguape - DZSJRP 2051, Brasil, São Paulo, Pariquera-Açu, Cedaval Setor Sul, riacho cercando arrozal alagado, BR $116 \mathrm{Km} \mathrm{461,} 24$ ex., 2 d\&c, 35,7 e 44,2 mm CP.

Grundulus cochae - Parátipos: DZSJRP 7498, Colômbia, Narino, Pasto, Laguna de La Cocha, 3 ex., 1 d\&c, 30,4 mm CP.

Gymnocorymbus ternetzi - DZSJRP 2808, Brasil, Mato Grosso do Sul, Miranda, rio Miranda, Morro do Azeite, fazenda Bodoquena, drenagem do rio Paraguai, 25 ex., 2 d\&c, 30,4 e 37,4 mm CP; DZSJRP 10858, Brasil, exemplares de aquário, 3 ex.

Hasemania crenuchoides - Holótipo: MZUSP 52732, Brasil, Distrito Federal, córrego Planaltina, rio São Bartolomeu, drenagem do rio Corumbá, rio Paranaíba, alto rio Paraná, 1 ex.; Parátipos: MHNG 2594.044, Brasil, Distrito Federal, córrego Planaltina, rio São Bartolomeu, drenagem do rio Corumbá, rio Paranaíba, alto rio Paraná, 2 ex.; DZSJRP 11039, Brasil, Distrito Federal, Brasília, córrego Paranoazinho, afluente do Sobradinho, rio São Bartolomeu, drenagem do rio Paranaíba, 47 ex., 4 d\&c, 33,8 - 50,7 mm CP .

Hasemania melanura - Parátipos: FMNH 54385, Brasil, Porto União, Rio Iguaçu, 39 ex., 3 d\&c, 28,2 - 31,7 mm CP.

Hasemania sp. n.- DZSJRP 8730, Brasil, Minas Gerais, Uberaba, nascente do rio Uberaba, BR 262, 133, 4 d\&c, 29,6 - 41,5 mm CP.

Hollandichthys aff. multifasciatus - DZSJRP 2293, Brasil, São Paulo, Jacupiranga, fazenda S.E.A.R.A. (riacho dentro da mata ), drenagem do rio Ribeira de lguape, 5 ex.; DZSJRP 5157, Brasil, sem dados de coleta, provavelmente rio Ribeira de lguape, $1 \mathrm{~d} \& \mathrm{c}, 76,8 \mathrm{~mm} \mathrm{CP}$.

Hyphessobrycon bentosi - MZUSP 77528, Brasil, Amazonas, rio Japurá em Serrinha, 18 ex., 2 d\&c, 22,5 e 23,6 mm CP. 
Hyphessobrycon coelestinus - DZSJRP 7905, Brasil, Distrito Federal, Planaltina, córrego Tabatinga, drenagem do rio Tocantins, reserva de Águas Emendadas, 8 ex., 1 d\&c, 22,9 mm CP.

Hyphessobrycon compressus - UFRGS 9683, Guatemala, Peten, Laguna de Peten along CAN airfield, near midlle of $S$ shore of Southern arm of lake, 6 de 50 ex.,2 d\&c, 30, 7 e 38,8 mm CP; MZUSP 18383, Belize R. Cayo, Belize Savanna pool (midle) mi 15 in Western Hwy behind D. Meyer's, 3 ex.

Hyphessobrycon diancistrus - Parátipos: MZUSP 13179, Colômbia, Vichada, San Jose de Ocune, rio Vichada, about $50 \mathrm{~km}$ West of San Jose de Ocune, 2 ex.. Material não tipo: MZUSP 29845, Brasil, Amazonas, rio Negro, Lago Central, Ilha de Buiu-açu, 19 ex.; MZUSP 17682, Brasil, Amazonas, Lagoa Central, margem esquerda do rio Negro, entre os rios Camaraú e Apaú, 3 ex.; MZUSP 96516, Venezuela, Bolívar, Cedeño, drenagem do rio Orinoco, rio Parguaza, comunidadde de Puente Parhueña, 16 ex.; MZUSP 29848, Brasil, Amazonas, Negro, rio Negro, confluência com o rio Urubaxi, 30 de 4223 ex., 4 d\&c, 20,7 - 24,6 mm CP.

Hyphessobrycon eilyos - DZSJRP 3092, Brasil, Mato Grosso, Alto Araguaia, lagoa a $16 \mathrm{Km}$ de Alto Araguaia, aos pés da Serra do Torto, rio Araguaia, 16 ex., $1 \mathrm{~d} \& \mathrm{c}, 22,9 \mathrm{~mm}$ CP.

Hyphessobrycon epicharis - Parátipos: MZUSP 42379, Venezuela, Terr. Fed. Amazonas, Dep. Rio Negro, small caño off Caño Urami, just upriver (rio Negro) from Santa Lucia, 3 d\&c de 55 ex.

Hyphessobrycon eques - DZSJRP 4754, Brasil, São Paulo, Rosana, Usina Hidrelétrica de Rosana, rio Paranapanema (limpeza de turbinas), 84 ex., 3 d\&c, 27,3-29,5 mm CP.

Hyphessobrycon erythrostigma - MZUSP 26325, Peru, lquitos, Moronacocha, prov. Mayana, Depto. Loreto, 3 d\&c de 18 ex.

Hyphessobrycon megalopterus - MZUSP 52462, Brasil, Mato Grosso, Cáceres, rio Paraguai em Cáceres e arredores, 11 ex., 1 d\&c., 15,1mm.

Hyphessobrycon micropterus - MZUSP 90825, Brasil, Minas Gerais, várzea da Palma, São Francisco, Lagoa da Olaria, lagoa marginal do rio das Velhas, 10 ex., 1 d\&c, 23,3 mm CP.

Hyphessobrycon takasei - MZUSP 29842, Brasil, Amapá, Araguari, rio Cupixi, ponte na Estrada para a Serra do Navio (boca do igarapé), 2 d\&c de 32 ex.

Jupiaba polylepis - MZUSP 52237, Brasil, Tocantins, Sandolândia, lagoas marginais do rio Água Fria, estrada entre Sandolândia e a barra do rio Verde, 10 ex., $1 \mathrm{~d} \& \mathrm{c}, 34,2 \mathrm{~mm}$ CP.

Microschemobrycon sp. - DZSJRP 8638, Brasil, Goiás, Doverlândia, rio Araguaia, 20 ex., 2 d\&c, 21,6 - 23,2 mm CP.

Moenkhausia bonita - DZSJRP 11372, Brasil, Mato Grosso, Tangará da Serra, afluente do rio Sepotuba, alto rio Paraguai, 193 ex., 3 d\&c, 32,8 - 34,5 mm CP.

Moenkhausia hemigrammoides - MZUSP 30531, Brasil, Roraima, rio Branco, cachoeira do Bem Querer, igarapé, 4 de 1586 ex.; MZUSP 92936, Brasil, Amazonas, Negro, igarapé Castanha, afl. Rio Tiquié, Sítio Belém pouco 
abaixo da comunidade de Santa Rosa, 20 de 59 ex., 1 d\&c, 30,7 mm CP.; MZUSP 30322, Rio Uraricoera, Maracá, 55 ex., 3 d\&c, 25 - 31, 8 mm CP.; MZUSP 30323, Brasil, Rio Branco, cachoeira Bem-querer, 12 ex.; MZUSP 38259, Suriname, Nickerie Dist. small stream entering stream located about 200 m upriver of Dalbana creek mouth (Corantijn), 14 ex.; MZUSP 32278, Suriname, Nickerie, Dist. small creek entering Lucie River, about $3 \mathrm{Km}$ upstream from junction of Lucie and Corantijn, 8 ex.; MZUSP 38275, Suriname, Nickerie Dist. stream entering south side of Lucie river about 200 $\mathrm{m}$ downstream of ferry crossing, 56 ex.

Moenkhausia intermedia - DZSJRP 420, Brasil, São Paulo, Mirassolândia, rio Preto, Macaúbas, rio Grande, 8 ex, 1 d\& c, 53,9 mm CP.

Moenkahusia sanctaefilomenae - DZSJRP 9217, Brasil, São Paulo, Nhandeara, ribeirão Bom Sucesso, abaixo da SP 461, 11 ex, 1 d\&c, 48,2 mm CP.; DZSJRP 3817, Brasil, São Paulo, Ipiguá, córrego da Barra Funda, drenagem do rio Preto, 2 d\&c, 27 e $41 \mathrm{~mm}$ CP.

Moenkhausia xinguensis - MZUSP 91690, Brasil, Mato Grosso, Paranatinga, Xingu, rio Culuene, na futura área da PHC Paranatinga II, 15 de 257 ex., 2 d\&c, 43,2 e 44,8 $\mathrm{mm}$ CP.

Oligosarcus pintoi - DZSJRP 7768, Brasil, São Paulo, Valparaíso, córrego da Queixada, afluente do córrego 15 de Janeiro, fazenda Paturi/Queixada, drenagem do rio Aguapeí, 21 ex., 2 d\&c, 44,4 e 51,4 mm CP.

Paracheirodon axelrodi - MZUSP 58337, Brasil, Amazonas, Negro, Castanhal Santa Rita, igarapé Anapichi, 19 ex., 2 d\&c, 25,4 e 25,9 mm CP.

Parapristella georgiae - MCP 14922, Brasil, Amazonas, Boa Vista, confluência do rio Negro com o rio Urubaxi, drenagem do Alto rio Negro, 8 ex., 1 d\&c, 30,7 mm CP; MZUSP 57493, Brasil, Amazonas, Santa Isabel do rio Negro, Paricatuba, lagoa na margem do rio Negro, 10 de 130 ex., 2 d\&c, 29,4 - 30,7 $\mathrm{mm}$ CP.

Petitella georgiae - MZUSP 59275, Brasil, Rondônia, Madeira, rio Machado, lago do Cururu, 20 de 176 ex., 2 d\&c, 30,5 e 30, 9 mm CP.

Pristella maxillaris - MZUSP 66678, Brasil, Amapá, savanas alagadas, Amazonas, 13 de 18 ex., 2 d\&c, 31,3 e 32,8 mm CP.

Psellogrammus kennedyi - MCP 16853, Brasil, Bahia, São Francisco, rio da Olaria, 30 km E de Malhada na estrada para Guanambi, 10 de 40 ex., 2 d\&c, 24 e 31 mm CP.; MNRJ 16341, Brasil, Minas Gerais, Manga, córrego do Retiro, cabeceira da lagoa do Sossego, margem direita do rio São Francisco, Mocambinho, 7 de 15 ex.; MNRJ 20554, Brasil, Mato Grosso, Cuiabá, pequeno tributário do rio Aricá-Açu, BR 364, a leste de Cuiabá, 5 de 11 ex.

Tetragonopterus argenteus - DZSJRP 526, Mato Grosso do Sul, Miranda, rio Miranda, Morro do Azeite, Fazenda Bodoquena, 1 ex.; DZSJRP 3834, Brasil, Mato Grosso do sul, Miranda, rio Miranda, Morro do Azeite, Fazenda Bodoquena, 2 d\&c, 44,5 e $52 \mathrm{~mm}$ CP.

Thayeria obliqua - MZUSP 29391, Brasil, Amazonas, confluência do rio Negro com o rio Urubaxi, 10 de 43 ex., 34,2 e 34,6 mm CP. 
2.2- Clado A (sensu Malabarba \& Weitzman, 2003)

Bryconamericus exodon - MZUSP 28026, Brasil, Mato Grosso, Poconé, rio Cuiabá (viveiro de pássaros), 98ex., 1 d\&c, 43,1 mm CP.; MZUSP 53947, Paraguai, Concepcion, rio Apa, em região de corredeira, 2ex.

Piabina argentea - DZSJRP 6338, Brasil, São Paulo, Nova Aliança, rio Borá, entre Nova Aliança e Potirendaba, drenagem do rio Tietê, 29 ex.; DZSJRP 443, Brasil, São Paulo, Irapuã, córrego afluente do rio Barra Mansa, afluente do rio Tietê, 1 d\&c, 45,2 $\mathrm{mm}$ CP.

Rhinopetitia myersi - DZSJRP 8499, Brasil, Goiás, Doverlândia, rio Araguaia, 3 ex., $1 \mathrm{~d} \& \mathrm{c}, 29,4 \mathrm{~mm}$ CP.

\section{3- Subfamília Aphyocharacinae}

Aphyocharax anisitsi - DZSJRP7573, Brasil, Mato Grosso do Sul, Nova Andradina, córrego do Laranjal, rio Ivinhema, 24 ex., 2 d\&c, 28,3 e 29 mm CP.

Aphyocharax pusillus - MZUSP 80044, Brasil, Amazonas, Tefé, Solimões, boca do Lago Caiambé, abaixo de Tefé, 6 de 25 ex., 1 d\&c, 29,6 mm CP

\section{4- Subfamília Characinae}

Roeboides bonariensis -DZSJRP 536, Brasil, Mato Grosso do Sul, Miranda, rio Miranda, Morro do Azeite, fazenda Bodoquena, drenagem do rio Paraguai, 6 ex., 1 d\&c, 50,7 mm CP.

Phenacogaster franciscoensis - MZUSP 83831, Brasil, Bahia, Formosa do Rio Preto, São Francisco, rio Sapão, bacia do rio Preto, à $1 \mathrm{Km}$ do povoado de São Marcelo, 6 de 10 ex.,2 d\&c, 29,6 e 30,4 mm CP.

\section{5- Subfamília Iguanodectinae}

Iguanodectes spilurus - DZSJRP 8365, Brasil, Mato Grosso, Barra do Garças, rio Corrente, bacia do rio das Mortes (Araguaia), ponte na BR158, 2 ex., 1 $\mathrm{d} \& \mathrm{c}, 61,7 \mathrm{~mm}$ CP.

\section{6- Subfamília Cheirodontinae}

Cheirodon interruptus - MCP 11613, Uruguai, Cerro Largo, Uruguay, lagoa a 10 $\mathrm{m}$ do rio Negro, 10 de 77 ex., 2 d\&c, 36,6 e 40,6 mm CP.

Serrapinnus heterodon - DZSJRP 2012, Brasil, São Paulo, Mirassolândia, rio Preto, Macaúbas, rio Grande, 69 ex., 30,4 - 32,1 mm CP.

Serrapinnus notomelas - DZSJRP 7707, Brasil, São Paulo, Lavínia, córrego Água do Tabajarinho (à jusante da represa), afluente do córrego 15 de Janeiro, drenagem do rio Aguapeí, 31 ex., 2 d\&c, 27,9 e 30 mm CP. 
Poptella paraguayensis - DZSJRP 627, Brasil, Mato Grosso do Sul, Miranda, rio Miranda, Morro do Azeite, Fazenda Bodoquena, 27 ex., 2 d\&c, 40 e 43,2 mm CP.

Stethaprion erythrops - MZUSP 30215, Brasil, Rondônia, rio Madeira, Calama (praia), 5 de 12 ex., 1 d\&c, 35,7 mm CP.

2.8- Grupos mais basais ou grupos irmãos de Characidae

Brycinus longipinnis - DZSJRP 3795, África, Ghana, Talkoradion, Tarkwa Road, state loc., $1 \mathrm{~d} \& \mathrm{c}, 76,2 \mathrm{~mm}$ CP.

Brycon cf. pesu - DZSJRP 3997, Brasil, Mato Grosso, Itaúba, rio Teles Pires, afluente do rio Tapajós, próximo a Canaã, 1ex.; DZSJRP 3803, Brasil, Mato Grosso, Itaúba, rio Teles Pires, afluente do rio Tapajós, próximo a Canaã, 1 $\mathrm{d} \& \mathrm{c}, 88,8 \mathrm{~mm}$ CP.

Bryconops melanurus - DZSJRP 6648, Brasil, Mato Grosso, Cuiabá, rio Aricá, afluente do rio Cuiabá, km 358 da BR 364, rio Paraguai, 1ex.; DZSJRP 6785, Brasil, Mato Grosso, Carlinda, riacho corgão, afluente do rio Teles Pires, Tapajós, 2 ex., 1 d\&c, 51,4 mm CP.

Chalceus spilogyros - MZUSP 19698, Brasil, Pará, lagoa Jacaré, rio Trombetas, 3 de 18 ex., 1 d\&c, 141,8 mm CP.

Lignobrycon myersi - MCP 36737, Brasil, Bahia, Leste, rio Cachoeira em Nova Ferradas, 2 de 12 ex., 1 d\&c, 51,7 mm CP.

Serrasalmus maculatus - DZSJRP 1903, Brasil, São Paulo, São José do Rio Preto, represa IPA, drenagem do rio Grande, 7 ex., 1 d\&c, 47,4 mm CP.

Salminus hilarii - DZSJRP 532, Brasil, São Paulo, divisa de Urupês e Irapuã, córrego da Figueira, afluente do rio Cubatão ou Barra Mansa, 3 ex.; DZSJRP 3833, Brasil, São Paulo, divisa de Urupês e Irapuã, córrego da Figueira, afluente do rio Cubatão ou Barra Mansa, 1 d\&c, 74,8 mm CP.

Triportheus nematurus - DZSJRP 623, Brasil, Mato Grosso do Sul, Miranda, Morro do Azeite, Fazenda Bodoquena, 18 ex., 1 d\&c, 49,9 mm CP. 


\title{
RESULTADOS E DISCUSSÃO
}

\section{1- Redescrição e descrição osteológica de Hemigrammus unilineatus}

\section{1- Redescrição}

\section{Hemigrammus unilineatus (Gill, 1858)}

\section{Figura 1}

Poecilurichthys (Hemigrammus) unilineatus Gill, 1858: 420 (descrição original; espécie-tipo do subgênero por designação original; localidade-tipo Western Portion of the Island of Trinidad, W. I. - Trinidad and Tobago - material tipo desconhecido); Günther, 1864: 317 (catálogo).

Tetragonopterus unilineatus - Eingemann \& Eigenmann, 1891: 54 (catálogo); Ulreyi 1895: 271 e 285.(lista de espécies, chave de identificação); Gilbert, 1900: 163 (catálogo)

Hemigrammus unilineatus - Eigenmann \& Ogle, 1907: 12; Eigenmann, 1910: 436; Ellis, 1911: 162 (lista de espécies); Eigenmann, 1918: 141 (redescrição, chave, lista sinonímica); Géry, 1977: 510 (chave); Lima et al, 2003: 133 (catálogo, lista sinonímica)

Hemigrammus unilineatus cayennensis Géry, 1959: 248 (descrição); Planquete,

Keith \& Le Bail, 1996: 282 (catálogo)

\begin{abstract}
Material examinado: ANSP 70182, West Indies, Trinidad/Tobago, sent from Port-of-Spain, 1 ex.; ANSP 147082, West Indies, Trinidad/Tobago, La Selva river, 2 ex.; UFPB 3958, Brasil, Paraíba, rio Caiana, afluente da margem direita do rio Camarutuba, Reserva Biológica Guaribas, 8 ex.; MZUSP 65409, Suriname, Distrito Brokopondo, Maykaboeka Creek, Gros Rosebel Area, Area de concessão Golden Star, 2 d\&c, 25,7 e 28,5 mm CP; MZUSP 63759, Brasil, Pernambuco, Igarassu, igarapé Jacoca (também chamado Tabatinga), Refúgio Ecológico Charles Darwin, 11 ex.; MZUSP 78011, Brasil, Rondônia, Calama, igarapé de água preta, 20 ex.; MZUSP 6278, Brasil, Amazonas, Lago Castro, boca do rio Purus, 6 ex.; MZUSP 17877, Brasil, Pará, igarapé Açu, perto de São Domingos do Capim, 1 ex.; MZUSP 16534, Brasil, Pará, Utinga, Belém, 2 ex.; MZUSP 16897, Brasil, Pará, Belém, 1 ex.; MZUSP 16543, Brasil, Pará, Castanhal, drenagem do rio Capim, rio Apeu, Boa Vista, 39 ex.; MZUSP 103617, Equador, drenagem do rio Napo, rio Tambococha, quebrada a $3.000 \mathrm{~m}$ de la confluencia em el rio Yasuní, 5 ex.; MZUSP 17417, Brasil, Amazonas, Fonte Boa, drenagem do rio Solimões, Igarapé em Jacaré, próximo a Fonte Boa, 64 ex.
\end{abstract}

\section{Diagnose}

Hemigrammus unilineatus se diferencia de todas as espécies de Characidae, exceto Moenkhausia hemigrammoides por apresentar: uma mancha 
negra, conspícua e oblíqua sobre a nadadeira anal, começando na base dos primeiros raios simples, atravessando a porção mediana dos raios seguintes e terminando na ponta do $2^{\circ}-4^{\circ}$ raio ramificado da nadadeira (vs. macha ausente ou não oblíqua em todas as demais espécies de Characidae); uma mancha na região ventral do corpo, anterior à nadadeira anal e contínua com a mancha da nadadeira anal (vs. mancha na região anterior do corpo ausente). Diferencia-se de Moenkhausia hemigrammoides por apresentar linha lateral incompleta (vs. linha lateral completa). Diferencia-se ainda das demais espécies de Hemigrammus, exceto $H$. ulreyi, $H$. coeruleus e $H$. elegans por apresentar uma mancha negra sobre os raios da nadadeira dorsal (vs. mancha negra na nadadeira dorsal ausente). Diferencia-se de $H$. ulreyi pela ausência de uma linha negra na base da nadadeira anal (vs. linha negra presente) e presença de uma mancha negra conspícua, delimitada e distribuída horizontalmente sobre a região mediana da nadadeira dorsal (vs. mancha negra mais clara, não delimitada e distribuída verticalmente sobre os primeiros raios da nadadeira, da base até a ponta). Diferencia-se ainda de $H$. coeruleus pela ausência de mancha negra na nadadeira peitoral (vs. presença); presença de uma mancha negra conspícua, delimitada e alongada horizontalmente sobre a região mediana da nadadeira dorsal (vs. mancha negra mais clara, não delimitada e alongada verticalmente sobre os raios da nadadeira em $H$. coeruleus) e 22-27 raios ramificados na nadadeira anal (vs. 20-22 raios ramificados). Finalmente, diferencia-se de $H$. elegans pela presença de mácula umeral (vs. ausência) e presença de uma mancha negra conspícua, delimitada e alongada horizontalmente sobre a região mediana da nadadeira dorsal ( $v s$. mancha negra mais clara, não delimitada e alongada verticalmente sobre os raios da nadadeira em $H$. elegans).

Descrição: Dados morfométricos e merísticos na tabela 2.

Corpo comprimido, moderadamente alongado, maior altura do corpo logo à frente da nadadeira dorsal. Perfil dorsal da cabeça reto, do focinho até o final do processo occipital. Perfil dorsal do corpo convexo, do final do processo occipital até o final da nadadeira dorsal; aproximadamente reto e inclinado ventralmente, do final da nadadeira dorsal até a nadadeira adiposa; levemente côncavo no pedúnculo caudal. Perfil ventral da cabeça convexo, do focinho até o 
final do istmo. Perfil ventral do corpo convexo, do ístmo até a origem da nadadeira anal, reto e inclinado dorsalmente da origem até o final da nadadeira anal; levemente côncavo no pedúnculo caudal.

Focinho arredondado. Boca terminal, maxilas iguais em tamanho (Figura 11). Pré-maxilar com duas séries de dentes; série externa com 3 (9), 4 (20) ou 5 (1) dentes tricuspidados e alinhados, série interna com 5 (26), 6 (3) ou 7 (2) dentes cônicos, tri, tetra ou pentacuspidados; o primeiro dente (próximo à junção dos pré-maxilares) e o $5^{\circ}$ ao $7^{\circ}$ dentes (próximos ao maxilar) com 0 menor número de cúspides (muitas vezes o 6ำ e 7ํㅗㅇo cônicos), e $02^{\circ}$ ao 4ํำ dentes 0 maior número de cúspides (Figura 11). Dentes da série interna do pré-maxilar diminuindo de tamanho do $1^{\circ}$ ao $6^{\circ}$ ou $7^{\circ}$; em muitos exemplares, cúspides laterais muito pouco desenvolvidas (especialmente nos dentes com mais de três cúspides), sendo melhor visualizadas em exemplares diafanizados (Figura 11). Maxilar com 3 (7), 4 (5), 5 (9), 6 (7) ou 7 (1) dentes cônicos, bi ou tricuspidados (Figura 11). Dentário com uma única série de dentes, 4 (27) ou 5 (3) dentes anteriores e maiores, tetra, penta ou hexacuspidados, seguidos por 10 a 11 (2) dentes menores, tricuspidados (geralmente 1 ou 2 dentes apenas) e/ou cônicos (a maioria) (Figura 11).

Nadadeira dorsal com ii, 9 (29) raios. Nadadeira adiposa presente. Extremidadade posterior da nadadeira peitoral com i, 10 (5), 11 (18) ou 12 (6) raios, atingindo os raios da nadadeira pélvica. Nadadeira pélvica com i, 7 (28) raios, extermidade posterior atingindo a origem da nadadeira anal. Nadadeira anal acentuadamente falcada, último raio simples e três a quatro primeiros ramificados muito mais longos que os demais (aproximadamente o dobro), a partir do $5^{\circ}$ a $6^{\circ}$ raio ramificado ocorre uma diminuição brusca de tamanho e à partir daí os raios seguintes diminuem gradualmente de tamanho em sentido posterior; anal com iii-iv, 22 (1), 23 (2), 24 (10), 25 (5), 26 (7) ou 27 (2) raios. Nadadeira caudal furcada, lobos iguais em tamanho e afilados, com $9+8$ (1) ou $10+9$ (22) raios principais.

Escamas ciclóides. Linha lateral incompleta, com 8 (3), 9 (1), 10 (3), 11 (5), 12 (4), 13 (5), 14 (3) ou 15 (1) escamas perfuradas e 18 (1), 19 (2), 20 (6), 21 (6), 22 (5) ou 23 (4) escamas não perfuradas. Cinco (18) ou 6 (7) séries longitudinais de escamas acima da linha lateral e 4 (21) ou 5 (4) séries longitudinais de escamas abaixo da linha lateral. Bainha de escamas ao longo 
da base da nadadeira anal com 3 (2), 4 (4), 5(4) ou 6 (7) escamas cobrindo a base do raios mais anteriores da nadadeira. Nadadeira caudal com escamas cobrindo a porção mais proximal dos raios e se estendendo sobre os lobos, especialmente nas bordas, por cerca de $1 / 3$ ou pouco mais de cada lobo. Primeiro arco branquial com 2 rastros no hipobranquial, 9-10 rastros no ceratobranquial, 1 rastro na cartilagem entre o ceratobranquial e o epibranquial e 6-7 rastros no epibranquial (2). Quatro raios branquiostégios (2).

\section{Coloração em álcool (Fig. 1)}

Corpo claro, castanho-amarelado. Cabeça com cromatóforos castanhoescuro pequenos distribuídos especialmente na região dorsal, no focinho e sobre o osso maxilar, diminuindo de concentração em sentido ventro-posterior, ausentes no ístmo. Cromatóforos castanho-escuro maiores que os do restante da cabeça e do corpo presentes em pequena quantidade sobre a região ventral do terceiro infra-orbital. Cromatóforos castanho-escuro em grande número sobre o opérculo. Guanina eventualmente presente sobre os ossos operculares e os infra-orbitais. Listra médio-dorsal castanho-escura da cabeça até o final do pedúnculo caudal Cromatóforos castanho-escuro dispersos por todo o corpo, em menor quantidade na região correspondente à cavidade visceral. Apesar de dispersos por toda a superfície da escama, os cromatóforos castanho-escuro aparecem também contornando a margem livre das escamas, condição mais acentuada na região latero-dorsal do corpo, onde a margem livre das escamas é contornada por um número maior de fileiras de cromatóforos castanho-escuro que as da região latero-ventral do corpo, porém sem formar um padrão reticulado. Mácula umeral pouco conspícua e verticalmente alongada. Listra médio-lateral estreita e relativamente incospícua, de pouco depois da mácula umeral até aproximadamente a metade do pedúnculo caudal. Cromatóforos castanho-escuro dispersos sobre o pedúnculo sem formar mancha característica. Nadadeiras peitorais, pélvicas e caudal sem manchas, com cromatóforos dispersos. Nadadeira dorsal com uma mancha negra, aproximadamente horizontal, muito conspícua, sobre a região mediana desde 0 primeiro raio simples até o $3^{\circ}$ ao $5^{\circ}$ ramificado; porções proximal e distal da nadadeira hialinas. Nadadeira anal com uma mancha negra muito conspícua e oblíqua, desde a base dos primeiros raios simples, atravessando obliquamente 
os raios seguintes e terminando na ponta do $2^{\circ}$ ao $4^{\circ}$ raios ramificados. Uma mancha negra horizontalmente alongada na região ventral, anterior à nadadeira anal e contínua com a mancha da nadadeira anal.

Distribuição: Ampla; exemplares são relatados para a América Central e América do Sul, em rios de Trinidad e Tobago, rios costeiros da Venezuela, rios da Guiana, Equador, Suriname, Guiana Francesa e bacia do rio Amazonas (Lima et al., 2003). Exemplares de H. unilineatus já foram também registrados em rios costeiros nos estados de Alagoas e Paraíba (Menezes et al., 2007) e no estado de Pernambuco (Gilbert, 1900).

Comentários: A distribuição de $H$. unilineatus é bastante ampla, ocorrendo, além dos rios de Trinidad e Tobago, região da localidade-tipo, em drenagens costeiras da Venezuela, Guiana, Suriname, Guiana Francesa e bacias dos rios Guaporé e Amazonas no Brasil; também registrados para rios costeiros nos estados de Alagoas, Paraíba e Pernambuco (Lima et al., 2003; Menezes et al., 2007, Gilbert, 1900). A grande amplitude geográfica da distribuição de $H$. unilineatus levanta dúvidas sobre o status taxonômico dessa espécie, não se sabendo se as diferentes populações realmente representam $H$. unilineatus ou se podem se tratar de espécies diferentes.

O padrão de colorido de exemplares coletados em diferentes localidades é praticamente idêntico, porém, dentre todos os exemplares que analisei, pude observar algumas diferenças em relação a número de escamas perfuradas na linha lateral, número de raios ramificados na nadadeira anal e especialmente número de cúspides nos dentes da série interna do pré-maxilar e número de dentes e cúpides dos dentes do maxilar. Em relação ao número de dentes e de cúspides dos dentes do maxilar (condição que mais me chamou a atenção dentre todas as diferenças encontradas), encontrei exemplares com cinco a sete dentes cônicos (ou a maioria cônico e apenas um ou dois bi ou tricuspidados) (exemplares de Trinidad e Tobago, Suriname e Pará) e exemplares com três a cinco dentes, todos tricuspidados ou cônicos e tricuspidados (exemplares de Pernambuco, Paraíba, Equador, Rondônia e Amazonas). As contagens de dentes das diferentes populações (bem como a de escamas perfuradas na linha lateral e raios ramificados na nadadeira 
dorsal), entretanto, se sobrepõem, tornando impossível a separação dessas populações em unidades diferentes; além disso, o número de exemplares analisados de $H$. unilineatus é pequeno frente a grande distribuição geográfica da espécie. Dessa forma todas as populações analisadas por mim foram aqui consideradas como sendo $H$. unilineatus. Weitzman \& Palmer (1997a) relatam a observação de diferenças em proporções corporais e algumas contagens de diferentes populações de $H$. unilineatus, porém também se disseram incapazases de separar completamente essas populações.

É interessante notar que algumas das principais características utilizadas por Géry (1965a) para diferenciar $M$. hemigrammoides de $H$. unilineatus são a presença de uma linha lateral completa em $M$. hemigrammoides (vs. incompleta em $H$. unilineatus); dois a três (raramente um) dentes tricuspidados no maxilar de M. hemigrammoides (vs. quatro a oito dentes em $H$. unilineatus) e 23 a 27 raios ramificados na nadadeira anal de $M$. hemigrammoides (vs. 22 a 25 raios ramificados em $H$. unilineatus). De acordo com as minhas observações e até mesmo com o apresentado por Géry (1965a) na descrição de $M$. hemigrammoides, a única característica efetiva para a separação total de $H$. unilineatus é a linha lateral incompleta na espécietipo de Hemigrammus e completa em $M$. hemigrammoides.

Infelizmente as séries de $H$. unilineatus depositadas em coleções são, na maioria das vezes, pequenas, especialmente aquelas referentes à Trinidad e Tobago, de onde a espécie foi descrita; além disso, os exemplares tipo da espécie estão perdidos. Entretanto, diante de todo o exposto é fácil constatar que uma revisão taxonômica de $H$. unilineatus é necessária, com o intuito de definir as características diagnósticas da espécie, esclarecer as diferenças observadas entre suas diferentes populações, definir sua real distribuição e separá-la mais adequadamente de M. hemigrammoides.

\section{2- Descricão Osteológica}

Material examinado: MZUSP 65409, Suriname, Distrito Brokopondo, Maykaboeka Creek, Gros Rosebel Area, Area de concessão Golden Star, 2 d\&c, 25,7 e 28,5 mm CP. 
Neurocrânio - Figuras 2 e 7

Mesetmóide com a porção anterior, até a origem das projeções laterais, triangular em vista dorsal; região mediana, entre as projeções laterais e o frontal, convexa em vista dorsal; projeções laterais triangulares. Vômer mais largo anteriormente, com uma leve reentrância na região mediana anterior, porém sem dividir essa região em duas partes distintas; porções laterais da região mais anterior do vômer visíveis em vista dorsal, lateralmente ao mesetmóide, com o pré-maxilar e nasal removidos. Frontal aproximadamente retangular, com a margem anterior arredondada. Fontanela craniana ampla, do mesetmóide ao supra-occipital, separando completamente os frontais e parietais medialmente, exceto na trabécula epifiseana. Etmóide lateral curto, com grande parte de sua superfície visível dorsalmente; ponte óssea entr o etmóide lateral e o vômer visível em vista dorsal com o pré-maxilar e o nasal removidos. Rinoesfenóide aproximadamente triangular, com duas regiões de ligação ao crânio, uma anterior, dirigida à junção dos etmóides laterais, e outra dorsal dirigida ao frontal. Órbito-esfenóide com uma ampla região de contato com o frontal, côncavo anteriormente e com uma projeção anterior alongada contactando o rinoesfenóide; porção posterior também com concavidade acentuada e uma projeção afilada em direção ao pteroesfenóide. Pteroesfenóide com a margem ventral ligeiramente côncava em vista lateral, desde o órbito-esfenóide até a região de contato com o esfenótico. Paresfenóide com curvatura ventral, terminando em bifurcação sob a região mediana do basioccipital. Processo lateral do esfenótico afilando e terminando em ponta, dirigida ventralmente. Pterótico com projeção lateral e posterior muito pequena e arredondada. Epoccipital delgado, com a porção mediana, entre as duas fossas pós-temporais, bastante estreitada em vista lateral; fossas amplas e completamente visíveis em vista lateral quando a cintura peitoral é removida. Exoccipital com a região posterior ventral em formato abaulado, bastante desenvolvida. Basioccipital com a região anterior, próximo à região de contato entre o pró-ótico e o exoccipital convexa e alta, região posterior, que fica abaixo do exoccipital côncava e mais baixa; porção posterior do basioccipital não atingindo a linha vertical que passa pela margem posterior do exoccipital. Processo posterior do supra-occipital ultrapassando a linha vertical 
que passa pela região posterior do exoccipital e atingindo a vertical que passa aproximadamente pelo meio do complexo neural.

Maxilas - Figura 11

Pré-maxilar mais longo que alto, com duas séries de dentes; série externa com três a cinco dentes tricuspidados e alinhados; série interna com cinco a sete dentes tri, tetra ou pentacuspidados, alinhados e maiores que os da série externa. Maxilar com porção anterior mais delgada; três a sete dentes cônicos, bi ou tricuspidados, na região mais anterior; porção posterior mais larga que a anterior; maxilar estendendo-se até a altura da região posterior do infra-orbital 2. Porção com dentes do maxilar mais curta que a porção sem dentes. Dentário relativamente curto, com a região posterior mais alta que a anterior; uma única série de dentes: quatro ou cinco dentes anteriore maiores, tetra, penta e/ou hexacuspidados, seguidos por 10 a 11 dentes tricuspidados (geralmente 1 ou 2 mais anteriores) e cônicos menores. Corono-meckeliano pouco desenvolvido, quase totalmente encoberto pela cartilagem do ânguloarticular em vista medial.

Infra-orbitais, Nasal e Antorbital - Figuras 15 A e 16

Supra-orbital ausente. Infra-orbitais 1 a 6 presentes; sendo o 3 o maior e o 6 o menor, muito reduzido, restrito quase que totalmente ao canal laterosensorial, com apenas uma lâmina óssea delgada. Canal latero-sensorial na porção interna de todos os infra-orbitais; infra-orbital $1 \mathrm{com}$ canal ausente na região mais anterior do osso; infra-orbitais 4 e 5 com canal um pouco deslocado para porção central do osso. Nasal delgado, com a mesma largura em toda a extensão, levemente convexo. Antorbital com a região dorsal delgada e a região ventral mais larga e abaulada.

\section{Arco Palatino, Suspensório e Ossos Operculares - Figuras 19 A e 20}

Palatino em forma de ampulheta, com uma constrição mediana e as porções anterior e posterior mais largas. Mesopterigóide triangular, com a região anterior, em contato com o palatino, mais estreita e a posterior (base do triângulo) mais larga, sobrepondo-se ao quadrado ventralmente. Ectopterigóide alongado, região anterior, em contato com o palatino, mais larga, região 
posterior delgada e apenas contatando o quadrado. Quadrado mais longo que alto. Simplético delgado, com aproximadamente a mesma largura em toda extensão, estendendo-se desde pouco depois da extremidade anterior do interopérculo até próximo ao hiomandibular. Metapterigóide mais baixo anteriormente e mais alto na região posterior; com uma projeção óssea laminar médio-dorsal bastante desenvolvida, acompanhando a altura do mesopterigóide. Metapterigóide com intersecção com o mesopterigóide anteriormente e com o hiomandibular posteriormente; ligado ao quadrado anteriormente por uma grande região cartilaginosa e ventro-posteriormente por uma cartilagem bastante delgada. Hiomandibular aproximadamente retangular até a região de contato com a porção dorsal do metapterigóide, onde ele forma uma superfície obliqua para encaixe do metapterigóide; leve concavidade na região médio-anterior. Pré-opérculo com canal sensorial largo e ramificado, atingindo a margem dorsal do hiomandibular e opérculo. Interopérculo baixo e ponteagudo anteriormente e alto e arredondado na região posterior, estendendo-se do ângulo-articular até pouco depois da margem posterior do pré-opérculo. Opérculo com a região posterior mais de duas vezes mais larga que a anterior, com uma concavidade dorso-posterior acentuada. Subopérculo mais alto na região anterior e afilado na região posterior, estendendo-se de pouco antes da margem anterior até o final da margem posterior do opérculo.

\section{Arco Hióideo e Arco Branquial - Figuras 22, 23 B e 24}

Cerato-hial posterior com uma fenestra alongada horizontalmente e localizada na região mais dorsal do osso. Quatro raios branquiostégios. Basihial triangular e alongado; porção anterior aproximadamente duas vezes mais larga que a posterior, alguns exemplares podem apresentar o basi-hial tendendo ao formato retangular, com a região anterior pouco mais larga que a posterior. Basibranquiais retangulares, com a região anterior pouco mais larga que a posterior, delgados e alongados, sendo o primeiro mais curto que os demais. Hipobranquiais cerca de $1 / 3$ a $1 / 4$ do comprimento dos ceratobranquiais. Hipobranquial 3 e ceratobranquial 4 com projeção óssea antero-dorsal afilada. Placa dentígera do ceratobranquial 5 triangular. Rastros branquiais setiformes, com cteniis, porém em pequeno número e esparsos. Rastros branquiais dos epibranquiais decrescendo de tamanho em sentido 
antero-posterior. Primeiro arco branquial com 2 rastros no hipobranquial, 9-10 rastros no ceratobranquial, 1 rastro na cartilagem entre o ceratobranquial e o epibranquial e 6-7 rastros no epibranquial. Ceratobranquiais 3 e 4 e epibranquiais 1 a 3 com duas séries de rastros, a segunda série é mais interna e os rastros são menores e menos ossificados. Faringo-branquial 1 triangular e pouco menor que o 2 e 3; faringo-branquial 2 aproximadamente retangular; faringo-branquial 3 também triangular, porém bem mais longo que o 1 e com dentes na face medial. Porção anterior do uro-hial bifurcada; abas laterais estreitas, bastante alongadas e fundidas medianamente. Eixo médio ventral do uro-hial se prolongando além das abas laterais.

\section{Aparelho de Weber e Supraneurais - Figura 26 e 33 A}

Complexo neural aproximadamente triangular, com a base voltada para os arcos neurais 3 e 4; região antero-dorsal convexa e póstero-dorsal côncava. Escáfio ovalado em vista lateral. Intercalário delgado em vista lateral, dirigido antero-lateralmente e localizado pouco acima do processo lateral do centro vertebral 2. Centro vertebral 1 quase totalmente visível em vista lateral, com apenas uma pequena região encoberta pelo processo lateral do centro vertebral 2 e pelo trípode. Arco neural 3 com um processo dorsal sobreposto ao arco neural 4 e um processo transverso, afilado e direcionado ao escáfio. Arco neural 4 com grande entalhe posterior e com espinho neural estendendo-se pouco além da porção posterior do complexo neural. Trípode triangular e amplo, com uma projeção delgada, posterior e medial à primeira costela pleural. Primeira costela pleural curta e larga. Quatro supraneurais distribuídos desde o aparelho de Weber, após o espinho neural da quarta vértebra, até a nadadeira dorsal, todos bastante delgados, alguns com a região dorsal pouco mais larga que a ventral.

\section{Cintura e Nadadeira Peitoral - Figura 27}

Coracóide com aproximadamente a mesma altura em toda extensão, formando uma fenestra grande e ovalada com o cleitro. Cleitro levemente inclinado para a frente. Supracleitro com um processo dorsal alongado que contata 0 pós-temporal medialmente. Pós-temporal largo e globoso ventralmente, afilando progressivamente e terminando em ponta. Extra- 
escapular alongado e com duas pequenas projeções laterais arredondadas. Pós-cleitro 1 com a região ventral arredondada e a dorsal arredondada também ou mais afilada; ligado à porção mais posterior do supra-cleitro e separado dos demais componentes da série. Pós-cleitro 2 oval e alongado verticalmente, sendo cerca de duas vezes mais comprido que o pós-celitro 1. Pós-cleitro 2 ligado dorsalmente à região mais posterior do cleitro e ventralmente ao póscleitro 3. Pós-cleitro 3 longo e sinuoso, com uma projeção óssea posterior globosa bastante desenvolvida, ocupando cerca de 1/3 do comprimento do osso. Pós-cleitro 3 localizado entre o pós-cleitro 2 e a escápula e estendendose pouco além da linha horizontal que passa pela margem ventral do coracóide. Escápula bifurcada dorsalmente em um ramo mais retangular, anterior, ligado ao cleitro e outro mais arredondado, posterior. Mesocoracóide delgado e com a base larga. Nadadeira peitoral com i, 10 a 12 raios.

\section{Cintura e Nadadeira Pélvica - Figura 31}

Osso pélvico aproximadamente triangular. Processo isquiático sinuoso formando um $\mathrm{V}$ invertido e com extremidade posterior cartilaginosa. Nadadeira pélvica com i, 7 raios.

Nadadeira Dorsal, Adiposa, Anal e Caudal - Figuras 32, 34 e 35

Primeiro radial proximal da nadadeira dorsal amplo e bifurcado anteriormente; demais radiais proximais simples e mais delgados, diminuindo progressivamente de tamanho em sentido posterior. Nadadeira dorsal com ii, 9 raios. Nadadeira adiposa presente.

Nadadeira anal longa com iii-iv, 22 a 27 raios; primeiro, segundo e terceiro radiais proximais quase do mesmo tamanho, sustentando todos os raios simples e os dois primeiros ramificados da nadadeira; demais radiais proximais progressivamente mais curtos em sentido posterior. Último raio simples e 1-3 primeiros ramificados muito longos, cerca de duas vezes mais longos que os demais raios; a partir do último ramificado longo ocorre uma diminuição brusca de tamanho, os demais raios são sub-iguais e diminuem de tamanho suavemente em sentido posterior. Nadadeira caudal com lobos iguais em tamanho; com sete hipurais, o primeiro e o segundo (fundidos e separados do centro composto) e o quarto são mais largos que os demais. Processo 
neural especializado bem desenvolvido, com uma projeção afilada na região dorsal. Com 10 raios procorrentes dorsais e 8 raios procorrentes ventrais. Nadadeira caudal com 9 a 10 raios principais no lobo superior e 8 a 9 raios principais no lobo inferior. 


\section{2- Análise Filogenética}

\section{1- Caracteres}

Segue abaixo uma relação com os 165 caracteres examinados obtidos através da observação de osteologia, de morfologia externa e de padrão de colorido, sintetizados na tabela 3. A busca por trabalhos que utilizaram o mesmo caráter não foi feita de modo exaustivo, tendo sido mais direcionada para aquelas produções com referência à Characidae, sendo assim, muitos trabalhos disponíveis podem não ter sido citados na presente análise. Além disso, na maioria das vezes, os caracteres utilizados por mim são codificados de forma diferente em relação àqueles citados nas referências.

\section{Crânio}

\section{1- Fontanela craniana (ic - 1,0)}

(Malabarba, 1998: 1; Buckup, 1998: 9; Vari, 1995: 26; Serra, 2003: 1; Mirande, 2009: 22):

\section{0 - ausente}

Brycon cf pesu

\section{1- presente (Figuras 2 - 6)}

Aphyocharax anisitsi, Aphyocharax pusillus, Astyanax altiparanae, Astyanax jacobinae, Astyanax mexicanus, Brycinus longipinnis, Bryconamericus exodon, Bryconella pallidifrons, Bryconexodon juruenae, Bryconops melanurus, Chalceus spilogyros, Cheirodon interruptus, Coptobrycon bilineatus, Deuterodon iguape, Grundulus cochae, Gymnocorymbus ternetzi, Hasemania crenuchoides, Hasemania melanura, Hasemania sp. n., Hemigrammus analis, Hemigrammus arua, Hemigrammus barrigonae, Hemigrammus bellottii, Hemigrammus bleheri, Hemigrammus boesemani, Hemigrammus brevis, Hemigrammus coeruleus, Hemigrammus cylindricus, Hemigrammus gracilis, Hemigrammus haraldi, Hemigrammus hyanuary, Hemigrammus levis, Hemigrammus lunatus, Hemigrammus marginatus, Hemigrammus mimus, Hemigrammus newboldi, Hemigrammus ocellifer, Hemigrammus orthus, Hemigrammus parana, Hemigrammus pretoensis, Hemigrammus pulcher, Hemigrammus rhodostomus, Hemigrammus rodwayi, Hemigrammus schmardae, Hemigrammus silimoni, Hemigrammus skolioplatus, Hemigrammus stictus, Hemigrammus taphorni, Hemigrammus tridens, Hemigrammus ulreyi, Hemigrammus unilineatus, Hemigrammus vorderwikleri, Hemigrammus yinyang, Hemigrammus sp. n., Hollandichthys aff. multifasciatus, Hyphessobrycon bentosi, Hyphessobrycon coelestinus, Hyphessobrycon compressus, Hyphessobrycon diancistrus, Hyphessobrycon eilyos, Hyphessobrycon epicharis, Hyphessobrycon eques, Hyphessobrycon erythrostigma, Hyphessobrycon megalopterus, Hyphessobrycon micropterus, Hyphessobrycon takasei, Iguanodectes spilurus, Jupiaba polylepis, Lignobrycon myersi, Microschemobrycon sp., Moenkhausia bonita, Moenkhausia hemigrammoides, Moenkhausia intermedia, Moenkhausia sanctaefilomenae, Moenkhausia xinguensis, Oligosarcus pintoi, Paracheirodon axelrodi, Parapristella georgiae, Petitella georgiae, Phenacogaster franciscoensis, Piabina argentea, Poptella paraguayensis, Psellogrammus kennedyi, Pristella maxillaris, Rhinopetitia myersi, Roeboides bonariensis, Salminus hilarii, Serrapinnus heterodon, Serrapinnus notomelas, Serrasalmus maculatus, Stethaprion erythrops, Tetragonopterus argenteus, Thayeria obliqua, Triportheus nematurus 


\section{2- Forma da fontanela do frontal (ic - 0,043)}

\section{0- triangular (Figuras 3 e 5)}

Aphyocharax anisitsi, Aphyocharax pusillus, Astyanax altiparanae, Astyanax jacobinae, Astyanax mexicanus, Bryconella pallidifrons, Bryconexodon juruenae, Chalceus spilogyros, Cheirodon interruptus, Coptobrycon bilineatus, Deuterodon iguape, Grundulus cochae, Gymnocorymbus ternetzi, Hasemania crenuchoides, Hasemania melanura, Hemigrammus analis, Hemigrammus bellottii, Hemigrammus bleheri, Hemigrammus boesemani, Hemigrammus coeruleus, Hemigrammus gracilis, Hemigrammus cylindricus, Hemigrammus haraldi, Hemigrammus hyanuary, Hemigrammus levis, Hemigrammus lunatus, Hemigrammus marginatus, Hemigrammus mimus, Hemigrammus newboldi, Hemigrammus ocellifer, Hemigrammus pretoensis, Hemigrammus rhodostomus, Hemigrammus rodwayi, Hemigrammus schmardae, Hemigrammus silimoni, Hemigrammus skolioplatus, Hemigrammus taphorni, Hemigrammus tridens, Hemigrammus ulreyi, Hemigrammus vorderwikleri, Hyphessobrycon bentosi, Hyphessobrycon diancistrus, Hyphessobrycon epicharis, Hyphessobrycon eques, Hyphessobrycon erythrostigma, Hyphessobrycon micropterus, Hyphessobrycon takasei, Iguanodectes spilurus, Microschemobrycon sp, Moenkhausia sanctaefilomenae, Moenkhausia xinguensis, Oligosarcus pintoi, Paracheirodon axelrodi, Parapristella georgiae, Petitella georgiae, Poptella paraguayensis, Pristella maxillaris, Salminus hilarii, Serrapinnus heterodon, Serrapinnus notomelas, Serrasalmus maculatus, Stethaprion erythrops, Thayeria obliqua, Triportheus nematurus

\section{1- retangular (Figuras 2 e 6 )}

Bryconamericus exodon, Bryconops melanurus, Hemigrammus arua, Hemigrammus barrigonae, Hemigrammus brevis, Hemigrammus orthus, Hemigrammus parana, Hemigrammus stictus, Hemigrammus sp $\mathrm{n}$. Hemigrammus unilineatus, Hemigrammus yinyang, Hollandichthys aff. multifasciatus, Hyphessobrycon coelestinus, Hyphessobrycon compressus, Hyphessobrycon eilyos, Hyphessobrycon megalopterus, Jupiaba polylepis, Lignobrycon myersi, Piabina argentea, Phenacogaster franciscoensis, Psellogrammus kennedyi, Roeboides bonariensis, Tetragonopterus argenteus, Moenkhausia bonita, Moenkhausia intermedia

\section{$\underline{\text { 9- Indeterminado }}$}

Brycon cf pesu, Brycinus longipinnis, Hasemania sp n., Hemigrammus pulcher, Rhinopetitia myersi, Salminus hilarii

\section{3- Fontanela do frontal (ic - 0,100)}

(Serra, 2003: 3; Moreira, 2007: 106):

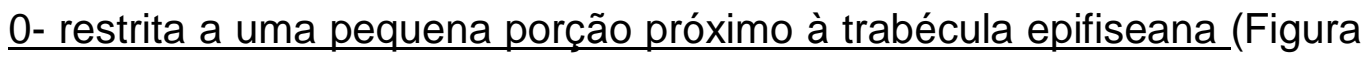

4)

Aphyocharax anisitsi, Brycinus longipinnis, Chalceus spilogyros, Cheirodon interruptus, Hasemania crenuchoides, Hasemania sp n., Hemigrammus analis, Iguanodectes spilurus, Microschemobrycon sp, Rhinopetitia myersi, Salminus hilarii, Serrapinnus heterodon, Serrapinnus notomelas, Stethaprion erythrops

\section{1- desenvolvida e alongada, maior que a metade da distância entre a}

\section{trabécula epifiseana e a extremidade anterior do frontal (Figuras 2 e 6)}

Aphyocharax pusillus, Astyanax altiparanae, Astyanax jacobinae, Astyanax mexicanus, Bryconamericus exodon, Bryconella pallidifrons, Bryconexodon juruenae, Bryconops melanurus, Coptobrycon bilineatus, Deuterodon iguape, Grundulus cochae, Gymnocorymbus 
ternetzi, Hasemania melanura, Hemigrammus arua, Hemigrammus barrigonae, Hemigrammus bellottii, Hemigrammus bleheri, Hemigrammus boesemani, Hemigrammus brevis, Hemigrammus coeruleus, Hemigrammus cylindricus, Hemigrammus gracilis, Hemigrammus haraldi, Hemigrammus hyanuary, Hemigrammus levis, Hemigrammus lunatus, Hemigrammus marginatus, Hemigrammus mimus, Hemigrammus newboldi, Hemigrammus ocellifer, Hemigrammus orthus, Hemigrammus parana, Hemigrammus pretoensis, Hemigrammus pulcher, Hemigrammus rhodostomus, Hemigrammus rodwayi, Hemigrammus schmardae, Hemigrammus silimoni, Hemigrammus skolioplatus Hemigrammus stictus, Hemigrammus taphorni, Hemigrammus tridens, Hemigrammus ulreyi, Hemigrammus unilineatus, Hemigrammus vorderwikleri, Hemigrammus yinyang, Hemigrammus sp n., Hollandichthys aff. multifasciatus, Hyphessobrycon bentosi, Hyphessobrycon coelestinus, Hyphessobrycon compressus, Hyphessobrycon diancistrus, Hyphessobrycon eilyos, Hyphessobrycon epicharis, Hyphessobrycon eques, Hyphessobrycon erythrostigma, Hyphessobrycon megalopterus, Hyphessobrycon micropterus, Hyphessobrycon takasei, Jupiaba polylepis, Lignobrycon myersi,Moenkhausia bonita, Moenkhausia hemigrammoides, Moenkhausia intermedia, Moenkhausia sanctaefilomenae, Moenkhausia xinguensis, Oligosarcus pintoi, Paracheirodon axelrodi, Parapristella georgiae, Petitella georgiae, Phenacogaster franciscoensis, Piabina argentea, Poptella paraguayensis, Psellogrammus kennedyi, Pristella maxillaris, Roeboides bonariensis, Serrasalmus maculatus, Tetragonopterus argenteus, Thayeria obliqua, Triportheus nematurus

\section{Indefinido/Indeterminado}

Brycon cf pesu

Dentro de Characidae o grau de desenvolvimento da fontanela craniana é muito variável. Nas espécies que apresentam a fontanela restrita a uma pequena porção próxima à trabécula epifiseana, as duas metades do frontal apresentam grande contato, ou o mesetmóide tem um prolongamento posterior, se aproximando muito da trabécula epifiseana. Já nas espécies que apresentam a fontanela bem desenvolvida, as duas metades do frontal também podem se sobrepor, mas em uma extensão muito menor, não reduzindo a fontanela a uma pequena região.

\section{4- Frontal (ic - 0,050)}

(Vari \& Harold, 2001: 37; Serra, 2003: 2; Benine, 2004: 9; Marinho, 2009: 33; Mirande, 2009: 21)

\section{0- metades não se contatam após o mesetmóide (Figuras 2, 5 - 6)}

Bryconamericus exodon, Bryconexodon juruenae, Bryconops melanurus, Coptobrycon bilineatus, Deuterodon iguape, Grundulus cochae, Gymnocorymbus ternetzi, Hasemania melanura, Hemigrammus arua, Hemigrammus barrigonae, Hemigrammus bellottii, Hemigrammus boesemani, Hemigrammus brevis, Hemigrammus gracilis, Hemigrammus haraldi, Hemigrammus levis, Hemigrammus lunatus, Hemigrammus newboldi, Hemigrammus ocellifer, Hemigrammus orthus, Hemigrammus parana, Hemigrammus pretoensis, Hemigrammus pulcher, Hemigrammus rodwayi, Hemigrammus silimoni, Hemigrammus stictus, Hemigrammus ulreyi, Hemigrammus unilineatus, Hemigrammus yinyang, Hemigrammus sp n., Hyphessobrycon bentosi, Hyphessobrycon coelestinus, Hyphessobrycon compressus, Hyphessobrycon diancistrus, Hyphessobrycon eilyos, Hyphessobrycon epicharis, Hyphessobrycon eques, Hyphessobrycon erythrostigma, Hyphessobrycon megalopterus, 
Hyphessobrycon micropterus, Jupiaba polylepis, Lignobrycon myersi, Microschemobrycon sp, Moenkhausia bonita, Moenkhausia hemigrammoides, Moenkhausia intermedia, Moenkhausia xinguensis, Paracheirodon axelrodi, Phenacogaster franciscoensis, Piabina argentea, Poptella paraguayensis, Psellogrammus kennedyi, Pristella maxillaris, Roeboides bonariensis, Stethaprion erythrops, Tetragonopterus argenteus

\section{1- metades se contatam ou sobrepõem após o mesetmóide (Figuras 3 -}

4)

Aphyocharax anisitsi, Aphyocharax pusillus, Astyanax altiparanae, Astyanax jacobinae,Astyanax mexicanus, Brycinus longipinnis, Brycon cf pesu, Bryconella pallidifrons, Chalceus spilogyros, Cheirodon interruptus, Hasemania crenuchoides, Hasemania sp n., Hemigrammus analis, Hemigrammus bleheri, Hemigrammus coeruleus, Hemigrammus cylindricus, Hemigrammus hyanuary, Hemigrammus marginatus, Hemigrammus mimus, Hemigrammus rhodostomus, Hemigrammus schmardae, Hemigrammus skolioplatus, Hemigrammus taphorni, Hemigrammus vorderwikleri, Hollandichthys aff. multifasciatus, Hyphessobrycon takasei, Iguanodectes spilurus, Moenkhausia sanctaefilomenae, Oligosarcus pintoi, Parapristella georgiae, Petitella georgiae, Rhinopetitia myersi, Salminus hilarii, Serrapinnus heterodon, Serrapinnus notomelas, Serrasalmus maculatus, Thayeria obliqua, Triportheus nematurus

\section{$\underline{0,1-\text { Polimorfico }}$ \\ Hemigrammus tridens}

\section{5- Fontanela do parietal (ic - 0,250 )}

\section{$\underline{0 \text { - retangular, com as margens laterais aproximadamente retas }}$}

Aphyocharax anisitsi, Aphyocharax pusillus, Astyanax altiparanae, Astyanax jacobinae, Astyanax mexicanus, Brycinus longipinnis, Brycon cf pesu, Bryconamericus exodon, Bryconella pallidifrons, Bryconexodon juruenae, Bryconops melanurus, Chalceus spilogyros, Cheirodon interruptus, Coptobrycon bilineatus, Deuterodon iguape, Grundulus cochae, Gymnocorymbus ternetzi, Hasemania crenuchoides, Hasemania melanura, Hasemania sp n., Hemigrammus analis, Hemigrammus arua, Hemigrammus barrigonae, Hemigrammus bellottii, Hemigrammus bleheri, Hemigrammus boesemani, Hemigrammus coeruleus, Hemigrammus cylindricus, Hemigrammus gracilis, Hemigrammus haraldi, Hemigrammus hyanuary, Hemigrammus levis, Hemigrammus lunatus, Hemigrammus marginatus, Hemigrammus mimus, Hemigrammus newboldi, Hemigrammus ocellifer, Hemigrammus parana, Hemigrammus pretoensis, Hemigrammus pulcher, Hemigrammus rhodostomus, Hemigrammus rodwayi, Hemigrammus schmardae, Hemigrammus silimoni, Hemigrammus skolioplatus, Hemigrammus stictus, Hemigrammus taphorni, Hemigrammus tridens, Hemigrammus ulreyi, Hemigrammus unilineatus, Hemigrammus vorderwikleri, Hemigrammus yinyang, Hemigrammus sp n., Hollandichthys aff. multifasciatus, Hyphessobrycon bentosi, Hyphessobrycon coelestinus, Hyphessobrycon compressus, Hyphessobrycon diancistrus, Hyphessobrycon eilyos, Hyphessobrycon epicharis, Hyphessobrycon eques, Hyphessobrycon erythrostigma, Hyphessobrycon takasei, Iguanodectes spilurus, Jupiaba polylepis, Lignobrycon myersi, Microschemobrycon sp, Moenkhausia bonita, Moenkhausia hemigrammoides, Moenkhausia intermedia, Moenkhausia sanctaefilomenae, Moenkhausia xinguensis, Oligosarcus pintoi, Paracheirodon axelrodi, Parapristella georgiae, Petitella georgiae, Phenacogaster franciscoensis, Piabina argentea, Poptella paraguayensis, Psellogrammus kennedyi, Pristella maxillaris, Rhinopetitia myersi, Roeboides bonariensis, Salminus hilarii, Serrapinnus heterodon, Serrapinnus notomelas, Serrasalmus maculatus, Tetragonopterus argenteus, Thayeria obliqua, Triportheus nematurus

\section{1- abaulada, com as margens laterais convexas}

Hemigrammus brevis, Hemigrammus orthus, Hyphessobrycon megalopterus, Hyphessobrycon micropterus, Stethaprion erythrops, 


\section{6- Ramo superior da linha lateral cefálica do frontal, próximo ao parietal} (ic $-0,167$ )

(Buckup, 1998: 16; Serra, 2003: 12; Moreira, 2007: 118).

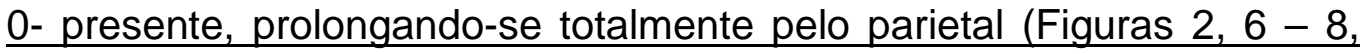

$\underline{10)}$

Astyanax altiparanae, Astyanax jacobinae, Astyanax mexicanus, Brycinus longipinnis, Brycon cf pesu, Bryconamericus exodon, Bryconexodon juruenae, Bryconops melanurus, Chalceus spilogyros, Deuterodon iguape, Gymnocorymbus ternetzi, Hasemania crenuchoides, Hemigrammus analis, Hemigrammus barrigonae, Hemigrammus bleheri, Hemigrammus coeruleus, Hemigrammus cylindricus, Hemigrammus gracilis, Hemigrammus levis, Hemigrammus lunatus, Hemigrammus marginatus, Hemigrammus mimus, Hemigrammus newboldi, Hemigrammus parana, Hemigrammus pretoensis, Hemigrammus rhodostomus, Hemigrammus rodwayi, Hemigrammus stictus, Hemigrammus taphorni, Hemigrammus ulreyi, Hemigrammus unilineatus, Hollandichthys aff. multifasciatus, Iguanodectes spilurus, Jupiaba polylepis, Microschemobrycon sp, Moenkhausia bonita, Moenkhausia hemigrammoides, Moenkhausia intermedia, Moenkhausia sanctaefilomenae, Moenkhausia xinguensis, Oligosarcus pintoi, Parapristella georgiae, Petitella georgiae, Phenacogaster franciscoensis, Piabina argentea, Poptella paraguayensis, Psellogrammus kennedyi, Roeboides bonariensis, Salminus hilarii, Serrapinnus heterodon, Serrapinnus notomelas, Serrasalmus maculatus, Stethaprion erythrops, Tetragonopterus argenteus, Thayeria obliqua, Triportheus nematurus

\section{1- presente, não se prolongando pelo parietal}

Aphyocharax pusillus, Grundulus cochae, Hasemania sp n., Hemigrammus boesemani, Hemigrammus ocellifer, Hemigrammus tridens, Hyphessobrycon compressus, Hyphessobrycon diancistrus

\section{2- presente, com apenas uma pequena porção no parietal, não se}

\section{estendendo ao longo de todo o osso}

Hasemania melanura, Hemigrammus bellottii, Hemigrammus brevis, Hemigrammus pulcher, Hemigrammus skolioplatus, Hemigrammus vorderwikleri, Lignobrycon myersi, Rhinopetitia myersi

\section{3- ausente (Figura 9)}

Aphyocharax anisitsi, Bryconella pallidifrons, Coptobrycon bilineatus, Hemigrammus haraldi, Hemigrammus orthus, Hemigrammus schmardae, Hemigrammus yinyang, Hyphessobrycon bentosi, Hyphessobrycon coelestinus, Hyphessobrycon eilyos, Hyphessobrycon epicharis, Hyphessobrycon eques, Hyphessobrycon erythrostigma, Hyphessobrycon megalopterus, Hyphessobrycon micropterus, Pristella maxillaris

\section{$\underline{0,1-P o l i m o ́ r f i c o ~}$}

Cheirodon interruptus, Hemigrammus arua, Hemigrammus silimoni, Hemigrammus sp n., Paracheirodon axelrodi

\section{1, 2- Polimórfico}

Hemigrammus hyanuary 


\title{
1, 3- Polimórfico
}

Hyphessobrycon takasei

\section{7- Projeção anterior do mesetmóide (ic - 0,125)}

\author{
0- triangular e larga, com as margens laterais confluentes (Figuras $2-3$,
} $5-6)$

Aphyocharax anisitsi, Aphyocharax pusillus, Astyanax jacobinae, Astyanax mexicanus, Brycinus longipinnis, Brycon cf pesu, Bryconamericus exodon, Bryconella pallidifrons, Bryconexodon juruenae, Chalceus spilogyros, Cheirodon interruptus, Coptobrycon bilineatus, Deuterodon iguape, Grundulus cochae, Gymnocorymbus ternetzi, Hemigrammus analis, Hemigrammus arua, Hemigrammus barrigonae, Hemigrammus bellottii, Hemigrammus bleheri, Hemigrammus boesemani, Hemigrammus brevis, Hemigrammus coeruleus, Hemigrammus cylindricus, Hemigrammus gracilis, Hemigrammus haraldi, Hemigrammus hyanuary, Hemigrammus levis, Hemigrammus lunatus, Hemigrammus marginatus, Hemigrammus mimus, Hemigrammus newboldi, Hemigrammus ocellifer, Hemigrammus orthus, Hemigrammus parana, Hemigrammus pretoensis, Hemigrammus pulcher, Hemigrammus rhodostomus, Hemigrammus rodwayi, Hemigrammus schmardae, Hemigrammus silimoni, Hemigrammus skolioplatus, Hemigrammus stictus, Hemigrammus taphorni, Hemigrammus tridens, Hemigrammus ulreyi, Hemigrammus unilineatus, Hemigrammus vorderwikleri, Hemigrammus yinyang, Hemigrammus $\mathrm{sp} n$., Hollandichthys aff. multifasciatus, Hyphessobrycon bentosi, Hyphessobrycon coelestinus, Hyphessobrycon compressus, Hyphessobrycon diancistrus, Hyphessobrycon eilyos, Hyphessobrycon eques, Hyphessobrycon erythrostigma, Hyphessobrycon megalopterus, Hyphessobrycon micropterus, Hyphessobrycon takasei, Iguanodectes spilurus, Jupiaba polylepis, Microschemobrycon sp, Moenkhausia bonita, Moenkhausia hemigrammoides, Moenkhausia intermedia, Moenkhausia sanctaefilomenae, Moenkhausia xinguensis, Paracheirodon axelrodi, Parapristella georgiae, Petitella georgiae, Phenacogaster franciscoensis, Piabina argentea, Poptella paraguayensis, Psellogrammus kennedyi, Pristella maxillaris, Rhinopetitia myersi, Roeboides bonariensis, Salminus hilarii, Serrapinnus heterodon, Serrapinnus notomelas, Tetragonopterus argenteus, Thayeria obliqua

\section{1- retangular e estreita, com as margens laterais aproximadamente}

\section{paralelas (Figura 4)}

Astyanax altiparanae, Bryconops melanurus, Hasemania crenuchoides, Hasemania melanura, Hasemania sp n., Lignobrycon myersi, Oligosarcus pintoi, Serrasalmus maculatus, Stethaprion erythrops, Triportheus nematurus

\section{9- Indeterminado}

Hyphessobrycon epicharis

\section{8- Asas laterais do mesetmóide em vista dorsal (ic - 0,222)}

(Serra, 2003: 6)

\section{0 - retangulares e estreitas (Figura 6)}

Brycinus longipinnis, Bryconamericus exodon, Bryconella pallidifrons, Grundulus cochae, Hemigrammus coeruleus, Hemigrammus mimus, Microschemobrycon sp, Paracheirodon axelrodi, Phenacogaster franciscoensis, Rhinopetitia myersi, Serrapinnus notomelas

\section{1- triangulares (Figuras $2-5$ )}


Aphyocharax anisitsi, Aphyocharax pusillus, Astyanax altiparanae, Astyanax jacobinae, Astyanax mexicanus, Brycon cf pesu, Bryconexodon juruenae, Bryconops melanurus, Cheirodon interruptus, Coptobrycon bilineatus, Deuterodon iguape, Gymnocorymbus ternetzi, Hasemania crenuchoides, Hasemania melanura, Hasemania sp n., Hemigrammus analis, Hemigrammus arua, Hemigrammus barrigonae, Hemigrammus bellottii, Hemigrammus bleheri, Hemigrammus boesemani, Hemigrammus brevis, Hemigrammus cylindricus, Hemigrammus gracilis, Hemigrammus haraldi, Hemigrammus hyanuary, Hemigrammus levis, Hemigrammus lunatus, Hemigrammus marginatus, Hemigrammus newboldi, Hemigrammus ocellifer, Hemigrammus orthus, Hemigrammus parana, Hemigrammus pretoensis, Hemigrammus pulcher, Hemigrammus rhodostomus, Hemigrammus rodwayi, Hemigrammus schmardae, Hemigrammus silimoni, Hemigrammus skolioplatus, Hemigrammus stictus, Hemigrammus taphorni, Hemigrammus tridens, Hemigrammus ulreyi, Hemigrammus unilineatus, Hemigrammus vorderwikleri, Hemigrammus yinyang, Hemigrammus sp n., Hyphessobrycon bentosi, Hyphessobrycon coelestinus, Hyphessobrycon diancistrus, Hyphessobrycon eilyos, Hyphessobrycon eques, Hyphessobrycon erythrostigma, Hyphessobrycon micropterus, Hyphessobrycon takasei, Iguanodectes spilurus, Jupiaba polylepis, Moenkhausia bonita, Moenkhausia hemigrammoides, Moenkhausia intermedia, Moenkhausia sanctaefilomenae, Moenkhausia xinguensis, Oligosarcus pintoi, Parapristella georgiae, Petitella georgiae, Piabina argentea, Poptella paraguayensis, Psellogrammus kennedyi, Pristella maxillaris, Roeboides bonariensis, Salminus hilarii, Serrapinnus heterodon, Serrasalmus maculatus, Stethaprion erythrops, Tetragonopterus argenteus, Thayeria obliqua, Triportheus nematurus

\section{2- retangulares e largas}

Hyphessobrycon compressus

\section{9- Indeterminado}

Chalceus spilogyros, Hollandichthys aff. multifasciatus, Hyphessobrycon epicharis, Hyphessobrycon megalopterus, Lignobrycon myersi,

\section{9- Asas laterais do mesetmóide (ic - 0,083)}

\section{$\underline{0 \text { - prolongando-se posteriormente ao longo do corpo do mesetmóide }}$}

\section{(Figura 4)}

Aphyocharax anisitsi, Aphyocharax pusillus, Astyanax altiparanae, Astyanax mexicanus, Brycon cf pesu, Chalceus spilogyros, Cheirodon interruptus, Hasemania crenuchoides, Hasemania sp n., Iguanodectes spilurus, Lignobrycon myersi, Oligosarcus pintoi, Piabina argentea, Salminus hilarii, Serrasalmus maculatus, Stethaprion erythrops, Tetragonopterus argenteus

\section{1- não se prolongando posteriormente, restritas à região mais anterior do} mesetmóide (Figuras 2 $-3,5-6$ )

Astyanax jacobinae, Brycinus longipinnis, Bryconamericus exodon, Bryconella pallidifrons, Bryconexodon juruenae, Bryconops melanurus, Coptobrycon bilineatus, Deuterodon iguape, Grundulus cochae, Gymnocorymbus ternetzi, Hasemania melanura, Hemigrammus analis, Hemigrammus arua, Hemigrammus barrigonae, Hemigrammus bellottii, Hemigrammus bleheri, Hemigrammus boesemani, Hemigrammus brevis, Hemigrammus coeruleus, Hemigrammus cylindricus, Hemigrammus gracilis, Hemigrammus haraldi, Hemigrammus hyanuary, Hemigrammus levis, Hemigrammus lunatus, Hemigrammus marginatus, Hemigrammus mimus, Hemigrammus newboldi, Hemigrammus ocellifer, Hemigrammus orthus, Hemigrammus parana, Hemigrammus pretoensis, Hemigrammus pulcher, Hemigrammus rhodostomus, Hemigrammus rodwayi, Hemigrammus schmardae, Hemigrammus silimoni, Hemigrammus skolioplatus, Hemigrammus stictus, Hemigrammus taphorni, Hemigrammus tridens, Hemigrammus ulreyi, Hemigrammus unilineatus, Hemigrammus vorderwikleri, Hemigrammus yinyang, Hemigrammus 
sp n., Hollandichthys aff. multifasciatus, Hyphessobrycon bentosi, Hyphessobrycon coelestinus, Hyphessobrycon compressus, Hyphessobrycon diancistrus, Hyphessobrycon eilyos, Hyphessobrycon epicharis, Hyphessobrycon eques, Hyphessobrycon erythrostigma, Hyphessobrycon megalopterus, Hyphessobrycon micropterus, Hyphessobrycon takasei, Jupiaba polylepis, Moenkhausia bonita, Moenkhausia hemigrammoides, Moenkhausia intermedia, Moenkhausia sanctaefilomenae, Moenkhausia xinguensis, Paracheirodon axelrodi, Parapristella georgiae, Petitella georgiae, Phenacogaster franciscoensis, Poptella paraguayensis, Psellogrammus kennedyi, Pristella maxillaris, Rhinopetitia myersi, Roeboides bonariensis, Serrapinnus heterodon, Serrapinnus notomelas, Thayeria obliqua, Triportheus nematurus

\section{9- Indeterminado}

Microschemobrycon sp

10- Asas laterais do mesetmóide em vista ventral (ic - 0,038)

(Serra, 2003: 7)

0- com $50 \%$ ou menos de sua superfície encoberta pelo vômer; em vista

ventral as asas tem uma porção muito grande exposta, sendo quase inteiramente visualizadas

Astyanax jacobinae, Brycinus longipinnis, Bryconella pallidifrons, Bryconexodon juruenae, Cheirodon interruptus, Coptobrycon bilineatus, Deuterodon iguape, Hemigrammus barrigonae, Hemigrammus bellottii, Hemigrammus boesemani, Hemigrammus cylindricus, Hemigrammus gracilis, Hemigrammus haraldi, Hemigrammus hyanuary, Hemigrammus lunatus, Hemigrammus marginatus, Hemigrammus mimus, Hemigrammus pretoensis, Hemigrammus pulcher, Hemigrammus taphorni, Hemigrammus skolioplatus, Hemigrammus sp n., Hemigrammus stictus, Hemigrammus tridens, Hollandichthys multifasciatus, Hyphessobrycon eilyos, Hyphessobrycon eques, Jupiaba polylepis, Microschemobrycon sp, Moenkhausia bonita, Paracheirodon axelrodi, Phenacogaster franciscoensis, Piabina argentea, Psellogrammus kennedyi, Rhinopetitia myersi, Salminus hilarii

1- com mais de $50 \%$ de sua superfície encoberta pelo vômer; em vista

\section{ventral as asas são quase ou totalmente encobertas}

Aphyocharax anisitsi, Aphyocharax pusillus, Astyanax altiparanae, Astyanax mexicanus, Brycon cf pesu, Bryconamericus exodon, Bryconops melanurus, Grundulus cochae, Gymnocorymbus ternetzi, Hasemania crenuchoides, Hasemania sp n., Hemigrammus analis, Hemigrammus arua, Hemigrammus bleheri, Hemigrammus brevis, Hemigrammus coeruleus, Hemigrammus levis, Hemigrammus newboldi, Hemigrammus ocellifer, Hemigrammus orthus, Hemigrammus parana, Hemigrammus rhodostomus, Hemigrammus rodwayi, Hemigrammus schmardae, Hemigrammus silimoni, Hemigrammus ulreyi, Hemigrammus unilineatus, Hemigrammus vorderwikleri, Hemigrammus yinyang. Hyphessobrycon bentosi, Hyphessobrycon coelestinus, Hyphessobrycon diancistrus, Hyphessobrycon epicharis, Hyphessobrycon erythrostigma, Hyphessobrycon megalopterus, Hyphessobrycon micropterus, Hyphessobrycon takasei, Iguanodectes spilurus, Moenkhausia hemigrammoides, Moenkhausia intermedia, Moenkhausia sanctaefilomenae, Moenkhausia xinguensis, Oligosarcus pintoi, Parapristella georgiae, Petitella georgiae, Poptella paraguayensis, Pristella maxillaris, Roeboides bonariensis, Serrapinnus heterodon, Serrapinnus notomelas, Serrasalmus maculatus, Stethaprion erythrops, Tetragonopterus argenteus, Thayeria obliqua, Triportheus nematurus

\section{9- Indeterminado}

Chalceus spilogyros, Hasemania melanura, Hyphessobrycon compressus, Lignobrycon myersi 
11- Lâmina dorsal do mesetmóide (ic - 0,200)

0- larga, com margens confluentes, lâmina mais larga que a fontanela do frontal e encobrindo total ou quase totalmente o vômer em vista dorsal (Figuras $2,4-6)$

Aphyocharax anisitsi, Aphyocharax pusillus, Astyanax altiparanae, Astyanax jacobinae, Astyanax mexicanus, Brycinus longipinnis, Brycon cf pesu, Bryconamericus exodon, Bryconella pallidifrons, Bryconexodon juruenae, Chalceus spilogyros, Cheirodon interruptus, Coptobrycon bilineatus, Deuterodon iguape, Gymnocorymbus ternetzi, Hasemania crenuchoides, Hasemania sp n., Hemigrammus analis, Hemigrammus arua, Hemigrammus barrigonae, Hemigrammus bellottii, Hemigrammus boesemani, Hemigrammus brevis, Hemigrammus coeruleus, Hemigrammus cylindricus, Hemigrammus gracilis, Hemigrammus haraldi, Hemigrammus hyanuary, Hemigrammus levis, Hemigrammus lunatus, Hemigrammus marginatus, Hemigrammus mimus, Hemigrammus newboldi, Hemigrammus ocellifer, Hemigrammus orthus, Hemigrammus parana, Hemigrammus pretoensis, Hemigrammus pulcher, Hemigrammus rodwayi, Hemigrammus schmardae, Hemigrammus silimoni, Hemigrammus skolioplatus, Hemigrammus stictus, Hemigrammus taphorni, Hemigrammus tridens, Hemigrammus ulreyi, Hemigrammus unilineatus, Hemigrammus vorderwikleri, Hemigrammus yinyang, Hemigrammus sp n., Hollandichthys aff. multifasciatus, Hyphessobrycon bentosi, Hyphessobrycon coelestinus, Hyphessobrycon compressus, Hyphessobrycon diancistrus, Hyphessobrycon eilyos, Hyphessobrycon epicharis, Hyphessobrycon eques, Hyphessobrycon erythrostigma, Hyphessobrycon megalopterus, Hyphessobrycon micropterus, Hyphessobrycon takasei, Iguanodectes spilurus, Jupiaba polylepis, Microschemobrycon sp, Moenkhausia bonita, Moenkhausia hemigrammoides, Moenkhausia intermedia, Moenkhausia sanctaefilomenae, Moenkhausia xinguensis, Oligosarcus pintoi, Paracheirodon axelrodi, Parapristella georgiae, Phenacogaster franciscoensis, Piabina argentea, Poptella paraguayensis, Psellogrammus kennedyi, Pristella maxillaris, Rhinopetitia myersi, Roeboides bonariensis, Salminus hilarii,Serrapinnus heterodon, Serrapinnus notomelas, Serrasalmus maculatus, Stethaprion erythrops, Tetragonopterus argenteus, Thayeria obliqua, Triportheus nematurus

\section{1- lâmina sempre com aspecto retangular, com margens paralelas e}

retas, ou às vezes levemente côncavas, bastante estreita, tão larga quanto ou mais estreita que a fontanela do frontal, deixando uma grande porção do vômer exposta em vista dorsal (Figura 3)

Bryconops melanurus, Grundulus cochae, Hasemania melanura, Hemigrammus bleheri, Hemigrammus rhodostomus, Lignobrycon myersi, Petitella georgiae

Segundo Buckup (1993), na maioria das espécies de Characiformes, a superfície dorsal do mesetmóide é formada por uma lâmina larga, que cobre uma porção considerável do focinho. O autor coloca ainda que em Characidiinae, a lâmina dorsal do mesetmóide é muito estreita e que suas margens laterais são mais ou menos paralelas. Lâmina dorsal do mesetmóide estreita foi observada na presente análise em $B$. melanurus, G. cochae, $H$. melanura, L. myersi e para o clado monofilético 138, que inclui $H$. rhodostomus, 
H. bleheri e P. georgiae. Sendo assim, essa característica parece ter aparecido mais de uma vez e independentemente dentro de Characiformes.

\section{2- Lâmina dorsal do mesetmóide (ic - 0,125 )}

\section{0- mais larga que a porção ventral do osso}

Aphyocharax anisitsi, Aphyocharax pusillus, Astyanax altiparanae, Astyanax jacobinae, Astyanax mexicanus, Brycon cf pesu, Bryconamericus exodon, Bryconella pallidifrons, Bryconexodon juruenae, Bryconops melanurus, Chalceus spilogyros, Cheirodon interruptus, Deuterodon iguape, Gymnocorymbus ternetzi, Hasemania crenuchoides, Hasemania sp n., Hemigrammus analis, Hemigrammus barrigonae, Hemigrammus bellottii, Hemigrammus brevis, Hemigrammus coeruleus, Hemigrammus cylindricus, Hemigrammus gracilis, Hemigrammus haraldi, Hemigrammus hyanuary, Hemigrammus levis, Hemigrammus lunatus, Hemigrammus marginatus, Hemigrammus newboldi, Hemigrammus ocellifer, Hemigrammus orthus, Hemigrammus parana, Hemigrammus pretoensis, Hemigrammus pulcher, Hemigrammus rodwayi, Hemigrammus schmardae, Hemigrammus silimoni, Hemigrammus skolioplatus, Hemigrammus stictus, Hemigrammus taphorni, Hemigrammus tridens, Hemigrammus ulreyi, Hemigrammus unilineatus, Hemigrammus vorderwikleri, Hemigrammus yinyang, Hemigrammus sp n., Hollandichthys aff. multifasciatus, Hyphessobrycon bentosi, Hyphessobrycon compressus, Hyphessobrycon diancistrus, Hyphessobrycon epicharis, Hyphessobrycon eques, Hyphessobrycon erythrostigma, Hyphessobrycon micropterus, Hyphessobrycon takasei, Jupiaba polylepis, Moenkhausia bonita, Moenkhausia hemigrammoides, Moenkhausia intermedia, Moenkhausia sanctaefilomenae, Moenkhausia xinguensis, Oligosarcus pintoi, Paracheirodon axelrodi, Parapristella georgiae, Phenacogaster franciscoensis, Piabina argentea, Poptella paraguayensis, Psellogrammus kennedyi, Pristella maxillaris, Rhinopetitia myersi, Roeboides bonariensis, Salminus hilarii, Serrapinnus heterodon, Serrapinnus notomelas, Serrasalmus maculatus, Tetragonopterus argenteus, Thayeria obliqua

\section{1- tão larga quanto a porção ventral do osso}

Coptobrycon bilineatus, Grundulus cochae, Hasemania melanura, Hemigrammus arua, Hemigrammus bleheri, Hemigrammus boesemani, Hemigrammus mimus, Hemigrammus rhodostomus, Hyphessobrycon coelestinus, Hyphessobrycon eilyos, Hyphessobrycon megalopterus, Iguanodectes spilurus, Lignobrycon myersi, Microschemobrycon sp, Petitella georgiae, Triportheus nematurus

\section{9- Indeterminado}

Brycinus longipinnis, Stethaprion erythrops

\section{3- Porção ventral do mesetmóide, abaixo da lâmina dorsal (ic - 0,143 )}

\section{$\underline{0 \text { - sem estreitamento na região médio-anterior }}$}

Astyanax altiparanae, Astyanax jacobinae, Astyanax mexicanus, Brycon cf pesu, Bryconella pallidifrons, Bryconexodon juruenae, Bryconops melanurus, Chalceus spilogyros, Deuterodon iguape, Gymnocorymbus ternetzi, Hasemania crenuchoides, Hasemania sp n., Hemigrammus analis, Hemigrammus arua, Hemigrammus barrigonae, Hemigrammus bellottii, Hemigrammus bleheri, Hemigrammus boesemani, Hemigrammus brevis, Hemigrammus coeruleus, Hemigrammus cylindricus, Hemigrammus gracilis, Hemigrammus hyanuary, Hemigrammus levis, Hemigrammus lunatus, Hemigrammus marginatus, Hemigrammus mimus, Hemigrammus newboldi, Hemigrammus ocellifer, Hemigrammus orthus, Hemigrammus parana, Hemigrammus pretoensis, Hemigrammus pulcher, Hemigrammus rodwayi, Hemigrammus schmardae, Hemigrammus silimoni, Hemigrammus skolioplatus, Hemigrammus stictus, Hemigrammus taphorni, Hemigrammus tridens, Hemigrammus ulreyi, Hemigrammus unilineatus, 
Hemigrammus yinyang, Hemigrammus sp n., Hollandichthys aff. multifasciatus, Hyphessobrycon bentosi, Hyphessobrycon coelestinus, Hyphessobrycon compressus, Hyphessobrycon diancistrus, Hyphessobrycon eilyos, Hyphessobrycon epicharis, Hyphessobrycon eques, Hyphessobrycon erythrostigma, Hyphessobrycon megalopterus, Hyphessobrycon micropterus, Hyphessobrycon takasei, lguanodectes spilurus, Jupiaba polylepis, Lignobrycon myersi, Moenkhausia bonita, Moenkhausia hemigrammoides, Moenkhausia intermedia, Moenkhausia sanctaefilomenae, Moenkhausia xinguensis, Oligosarcus pintoi, Parapristella georgiae, Phenacogaster franciscoensis, Piabina argentea, Poptella paraguayensis, Psellogrammus kennedyi, Pristella maxillaris, Roeboides bonariensis, Salminus hilarii, Serrasalmus maculatus, Stethaprion erythrops, Tetragonopterus argenteus, Thayeria obliqua, Triportheus nematurus

\section{1- com estreitamento na região médio-anterior}

Bryconamericus exodon, Cheirodon interruptus, Coptobrycon bilineatus, Hasemania melanura, Hemigrammus haraldi, Hemigrammus rhodostomus, Microschemobrycon sp, Paracheirodon axelrodi, Petitella georgiae, Serrapinnus heterodon, Serrapinnus notomelas

\section{9-Indeterminado}

Aphyocharax anisitsi, Aphyocharax pusillus, Brycinus longipinnis, Grundulus cochae, Hemigrammus vorderwikleri, Rhinopetitia myersi

\section{4- Região posterior do mesetmóide (ic - 0,056)}

(Serra, 2003: 8)

\section{$\underline{0 \text { - terminando após a região de inserção do etmóide lateral }}$}

Astyanax altiparanae, Astyanax jacobinae, Astyanax mexicanus, Brycinus longipinnis, Brycon cf pesu, Bryconamericus exodon, Bryconexodon juruenae, Bryconops melanurus, Chalceus spilogyros, Coptobrycon bilineatus, Deuterodon iguape, Gymnocorymbus ternetzi, Hemigrammus barrigonae, Hemigrammus bellottii, Hemigrammus coeruleus, Hemigrammus hyanuary, Hemigrammus newboldi, Hemigrammus ocellifer, Hemigrammus orthus, Hemigrammus pretoensis, Hemigrammus pulcher, Hemigrammus schmardae, Hemigrammus skolioplatus, Hemigrammus taphorni, Hemigrammus tridens, Hemigrammus unilineatus, Hemigrammus vorderwikleri, Hemigrammus yinyang, Hyphessobrycon bentosi, Hyphessobrycon coelestinus, Hyphessobrycon compressus, Hyphessobrycon eilyos, Hyphessobrycon epicharis, Hyphessobrycon erythrostigma, Iguanodectes spilurus, Jupiaba polylepis, Microschemobrycon sp, Moenkhausia bonita, Moenkhausia hemigrammoides, Moenkhausia intermedia, Moenkhausia sanctaefilomenae, Moenkhausia xinguensis, Oligosarcus pintoi, Paracheirodon axelrodi, Parapristella georgiae, Phenacogaster franciscoensis, Piabina argentea, Psellogrammus kennedyi, Rhinopetitia myersi, Roeboides bonariensis, Salminus hilarii, Stethaprion erythrops, Tetragonopterus argenteus, Thayeria obliqua, Triportheus nematurus

\section{$\underline{1-\text { terminando antes ou aproximadamente na mesma altura da região de }}$}

\section{inserção do etmóide lateral.}

Aphyocharax anisitsi, Aphyocharax pusillus, Bryconella pallidifrons, Cheirodon interruptus, Hasemania crenuchoides, Hemigrammus analis, Hemigrammus arua, Hemigrammus bleheri, Hemigrammus boesemani, Hemigrammus brevis, Hemigrammus cylindricus, Hemigrammus gracilis, Hemigrammus haraldi, Hemigrammus levis, Hemigrammus lunatus, Hemigrammus marginatus, Hemigrammus mimus, Hemigrammus parana, Hemigrammus rhodostomus, Hemigrammus rodwayi, Hemigrammus silimoni, Hemigrammus stictus, Hemigrammus sp n., Hemigrammus ulreyi, Hollandichthys aff. multifasciatus, Hyphessobrycon diancistrus, Hyphessobrycon eques, Hyphessobrycon megalopterus, Hyphessobrycon micropterus, 
Serra, J. P. - Análise Filogenética das Espécies de Hemigrammus Gill, 1858 (Characiformes, Characidae)

Hyphessobrycon takasei, Lignobrycon myersi, Petitella georgiae, Poptella paraguayensis, Pristella maxillaris, Serrapinnus heterodon, Serrapinnus notomelas, Serrasalmus maculatus

\section{9- Indeterminado}

Grundulus cochae, Hasemania melanura, Hasemania sp n.

15- Etmóide lateral (ic $-0,333$ )

(Serra, 2003: 9)

$\underline{0 \text { - sem inclinação anterior, totalmente dirigido ventralmente, em vista }}$

dorsal apresenta apenas uma pequena porção proximal visível (menos de 50\% $\underline{\text { do osso) }}$

Aphyocharax anisitsi, Aphyocharax pusillus, Astyanax altiparanae, Astyanax jacobinae, Astyanax mexicanus, Brycinus longipinnis, Brycon cf pesu, Bryconamericus exodon, Bryconella pallidifrons, Bryconexodon juruenae, Bryconops melanurus, Chalceus spilogyros, Cheirodon interruptus, Coptobrycon bilineatus, Deuterodon iguape, Grundulus cochae, Hemigrammus analis, Hemigrammus arua, Hemigrammus barrigonae, Hemigrammus bellottii, Hemigrammus bleheri, Hemigrammus boesemani, Hemigrammus brevis, Hemigrammus coeruleus, Hemigrammus cylindricus, Hemigrammus gracilis, Hemigrammus haraldi, Hemigrammus hyanuary, Hemigrammus levis, Hemigrammus lunatus, Hemigrammus marginatus, Hemigrammus mimus, Hemigrammus newboldi, Hemigrammus ocellifer, Hemigrammus orthus, Hemigrammus parana, Hemigrammus pretoensis, Hemigrammus pulcher, Hemigrammus rhodostomus, Hemigrammus rodwayi, Hemigrammus schmardae, Hemigrammus silimoni, Hemigrammus skolioplatus, Hemigrammus stictus, Hemigrammus taphorni, Hemigrammus tridens, Hemigrammus ulreyi, Hemigrammus unilineatus, Hemigrammus vorderwikleri, Hemigrammus yinyang, Hemigrammus sp n., Hollandichthys multifasciatus, Hyphessobrycon bentosi, Hyphessobrycon coelestinus, Hyphessobrycon compressus, Hyphessobrycon diancistrus, Hyphessobrycon eilyos, Hyphessobrycon epicharis, Hyphessobrycon eques, Hyphessobrycon erythrostigma, Hyphessobrycon megalopterus, Hyphessobrycon micropterus, Hyphessobrycon takasei, Iguanodectes spilurus, Jupiaba polylepis, Lignobrycon myersi, Microschemobrycon sp, Moenkhausia bonita, Moenkhausia hemigrammoides, Moenkhausia intermedia, Moenkhausia sanctaefilomenae, Moenkhausia xinguensis, Oligosarcus pintoi, Paracheirodon axelrodi, Parapristella georgiae, Petitella georgiae, Phenacogaster franciscoensis, Piabina argentea, Poptella paraguayensis, Psellogrammus kennedyi, Pristella maxillaris, Rhinopetitia myersi, Roeboides bonariensis, Salminus hilarii, Serrapinnus heterodon, Serrapinnus notomelas, Serrasalmus maculatus, Stethaprion erythrops, Thayeria obliqua

1- com inclinação anterior, em vista dorsal uma grande parte da superfície do osso é visível (mais de $50 \%$ do osso)

Gymnocorymbus ternetzi, Hasemania crenuchoides, Hasemania melanura, Hasemania sp n., Tetragonopterus argenteus, Triportheus nematurus

\section{6- Ponte óssea que liga o etmóide lateral ao vômer (ic - 0,048)}

$\underline{0 \text { - totalmente encoberta pelo frontal, não visível ou com apenas uma }}$ pequena porção visível em vista dorsal (Figuras 3 e 6)

Aphyocharax anisitsi, Aphyocharax pusillus, Brycinus longipinnis, Brycon cf pesu, Bryconamericus exodon, Bryconexodon juruenae, Chalceus spilogyros, Hemigrammus analis, Hemigrammus barrigonae, Hemigrammus bellottii, Hemigrammus bleheri, Hemigrammus coeruleus, Hemigrammus cylindricus, Hemigrammus gracilis, Hemigrammus hyanuary, 
Hemigrammus mimus, Hemigrammus pretoensis, Hemigrammus pulcher, Hemigrammus skolioplatus, Hemigrammus taphorni, Hyphessobrycon takasei, Microschemobrycon sp, Moenkhausia hemigrammoides, Moenkhausia sanctaefilomenae, Oligosarcus pintoi, Paracheirodon axelrodi, Phenacogaster franciscoensis, Piabina argentea, Rhinopetitia myersi, Salminus hilarii, Serrapinnus heterodon, Serrapinnus notomelas, Thayeria obliqua

\section{1 - totalmente exposta ou com apenas uma pequena porção mais basal} encoberta pelo frontal, inteiramente visível em vista dorsal (Figuras 2, 4 - 5)

Astyanax altiparanae, Astyanax mexicanus, Bryconella pallidifrons, Bryconops melanurus, Cheirodon interruptus, Coptobrycon bilineatus, Deuterodon iguape, Grundulus cochae, Gymnocorymbus ternetzi, Hasemania crenuchoides, Hasemania melanura, Hasemania sp n., Hemigrammus arua, Hemigrammus boesemani, Hemigrammus brevis, Hemigrammus haraldi, Hemigrammus levis, Hemigrammus lunatus, Hemigrammus marginatus, Hemigrammus newboldi, Hemigrammus ocellifer Hemigrammus orthus, Hemigrammus parana, Hemigrammus rhodostomus, Hemigrammus rodwayi, Hemigrammus schmardae, Hemigrammus silimoni, Hemigrammus stictus, Hemigrammus tridens, Hemigrammus ulreyi, Hemigrammus unilineatus, Hemigrammus vorderwikleri, Hemigrammus yinyang, Hemigrammus sp n., Hollandichthys aff. multifasciatus, Hyphessobrycon bentosi, Hyphessobrycon coelestinus, Hyphessobrycon compressus, Hyphessobrycon diancistrus, Hyphessobrycon eilyos, Hyphessobrycon epicharis, Hyphessobrycon eques, Hyphessobrycon erythrostigma, Hyphessobrycon megalopterus, Hyphessobrycon micropterus, Iguanodectes spilurus, Jupiaba polylepis, Lignobrycon myersi, Moenkhausia bonita, Moenkhausia intermedia, Moenkhausia xinguensis, Parapristella georgiae, Petitella georgiae, Poptella paraguayensis, Psellogrammus kennedyi, Pristella maxillaris, Roeboides bonariensis, Serrasalmus maculatus, Stethaprion erythrops, Tetragonopterus argenteus, Triportheus nematurus

\section{9- Indeterminado}

Astyanax jacobinae

17- Órbito-esfenóide (ic - 0,250)

0- região anterior aproximadamente reta

Brycon cf pesu, Grundulus cochae, Hasemania sp n., Tetragonopterus argenteus

\section{1- região anterior côncava (Figura 7 - 10)}

Aphyocharax anisitsi, Aphyocharax pusillus, Astyanax altiparanae, Astyanax mexicanus, Brycinus longipinnis, Bryconamericus exodon, Bryconella pallidifrons, Bryconexodon juruenae, Bryconops melanurus, Chalceus spilogyros, Cheirodon interruptus, Coptobrycon bilineatus, Deuterodon iguape, Gymnocorymbus ternetzi, Hasemania crenuchoides, Hasemania melanura, Hemigrammus analis, Hemigrammus arua, Hemigrammus barrigonae, Hemigrammus bellottii, Hemigrammus bleheri, Hemigrammus boesemani, Hemigrammus brevis, Hemigrammus coeruleus, Hemigrammus cylindricus, Hemigrammus gracilis, Hemigrammus haraldi, Hemigrammus hyanuary, Hemigrammus levis, Hemigrammus lunatus, Hemigrammus marginatus, Hemigrammus mimus, Hemigrammus newboldi, Hemigrammus ocellifer, Hemigrammus orthus, Hemigrammus parana, Hemigrammus pretoensis, Hemigrammus pulcher, Hemigrammus rhodostomus, Hemigrammus rodwayi, Hemigrammus schmardae, Hemigrammus silimoni, Hemigrammus skolioplatus, Hemigrammus stictus, Hemigrammus taphorni, Hemigrammus tridens, Hemigrammus ulreyi, Hemigrammus unilineatus, Hemigrammus vorderwikleri, Hemigrammus yinyang, Hemigrammus sp n., Hollandichthys aff. multifasciatus, Hyphessobrycon bentosi, Hyphessobrycon coelestinus, Hyphessobrycon compressus, Hyphessobrycon diancistrus, Hyphessobrycon eilyos, Hyphessobrycon epicharis, Hyphessobrycon eques, Hyphessobrycon erythrostigma, Hyphessobrycon megalopterus, Hyphessobrycon micropterus, Hyphessobrycon takasei, Iguanodectes spilurus, Jupiaba 
polylepis, Lignobrycon myersi, Microschemobrycon sp, Moenkhausia bonita, Moenkhausia hemigrammoides, Moenkhausia intermedia, Moenkhausia sanctaefilomenae, Moenkhausia xinguensis, Oligosarcus pintoi, Paracheirodon axelrodi, Parapristella georgiae, Petitella georgiae, Phenacogaster franciscoensis, Piabina argentea, Poptella paraguayensis, Psellogrammus kennedyi, Pristella maxillaris, Rhinopetitia myersi, Roeboides bonariensis, Salminus hilarii, Serrapinnus heterodon, Serrapinnus notomelas, Serrasalmus maculatus, Stethaprion erythrops, Thayeria obliqua, Triportheus nematurus

\section{$\underline{\text { 9- Indeterminado }}$}

Astyanax jacobinae

18- Órbito-esfenóide (ic - 0,100)

(Serra, 2003: 13)

\section{$\underline{0 \text { - sem concavidade próximo à união com o pteroesfenóide }}$}

Astyanax altiparanae, Astyanax mexicanus, Brycinus longipinnis, Brycon cf pesu, Bryconexodon juruenae, Bryconops melanurus, Chalceus spilogyros, Hemigrammus ulreyi, Iguanodectes spilurus, Lignobrycon myersi, Moenkhausia intermedia, Moenkhausia xinguensis, Piabina argentea, Poptella paraguayensis, Salminus hilarii, Serrasalmus maculatus, Stethaprion erythrops

\section{1- com concavidade pouco característica, formando uma região}

\section{levemente côncava (Figuras 8 e 10)}

Aphyocharax anisitsi, Aphyocharax pusillus, Astyanax jacobinae, Bryconamericus exodon, Grundulus cochae, Gymnocorymbus ternetzi, Hasemania crenuchoides, Hasemania sp n., Hemigrammus cylindricus, Hemigrammus levis, Hemigrammus marginatus, Hemigrammus newboldi, Hemigrammus pretoensis, Hemigrammus skolioplatus, Hemigrammus stictus, Hollandichthys aff. multifasciatus, Moenkhausia hemigrammoides, Moenkhausia sanctaefilomenae, Oligosarcus pintoi, Rhinopetitia myersi, Roeboides bonariensis, Tetragonopterus argenteus, Thayeria obliqua, Triportheus nematurus

\section{2- com concavidade bastante acentuada próximo à união com o} pteroesfenóide, formando uma região com uma forte invaginação (Figuras 7 e 9)

Bryconella pallidifrons, Cheirodon interruptus, Coptobrycon bilineatus, Deuterodon iguape, Hasemania melanura, Hemigrammus analis, Hemigrammus arua, Hemigrammus barrigonae, Hemigrammus bellottii, Hemigrammus bleheri, Hemigrammus boesemani, Hemigrammus brevis, Hemigrammus coeruleus, Hemigrammus gracilis, Hemigrammus haraldi, Hemigrammus hyanuary, Hemigrammus lunatus, Hemigrammus mimus, Hemigrammus ocellifer, Hemigrammus orthus, Hemigrammus parana, Hemigrammus pulcher, Hemigrammus rhodostomus, Hemigrammus rodwayi, Hemigrammus schmardae, Hemigrammus silimoni, Hemigrammus taphorni, Hemigrammus tridens, Hemigrammus unilineatus, Hemigrammus vorderwikleri, Hemigrammus yinyang, Hemigrammus sp n., Hyphessobrycon bentosi, Hyphessobrycon coelestinus, Hyphessobrycon compressus, Hyphessobrycon diancistrus, Hyphessobrycon eilyos, Hyphessobrycon epicharis, Hyphessobrycon eques, Hyphessobrycon erythrostigma, Hyphessobrycon megalopterus, Hyphessobrycon micropterus, Hyphessobrycon takasei, Jupiaba polylepis, Microschemobrycon sp, Moenkhausia bonita, Paracheirodon axelrodi, Parapristella georgiae, Petitella georgiae, Phenacogaster franciscoensis, Pristella maxillaris, Serrapinnus heterodon, Serrapinnus notomelas 
1, 2- Polimórfico

Psellogrammus kennedyi

19- Órbito-esfenóide (ic - 0,083)

(Serra, 2003: 14; Benine, 2004: 22; Ferreira, 2007: 31, Marinho, 2009: 37)

$\underline{0 \text { - sem projeção posterior dirigida ao pteroesfenóide, ou com apenas }}$

uma ponta (Figuras 8 e 10)

Aphyocharax anisitsi, Aphyocharax pusillus, Astyanax altiparanae, Astyanax jacobinae, Astyanax mexicanus, Brycinus longipinnis, Brycon cf pesu, Bryconamericus exodon, Bryconexodon juruenae, Bryconops melanurus, Chalceus spilogyros, Cheirodon interruptus, Grundulus cochae, Gymnocorymbus ternetzi, Hasemania crenuchoides, Hasemania melanura, Hasemania sp n., Hemigrammus analis, Hemigrammus barrigonae, Hemigrammus bleheri, Hemigrammus coeruleus, Hemigrammus cylindricus, Hemigrammus levis, Hemigrammus lunatus, Hemigrammus marginatus, Hemigrammus mimus, Hemigrammus newboldi, Hemigrammus ocellifer, Hemigrammus parana, Hemigrammus pretoensis, Hemigrammus pulcher, Hemigrammus skolioplatus, Hemigrammus sp n., Hemigrammus stictus, Hemigrammus taphorni, Hemigrammus ulreyi, Hollandichthys aff. multifasciatus, Hyphessobrycon diancistrus, Hyphessobrycon eilyos, Hyphessobrycon micropterus, Iguanodectes spilurus, Jupiaba polylepis, Lignobrycon myersi, Microschemobrycon sp, Moenkhausia bonita, Moenkhausia hemigrammoides, Moenkhausia intermedia, Moenkhausia sanctaefilomenae, Moenkhausia xinguensis, Oligosarcus pintoi, Phenacogaster franciscoensis, Piabina argentea, Poptella paraguayensis, Psellogrammus kennedyi, Pristella maxillaris, Rhinopetitia myersi, Roeboides bonariensis, Salminus hilarii, Serrasalmus maculatus, Stethaprion erythrops, Tetragonopterus argenteus, Thayeria obliqua, Triportheus nematurus

\section{1- com uma projeção posterior alongada, dirigida ao pteroesfenóide}

\section{(Figura 7 e 9 )}

Bryconella pallidifrons, Hemigrammus arua, Hemigrammus bellottii, Hemigrammus boesemani, Hemigrammus brevis, Hemigrammus gracilis, Hemigrammus haraldi, Hemigrammus hyanuary, Hemigrammus orthus, Hemigrammus rhodostomus, Hemigrammus rodwayi, Hemigrammus schmardae, Hemigrammus silimoni, Hemigrammus tridens, Hemigrammus unilineatus, Hemigrammus vorderwikleri, Hemigrammus yinyang, Hyphessobrycon bentosi, Hyphessobrycon coelestinus, Hyphessobrycon compressus, Hyphessobrycon epicharis, Hyphessobrycon eques, Hyphessobrycon erythrostigma, Hyphessobrycon megalopterus, Hyphessobrycon takasei, Paracheirodon axelrodi, Parapristella georgiae, Petitella georgiae,Serrapinnus heterodon, Serrapinnus notomelas

\section{0,1-Polimórfico}

Coptobrycon bilineatus, Deuterodon iguape

20- Rinoesfenóide (ic - 0,143)

(Buckup, 1998: 7; Toledo-Piza, 2000: 16; Serra, 2003: 15; Benine, 2004: 20; Moreira, 2007: 101; Bertaco, 2008: 32; Mirande, 2009: 47).

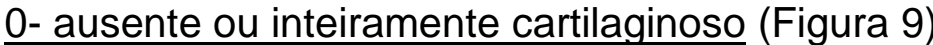

Astyanax altiparanae, Brycinus longipinnis, Chalceus spilogyros, Coptobrycon bilineatus, Grundulus cochae, Hasemania crenuchoides, Hasemania melanura, Hasemania sp n., Hemigrammus skolioplatus, Psellogrammus kennedyi, Roeboides bonariensis, Salminus hilarii, Serrasalmus maculatus 


\section{1- presente inteiramente ósseo ou com porções ósseas e cartilaginosas}

(Figuras $7-8$ e 10)

Aphyocharax anisitsi, Aphyocharax pusillus, Astyanax jacobinae, Astyanax mexicanus, Brycon cf pesu, Bryconamericus exodon, Bryconella pallidifrons, Bryconexodon juruenae, Bryconops melanurus, Cheirodon interruptus, Deuterodon iguape, Gymnocorymbus ternetzi, Hemigrammus analis, Hemigrammus barrigonae, Hemigrammus bellottii, Hemigrammus bleheri, Hemigrammus boesemani, Hemigrammus brevis, Hemigrammus coeruleus, Hemigrammus cylindricus, Hemigrammus gracilis, Hemigrammus haraldi, Hemigrammus hyanuary, Hemigrammus levis, Hemigrammus lunatus, Hemigrammus marginatus, Hemigrammus mimus, Hemigrammus newboldi, Hemigrammus ocellifer, Hemigrammus orthus, Hemigrammus parana, Hemigrammus pretoensis, Hemigrammus pulcher, Hemigrammus rhodostomus, Hemigrammus rodwayi, Hemigrammus schmardae, Hemigrammus silimoni, Hemigrammus stictus, Hemigrammus taphorni, Hemigrammus tridens, Hemigrammus ulreyi, Hemigrammus unilineatus, Hemigrammus vorderwikleri, Hemigrammus sp n., Hollandichthys aff. multifasciatus, Hyphessobrycon bentosi, Hyphessobrycon coelestinus, Hyphessobrycon compressus, Hyphessobrycon diancistrus, Hyphessobrycon eilyos, Hyphessobrycon epicharis, Hyphessobrycon eques, Hyphessobrycon erythrostigma, Hyphessobrycon megalopterus, Hyphessobrycon micropterus, Hyphessobrycon takasei, Iguanodectes spilurus, Jupiaba polylepis, Lignobrycon myersi, Microschemobrycon sp, Moenkhausia bonita, Moenkhausia hemigrammoides, Moenkhausia intermedia, Moenkhausia sanctaefilomenae, Moenkhausia xinguensis, Paracheirodon axelrodi, Parapristella georgiae, Petitella georgiae, Phenacogaster franciscoensis, Piabina argentea, Poptella paraguayensis, Pristella maxillaris, Rhinopetitia myersi, Serrapinnus heterodon, Serrapinnus notomelas, Stethaprion erythrops, Tetragonopterus argenteus, Thayeria obliqua, Triportheus nematurus

\section{0, 1- Polimórfico}

Hemigrammus arua, Hemigrammus yinyang, Oligosarcus pintoi

O rinosfenóide é um osso observado apenas em Characiformes (Weitzman, 1962; Toledo-Pizza, 2000) e está tipicamente presente na maioria dos representantes de Characidae (Zanata \& Vari, 2005). Dentre as espécies analisadas pude observar que em alguns casos um rinoesfenóide ósseo está completamente ausente, com uma grande porção de cartilagem, geralmente sem forma definida ocupando o espaço entre o órbito-esfenóide e 0 paresfenóide/etmóide lateral. Em outro caso, entretanto o rinoesfenóide ósseo também está ausente, porém no seu lugar está presente uma cartilagem não muito grande e que apresenta um formato semelhante ao do rinoesfenóide ósseo. As duas condições, entretanto são muito difíceis de serem completamente separadas, e para isso seria necessária uma análise de muitos outros terminais externos a Characidae para entender melhor essa série de transformação. Sendo assim, as duas condições citadas acima foram incluídas no estado 0 . No estado 1 estão incluídas espécies que sempre apresentam o rinoesfenóide ósseo, entretanto algumas porções desse osso (geralmente a 
porção de contato com o órbito-esfenóide ou a extensão dorsal) permanecem cartilaginosas.

21- Rinoesfenóide (ic - 0,143)

(Serra, 2003: 17; Benine, 2004: 21; Ferreira, 2007: 30, Marinho, 2009: 38; Mirande, 2009: 48)

$\underline{0 \text { - sem extensão dorsal, contactando o crânio apenas anteriormente e o }}$ pterosfenóide posteriormente, osso aproximadamente retangular ou mais arredondado (Figuras 8 e 10)

Aphyocharax anisitsi, Aphyocharax pusillus, Astyanax altiparanae, Astyanax jacobinae, Astyanax mexicanus, Brycinus longipinnis, Brycon cf pesu, Bryconamericus exodon, Bryconexodon juruenae, Cheirodon interruptus, Grundulus cochae, Gymnocorymbus ternetzi, Hasemania crenuchoides, Hasemania sp n., Lignobrycon myersi, Oligosarcus pintoi, Piabina argentea, Phenacogaster franciscoensis, Psellogrammus kennedyi, Rhinopetitia myersi, Serrapinnus heterodon, Serrapinnus notomelas, Tetragonopterus argenteus, Triportheus nematurus

1- com extensão dorsal, contactando o crânio anteriormente e dorsalmente e o pterosfenóide posteriormente, osso com formato mais triangular (3 pontas) (Figura 7 )

Bryconella pallidifrons, Bryconops melanurus, Deuterodon iguape, Hemigrammus analis, Hemigrammus arua, Hemigrammus barrigonae, Hemigrammus bellottii, Hemigrammus bleheri, Hemigrammus boesemani, Hemigrammus brevis, Hemigrammus coeruleus, Hemigrammus cylindricus, Hemigrammus gracilis, Hemigrammus haraldi, Hemigrammus hyanuary, Hemigrammus levis, Hemigrammus lunatus, Hemigrammus marginatus, Hemigrammus mimus, Hemigrammus newboldi, Hemigrammus ocellifer, Hemigrammus orthus, Hemigrammus parana, Hemigrammus pretoensis, Hemigrammus pulcher, Hemigrammus rhodostomus, Hemigrammus rodwayi, Hemigrammus schmardae, Hemigrammus silimoni, Hemigrammus skolioplatus, Hemigrammus stictus, Hemigrammus taphorni, Hemigrammus tridens, Hemigrammus ulreyi, Hemigrammus unilineatus, Hemigrammus vorderwikleri, Hemigrammus sp n., Hyphessobrycon bentosi, Hyphessobrycon coelestinus, Hyphessobrycon compressus, Hyphessobrycon diancistrus, Hyphessobrycon eilyos, Hyphessobrycon epicharis, Hyphessobrycon eques, Hyphessobrycon erythrostigma, Hyphessobrycon megalopterus, Hyphessobrycon micropterus, Hyphessobrycon takasei, Iguanodectes spilurus, Jupiaba polylepis, Moenkhausia bonita, Moenkhausia hemigrammoides, Moenkhausia intermedia, Moenkhausia sanctaefilomenae, Moenkhausia xinguensis, Paracheirodon axelrodi, Parapristella georgiae, Petitella georgiae, Poptella paraguayensis, Pristella maxillaris, Stethaprion erythrops, Thayeria obliqua

\section{9- Indeterminado}

Chalceus spilogyros, Coptobrycon bilineatus, Hasemania melanura, Hemigrammus yinyang, Hollandichthys aff. multifasciatus, Microschemobrycon sp, Roeboides bonariensis, Salminus hilarii, Serrasalmus maculatus 
O osso rinoesfenóide pode apresentar diferentes formatos de acordo com o táxon analisado, porém o que chama mais atenção é a presença ou não de uma extensão dorsal, ligando o rinoesfenóide à parte ventral do teto do crânio. Alguns autores já relataram a presença da referida extensão para alguns grupos dentro de Characiformes (Lucena \& Menezes, 1998; ToledoPizza, 2000; Serra, 2003; Benine, 2004; Ferreira, 2007; Marinho, 2009; Mirande, 2009). Nos exemplares onde a extensão foi observada, pude constatar que ela pode ser total ou parcialmente ossificada, ou ainda completamente cartilaginosa (condições essas consideradas homólogas) e ainda pode apresentar formato e largura diferentes. Entretanto, as variações observadas nessa extensão não puderam ser avaliadas de forma adequada a ponto de serem incluídas na análise. De acordo com os resultados obtidos, a presença de uma extensão dorsal no rinoesfenóide aparece como sinapomórfica para Characidae, sustentando o clado 173 , onde estão reunidas a maior parte das espécies de pequeno porte analisadas e dentre elas, boa parte dos Tetragonopterinae (sensu Géry, 1977).

Segundo Toledo-Pizza (2000), a extensão dorsal do rinoesfenóide se desenvolve mais tarde ontogeneticamente que o restante da ossificação, a autora coloca ainda que todas as espécies de Hydrolycus (exceto $H$. scomberoides) possuem uma extensão dorsal no rinosfenóide. Lucena \& Menezes (1998), colocam que um rinosfenóide com extensão dorsal pode ser observado em outros táxons, porém trataram a presença da referida extensão como uma autapomorfia para Gilbertolus maracaiboensis. Provavelmente a extensão dorsal do rinoesfenóide presente em Hydrolycus e Gilbertolus não é homóloga àquela observada nos táxons examinados nesta análise, entretando a distribuição desse caráter e as diferentes morfologias da extensão dorsal precisam ser melhor avaliadas dentro de Characiformes.

\section{2- Paresfenóide (ic - 0,091)}

(Moreira, 2007: 95; Serra, 2003: 20).

\section{$\underline{0 \text { - retilíneo em toda a sua extensão }}$}

Aphyocharax anisitsi, Aphyocharax pusillus, Chalceus spilogyros, Grundulus cochae, Hasemania crenuchoides, Hasemania sp n., Hemigrammus mimus, Hemigrammus skolioplatus, Hollandichthys aff. multifasciatus, Hyphessobrycon compressus, Hyphessobrycon micropterus, 
Microschemobrycon sp, Oligosarcus pintoi, Phenacogaster franciscoensis, Psellogrammus kennedyi, Roeboides bonariensis, Salminus hilarii, Serrasalmus maculatus

\section{1 - com curvatura ventral (Figuras 7 - 10)}

Astyanax altiparanae, Astyanax jacobinae, Astyanax mexicanus, Brycinus longipinnis, Brycon cf pesu, Bryconamericus exodon, Bryconella pallidifrons, Bryconexodon juruenae, Bryconops melanurus, Cheirodon interruptus, Coptobrycon bilineatus, Deuterodon iguape, Gymnocorymbus ternetzi, Hasemania melanura, Hemigrammus analis, Hemigrammus arua, Hemigrammus barrigonae, Hemigrammus bellottii, Hemigrammus bleheri, Hemigrammus boesemani, Hemigrammus brevis, Hemigrammus coeruleus, Hemigrammus cylindricus, Hemigrammus gracilis, Hemigrammus haraldi, Hemigrammus hyanuary, Hemigrammus levis, Hemigrammus lunatus, Hemigrammus marginatus, Hemigrammus newboldi, Hemigrammus ocellifer, Hemigrammus orthus, Hemigrammus parana, Hemigrammus pretoensis, Hemigrammus pulcher, Hemigrammus rhodostomus, Hemigrammus rodwayi, Hemigrammus schmardae, Hemigrammus silimoni, Hemigrammus stictus, Hemigrammus taphorni, Hemigrammus tridens, Hemigrammus ulreyi, Hemigrammus unilineatus, Hemigrammus vorderwikleri, Hemigrammus yinyang, Hemigrammus sp n., Hyphessobrycon bentosi, Hyphessobrycon coelestinus, Hyphessobrycon diancistrus, Hyphessobrycon eilyos, Hyphessobrycon epicharis, Hyphessobrycon eques, Hyphessobrycon erythrostigma, Hyphessobrycon megalopterus, Hyphessobrycon takasei, Iguanodectes spilurus, Jupiaba polylepis, Lignobrycon myersi, Moenkhausia bonita, Moenkhausia hemigrammoides, Moenkhausia intermedia, Moenkhausia sanctaefilomenae, Moenkhausia xinguensis, Paracheirodon axelrodi, Parapristella georgiae, Petitella georgiae, Piabina argentea, Poptella paraguayensis, Pristella maxillaris, Rhinopetitia myersi, Serrapinnus heterodon, Serrapinnus notomelas, Stethaprion erythrops, Tetragonopterus argenteus, Thayeria obliqua, Triportheus nematurus

\section{3- Carena médio ventral na região posterior do paresfenóide (ic - 0,500)}

(Moreira, 2007: 96)

\section{0- ausente (Figura 8)}

Bryconamericus exodon, Chalceus spilogyros, Piabina argentea

\section{1- presente (Figura 7,9-10)}

Aphyocharax anisitsi, Aphyocharax pusillus, Astyanax altiparanae, Astyanax jacobinae, Astyanax mexicanus, Brycinus longipinnis, Brycon cf pesu, Bryconella pallidifrons, Bryconexodon juruenae, Bryconops melanurus, Cheirodon interruptus, Coptobrycon bilineatus, Deuterodon iguape, Grundulus cochae, Gymnocorymbus ternetzi, Hasemania crenuchoides, Hasemania melanura, Hasemania sp n., Hemigrammus analis, Hemigrammus arua, Hemigrammus barrigonae, Hemigrammus bellottii, Hemigrammus bleheri, Hemigrammus boesemani, Hemigrammus brevis, Hemigrammus coeruleus, Hemigrammus cylindricus, Hemigrammus gracilis, Hemigrammus haraldi, Hemigrammus hyanuary, Hemigrammus levis, Hemigrammus lunatus, Hemigrammus marginatus, Hemigrammus mimus, Hemigrammus newboldi, Hemigrammus ocellifer, Hemigrammus orthus, Hemigrammus parana, Hemigrammus pretoensis, Hemigrammus pulcher, Hemigrammus rhodostomus, Hemigrammus rodwayi, Hemigrammus schmardae, Hemigrammus silimoni, Hemigrammus skolioplatus, Hemigrammus stictus, Hemigrammus taphorni, Hemigrammus tridens, Hemigrammus ulreyi, Hemigrammus unilineatus, Hemigrammus vorderwikleri, Hemigrammus yinyang, Hemigrammus sp n., Hollandichthys aff. multifasciatus, Hyphessobrycon bentosi, Hyphessobrycon coelestinus, Hyphessobrycon compressus, Hyphessobrycon diancistrus, Hyphessobrycon eilyos, Hyphessobrycon epicharis, Hyphessobrycon eques, Hyphessobrycon erythrostigma, Hyphessobrycon megalopterus, Hyphessobrycon micropterus, Hyphessobrycon takasei, Iguanodectes spilurus, Jupiaba polylepis, Lignobrycon myersi, Microschemobrycon sp, Moenkhausia bonita, Moenkhausia hemigrammoides, Moenkhausia intermedia, Moenkhausia 
sanctaefilomenae, Moenkhausia xinguensis, Oligosarcus pintoi, Paracheirodon axelrodi, Parapristella georgiae, Petitella georgiae, Phenacogaster franciscoensis, Poptella paraguayensis, Psellogrammus kennedyi, Pristella maxillaris, Rhinopetitia myersi, Roeboides bonariensis, Salminus hilarii, Serrapinnus heterodon, Serrapinnus notomelas, Serrasalmus maculatus, Stethaprion erythrops, Tetragonopterus argenteus, Thayeria obliqua, Triportheus nematurus

\section{4- Processo lateral do esfenótico (ic $-0,071$ )}

(Serra, 2003: 10)

\section{0 - sobreposto pelo frontal}

Astyanax altiparanae, Astyanax mexicanus, Brycon cf pesu, Bryconamericus exodon, Bryconexodon juruenae, Bryconops melanurus, Gymnocorymbus ternetzi, Hasemania crenuchoides, Hasemania melanura, Hasemania sp n., Hemigrammus analis, Hemigrammus bellottii, Hemigrammus bleheri, Hemigrammus boesemani, Hemigrammus brevis, Hemigrammus cylindricus, Hemigrammus haraldi, Hemigrammus lunatus, Hemigrammus newboldi, Hemigrammus orthus, Hemigrammus parana, Hemigrammus pretoensis, Hemigrammus rodwayi, Hemigrammus schmardae, Hollandichthys aff. multifasciatus, Hyphessobrycon compressus, Hyphessobrycon epicharis, Hyphessobrycon eques, Hyphessobrycon erythrostigma, Iguanodectes spilurus, Microschemobrycon sp, Moenkhausia bonita, Moenkhausia hemigrammoides, Moenkhausia intermedia, Oligosarcus pintoi, Phenacogaster franciscoensis, Poptella paraguayensis, Pristella maxillaris, Psellogrammus kennedyi, Roeboides bonariensis, Salminus hilarii, Serrasalmus maculatus, Tetragonopterus argenteus, Triportheus nematurus

\section{1- frontal apenas contacta (toca) o processo lateral do esfenótico}

Brycinus longipinnis, Bryconella pallidifrons, Chalceus spilogyros, Cheirodon interruptus, Coptobrycon bilineatus, Deuterodon iguape, Hemigrammus arua, Hemigrammus barrigonae, Hemigrammus coeruleus, Hemigrammus gracilis, Hemigrammus hyanuary, Hemigrammus levis, Hemigrammus marginatus, Hemigrammus mimus, Hemigrammus ocellifer, Hemigrammus pulcher, Hemigrammus rhodostomus, Hemigrammus silimoni, Hemigrammus skolioplatus, Hemigrammus sp n., Hemigrammus stictus, Hemigrammus taphorni, Hemigrammus ulreyi, Hemigrammus unilineatus, Hemigrammus vorderwikleri, Hemigrammus yinyang, Hyphessobrycon bentosi, Hyphessobrycon coelestinus, Hyphessobrycon diancistrus, Hyphessobrycon eilyos, Hyphessobrycon megalopterus, Hyphessobrycon micropterus, Hyphessobrycon takasei, Jupiaba polylepis, Lignobrycon myersi, Moenkhausia sanctaefilomenae, Moenkhausia xinguensis, Paracheirodon axelrodi, Parapristella georgiae, Petitella georgiae, Piabina argentea, Rhinopetitia myersi, Serrapinnus heterodon, Serrapinnus notomelas, Stethaprion erythrops, Thayeria obliqua

\section{2- sem contato com o frontal}

Aphyocharax anisitsi, Aphyocharax pusillus, Astyanax jacobinae, Hemigrammus tridens, Paracheirodon axelrodi

\section{9- Indeterminado}

Grundulus cochae

\section{5- Processo lateral do esfenótico (ic - 0,222)}

\section{$\underline{0 \text { - delgado e afilado, pontiagudo (Figura } 7 \text { - } 8 \text { e 10) }}$}

Aphyocharax anisitsi, Astyanax altiparanae, Astyanax jacobinae, Brycinus longipinnis, Brycon cf pesu, Bryconamericus exodon, Bryconella pallidifrons, Bryconexodon juruenae, Bryconops 
melanurus, Chalceus spilogyros, Deuterodon iguape, Gymnocorymbus ternetzi, Hemigrammus analis, Hemigrammus arua, Hemigrammus barrigonae, Hemigrammus bellottii, Hemigrammus bleheri, Hemigrammus brevis, Hemigrammus coeruleus, Hemigrammus cylindricus, Hemigrammus gracilis, Hemigrammus haraldi, Hemigrammus hyanuary, Hemigrammus levis, Hemigrammus lunatus, Hemigrammus marginatus, Hemigrammus mimus, Hemigrammus newboldi, Hemigrammus ocellifer, Hemigrammus orthus, Hemigrammus parana, Hemigrammus pretoensis, Hemigrammus pulcher, Hemigrammus rhodostomus, Hemigrammus rodwayi, Hemigrammus schmardae, Hemigrammus silimoni, Hemigrammus skolioplatus, Hemigrammus stictus, Hemigrammus taphorni, Hemigrammus tridens, Hemigrammus ulreyi, Hemigrammus unilineatus, Hemigrammus vorderwikleri, Hemigrammus yinyang, Hemigrammus sp n., Hyphessobrycon bentosi, Hyphessobrycon coelestinus, Hyphessobrycon compressus, Hyphessobrycon diancistrus, Hyphessobrycon eilyos, Hyphessobrycon epicharis, Hyphessobrycon eques, Hyphessobrycon erythrostigma, Hyphessobrycon megalopterus, Hyphessobrycon micropterus, Hyphessobrycon takasei, Iguanodectes spilurus, Jupiaba polylepis, Moenkhausia bonita, Moenkhausia hemigrammoides, Moenkhausia intermedia, Moenkhausia sanctaefilomenae, Moenkhausia xinguensis, Oligosarcus pintoi, Parapristella georgiae, Petitella georgiae, Phenacogaster franciscoensis, Piabina argentea, Poptella paraguayensis, Psellogrammus kennedyi, Pristella maxillaris, Rhinopetitia myersi, Roeboides bonariensis, Salminus hilarii, Serrapinnus heterodon, Serrapinnus notomelas, Stethaprion erythrops, Tetragonopterus argenteus, Thayeria obliqua, Triportheus nematurus

\title{
1- retangular a quadrado e bastante largo (Figura 9)
}

Aphyocharax pusillus, Cheirodon interruptus, Coptobrycon bilineatus, Grundulus cochae, Hasemania crenuchoides, Hasemania melanura, Hasemania sp n., Hollandichthys aff. multifasciatus

\section{2- retangular e estreito (delgado)}

Astyanax mexicanus, Hemigrammus boesemani, Microschemobrycon sp, Paracheirodon axelrodi

\section{9- Indeterminado}

Lignobrycon myersi, Serrasalmus maculatus

\section{6- Processo posterior do pterótico em vista dorsal (ic - 0,158 )}

\author{
(Serra, 2003: 11; Benine, 2004: 18; Moreira, 2007: 114)
}

\section{$\underline{0 \text { - presente e muito alongado }}$}

Brycinus longipinnis, Brycon cf pesu, Bryconexodon juruenae, Chalceus spilogyros, Lignobrycon myersi, Salminus hilarii, Serrasalmus maculatus, Triportheus nematurus

\section{1 - presente e curto}

Astyanax altiparanae, Astyanax mexicanus, Bryconops melanurus, Hasemania crenuchoides, Hasemania sp n., Poptella paraguayensis, Hemigrammus taphorni, Hemigrammus ulreyi, Hollandichthys aff. multifasciatus, Moenkhausia intermedia, Moenkhausia sanctaefilomenae, Moenkhausia xinguensis, Oligosarcus pintoi, Roeboides bonariensis, Stethaprion erythrops, Tetragonopterus argenteus 
2- presente, porém pouco desenvolvido, não formando uma projeção característica apenas uma protuberância arredondada ou pontiaguda (Figura 2

e 7)

Aphyocharax anisitsi, Astyanax jacobinae, Bryconamericus exodon, Cheirodon interruptus, Deuterodon iguape, Gymnocorymbus ternetzi, Hasemania melanura, Hemigrammus analis, Hemigrammus barrigonae, Hemigrammus bellottii, Hemigrammus coeruleus, Hemigrammus cylindricus, Hemigrammus gracilis, Hemigrammus haraldi, Hemigrammus levis, Hemigrammus lunatus, Hemigrammus marginatus, Hemigrammus newboldi, Hemigrammus ocellifer, Hemigrammus orthus, Hemigrammus parana, Hemigrammus pretoensis, Hemigrammus pulcher, Hemigrammus rodwayi, Hemigrammus schmardae, Hemigrammus silimoni, Hemigrammus skolioplatus, Hemigrammus stictus, Hemigrammus unilineatus, Hemigrammus sp n., Hyphessobrycon coelestinus, Hyphessobrycon compressus, Hyphessobrycon diancistrus, Hyphessobrycon epicharis, Hyphessobrycon eques, Hyphessobrycon erythrostigma, Hyphessobrycon takasei, Jupiaba polylepis, Moenkhausia bonita, Moenkhausia hemigrammoides, Parapristella georgiae, Piabina argentea, Pristella maxillaris, Rhinopetitia myersi, Thayeria obliqua

\section{3- ausente}

Bryconella pallidifrons, Coptobrycon bilineatus, Grundulus cochae, Hemigrammus arua, Hemigrammus bleheri, Hemigrammus boesemani, Hemigrammus brevis, Hemigrammus hyanuary, Hemigrammus mimus, Hemigrammus rhodostomus, Hemigrammus tridens, Hemigrammus vorderwikleri, Hemigrammus yinyang, Hyphessobrycon bentosi, Hyphessobrycon eilyos, Hyphessobrycon megalopterus, Hyphessobrycon micropterus, Microschemobrycon sp, Paracheirodon axelrodi, Petitella georgiae, Phenacogaster franciscoensis, Psellogrammus kennedyi, Serrapinnus heterodon

\section{9- Indeterminado}

Aphyocharax pusillus, Iguanodectes spilurus, Serrapinnus notomelas

\section{7- Direcionamento da projeção posterior do pterótico (ic - 0,200)}

\section{0- para trás}

Astyanax altiparanae, Astyanax mexicanus, Brycinus longipinnis, Bryconops melanurus, Chalceus spilogyros, Hemigrammus marginatus, Lignobrycon myersi, Moenkhausia xinguensis, Roeboides bonariensis, Salminus hilarii, Triportheus nematurus

\section{1- para baixo (Figura 7)}

Aphyocharax anisitsi, Astyanax jacobinae, Bryconamericus exodon, Bryconexodon juruenae, Cheirodon interruptus, Deuterodon iguape, Gymnocorymbus ternetzi, Hasemania crenuchoides, Hasemania melanura, Hasemania sp n., Hemigrammus analis, Hemigrammus barrigonae, Hemigrammus bellottii, Hemigrammus coeruleus, Hemigrammus cylindricus, Hemigrammus gracilis, Hemigrammus haraldi, Hemigrammus levis, Hemigrammus lunatus, Hemigrammus newboldi, Hemigrammus ocellifer, Hemigrammus orthus, Hemigrammus parana, Hemigrammus pretoensis, Hemigrammus pulcher, Hemigrammus rodwayi, Hemigrammus schmardae, Hemigrammus silimoni, Hemigrammus skolioplatus, Hemigrammus stictus, Hemigrammus taphorni, Hemigrammus ulreyi, Hemigrammus unilineatus, Hemigrammus sp n., Hollandichthys aff. multifasciatus, Hyphessobrycon coelestinus, Hyphessobrycon compressus, Hyphessobrycon diancistrus, Hyphessobrycon epicharis, Hyphessobrycon eques, Hyphessobrycon erythrostigma, Hyphessobrycon takasei, Jupiaba polylepis, Moenkhausia bonita, Moenkhausia hemigrammoides, Moenkhausia intermedia, Moenkhausia sanctaefilomenae, Oligosarcus pintoi, Parapristella georgiae, Piabina argentea, Poptella 
paraguayensis, Pristella maxillaris, Rhinopetitia myersi, Serrasalmus maculatus, Stethaprion erythrops, Tetragonopterus argenteus, Thayeria obliqua

\section{9- Indeterminado}

Aphyocharax pusillus, Brycon cf pesu, Bryconella pallidifrons, Coptobrycon bilineatus, Grundulus cochae, Hemigrammus arua, Hemigrammus bleheri, Hemigrammus boesemani, Hemigrammus brevis, Hemigrammus hyanuary, Hemigrammus mimus, Hemigrammus rhodostomus, Hemigrammus tridens, Hemigrammus vorderwikleri, Hemigrammus yinyang, Hyphessobrycon bentosi, Hyphessobrycon eilyos, Hyphessobrycon megalopterus, Hyphessobrycon micropterus, Iguanodectes spilurus, Microschemobrycon sp, Paracheirodon axelrodi, Petitella georgiae, Phenacogaster franciscoensis, Psellogrammus kennedyi, Serrapinnus heterodon, Serrapinnus notomelas

\section{8- Processo do supraoccipital (ic - 0,105 )}

(Malabarba, 1998: 8; Serra, 2003: 18; Moreira, 2007: 117; Bertaco, 2008: 27; Mirande, 2009: 52-53).

$\underline{0 \text { - ultrapassando a vertical que passa pela porção final do basioccipital, }}$ atingindo a região posterior do complexo neural ou ultrapassando-a

Astyanax altiparanae, Bryconexodon juruenae, Moenkhausia hemigrammoides, Poptella paraguayensis, Pristella maxillaris, Psellogrammus kennedyi, Roeboides bonariensis, Serrasalmus maculatus, Tetragonopterus argenteus

\section{1- ultrapassando a vertical que passa pela porção final do basioccipital,} terminando na altura da região mais anterior ou mediana do complexo neural

Astyanax jacobinae, Astyanax mexicanus, Brycinus longipinnis, Cheirodon interruptus, Deuterodon iguape, Gymnocorymbus ternetzi, Hasemania crenuchoides, Hasemania sp n., Hemigrammus arua, Hemigrammus barrigonae, Hemigrammus bellottii, Hemigrammus boesemani, Hemigrammus gracilis, Hemigrammus lunatus, Hemigrammus marginatus, Hemigrammus ocellifer, Hemigrammus parana, Hemigrammus pretoensis, Hemigrammus pulcher, Hemigrammus rodwayi, Hemigrammus schmardae, Hemigrammus sp n., Hemigrammus stictus, Hemigrammus taphorni, Hemigrammus ulreyi, Hemigrammus unilineatus, Hollandichthys aff. multifasciatus, Hyphessobrycon bentosi, Hyphessobrycon coelestinus, Hyphessobrycon compressus, Hyphessobrycon epicharis, Hyphessobrycon eques, Hyphessobrycon erythrostigma, Hyphessobrycon megalopterus, Hyphessobrycon micropterus, Hyphessobrycon takasei, Jupiaba polylepis, Moenkhausia bonita, Moenkhausia intermedia, Moenkhausia sanctaefilomenae, Moenkhausia xinguensis, Oligosarcus pintoi, Phenacogaster franciscoensis, Serrapinnus heterodon, Serrapinnus notomelas, Stethaprion erythrops

\section{2- não ultrapassando a vertical que passa pela porção final do}

\section{basioccipital}

Aphyocharax anisitsi, Aphyocharax pusillus, Brycon cf pesu, Bryconamericus exodon, Bryconella pallidifrons, Bryconops melanurus, Chalceus spilogyros, Coptobrycon bilineatus, Grundulus cochae, Hasemania melanura, Hemigrammus analis, Hemigrammus bleheri, Hemigrammus brevis, Hemigrammus coeruleus, Hemigrammus cylindricus, Hemigrammus haraldi, Hemigrammus hyanuary, Hemigrammus levis, Hemigrammus mimus, Hemigrammus newboldi, Hemigrammus orthus, Hemigrammus rhodostomus, Hemigrammus silimoni, Hemigrammus skolioplatus, Hemigrammus tridens, Hemigrammus vorderwikleri, Hemigrammus yinyang, Hyphessobrycon diancistrus, Hyphessobrycon eilyos, lguanodectes spilurus, Lignobrycon myersi, Microschemobrycon sp, Paracheirodon axelrodi, Parapristella georgiae, 
Petitella georgiae, Piabina argentea, Rhinopetitia myersi, Salminus hilarii, Thayeria obliqua, Triportheus nematurus

\section{9- Processo do supraoccipital (ic - 0,200)}

(Serra, 2003: 19)

$\underline{0-\text { paralelo ou apenas ligeiramente inclinado dorsalmente em relação ao }}$ eixo longitudinal do crânio (Figuras 7 - 9)

Aphyocharax anisitsi, Aphyocharax pusillus, Astyanax jacobinae, Astyanax mexicanus, Brycinus longipinnis, Brycon of pesu, Bryconamericus exodon, Bryconella pallidifrons, Bryconexodon juruenae, Bryconops melanurus, Chalceus spilogyros, Cheirodon interruptus, Coptobrycon bilineatus, Deuterodon iguape, Grundulus cochae, Hasemania crenuchoides, Hasemania melanura, Hasemania sp n., Hemigrammus analis, Hemigrammus arua, Hemigrammus barrigonae, Hemigrammus bellottii, Hemigrammus bleheri, Hemigrammus boesemani, Hemigrammus brevis, Hemigrammus coeruleus, Hemigrammus cylindricus, Hemigrammus gracilis, Hemigrammus haraldi, Hemigrammus hyanuary, Hemigrammus levis, Hemigrammus lunatus, Hemigrammus marginatus, Hemigrammus mimus, Hemigrammus newboldi, Hemigrammus ocellifer, Hemigrammus orthus, Hemigrammus parana, Hemigrammus pretoensis, Hemigrammus pulcher, Hemigrammus rhodostomus, Hemigrammus rodwayi, Hemigrammus schmardae, Hemigrammus silimoni, Hemigrammus skolioplatus, Hemigrammus stictus, Hemigrammus tridens, Hemigrammus ulreyi, Hemigrammus unilineatus, Hemigrammus vorderwikleri, Hemigrammus yinyang, Hemigrammus sp $\mathrm{n}$., Hollandichthys aff. multifasciatus, Hyphessobrycon bentosi, Hyphessobrycon coelestinus, Hyphessobrycon compressus, Hyphessobrycon diancistrus, Hyphessobrycon eilyos, Hyphessobrycon eques, Hyphessobrycon megalopterus, Hyphessobrycon micropterus, Hyphessobrycon takasei, Iguanodectes spilurus, Lignobrycon myersi, Jupiaba polylepis, Microschemobrycon sp, Moenkhausia bonita, Moenkhausia hemigrammoides, Moenkhausia intermedia, Moenkhausia sanctaefilomenae, Moenkhausia xinguensis, Paracheirodon axelrodi, Parapristella georgiae, Petitella georgiae, Piabina argentea, Pristella maxillaris, Rhinopetitia myersi, Salminus hilarii, Serrapinnus heterodon, Serrapinnus notomelas, Thayeria obliqua, Triportheus nematurus

\section{1- muito inclinado dorsalmente em relação ao eixo longitudinal do crânio}

(Figura 10)

Astyanax altiparanae, Gymnocorymbus ternetzi, Hemigrammus taphorni, Hyphessobrycon epicharis, Hyphessobrycon erythrostigma, Oligosarcus pintoi, Poptella paraguayensis, Phenacogaster franciscoensis, Psellogrammus kennedyi, Roeboides bonariensis, Serrasalmus maculatus, Stethaprion erythrops, Tetragonopterus argenteus

\section{Maxilas}

30- Maxilas (ic - 0,500)

(Benine, 2004: 38; Bertaco, 2008: 25)

\section{0- ambas extremidades na mesma linha vertical}

Aphyocharax anisitsi, Aphyocharax pusillus, Astyanax altiparanae, Astyanax jacobinae, Astyanax mexicanus, Brycinus longipinnis, Bryconella pallidifrons, Bryconexodon juruenae, Bryconops melanurus, Chalceus spilogyros, Cheirodon interruptus, Coptobrycon bilineatus, Deuterodon iguape, Grundulus cochae, Gymnocorymbus ternetzi, Hasemania crenuchoides, Hasemania melanura, Hasemania sp n., Hemigrammus analis, Hemigrammus arua, Hemigrammus barrigonae, Hemigrammus bellottii, Hemigrammus bleheri, Hemigrammus boesemani, Hemigrammus brevis, Hemigrammus coeruleus, Hemigrammus cylindricus, Hemigrammus gracilis, Hemigrammus haraldi, Hemigrammus hyanuary, Hemigrammus levis, Hemigrammus lunatus, Hemigrammus marginatus, Hemigrammus mimus, Hemigrammus newboldi, Hemigrammus ocellifer, Hemigrammus orthus, Hemigrammus parana, Hemigrammus 
pretoensis, Hemigrammus pulcher, Hemigrammus rhodostomus, Hemigrammus rodwayi, Hemigrammus schmardae, Hemigrammus silimoni, Hemigrammus skolioplatus, Hemigrammus stictus, Hemigrammus taphorni, Hemigrammus tridens, Hemigrammus ulreyi, Hemigrammus unilineatus, Hemigrammus vorderwikleri, Hemigrammus yinyang, Hemigrammus sp n., Hollandichthys aff. multifasciatus, Hyphessobrycon bentosi, Hyphessobrycon coelestinus, Hyphessobrycon compressus, Hyphessobrycon diancistrus, Hyphessobrycon eilyos, Hyphessobrycon eques, Hyphessobrycon erythrostigma, Hyphessobrycon megalopterus, Hyphessobrycon micropterus, Hyphessobrycon takasei, lguanodectes spilurus, Jupiaba polylepis, Lignobrycon myersi, Microschemobrycon sp, Moenkhausia bonita, Moenkhausia hemigrammoides, Moenkhausia intermedia, Moenkhausia sanctaefilomenae, Moenkhausia xinguensis, Oligosarcus pintoi, Paracheirodon axelrodi, Parapristella georgiae, Petitella georgiae, Phenacogaster franciscoensis, Poptella paraguayensis, Psellogrammus kennedyi, Pristella maxillaris, Roeboides bonariensis, Salminus hilarii, Serrapinnus heterodon, Serrapinnus notomelas, Stethaprion erythrops, Tetragonopterus argenteus, Thayeria obliqua, Triportheus nematurus

\section{$\underline{1 \text { - superior mais pronunciada anteriormente que a inferior }}$}

Brycon cf pesu, Bryconamericus exodon, Piabina argentea, Rhinopetitia myersi

\section{2- inferior mais pronunciada anteriormente que superior}

Hyphessobrycon epicharis, Serrasalmus maculatus

\section{1- Forma dos dentes (ic - 0,375)}

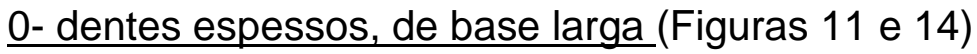

Aphyocharax anisitsi, Astyanax altiparanae, Astyanax jacobinae, Astyanax mexicanus, Brycinus longipinnis, Brycon cf pesu, Bryconamericus exodon, Bryconella pallidifrons, Bryconops melanurus, Chalceus spilogyros, Deuterodon iguape, Gymnocorymbus ternetzi, Hasemania crenuchoides, Hasemania melanura, Hasemania sp n., Hemigrammus analis, Hemigrammus barrigonae, Hemigrammus bellottii, Hemigrammus bleheri, Hemigrammus boesemani, Hemigrammus brevis, Hemigrammus coeruleus, Hemigrammus cylindricus, Hemigrammus gracilis, Hemigrammus haraldi, Hemigrammus hyanuary, Hemigrammus levis, Hemigrammus lunatus, Hemigrammus marginatus, Hemigrammus mimus, Hemigrammus newboldi, Hemigrammus ocellifer, Hemigrammus orthus, Hemigrammus parana, Hemigrammus pretoensis, Hemigrammus pulcher, Hemigrammus rhodostomus, Hemigrammus rodwayi, Hemigrammus schmardae, Hemigrammus silimoni, Hemigrammus skolioplatus, Hemigrammus stictus, Hemigrammus taphorni, Hemigrammus tridens, Hemigrammus ulreyi, Hemigrammus unilineatus, Hemigrammus vorderwikleri, Hemigrammus yinyang, Hemigrammus sp n., Hollandichthys aff. multifasciatus, Hyphessobrycon bentosi, Hyphessobrycon coelestinus, Hyphessobrycon compressus, Hyphessobrycon diancistrus, Hyphessobrycon eilyos, Hyphessobrycon epicharis, Hyphessobrycon eques, Hyphessobrycon erythrostigma, Hyphessobrycon megalopterus, Hyphessobrycon micropterus, Hyphessobrycon takasei, Jupiaba polylepis, Lignobrycon myersi, Microschemobrycon sp, Moenkhausia bonita, Moenkhausia hemigrammoides, Moenkhausia intermedia, Moenkhausia sanctaefilomenae, Moenkhausia xinguensis, Oligosarcus pintoi, Paracheirodon axelrodi, Parapristella georgiae, Petitella georgiae, Phenacogaster franciscoensis, Piabina argentea, Poptella paraguayensis, Psellogrammus kennedyi, Pristella maxillaris, Rhinopetitia myersi, Serrasalmus maculatus, Stethaprion erythrops, Tetragonopterus argenteus, Thayeria obliqua, Triportheus nematurus

\section{1- dentes comprimidos, pedunculados e com coroa expandida (Figuras}

$12-13)$

Cheirodon interruptus, Coptobrycon bilineatus, Iguanodectes spilurus, Serrapinnus heterodon, Serrapinnus notomelas 


\section{2- dentes cônicos}

Aphyocharax pusillus, Grundulus cochae, Salminus hilarii

\section{3- dentes cônicos e mamiliformes}

Bryconexodon juruenae, Roeboides bonariensis

\section{$\underline{\text { 9- Indeterminado }}$}

Hemigrammus arua

32- Maxilar (ic - 0,200)

(Serra, 2003: 46).

\section{0- com dentes distribuídos por quase todo o osso}

Brycon cf pesu, Bryconexodon juruenae, Chalceus spilogyros, Hollandichthys aff. multifasciatus, Lignobrycon myersi, Oligosarcus pintoi, Phenacogaster franciscoensis, Pristella maxillaris, Salminus hilarii

\section{1 - com dentes apenas na região proximal ou mediana (Figura 11)}

Aphyocharax anisitsi, Aphyocharax pusillus, Astyanax jacobinae, Astyanax mexicanus, Bryconamericus exodon, Bryconella pallidifrons, Bryconops melanurus, Cheirodon interruptus, Deuterodon iguape, Grundulus cochae, Gymnocorymbus ternetzi, Hasemania crenuchoides, Hemigrammus analis, Hemigrammus arua, Hemigrammus barrigonae, Hemigrammus bellottii, Hemigrammus bleheri, Hemigrammus boesemani, Hemigrammus brevis, Hemigrammus coeruleus, Hemigrammus cylindricus, Hemigrammus gracilis, Hemigrammus haraldi, Hemigrammus hyanuary, Hemigrammus levis, Hemigrammus lunatus, Hemigrammus marginatus, Hemigrammus mimus, Hemigrammus newboldi, Hemigrammus ocellifer, Hemigrammus orthus, Hemigrammus parana, Hemigrammus pretoensis, Hemigrammus pulcher, Hemigrammus rhodostomus, Hemigrammus rodwayi, Hemigrammus schmardae, Hemigrammus silimoni, Hemigrammus skolioplatus, Hemigrammus stictus, Hemigrammus taphorni, Hemigrammus tridens, Hemigrammus ulreyi, Hemigrammus unilineatus, Hemigrammus vorderwikleri, Hemigrammus yinyang, Hemigrammus sp n., Hyphessobrycon bentosi, Hyphessobrycon coelestinus, Hyphessobrycon compressus, Hyphessobrycon eilyos, Hyphessobrycon epicharis, Hyphessobrycon eques, Hyphessobrycon erythrostigma, Hyphessobrycon megalopterus, Hyphessobrycon micropterus, Hyphessobrycon takasei, Iguanodectes spilurus, Jupiaba polylepis, Microschemobrycon sp, Moenkhausia bonita, Moenkhausia hemigrammoides, Moenkhausia intermedia, Moenkhausia sanctaefilomenae, Moenkhausia xinguensis, Paracheirodon axelrodi, Parapristella georgiae, Petitella georgiae, Piabina argentea, Poptella paraguayensis, Psellogrammus kennedyi, Rhinopetitia myersi, Roeboides bonariensis, Serrapinnus heterodon, Serrapinnus notomelas, Stethaprion erythrops, Tetragonopterus argenteus, Thayeria obliqua, Triportheus nematurus

\section{2- sem dentes (Figuras $12-13$ )}

Astyanax altiparanae, Brycinus longipinnis, Coptobrycon bilineatus, Hasemania melanura, Hasemania sp n., Hyphessobrycon diancistrus, Serrasalmus maculatus

33- Dentes do maxilar (ic - 0,125)

(Serra, 2003: 47) 


\section{0- predominantemente retilíneos (posicionados aproximadamente $90^{\circ}$}

\section{em relação ao osso)}

Aphyocharax anisitsi, Aphyocharax pusillus, Astyanax jacobinae, Astyanax mexicanus, Brycon cf pesu, Bryconamericus exodon, Bryconella pallidifrons, Bryconops melanurus, Chalceus spilogyros, Cheirodon interruptus, Deuterodon iguape, Grundulus cochae, Gymnocorymbus ternetzi, Hasemania crenuchoides, Hemigrammus analis, Hemigrammus arua, Hemigrammus barrigonae, Hemigrammus bellottii, Hemigrammus bleheri, Hemigrammus coeruleus, Hemigrammus cylindricus, Hemigrammus hyanuary, Hemigrammus lunatus, Hemigrammus marginatus, Hemigrammus mimus, Hemigrammus newboldi, Hemigrammus ocellifer, Hemigrammus orthus, Hemigrammus parana, Hemigrammus pretoensis, Hemigrammus rodwayi, Hemigrammus schmardae, Hemigrammus skolioplatus, Hemigrammus stictus, Hemigrammus taphorni, Hemigrammus tridens, Hemigrammus vorderwikleri, Hemigrammus yinyang, Hemigrammus sp n., Hollandichthys aff. multifasciatus, Hyphessobrycon bentosi, Hyphessobrycon coelestinus, Hyphessobrycon compressus, Hyphessobrycon eilyos, Hyphessobrycon epicharis, Hyphessobrycon eques, Hyphessobrycon erythrostigma, Hyphessobrycon megalopterus, Hyphessobrycon micropterus, Hyphessobrycon takasei, Iguanodectes spilurus, Jupiaba polylepis, Lignobrycon myersi, Microschemobrycon sp, Moenkhausia bonita, Moenkhausia hemigrammoides, Moenkhausia intermedia, Moenkhausia sanctaefilomenae, Moenkhausia xinguensis, Oligosarcus pintoi, Paracheirodon axelrodi, Parapristella georgiae, Petitella georgiae, Phenacogaster franciscoensis, Piabina argentea, Psellogrammus kennedyi, Pristella maxillaris, Rhinopetitia myersi, Roeboides bonariensis, Salminus hilarii, Serrapinnus heterodon, Serrapinnus notomelas, Stethaprion erythrops, Tetragonopterus argenteus, Thayeria obliqua

\section{1- predominantemente curvos posteriormente}

Hemigrammus boesemani, Hemigrammus gracilis, Hemigrammus haraldi, Hemigrammus levis, Hemigrammus pulcher, Hemigrammus rhodostomus, Hemigrammus silimoni, Hemigrammus ulreyi, Poptella paraguayensis

\section{9- Indeterminado}

Astyanax altiparanae, Brycinus longipinnis, Bryconexodon juruenae, Coptobrycon bilineatus, Hasemania melanura, Hasemania sp n., Hemigrammus brevis, Hyphessobrycon diancistrus, Serrasalmus maculatus, Triportheus nematurus

\section{4- Número de cúspides dos dentes do maxilar (ic - 0,061)}

(Serra, 2003: 48; Benine, 2004: 35; Ferreira, 2007: 48; Bertaco, 2008: 9; Marinho, 2009: 10)

\section{0- maioria com 4 ou mais cúspides}

Astyanax jacobinae, Astyanax mexicanus, Chalceus spilogyros, Cheirodon interruptus, Deuterodon iguape, Hasemania sp n., Hemigrammus analis, Hemigrammus arua, Hemigrammus barrigonae, Hemigrammus bleheri, Hemigrammus ocellifer, Hemigrammus schmardae, Hemigrammus stictus, Hemigrammus taphorni, Hemigrammus tridens, Hemigrammus vorderwikleri, Hollandichthys aff. multifasciatus, Hyphessobrycon bentosi, Hyphessobrycon takasei, Iguanodectes spilurus, Moenkhausia sanctaefilomenae, Moenkhausia xinguensis, Paracheirodon axelrodi, Parapristella georgiae, Rhinopetitia myersi, Serrapinnus heterodon, Serrapinnus notomelas

\section{1- maioria com três cúspides}

Brycon cf pesu, Bryconamericus exodon, Bryconella pallidifrons, Bryconops melanurus, Hasemania crenuchoides, Hemigrammus coeruleus, Hemigrammus cylindricus, Hemigrammus levis, Hemigrammus marginatus, Hemigrammus newboldi, Hemigrammus pretoensis, 
Hemigrammus rodwayi, Hemigrammus sp n., Hemigrammus skolioplatus, Hemigrammus ulreyi, Hyphessobrycon coelestinus, Hyphessobrycon compressus, Hyphessobrycon eilyos, Hyphessobrycon epicharis, Hyphessobrycon eques, Hyphessobrycon micropterus, Jupiaba polylepis, Lignobrycon myersi, Moenkhausia bonita, Moenkhausia hemigrammoides, Moenkhausia intermedia, Oligosarcus pintoi, Piabina argentea, Poptella paraguayensis, Tetragonopterus argenteus, Thayeria obliqua

\section{2- maioria unicúspides}

Aphyocharax anisitsi, Aphyocharax pusillus, Bryconexodon juruenae, Coptobrycon bilineatus, Grundulus cochae, Gymnocorymbus ternetzi, Hasemania melanura, Hemigrammus boesemani, Hemigrammus brevis, Hemigrammus gracilis, Hemigrammus lunatus, Hemigrammus mimus, Hemigrammus orthus, Hemigrammus silimoni, Hemigrammus unilineatus, Hemigrammus yinyang, Hyphessobrycon erythrostigma, Hyphessobrycon megalopterus, Microschemobrycon $\mathrm{sp}$, Phenacogaster franciscoensis, Pristella maxillaris, Roeboides bonariensis, Salminus hilarii

\section{$\underline{0,1-P o l i m o ́ r f i c o ~}$}

Hemigrammus hyanuary, Psellogrammus kennedyi

\section{1, 2- Polimórfico}

Hemigrammus haraldi, Hemigrammus parana, Hemigrammus rhodostomus

\section{9- Indeterminado}

Astyanax altiparanae, Brycinus longipinnis, Hemigrammus bellottii, Hyphessobrycon diancistrus, Hemigrammus pulcher, Petitella georgiae, Serrasalmus maculatus, Stethaprion erythrops, Triportheus nematurus

\section{5- Tamanho das cúspides dos dentes do maxilar (ic - 0,125)}

(Bertaco, 2008: 10).

\section{0 - todas com aproximadamente o mesmo tamanho}

Astyanax jacobinae, Cheirodon interruptus, Deuterodon iguape, Hemigrammus arua, Hemigrammus bleheri, Hemigrammus stictus, Iguanodectes spilurus, Paracheirodon axelrodi, Rhinopetitia myersi, Serrapinnus heterodon, Serrapinnus notomelas

\section{1- uma das cúspides mais desenvolvida que as demais (geralmente a}

\section{$\underline{\text { mediana) }}$}

Astyanax mexicanus, Brycon cf pesu, Bryconamericus exodon, Bryconella pallidifrons, Bryconops melanurus, Chalceus spilogyros, Hasemania crenuchoides, Hasemania sp n., Hemigrammus analis, Hemigrammus barrigonae, Hemigrammus coeruleus, Hemigrammus cylindricus, Hemigrammus hyanuary, Hemigrammus marginatus, Hemigrammus newboldi, Hemigrammus ocellifer, Hemigrammus parana, Hemigrammus pretoensis, Hemigrammus pulcher, Hemigrammus rodwayi, Hemigrammus schmardae, Hemigrammus skolioplatus, Hemigrammus taphorni, Hemigrammus tridens, Hemigrammus ulreyi, Hemigrammus vorderwikleri, Hemigrammus sp n., Hollandichthys aff. multifasciatus, Hyphessobrycon bentosi, Hyphessobrycon coelestinus, Hyphessobrycon compressus, Hyphessobrycon eilyos, Hyphessobrycon epicharis, Hyphessobrycon eques, Hyphessobrycon micropterus, Hyphessobrycon takasei, Jupiaba polylepis, Lignobrycon myersi, Moenkhausia bonita, Moenkhausia hemigrammoides, Moenkhausia intermedia, Moenkhausia sanctaefilomenae, 
Moenkhausia xinguensis, Oligosarcus pintoi, Parapristella georgiae, Petitella georgiae, Piabina argentea, Psellogrammus kennedyi, Roeboides bonariensis, Tetragonopterus argenteus, Thayeria obliqua

\section{9- Indeterminado}

Aphyocharax anisitsi, Aphyocharax pusillus, Astyanax altiparanae, Brycinus longipinnis, Bryconexodon juruenae, Coptobrycon bilineatus, Grundulus cochae, Gymnocorymbus ternetzi, Hasemania melanura, Hemigrammus bellottii, Hemigrammus boesemani, Hemigrammus brevis, Hemigrammus gracilis, Hemigrammus haraldi, Hemigrammus levis, Hemigrammus lunatus, Hemigrammus mimus, Hemigrammus orthus, Hemigrammus unilineatus, Hemigrammus rhodostomus, Hemigrammus silimoni, Hemigrammus yinyang, Hyphessobrycon diancistrus, Hyphessobrycon erythrostigma, Hyphessobrycon megalopterus, Microschemobrycon sp, Phenacogaster franciscoensis, Poptella paraguayensis, Pristella maxillaris, Salminus hilarii, Serrasalmus maculatus, Stethaprion erythrops, Triportheus nematurus

\section{6- Dentes do maxilar (ic - 0,056)}

(Serra, 2003: 49)

$\underline{0 \text { - semelhantes aos dentes do pré-maxilar, aproximadamente do mesmo }}$

\section{$\underline{\text { tamanho }}$}

Aphyocharax anisitsi, Aphyocharax pusillus, Astyanax jacobinae, Astyanax mexicanus, Brycon cf pesu, Bryconamericus exodon, Bryconella pallidifrons, Bryconexodon juruenae, Bryconops melanurus, Chalceus spilogyros, Cheirodon interruptus, Deuterodon iguape, Grundulus cochae, Hasemania crenuchoides, Hasemania sp n., Hemigrammus analis, Hemigrammus arua, Hemigrammus barrigonae, Hemigrammus bleheri, Hemigrammus coeruleus, Hemigrammus cylindricus, Hemigrammus marginatus, Hemigrammus newboldi, Hemigrammus ocellifer, Hemigrammus pretoensis, Hemigrammus schmardae, Hemigrammus stictus, Hemigrammus taphorni, Hemigrammus tridens, Hemigrammus vorderwikleri, Hemigrammus sp n., Hollandichthys aff. multifasciatus, Hyphessobrycon bentosi, Hyphessobrycon coelestinus, Hyphessobrycon compressus, Hyphessobrycon eilyos, Hyphessobrycon epicharis, Hyphessobrycon eques, Hyphessobrycon erythrostigma, Hyphessobrycon micropterus, Hyphessobrycon takasei, Iguanodectes spilurus, Lignobrycon myersi,Microschemobrycon sp, Moenkhausia intermedia, Moenkhausia sanctaefilomenae, Moenkhausia xinguensis, Oligosarcus pintoi, Paracheirodon axelrodi, Parapristella georgiae, Piabina argentea, Rhinopetitia myersi, Roeboides bonariensis, Salminus hilarii, Serrapinnus heterodon, Serrapinnus notomelas, Tetragonopterus argenteus, Thayeria obliqua

\section{1- consideravelmente menores que os do pré-maxilar, geralmente}

\section{pequenos e estreitos (Figura 11)}

Hemigrammus bellottii, Hemigrammus boesemani, Hemigrammus brevis, Hemigrammus gracilis, Hemigrammus haraldi, Hemigrammus levis, Hemigrammus lunatus, Hemigrammus mimus, Hemigrammus orthus, Hemigrammus parana, Hemigrammus pulcher, Hemigrammus rhodostomus, Hemigrammus rodwayi, Hemigrammus silimoni, Hemigrammus skolioplatus, Hemigrammus ulreyi, Hemigrammus unilineatus, Hemigrammus yinyang, Hyphessobrycon megalopterus, Jupiaba polylepis, Moenkhausia bonita, Moenkhausia hemigrammoides, Phenacogaster franciscoensis, Poptella paraguayensis, Pristella maxillaris, Psellogrammus kennedyi, Stethaprion erythrops, Triportheus nematurus

\section{9- Indeterminado}

Astyanax altiparanae, Brycinus longipinnis, Coptobrycon bilineatus, Gymnocorymbus ternetzi, Hasemania melanura, Hemigrammus hyanuary, Hyphessobrycon diancistrus, Petitella georgiae, Serrasalmus maculatus 
37- Número de dentes do maxilar (ic - 0,136)

(Serra, 2003: 50; Benine, 2004: 34; Ferreira, 2007: 47; Bertaco, 2008: 8; Marinho, 2009: 9)

0 - vinte a quarenta dentes

Brycon cf pesu, Lignobrycon myersi, Hollandichthys aff. multifasciatus, Phenacogaster franciscoensis, Salminus hilarii

\section{1- onze a dezenove dentes}

Chalceus spilogyros, Oligosarcus pintoi, Roeboides bonariensis

\section{2- quatro a dez dentes}

Aphyocharax pusillus, Bryconexodon juruenae, Grundulus cochae, Hemigrammus arua, Hemigrammus coeruleus, Hemigrammus cylindricus, Hemigrammus lunatus, Hemigrammus mimus, Hemigrammus orthus, Hemigrammus pretoensis, Hemigrammus skolioplatus, Hemigrammus ulreyi, Hyphessobrycon coelestinus, Hyphessobrycon eilyos, Hyphessobrycon epicharis, Hyphessobrycon erythrostigma, Microschemobrycon sp, Parapristella georgiae, Tetragonopterus argenteus

\section{3- um a três dentes.}

Aphyocharax anisitsi, Astyanax mexicanus, Bryconamericus exodon, Bryconella pallidifrons, Bryconops melanurus, Cheirodon interruptus, Deuterodon iguape, Gymnocorymbus ternetzi, Hasemania crenuchoides, Hasemania sp n., Hemigrammus analis, Hemigrammus barrigonae, Hemigrammus bellottii, Hemigrammus bleheri, Hemigrammus boesemani, Hemigrammus brevis, Hemigrammus gracilis, Hemigrammus haraldi, Hemigrammus hyanuary, Hemigrammus levis, Hemigrammus marginatus, Hemigrammus newboldi, Hemigrammus ocellifer, Hemigrammus pulcher, Hemigrammus rhodostomus, Hemigrammus rodwayi, Hemigrammus schmardae, Hemigrammus stictus, Hemigrammus tridens, Hemigrammus vorderwikleri, Hemigrammus sp n., Hyphessobrycon bentosi, Hyphessobrycon eques, Hyphessobrycon takasei, Iguanodectes spilurus, Jupiaba polylepis, Moenkhausia bonita, Moenkhausia hemigrammoides, Moenkhausia intermedia, Moenkhausia sanctaefilomenae, Moenkhausia xinguensis, Paracheirodon axelrodi, Petitella georgiae, Piabina argentea, Poptella paraguayensis, Psellogrammus kennedyi, Rhinopetitia myersi, Serrapinnus heterodon, Serrapinnus notomelas, Stethaprion erythrops, Thayeria obliqua, Triportheus nematurus

$$
\text { 1,0-Polimórfico }
$$

Pristella maxillaris

\section{1, 2- Polimórfico}

Hyphessobrycon megalopterus

\section{2, 3- Polimórfico}

Astyanax jacobinae, Hemigrammus parana, Hemigrammus silimoni, Hemigrammus taphorni, Hemigrammus yinyang, Hyphessobrycon compressus, Hyphessobrycon micropterus

\section{9- Indeterminado}


Astyanax altiparanae, Brycinus longipinnis, Coptobrycon bilineatus, Hasemania melanura, Hyphessobrycon diancistrus, Serrasalmus maculatus

\section{8- Região do maxilar com dentes (ic - 0,250 )}

(Serra, 2003: 51; Bertaco, 2008: 15).

\section{0-mais longa que a região sem dentes}

Brycon cf pesu, Chalceus spilogyros, Hollandichthys aff. multifasciatus, Lignobrycon myersi, Oligosarcus pintoi, Phenacogaster franciscoensis, Pristella maxillaris

\section{1- mais curta ou igual à região sem dentes (Figura 11)}

Aphyocharax anisitsi, Aphyocharax pusillus, Astyanax jacobinae, Astyanax mexicanus, Bryconamericus exodon, Bryconella pallidifrons, Bryconexodon juruenae, Bryconops melanurus, Cheirodon interruptus, Deuterodon iguape, Grundulus cochae, Gymnocorymbus ternetzi, Hasemania crenuchoides, Hasemania sp n., Hemigrammus analis, Hemigrammus arua, Hemigrammus barrigonae, Hemigrammus bellottii, Hemigrammus bleheri, Hemigrammus boesemani, Hemigrammus brevis, Hemigrammus coeruleus, Hemigrammus cylindricus, Hemigrammus gracilis, Hemigrammus haraldi, Hemigrammus hyanuary, Hemigrammus levis, Hemigrammus lunatus, Hemigrammus marginatus, Hemigrammus mimus, Hemigrammus newboldi, Hemigrammus ocellifer, Hemigrammus orthus, Hemigrammus parana, Hemigrammus pretoensis, Hemigrammus pulcher, Hemigrammus rhodostomus, Hemigrammus rodwayi, Hemigrammus schmardae, Hemigrammus silimoni, Hemigrammus skolioplatus, Hemigrammus stictus, Hemigrammus taphorni, Hemigrammus tridens, Hemigrammus ulreyi, Hemigrammus unilineatus, Hemigrammus vorderwikleri, Hemigrammus yinyang, Hemigrammus sp n., Hyphessobrycon bentosi, Hyphessobrycon coelestinus, Hyphessobrycon compressus, Hyphessobrycon eilyos, Hyphessobrycon epicharis, Hyphessobrycon eques, Hyphessobrycon erythrostigma, Hyphessobrycon megalopterus, Hyphessobrycon micropterus, Hyphessobrycon takasei, Iguanodectes spilurus, Jupiaba polylepis, Microschemobrycon sp, Moenkhausia bonita, Moenkhausia hemigrammoides, Moenkhausia intermedia, Moenkhausia sanctaefilomenae, Moenkhausia xinguensis, Paracheirodon axelrodi, Parapristella georgiae, Petitella georgiae, Piabina argentea, Poptella paraguayensis, Psellogrammus kennedyi, Rhinopetitia myersi, Roeboides bonariensis, Serrapinnus heterodon, Serrapinnus notomelas, Stethaprion erythrops, Tetragonopterus argenteus, Thayeria obliqua, Triportheus nematurus

\section{9- Indeterminado}

Astyanax altiparanae, Brycinus longipinnis, Coptobrycon bilineatus, Hasemania melanura, Hyphessobrycon diancistrus, Serrasalmus maculatus

\section{9- Largura do corpo principal do maxilar (ic - 0,037 )}

(Serra, 2003: 52).

0- com a região mediana ou posterior mais larga (nas espécies que apresentam dentes na maxila, geralmente, a região mais larga começa depois da região com dentes)

Aphyocharax anisitsi, Aphyocharax pusillus, Astyanax altiparanae, Astyanax mexicanus, Brycinus longipinnis, Bryconops melanurus, Deuterodon iguape, Grundulus cochae, Gymnocorymbus ternetzi, Hasemania melanura, Hasemania sp n., Hemigrammus bleheri, Hemigrammus haraldi, Hemigrammus mimus, Hemigrammus parana, Hemigrammus rhodostomus, Hemigrammus rodwayi, Hemigrammus stictus, Hyphessobrycon bentosi, Hyphessobrycon diancistrus, Hyphessobrycon eilyos, Hyphessobrycon eques, Hyphessobrycon erythrostigma, Hyphessobrycon micropterus, Iguanodectes spilurus, Jupiaba polylepis, Lignobrycon myersi, Microschemobrycon sp, Moenkhausia bonita, Moenkhausia intermedia, 
Oligosarcus pintoi, Parapristella georgiae, Petitella georgiae, Phenacogaster franciscoensis, Piabina argentea, Poptella paraguayensis, Psellogrammus kennedyi, Serrapinnus heterodon, Serrapinnus notomelas, Thayeria obliqua, Triportheus nematurus

\section{1- com aproximadamente a mesma largura em todo o osso}

Astyanax jacobinae, Brycon cf pesu, Bryconamericus exodon, Bryconella pallidifrons, Bryconexodon juruenae, Chalceus spilogyros, Cheirodon interruptus, Coptobrycon bilineatus, Hasemania crenuchoides, Hemigrammus analis, Hemigrammus arua, Hemigrammus barrigonae, Hemigrammus bellottii, Hemigrammus boesemani, Hemigrammus brevis, Hemigrammus coeruleus, Hemigrammus cylindricus, Hemigrammus gracilis, Hemigrammus hyanuary, Hemigrammus levis, Hemigrammus lunatus, Hemigrammus marginatus, Hemigrammus newboldi, Hemigrammus ocellifer, Hemigrammus orthus, Hemigrammus pretoensis, Hemigrammus pulcher, Hemigrammus schmardae, Hemigrammus silimoni, Hemigrammus skolioplatus, Hemigrammus sp. n., Hemigrammus taphorni, Hemigrammus tridens, Hemigrammus ulreyi, Hemigrammus unilineatus, Hemigrammus vorderwikleri, Hemigrammus yinyang, Hemigrammus $\mathrm{sp} \mathrm{n}$. ., Hollandichthys aff. multifasciatus, Hyphessobrycon coelestinus, Hyphessobrycon compressus, Hyphessobrycon epicharis, Hyphessobrycon megalopterus, Hyphessobrycon takasei, Moenkhausia hemigrammoides, Moenkhausia sanctaefilomenae, Moenkhausia xinguensis, Paracheirodon axelrodi, Pristella maxillaris, Rhinopetitia myersi, Roeboides bonariensis Salminus hilarii, Serrasalmus maculatus, Stethaprion erythrops, Tetragonopterus argenteus

\section{0- Forma do maxilar (ic - 1,0)}

\section{$\underline{0 \text { - convexo dorsalmente, dirigido ventralmente }}$}

Brycinus longipinnis

\section{1- retilíneo}

Aphyocharax anisitsi, Aphyocharax pusillus, Astyanax altiparanae, Astyanax jacobinae, Astyanax mexicanus, Brycon cf pesu, Bryconamericus exodon, Bryconella pallidifrons, Bryconexodon juruenae, Bryconops melanurus, Chalceus spilogyros, Cheirodon interruptus, Coptobrycon bilineatus, Deuterodon iguape, Grundulus cochae, Gymnocorymbus ternetzi, Hasemania crenuchoides, Hasemania melanura, Hasemania sp n., Hemigrammus analis, Hemigrammus arua, Hemigrammus barrigonae, Hemigrammus bellottii, Hemigrammus bleheri, Hemigrammus boesemani, Hemigrammus brevis, Hemigrammus coeruleus, Hemigrammus cylindricus, Hemigrammus gracilis, Hemigrammus haraldi, Hemigrammus hyanuary, Hemigrammus levis, Hemigrammus lunatus, Hemigrammus marginatus, Hemigrammus mimus, Hemigrammus newboldi, Hemigrammus ocellifer, Hemigrammus orthus, Hemigrammus parana, Hemigrammus pretoensis, Hemigrammus pulcher, Hemigrammus rhodostomus, Hemigrammus rodwayi, Hemigrammus schmardae, Hemigrammus silimoni, Hemigrammus skolioplatus, Hemigrammus stictus, Hemigrammus taphorni, Hemigrammus tridens, Hemigrammus ulreyi, Hemigrammus unilineatus, Hemigrammus vorderwikleri, Hemigrammus yinyang, Hemigrammus sp n., Hollandichthys aff. multifasciatus, Hyphessobrycon bentosi, Hyphessobrycon coelestinus, Hyphessobrycon compressus, Hyphessobrycon diancistrus, Hyphessobrycon eilyos, Hyphessobrycon epicharis, Hyphessobrycon eques, Hyphessobrycon erythrostigma, Hyphessobrycon megalopterus, Hyphessobrycon micropterus, Hyphessobrycon takasei, Iguanodectes spilurus, Jupiaba polylepis, Lignobrycon myersi, Microschemobrycon sp, Moenkhausia bonita, Moenkhausia hemigrammoides, Moenkhausia intermedia, Moenkhausia sanctaefilomenae, Moenkhausia xinguensis, Oligosarcus pintoi, Paracheirodon axelrodi, Parapristella georgiae, Petitella georgiae, Phenacogaster franciscoensis, Piabina argentea, Poptella paraguayensis, Psellogrammus kennedyi, Pristella maxillaris, Rhinopetitia myersi, Roeboides bonariensis, Salminus hilarii, Serrapinnus heterodon, Serrapinnus notomelas, Serrasalmus maculatus, Stethaprion erythrops, Tetragonopterus argenteus, Thayeria obliqua, Triportheus nematurus 


\section{1- Posição da margem posterior do maxilar (ic - 0,067)}

(Serra, 2003: 54).

\section{$\underline{0 \text { - terminando na vertical que passa posteriormente ao etmóide lateral }}$}

Brycon cf pesu, Bryconexodon juruenae, Bryconops melanurus, Hasemania crenuchoides, Hemigrammus arua, Hemigrammus bellottii, Hemigrammus brevis, Hemigrammus coeruleus, Hemigrammus gracilis, Hemigrammus haraldi, Hemigrammus marginatus, Hemigrammus orthus, Hemigrammus pretoensis, Hemigrammus skolioplatus, Hemigrammus taphorni, Hemigrammus unilineatus, Hemigrammus yinyang, Hyphessobrycon bentosi, Hyphessobrycon eilyos, Hyphessobrycon epicharis, Hyphessobrycon erythrostigma, Hyphessobrycon takasei, Jupiaba polylepis, Moenkhausia sanctaefilomenae, Oligosarcus pintoi, Parapristella georgiae, Phenacogaster franciscoensis, Poptella paraguayensis, Roeboides bonariensis, Salminus hilarii, Serrasalmus maculatus, Tetragonopterus argenteus, Thayeria obliqua

\section{1- terminando aproximadamente na vertical que passa pelo etmóide}

\section{lateral}

Aphyocharax anisitsi, Aphyocharax pusillus, Astyanax altiparanae, Astyanax jacobinae, Astyanax mexicanus, Bryconella pallidifrons, Chalceus spilogyros, Deuterodon iguape, Gymnocorymbus ternetzi, Hasemania melanura, Hasemania sp n., Hemigrammus analis, Hemigrammus barrigonae, Hemigrammus bleheri, Hemigrammus boesemani, Hemigrammus cylindricus, Hemigrammus hyanuary, Hemigrammus levis, Hemigrammus lunatus, Hemigrammus newboldi, Hemigrammus ocellifer, Hemigrammus parana, Hemigrammus pulcher, Hemigrammus rodwayi, Hemigrammus schmardae, Hemigrammus silimoni, Hemigrammus stictus, Hemigrammus tridens, Hemigrammus ulreyi, Hemigrammus vorderwikleri, Hyphessobrycon coelestinus, Hyphessobrycon compressus, Hyphessobrycon diancistrus, Hyphessobrycon eques, Hyphessobrycon megalopterus, Hyphessobrycon micropterus, Lignobrycon myersi, Moenkhausia bonita, Moenkhausia hemigrammoides, Moenkhausia intermedia, Moenkhausia xinguensis, Paracheirodon axelrodi, Petitella georgiae, Piabina argentea, Pristella maxillaris, Rhinopetitia myersi, Stethaprion erythrops

\section{2- terminando antes da vertical que passa pelo etmóide lateral}

Brycinus longipinnis, Cheirodon interruptus, Coptobrycon bilineatus, Grundulus cochae, Hemigrammus mimus, Hemigrammus rhodostomus, lguanodectes spilurus, Microschemobrycon sp, Psellogrammus kennedyi, Serrapinnus heterodon, Serrapinnus notomelas

\section{$\underline{0,1-\text { Polimórfico }}$}

Hemigrammus sp $\mathrm{n}$.

\section{9- Indeterminado}

Bryconamericus exodon, Hollandichthys aff. multifasciatus

\section{2- Número de séries de dentes no pré-maxilar (ic - 0,167)}

(Buckup, 1998: 32; Serra, 2003: 55; Benine, 2004: 27; Ferreira, 2007: 36, Moreira, 2007: 6; Bertaco, 2008: 1; Marinho, 2009: 2; Mirande, 2009: 122-123). 0- três séries

Brycon cf pesu, Chalceus spilogyros, Triportheus nematurus 


\section{1- uma série}

Aphyocharax anisitsi, Aphyocharax pusillus, Cheirodon interruptus, Grundulus cochae, Iguanodectes spilurus, Microschemobrycon sp, Oligosarcus pintoi, Paracheirodon axelrodi, Petitella georgiae, Salminus hilarii, Serrapinnus heterodon, Serrapinnus notomelas, Serrasalmus maculatus

\section{2- duas séries}

Astyanax altiparanae, Astyanax jacobinae, Astyanax mexicanus, Brycinus longipinnis, Bryconamericus exodon, Bryconella pallidifrons, Bryconops melanurus, Coptobrycon bilineatus, Deuterodon iguape, Gymnocorymbus ternetzi, Hasemania crenuchoides, Hasemania melanura, Hasemania sp n., Hemigrammus analis, Hemigrammus arua, Hemigrammus barrigonae, Hemigrammus bellottii, Hemigrammus bleheri, Hemigrammus boesemani, Hemigrammus brevis, Hemigrammus coeruleus, Hemigrammus cylindricus, Hemigrammus gracilis, Hemigrammus haraldi, Hemigrammus hyanuary, Hemigrammus levis, Hemigrammus lunatus, Hemigrammus marginatus, Hemigrammus mimus, Hemigrammus newboldi, Hemigrammus ocellifer, Hemigrammus orthus, Hemigrammus parana, Hemigrammus pretoensis, Hemigrammus pulcher, Hemigrammus rhodostomus, Hemigrammus rodwayi, Hemigrammus schmardae, Hemigrammus silimoni, Hemigrammus skolioplatus, Hemigrammus stictus, Hemigrammus taphorni, Hemigrammus tridens, Hemigrammus ulreyi, Hemigrammus unilineatus, Hemigrammus vorderwikleri, Hemigrammus yinyang, Hemigrammus sp n., Hollandichthys multifasciatus, Hyphessobrycon bentosi, Hyphessobrycon coelestinus, Hyphessobrycon compressus, Hyphessobrycon diancistrus, Hyphessobrycon eilyos, Hyphessobrycon epicharis, Hyphessobrycon eques, Hyphessobrycon erythrostigma, Hyphessobrycon megalopterus, Hyphessobrycon micropterus, Hyphessobrycon takasei, Jupiaba polylepis, Lignobrycon myersi, Moenkhausia bonita, Moenkhausia hemigrammoides, Moenkhausia intermedia, Moenkhausia sanctaefilomenae, Moenkhausia xinguensis, Parapristella georgiae, Phenacogaster franciscoensis, Piabina argentea, Poptella paraguayensis, Psellogrammus kennedyi, Rhinopetitia myersi, Stethaprion erythrops, Tetragonopterus argenteus, Thayeria obliqua

\section{1, 2- Polimórfico}

Pristella maxillaris

\section{9- Indeterminado}

Bryconexodon juruenae, Roeboides bonariensis

Tradicionalmente as espécies de Characidae eram agrupadas de acordo com a morfologia e o número dos dentes ou das séries de dentes. Weitzman \& Malabarba (1998) colocam que a utilização da morfologia e número de dentes, utlizadas ao longo dos anos para definir grupos dentro de Characidae têm levado a uma série de agrupamentos reconhecidamente polifiléticos, entretanto, os autores estimulam a utilização desses caracteres dentro de um contexto filogenético e associado à análise de outros caracteres.

Segundo Malabarba (1998) a redução no número de séries de dentes no pré-maxilar é vista como sinapomórfica, porém não ocorreu uma única vez 
dentro de Characidae. Malabarba (1998) também coloca que a melhor evidência para a redução independente do número de séries de dentes no prémaxilar dentro de Characidae, é provida por recentes hipoteses filogenéticas que agrupam espécies com uma única série de dentes no pré-maxilar com outras apresentando duas séries de dentes. Na presente análise, alguns clados monofiléticos formados, englobam tanto espécies como uma única série de dentes no pré-maxilar quanto espécies com duas séries, como é o caso de $H$. rhodostomus e P. georgiae (clado 137) e G. cochae e C. bilineatus (clado 122).

Weitman \& Palmer (1997a) colocam que para várias espécies de "rosy tetra" existe mudança ontogenética tanto no número de dentes quanto no número de séries de dentes no pré-maxilar. Dessa forma, devido à ausência de séries representativas de indivíduos variando de juvenis a adultos de cada uma das espécies aqui analisadas, o número dentes e de séries de dentes no prémaxilar foi examinado apenas em indivíduos considerados adultos.

\section{3- Dentes da série externa do pré-maxilar (para táxons que apresentam} duas séries de dentes) (ic - 0,077)

(Serra, 2003: 56; Bertaco, 2008: 4; Mirande, 2009: 124)

\section{$\underline{0 \text { - desalinhados }}$}

Bryconamericus exodon, Hemigrammus analis, Hemigrammus arua, Hemigrammus haraldi, Hemigrammus newboldi, Hemigrammus ocellifer, Hemigrammus parana, Hemigrammus pulcher, Hemigrammus skolioplatus, Hemigrammus taphorni, Hyphessobrycon compressus, Jupiaba polylepis, Moenkhausia bonita, Moenkhausia hemigrammoides, Parapristella georgiae, Piabina argentea, Thayeria obliqua

\section{1- alinhados}

Astyanax altiparanae, Astyanax jacobinae, Astyanax mexicanus, Brycinus longipinnis, Bryconella pallidifrons, Bryconops melanurus, Deuterodon iguape, Gymnocorymbus ternetzi, Hasemania crenuchoides, Hasemania melanura, Hasemania sp n., Hemigrammus barrigonae, Hemigrammus bellottii, Hemigrammus bleheri, Hemigrammus boesemani, Hemigrammus brevis, Hemigrammus coeruleus, Hemigrammus cylindricus, Hemigrammus gracilis, Hemigrammus hyanuary, Hemigrammus levis, Hemigrammus lunatus, Hemigrammus marginatus, Hemigrammus orthus, Hemigrammus pretoensis, Hemigrammus rhodostomus, Hemigrammus rodwayi, Hemigrammus schmardae, Hemigrammus silimoni, Hemigrammus sp n., Hemigrammus stictus, Hemigrammus tridens, Hemigrammus ulreyi, Hemigrammus unilineatus, Hemigrammus vorderwikleri, Hemigrammus yinyang, Hollandichthys aff. multifasciatus, Hyphessobrycon bentosi, Hyphessobrycon coelestinus, Hyphessobrycon diancistrus, Hyphessobrycon eilyos, Hyphessobrycon epicharis, Hyphessobrycon eques, Hyphessobrycon megalopterus, Hyphessobrycon takasei, Lignobrycon myersi, Moenkhausia intermedia, Moenkhausia sanctaefilomenae, Moenkhausia xinguensis, Phenacogaster franciscoensis, Poptella paraguayensis, Psellogrammus kennedyi, Rhinopetitia myersi, Serrasalmus maculatus, Stethaprion erythrops, Tetragonopterus argenteus 


\section{9- Indeterminado}

Aphyocharax anisitsi, Aphyocharax pusillus, Brycon cf pesu, Bryconexodon juruenae, Chalceus spilogyros, Cheirodon interruptus, Coptobrycon bilineatus, Grundulus cochae, Hemigrammus mimus, Hyphessobrycon erythrostigma, Hyphessobrycon micropterus, Iguanodectes spilurus, Microschemobrycon sp, Oligosarcus pintoi, Paracheirodon axelrodi, Petitella georgiae, Pristella maxillaris, Roeboides bonariensis, Salminus hilarii, Serrapinnus heterodon, Serrapinnus notomelas, Triportheus nematurus

\section{4- Número de cúspides dos dentes da série externa do pré-maxilar (para táxons que apresentam duas séries de dentes) (ic - 0,167)}

(Benine, 2004: 28, Marinho, 2009: 4; Mirande, 2009: 125)

\section{0- com mais de 3 cúspides}

Brycinus longipinnis, Coptobrycon bilineatus, Deuterodon iguape, Hasemania melanura, Hemigrammus barrigonae, Hemigrammus stictus, Hemigrammus ulreyi, Parapristella georgiae, Rhinopetitia myersi

\section{1 - com 3 cúspides}

Astyanax altiparanae, Astyanax mexicanus, Bryconamericus exodon, Bryconella pallidifrons, Bryconops melanurus, Gymnocorymbus ternetzi, Hasemania crenuchoides, Hasemania sp n., Hemigrammus bellottii, Hemigrammus bleheri, Hemigrammus boesemani, Hemigrammus brevis, Hemigrammus coeruleus, Hemigrammus cylindricus, Hemigrammus gracilis, Hemigrammus haraldi, Hemigrammus hyanuary, Hemigrammus levis, Hemigrammus lunatus, Hemigrammus marginatus, Hemigrammus mimus, Hemigrammus newboldi, Hemigrammus ocellifer, Hemigrammus orthus, Hemigrammus parana, Hemigrammus pretoensis, Hemigrammus pulcher, Hemigrammus rhodostomus, Hemigrammus rodwayi, Hemigrammus schmardae, Hemigrammus silimoni, Hemigrammus skolioplatus, Hemigrammus taphorni, Hemigrammus tridens, Hemigrammus unilineatus, Hemigrammus vorderwikleri, Hemigrammus sp n., Hollandichthys aff. multifasciatus, Hyphessobrycon bentosi, Hyphessobrycon coelestinus, Hyphessobrycon compressus, Hyphessobrycon diancistrus, Hyphessobrycon eilyos, Hyphessobrycon epicharis, Hyphessobrycon eques, Hyphessobrycon micropterus, Hyphessobrycon takasei, Jupiaba polylepis, Lignobrycon myersi, Moenkhausia bonita, Moenkhausia hemigrammoides, Moenkhausia intermedia, Moenkhausia sanctaefilomenae, Moenkhausia xinguensis, Piabina argentea, Poptella paraguayensis, Psellogrammus kennedyi, Pristella maxillaris, Stethaprion erythrops, Tetragonopterus argenteus, Thayeria obliqua

\section{2- unicúspides}

Hyphessobrycon erythrostigma, Hyphessobrycon megalopterus, Salminus hilarii, Triportheus nematurus

\section{$\underline{0,1-\text { Polimórfico }}$}

Astyanax jacobinae, Hemigrammus arua, Phenacogaster franciscoensis

\section{1, 2- Polimórfico}

Hemigrammus yinyang

\section{$\underline{\text { 9- Indeterminado }}$}


Aphyocharax anisitsi, Aphyocharax pusillus, Brycon cf pesu, Bryconexodon juruenae, Chalceus spilogyros, Cheirodon interruptus, Grundulus cochae, Hemigrammus analis, Iguanodectes spilurus, Microschemobrycon sp, Oligosarcus pintoi, Paracheirodon axelrodi, Petitella georgiae, Roeboides bonariensis, Serrapinnus heterodon, Serrapinnus notomelas, Serrasalmus maculatus

\section{5- Número de dentes da série interna do pré-maxilar (para táxons que apresentam duas séries de dentes) (ic - 0,250)}

(Serra, 2003: 57; Benine, 2004: 29; Ferreira, 2007: 37; Moreira, 2007: 8;

Bertaco, 2008: 2; Marinho, 2009: 3; Mirande, 2009: 129-130).

\section{$\underline{0 \text { - seis ou mais dentes }}$}

Lignobrycon myersi, Hemigrammus cylindricus, Hemigrammus mimus, Hyphessobrycon compressus, Hyphessobrycon eilyos, Hyphessobrycon epicharis, Hyphessobrycon eques, Hyphessobrycon erythrostigma, Hyphessobrycon megalopterus, Hyphessobrycon micropterus, Phenacogaster franciscoensis, Pristella maxillaris, Salminus hilarii

\section{1- quatro dentes}

Brycinus longipinnis, Bryconamericus exodon, Hasemania sp n., Piabina argentea, Rhinopetitia myersi

\section{2- cinco dentes}

Astyanax altiparanae, Astyanax jacobinae, Astyanax mexicanus, Bryconella pallidifrons, Bryconops melanurus, Deuterodon iguape, Gymnocorymbus ternetzi, Hasemania crenuchoides, Hemigrammus analis, Hemigrammus barrigonae, Hemigrammus bellottii, Hemigrammus bleheri, Hemigrammus boesemani, Hemigrammus brevis, Hemigrammus coeruleus, Hemigrammus gracilis, Hemigrammus haraldi, Hemigrammus levis, Hemigrammus lunatus, Hemigrammus marginatus, Hemigrammus newboldi, Hemigrammus ocellifer, Hemigrammus orthus, Hemigrammus parana, Hemigrammus pretoensis, Hemigrammus pulcher, Hemigrammus rhodostomus, Hemigrammus rodwayi, Hemigrammus schmardae, Hemigrammus silimoni, Hemigrammus stictus, Hemigrammus taphorni, Hemigrammus tridens, Hemigrammus ulreyi, Hemigrammus vorderwikleri, Hemigrammus sp n., Hollandichthys aff. multifasciatus, Hyphessobrycon bentosi, Hyphessobrycon coelestinus, Hyphessobrycon diancistrus, Hyphessobrycon takasei, Jupiaba polylepis, Moenkhausia bonita, Moenkhausia intermedia, Moenkhausia sanctaefilomenae, Moenkhausia xinguensis, Parapristella georgiae, Poptella paraguayensis, Psellogrammus kennedyi, Stethaprion erythrops, Tetragonopterus argenteus, Thayeria obliqua

\section{0, 2- Polimórfico}

Bryconexodon juruenae, Hemigrammus arua, Hemigrammus hyanuary, Hemigrammus skolioplatus, Hemigrammus unilineatus, Hemigrammus yinyang, Moenkhausia hemigrammoides

\section{1, 2- Polimórfico}

Coptobrycon bilineatus, Hasemania melanura

\section{$\underline{\text { 9- Indeterminado }}$}


Aphyocharax anisitsi, Aphyocharax pusillus, Brycon cf pesu, Chalceus spilogyros, Cheirodon interruptus, Grundulus cochae, Iguanodectes spilurus, Microschemobrycon sp, Oligosarcus pintoi, Paracheirodon axelrodi, Petitella georgiae, Roeboides bonariensis, Serrapinnus heterodon, Serrapinnus notomelas, Serrasalmus maculatus, Triportheus nematurus

O número de dentes na série interna do pré-maxilar tem sido usado desde Eigenmann (1917) para a definição de diferentes gêneros dentro de Characidae. Recentemente, a presença de quatro dentes na série interna do pré-maxilar foi proposta por Malabarba \& Weitzman (2003) como uma sinapomorfia para um clado monofilético dentro da família Characide, juntamente com a presença de ii, 8 raios na nadadeira dorsal. Essa hipótese é corroborada na presente análise, com a presença de quatro dentes na série interna do pré-maxilar aparecendo como uma sinpomorfia para o clado 183, que inclui $B$. exodon, $P$. argentea e $R$. myersi. Quatro dentes na série interna do pré-maxilar também aparecem na presente análise como uma autapomorfia adquirida independentemente para uma nova espécie de Hasemania (clado 174). A ocorrência de quatro dentes na série interna do pré-maxilar em Brycinus longipinnnis e alguns outros membros de Alestidae é considerada homoplástica por \& Vari (2005).

\section{6- Número de cúspides dos dentes da série interna do pré-maxilar (para táxons que apresentam duas séries de dentes) (ic - 0,167)}

(Serra, 2003: 58; Bertaco, 2008: 3)

\section{0 - com cinco, seis, sete e/ou oito cúspides}

Astyanax jacobinae, Astyanax mexicanus, Brycinus longipinnis, Bryconamericus exodon, Bryconops melanurus, Coptobrycon bilineatus, Deuterodon iguape, Hasemania melanura, Hasemania sp n., Hemigrammus analis, Hemigrammus arua, Hemigrammus barrigonae, Hemigrammus bleheri, Hemigrammus levis, Hemigrammus pulcher, Hemigrammus stictus, Parapristella georgiae, Rhinopetitia myersi, Thayeria obliqua

\section{1- com três, quatro e/ou cinco cúspides}

Astyanax altiparanae, Gymnocorymbus ternetzi, Hasemania crenuchoides, Hemigrammus bellottii, Hemigrammus boesemani, Hemigrammus brevis, Hemigrammus coeruleus, Hemigrammus gracilis, Hemigrammus haraldi, Hemigrammus hyanuary, Hemigrammus lunatus, Hemigrammus marginatus, Hemigrammus newboldi, Hemigrammus ocellifer, Hemigrammus orthus, Hemigrammus parana, Hemigrammus pretoensis, Hemigrammus rhodostomus, Hemigrammus rodwayi, Hemigrammus schmardae, Hemigrammus silimoni, Hemigrammus skolioplatus, Hemigrammus taphorni, Hemigrammus tridens, Hemigrammus ulreyi, Hemigrammus unilineatus, Hemigrammus vorderwikleri, Hemigrammus sp n., Hollandichthys aff. multifasciatus, Hyphessobrycon bentosi, Hyphessobrycon coelestinus, Hyphessobrycon diancistrus, Hyphessobrycon eques, Hyphessobrycon takasei, Jupiaba polylepis, Moenkhausia bonita, Moenkhausia hemigrammoides, Moenkhausia intermedia, Moenkhausia 
sanctaefilomenae, Moenkhausia xinguensis, Piabina argentea, Poptella paraguayensis, Psellogrammus kennedyi, Stethaprion erythrops, Tetragonopterus argenteus

\section{2- cônicos}

Bryconella pallidifrons, Hemigrammus mimus, Hyphessobrycon eilyos, Hyphessobrycon megalopterus, Salminus hilarii

\section{3-somente com 3 cúspides}

Hemigrammus cylindricus, Hemigrammus yinyang, Hyphessobrycon compressus, Hyphessobrycon epicharis, Hyphessobrycon micropterus, Lignobrycon myersi, Pristella maxillaris

\section{2, 3-Polimórfico}

Hyphessobrycon erythrostigma, Phenacogaster franciscoensis

\section{9- Indeterminado}

Aphyocharax anisitsi, Aphyocharax pusillus, Brycon cf pesu, Bryconexodon juruenae, Chalceus spilogyros, Cheirodon interruptus, Grundulus cochae, Iguanodectes spilurus, Microschemobrycon sp, Oligosarcus pintoi, Paracheirodon axelrodi, Petitella georgiae, Roeboides bonariensis, Serrasalmus maculatus, Serrapinnus heterodon, Serrapinnus notomelas, Triportheus nematurus

Os dentes da série interna da pré-maxila apresentam número variável de cúspides de acordo com a espécie e para aquelas com dentes com mais de três cúspides também é comum que esses dentes apresentem número de cúspides variável em um mesmo indivíduo. Nesse caso, os primeiros dentes (próximos à sínfise) e os dentes medianos apresentam maior número de cúspides e os últimos dentes (aqueles mais próximos do maxilar) são os que apresentam o menor número. Dessa forma, os estados 0 e 1 consideram a variação observada no número de cúspides encontrado em um mesmo indivíduo.

\section{7- Tamanho do pré-maxilar (ic - 0,200)}

(Serra, 2003: 60; Marinho, 2009: 0)

\section{0 - mais longo que alto}

Aphyocharax anisitsi, Aphyocharax pusillus, Astyanax jacobinae, Astyanax mexicanus, Brycinus longipinnis, Brycon cf pesu, Bryconamericus exodon, Bryconella pallidifrons, Bryconops melanurus, Chalceus spilogyros, Cheirodon interruptus, Coptobrycon bilineatus, Deuterodon iguape, Grundulus cochae, Hasemania melanura, n., Hemigrammus analis, Hemigrammus arua, Hemigrammus barrigonae, Hemigrammus bellottii, Hemigrammus bleheri, Hemigrammus boesemani, Hemigrammus brevis, Hemigrammus coeruleus, Hemigrammus cylindricus, Hemigrammus gracilis, Hemigrammus haraldi, Hemigrammus hyanuary, Hemigrammus levis, 
Hemigrammus lunatus, Hemigrammus marginatus, Hemigrammus mimus, Hemigrammus newboldi, Hemigrammus ocellifer, Hemigrammus orthus, Hemigrammus parana, Hemigrammus pretoensis, Hemigrammus pulcher, Hemigrammus rhodostomus, Hemigrammus rodwayi, Hemigrammus schmardae, Hemigrammus silimoni, Hemigrammus skolioplatus, Hemigrammus stictus, Hemigrammus taphorni, Hemigrammus tridens, Hemigrammus ulreyi, Hemigrammus vorderwikleri, Hemigrammus yinyang, Hemigrammus sp n., Hollandichthys aff. multifasciatus, Hyphessobrycon bentosi, Hyphessobrycon compressus, Hyphessobrycon diancistrus, Hyphessobrycon eilyos, Hyphessobrycon epicharis, Hyphessobrycon eques, Hyphessobrycon erythrostigma, Hyphessobrycon megalopterus, Hyphessobrycon micropterus, Hyphessobrycon takasei, Iguanodectes spilurus, Jupiaba polylepis, Lignobrycon myersi, Microschemobrycon sp, Moenkhausia bonita, Moenkhausia hemigrammoides, Moenkhausia sanctaefilomenae, Moenkhausia xinguensis, Oligosarcus pintoi, Paracheirodon axelrodi, Parapristella georgiae, Petitella georgiae, Phenacogaster franciscoensis, Psellogrammus kennedyi, Pristella maxillaris, Rhinopetitia myersi, Roeboides bonariensis, Serrapinnus heterodon, Serrapinnus notomelas, Serrasalmus maculatus, Stethaprion erythrops, Tetragonopterus argenteus, Thayeria obliqua, Triportheus nematurus

\section{1- aproximadamente tão alto quanto longo}

Astyanax altiparanae, Gymnocorymbus ternetzi, Hasemania crenuchoides, Hasemania sp, Hemigrammus unilineatus, Hyphessobrycon coelestinus, Moenkhausia intermedia, Poptella paraguayensis, Salminus hilarii

\section{2- mais alto que longo}

Bryconexodon juruenae, Piabina argentea

\section{8- Tamanho do dentário (ic - 0,500)}

(Vari \& Harold, 1998; Serra, 2003: 61)

$\underline{0-}$ curto, com poucos dentes cobrindo uma grande porcentagem da superfície do osso (aproximadamente metade) (Figuras 12 - 13)

Coptobrycon bilineatus, Piabina argentea

1- não encurtado, com uma pequena superfície do osso recoberta por dentes ou sendo necessários muitos dentes para encobrir metade da superfície (Figuras 11 e 14)

Aphyocharax anisitsi, Aphyocharax pusillus, Astyanax altiparanae, Astyanax jacobinae, Astyanax mexicanus, Brycinus longipinnis, Brycon cf pesu, Bryconamericus exodon, Bryconella pallidifrons, Bryconexodon juruenae, Bryconops melanurus, Chalceus spilogyros, Cheirodon interruptus, Deuterodon iguape, Grundulus cochae, Gymnocorymbus ternetzi, Hasemania crenuchoides, Hasemania melanura, Hasemania sp n., Hemigrammus analis, Hemigrammus arua, Hemigrammus barrigonae, Hemigrammus bellottii, Hemigrammus bleheri, Hemigrammus boesemani, Hemigrammus brevis, Hemigrammus coeruleus, Hemigrammus cylindricus, Hemigrammus gracilis, Hemigrammus haraldi, Hemigrammus hyanuary, Hemigrammus levis, Hemigrammus lunatus, Hemigrammus marginatus, Hemigrammus mimus, Hemigrammus newboldi, Hemigrammus ocellifer, Hemigrammus orthus, Hemigrammus parana, Hemigrammus pretoensis, Hemigrammus pulcher, Hemigrammus rhodostomus, Hemigrammus rodwayi, Hemigrammus schmardae, Hemigrammus silimoni, Hemigrammus skolioplatus, Hemigrammus 
stictus, Hemigrammus taphorni, Hemigrammus tridens, Hemigrammus ulreyi, Hemigrammus unilineatus, Hemigrammus vorderwikleri, Hemigrammus yinyang, Hemigrammus sp n., Hollandichthys aff. multifasciatus, Hyphessobrycon bentosi, Hyphessobrycon coelestinus, Hyphessobrycon compressus, Hyphessobrycon diancistrus, Hyphessobrycon eilyos, Hyphessobrycon epicharis, Hyphessobrycon eques, Hyphessobrycon erythrostigma, Hyphessobrycon megalopterus, Hyphessobrycon micropterus, Hyphessobrycon takasei, Iguanodectes spilurus, Jupiaba polylepis, Lignobrycon myersi, Microschemobrycon sp, Moenkhausia bonita, Moenkhausia hemigrammoides, Moenkhausia intermedia, Moenkhausia sanctaefilomenae, Moenkhausia xinguensis, Oligosarcus pintoi, Paracheirodon axelrodi, Parapristella georgiae, Petitella georgiae, Phenacogaster franciscoensis, Poptella paraguayensis, Psellogrammus kennedyi, Pristella maxillaris, Rhinopetitia myersi, Roeboides bonariensis, Salminus hilarii, Serrapinnus heterodon, Serrapinnus notomelas, Serrasalmus maculatus, Stethaprion erythrops, Tetragonopterus argenteus, Thayeria obliqua, Triportheus nematurus

\section{9- Dentário (ic - 0,100)}

(Serra, 2003: 62)

\section{$\underline{0 \text { - retangular, com aproximadamente a mesma altura em toda extensão }}$}

Bryconops melanurus, Hemigrammus arua, Hemigrammus bellottii, Hemigrammus gracilis, Hemigrammus ocellifer, Hemigrammus silimoni, Hemigrammus sp n., Hyphessobrycon diancistrus, Oligosarcus pintoi, Parapristella georgiae, Serrasalmus maculatus

\section{1- com a região posterior mais alta que a anterior, geralmente abaulado}

Aphyocharax anisitsi, Aphyocharax pusillus, Astyanax altiparanae, Astyanax jacobinae, Astyanax mexicanus, Brycinus longipinnis, Brycon cf pesu, Bryconamericus exodon, Bryconella pallidifrons, Bryconexodon juruenae, Chalceus spilogyros, Cheirodon interruptus, Coptobrycon bilineatus, Deuterodon iguape, Grundulus cochae Gymnocorymbus ternetzi, Hasemania crenuchoides, Hasemania melanura, Hasemania sp n., Hemigrammus analis, Hemigrammus barrigonae, Hemigrammus bleheri, Hemigrammus boesemani, Hemigrammus brevis, Hemigrammus coeruleus, Hemigrammus cylindricus, Hemigrammus haraldi, Hemigrammus hyanuary, Hemigrammus levis, Hemigrammus lunatus, Hemigrammus marginatus, Hemigrammus mimus, Hemigrammus newboldi, Hemigrammus orthus, Hemigrammus parana, Hemigrammus pretoensis, Hemigrammus pulcher, Hemigrammus rhodostomus, Hemigrammus rodwayi, Hemigrammus schmardae, Hemigrammus skolioplatus, Hemigrammus stictus, Hemigrammus taphorni, Hemigrammus tridens, Hemigrammus ulreyi, Hemigrammus unilineatus, Hemigrammus vorderwikleri, Hemigrammus yinyang, Hollandichthys aff. multifasciatus, Hyphessobrycon bentosi, Hyphessobrycon coelestinus, Hyphessobrycon compressus, Hyphessobrycon eilyos, Hyphessobrycon epicharis, Hyphessobrycon eques, Hyphessobrycon erythrostigma, Hyphessobrycon megalopterus, Hyphessobrycon micropterus, Hyphessobrycon takasei, Iguanodectes spilurus, Jupiaba polylepis, Lignobrycon myersi, Microschemobrycon sp, Moenkhausia bonita, Moenkhausia hemigrammoides, Moenkhausia intermedia, Moenkhausia sanctaefilomenae, Moenkhausia xinguensis, Paracheirodon axelrodi, Petitella georgiae, Phenacogaster franciscoensis, Piabina argentea, Poptella paraguayensis, Psellogrammus kennedyi, Pristella maxillaris, Rhinopetitia myersi, Roeboides bonariensis, Salminus hilarii, Serrapinnus heterodon, Serrapinnus notomelas, Stethaprion erythrops, Tetragonopterus argenteus, Thayeria obliqua, Triportheus nematurus

50- Forma da região anterior do dentário (ic - 0,083) 


\section{0- com declividade acentuada deixando a região anterior bem mais}

\section{afilada que a posterior (Figura 14)}

Aphyocharax anisitsi, Aphyocharax pusillus, Bryconella pallidifrons, Cheirodon interruptus, Grundulus cochae, Hemigrammus mimus, Hyphessobrycon eques, Hyphessobrycon megalopterus, Hyphessobrycon micropterus, Hyphessobrycon takasei, Microschemobrycon sp, Petitella georgiae, Phenacogaster franciscoensis, Rhinopetitia myersi, Roeboides bonariensis

1- sem declividade, ou com esta pouco acentuada, deixando a região anterior do dentário aproximadamente da mesma altura que a região posterior

(Figuras 11, $12-13$ )

Astyanax altiparanae, Astyanax jacobinae, Astyanax mexicanus, Brycinus longipinnis, Brycon cf pesu, Bryconamericus exodon, Bryconexodon juruenae, Bryconops melanurus, Chalceus spilogyros, Coptobrycon bilineatus, Deuterodon iguape, Gymnocorymbus ternetzi, Hasemania crenuchoides, Hasemania melanura, Hasemania sp n., Hemigrammus analis, Hemigrammus arua, Hemigrammus barrigonae, Hemigrammus bellottii, Hemigrammus bleheri, Hemigrammus boesemani, Hemigrammus brevis, Hemigrammus coeruleus, Hemigrammus cylindricus, Hemigrammus gracilis, Hemigrammus haraldi, Hemigrammus hyanuary, Hemigrammus levis, Hemigrammus lunatus, Hemigrammus marginatus, Hemigrammus newboldi, Hemigrammus ocellifer, Hemigrammus orthus, Hemigrammus parana, Hemigrammus pretoensis, Hemigrammus pulcher, Hemigrammus rhodostomus, Hemigrammus rodwayi, Hemigrammus schmardae, Hemigrammus silimoni, Hemigrammus skolioplatus, Hemigrammus stictus, Hemigrammus taphorni, Hemigrammus tridens, Hemigrammus ulreyi, Hemigrammus unilineatus, Hemigrammus vorderwikleri, Hemigrammus yinyang, Hemigrammus sp n., Hollandichthys aff. multifasciatus, Hyphessobrycon bentosi, Hyphessobrycon coelestinus, Hyphessobrycon compressus, Hyphessobrycon diancistrus, Hyphessobrycon eilyos, Hyphessobrycon epicharis, Hyphessobrycon erythrostigma, Iguanodectes spilurus Jupiaba polylepis, Lignobrycon myersi, Moenkhausia bonita, Moenkhausia hemigrammoides, Moenkhausia intermedia, Moenkhausia sanctaefilomenae, Moenkhausia xinguensis, Oligosarcus pintoi, Paracheirodon axelrodi, Parapristella georgiae, Piabina argentea, Poptella paraguayensis, Psellogrammus kennedyi, Pristella maxillaris, Salminus hilarii, Serrapinnus heterodon, Serrapinnus notomelas, Serrasalmus maculatus, Stethaprion erythrops, Tetragonopterus argenteus, Thayeria obliqua, Triportheus nematurus

51- Número de séries de dentes do dentário (ic - 0,750)

(Malabarba, 1998: 41; Buckup, 1998: 36; Serra, 2003: 63).

$\underline{0 \text { - duas, a segunda formada apenas por um par de dentes sinfisianos }}$

Brycinus longipinnis, Triportheus nematurus

1- duas, a segunda com um par de dentes sinfisianos mais uma série de dentes menores localizados na região mais posterior do osso, separados por um espaço sem dentes

Brycon cf pesu, Chalceus spilogyros

\section{2- duas, a segunda com dentes por toda a superfície do osso}

Salminus hilarii 


\section{3- uma}

Aphyocharax anisitsi, Aphyocharax pusillus, Astyanax altiparanae, Astyanax jacobinae, Astyanax mexicanus, Bryconamericus exodon, Bryconella pallidifrons, Bryconops melanurus, Cheirodon interruptus, Coptobrycon bilineatus, Deuterodon iguape, Grundulus cochae, Gymnocorymbus ternetzi, Hasemania crenuchoides, Hasemania melanura, Hasemania sp n., Hemigrammus analis, Hemigrammus arua, Hemigrammus barrigonae, Hemigrammus bellottii, Hemigrammus bleheri, Hemigrammus boesemani, Hemigrammus brevis, Hemigrammus coeruleus, Hemigrammus cylindricus, Hemigrammus gracilis, Hemigrammus haraldi, Hemigrammus hyanuary, Hemigrammus levis, Hemigrammus lunatus, Hemigrammus marginatus, Hemigrammus mimus, Hemigrammus newboldi, Hemigrammus ocellifer, Hemigrammus orthus, Hemigrammus parana, Hemigrammus pretoensis, Hemigrammus pulcher, Hemigrammus rhodostomus, Hemigrammus rodwayi, Hemigrammus schmardae, Hemigrammus silimoni, Hemigrammus skolioplatus, Hemigrammus stictus, Hemigrammus taphorni, Hemigrammus tridens, Hemigrammus ulreyi, Hemigrammus unilineatus, Hemigrammus vorderwikleri, Hemigrammus yinyang, Hemigrammus sp n., Hollandichthys aff. multifasciatus, Hyphessobrycon bentosi, Hyphessobrycon coelestinus, Hyphessobrycon compressus, Hyphessobrycon diancistrus, Hyphessobrycon eilyos, Hyphessobrycon epicharis, Hyphessobrycon eques, Hyphessobrycon erythrostigma, Hyphessobrycon megalopterus, Hyphessobrycon micropterus, Hyphessobrycon takasei, Iguanodectes spilurus, Jupiaba polylepis, Lignobrycon myersi, Microschemobrycon sp, Moenkhausia bonita, Moenkhausia hemigrammoides, Moenkhausia intermedia, Moenkhausia sanctaefilomenae, Moenkhausia xinguensis, Oligosarcus pintoi, Paracheirodon axelrodi, Parapristella georgiae, Petitella georgiae, Phenacogaster franciscoensis, Piabina argentea, Poptella paraguayensis, Psellogrammus kennedyi, Pristella maxillaris, Rhinopetitia myersi, Serrapinnus heterodon, Serrapinnus notomelas, Serrasalmus maculatus, Stethaprion erythrops, Tetragonopterus argenteus, Thayeria obliqua

\section{9- Indeterminado}

Bryconexodon juruenae, Roeboides bonariensis

52- Dentes do dentário (ic - 0,143)

(Serra, 2003: 67; Bertaco, 2008: 21)

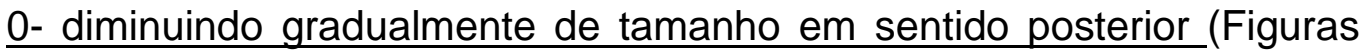

14)

Aphyocharax anisitsi, Aphyocharax pusillus, Astyanax jacobinae, Deuterodon iguape, Grundulus cochae, Hemigrammus mimus, Hyphessobrycon epicharis, Hyphessobrycon erythrostigma, Microschemobrycon sp, Phenacogaster franciscoensis, Serrasalmus maculatus

\section{1- diminuindo abruptamente de tamanho em sentido posterior (Figuras}

11 e 13)

Astyanax altiparanae, Astyanax mexicanus, Brycinus longipinnis, Brycon cf pesu, Bryconamericus exodon, Bryconella pallidifrons, Bryconops melanurus, Cheirodon interruptus, Coptobrycon bilineatus, Gymnocorymbus ternetzi, Hasemania crenuchoides, Hasemania melanura, Hasemania sp n., Hemigrammus analis, Hemigrammus arua, Hemigrammus barrigonae, Hemigrammus bellottii, Hemigrammus bleheri, Hemigrammus boesemani, Hemigrammus brevis, Hemigrammus coeruleus, Hemigrammus cylindricus, Hemigrammus gracilis, Hemigrammus haraldi, Hemigrammus hyanuary, Hemigrammus levis, Hemigrammus lunatus, Hemigrammus marginatus, Hemigrammus newboldi, Hemigrammus ocellifer, Hemigrammus orthus, Hemigrammus parana, Hemigrammus pretoensis, Hemigrammus pulcher, Hemigrammus rhodostomus, Hemigrammus rodwayi, Hemigrammus schmardae, Hemigrammus silimoni, Hemigrammus skolioplatus, Hemigrammus stictus, Hemigrammus taphorni, Hemigrammus tridens, Hemigrammus ulreyi, Hemigrammus unilineatus, 
Hemigrammus vorderwikleri, Hemigrammus yinyang, Hemigrammus sp n., Hollandichthys aff. multifasciatus, Hyphessobrycon bentosi, Hyphessobrycon coelestinus, Hyphessobrycon compressus, Hyphessobrycon diancistrus, Hyphessobrycon eilyos, Hyphessobrycon eques, Hyphessobrycon megalopterus, Hyphessobrycon micropterus, Hyphessobrycon takasei, Iguanodectes spilurus, Jupiaba polylepis, Lignobrycon myersi, Moenkhausia bonita, Moenkhausia hemigrammoides, Moenkhausia intermedia, Moenkhausia sanctaefilomenae, Moenkhausia xinguensis, Oligosarcus pintoi, Paracheirodon axelrodi, Parapristella georgiae, Petitella georgiae, Piabina argentea, Poptella paraguayensis, Psellogrammus kennedyi, Pristella maxillaris, Rhinopetitia myersi, Serrapinnus heterodon, Serrapinnus notomelas, Stethaprion erythrops, Tetragonopterus argenteus, Thayeria obliqua

\section{9- Indeterminado}

Bryconexodon juruenae, Chalceus spilogyros, Roeboides bonariensis, Salminus hilarii, Triportheus nematurus

A condição comumente observda dentro de Characide é a presença, no dentário, de alguns dentes anteriores e maiores, seguidos por outros dentes considerávelmente menores, ou seja existe uma mudança brusca de tamanho nos dentes em sentido posterior, sendo que aqueles anteriores e maiores são claramente distinguíveis dos demais (estado 1). Em alguns táxons, entretanto, os dentes diminuem gradualmente de tamanho em sentido posterior (estado 0), não sendo distinguíveis dentes anteriores e maiores daqueles posteriores e menores.

\section{3- Número de dentes anteriores e mais largos do dentário (para táxons que apresentam uma série de dentes) (ic - 0,154)}

(Serra, 2003: 64; Bertaco, 2008: 18)

\section{$\underline{0 \text { - seis ou mais dentes }}$}

Hemigrammus arua, Hemigrammus stictus, Hollandichthys aff. multifasciatus, Hyphessobrycon eilyos, Hyphessobrycon eques, Hyphessobrycon micropterus, Hyphessobrycon takasei, Iguanodectes spilurus, Rhinopetitia myersi, Serrapinnus heterodon, Serrapinnus notomelas

\section{1- dois a três dentes}

Brycinus longipinnis, Coptobrycon bilineatus, Hasemania crenuchoides, Piabina argentea

\section{2- quatro a cinco dentes}

Astyanax altiparanae, Astyanax mexicanus, Bryconamericus exodon, Bryconella pallidifrons, Bryconops melanurus, Cheirodon interruptus, Gymnocorymbus ternetzi, Hasemania melanura, Hasemania sp n., Hemigrammus analis, Hemigrammus barrigonae, Hemigrammus bellottii, Hemigrammus bleheri, Hemigrammus boesemani, Hemigrammus brevis, Hemigrammus coeruleus, Hemigrammus cylindricus, Hemigrammus gracilis, Hemigrammus haraldi, Hemigrammus hyanuary, Hemigrammus levis, Hemigrammus lunatus, Hemigrammus marginatus, Hemigrammus newboldi, Hemigrammus ocellifer, Hemigrammus orthus, Hemigrammus parana, Hemigrammus pretoensis, Hemigrammus pulcher, Hemigrammus rhodostomus, Hemigrammus rodwayi, Hemigrammus schmardae, Hemigrammus silimoni, 
Hemigrammus skolioplatus, Hemigrammus taphorni, Hemigrammus tridens, Hemigrammus ulreyi, Hemigrammus unilineatus, Hemigrammus vorderwikleri, Hemigrammus yinyang, Hemigrammus sp n., Hyphessobrycon bentosi, Hyphessobrycon coelestinus, Hyphessobrycon compressus, Hyphessobrycon diancistrus, Jupiaba polylepis, Lignobrycon myersi, Moenkhausia bonita, Moenkhausia hemigrammoides, Moenkhausia intermedia, Moenkhausia sanctaefilomenae, Moenkhausia xinguensis, Oligosarcus pintoi, Parapristella georgiae, Petitella georgiae, Poptella paraguayensis, Psellogrammus kennedyi, Pristella maxillaris, Stethaprion erythrops, Tetragonopterus argenteus, Thayeria obliqua

\section{$\underline{0,2-\text { Polimórfico }}$}

Hyphessobrycon megalopterus, Paracheirodon axelrodi, Phenacogaster franciscoensis

\section{9- Indeterminado}

Aphyocharax anisitsi, Aphyocharax pusillus, Astyanax jacobinae, Brycon cf pesu, Bryconexodon juruenae, Chalceus spilogyros, Deuterodon iguape, Grundulus cochae, Hemigrammus mimus, Hyphessobrycon epicharis, Hyphessobrycon erythrostigma, Microschemobrycon sp, Roeboides bonariensis, Salminus hilarii, Serrasalmus maculatus, Triportheus nematurus

Ver comentários do caráter 52.

\section{4- Dentes anteriores do dentário (para táxons que apresentam uma série de dentes) (ic $-0,250$ )}

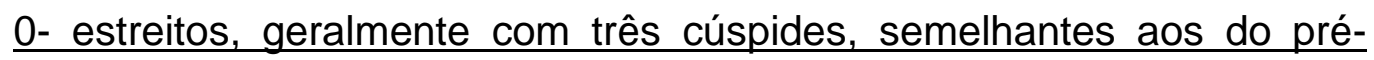
$\underline{\text { maxilar }}$

Hemigrammus cylindricus, Hyphessobrycon eilyos, Hyphessobrycon megalopterus, Hyphessobrycon micropterus, Petitella georgiae

\section{1- largos, com número de cúspides variável (geralmente mais de três), e}

\section{maiores que os do pré-maxilar}

Astyanax altiparanae, Astyanax mexicanus, Brycinus longipinnis, Bryconamericus exodon, Bryconella pallidifrons, Bryconops melanurus, Coptobrycon bilineatus, Gymnocorymbus ternetzi, Hasemania crenuchoides, Hasemania melanura, Hasemania sp n., Hemigrammus analis, Hemigrammus arua, Hemigrammus barrigonae, Hemigrammus bellottii, Hemigrammus bleheri, Hemigrammus boesemani, Hemigrammus brevis, Hemigrammus coeruleus, Hemigrammus gracilis, Hemigrammus haraldi, Hemigrammus hyanuary, Hemigrammus levis, Hemigrammus lunatus, Hemigrammus marginatus, Hemigrammus newboldi, Hemigrammus ocellifer, Hemigrammus orthus, Hemigrammus parana, Hemigrammus pretoensis, Hemigrammus pulcher, Hemigrammus rhodostomus, Hemigrammus rodwayi, Hemigrammus schmardae, Hemigrammus silimoni, Hemigrammus skolioplatus, Hemigrammus stictus, Hemigrammus taphorni, Hemigrammus tridens, Hemigrammus ulreyi, Hemigrammus unilineatus, Hemigrammus vorderwikleri, Hemigrammus yinyang, Hemigrammus sp n., Hollandichthys aff. multifasciatus, Hyphessobrycon bentosi, Hyphessobrycon coelestinus, Hyphessobrycon compressus, Hyphessobrycon diancistrus, Hyphessobrycon eques, Hyphessobrycon takasei, Jupiaba polylepis, Lignobrycon myersi, Moenkhausia bonita, Moenkhausia hemigrammoides, Moenkhausia intermedia, Moenkhausia sanctaefilomenae, Moenkhausia xinguensis, Oligosarcus pintoi, Paracheirodon axelrodi, Parapristella georgiae, Piabina argentea, Poptella paraguayensis, Psellogrammus kennedyi, Pristella maxillaris, Rhinopetitia myersi, Serrapinnus heterodon, Serrapinnus notomelas, Stethaprion erythrops, Tetragonopterus argenteus, Thayeria obliqua 


\section{9- Indeterminado}

Aphyocharax anisitsi, Aphyocharax pusillus, Astyanax jacobinae, Brycon cf pesu, Bryconexodon juruenae, Chalceus spilogyros, Cheirodon interruptus, Deuterodon iguape, Grundulus cochae, Hemigrammus mimus, Hyphessobrycon epicharis, Hyphessobrycon erythrostigma, Iguanodectes spilurus, Microschemobrycon sp, Phenacogaster franciscoensis, Roeboides bonariensis, Salminus hilarii, Serrasalmus maculatus, Triportheus nematurus

\section{5- Número total de dentes no dentário (para táxons que apresentam uma série de dentes) (ic $-0,071$ )}

(Serra, 2003: 65)

\section{$\underline{0-11 \text { a } 20 \text { dentes }}$}

Aphyocharax anisitsi, Aphyocharax pusillus, Astyanax jacobinae, Bryconamericus exodon, Grundulus cochae, Hasemania sp n., Hemigrammus arua, Hemigrammus bellottii, Hemigrammus boesemani, Hemigrammus brevis, Hemigrammus coeruleus, Hemigrammus cylindricus, Hemigrammus gracilis, Hemigrammus haraldi, Hemigrammus hyanuary, Hemigrammus levis, Hemigrammus lunatus, Hemigrammus marginatus, Hemigrammus mimus, Hemigrammus newboldi, Hemigrammus ocellifer, Hemigrammus orthus, Hemigrammus parana, Hemigrammus pretoensis, Hemigrammus pulcher, Hemigrammus rodwayi, Hemigrammus silimoni, Hemigrammus skolioplatus, Hemigrammus taphorni, Hemigrammus ulreyi, Hemigrammus unilineatus, Hemigrammus yinyang, Hemigrammus sp n., Hollandichthys aff. multifasciatus, Hyphessobrycon bentosi, Hyphessobrycon coelestinus, Hyphessobrycon compressus, Hyphessobrycon eilyos, Hyphessobrycon epicharis, Hyphessobrycon eques, Hyphessobrycon erythrostigma, Hyphessobrycon megalopterus, Hyphessobrycon micropterus, Hyphessobrycon takasei, Jupiaba polylepis, Lignobrycon myersi, Microschemobrycon sp, Moenkhausia bonita, Moenkhausia intermedia, Moenkhausia sanctaefilomenae, Moenkhausia xinguensis, Oligosarcus pintoi, Parapristella georgiae, Phenacogaster franciscoensis, Poptella paraguayensis, Pristella maxillaris

\section{1- 1 a 10 dentes}

Astyanax altiparanae, Astyanax mexicanus, Brycinus longipinnis, Bryconella pallidifrons, Bryconops melanurus, Cheirodon interruptus, Coptobrycon bilineatus, Deuterodon iguape, Gymnocorymbus ternetzi, Hasemania crenuchoides, Hasemania melanura, Hemigrammus analis, Hemigrammus barrigonae, Hemigrammus bleheri, Hemigrammus stictus, Hemigrammus tridens, Hemigrammus vorderwikleri, Hyphessobrycon diancistrus, Iguanodectes spilurus, Paracheirodon axelrodi, Petitella georgiae, Piabina argentea, Psellogrammus kennedyi, Rhinopetitia myersi, Serrapinnus heterodon, Serrapinnus notomelas, Serrasalmus maculatus, Tetragonopterus argenteus

\section{$\underline{0,1-\text { Polimórfico }}$}

Hemigrammus rhodostomus, Hemigrammus schmardae, Moenkhausia hemigrammoides, Stethaprion erythrops, Thayeria obliqua

\section{9- Indeterminado}

Brycon cf pesu, Bryconexodon juruenae, Chalceus spilogyros, Roeboides bonariensis, Salminus hilarii, Triportheus nematurus

\section{6- Dentes do dentário após os dentes anteriores maiores (para táxons} que apresentam uma série de dentes) (ic - 0,067) 
(Serra, 2003: 66)

\section{0 - maioria cuspidados}

Astyanax mexicanus, Brycinus longipinnis, Bryconamericus exodon, Bryconella pallidifrons, Bryconops melanurus, Cheirodon interruptus, Hasemania crenuchoides, Hasemania sp n., Hemigrammus analis, Hemigrammus barrigonae, Hemigrammus bleheri, Hemigrammus coeruleus, Hemigrammus newboldi, Hemigrammus ocellifer, Hemigrammus orthus, Hemigrammus taphorni, Hemigrammus schmardae, Hemigrammus stictus, Hemigrammus tridens, Hemigrammus vorderwikleri, Hollandichthys aff. multifasciatus, Hyphessobrycon eques, Hyphessobrycon takasei, Moenkhausia sanctaefilomenae, Oligosarcus pintoi, Parapristella georgiae, Piabina argentea, Rhinopetitia myersi, Serrapinnus heterodon, Serrapinnus notomelas

\section{1- maioria cônicos}

Astyanax altiparanae, Coptobrycon bilineatus, Gymnocorymbus ternetzi, Hasemania melanura, Hemigrammus arua, Hemigrammus bellottii, Hemigrammus boesemani, Hemigrammus brevis, Hemigrammus cylindricus, Hemigrammus gracilis, Hemigrammus haraldi, Hemigrammus hyanuary, Hemigrammus levis, Hemigrammus lunatus, Hemigrammus marginatus, Hemigrammus parana, Hemigrammus pretoensis, Hemigrammus pulcher, Hemigrammus rhodostomus, Hemigrammus rodwayi, Hemigrammus silimoni, Hemigrammus skolioplatus, Hemigrammus ulreyi, Hemigrammus unilineatus, Hemigrammus yinyang, Hemigrammus sp n., Hyphessobrycon bentosi, Hyphessobrycon coelestinus, Hyphessobrycon compressus, Hyphessobrycon diancistrus, Hyphessobrycon eilyos, Hyphessobrycon megalopterus, Hyphessobrycon micropterus, Jupiaba polylepis, Lignobrycon myersi, Moenkhausia bonita, Moenkhausia hemigrammoides, Moenkhausia intermedia, Moenkhausia xinguensis, Paracheirodon axelrodi, Petitella georgiae, Poptella paraguayensis, Psellogrammus kennedyi, Pristella maxillaris, Stethaprion erythrops, Tetragonopterus argenteus, Thayeria obliqua

\section{9- Indeterminado}

Aphyocharax anisitsi, Astyanax jacobinae, Aphyocharax pusillus, Brycon cf pesu, Bryconexodon juruenae, Chalceus spilogyros, Deuterodon iguape, Grundulus cochae, Hemigrammus mimus, Hyphessobrycon epicharis, Hyphessobrycon erythrostigma, Iguanodectes spilurus, Microschemobrycon sp, Phenacogaster franciscoensis, Roeboides bonariensis, Salminus hilarii, Serrasalmus maculatus, Triportheus nematurus

\section{7- Tamanho das cúspides dos dentes do dentário (ic - 0,333)}

\section{0- subiguais, todas com aproximadamente o mesmo tamanho (Figuras} $12-13)$

Cheirodon interruptus, Coptobrycon bilineatus, Iguanodectes spilurus, Paracheirodon axelrodi, Serrapinnus heterodon, Serrapinnus notomelas

\section{1- uma das cúspides claramente maior que as demais (cúspide}

\section{mediana)(Figuras 11 e 14)}

Aphyocharax anisitsi, Aphyocharax pusillus, Astyanax altiparanae, Astyanax jacobinae, Astyanax mexicanus, Brycinus longipinnis, Brycon cf pesu, Bryconamericus exodon, Bryconella pallidifrons, Bryconops melanurus, Chalceus spilogyros, Deuterodon iguape, Gymnocorymbus ternetzi, Hasemania crenuchoides, Hasemania melanura, Hasemania sp n., Hemigrammus analis, Hemigrammus arua, Hemigrammus barrigonae, Hemigrammus bellottii, Hemigrammus bleheri, Hemigrammus boesemani, Hemigrammus brevis, Hemigrammus coeruleus, Hemigrammus cylindricus, Hemigrammus gracilis, Hemigrammus haraldi, Hemigrammus hyanuary, Hemigrammus levis, Hemigrammus lunatus, Hemigrammus marginatus, 
Hemigrammus mimus, Hemigrammus newboldi, Hemigrammus ocellifer, Hemigrammus orthus, Hemigrammus parana, Hemigrammus pretoensis, Hemigrammus pulcher, Hemigrammus rhodostomus, Hemigrammus rodwayi, Hemigrammus schmardae, Hemigrammus silimoni, Hemigrammus skolioplatus, Hemigrammus stictus, Hemigrammus taphorni, Hemigrammus tridens, Hemigrammus ulreyi, Hemigrammus unilineatus, Hemigrammus vorderwikleri, Hemigrammus yinyang, Hemigrammus sp n., Hollandichthys aff. multifasciatus, Hyphessobrycon bentosi, Hyphessobrycon coelestinus, Hyphessobrycon compressus, Hyphessobrycon diancistrus, Hyphessobrycon eilyos, Hyphessobrycon epicharis, Hyphessobrycon eques, Hyphessobrycon erythrostigma, Hyphessobrycon megalopterus, Hyphessobrycon micropterus, Hyphessobrycon takasei, Jupiaba polylepis, Lignobrycon myersi, Microschemobrycon sp, Moenkhausia bonita, Moenkhausia hemigrammoides, Moenkhausia intermedia, Moenkhausia sanctaefilomenae, Moenkhausia xinguensis, Oligosarcus pintoi, Parapristella georgiae, Petitella georgiae, Phenacogaster franciscoensis, Piabina argentea, Poptella paraguayensis, Psellogrammus kennedyi, Pristella maxillaris, Rhinopetitia myersi, Serrasalmus maculatus, Stethaprion erythrops, Tetragonopterus argenteus, Thayeria obliqua, Triportheus nematurus

\section{9- Indeterminado}

Bryconexodon juruenae, Grundulus cochae, Roeboides bonariensis, Salminus hilarii

\section{8- Articulação entre o quadrado e o ângulo-articular (ic - 0,125)}

(Serra, 2003: 42; Moreira, 2007: 68; Bertaco, 2008: 49; Mirande, 2009: 153154).

\section{$\underline{0-\text { na vertical que passa pelo meio da órbita ou posteriormente a ela }}$}

Aphyocharax pusillus, Brycon cf pesu, Bryconella pallidifrons, Bryconexodon juruenae, Bryconops melanurus, Hasemania crenuchoides, Hemigrammus analis, Hemigrammus cylindricus, Hemigrammus marginatus, Hemigrammus skolioplatus, Hemigrammus taphorni, Hyphessobrycon coelestinus, Hyphessobrycon eilyos, Hyphessobrycon epicharis, Oligosarcus pintoi, Parapristella georgiae, Phenacogaster franciscoensis, Roeboides bonariensis, Salminus hilarii, Serrasalmus maculatus

\section{1- na vertical que passa pelo etmóide lateral ou anterior à porção}

\section{mediana da órbita}

Aphyocharax anisitsi, Astyanax altiparanae, Astyanax jacobinae, Astyanax mexicanus, Brycinus longipinnis, Chalceus spilogyros, Cheirodon interruptus, Coptobrycon bilineatus, Deuterodon iguape, Grundulus cochae, Gymnocorymbus ternetzi, Hasemania melanura, Hasemania sp n., Hemigrammus arua, Hemigrammus barrigonae, Hemigrammus bellottii, Hemigrammus bleheri, Hemigrammus boesemani, Hemigrammus brevis, Hemigrammus coeruleus, Hemigrammus gracilis, Hemigrammus haraldi, Hemigrammus hyanuary, Hemigrammus levis, Hemigrammus lunatus, Hemigrammus mimus, Hemigrammus newboldi, Hemigrammus ocellifer, Hemigrammus orthus, Hemigrammus parana, Hemigrammus pretoensis, Hemigrammus pulcher, Hemigrammus rhodostomus, Hemigrammus rodwayi, Hemigrammus schmardae, Hemigrammus silimoni, Hemigrammus stictus, Hemigrammus tridens, Hemigrammus ulreyi, Hemigrammus unilineatus, Hemigrammus vorderwikleri, Hemigrammus yinyang, Hemigrammus sp n., Hyphessobrycon bentosi, Hyphessobrycon compressus, Hyphessobrycon diancistrus, Hyphessobrycon eques, Hyphessobrycon erythrostigma, Hyphessobrycon megalopterus, Hyphessobrycon micropterus, Hyphessobrycon takasei, Jupiaba polylepis, Lignobrycon myersi, Microschemobrycon sp, Moenkhausia bonita, Moenkhausia hemigrammoides, Moenkhausia intermedia, Moenkhausia sanctaefilomenae, Moenkhausia xinguensis, Paracheirodon axelrodi, Petitella georgiae, Piabina argentea, Poptella paraguayensis, Psellogrammus kennedyi, Pristella maxillaris, Rhinopetitia myersi, Serrapinnus heterodon, Serrapinnus notomelas, Stethaprion erythrops, Tetragonopterus argenteus, Thayeria obliqua 


\section{2- na vertical que passa anteriormente ao etmóide lateral}

Iguanodectes spilurus, Triportheus nematurus

\section{9- Indeterminado}

Bryconamericus exodon, Hollandichthys aff. multifasciatus

\section{9- Desenvolvimento do osso corono-meckeliano (ic - 0,056 )}

(Serra, 2003: 43)

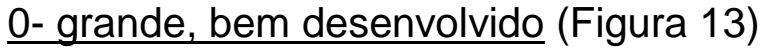

Aphyocharax anisitsi, Astyanax altiparanae, Astyanax mexicanus, Brycinus longipinnis, Brycon cf pesu, Bryconamericus exodon, Bryconella pallidifrons, Bryconexodon juruenae, Bryconops melanurus, Chalceus spilogyros, Cheirodon interruptus, Coptobrycon bilineatus, Deuterodon iguape, Grundulus cochae, Hasemania crenuchoides, Hasemania melanura, Hasemania sp n., Hemigrammus analis, Hemigrammus barrigonae, Hemigrammus coeruleus, Hemigrammus levis, Hemigrammus lunatus, Hemigrammus newboldi, Hemigrammus ocellifer, Hemigrammus pretoensis, Hemigrammus rhodostomus, Hemigrammus taphorni, Hollandichthys aff. multifasciatus, Hyphessobrycon compressus, Hyphessobrycon micropterus, Hyphessobrycon takasei, Iguanodectes spilurus, Lignobrycon myersi, Moenkhausia sanctaefilomenae, Moenkhausia xinguensis, Oligosarcus pintoi, Paracheirodon axelrodi, Parapristella georgiae, Phenacogaster franciscoensis, Piabina argentea, Psellogrammus kennedyi, Rhinopetitia myersi, Salminus hilarii, Serrapinnus notomelas, Serrasalmus maculatus, Stethaprion erythrops, Tetragonopterus argenteus

\section{1- muito pequeno, reduzido (Figura 14)}

Aphyocharax pusillus, Gymnocorymbus ternetzi, Hemigrammus arua, Hemigrammus bellottii, Hemigrammus bleheri, Hemigrammus boesemani, Hemigrammus brevis, Hemigrammus cylindricus, Hemigrammus gracilis, Hemigrammus haraldi, Hemigrammus hyanuary, Hemigrammus marginatus, Hemigrammus mimus, Hemigrammus orthus, Hemigrammus parana, Hemigrammus pulcher, Hemigrammus rodwayi, Hemigrammus schmardae, Hemigrammus silimoni, Hemigrammus skolioplatus, Hemigrammus stictus, Hemigrammus tridens, Hemigrammus ulreyi, Hemigrammus unilineatus, Hemigrammus vorderwikleri, Hemigrammus yinyang, Hemigrammus sp n., Hyphessobrycon bentosi, Hyphessobrycon coelestinus, Hyphessobrycon diancistrus, Hyphessobrycon eilyos, Hyphessobrycon epicharis, Hyphessobrycon eques, Hyphessobrycon erythrostigma, Hyphessobrycon megalopterus, Jupiaba polylepis, Microschemobrycon sp, Moenkhausia bonita, Moenkhausia hemigrammoides, Moenkhausia intermedia, Petitella georgiae, Poptella paraguayensis, Pristella maxillaris, Roeboides bonariensis, Serrapinnus heterodon, Thayeria obliqua, Triportheus nematurus

\section{9- Indeterminado}

Astyanax jacobinae

60- Osso corono-meckeliano em vista medial (ic - 0,063)

(Serra, 2003: 44) 


\section{0- exposto, com $50 \%$ ou menos de sua superfície encoberta pela}

\section{cartilagem de Meckel (Figura 13)}

Astyanax altiparanae, Astyanax mexicanus, Brycinus longipinnis, Brycon cf pesu, Bryconamericus exodon, Bryconexodon juruenae, Bryconops melanurus, Chalceus spilogyros, Cheirodon interruptus, Coptobrycon bilineatus, Deuterodon iguape, Grundulus cochae, Hasemania crenuchoides, Hasemania sp n., Hemigrammus analis, Hemigrammus barrigonae, Hemigrammus bleheri, Hemigrammus coeruleus, Hemigrammus cylindricus, Hemigrammus hyanuary, Hemigrammus lunatus, Hemigrammus newboldi, Hemigrammus ocellifer, Hemigrammus pretoensis, Hemigrammus pulcher, Hemigrammus skolioplatus, Hemigrammus taphorni, Hemigrammus ulreyi, Hollandichthys aff. multifasciatus, Iguanodectes spilurus, Moenkhausia intermedia, Moenkhausia xinguensis, Oligosarcus pintoi, Paracheirodon axelrodi, Parapristella georgiae, Piabina argentea, Psellogrammus kennedyi, Rhinopetitia myersi, Salminus hilarii, Serrasalmus maculatus, Tetragonopterus argenteus, Thayeria obliqua, Triportheus nematurus

\section{1- completamente encoberto pela cartilagem de Meckel ou com apenas} uma pequena porção exposta, não atingindo 50\% (Figura 14)

Aphyocharax anisitsi, Aphyocharax pusillus, Bryconella pallidifrons, Gymnocorymbus ternetzi, Hemigrammus arua, Hemigrammus bellottii, Hemigrammus boesemani, Hemigrammus brevis, Hemigrammus gracilis, Hemigrammus haraldi, Hemigrammus levis, Hemigrammus marginatus, Hemigrammus mimus, Hemigrammus orthus, Hemigrammus parana, Hemigrammus rhodostomus, Hemigrammus rodwayi, Hemigrammus schmardae, Hemigrammus silimoni, Hemigrammus stictus, Hemigrammus tridens, Hemigrammus unilineatus, Hemigrammus vorderwikleri, Hemigrammus yinyang, Hemigrammus sp n., Hyphessobrycon bentosi, Hyphessobrycon coelestinus, Hyphessobrycon compressus, Hyphessobrycon diancistrus, Hyphessobrycon eilyos, Hyphessobrycon epicharis, Hyphessobrycon eques, Hyphessobrycon erythrostigma, Hyphessobrycon megalopterus, Hyphessobrycon micropterus, Hyphessobrycon takasei, Jupiaba polylepis, Lignobrycon myersi, Microschemobrycon sp, Moenkhausia bonita, Moenkhausia hemigrammoides, Moenkhausia sanctaefilomenae, Petitella georgiae, Phenacogaster franciscoensis, Poptella paraguayensis, Pristella maxillaris, Roeboides bonariensis, Serrapinnus heterodon, Serrapinnus notomelas, Stethaprion erythrops

\section{9- Indeterminado}

Astyanax jacobinae, Hasemania melanura

\section{Antorbital, Nasal, Supraorbital e Infra-orbitais}

61- Antorbital (ic - 0,333)

(Malabarba, 1998: 4; Benine, 2004: 1; Moreira, 207: 122 e 125; Mirande, 2009: 55 e 72).

\section{$\underline{0 \text { - presente, com canal sensorial ou com resquícios dele }}$}

Bryconops melanurus, Iguanodectes spilurus, Salminus hilarii

\section{1 - presente, sem canal sensorial (Figura 15)}

Aphyocharax anisitsi, Aphyocharax pusillus, Astyanax altiparanae, Astyanax jacobinae, Astyanax mexicanus, Brycinus longipinnis, Brycon cf pesu, Bryconamericus exodon, Bryconella pallidifrons, Bryconexodon juruenae, Chalceus spilogyros, Cheirodon interruptus, Coptobrycon bilineatus, Deuterodon iguape, Grundulus cochae, Gymnocorymbus ternetzi, Hasemania 
crenuchoides, Hasemania melanura, Hasemania sp n., Hemigrammus analis, Hemigrammus arua, Hemigrammus barrigonae, Hemigrammus bellottii, Hemigrammus bleheri, Hemigrammus boesemani, Hemigrammus brevis, Hemigrammus coeruleus, Hemigrammus cylindricus, Hemigrammus gracilis, Hemigrammus haraldi, Hemigrammus hyanuary, Hemigrammus levis, Hemigrammus lunatus, Hemigrammus marginatus, Hemigrammus mimus, Hemigrammus newboldi, Hemigrammus ocellifer, Hemigrammus orthus, Hemigrammus parana, Hemigrammus pretoensis, Hemigrammus pulcher, Hemigrammus rhodostomus, Hemigrammus rodwayi, Hemigrammus schmardae, Hemigrammus silimoni, Hemigrammus skolioplatus, Hemigrammus stictus, Hemigrammus taphorni, Hemigrammus tridens, Hemigrammus ulreyi, Hemigrammus unilineatus, Hemigrammus vorderwikleri, Hemigrammus yinyang, Hemigrammus sp n., Hollandichthys aff. multifasciatus, Hyphessobrycon bentosi, Hyphessobrycon coelestinus, Hyphessobrycon compressus, Hyphessobrycon diancistrus, Hyphessobrycon eilyos, Hyphessobrycon epicharis, Hyphessobrycon eques, Hyphessobrycon erythrostigma, Hyphessobrycon megalopterus, Hyphessobrycon micropterus, Hyphessobrycon takasei, Jupiaba polylepis, Lignobrycon myersi, Microschemobrycon sp, Moenkhausia bonita, Moenkhausia hemigrammoides, Moenkhausia intermedia, Moenkhausia sanctaefilomenae, Moenkhausia xinguensis, Oligosarcus pintoi, Paracheirodon axelrodi, Parapristella georgiae, Petitella georgiae, Phenacogaster franciscoensis, Piabina argentea, Poptella paraguayensis, Psellogrammus kennedyi, Pristella maxillaris, Rhinopetitia myersi, Roeboides bonariensis, Serrapinnus heterodon, Serrapinnus notomelas, Serrasalmus maculatus, Stethaprion erythrops, Tetragonopterus argenteus, Thayeria obliqua, Triportheus nematurus

\section{2- Posição do Antorbital (ic - 0,167)}

(Ferreira, 2007: 1; Bertaco, 2008: 39; Marinho, 2009: 19).

\section{0 - em contato com o infra-orbital 1}

Aphyocharax anisitsi, Brycinus longipinnis, Bryconops melanurus, Iguanodectes spilurus, Salminus hilarii, Serrapinnus notomelas

\section{1- separado do infra-orbital 1}

Aphyocharax pusillus, Astyanax altiparanae, Astyanax jacobinae, Astyanax mexicanus, Brycon cf pesu, Bryconamericus exodon, Bryconella pallidifrons, Chalceus spilogyros, Cheirodon interruptus, Coptobrycon bilineatus, Deuterodon iguape, Grundulus cochae, Gymnocorymbus ternetzi, Hasemania crenuchoides, Hasemania melanura, Hasemania sp n., Hemigrammus analis, Hemigrammus arua, Hemigrammus barrigonae, Hemigrammus bellottii, Hemigrammus bleheri, Hemigrammus boesemani, Hemigrammus brevis, Hemigrammus coeruleus, Hemigrammus cylindricus, Hemigrammus gracilis, Hemigrammus haraldi, Hemigrammus levis, Hemigrammus lunatus, Hemigrammus marginatus, Hemigrammus mimus, Hemigrammus newboldi, Hemigrammus ocellifer, Hemigrammus orthus, Hemigrammus parana, Hemigrammus pretoensis, Hemigrammus pulcher, Hemigrammus rhodostomus, Hemigrammus rodwayi, Hemigrammus schmardae, Hemigrammus silimoni, Hemigrammus skolioplatus, Hemigrammus stictus, Hemigrammus taphorni, Hemigrammus tridens, Hemigrammus ulreyi, Hemigrammus unilineatus, Hemigrammus vorderwikleri, Hemigrammus yinyang, Hemigrammus sp n., Hollandichthys aff. multifasciatus, Hyphessobrycon bentosi, Hyphessobrycon coelestinus, Hyphessobrycon compressus, Hyphessobrycon diancistrus, Hyphessobrycon eilyos, Hyphessobrycon epicharis, Hyphessobrycon eques, Hyphessobrycon erythrostigma, Hyphessobrycon megalopterus, Hyphessobrycon micropterus, Hyphessobrycon takasei, Jupiaba polylepis, Lignobrycon myersi, Microschemobrycon sp, Moenkhausia hemigrammoides, Moenkhausia intermedia, Moenkhausia sanctaefilomenae, Moenkhausia xinguensis, Oligosarcus pintoi, Paracheirodon axelrodi, Parapristella georgiae, Petitella georgiae, Phenacogaster franciscoensis, Piabina argentea, Poptella paraguayensis, Psellogrammus kennedyi, Pristella maxillaris, Rhinopetitia myersi, Roeboides bonariensis, Serrapinnus heterodon, Serrasalmus maculatus, Stethaprion erythrops, Tetragonopterus argenteus, Thayeria obliqua, Triportheus nematurus 
63- Forma do Antorbital (ic - 0,136)

(Ferreira, 2007: 3; Bertaco, 2008: 40; Marinho, 2009: 17)

0- alongado, com a largura não mudando muito ao longo do comprimento (osso pode ser estreito ou largo) (Figura $15 \mathrm{C}$ )

Aphyocharax pusillus, Bryconexodon juruenae, Bryconops melanurus, Chalceus spilogyros, Hemigrammus mimus, Hemigrammus skolioplatus, Hollandichthys aff. multifasciatus, Hyphessobrycon epicharis, Hyphessobrycon erythrostigma. Hyphessobrycon megalopterus, Hyphessobrycon takasei, Microschemobrycon sp, Phenacogaster franciscoensis, Roeboides bonariensis, Serrasalmus maculatus, Stethaprion erythrops

\section{1- porção dorsal afilada e ventral mais larga (Figura $15 \mathrm{~A}$ )}

Aphyocharax anisitsi, Astyanax altiparanae, Astyanax jacobinae, Astyanax mexicanus, Bryconamericus exodon, Cheirodon interruptus, Deuterodon iguape, Gymnocorymbus ternetzi, Hasemania crenuchoides, Hasemania melanura, Hasemania sp n., Hemigrammus analis, Hemigrammus arua, Hemigrammus barrigonae, Hemigrammus bellottii, Hemigrammus bleheri, Hemigrammus boesemani, Hemigrammus brevis, Hemigrammus coeruleus, Hemigrammus cylindricus, Hemigrammus gracilis, Hemigrammus haraldi, Hemigrammus levis, Hemigrammus lunatus, Hemigrammus marginatus, Hemigrammus newboldi, Hemigrammus ocellifer, Hemigrammus orthus, Hemigrammus parana, Hemigrammus pretoensis, Hemigrammus pulcher, Hemigrammus rhodostomus, Hemigrammus rodwayi, Hemigrammus schmardae, Hemigrammus silimoni, Hemigrammus stictus, Hemigrammus taphorni, Hemigrammus ulreyi, Hemigrammus unilineatus, Hemigrammus vorderwikleri, Hemigrammus yinyang, Hemigrammus $\mathrm{sp}$ n., Hyphessobrycon bentosi, Hyphessobrycon coelestinus, Hyphessobrycon compressus, Hyphessobrycon diancistrus, Hyphessobrycon eilyos, Hyphessobrycon eques, Jupiaba polylepis, Moenkhausia bonita, Moenkhausia hemigrammoides, Moenkhausia intermedia, Moenkhausia sanctaefilomenae, Moenkhausia xinguensis, Oligosarcus pintoi, Paracheirodon axelrodi, Parapristella georgiae, Petitella georgiae, Piabina argentea, Poptella paraguayensis, Pristella maxillaris, Rhinopetitia myersi, Serrapinnus heterodon, Serrapinnus notomelas, Tetragonopterus argenteus

\section{2- osso bastante largo com a porção ventral pouco mais larga que 0} restante do osso (Figura $15 \mathrm{~B}$ )

Brycinus longipinnis, Brycon cf pesu, Bryconella pallidifrons, Hemigrammus hyanuary, Hemigrammus tridens, Hyphessobrycon micropterus, Iguanodectes spilurus, Salminus hilarii, Thayeria obliqua, Triportheus nematurus

3- osso largo com a porção ventral afilada, ou com as porções dorsal e ventral afiladas (Figura $15 \mathrm{D}-\mathrm{E}$ )

Coptobrycon bilineatus, Lignobrycon myersi

\section{9- Indeterminado}

Grundulus cochae, Psellogrammus kennedyi

64- Nasal (ic $-0,400)$ 
(Mirande, 2009: 33)

$\underline{0 \text { - presente e alongado, ocupando a maior parte da distância entre a }}$ margem anterior do frontal e as asas laterais do mesetmóide

Aphyocharax anisitsi, Aphyocharax pusillus, Astyanax altiparanae, Astyanax jacobinae, Astyanax mexicanus, Brycinus longipinnis, Brycon cf pesu, Bryconamericus exodon, Bryconella pallidifrons, Bryconexodon juruenae, Bryconops melanurus, Chalceus spilogyros, Cheirodon interruptus, Deuterodon iguape, Gymnocorymbus ternetzi, Hasemania crenuchoides, Hasemania melanura, Hasemania sp n., Hemigrammus analis, Hemigrammus arua, Hemigrammus barrigonae, Hemigrammus bellottii, Hemigrammus bleheri, Hemigrammus boesemani, Hemigrammus brevis, Hemigrammus coeruleus, Hemigrammus cylindricus, Hemigrammus gracilis, Hemigrammus haraldi, Hemigrammus hyanuary, Hemigrammus levis, Hemigrammus lunatus, Hemigrammus marginatus, Hemigrammus mimus, Hemigrammus newboldi, Hemigrammus ocellifer, Hemigrammus orthus, Hemigrammus parana, Hemigrammus pretoensis, Hemigrammus pulcher, Hemigrammus rhodostomus, Hemigrammus rodwayi, Hemigrammus schmardae, Hemigrammus silimoni, Hemigrammus skolioplatus, Hemigrammus stictus, Hemigrammus taphorni, Hemigrammus tridens, Hemigrammus ulreyi, Hemigrammus unilineatus, Hemigrammus vorderwikleri, Hemigrammus yinyang, Hemigrammus sp n., Hollandichthys aff. multifasciatus, Hyphessobrycon bentosi, Hyphessobrycon coelestinus, Hyphessobrycon compressus, Hyphessobrycon diancistrus, Hyphessobrycon epicharis, Hyphessobrycon eques, Hyphessobrycon erythrostigma, Hyphessobrycon takasei, Iguanodectes spilurus, Jupiaba polylepis, Lignobrycon myersi, Microschemobrycon sp, Moenkhausia bonita, Moenkhausia hemigrammoides, Moenkhausia intermedia, Moenkhausia sanctaefilomenae, Moenkhausia xinguensis, Oligosarcus pintoi, Paracheirodon axelrodi, Parapristella georgiae, Petitella georgiae, Phenacogaster franciscoensis, Piabina argentea, Poptella paraguayensis, Psellogrammus kennedyi, Pristella maxillaris, Rhinopetitia myersi, Roeboides bonariensis, Salminus hilarii, Serrapinnus heterodon, Serrapinnus notomelas, Serrasalmus maculatus, Stethaprion erythrops, Tetragonopterus argenteus, Thayeria obliqua, Triportheus nematurus

\section{$\underline{1 \text { - ausente }}$}

Coptobrycon bilineatus, Hyphessobrycon megalopterus

2- presente e muito reduzido, restrito à porção mediana da distância entre a margem anterior do frontal e as asas laterais do mesetmóide

Grundulus cochae, Hyphessobrycon eilyos, Hyphessobrycon micropterus

$\mathrm{Na}$ imensa maioria dos Characiformes o osso nasal está presente e é bem desenvolvido e alongado, ocupando a maior parte do espaço entre as asas laterais do mesetmóide e a margem anterior do frontal. Em G. cochae, $H$. eilyos e $H$. micropterus, entretanto o osso nasal é bastante reduzido, sendo muito curto e estreito, e ocupando apenas a região mediana do espaço entre as asas laterais do mesetmóide e a margem anterior do frontal. Já em $C$. bilineatus e $H$. megalopterus o osso nasal está completamente ausente.

65- Nasal (ic - 0,063) 
(Ferreira, 2007: 17; Vari, 1995, caráter 17; Oyakawa, 1998, caráter 3; Moreira, 2007: 175; Bertaco, 2008: 28; Marinho, 2009: 14).

\section{$\underline{0 \text { - tubular, com expansões ósseas laminares laterais }}$}

Brycinus longipinnis, Brycon cf pesu, Bryconamericus exodon, Bryconexodon juruenae, Bryconops melanurus, Chalceus spilogyros, Hasemania crenuchoides, Hasemania melanura, Hemigrammus analis, Hemigrammus levis, Hemigrammus mimus, Hemigrammus ocellifer, Hemigrammus taphorni, Hollandichthys aff. multifasciatus, Hyphessobrycon eques, Iguanodectes spilurus, Moenkhausia sanctaefilomenae, Salminus hilarii, Serrasalmus maculatus

\section{1- tubular, sem expansões ósseas}

Aphyocharax anisitsi, Aphyocharax pusillus, Astyanax altiparanae, Astyanax jacobinae, Astyanax mexicanus, Bryconella pallidifrons, Cheirodon interruptus, Deuterodon iguape, Gymnocorymbus ternetzi, , Hasemania sp n., Hemigrammus arua, Hemigrammus barrigonae, Hemigrammus bellottii, Hemigrammus bleheri, Hemigrammus boesemani, Hemigrammus brevis, Hemigrammus coeruleus, Hemigrammus cylindricus, Hemigrammus gracilis, Hemigrammus hyanuary, Hemigrammus lunatus, Hemigrammus marginatus, Hemigrammus newboldi, Hemigrammus orthus, Hemigrammus parana, Hemigrammus pulcher, Hemigrammus rhodostomus, Hemigrammus rodwayi, Hemigrammus schmardae, Hemigrammus silimoni, Hemigrammus skolioplatus, Hemigrammus stictus, Hemigrammus tridens, Hemigrammus ulreyi, Hemigrammus unilineatus, Hemigrammus vorderwikleri, Hemigrammus yinyang, Hemigrammus sp n., Hyphessobrycon bentosi, Hyphessobrycon coelestinus, Hyphessobrycon compressus, Hyphessobrycon diancistrus, Hyphessobrycon epicharis, Hyphessobrycon erythrostigma, Hyphessobrycon takasei, Jupiaba polylepis, Lignobrycon myersi, Microschemobrycon sp, Moenkhausia bonita, Moenkhausia intermedia, Moenkhausia xinguensis, Oligosarcus pintoi, Paracheirodon axelrodi, Parapristella georgiae, Petitella georgiae, Phenacogaster franciscoensis, Piabina argentea, Poptella paraguayensis, Psellogrammus kennedyi, Pristella maxillaris, Rhinopetitia myersi, Roeboides bonariensis, Serrapinnus heterodon, Serrapinnus notomelas, Stethaprion erythrops, Tetragonopterus argenteus, Thayeria obliqua, Triportheus nematurus

\section{$\underline{0,1-\text { Polimórfico }}$}

Hemigrammus haraldi, Hemigrammus pretoensis, Moenkhausia hemigrammoides

\section{9- Indeterminado}

Coptobrycon bilineatus, Grundulus cochae, Hyphessobrycon eilyos, Hyphessobrycon megalopterus, Hyphessobrycon micropterus

\section{6- Supra-orbital (ic - 0,333)}

(Vari, 1995: 9; Fink \& Fink, 1996: 19; Serra, 2003: 21; Benine, 2004: 5; Moreira, 2007: 168; Ferreira, 2007: 5; Bertaco, 2008: 34; Marinho, 2009: 12; Mirande, 2099: 70).

\section{$\underline{0 \text { - presente }}$}

Brycinus longipinnis, Brycon cf pesu, Bryconops melanurus, Chalceus spilogyros, Lignobrycon myersi, Salminus hilarii, Serrasalmus maculatus, Triportheus nematurus

$$
\underline{1-\text { ausente (Figuras } 16-18)}
$$


Aphyocharax anisitsi, Aphyocharax pusillus, Astyanax altiparanae, Astyanax jacobinae, Astyanax mexicanus, Bryconamericus exodon, Bryconella pallidifrons, Bryconexodon juruenae, Cheirodon interruptus, Coptobrycon bilineatus, Deuterodon iguape, Grundulus cochae, Gymnocorymbus ternetzi, Hasemania crenuchoides, Hasemania melanura, Hasemania sp n., Hemigrammus analis, Hemigrammus arua, Hemigrammus barrigonae, Hemigrammus bellottii, Hemigrammus bleheri, Hemigrammus boesemani, Hemigrammus brevis, Hemigrammus coeruleus, Hemigrammus cylindricus, Hemigrammus gracilis, Hemigrammus haraldi, Hemigrammus hyanuary, Hemigrammus levis, Hemigrammus lunatus, Hemigrammus marginatus, Hemigrammus mimus, Hemigrammus newboldi, Hemigrammus ocellifer, Hemigrammus orthus, Hemigrammus parana, Hemigrammus pretoensis, Hemigrammus pulcher, Hemigrammus rhodostomus, Hemigrammus rodwayi, Hemigrammus schmardae, Hemigrammus silimoni, Hemigrammus skolioplatus, Hemigrammus stictus, Hemigrammus taphorni, Hemigrammus tridens, Hemigrammus ulreyi, Hemigrammus unilineatus, Hemigrammus vorderwikleri, Hemigrammus yinyang, Hemigrammus sp n., Hollandichthys aff. multifasciatus, Hyphessobrycon bentosi, Hyphessobrycon coelestinus, Hyphessobrycon compressus, Hyphessobrycon diancistrus, Hyphessobrycon eilyos, Hyphessobrycon epicharis, Hyphessobrycon eques, Hyphessobrycon erythrostigma, Hyphessobrycon megalopterus, Hyphessobrycon micropterus, Hyphessobrycon takasei, Iguanodectes spilurus, Jupiaba polylepis, Microschemobrycon sp, Moenkhausia bonita, Moenkhausia hemigrammoides, Moenkhausia intermedia, Moenkhausia sanctaefilomenae, Moenkhausia xinguensis, Oligosarcus pintoi, Paracheirodon axelrodi, Parapristella georgiae, Petitella georgiae, Phenacogaster franciscoensis, Piabina argentea, Poptella paraguayensis, Psellogrammus kennedyi, Pristella maxillaris, Rhinopetitia myersi, Roeboides bonariensis, Serrapinnus heterodon, Serrapinnus notomelas, Stethaprion erythrops, Tetragonopterus argenteus, Thayeria obliqua

Roberts (1969) coloca a presença de um anel orbital completo, que inclue o supra-orbital, como uma condição primitiva em Characiformes; Zanata \& Vari (2005), também colocam que supra-orbital localizado dorsalmente na margem da órbita, está presente em grupos basais de Characiformes. Weitzman \& Malabarba (1998) e Malabarba \& Weitzmann (2003) usam a ausência de supra-orbitail para delimitar um agrupamento monofilético formado por Aphyocharacinae, Characinae, Cheirodontinae, Glandulocaudinae, Paragoniatinae, Rhoadsiinae, Stethaprioninae e Tetragonopterinae (sensu Géry, 1977). Malabarba \& Weitzman (2003) colocam ainda que a ausência de supra-orbital em Erythrinidae e Lebiasinidae é homoplástica com a condição encontrada em Characidae. Supra-orbital está ausente na maioria das espécies examinadas na presente análise, sendo sua ausência uma condição sinapomórfica para as espécies que compõe o clado 184, dentre elas, representantes de Aphyocharacinae, Characinae, Cheirodontinae, Stethaprioninae e Tetragonopterinae (sensu Géry, 1977) (com reversão para supra-orbital presente em $S$. maculatus).

67- Infra-orbital 1 (ic - 0,100)

(Ferreira, 2007: 7; Marinho, 2009: 21). 


\title{
0- com comprimento menor que o do infra-orbital 2 (Figuras 17 - 18)
}

Aphyocharax anisitsi, Aphyocharax pusillus, Astyanax altiparanae, Astyanax jacobinae, Astyanax mexicanus, Brycon cf pesu, Bryconops melanurus, Chalceus spilogyros, Coptobrycon bilineatus, Deuterodon iguape, Grundulus cochae, Gymnocorymbus ternetzi, Hasemania crenuchoides, Hasemania melanura, Hasemania sp n., Hemigrammus analis, Hemigrammus arua, Hemigrammus barrigonae, Hemigrammus bellottii, Hemigrammus bleheri, Hemigrammus boesemani, Hemigrammus brevis, Hemigrammus cylindricus, Hemigrammus gracilis, Hemigrammus hyanuary, Hemigrammus lunatus, Hemigrammus mimus, Hemigrammus orthus, Hemigrammus parana, Hemigrammus pretoensis, Hemigrammus rhodostomus, Hemigrammus rodwayi, Hemigrammus schmardae, Hemigrammus skolioplatus, Hemigrammus sp n., Hemigrammus stictus, Hemigrammus taphorni, Hemigrammus tridens, Hemigrammus ulreyi, Hemigrammus vorderwikleri, Hollandichthys aff. multifasciatus, Hyphessobrycon bentosi, Hyphessobrycon coelestinus, Hyphessobrycon compressus, Hyphessobrycon diancistrus, Hyphessobrycon eilyos, Hyphessobrycon epicharis, Hyphessobrycon eques, Hyphessobrycon erythrostigma, Hyphessobrycon megalopterus, Hyphessobrycon micropterus, Hyphessobrycon takasei, Iguanodectes spilurus, Lignobrycon myersi, Microschemobrycon sp, Moenkhausia hemigrammoides, Moenkhausia intermedia, Moenkhausia xinguensis, Oligosarcus pintoi, Paracheirodon axelrodi, Parapristella georgiae, Petitella georgiae, Phenacogaster franciscoensis, Piabina argentea, Psellogrammus kennedyi, Pristella maxillaris, Roeboides bonariensis, Salminus hilarii, Serrapinnus heterodon, Serrapinnus notomelas, Serrasalmus maculatus, Stethaprion erythrops, Tetragonopterus argenteus

\section{1- com comprimento igual ao do infra-orbital 2 (Figura 16)}

Brycinus longipinnis, Bryconamericus exodon, Bryconella pallidifrons, Hemigrammus coeruleus, Hemigrammus haraldi, Hemigrammus levis, Hemigrammus marginatus, Hemigrammus newboldi, Hemigrammus ocellifer, Hemigrammus pulcher, Hemigrammus unilineatus, Hemigrammus yinyang, Jupiaba polylepis, Moenkhausia bonita, Moenkhausia sanctaefilomenae, Poptella paraguayensis, Rhinopetitia myersi, Thayeria obliqua

\section{2- com comprimento maior que o do infra-orbital 2}

Bryconexodon juruenae, Cheirodon interruptus, Hemigrammus silimoni, Triportheus nematurus

\section{8- Margem posterior do infra-orbital 1 (ic - 0,095)}

\author{
(Ferreira, 2007: 9; Moreira, 2007: 139; Marinho, 2009: 22). \\ 0- aproximadamente do mesmo tamanho que a margem anterior do
}

\section{infra-orbital 2}

Astyanax altiparanae, Astyanax jacobinae, Astyanax mexicanus, Brycon cf pesu, Bryconops melanurus, Chalceus spilogyros, Cheirodon interruptus, Deuterodon iguape, Grundulus cochae, Hasemania melanura, Hemigrammus analis, Hemigrammus arua, Hemigrammus barrigonae, Hemigrammus bellottii, Hemigrammus bleheri, Hemigrammus brevis, Hemigrammus cylindricus, Hemigrammus gracilis, Hemigrammus haraldi, Hemigrammus hyanuary, Hemigrammus levis, Hemigrammus lunatus, Hemigrammus marginatus, Hemigrammus newboldi, Hemigrammus ocellifer, Hemigrammus orthus, Hemigrammus pretoensis, Hemigrammus pulcher, Hemigrammus rhodostomus, Hemigrammus rodwayi, Hemigrammus schmardae, Hemigrammus silimoni, Hemigrammus skolioplatus, Hemigrammus stictus, Hemigrammus tridens, Hemigrammus ulreyi, Hemigrammus unilineatus, Hemigrammus vorderwikleri, Hemigrammus sp n., Hollandichthys aff. multifasciatus, Hyphessobrycon coelestinus, Hyphessobrycon compressus, Hyphessobrycon diancistrus, Hyphessobrycon eilyos, Hyphessobrycon epicharis, Hyphessobrycon eques, Hyphessobrycon erythrostigma, Hyphessobrycon megalopterus, Hyphessobrycon micropterus, Hyphessobrycon takasei, Jupiaba polylepis, Lignobrycon myersi, Microschemobrycon sp, Moenkhausia bonita, Moenkhausia hemigrammoides, Moenkhausia intermedia, Moenkhausia sanctaefilomenae, 
Oligosarcus pintoi, Paracheirodon axelrodi, Parapristella georgiae, Petitella georgiae, Phenacogaster franciscoensis, Piabina argentea, Psellogrammus kennedyi, Pristella maxillaris, Rhinopetitia myersi, Roeboides bonariensis, Salminus hilarii, Serrapinnus notomelas, Stethaprion erythrops, Thayeria obliqua, Triportheus nematurus

\section{1- menor que a margem anterior do infra-orbital 2}

Brycinus longipinnis, Bryconella pallidifrons, Coptobrycon bilineatus, Hasemania crenuchoides, Hasemania sp n., Hemigrammus boesemani, Hemigrammus yinyang, Hyphessobrycon bentosi, Iguanodectes spilurus, Moenkhausia xinguensis, Serrasalmus maculatus, Tetragonopterus argenteus

\section{2- maior que a margem anterior do infra-orbital 2}

Aphyocharax anisitsi, Aphyocharax pusillus, Bryconamericus exodon, Bryconexodon juruenae, Gymnocorymbus ternetzi, Hemigrammus coeruleus, Hemigrammus mimus, Hemigrammus parana, Hemigrammus taphorni, Poptella paraguayensis, Serrapinnus heterodon

\section{9- Canal sensorial do infra-orbital 1 (ic $-0,182$ )}

(Serra, 2003: 23; Moreira, 2007: 138)

\section{0 - presente ao longo de todo o osso ou em mais de $50 \%$ de sua}

\section{extensão (Figuras 16 e 18)}

Aphyocharax anisitsi, Aphyocharax pusillus, Astyanax altiparanae, Astyanax jacobinae, Astyanax mexicanus, Brycinus longipinnis, Brycon cf pesu, Bryconamericus exodon, Bryconexodon juruenae, Bryconops melanurus, Chalceus spilogyros, Deuterodon iguape Gymnocorymbus ternetzi, Hasemania crenuchoides, Hasemania melanura, Hasemania sp n., Hemigrammus analis, Hemigrammus barrigonae, Hemigrammus bleheri, Hemigrammus coeruleus, Hemigrammus cylindricus, Hemigrammus gracilis, Hemigrammus levis, Hemigrammus marginatus, Hemigrammus mimus, Hemigrammus newboldi, Hemigrammus ocellifer, Hemigrammus parana, Hemigrammus pretoensis, Hemigrammus rhodostomus, Hemigrammus rodwayi, Hemigrammus silimoni, Hemigrammus skolioplatus, Hemigrammus stictus, Hemigrammus taphorni, Hemigrammus ulreyi, Hemigrammus unilineatus, Hemigrammus sp n., Hollandichthys aff. multifasciatus, Hyphessobrycon bentosi, Hyphessobrycon epicharis, Hyphessobrycon eques, Hyphessobrycon erythrostigma, Hyphessobrycon takasei, Iguanodectes spilurus, Jupiaba polylepis, Lignobrycon myersi, Moenkhausia bonita, Moenkhausia hemigrammoides, Moenkhausia intermedia, Moenkhausia sanctaefilomenae, Moenkhausia xinguensis, Oligosarcus pintoi, Parapristella georgiae, Petitella georgiae, Phenacogaster franciscoensis, Piabina argentea, Poptella paraguayensis, Psellogrammus kennedyi, Rhinopetitia myersi, Roeboides bonariensis, Salminus hilarii, Serrapinnus heterodon, Serrasalmus maculatus, Stethaprion erythrops, Tetragonopterus argenteus, Thayeria obliqua, Triportheus nematurus

\section{1- ausente (Figura 17)}

Bryconella pallidifrons, Cheirodon interruptus, Coptobrycon bilineatus, Grundulus cochae, Hemigrammus bellottii, Hemigrammus orthus, Hemigrammus schmardae, Hemigrammus tridens, Hemigrammus vorderwikleri, Hyphessobrycon compressus, Hyphessobrycon megalopterus, Microschemobrycon sp, Paracheirodon axelrodi, Pristella maxillaris, Hemigrammus yinyang

\section{2- presente e curto, atingindo no máximo $50 \%$ da extensão do osso}

Hemigrammus arua, Hemigrammus boesemani, Hemigrammus brevis, Hemigrammus haraldi, Hemigrammus hyanuary, Hemigrammus lunatus, Hemigrammus pulcher, Hyphessobrycon 
coelestinus, Hyphessobrycon diancistrus, Hyphessobrycon eilyos, Hyphessobrycon micropterus, Serrapinnus notomelas

70- Infra-orbitais 1 e 2 (ic - 0,40)

(Moreira, 2007: 142)

\section{0- sem contato ou sobreposição}

Aphyocharax anisitsi, Aphyocharax pusillus, Bryconella pallidifrons, Grundulus cochae, Gymnocorymbus ternetzi, Hemigrammus analis, Hemigrammus arua, Hemigrammus barrigonae, Hemigrammus bellottii, Hemigrammus bleheri, Hemigrammus brevis, Hemigrammus coeruleus, Hemigrammus cylindricus, Hemigrammus haraldi, Hemigrammus hyanuary, Hemigrammus lunatus, Hemigrammus orthus, Hemigrammus parana, Hemigrammus silimoni, Hemigrammus taphorni, Hemigrammus tridens, Hemigrammus vorderwikleri, Hemigrammus sp n., Hyphessobrycon bentosi, Hyphessobrycon coelestinus, Hyphessobrycon compressus, Hyphessobrycon eilyos, Hyphessobrycon megalopterus, Hyphessobrycon takasei, Microschemobrycon sp, Moenkhausia hemigrammoides, Parapristella georgiae, Phenacogaster franciscoensis, Piabina argentea, Poptella paraguayensis, Pristella maxillaris, Roeboides bonariensis, Salminus hilarii

\section{1- em contato ou com sobreposição}

Astyanax altiparanae, Astyanax jacobinae, Astyanax mexicanus, Brycinus longipinnis, Brycon cf pesu, Bryconamericus exodon, Bryconexodon juruenae, Bryconops melanurus, Chalceus spilogyros, Cheirodon interruptus, Coptobrycon bilineatus, Deuterodon iguape, Hasemania crenuchoides, Hasemania melanura, Hasemania sp n., Hemigrammus gracilis, Hemigrammus levis, Hemigrammus newboldi, Hemigrammus marginatus, Hemigrammus mimus, Hemigrammus ocellifer, Hemigrammus pretoensis, Hemigrammus pulcher, Hemigrammus rhodostomus, Hemigrammus rodwayi, Hemigrammus skolioplatus, Hemigrammus stictus, Hemigrammus ulreyi, Hemigrammus unilineatus, Hollandichthys aff. multifasciatus, Hyphessobrycon epicharis, Hyphessobrycon eques, Hyphessobrycon erythrostigma, Hyphessobrycon micropterus, Iguanodectes spilurus, Jupiaba polylepis, Lignobrycon myersi, Moenkhausia intermedia, Moenkhausia sanctaefilomenae, Moenkhausia xinguensis, Oligosarcus pintoi, Petitella georgiae, Psellogrammus kennedyi, Rhinopetitia myersi, Serrapinnus heterodon, Serrapinnus notomelas, Serrasalmus maculatus, Stethaprion erythrops, Tetragonopterus argenteus, Thayeria obliqua, Triportheus nematurus

\section{0, 1- Polimórfico}

Hemigrammus boesemani, Hemigrammus schmardae, Hemigrammus yinyang, Hyphessobrycon diancistrus, Paracheirodon axelrodi, Moenkhausia bonita

\section{1- Forma do Infra-orbital 2 (ic - 0,083)}

(Ferreira, 2007: 10; Marinho, 2009: 26)

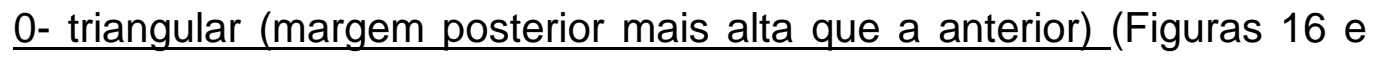
18)

Aphyocharax anisitsi, Aphyocharax pusillus, Astyanax altiparanae, Astyanax jacobinae, Astyanax mexicanus, Brycon cf pesu, Bryconamericus exodon, Bryconella pallidifrons, Bryconexodon juruenae, Cheirodon interruptus, Deuterodon iguape, Gymnocorymbus ternetzi, Hasemania crenuchoides, Hasemania melanura, Hasemania sp n., Hemigrammus analis, Hemigrammus arua, Hemigrammus barrigonae, Hemigrammus bellottii, Hemigrammus boesemani, Hemigrammus brevis, Hemigrammus coeruleus, Hemigrammus cylindricus, Hemigrammus gracilis, Hemigrammus haraldi, Hemigrammus hyanuary, Hemigrammus lunatus, Hemigrammus mimus, Hemigrammus ocellifer, Hemigrammus orthus, Hemigrammus parana, 
Hemigrammus pretoensis, Hemigrammus pulcher, Hemigrammus rhodostomus, Hemigrammus rodwayi, Hemigrammus schmardae, Hemigrammus silimoni, Hemigrammus skolioplatus, Hemigrammus stictus, Hemigrammus taphorni, Hemigrammus tridens, Hemigrammus ulreyi, Hemigrammus unilineatus, Hemigrammus vorderwikleri, Hemigrammus yinyang, Hyphessobrycon bentosi, Hyphessobrycon coelestinus, Hyphessobrycon compressus, Hyphessobrycon eilyos, Hyphessobrycon epicharis, Hyphessobrycon eques, Hyphessobrycon erythrostigma, Hyphessobrycon megalopterus, Hyphessobrycon micropterus, Hyphessobrycon takasei, Jupiaba polylepis, Microschemobrycon sp, Moenkhausia bonita, Moenkhausia hemigrammoides, Moenkhausia intermedia, Moenkhausia sanctaefilomenae, Moenkhausia xinguensis, Oligosarcus pintoi, Parapristella georgiae, Piabina argentea, Poptella paraguayensis, Psellogrammus kennedyi, Rhinopetitia myersi, Salminus hilarii, Serrapinnus heterodon, Serrapinnus notomelas, Stethaprion erythrops, Tetragonopterus argenteus, Thayeria obliqua, Triportheus nematurus

\section{1- retangular, quadrado ou mais arredondado (margens anterior $\mathrm{e}$}

\section{posterior aproximadamente da mesma altura) (Figura 17)}

Brycinus longipinnis, Bryconops melanurus, Chalceus spilogyros, Coptobrycon bilineatus, Grundulus cochae, Hemigrammus bleheri, Hemigrammus levis, Hemigrammus marginatus, Hemigrammus sp n., Hollandichthys aff. multifasciatus, Hyphessobrycon diancistrus, Iguanodectes spilurus, Lignobrycon myersi, Paracheirodon axelrodi, Petitella georgiae, Phenacogaster franciscoensis, Pristella maxillaris, Roeboides bonariensis

\section{9- Indeterminado}

Hemigrammus newboldi, Serrasalmus maculatus

\section{2- Infra-orbital 2 (ic - 0,059)}

\section{0- sem projecão antero-dorsal}

Aphyocharax anisitsi, Aphyocharax pusillus, Astyanax mexicanus, Brycinus longipinnis, Brycon cf pesu, Bryconamericus exodon, Bryconella pallidifrons, Bryconexodon juruenae, Bryconops melanurus, Chalceus spilogyros, Cheirodon interruptus, Coptobrycon bilineatus, Grundulus cochae, Hasemania melanura, Hemigrammus analis, Hemigrammus arua, Hemigrammus barrigonae, Hemigrammus bellottii, Hemigrammus bleheri, Hemigrammus boesemani, Hemigrammus brevis, Hemigrammus coeruleus, Hemigrammus cylindricus, Hemigrammus gracilis, Hemigrammus haraldi, Hemigrammus hyanuary, Hemigrammus levis, Hemigrammus lunatus, Hemigrammus marginatus, Hemigrammus mimus, Hemigrammus ocellifer, Hemigrammus orthus, Hemigrammus parana, Hemigrammus pretoensis, Hemigrammus rhodostomus, Hemigrammus rodwayi, Hemigrammus schmardae, Hemigrammus silimoni, Hemigrammus stictus, Hemigrammus taphorni, Hemigrammus tridens, Hemigrammus ulreyi, Hemigrammus unilineatus, Hemigrammus vorderwikleri, Hemigrammus yinyang, Hemigrammus $\mathrm{sp}$ n., Hollandichthys multifasciatus, Hyphessobrycon compressus, Hyphessobrycon diancistrus, Hyphessobrycon eilyos, Hyphessobrycon epicharis, Hyphessobrycon eques, Hyphessobrycon erythrostigma, Hyphessobrycon megalopterus, Hyphessobrycon micropterus, Hyphessobrycon takasei, Iguanodectes spilurus, Jupiaba polylepis, Microschemobrycon sp, Moenkhausia bonita, Moenkhausia sanctaefilomenae, Moenkhausia xinguensis, Oligosarcus pintoi, Paracheirodon axelrodi, Petitella georgiae, Phenacogaster franciscoensis, Piabina argentea, Pristella maxillaris, Rhinopetitia myersi, Roeboides bonariensis, Salminus hilarii, Serrapinnus notomelas, Serrasalmus maculatus, Stethaprion erythrops, Triportheus nematurus

\section{1- com uma projeção antero-dorsal sobre o infra-orbital 1}

Astyanax altiparanae, Deuterodon iguape, Gymnocorymbus ternetzi, Hasemania crenuchoides, Hasemania sp n., Hemigrammus newboldi, Hemigrammus pulcher, Hemigrammus skolioplatus, Hyphessobrycon bentosi, Hyphessobrycon coelestinus, Lignobrycon myersi, Moenkhausia 
hemigrammoides, Moenkhausia intermedia, Parapristella georgiae, Poptella paraguayensis, Psellogrammus kennedyi, Serrapinnus heterodon, Tetragonopterus argenteus, Thayeria obliqua

\section{9- Indeterminado}

Astyanax jacobinae

73- Infra-orbitais 2 e 3 (ic - 0,083)

\section{0- sem sobreposição}

Grundulus cochae, Gymnocorymbus ternetzi, Hasemania melanura, Hemigrammus arua, Hemigrammus brevis, Hemigrammus coeruleus, Hemigrammus haraldi, Hemigrammus newboldi, Hemigrammus pulcher, Hemigrammus silimoni, Hemigrammus taphorni, Hemigrammus sp n., Hemigrammus unilineatus, Hyphessobrycon compressus, Oligosarcus pintoi, Psellogrammus kennedyi, Roeboides bonariensis, Stethaprion erythrops

\section{1- com sobreposicão pequena}

Aphyocharax anisitsi, Astyanax altiparanae, Astyanax jacobinae, Astyanax mexicanus, Brycinus longipinnis, Brycon cf pesu, Bryconella pallidifrons, Chalceus spilogyros, Cheirodon interruptus, Deuterodon iguape, Hemigrammus analis, Hemigrammus barrigonae, Hemigrammus bellottii, Hemigrammus bleheri, Hemigrammus cylindricus, Hemigrammus gracilis, Hemigrammus hyanuary, Hemigrammus levis, Hemigrammus lunatus, Hemigrammus marginatus, Hemigrammus mimus, Hemigrammus ocellifer, Hemigrammus orthus, Hemigrammus parana, Hemigrammus pretoensis, Hemigrammus rodwayi, Hemigrammus schmardae, Hemigrammus skolioplatus, Hemigrammus stictus, Hemigrammus tridens, Hemigrammus ulreyiHemigrammus vorderwikleri, Hemigrammus yinyang, Hollandichthys aff. multifasciatus, Hyphessobrycon bentosi, Hyphessobrycon coelestinus, Hyphessobrycon eilyos, Hyphessobrycon epicharis, Hyphessobrycon eques, Hyphessobrycon erythrostigma, Hyphessobrycon megalopterus, Hyphessobrycon micropterus, Hyphessobrycon takasei, Iguanodectes spilurus, Jupiaba polylepis, Lignobrycon myersi, Microschemobrycon sp, Moenkhausia bonita, Moenkhausia intermedia, Moenkhausia sanctaefilomenae, Moenkhausia xinguensis, Paracheirodon axelrodi, Petitella georgiae, Phenacogaster franciscoensis, Poptella paraguayensis, Pristella maxillaris, Serrasalmus maculatus, Thayeria obliqua, Triportheus nematurus

\section{2- com sobreposição grande}

Aphyocharax pusillus, Bryconamericus exodon, Bryconexodon juruenae, Bryconops melanurus, Hasemania crenuchoides, Hasemania sp n., Hemigrammus rhodostomus, Piabina argentea, Rhinopetitia myersi, Salminus hilarii, Serrapinnus heterodon, Serrapinnus notomelas

\section{0,1- Polimórfico}

Coptobrycon bilineatus, Hemigrammus boesemani, Hyphessobrycon diancistrus, Moenkhausia hemigrammoides, Parapristella georgiae

\section{1, 2- Polimórfico}

Tetragonopterus argenteus

74- Infra-orbital 4 (ic - 0,143)

(Mirande, 2009: 66)

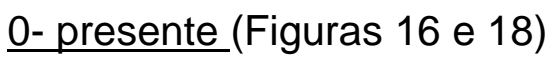


Astyanax altiparanae, Astyanax mexicanus, Brycinus longipinnis, Brycon cf pesu, Bryconamericus exodon, Bryconexodon juruenae, Bryconops melanurus, Chalceus spilogyros, Cheirodon interruptus, Deuterodon iguape, Gymnocorymbus ternetzi, Hasemania crenuchoides, Hasemania melanura, Hemigrammus analis, Hemigrammus arua, Hemigrammus barrigonae, Hemigrammus bellottii, Hemigrammus bleheri, Hemigrammus boesemani, Hemigrammus brevis, Hemigrammus coeruleus, Hemigrammus cylindricus, Hemigrammus gracilis, Hemigrammus haraldi, Hemigrammus hyanuary, Hemigrammus levis, Hemigrammus lunatus, Hemigrammus marginatus, Hemigrammus mimus, Hemigrammus newboldi, Hemigrammus ocellifer, Hemigrammus orthus, Hemigrammus parana, Hemigrammus pretoensis, Hemigrammus pulcher, Hemigrammus rhodostomus, Hemigrammus rodwayi, Hemigrammus schmardae, Hemigrammus silimoni, Hemigrammus stictus, Hemigrammus taphorni, Hemigrammus tridens, Hemigrammus ulreyi, Hemigrammus unilineatus, Hemigrammus vorderwikleri, Hemigrammus sp n., Hyphessobrycon bentosi, Hyphessobrycon coelestinus, Hyphessobrycon diancistrus, Hyphessobrycon eilyos, Hyphessobrycon epicharis, Hyphessobrycon eques, Hyphessobrycon erythrostigma, Hyphessobrycon micropterus, Hyphessobrycon takasei, Iguanodectes spilurus, Jupiaba polylepis, Lignobrycon myersi, Microschemobrycon sp, Moenkhausia bonita, Moenkhausia hemigrammoides, Moenkhausia intermedia, Moenkhausia sanctaefilomenae, Moenkhausia xinguensis, Oligosarcus pintoi, Parapristella georgiae, Petitella georgiae, Phenacogaster franciscoensis, Piabina argentea, Poptella paraguayensis, Psellogrammus kennedyi, Pristella maxillaris, Rhinopetitia myersi, Salminus hilarii, Serrapinnus heterodon, Serrapinnus notomelas, Serrasalmus maculatus, Stethaprion erythrops, Tetragonopterus argenteus, Thayeria obliqua, Triportheus nematurus

\section{1- ausente (Figura 17)}

Aphyocharax anisitsi, Aphyocharax pusillus, Astyanax jacobinae, Bryconella pallidifrons, Coptobrycon bilineatus, Grundulus cochae, Hollandichthys aff. multifasciatus, Hyphessobrycon compressus, Hyphessobrycon megalopterus, Paracheirodon axelrodi, Roeboides bonariensis

\section{$\underline{0,1-\text { Polimórfico }}$}

Hasemania sp n., Hemigrammus yinyang

\section{9- Indeterminado}

Hemigrammus skolioplatus

A maioria dos Characidae apresenta o infra-orbital 4 compondo a série de ossos que circunda a órbita. Porém em algumas espécies esse osso está ausente, ou encontra-se fundido com outro componente da série (infra-orbital 3 ou 5). Por ser difícil de identificar sem margem de erro quando o infra-orbital 4 encontra-se fundido com outro elemento da série infra-orbital, qualquer condição de ausência de uma unidade isolada reconhecida como o infra-orbital 4 foi interpretada como ausência do referido osso.

75- Infra-orbital 4 (ic - 0,083)

(Serra, 2003: 24; Moreira, 2007: 155). 
Serra, J. P. - Análise Filogenética das Espécies de Hemigrammus Gill, 1858 (Characiformes, Characidae)

\section{0- com as margens anterior e posterior aproximadamente da mesma}

$\underline{\text { altura }}$

Astyanax jacobinae, Bryconamericus exodon, Bryconops melanurus, Chalceus spilogyros, Cheirodon interruptus, Hasemania melanura, Hemigrammus analis, Hemigrammus arua, Hemigrammus barrigonae, Hemigrammus bleheri, Hemigrammus boesemani, Hemigrammus cylindricus, Hemigrammus gracilis, Hemigrammus haraldi, Hemigrammus hyanuary, Hemigrammus levis, Hemigrammus mimus, Hemigrammus newboldi, Hemigrammus pretoensis, Hemigrammus stictus, Hemigrammus tridens, Hemigrammus $\mathrm{sp}$ n., Hyphessobrycon bentosi, Hyphessobrycon epicharis, Hyphessobrycon erythrostigma, Hyphessobrycon micropterus, Lignobrycon myersi,Moenkhausia sanctaefilomenae, Moenkhausia xinguensis, Parapristella georgiae, Petitella georgiae, Phenacogaster franciscoensis, Psellogrammus kennedyi, Pristella maxillaris, Rhinopetitia myersi, Salminus hilarii, Serrapinnus heterodon, Serrapinnus notomelas, Serrasalmus maculatus, Tetragonopterus argenteus, Triportheus nematurus

\section{1 - com a margem anterior mais alta que a posterior (Figuras 16 e 18)}

Astyanax altiparanae, Astyanax mexicanus, Brycon cf pesu, Bryconexodon juruenae, Deuterodon iguape, Gymnocorymbus ternetzi, Hemigrammus bellottii, Hemigrammus brevis, Hemigrammus coeruleus, Hemigrammus lunatus, Hemigrammus marginatus, Hemigrammus ocellifer, Hemigrammus orthus, Hemigrammus parana, Hemigrammus pulcher, Hemigrammus rhodostomus, Hemigrammus rodwayi, Hemigrammus schmardae, Hemigrammus silimoni, Hemigrammus taphorni, Hemigrammus ulreyi, Hemigrammus unilineatus, Hemigrammus vorderwikleri, Hemigrammus yinyang, Hyphessobrycon diancistrus, Hyphessobrycon eilyos, Hyphessobrycon eques, Hyphessobrycon takasei, Jupiaba polylepis, Microschemobrycon sp, Moenkhausia bonita, Moenkhausia hemigrammoides, Moenkhausia intermedia, Piabina argentea, Poptella paraguayensis, Stethaprion erythrops, Thayeria obliqua

\section{2- com a margem posterior mais alta que a anterior}

Brycinus longipinnis, Iguanodectes spilurus, Oligosarcus pintoi

\section{9- Indeterminado}

Aphyocharax anisitsi, Aphyocharax pusillus, Bryconella pallidifrons, Coptobrycon bilineatus, Grundulus cochae, Hasemania crenuchoides, Hasemania sp n., Hemigrammus skolioplatus, Hollandichthys aff. multifasciatus, Hyphessobrycon coelestinus, Hyphessobrycon compressus, Hyphessobrycon megalopterus, Paracheirodon axelrodi, Roeboides bonariensis

76- Infra-orbital 4 (ic - 0,100)

(Serra, 2003: 25; Benine, 2004: 2; Moreira, 2007: 153; Ferreira, 2007: 11; Marinho, 2009: 27; Mirande, 2009: 67)

\section{0 - mais alto que longo (Figuras 16 e a18)}

Astyanax altiparanae, Brycinus longipinnis, Bryconexodon juruenae, Cheirodon interruptus, Deuterodon iguape, Gymnocorymbus ternetzi, Hemigrammus analis, Hemigrammus arua, Hemigrammus barrigonae, Hemigrammus bellottii, Hemigrammus bleheri, Hemigrammus boesemani, Hemigrammus brevis, Hemigrammus coeruleus, Hemigrammus gracilis, Hemigrammus haraldi, Hemigrammus hyanuary, Hemigrammus levis, Hemigrammus lunatus, Hemigrammus marginatus, Hemigrammus mimus, Hemigrammus newboldi, Hemigrammus ocellifer, Hemigrammus orthus, Hemigrammus parana, Hemigrammus pulcher, Hemigrammus rhodostomus, Hemigrammus rodwayi, Hemigrammus schmardae, Hemigrammus silimoni, Hemigrammus stictus, Hemigrammus tridens, Hemigrammus ulreyi, Hemigrammus unilineatus, 
Hemigrammus vorderwikleri, Hemigrammus yinyang, Hemigrammus sp n., Hyphessobrycon bentosi, Hyphessobrycon eilyos, Hyphessobrycon epicharis, Hyphessobrycon eques, Hyphessobrycon erythrostigma, Hyphessobrycon takasei, Iguanodectes spilurus, Moenkhausia hemigrammoides, Moenkhausia intermedia, Moenkhausia xinguensis, Oligosarcus pintoi, Petitella georgiae, Poptella paraguayensis, Psellogrammus kennedyi, Pristella maxillaris, Serrapinnus notomelas, Stethaprion erythrops, Tetragonopterus argenteus, Thayeria obliqua, Triportheus nematurus

\section{1- mais longo que alto}

Astyanax mexicanus, Brycon cf pesu, Chalceus spilogyros, Hasemania crenuchoides, Hasemania melanura, Hemigrammus taphorni, Moenkhausia bonita, Phenacogaster franciscoensis, Piabina argentea, Salminus hilarii, Serrasalmus maculatus

\section{2- aproximadamente tão longo quanto alto}

Bryconamericus exodon, Bryconops melanurus, Hemigrammus cylindricus, Hemigrammus pretoensis, Hyphessobrycon diancistrus, Hyphessobrycon micropterus, Jupiaba polylepis, Lignobrycon myersi, Microschemobrycon sp, Moenkhausia sanctaefilomenae, Parapristella georgiae, Rhinopetitia myersi, Serrapinnus heterodon

\section{9- Indeterminado}

Aphyocharax anisitsi, Aphyocharax pusillus, Astyanax jacobinae, Bryconella pallidifrons, Coptobrycon bilineatus, Grundulus cochae, Hasemania sp n., Hemigrammus skolioplatus, Hollandichthys aff. multifasciatus, Hyphessobrycon coelestinus, Hyphessobrycon compressus, Hyphessobrycon megalopterus, Paracheirodon axelrodi, Roeboides bonariensis

77- Infra-orbital 4 (ic - 0,182)

(Serra, 2003: 26)

\section{0- tão alto quanto o infra-orbital 5 ou maior que a metade dele (Figuras}

\section{6 e 18)}

Astyanax altiparanae, Astyanax mexicanus, Bryconamericus exodon, Bryconexodon juruenae, Bryconops melanurus, Chalceus spilogyros,Cheirodon interruptus, Deuterodon iguape, Gymnocorymbus ternetzi, Hasemania crenuchoides, Hasemania melanura, Hemigrammus analis, Hemigrammus arua, Hemigrammus barrigonae, Hemigrammus bellottii, Hemigrammus bleheri, Hemigrammus boesemani, Hemigrammus brevis, Hemigrammus coeruleus, Hemigrammus cylindricus, Hemigrammus gracilis, Hemigrammus haraldi, Hemigrammus hyanuary, Hemigrammus levis, Hemigrammus lunatus, Hemigrammus marginatus, Hemigrammus newboldi, Hemigrammus ocellifer, Hemigrammus orthus, Hemigrammus parana, Hemigrammus pretoensis, Hemigrammus rhodostomus, Hemigrammus rodwayi, Hemigrammus schmardae, Hemigrammus silimoni, Hemigrammus stictus, Hemigrammus ulreyi, Hemigrammus unilineatus, Hemigrammus vorderwikleri, Hemigrammus sp n., Hyphessobrycon diancistrus, Hyphessobrycon epicharis, Hyphessobrycon eques, Hyphessobrycon erythrostigma, Hyphessobrycon micropterus, Hyphessobrycon takasei, Iguanodectes spilurus, Jupiaba polylepis, Microschemobrycon sp, Moenkhausia bonita, Moenkhausia hemigrammoides, Moenkhausia intermedia, Moenkhausia sanctaefilomenae, Moenkhausia xinguensis, Parapristella georgiae, Petitella georgiae, Phenacogaster franciscoensis, Piabina argentea, Poptella paraguayensis, Psellogrammus kennedyi, Pristella maxillaris, Serrapinnus heterodon, Serrapinnus notomelas, Stethaprion erythrops, Tetragonopterus argenteus, Thayeria obliqua, Triportheus nematurus

\section{1- mais baixo que a metade do infra-orbital 5}


Brycon cf pesu, Hemigrammus mimus, Hemigrammus pulcher, Hemigrammus taphorni, Hemigrammus tridens, Hyphessobrycon bentosi, Hyphessobrycon eilyos, Lignobrycon myersi, Rhinopetitia myersi, Salminus hilarii, Serrasalmus maculatus

\section{2- mais alto que o infra-orbital 5}

Brycinus longipinnis, Oligosarcus pintoi

\section{9- Indeterminado}

Aphyocharax anisitsi, Aphyocharax pusillus, Astyanax jacobinae, Bryconella pallidifrons, Coptobrycon bilineatus, Grundulus cochae, Hasemania sp n., Hemigrammus skolioplatus, Hemigrammus yinyang, Hollandichthys aff. multifasciatus, Hyphessobrycon coelestinus, Hyphessobrycon compressus, Hyphessobrycon megalopterus, Paracheirodon axelrodi, Roeboides bonariensis

\section{8- Infra-orbital 5 (ic - 0,500)}

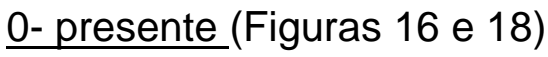

Aphyocharax anisitsi, Aphyocharax pusillus, Astyanax altiparanae, Astyanax jacobinae, Astyanax mexicanus, Brycinus longipinnis, Brycon cf pesu, Bryconamericus exodon, Bryconexodon juruenae, Bryconops melanurus, Chalceus spilogyros, Cheirodon interruptus, Deuterodon iguape, Gymnocorymbus ternetzi, Hasemania crenuchoides, Hasemania melanura, Hemigrammus analis, Hemigrammus arua, Hemigrammus barrigonae, Hemigrammus bellottii, Hemigrammus bleheri, Hemigrammus boesemani, Hemigrammus brevis, Hemigrammus coeruleus, Hemigrammus cylindricus, Hemigrammus gracilis, Hemigrammus haraldi, Hemigrammus hyanuary, Hemigrammus levis, Hemigrammus lunatus, Hemigrammus marginatus, Hemigrammus mimus, Hemigrammus newboldi, Hemigrammus ocellifer, Hemigrammus orthus, Hemigrammus parana, Hemigrammus pretoensis, Hemigrammus pulcher, Hemigrammus rhodostomus, Hemigrammus rodwayi, Hemigrammus schmardae, Hemigrammus silimoni, Hemigrammus skolioplatus, Hemigrammus stictus, Hemigrammus taphorni, Hemigrammus tridens, Hemigrammus ulreyi, Hemigrammus unilineatus, Hemigrammus vorderwikleri, Hemigrammus $\mathrm{sp} \mathrm{n}$., Hollandichthys aff. multifasciatus, Hyphessobrycon bentosi, Hyphessobrycon compressus, Hyphessobrycon diancistrus, Hyphessobrycon eilyos, Hyphessobrycon epicharis, Hyphessobrycon eques, Hyphessobrycon erythrostigma, Hyphessobrycon micropterus, Hyphessobrycon takasei, Iguanodectes spilurus, Jupiaba polylepis, Lignobrycon myersi, Microschemobrycon sp, Moenkhausia bonita, Moenkhausia hemigrammoides, Moenkhausia intermedia, Moenkhausia sanctaefilomenae, Moenkhausia xinguensis, Oligosarcus pintoi, Parapristella georgiae, Petitella georgiae, Phenacogaster franciscoensis, Piabina argentea, Poptella paraguayensis, Psellogrammus kennedyi, Pristella maxillaris, Rhinopetitia myersi, Roeboides bonariensis, Salminus hilarii, Serrapinnus heterodon, Serrapinnus notomelas, Serrasalmus maculatus, Stethaprion erythrops, Tetragonopterus argenteus, Thayeria obliqua, Triportheus nematurus

\section{1- ausente (Figura 17)}

Bryconella pallidifrons, Grundulus cochae, Hemigrammus yinyang, Hyphessobrycon coelestinus, Hyphessobrycon megalopterus, Paracheirodon axelrodi

\section{$\underline{0,1-\text { Polimórfico }}$}

Coptobrycon bilineatus 
A maioria dos Characidae apresenta o infra-orbital 5 compondo a série de ossos que circunda a órbita. Porém em algumas espécies esse osso está ausente, ou encontra-se fundido com outro componente da série (infra-orbital 4). Por ser difícil de identificar sem margem de erro quando o infra-orbital 5 encontra-se fundido com outro elemento da série infra-orbital, qualquer condição de ausência de uma unidade isolada reconhecida como o infra-orbital 5 foi interpretada como ausência do referido osso.

79- Infra-orbital 5 (ic - 0,333)

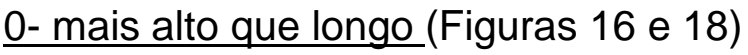

Aphyocharax anisitsi, Aphyocharax pusillus, Astyanax altiparanae, Astyanax jacobinae, Brycinus longipinnis, Brycon cf pesu, Bryconamericus exodon, Bryconexodon juruenae, Bryconops melanurus, Chalceus spilogyros, Cheirodon interruptus, Coptobrycon bilineatus, Deuterodon iguape, Gymnocorymbus ternetzi, Hasemania melanura, Hemigrammus analis, Hemigrammus arua, Hemigrammus barrigonae, Hemigrammus bellottii, Hemigrammus bleheri, Hemigrammus boesemani, Hemigrammus brevis, Hemigrammus coeruleus, Hemigrammus cylindricus, Hemigrammus gracilis, Hemigrammus haraldi, Hemigrammus hyanuary, Hemigrammus levis, Hemigrammus lunatus, Hemigrammus marginatus, Hemigrammus mimus, Hemigrammus newboldi, Hemigrammus ocellifer, Hemigrammus orthus, Hemigrammus parana, Hemigrammus pretoensis, Hemigrammus pulcher, Hemigrammus rhodostomus, Hemigrammus rodwayi, Hemigrammus schmardae, Hemigrammus silimoni, Hemigrammus stictus, Hemigrammus taphorni, Hemigrammus tridens, Hemigrammus ulreyi, Hemigrammus unilineatus, Hemigrammus vorderwikleri, Hemigrammus sp n., Hollandichthys aff. multifasciatus, Hyphessobrycon bentosi, Hyphessobrycon compressus, Hyphessobrycon diancistrus, Hyphessobrycon eilyos, Hyphessobrycon epicharis, Hyphessobrycon eques, Hyphessobrycon erythrostigma, Hyphessobrycon micropterus, Hyphessobrycon takasei, Iguanodectes spilurus, Jupiaba polylepis, Lignobrycon myersi, Microschemobrycon sp, Moenkhausia bonita, Moenkhausia hemigrammoides, Moenkhausia intermedia, Moenkhausia sanctaefilomenae, Moenkhausia xinguensis, Parapristella georgiae, Petitella georgiae, Phenacogaster franciscoensis, Piabina argentea, Poptella paraguayensis, Psellogrammus kennedyi, Pristella maxillaris, Rhinopetitia myersi, Roeboides bonariensis, Serrapinnus heterodon, Serrapinnus notomelas, Stethaprion erythrops, Tetragonopterus argenteus, Thayeria obliqua

\section{1- mais longo que alto}

Salminus hilarii, Serrasalmus maculatus

\section{2- aproximadamente tão alto quanto longo}

Astyanax mexicanus, Hasemania crenuchoides, Oligosarcus pintoi, Triportheus nematurus

\section{9- Indeterminado}

Bryconella pallidifrons, Grundulus cochae, Hasemania sp n., Hemigrammus skolioplatus, Hemigrammus yinyang, Hyphessobrycon coelestinus, Hyphessobrycon megalopterus, Paracheirodon axelrodi

80- Infra-orbital 6 (ic $-0,333$ ) 
(Serra, 2003: 27; Moreira, 2007: 162; Bertaco, 2008: 36)

\section{0- ausente (Figura 17)}

Bryconella pallidifrons, Coptobrycon bilineatus, Grundulus cochae, Hasemania sp n., Hemigrammus yinyang, Hyphessobrycon megalopterus, Hyphessobrycon micropterus, Paracheirodon axelrodi

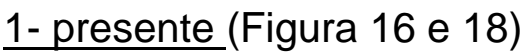

Aphyocharax anisitsi, Aphyocharax pusillus, Astyanax altiparanae, Astyanax jacobinae, Astyanax mexicanus, Brycinus longipinnis, Brycon of pesu, Bryconamericus exodon, Bryconexodon juruenae, Bryconops melanurus, Chalceus spilogyros, Cheirodon interruptus, Deuterodon iguape, Gymnocorymbus ternetzi, Hasemania crenuchoides, Hasemania melanura, Hemigrammus analis, Hemigrammus barrigonae, Hemigrammus bellottii, Hemigrammus bleheri, Hemigrammus boesemani, Hemigrammus brevis, Hemigrammus coeruleus, Hemigrammus cylindricus, Hemigrammus gracilis, Hemigrammus haraldi, Hemigrammus hyanuary, Hemigrammus levis, Hemigrammus lunatus, Hemigrammus marginatus, Hemigrammus mimus, Hemigrammus newboldi, Hemigrammus ocellifer, Hemigrammus orthus, Hemigrammus parana, Hemigrammus pretoensis, Hemigrammus pulcher, Hemigrammus rhodostomus, Hemigrammus rodwayi, Hemigrammus schmardae, Hemigrammus silimoni, Hemigrammus skolioplatus, Hemigrammus stictus, Hemigrammus taphorni, Hemigrammus tridens, Hemigrammus ulreyi, Hemigrammus unilineatus, Hemigrammus vorderwikleri, Hemigrammus sp n., Hollandichthys aff. multifasciatus, Hyphessobrycon bentosi, Hyphessobrycon coelestinus, Hyphessobrycon compressus, Hyphessobrycon diancistrus, Hyphessobrycon eilyos, Hyphessobrycon epicharis, Hyphessobrycon eques, Hyphessobrycon erythrostigma, Hyphessobrycon takasei, Iguanodectes spilurus, Jupiaba polylepis, Lignobrycon myersi, Microschemobrycon sp, Moenkhausia bonita, Moenkhausia hemigrammoides, Moenkhausia intermedia, Moenkhausia sanctaefilomenae, Moenkhausia xinguensis, Oligosarcus pintoi, Parapristella georgiae, Petitella georgiae, Phenacogaster franciscoensis, Piabina argentea, Poptella paraguayensis, Psellogrammus kennedyi, Pristella maxillaris, Rhinopetitia myersi, Roeboides bonariensis, Salminus hilarii, Serrapinnus heterodon, Serrapinnus notomelas, Serrasalmus maculatus, Stethaprion erythrops, Tetragonopterus argenteus, Thayeria obliqua, Triportheus nematurus

\section{$\underline{0,1-P o l i m o ́ r f i c o ~}$}

Hemigrammus arua

A maior parte das espécies componentes de Characidae apresentam o infra-orbital 6 como um osso bem desenvolvido e que compõe a série que circunda a órbita. Algumas espécies, porém, apresentam o infra-orbital 6 muito reduzido ou ausente; segundo Weitzman \& Fink (1983), redução e perda dos ossos infra-orbitais são comuns em espécies de Characidae de pequeno porte e espécies miniatura. Weitzman \& Malabarba (1999) segerem a perda do infraorbital 6 como uma sinapomorfia para Spintherobolus. Na presente análise a perda do referido osso aparece como uma condição derivada para alguns agrupamentos de espécies de pequeno porte dentro de Characidae, como o clado 125 (que inclui $H$. yinyang, B. pallidifrons, $P$. axelrodi, $C$. bilineatus e $G$. cochae) e o clado 154 (que inclui $H$. megalopterus e $H$. micropterus). Ausência 
do infra-orbital 6 aparece também como uma autapomorfia para Hasemania sp.n.

81- Infra-orbital 6 (ic - 0,154)

(Serra, 2003: 28; Moreira, 2007: 162)

\section{$\underline{0-\text { com a porção lamelar desenvolvida, canal sensorial completo e }}$}

\section{totalmente cercado por osso (geralmente osso grande) (Figura 18)}

Astyanax altiparanae, Astyanax jacobinae, Astyanax mexicanus, Brycinus longipinnis, Brycon cf pesu, Bryconamericus exodon, Bryconexodon juruenae, Bryconops melanurus, Chalceus spilogyros, Deuterodon iguape, Gymnocorymbus ternetzi, Hasemania crenuchoides, Hasemania melanura, Hemigrammus cylindricus, Hemigrammus levis, Hemigrammus newboldi, Hemigrammus pretoensis, Hemigrammus rhodostomus, Hemigrammus skolioplatus, Hemigrammus taphorni, Hollandichthys aff. multifasciatus, Iguanodectes spilurus, Jupiaba polylepis, Lignobrycon myersi, Moenkhausia intermedia, Moenkhausia sanctaefilomenae, Moenkhausia xinguensis, Oligosarcus pintoi, Parapristella georgiae, Phenacogaster franciscoensis, Piabina argentea, Poptella paraguayensis, Psellogrammus kennedyi, Roeboides bonariensis, Salminus hilarii, Serrapinnus heterodon, Serrapinnus notomelas, Stethaprion erythrops, Tetragonopterus argenteus, Triportheus nematurus

\section{1- com a porção lamelar reduzida, restrito quase que totalmente à} porção tubular do canal sensorial cercado apenas por uma lâmina óssea delgada ou com regiões do canal totalmente expostas (Figura 16)

Aphyocharax anisitsi, Aphyocharax pusillus, Cheirodon interruptus, Hemigrammus analis, Hemigrammus arua, Hemigrammus barrigonae, Hemigrammus bellottii, Hemigrammus bleheri, Hemigrammus boesemani, Hemigrammus brevis, Hemigrammus coeruleus, Hemigrammus gracilis, Hemigrammus haraldi, Hemigrammus hyanuary, Hemigrammus lunatus, Hemigrammus marginatus, Hemigrammus mimus, Hemigrammus ocellifer, Hemigrammus parana, Hemigrammus pulcher, Hemigrammus rodwayi, Hemigrammus schmardae, Hemigrammus silimoni, Hemigrammus stictus, Hemigrammus sp n., Hemigrammus ulreyi, Hemigrammus unilineatus, Hyphessobrycon bentosi, Hyphessobrycon coelestinus, Hyphessobrycon diancistrus, Hyphessobrycon epicharis, Hyphessobrycon eques, Hyphessobrycon erythrostigma, Hyphessobrycon takasei, Moenkhausia bonita, Moenkhausia hemigrammoides, Petitella georgiae, Pristella maxillaris, Rhinopetitia myersi, Serrasalmus maculatus, Thayeria obliqua

2- sem canal sensorial (ou muito teduzido), restando apenas uma pequena porção óssea (geralmente osso pequeno)

Hemigrammus orthus, Hemigrammus tridens, Hemigrammus vorderwikleri, Hyphessobrycon compressus, Hyphessobrycon eilyos, Microschemobrycon sp

\section{9- Indeterminado}

Bryconella pallidifrons, Coptobrycon bilineatus, Grundulus cochae, Hasemania sp n., Hemigrammus yinyang, Hyphessobrycon megalopterus, Hyphessobrycon micropterus, Paracheirodon axelrodi 
A maior parte das espécies componentes de Characidae apresenta 0 infra-orbital 6 como um osso bem desenvolvido, formado por uma grande lâmina óssea que circunda o canal sensorial. Em algumas espécies da família o infra-orbital 6 está ausente (como apresentado no caráter 80); em outras espécies ainda, embora presente, o infra-orbital 6 é bastante reduzido, ou com uma lâmina óssea muito pequena (estreita e curta), ficando quase que restrito somente ao canal sensorial (estado 1) ou formado somente por uma pequena lâmina óssea desprovida de canal sensorial (estado 2). Segundo Weitzman \& Fink (1983), redução e perda dos ossos infra-orbitais são comuns em espécies de Characidae de pequeno porte e espécies miniatura. Os estados 1 e 2 descritos acima são observados na grande maioria das espécies de pequeno porte incluídas na presente análise (especialmente componentes do clado 172 , com reversão para o estado 0 no clado 121, onde estão as espécies de maior porte). Buckup (1993) coloca que em Characidiinae também ocorre redução do infra-orbital 6, sendo que as porções lamelares do osso são muito reduzidas, e o infra-orbital 6 é representado apenas pela porção tubular do canal sensorial, condição provavelmente adquirida independentemente das espécies incluídas na presente análise.

\section{Arco palatino, Suspensório e Ossos operculares}

82- Palatino (ic - 0,500)

$$
\text { 0- mais longo que largo (Figura } 19 \mathrm{~A} \text { e B) }
$$

Aphyocharax anisitsi, Aphyocharax pusillus, Astyanax jacobinae, Brycinus longipinnis, Brycon cf pesu, Bryconamericus exodon, Bryconella pallidifrons, Bryconexodon juruenae, Bryconops melanurus, Chalceus spilogyros, Cheirodon interruptus, Coptobrycon bilineatus, Deuterodon iguape, Grundulus cochae, Gymnocorymbus ternetzi, Hasemania melanura, Hemigrammus analis, Hemigrammus arua, Hemigrammus barrigonae, Hemigrammus bellottii, Hemigrammus bleheri, Hemigrammus boesemani, Hemigrammus brevis, Hemigrammus coeruleus, Hemigrammus cylindricus, Hemigrammus gracilis, Hemigrammus haraldi, Hemigrammus hyanuary, Hemigrammus levis, Hemigrammus lunatus, Hemigrammus marginatus, Hemigrammus mimus, Hemigrammus newboldi, Hemigrammus ocellifer, Hemigrammus orthus, Hemigrammus parana, Hemigrammus pretoensis, Hemigrammus pulcher, Hemigrammus rhodostomus, Hemigrammus rodwayi, Hemigrammus schmardae, Hemigrammus silimoni, Hemigrammus skolioplatus, Hemigrammus stictus, Hemigrammus taphorni, Hemigrammus tridens, Hemigrammus ulreyi, Hemigrammus unilineatus, Hemigrammus vorderwikleri, Hemigrammus yinyang, Hemigrammus $\mathrm{sp} \mathrm{n}$., Hollandichthys aff. multifasciatus, Hyphessobrycon bentosi, Hyphessobrycon coelestinus, Hyphessobrycon compressus, Hyphessobrycon diancistrus, Hyphessobrycon eilyos, Hyphessobrycon epicharis, Hyphessobrycon eques, Hyphessobrycon erythrostigma, Hyphessobrycon megalopterus, Hyphessobrycon micropterus, Hyphessobrycon takasei, Iguanodectes spilurus, Jupiaba polylepis, Lignobrycon myersi, Microschemobrycon sp, Moenkhausia bonita, Moenkhausia hemigrammoides, Moenkhausia intermedia, Moenkhausia sanctaefilomenae, Moenkhausia xinguensis, Oligosarcus pintoi, Paracheirodon axelrodi, Parapristella georgiae, Petitella 
georgiae, Phenacogaster franciscoensis, Piabina argentea, Poptella paraguayensis, Psellogrammus kennedyi, Pristella maxillaris, Rhinopetitia myersi, Roeboides bonariensis, Salminus hilarii, Serrapinnus heterodon, Serrapinnus notomelas, Serrasalmus maculatus, Stethaprion erythrops, Tetragonopterus argenteus, Thayeria obliqua, Triportheus nematurus

\section{1- aproximadamente tão largo quanto longo (Figura $19 \mathrm{C}$ )}

Astyanax altiparanae, Astyanax mexicanus, Hasemania crenuchoides, Hasemania sp n.

83- Forma do palatino (ic - 0,143)

(Ferreira, 2007: 76; Bertaco, 2008: 41)

$\underline{0 \text { - largo, geralmente curto, com a largura não mudando muito ao longo }}$ do comprimento (Figura $19 \mathrm{C}$ )

Astyanax altiparanae, Astyanax mexicanus, Brycon cf pesu, Bryconamericus exodon, Bryconops melanurus, Cheirodon interruptus, Gymnocorymbus ternetzi, Hasemania crenuchoides, Hasemania melanura, Hasemania sp n., Hemigrammus barrigonae, Hemigrammus ulreyi, Hollandichthys aff. multifasciatus, Hyphessobrycon bentosi, Moenkhausia sanctaefilomenae, Oligosarcus pintoi, Phenacogaster franciscoensis, Piabina argentea, Psellogrammus kennedyi, Rhinopetitia myersi, Salminus hilarii, Serrapinnus heterodon, Serrapinnus notomelas, Tetragonopterus argenteus, Triportheus nematurus

\section{1- mais estreito e geralmente mais alongado, com a porção mediana} estreita e as porções anterior e posterior mais largas (Figura 19 A)

Astyanax jacobinae, Bryconella pallidifrons, Bryconexodon juruenae, Coptobrycon bilineatus, Deuterodon iguape, Grundulus cochae, Hemigrammus analis, Hemigrammus arua, Hemigrammus bellottii, Hemigrammus bleheri, Hemigrammus boesemani, Hemigrammus brevis, Hemigrammus coeruleus, Hemigrammus cylindricus, Hemigrammus gracilis, Hemigrammus haraldi, Hemigrammus hyanuary, Hemigrammus levis, Hemigrammus lunatus, Hemigrammus marginatus, Hemigrammus newboldi, Hemigrammus ocellifer, Hemigrammus orthus, Hemigrammus parana, Hemigrammus pretoensis, Hemigrammus pulcher, Hemigrammus rhodostomus, Hemigrammus rodwayi, Hemigrammus schmardae, Hemigrammus silimoni, Hemigrammus skolioplatus, Hemigrammus stictus, Hemigrammus taphorni, Hemigrammus tridens, Hemigrammus unilineatus, Hemigrammus yinyang, Hemigrammus sp n., Hyphessobrycon coelestinus, Hyphessobrycon compressus, Hyphessobrycon diancistrus, Hyphessobrycon eilyos, Hyphessobrycon epicharis, Hyphessobrycon eques, Hyphessobrycon erythrostigma, Hyphessobrycon megalopterus, Hyphessobrycon micropterus, Hyphessobrycon takasei, lguanodectes spilurus, Jupiaba polylepis, Lignobrycon myersi, Moenkhausia bonita, Moenkhausia hemigrammoides, Moenkhausia intermedia, Moenkhausia xinguensis, Paracheirodon axelrodi, Parapristella georgiae, Petitella georgiae, Poptella paraguayensis, Pristella maxillaris, Roeboides bonariensis, Stethaprion erythrops, Thayeria obliqua

\section{2- largo, com a porção anterior bem mais larga que o restante do osso} (Figura 19 B)

Aphyocharax anisitsi, Aphyocharax pusillus, Brycinus longipinnis, Hemigrammus mimus, Microschemobrycon sp

\section{9- Indeterminado}

Chalceus spilogyros, Hemigrammus vorderwikleri, Serrasalmus maculatus 


\title{
84- Forma das margens laterais do palatino (ic - 0,083)
}

\author{
(Serra, 2003: 39; Moreira, 2007: 49)
}

\section{$\underline{0 \text { - com ambas as margens laterais aproximadamente retas ou convexas }}$} (Figura $19 \mathrm{~B}$ e C)

Aphyocharax anisitsi, Aphyocharax pusillus, Astyanax altiparanae, Astyanax mexicanus, Brycon cf pesu, Bryconamericus exodon, Bryconella pallidifrons, Bryconops melanurus, Cheirodon interruptus, Gymnocorymbus ternetzi, Hasemania crenuchoides, Hasemania melanura, Hasemania sp n., Hemigrammus levis, Hemigrammus mimus, Hemigrammus vorderwikleri, Hyphessobrycon compressus, Microschemobrycon sp, Paracheirodon axelrodi, Phenacogaster franciscoensis, Piabina argentea, Rhinopetitia myersi, Salminus hilarii, Serrapinnus heterodon, Serrapinnus notomelas, Serrasalmus maculatus, Tetragonopterus argenteus

\section{1- com uma ou ambas as margens laterais côncavas (Figura 19 A)}

Astyanax jacobinae, Brycinus longipinnis, Bryconexodon juruenae, Chalceus spilogyros, Coptobrycon bilineatus, Deuterodon iguape, Grundulus cochae, Hemigrammus analis, Hemigrammus arua, Hemigrammus barrigonae, Hemigrammus bellottii, Hemigrammus bleheri, Hemigrammus boesemani, Hemigrammus brevis, Hemigrammus coeruleus, Hemigrammus cylindricus, Hemigrammus gracilis, Hemigrammus haraldi, Hemigrammus hyanuary, Hemigrammus lunatus, Hemigrammus marginatus, Hemigrammus newboldi, Hemigrammus ocellifer, Hemigrammus orthus, Hemigrammus parana, Hemigrammus pretoensis, Hemigrammus pulcher, Hemigrammus rhodostomus, Hemigrammus rodwayi, Hemigrammus schmardae, Hemigrammus silimoni, Hemigrammus stictus, Hemigrammus taphorni, Hemigrammus tridens, Hemigrammus ulreyi, Hemigrammus unilineatus, Hemigrammus yinyang, Hemigrammus sp n., Hollandichthys aff. multifasciatus, Hyphessobrycon bentosi, Hyphessobrycon coelestinus, Hyphessobrycon diancistrus, Hyphessobrycon eilyos, Hyphessobrycon epicharis, Hyphessobrycon eques, Hyphessobrycon erythrostigma, Hyphessobrycon megalopterus, Hyphessobrycon micropterus, Hyphessobrycon takasei, Iguanodectes spilurus, Jupiaba polylepis, Lignobrycon myersi, Moenkhausia bonita, Moenkhausia hemigrammoides, Moenkhausia intermedia, Moenkhausia sanctaefilomenae, Moenkhausia xinguensis, Oligosarcus pintoi, Parapristella georgiae, Petitella georgiae, Poptella paraguayensis, Psellogrammus kennedyi, Pristella maxillaris, Roeboides bonariensis, Stethaprion erythrops, Thayeria obliqua, Triportheus nematurus

\section{9- Indeterminado}

Hemigrammus skolioplatus

\section{5- Fenestra antero-medial no palatino (ic - 1,0)}

(Serra \& Langeani, 2006; Ferreira, 2007: 75; Bertaco, 2008: 42))

\section{0- ausente (Figura 19 A - C)}

Aphyocharax anisitsi, Aphyocharax pusillus, Astyanax altiparanae, Astyanax jacobinae, Astyanax mexicanus, Brycinus longipinnis, Brycon cf pesu, Bryconella pallidifrons, Bryconexodon juruenae, Bryconops melanurus, Chalceus spilogyros, Cheirodon interruptus, Coptobrycon bilineatus, Deuterodon iguape, Grundulus cochae, Gymnocorymbus ternetzi, Hasemania crenuchoides, Hasemania melanura, Hasemania sp n., Hemigrammus analis, Hemigrammus arua, Hemigrammus barrigonae, Hemigrammus bellottii, Hemigrammus bleheri, Hemigrammus boesemani, Hemigrammus brevis, Hemigrammus coeruleus, Hemigrammus cylindricus, Hemigrammus gracilis, Hemigrammus haraldi, Hemigrammus hyanuary, Hemigrammus levis, Hemigrammus lunatus, Hemigrammus marginatus, Hemigrammus mimus, Hemigrammus newboldi, Hemigrammus ocellifer, Hemigrammus orthus, Hemigrammus parana, 
Hemigrammus pretoensis, Hemigrammus pulcher, Hemigrammus rhodostomus, Hemigrammus rodwayi, Hemigrammus schmardae, Hemigrammus silimoni, Hemigrammus skolioplatus, Hemigrammus stictus, Hemigrammus taphorni, Hemigrammus tridens, Hemigrammus ulreyi, Hemigrammus unilineatus, Hemigrammus vorderwikleri, Hemigrammus yinyang, Hemigrammus sp n., Hollandichthys aff. multifasciatus, Hyphessobrycon bentosi, Hyphessobrycon coelestinus, Hyphessobrycon compressus, Hyphessobrycon diancistrus, Hyphessobrycon eilyos, Hyphessobrycon epicharis, Hyphessobrycon eques, Hyphessobrycon erythrostigma, Hyphessobrycon megalopterus, Hyphessobrycon micropterus, Hyphessobrycon takasei, Iguanodectes spilurus, Jupiaba polylepis, Lignobrycon myersi, Microschemobrycon sp, Moenkhausia bonita, Moenkhausia hemigrammoides, Moenkhausia intermedia, Moenkhausia sanctaefilomenae, Moenkhausia xinguensis, Oligosarcus pintoi, Paracheirodon axelrodi, Parapristella georgiae, Petitella georgiae, Phenacogaster franciscoensis, Piabina argentea, Poptella paraguayensis, Psellogrammus kennedyi, Pristella maxillaris, Rhinopetitia myersi, Roeboides bonariensis, Salminus hilarii, Serrapinnus heterodon, Serrapinnus notomelas, Serrasalmus maculatus, Stethaprion erythrops, Tetragonopterus argenteus, Thayeria obliqua, Triportheus nematurus

\section{1- presente}

Bryconamericus exodon

Segundo Serra \& Langeani (2006) o palatino na grande maioria dos Characiformes é um osso relativamente retangular, inteiriço e sem nenhuma fenestra ou perfuração. Os autores, entretanto, apresentam um palatino com uma fenestra grande, anterior e medial para Bryconamericus exodon e algumas outras espécies do "clado A" de Malabarba \& Weitzman (2003). Além da fenestra própriamente dita, os autores também consideram homóloga a presença, na mesma região, de uma perfuração pequena ou um adelgaçamento. Essas condições são sugeridas por Serra \& Langeani (2006) como apomórficas em Characidae e como uma sinapomorfia adicional para o "clado A" de Malabarba \& Weitzman (2003).

86- Mesopterigóide (ic - 0,500)

(Vari \& Harold, 1998; Langeani, 1998; Serra, 2003: 36)

\section{0 - separado do quadrado}

Piabina argentea, Rhinopetitia myersi, Serrapinnus heterodon, Serrapinnus notomelas

\section{1- apenas contatando o quadrado}

Bryconamericus exodon, Chalceus spilogyros

\section{2- sobrepondo-se ao quadrado (Figura 20)}

Aphyocharax anisitsi, Aphyocharax pusillus, Astyanax altiparanae, Astyanax jacobinae, Astyanax mexicanus, Brycinus longipinnis, Brycon cf pesu, Bryconella pallidifrons, 
Bryconexodon juruenae, Bryconops melanurus, Cheirodon interruptus, Coptobrycon bilineatus, Deuterodon iguape, Grundulus cochae, Gymnocorymbus ternetzi, Hasemania crenuchoides, Hasemania melanura, Hasemania sp n., Hemigrammus analis, Hemigrammus arua, Hemigrammus barrigonae, Hemigrammus bellottii, Hemigrammus bleheri, Hemigrammus boesemani, Hemigrammus brevis, Hemigrammus coeruleus, Hemigrammus cylindricus, Hemigrammus gracilis, Hemigrammus haraldi, Hemigrammus hyanuary, Hemigrammus levis, Hemigrammus lunatus, Hemigrammus marginatus, Hemigrammus mimus, Hemigrammus newboldi, Hemigrammus ocellifer, Hemigrammus orthus, Hemigrammus parana, Hemigrammus pretoensis, Hemigrammus pulcher, Hemigrammus rhodostomus, Hemigrammus rodwayi, Hemigrammus schmardae, Hemigrammus silimoni, Hemigrammus skolioplatus, Hemigrammus stictus, Hemigrammus taphorni, Hemigrammus tridens, Hemigrammus ulreyi, Hemigrammus unilineatus, Hemigrammus vorderwikleri, Hemigrammus yinyang, Hemigrammus sp n., Hollandichthys aff. multifasciatus, Hyphessobrycon bentosi, Hyphessobrycon coelestinus, Hyphessobrycon compressus, Hyphessobrycon diancistrus, Hyphessobrycon eilyos, Hyphessobrycon epicharis, Hyphessobrycon eques, Hyphessobrycon erythrostigma, Hyphessobrycon megalopterus, Hyphessobrycon micropterus, Hyphessobrycon takasei, Iguanodectes spilurus, Jupiaba polylepis, Lignobrycon myersi, Microschemobrycon sp, Moenkhausia bonita, Moenkhausia hemigrammoides, Moenkhausia intermedia, Moenkhausia sanctaefilomenae, Moenkhausia xinguensis, Oligosarcus pintoi, Paracheirodon axelrodi, Parapristella georgiae, Petitella georgiae, Phenacogaster franciscoensis, Poptella paraguayensis, Psellogrammus kennedyi, Pristella maxillaris, Roeboides bonariensis, Salminus hilarii, Serrasalmus maculatus, Stethaprion erythrops, Tetragonopterus argenteus, Thayeria obliqua, Triportheus nematurus

No estado 0 o mesopterigóide está distante do quadrado, sem nenhuma forma de contato; no estado 1, o mesopterigóide apenas encosta no quadrado; já no estado 2 o mesopterigóide apresenta sobreposição com o quadrado, essa sobreposição pode ser de diferentes graus, desde muito pequena, apenas na região mais dorsal do osso, até muito grande, podendo alcançar a região ventral do quadrado.

\section{7- Margem posterior do mesopterigóide (ic - 0,100)}

\section{$\underline{0 \text { - com concavidade acentuada }}$}

Astyanax altiparanae, Astyanax mexicanus, Brycon cf pesu, Bryconexodon juruenae, Hemigrammus coeruleus, Hemigrammus newboldi, Hemigrammus pretoensis, Hemigrammus schmardae, Hemigrammus taphorni, Moenkhausia sanctaefilomenae, Moenkhausia xinguensis, Piabina argentea, Pristella maxillaris, Salminus hilarii, Tetragonopterus argenteus

\section{1- aproximadamente reta ou levemente convexa, sem concavidade}

Aphyocharax anisitsi, Aphyocharax pusillus, Brycinus longipinnis, Bryconamericus exodon, Bryconella pallidifrons, Bryconops melanurus, Chalceus spilogyros, Cheirodon interruptus, Coptobrycon bilineatus, Deuterodon iguape, Grundulus cochae, Gymnocorymbus ternetzi, Hasemania crenuchoides, Hasemania sp n., Hemigrammus analis, Hemigrammus arua, Hemigrammus barrigonae, Hemigrammus bellottii, Hemigrammus bleheri, Hemigrammus boesemani, Hemigrammus brevis, Hemigrammus cylindricus, Hemigrammus gracilis, Hemigrammus haraldi, Hemigrammus hyanuary, Hemigrammus levis, Hemigrammus lunatus, Hemigrammus marginatus, Hemigrammus mimus, Hemigrammus ocellifer, Hemigrammus orthus, Hemigrammus parana, Hemigrammus pulcher, Hemigrammus rhodostomus, Hemigrammus rodwayi, Hemigrammus silimoni, Hemigrammus skolioplatus, Hemigrammus stictus, Hemigrammus tridens, Hemigrammus ulreyi, Hemigrammus unilineatus, Hemigrammus vorderwikleri, Hemigrammus yinyang, Hemigrammus sp n., Hollandichthys aff. multifasciatus, 
Hyphessobrycon bentosi, Hyphessobrycon coelestinus, Hyphessobrycon compressus, Hyphessobrycon diancistrus, Hyphessobrycon eilyos, Hyphessobrycon epicharis, Hyphessobrycon eques, Hyphessobrycon erythrostigma, Hyphessobrycon megalopterus, Hyphessobrycon micropterus, Hyphessobrycon takasei, lguanodectes spilurus, Jupiaba polylepis, Lignobrycon myersi, Microschemobrycon sp, Moenkhausia bonita, Moenkhausia hemigrammoides, Moenkhausia intermedia, Oligosarcus pintoi, Paracheirodon axelrodi, Parapristella georgiae, Petitella georgiae, Phenacogaster franciscoensis, Poptella paraguayensis, Psellogrammus kennedyi, Rhinopetitia myersi, Roeboides bonariensis, Serrapinnus heterodon, Serrapinnus notomelas, Serrasalmus maculatus, Stethaprion erythrops, Thayeria obliqua, Triportheus nematurus

\section{9- Indeterminado}

Astyanax jacobinae, Hasemania melanura

\section{8- Ectopterigóide (ic - 0,054)}

(Vari \& Harold, 1998; Vari \& Harold, 2001: 21; Serra, 2003: 37; Benine, 2004: 42; Moreira, 2007: 53; Mirande, 2009: 162).

\section{$\underline{0 \text { - contacta o quadrado }}$}

Aphyocharax anisitsi, Bryconella pallidifrons, Cheirodon interruptus, Gymnocorymbus ternetzi, Hasemania crenuchoides, Hemigrammus barrigonae, Hemigrammus bleheri, Hemigrammus brevis, Hemigrammus coeruleus, Hemigrammus haraldi, Hemigrammus lunatus, Hemigrammus marginatus, Hemigrammus ocellifer, Hemigrammus pulcher, Hemigrammus rodwayi, Hemigrammus taphorni, Hemigrammus unilineatus, Hollandichthys aff. multifasciatus, Hyphessobrycon epicharis, Hyphessobrycon megalopterus, Iguanodectes spilurus, Moenkhausia intermedia, Moenkhausia sanctaefilomenae, Parapristella georgiae, Petitella georgiae, Pristella maxillaris, Psellogrammus kennedyi, Roeboides bonariensis, Tetragonopterus argenteus

\section{1 - não contacta o quadrado}

Astyanax jacobinae, Brycinus longipinnis, Bryconamericus exodon, Coptobrycon bilineatus, Hemigrammus analis, Hemigrammus bellottii, Hemigrammus gracilis, Hemigrammus mimus, Hemigrammus orthus, Hyphessobrycon diancistrus, Microschemobrycon sp, Moenkhausia bonita, Moenkhausia hemigrammoides, Phenacogaster franciscoensis, Piabina argentea, Rhinopetitia myersi, Serrapinnus heterodon, Serrapinnus notomelas, Serrasalmus maculatus, Thayeria obliqua

\section{2- sobrepõe-se ao quadrado}

Astyanax altiparanae, Brycon cf pesu, Bryconexodon juruenae, Bryconops melanurus, Chalceus spilogyros, Deuterodon iguape, Grundulus cochae, Hasemania melanura, Hasemania sp n., Hemigrammus arua, Hemigrammus cylindricus, Hemigrammus hyanuary, Hemigrammus levis, Hemigrammus pretoensis, Hemigrammus stictus, Hemigrammus tridens, Hemigrammus ulreyi, Hyphessobrycon coelestinus, Hyphessobrycon compressus, Hyphessobrycon eilyos, Hyphessobrycon eques, Hyphessobrycon erythrostigma, Hyphessobrycon micropterus, Hyphessobrycon takasei, Jupiaba polylepis, Lignobrycon myersi, Oligosarcus pintoi, Paracheirodon axelrodi, Poptella paraguayensis, Salminus hilarii, Stethaprion erythrops, Triportheus nematurus

\section{$\underline{0,1-P o l i m o ́ r f i c o ~}$}


Astyanax mexicanus, Hemigrammus newboldi, Hemigrammus parana, Hemigrammus rhodostomus, Hemigrammus silimoni, Hemigrammus sp n., Hemigrammus vorderwikleri, Hemigrammus yinyang

\section{$\underline{0,2-\text { Polimórfico }}$}

Hemigrammus schmardae, Hyphessobrycon bentosi

\section{1,2- Polimórfico}

Hemigrammus boesemani

\section{9- Indeterminado}

Aphyocharax pusillus, Hemigrammus skolioplatus, Moenkhausia xinguensis

\section{9- Concavidade na região dorso-posterior do metapterigóide próxima à união com o hiomandibular (ic - 0,043)}

\section{$\underline{0 \text { - ausente ou pouco conspícua }}$}

Aphyocharax anisitsi, Astyanax jacobinae, Brycinus longipinnis, Bryconamericus exodon, Bryconops melanurus, Chalceus spilogyros, Coptobrycon bilineatus, Hasemania crenuchoides, Hasemania melanura, Hasemania sp n., Hemigrammus analis, Hemigrammus barrigonae, Hemigrammus bellottii, Hemigrammus bleheri, Hemigrammus boesemani, Hemigrammus brevis, Hemigrammus coeruleus, Hemigrammus cylindricus, Hemigrammus hyanuary, Hemigrammus levis, Hemigrammus marginatus, Hemigrammus mimus, Hemigrammus orthus, Hemigrammus rodwayi, Hemigrammus silimoni, Hemigrammus skolioplatus, Hemigrammus sp n., Hemigrammus stictus, Hemigrammus taphorni, Hemigrammus ulreyi, Hemigrammus unilineatus, Hyphessobrycon bentosi, Hyphessobrycon coelestinus, Hyphessobrycon diancistrus, Hyphessobrycon eilyos, Hyphessobrycon epicharis, Hyphessobrycon eques, Hyphessobrycon erythrostigma, Hyphessobrycon micropterus, Hyphessobrycon takasei, Iguanodectes spilurus, Lignobrycon myersi, Microschemobrycon sp, Paracheirodon axelrodi, Petitella georgiae, Pristella maxillaris, Rhinopetitia myersi, Roeboides bonariensis, Serrapinnus heterodon, Serrapinnus notomelas, Serrasalmus maculatus, Thayeria obliqua, Triportheus nematurus

\section{1 - presente e acentuada}

Aphyocharax pusillus, Astyanax altiparanae, Astyanax mexicanus, Brycon cf pesu, Bryconella pallidifrons, Bryconexodon juruenae, Cheirodon interruptus, Deuterodon iguape, Grundulus cochae, Gymnocorymbus ternetzi, Hemigrammus haraldi, Hemigrammus lunatus, Hemigrammus newboldi, Hemigrammus ocellifer, Hemigrammus parana, Hemigrammus pretoensis, Hemigrammus pulcher, Hemigrammus rhodostomus, Hemigrammus schmardae, Hemigrammus tridens, Hemigrammus vorderwikleri, Hemigrammus yinyang, Hollandichthys aff. multifasciatus, Hyphessobrycon compressus, Hyphessobrycon megalopterus, Jupiaba polylepis, Moenkhausia bonita, Moenkhausia hemigrammoides, Moenkhausia intermedia, Moenkhausia sanctaefilomenae, Moenkhausia xinguensis, Oligosarcus pintoi, Parapristella georgiae, Phenacogaster franciscoensis, Piabina argentea, Poptella paraguayensis, Psellogrammus kennedyi, Salminus hilarii, Stethaprion erythrops, Tetragonopterus argenteus

\section{$\underline{0,1-\text { Polimórfico }}$}

Hemigrammus arua 


\section{9- Indeterminado}

Hemigrammus gracilis

\section{0- Projeção ântero-dorsal do metapterigóide (ic - 0,143)}

(Serra, 2003: 31; Ferreira, 2007: 66; Marinho, 2009: 43)

\section{0 - ausente}

Bryconexodon juruenae, Chalceus spilogyros, Hemigrammus taphorni, Hollandichthys aff. multifasciatus, Roeboides bonariensis

\section{1- presente, pouco desenvolvida}

Astyanax jacobinae, Brycinus longipinnis, Brycon cf pesu, Bryconamericus exodon, Bryconops melanurus, Hasemania sp n., Hemigrammus cylindricus, Hemigrammus skolioplatus, Hemigrammus ulreyi, Moenkhausia sanctaefilomenae, Piabina argentea, Pristella maxillaris, Rhinopetitia myersi, Salminus hilarii

\section{2- presente, desenvolvida, acompanhando a altura do mesopterigóide} (Figura 20)

Aphyocharax anisitsi, Aphyocharax pusillus, Astyanax altiparanae, Astyanax mexicanus, Bryconella pallidifrons, Cheirodon interruptus, Deuterodon iguape, Grundulus cochae, Gymnocorymbus ternetzi, Hasemania crenuchoides, Hasemania melanura, Hemigrammus analis, Hemigrammus arua, Hemigrammus barrigonae, Hemigrammus bellottii, Hemigrammus bleheri, Hemigrammus boesemani, Hemigrammus brevis, Hemigrammus coeruleus, Hemigrammus gracilis, Hemigrammus haraldi, Hemigrammus hyanuary, Hemigrammus levis, Hemigrammus lunatus, Hemigrammus marginatus, Hemigrammus mimus, Hemigrammus newboldi, Hemigrammus ocellifer, Hemigrammus orthus, Hemigrammus parana, Hemigrammus pretoensis, Hemigrammus pulcher, Hemigrammus rhodostomus, Hemigrammus rodwayi, Hemigrammus schmardae, Hemigrammus silimoni, Hemigrammus stictus, Hemigrammus tridens, Hemigrammus unilineatus, Hemigrammus vorderwikleri, Hemigrammus yinyang, Hemigrammus sp n., Hyphessobrycon bentosi, Hyphessobrycon coelestinus, Hyphessobrycon compressus, Hyphessobrycon diancistrus, Hyphessobrycon eilyos, Hyphessobrycon epicharis, Hyphessobrycon eques, Hyphessobrycon erythrostigma, Hyphessobrycon megalopterus, Hyphessobrycon micropterus, Hyphessobrycon takasei, Iguanodectes spilurus, Jupiaba polylepis, Lignobrycon myersi, Microschemobrycon sp, Moenkhausia bonita, Moenkhausia hemigrammoides, Moenkhausia intermedia, Moenkhausia xinguensis, Oligosarcus pintoi, Paracheirodon axelrodi, Parapristella georgiae, Petitella georgiae, Phenacogaster franciscoensis, Poptella paraguayensis, Psellogrammus kennedyi, Serrapinnus heterodon, Serrapinnus notomelas, Serrasalmus maculatus, Stethaprion erythrops, Tetragonopterus argenteus, Thayeria obliqua, Triportheus nematurus

$$
\underline{0,1-\text { Polimórfico }}
$$

\section{Coptobrycon bilineatus}

91- Metapterigóide (ic - 0,100)

(Serra, 2003: 32)

\section{$\underline{0 \text { - com uma pequena região direcionada ao simplético }}$}

Brycinus longipinnis, Brycon of pesu, Bryconexodon juruenae, Gymnocorymbus ternetzi, Hasemania crenuchoides, Hasemania sp n., Hemigrammus coeruleus, Hemigrammus 
skolioplatus, Roeboides bonariensis, Poptella paraguayensis, Rhinopetitia myersi, Salminus hilarii, Serrasalmus maculatus, Stethaprion erythrops

\section{1 - com uma grande região direcionada ao simplético}

Aphyocharax anisitsi, Aphyocharax pusillus, Astyanax altiparanae, Astyanax jacobinae, Astyanax mexicanus, Bryconamericus exodon, Bryconella pallidifrons, Bryconops melanurus, Chalceus spilogyros, Cheirodon interruptus, Coptobrycon bilineatus, Deuterodon iguape, Grundulus cochae, Hasemania melanura, Hemigrammus analis, Hemigrammus arua, Hemigrammus barrigonae, Hemigrammus bellottii, Hemigrammus bleheri, Hemigrammus boesemani, Hemigrammus brevis, Hemigrammus cylindricus, Hemigrammus gracilis, Hemigrammus haraldi, Hemigrammus hyanuary, Hemigrammus levis, Hemigrammus lunatus, Hemigrammus mimus, Hemigrammus newboldi, Hemigrammus ocellifer, Hemigrammus orthus, Hemigrammus parana, Hemigrammus pretoensis, Hemigrammus pulcher, Hemigrammus rhodostomus, Hemigrammus rodwayi, Hemigrammus schmardae, Hemigrammus silimoni, Hemigrammus stictus, Hemigrammus taphorni, Hemigrammus tridens, Hemigrammus ulreyi, Hemigrammus unilineatus, Hemigrammus vorderwikleri, Hemigrammus yinyang, Hemigrammus sp n., Hollandichthys aff. multifasciatus, Hyphessobrycon bentosi, Hyphessobrycon coelestinus, Hyphessobrycon compressus, Hyphessobrycon diancistrus, Hyphessobrycon eilyos, Hyphessobrycon epicharis, Hyphessobrycon eques, Hyphessobrycon erythrostigma, Hyphessobrycon megalopterus, Hyphessobrycon micropterus, Hyphessobrycon takasei, Iguanodectes spilurus, Jupiaba polylepis, Lignobrycon myersi, Microschemobrycon sp, Moenkhausia bonita, Moenkhausia hemigrammoides, Moenkhausia intermedia, Moenkhausia sanctaefilomenae, Moenkhausia xinguensis, Oligosarcus pintoi, Paracheirodon axelrodi, Parapristella georgiae, Petitella georgiae, Phenacogaster franciscoensis, Piabina argentea, Psellogrammus kennedyi, Pristella maxillaris, Serrapinnus heterodon, Serrapinnus notomelas, Tetragonopterus argenteus, Thayeria obliqua, Triportheus nematurus

\section{9- Indeterminado}

Hemigrammus marginatus

\section{2- Projeção óssea ventral do metapterigóide direcionada ao quadrado (ic $-0,043)$}

(Serra, 2003: 33)

\section{$\underline{0 \text { - presente }}$}

Aphyocharax anisitsi, Aphyocharax pusillus, Astyanax mexicanus, Brycinus longipinnis, Bryconamericus exodon, Bryconexodon juruenae, Bryconops melanurus, Cheirodon interruptus, Coptobrycon bilineatus, Deuterodon iguape, Hasemania melanura, Hemigrammus analis, Hemigrammus barrigonae, Hemigrammus bellottii, Hemigrammus bleheri, Hemigrammus boesemani, Hemigrammus coeruleus, Hemigrammus cylindricus, Hemigrammus gracilis, Hemigrammus lunatus, Hemigrammus marginatus, Hemigrammus newboldi, Hemigrammus ocellifer, Hemigrammus parana, Hemigrammus pretoensis, Hemigrammus rhodostomus, Hemigrammus schmardae, Hemigrammus silimoni, Hemigrammus sp n., Hemigrammus stictus, Hemigrammus taphorni, Hemigrammus tridens, Hemigrammus ulreyi, Hemigrammus vorderwikleri, Hyphessobrycon compressus, Hyphessobrycon eques, Hyphessobrycon takasei, Jupiaba polylepis, Lignobrycon myersi, Moenkhausia bonita, Moenkhausia intermedia, Moenkhausia sanctaefilomenae, Moenkhausia xinguensis, Oligosarcus pintoi, Paracheirodon axelrodi, Parapristella georgiae, Petitella georgiae, Phenacogaster franciscoensis, Piabina argentea, Serrapinnus heterodon, Serrapinnus notomelas, Tetragonopterus argenteus, Thayeria obliqua

\section{1- ausente}


Astyanax altiparanae, Astyanax jacobinae, Brycon cf pesu, Bryconella pallidifrons, Chalceus spilogyros, Grundulus cochae, Gymnocorymbus ternetzi, Hasemania crenuchoides, Hasemania $\mathrm{sp}$ n., Hemigrammus arua, Hemigrammus brevis, Hemigrammus haraldi, Hemigrammus hyanuary, Hemigrammus levis, Hemigrammus mimus, Hemigrammus orthus, Hemigrammus pulcher, Hemigrammus rodwayi, Hemigrammus skolioplatus, Hemigrammus unilineatus, Hemigrammus yinyang, Hollandichthys aff. multifasciatus, Hyphessobrycon coelestinus, Hyphessobrycon diancistrus, Hyphessobrycon eilyos, Hyphessobrycon epicharis, Hyphessobrycon erythrostigma, Hyphessobrycon megalopterus, Hyphessobrycon micropterus, Iguanodectes spilurus, Microschemobrycon sp, Poptella paraguayensis, Pristella maxillaris, Psellogrammus kennedyi, Rhinopetitia myersi, Roeboides bonariensis, Salminus hilarii, Serrasalmus maculatus, Stethaprion erythrops, Triportheus nematurus

\section{$\underline{0,1-\text { Polimórfico }}$}

Hyphessobrycon bentosi, Moenkhausia hemigrammoides

\section{3- Fenestra na região posterior do metapterigóide (ic - 0,059)}

(Serra, 2003: 35; Benine, 2004: 43; Ferreira, 2007: 67; Bertaco, 2008: 44; Marinho, 2009: 42; Mirande, 2009: 168).

\section{0- aberta posteriormente}

Brycinus longipinnis, Brycon cf pesu, Bryconella pallidifrons, Bryconops melanurus, Chalceus spilogyros, Cheirodon interruptus, Hasemania crenuchoides, Hasemania sp n., Hemigrammus bleheri, Hemigrammus cylindricus, Hemigrammus haraldi, Hemigrammus orthus, Hemigrammus rhodostomus, Hemigrammus skolioplatus, Hemigrammus stictus, Hemigrammus yinyang, Hyphessobrycon bentosi, Hyphessobrycon compressus, Hyphessobrycon diancistrus, Hyphessobrycon megalopterus, Hyphessobrycon micropterus, Hyphessobrycon takasei, Iguanodectes spilurus, Lignobrycon myersi, Microschemobrycon sp, Paracheirodon axelrodi, Petitella georgiae, Salminus hilarii, Serrapinnus notomelas, Serrasalmus maculatus, Triportheus nematurus

\section{1- totalmente fechada (Figura 20)}

Aphyocharax anisitsi, Aphyocharax pusillus, Astyanax altiparanae, Astyanax jacobinae, Astyanax mexicanus, Bryconamericus exodon, Bryconexodon juruenae, Coptobrycon bilineatus, Deuterodon iguape, Grundulus cochae, Gymnocorymbus ternetzi, Hasemania melanura, Hemigrammus analis, Hemigrammus arua, Hemigrammus barrigonae, Hemigrammus bellottii, Hemigrammus boesemani, Hemigrammus brevis, Hemigrammus coeruleus, Hemigrammus gracilis, Hemigrammus hyanuary, Hemigrammus levis, Hemigrammus lunatus, Hemigrammus marginatus, Hemigrammus mimus, Hemigrammus newboldi, Hemigrammus ocellifer, Hemigrammus parana, Hemigrammus pretoensis, Hemigrammus pulcher, Hemigrammus rodwayi, Hemigrammus schmardae, Hemigrammus silimoni, Hemigrammus taphorni, Hemigrammus tridens, Hemigrammus ulreyi, Hemigrammus unilineatus, Hemigrammus vorderwikleri, Hemigrammus sp n., Hollandichthys aff. multifasciatus, Hyphessobrycon coelestinus, Hyphessobrycon eilyos, Hyphessobrycon epicharis, Hyphessobrycon eques, Hyphessobrycon erythrostigma, Moenkhausia bonita, Moenkhausia hemigrammoides, Moenkhausia sanctaefilomenae, Moenkhausia xinguensis, Parapristella georgiae, Phenacogaster franciscoensis, Piabina argentea, Poptella paraguayensis, Psellogrammus kennedyi, Pristella maxillaris, Rhinopetitia myersi, Roeboides bonariensis, Serrapinnus heterodon, Tetragonopterus argenteus, Thayeria obliqua

\section{$\underline{0,1-\text { Polimórfico }}$}

Jupiaba polylepis, Oligosarcus pintoi, Moenkhausia intermedia, Stethaprion erythrops 


\section{4- Fenestra formada pelo metapterigóide e quadrado (ic - 0,250)}

(Buckup, 1998: 24; Benine, 2004: 45; Ferreira, 2007: 70; Moreira, 2007: 69;

Bertaco, 2008: 52)

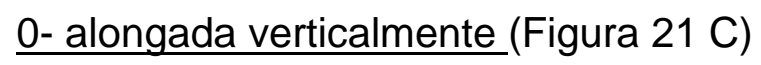

Salminus hilarii, Serrasalmus maculatus

\section{1- alongada horizontalmente (aproximadamente oval) (Figura 20)}

Aphyocharax anisitsi, Aphyocharax pusillus, Astyanax jacobinae, Brycinus longipinnis, Bryconamericus exodon, Bryconella pallidifrons, Bryconops melanurus, Cheirodon interruptus, Coptobrycon bilineatus, Deuterodon iguape, Hasemania melanura, Hemigrammus analis, Hemigrammus arua, Hemigrammus barrigonae, Hemigrammus bellottii, Hemigrammus bleheri, Hemigrammus boesemani, Hemigrammus brevis, Hemigrammus coeruleus, Hemigrammus cylindricus, Hemigrammus gracilis, Hemigrammus haraldi, Hemigrammus hyanuary, Hemigrammus levis, Hemigrammus lunatus, Hemigrammus marginatus, Hemigrammus mimus, Hemigrammus newboldi, Hemigrammus ocellifer, Hemigrammus orthus, Hemigrammus parana, Hemigrammus pulcher, Hemigrammus rhodostomus, Hemigrammus rodwayi, Hemigrammus schmardae, Hemigrammus silimoni, Hemigrammus stictus, Hemigrammus tridens, Hemigrammus ulreyi, Hemigrammus unilineatus, Hemigrammus vorderwikleri, Hemigrammus yinyang, Hemigrammus sp n., Hyphessobrycon bentosi, Hyphessobrycon compressus, Hyphessobrycon diancistrus, Hyphessobrycon epicharis, Hyphessobrycon eques, Hyphessobrycon erythrostigma, Hyphessobrycon megalopterus, Hyphessobrycon micropterus, Hyphessobrycon takasei, Iguanodectes spilurus, Lignobrycon myersi, Microschemobrycon sp, Moenkhausia bonita, Moenkhausia hemigrammoides, Moenkhausia intermedia, Moenkhausia xinguensis, Paracheirodon axelrodi, Parapristella georgiae, Petitella georgiae, Phenacogaster franciscoensis, Piabina argentea, Poptella paraguayensis, Psellogrammus kennedyi, Pristella maxillaris, Rhinopetitia myersi, Serrapinnus heterodon, Serrapinnus notomelas, Stethaprion erythrops, Thayeria obliqua, Triportheus nematurus

\section{2- globosa, arredondada (Figura $21 \mathrm{~B})$}

Astyanax altiparanae, Grundulus cochae, Hasemania crenuchoides, Hasemania sp n., Hemigrammus pretoensis, Hemigrammus taphorni, Hollandichthys aff. multifasciatus, Hyphessobrycon coelestinus, Moenkhausia sanctaefilomenae, Roeboides bonariensis, Tetragonopterus argenteus

\section{3- aproximadamente quadrada (Figura $21 \mathrm{~A}$ )}

Hemigrammus skolioplatus, Hyphessobrycon eilyos, Oligosarcus pintoi

\section{9- Indeterminado}

Astyanax mexicanus, Brycon cf pesu, Bryconexodon juruenae, Chalceus spilogyros, Gymnocorymbus ternetzi, Jupiaba polylepis

\section{5- Tamanho do quadrado (ic - 0,250)}

(Serra, 2003: 41)

\section{$\underline{0 \text { - aproximadamente tão longo quanto alto }}$}

Bryconexodon juruenae, Hollandichthys aff. multifasciatus, Roeboides bonariensis, Salminus hilarii 


\title{
1- mais longo que alto
}

Aphyocharax anisitsi, Aphyocharax pusillus, Astyanax altiparanae, Astyanax jacobinae, Astyanax mexicanus, Brycinus longipinnis, Brycon cf pesu, Bryconamericus exodon, Bryconella pallidifrons, Bryconops melanurus, Chalceus spilogyros, Cheirodon interruptus, Coptobrycon bilineatus, Deuterodon iguape, Grundulus cochae, Gymnocorymbus ternetzi, Hasemania crenuchoides, Hasemania melanura, Hasemania sp n., Hemigrammus analis, Hemigrammus arua, Hemigrammus barrigonae, Hemigrammus bellottii, Hemigrammus bleheri, Hemigrammus boesemani, Hemigrammus brevis, Hemigrammus coeruleus, Hemigrammus cylindricus, Hemigrammus gracilis, Hemigrammus haraldi, Hemigrammus hyanuary, Hemigrammus levis, Hemigrammus lunatus, Hemigrammus marginatus, Hemigrammus mimus, Hemigrammus newboldi, Hemigrammus ocellifer, Hemigrammus orthus, Hemigrammus parana, Hemigrammus pretoensis, Hemigrammus pulcher, Hemigrammus rhodostomus, Hemigrammus rodwayi, Hemigrammus schmardae, Hemigrammus silimoni, Hemigrammus skolioplatus, Hemigrammus stictus, Hemigrammus taphorni, Hemigrammus tridens, Hemigrammus ulreyi, Hemigrammus unilineatus, Hemigrammus vorderwikleri, Hemigrammus yinyang, Hemigrammus sp n., Hyphessobrycon bentosi, Hyphessobrycon coelestinus, Hyphessobrycon compressus, Hyphessobrycon diancistrus, Hyphessobrycon eilyos, Hyphessobrycon epicharis, Hyphessobrycon eques, Hyphessobrycon erythrostigma, Hyphessobrycon megalopterus, Hyphessobrycon micropterus, Hyphessobrycon takasei, Iguanodectes spilurus, Jupiaba polylepis, Lignobrycon myersi, Microschemobrycon sp, Moenkhausia bonita, Moenkhausia hemigrammoides, Moenkhausia intermedia, Moenkhausia sanctaefilomenae, Moenkhausia xinguensis, Oligosarcus pintoi, Paracheirodon axelrodi, Parapristella georgiae, Petitella georgiae, Phenacogaster franciscoensis, Piabina argentea, Poptella paraguayensis, Psellogrammus kennedyi, Pristella maxillaris, Rhinopetitia myersi, Serrapinnus heterodon, Serrapinnus notomelas, Serrasalmus maculatus, Stethaprion erythrops, Tetragonopterus argenteus, Thayeria obliqua, Triportheus nematurus

\section{6- Região póstero-ventral do quadrado (ic - 0,034)}

\author{
(Serra, 2003: 34)
}

\section{0- próxima ou em contato com o metapterigóide}

Aphyocharax anisitsi, Astyanax altiparanae, Astyanax mexicanus, Brycon cf pesu, Bryconella pallidifrons, Bryconexodon juruenae, Bryconops melanurus, Chalceus spilogyros, Coptobrycon bilineatus, Deuterodon iguape, Hemigrammus analis, Hemigrammus arua, Hemigrammus barrigonae, Hemigrammus bellottii, Hemigrammus bleheri, Hemigrammus coeruleus, Hemigrammus gracilis, Hemigrammus lunatus, Hemigrammus marginatus, Hemigrammus ocellifer, Hemigrammus orthus, Hemigrammus parana, Hemigrammus pretoensis, Hemigrammus rodwayi, Hemigrammus silimoni, Hemigrammus skolioplatus, Hemigrammus stictus, Hemigrammus taphorni, Hemigrammus tridens, Hemigrammus ulreyi, Hemigrammus sp n., Hollandichthys aff. multifasciatus, Hyphessobrycon coelestinus, Hyphessobrycon compressus, Hyphessobrycon eilyos, Hyphessobrycon eques, Hyphessobrycon megalopterus, Hyphessobrycon micropterus, Hyphessobrycon takasei, Jupiaba polylepis, Microschemobrycon sp, Moenkhausia bonita, Moenkhausia sanctaefilomenae, Oligosarcus pintoi, Paracheirodon axelrodi, Parapristella georgiae, Petitella georgiae, Phenacogaster franciscoensis, Piabina argentea, Roeboides bonariensis, Salminus hilarii, Serrapinnus heterodon, Serrasalmus maculatus, Tetragonopterus argenteus

\section{1- distante do metapterigóide}

Aphyocharax pusillus, Astyanax jacobinae, Brycinus longipinnis, Bryconamericus exodon, Cheirodon interruptus, Grundulus cochae, Gymnocorymbus ternetzi, Hasemania crenuchoides, Hasemania melanura, Hasemania sp n., Hemigrammus brevis, Hemigrammus cylindricus, Hemigrammus haraldi, Hemigrammus hyanuary, Hemigrammus levis, Hemigrammus mimus, Hemigrammus newboldi, Hemigrammus pulcher, Hemigrammus rhodostomus, Hemigrammus schmardae, Hemigrammus unilineatus, Hemigrammus vorderwikleri, Hemigrammus yinyang, 
Hyphessobrycon bentosi, Hyphessobrycon diancistrus, Hyphessobrycon epicharis, Hyphessobrycon erythrostigma, Iguanodectes spilurus, Lignobrycon myersi, Moenkhausia intermedia, Moenkhausia xinguensis, Poptella paraguayensis, Pristella maxillaris, Psellogrammus kennedyi, Rhinopetitia myersi, Serrapinnus notomelas, Stethaprion erythrops, Thayeria obliqua, Triportheus nematurus

\section{$\underline{0,1-P o l i m o ́ r f i c o ~}$}

Hemigrammus boesemani, Moenkhausia hemigrammoides

97- Hiomandibular (ic - 0,333)

(Serra, 2003: 40)

$\underline{0 \text { - largo e abaulado, com a região dorsal às vezes mais larga que } 0}$ restante do osso e a região mediana convexa

Hasemania crenuchoides, Hasemania sp n., Hollandichthys aff. multifasciatus, Piabina argentea

\section{1- estreito e mais retangular, com a região dorsal aproximadamente da} mesma largura do restante do osso e a região mediana aproximadamente reta (Figura 20)

Aphyocharax anisitsi, Aphyocharax pusillus, Astyanax altiparanae, Astyanax jacobinae, Astyanax mexicanus, Brycinus longipinnis, Brycon cf pesu, Bryconamericus exodon, Bryconella pallidifrons, Bryconexodon juruenae, Bryconops melanurus, Chalceus spilogyros, Cheirodon interruptus, Coptobrycon bilineatus, Deuterodon iguape, Grundulus cochae, Gymnocorymbus ternetzi, Hasemania melanura, Hemigrammus analis, Hemigrammus arua, Hemigrammus barrigonae, Hemigrammus bellottii, Hemigrammus bleheri, Hemigrammus boesemani, Hemigrammus brevis, Hemigrammus coeruleus, Hemigrammus cylindricus, Hemigrammus gracilis, Hemigrammus haraldi, Hemigrammus hyanuary, Hemigrammus levis, Hemigrammus lunatus, Hemigrammus marginatus, Hemigrammus mimus, Hemigrammus newboldi, Hemigrammus ocellifer, Hemigrammus orthus, Hemigrammus parana, Hemigrammus pretoensis, Hemigrammus pulcher, Hemigrammus rhodostomus, Hemigrammus rodwayi, Hemigrammus schmardae, Hemigrammus silimoni, Hemigrammus skolioplatus, Hemigrammus stictus, Hemigrammus taphorni, Hemigrammus tridens, Hemigrammus ulreyi, Hemigrammus unilineatus, Hemigrammus vorderwikleri, Hemigrammus yinyang, Hemigrammus sp n., Hyphessobrycon bentosi, Hyphessobrycon coelestinus, Hyphessobrycon compressus, Hyphessobrycon diancistrus, Hyphessobrycon eilyos, Hyphessobrycon epicharis, Hyphessobrycon eques, Hyphessobrycon erythrostigma, Hyphessobrycon megalopterus, Hyphessobrycon micropterus, Hyphessobrycon takasei, Iguanodectes spilurus, Jupiaba polylepis, Lignobrycon myersi, Microschemobrycon sp, Moenkhausia bonita, Moenkhausia hemigrammoides, Moenkhausia intermedia, Moenkhausia sanctaefilomenae, Moenkhausia xinguensis, Oligosarcus pintoi, Paracheirodon axelrodi, Parapristella georgiae, Petitella georgiae, Phenacogaster franciscoensis, Poptella paraguayensis, Psellogrammus kennedyi, Pristella maxillaris, Rhinopetitia myersi, Roeboides bonariensis, Serrapinnus heterodon, Serrapinnus notomelas, Stethaprion erythrops, Tetragonopterus argenteus, Thayeria obliqua, Triportheus nematurus

\section{9- Indeterminado}

Salminus hilarii, Serrasalmus maculatus 


\section{8- Canal latero-sensorial do pré-opérculo (ic - 0,083)}

(Serra, 2003: 29)

\section{$\underline{0 \text { - próximo ou atingindo a margem dorsal formada pelo hiomandibular } \mathrm{e}}$}

\section{o opérculo}

Aphyocharax anisitsi, Aphyocharax pusillus, Astyanax altiparanae, Astyanax jacobinae, Astyanax mexicanus, Brycinus longipinnis, Brycon cf pesu, Bryconamericus exodon, Bryconexodon juruenae, Bryconops melanurus, Chalceus spilogyros, Gymnocorymbus ternetzi, Hemigrammus analis, Hemigrammus arua, Hemigrammus barrigonae, Hemigrammus bleheri, Hemigrammus boesemani, Hemigrammus brevis, Hemigrammus coeruleus, Hemigrammus cylindricus, Hemigrammus gracilis, Hemigrammus hyanuary, Hemigrammus levis, Hemigrammus lunatus, Hemigrammus marginatus, Hemigrammus mimus, Hemigrammus newboldi, Hemigrammus ocellifer, Hemigrammus parana, Hemigrammus pretoensis, Hemigrammus pulcher, Hemigrammus rhodostomus, Hemigrammus rodwayi, Hemigrammus silimoni, Hemigrammus skolioplatus, Hemigrammus stictus, Hemigrammus taphorni, Hemigrammus ulreyi, Hemigrammus unilineatus, Hemigrammus sp n., Hyphessobrycon bentosi, Hyphessobrycon diancistrus, Hyphessobrycon erythrostigma, Hyphessobrycon takasei, Iguanodectes spilurus, Jupiaba polylepis, Microschemobrycon sp, Moenkhausia bonita, Moenkhausia hemigrammoides, Moenkhausia intermedia, Moenkhausia sanctaefilomenae, Moenkhausia xinguensis, Oligosarcus pintoi, Parapristella georgiae, Petitella georgiae, Phenacogaster franciscoensis, Piabina argentea, Poptella paraguayensis, Pristella maxillaris, Rhinopetitia myersi, Roeboides bonariensis, Salminus hilarii, Serrapinnus heterodon, Serrapinnus notomelas, Serrasalmus maculatus, Stethaprion erythrops, Tetragonopterus argenteus, Triportheus nematurus

\section{1- não atingindo essa margem, geralmente ficando restrito a região d a}

\section{articulação entre hiomandibular e opérculo}

Bryconella pallidifrons, Cheirodon interruptus, Coptobrycon bilineatus, Deuterodon iguape, Grundulus cochae, Hasemania crenuchoides, Hasemania melanura, Hasemania sp n., Hemigrammus bellottii, Hemigrammus haraldi, Hemigrammus orthus, Hemigrammus schmardae, Hemigrammus tridens, Hemigrammus vorderwikleri, Hemigrammus yinyang, Hyphessobrycon coelestinus, Hyphessobrycon compressus, Hyphessobrycon eilyos, Hyphessobrycon epicharis, Hyphessobrycon eques, Hyphessobrycon megalopterus, Hyphessobrycon micropterus, Lignobrycon myersi, Paracheirodon axelrodi, Psellogrammus kennedyi, Thayeria obliqua

\section{9- Indeterminado}

Hollandichthys aff. multifasciatus

\section{Arco Hióideo}

99- Abas laterais do uro-hial (ic - 0,125)

(Moreira, 2007: 197)

\section{0- reduzidas (fundidas com o eixo médio) ou ausentes (Figura $23 \mathrm{~A}$ )}

Hemigrammus hyanuary, Hemigrammus schmardae, Hyphessobrycon bentosi, Hyphessobrycon eques, Hyphessobrycon erythrostigma, Paracheirodon axelrodi, Petitella georgiae, Thayeria obliqua 
1- desenvolvidas, formando superfícies aplanadas ventro-laterais ao longo do eixo médio (Figura 23 B - D)

Aphyocharax anisitsi, Aphyocharax pusillus, Astyanax altiparanae, Astyanax jacobinae, Astyanax mexicanus, Brycinus longipinnis, Brycon cf pesu, Bryconamericus exodon, Bryconella pallidifrons, Bryconexodon juruenae, Bryconops melanurus, Chalceus spilogyros, Cheirodon interruptus, Coptobrycon bilineatus, Deuterodon iguape, Grundulus cochae, Gymnocorymbus ternetzi, Hasemania crenuchoides, Hasemania melanura, Hasemania sp n., Hemigrammus analis, Hemigrammus arua, Hemigrammus barrigonae, Hemigrammus bellottii, Hemigrammus bleheri, Hemigrammus boesemani, Hemigrammus brevis, Hemigrammus coeruleus, Hemigrammus cylindricus, Hemigrammus gracilis, Hemigrammus haraldi, Hemigrammus levis, Hemigrammus lunatus, Hemigrammus marginatus, Hemigrammus mimus, Hemigrammus newboldi, Hemigrammus ocellifer, Hemigrammus orthus, Hemigrammus parana, Hemigrammus pretoensis, Hemigrammus pulcher, Hemigrammus rhodostomus, Hemigrammus rodwayi, Hemigrammus silimoni, Hemigrammus skolioplatus, Hemigrammus stictus, Hemigrammus taphorni, Hemigrammus tridens, Hemigrammus ulreyi, Hemigrammus unilineatus, Hemigrammus vorderwikleri, Hemigrammus yinyang, Hemigrammus sp n., Hollandichthys aff. multifasciatus, Hyphessobrycon coelestinus, Hyphessobrycon compressus, Hyphessobrycon diancistrus, Hyphessobrycon eilyos, Hyphessobrycon epicharis, Hyphessobrycon megalopterus, Hyphessobrycon micropterus, Hyphessobrycon takasei, lguanodectes spilurus, Jupiaba polylepis, Lignobrycon myersi, Microschemobrycon sp, Moenkhausia bonita, Moenkhausia hemigrammoides, Moenkhausia intermedia, Moenkhausia sanctaefilomenae, Moenkhausia xinguensis, Oligosarcus pintoi, Parapristella georgiae, Phenacogaster franciscoensis, Piabina argentea, Poptella paraguayensis, Psellogrammus kennedyi, Pristella maxillaris, Rhinopetitia myersi, Roeboides bonariensis, Salminus hilarii, Serrapinnus heterodon, Serrapinnus notomelas, Serrasalmus maculatus, Stethaprion erythrops, Tetragonopterus argenteus, Triportheus nematurus

Segundo Zanata e Vari (2005) em vários grupos dentro de Characiformes o uro-hial é tripartido em corte transversal, com uma asa mediana dorsalmente direcionada (tratada aqui como eixo médio) e um processo ventro-lateralmente dirigido de cada lado (chamado aqui de aba lateral). Ainda segundo os autores essas abas laterais apresentam graus diferentes de desenvolvimento dentro de Characiformes. Dentre as espécies examinadas, todas apresentam o uro-hial tripartido, entretanto, o grau de desenvolvimento das asbas laterais apresenta diferença, especialmente comparando-se as espécies incluídas no clado 184 (abas menos desenvolvidas) com aquelas que ficaram fora desse clado (abas mais desenvolvidas. Dentro ainda das espécies incluídas no clado 184, algumas apresentam alto grau de redução das abas laterais do uro-hial, nesses casos as abas são muito estreitas e curtas e em alguns casos completamente fundidas como o eixo médio.

100- Tamanho das abas laterais do uro-hial em vista dorsal (ic - 0,333)

(Moreira, 2007: 200) 
0- aproximadamente tão longas quanto a extremidade posterior do eixo médio ou mais longas (Figura 23 D)

Brycinus longipinnis, Brycon cf pesu, Chalceus spilogyros, Serrasalmus maculatus

1- mais curtas que a extremidade posterior do eixo médio (Figura $23 \mathrm{~A}$ -

C)

Aphyocharax anisitsi, Aphyocharax pusillus, Astyanax altiparanae, Astyanax jacobinae, Astyanax mexicanus, Bryconamericus exodon, Bryconella pallidifrons, Bryconexodon juruenae, Bryconops melanurus, Cheirodon interruptus, Coptobrycon bilineatus, Deuterodon iguape, Grundulus cochae, Gymnocorymbus ternetzi, Hasemania crenuchoides, Hasemania melanura, Hasemania sp n., Hemigrammus analis, Hemigrammus arua, Hemigrammus barrigonae, Hemigrammus bellottii, Hemigrammus bleheri, Hemigrammus boesemani, Hemigrammus brevis, Hemigrammus coeruleus, Hemigrammus cylindricus, Hemigrammus gracilis, Hemigrammus haraldi, Hemigrammus hyanuary, Hemigrammus levis, Hemigrammus lunatus, Hemigrammus marginatus, Hemigrammus mimus, Hemigrammus newboldi, Hemigrammus ocellifer, Hemigrammus orthus, Hemigrammus parana, Hemigrammus pretoensis, Hemigrammus pulcher, Hemigrammus rhodostomus, Hemigrammus rodwayi, Hemigrammus schmardae, Hemigrammus silimoni, Hemigrammus skolioplatus, Hemigrammus stictus, Hemigrammus taphorni, Hemigrammus tridens, Hemigrammus ulreyi, Hemigrammus unilineatus, Hemigrammus vorderwikleri, Hemigrammus yinyang, Hemigrammus sp n., Hollandichthys aff. multifasciatus, Hyphessobrycon bentosi, Hyphessobrycon coelestinus, Hyphessobrycon compressus, Hyphessobrycon diancistrus, Hyphessobrycon eilyos, Hyphessobrycon epicharis, Hyphessobrycon eques, Hyphessobrycon erythrostigma, Hyphessobrycon megalopterus, Hyphessobrycon micropterus, Hyphessobrycon takasei, Iguanodectes spilurus, Jupiaba polylepis, Lignobrycon myersi, Microschemobrycon sp, Moenkhausia bonita, Moenkhausia hemigrammoides, Moenkhausia intermedia, Moenkhausia sanctaefilomenae, Moenkhausia xinguensis, Oligosarcus pintoi, Paracheirodon axelrodi, Parapristella georgiae, Petitella georgiae, Phenacogaster franciscoensis, Piabina argentea, Poptella paraguayensis, Psellogrammus kennedyi, Pristella maxillaris, Rhinopetitia myersi, Roeboides bonariensis, Salminus hilarii, Serrapinnus heterodon, Serrapinnus notomelas, Stethaprion erythrops, Tetragonopterus argenteus, Thayeria obliqua, Triportheus nematurus

As abas laterais do uro-hial, na grande maioria das espécies analisadas, são mais curtas que a extremidade posterior do eixo médio. Entretanto, em algumas espécies posicionadas como mais basais na análise ( $B$. longipinnis, $C$. spilogyros e Brycon cf pesu.), essas abas são bastante longas, atingindo ou ultrapassando a extremidade posterior do eixo médio. Condição semelhante aparece como adquirida independentemente para S. maculatus.

\section{1- Margem posterior das abas do uro-hial (ic - 0,143)}

(Serra, 2003: 78)

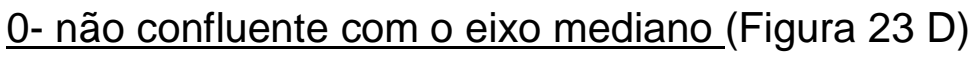

Astyanax jacobinae, Brycinus longipinnis, Brycon cf pesu, Chalceus spilogyros, Deuterodon iguape, Hemigrammus coeruleus, Hollandichthys aff. multifasciatus, Hyphessobrycon compressus, Iguanodectes spilurus, Oligosarcus pintoi, Salminus hilarii, Serrapinnus heterodon, Serrapinnus notomelas, Triportheus nematurus 
Serra, J. P. - Análise Filogenética das Espécies de Hemigrammus Gill, 1858 (Characiformes, Characidae)

1- afilada medianamente, confluente com o eixo mediano (Figura $23 \mathrm{~A}$ -

C)

Aphyocharax anisitsi, Aphyocharax pusillus, Astyanax altiparanae, Astyanax mexicanus, Bryconamericus exodon, Bryconella pallidifrons, Bryconexodon juruenae, Bryconops melanurus, Gymnocorymbus ternetzi, Hasemania crenuchoides, Hasemania melanura, Hasemania sp n., Hemigrammus analis, Hemigrammus arua, Hemigrammus barrigonae, Hemigrammus bellottii, Hemigrammus bleheri, Hemigrammus boesemani, Hemigrammus brevis, Hemigrammus cylindricus, Hemigrammus gracilis, Hemigrammus haraldi, Hemigrammus hyanuary, Hemigrammus levis, Hemigrammus lunatus, Hemigrammus marginatus, Hemigrammus mimus, Hemigrammus newboldi, Hemigrammus orthus, Hemigrammus parana, Hemigrammus pretoensis, Hemigrammus pulcher, Hemigrammus rhodostomus, Hemigrammus rodwayi, Hemigrammus schmardae, Hemigrammus silimoni, Hemigrammus skolioplatus, Hemigrammus stictus, Hemigrammus taphorni, Hemigrammus tridens, Hemigrammus ulreyi, Hemigrammus unilineatus, Hemigrammus vorderwikleri, Hemigrammus yinyang, Hemigrammus sp n., Hyphessobrycon bentosi, Hyphessobrycon coelestinus, Hyphessobrycon diancistrus, Hyphessobrycon eilyos, Hyphessobrycon epicharis, Hyphessobrycon eques, Hyphessobrycon erythrostigma, Hyphessobrycon megalopterus, Hyphessobrycon micropterus, Hyphessobrycon takasei, Jupiaba polylepis, Microschemobrycon sp, Moenkhausia bonita, Moenkhausia hemigrammoides, Moenkhausia intermedia, Moenkhausia xinguensis, Paracheirodon axelrodi, Parapristella georgiae, Petitella georgiae, Phenacogaster franciscoensis, Piabina argentea, Poptella paraguayensis, Psellogrammus kennedyi, Pristella maxillaris, Rhinopetitia myersi, Roeboides bonariensis, Serrasalmus maculatus, Stethaprion erythrops, Tetragonopterus argenteus, Thayeria obliqua

\section{0, 1- Polimórfico}

Coptobrycon bilineatus, Hemigrammus ocellifer, Moenkhausia sanctaefilomenae

\section{9- Indeterminado}

Cheirodon interruptus, Grundulus cochae, Lignobrycon myersi

102- Uro-hial (ic - 0,333)

0- porção externa da margem posterior das abas projetando-se posteriormente (Figura $23 \mathrm{D}$ )

Brycinus longipinnis, Brycon cf pesu, Iguanodectes spilurus, Triportheus nematurus

\section{1- porção externa da margem posterior das abas não se projetando} posteriormente, terminando na mesma altura da porção mediana da margem posterior das abas (Figura $23 \mathrm{~A}-\mathrm{C}$ )

Aphyocharax anisitsi, Aphyocharax pusillus, Astyanax altiparanae, Astyanax jacobinae, Astyanax mexicanus, Bryconamericus exodon, Bryconella pallidifrons, Bryconexodon juruenae, Bryconops melanurus, Chalceus spilogyros, Cheirodon interruptus, Coptobrycon bilineatus, Deuterodon iguape, Grundulus cochae, Gymnocorymbus ternetzi, Hasemania crenuchoides, Hasemania melanura, Hasemania sp n., Hemigrammus analis, Hemigrammus arua, Hemigrammus barrigonae, Hemigrammus bellottii, Hemigrammus bleheri, Hemigrammus boesemani, Hemigrammus brevis, Hemigrammus coeruleus, Hemigrammus cylindricus, Hemigrammus gracilis, Hemigrammus haraldi, Hemigrammus hyanuary, Hemigrammus levis, Hemigrammus lunatus, Hemigrammus marginatus, Hemigrammus mimus, Hemigrammus newboldi, Hemigrammus ocellifer, Hemigrammus orthus, Hemigrammus parana, Hemigrammus 
pretoensis, Hemigrammus pulcher, Hemigrammus rhodostomus, Hemigrammus rodwayi, Hemigrammus schmardae, Hemigrammus silimoni, Hemigrammus skolioplatus, Hemigrammus stictus, Hemigrammus taphorni, Hemigrammus tridens, Hemigrammus ulreyi, Hemigrammus unilineatus, Hemigrammus vorderwikleri, Hemigrammus yinyang, Hemigrammus sp n., Hollandichthys aff. multifasciatus, Hyphessobrycon bentosi, Hyphessobrycon coelestinus, Hyphessobrycon diancistrus, Hyphessobrycon eilyos, Hyphessobrycon epicharis, Hyphessobrycon eques, Hyphessobrycon erythrostigma, Hyphessobrycon megalopterus, Hyphessobrycon micropterus, Hyphessobrycon takasei, Jupiaba polylepis, Lignobrycon myersi, Microschemobrycon sp, Moenkhausia bonita, Moenkhausia hemigrammoides, Moenkhausia intermedia, Moenkhausia sanctaefilomenae, Moenkhausia xinguensis, Oligosarcus pintoi, Paracheirodon axelrodi, Parapristella georgiae, Petitella georgiae, Phenacogaster franciscoensis, Piabina argentea, Poptella paraguayensis, Psellogrammus kennedyi, Pristella maxillaris, Rhinopetitia myersi, Roeboides bonariensis, Salminus hilarii, Serrapinnus heterodon, Serrapinnus notomelas, Serrasalmus maculatus, Stethaprion erythrops, Tetragonopterus argenteus, Thayeria obliqua

\author{
$\underline{0,1-\text { Polimórfico }}$
}

Hyphessobrycon compressus

103- Margem posterior do eixo médio do uro-hial em vista lateral(ic 0,036)

(Serra, 2003:79)

\title{
0- com uma ou várias pontas, ou com reentrâncias pronunciadas (Figura
}

$23 \mathrm{~A}-\mathrm{B})$

Aphyocharax anisitsi, Aphyocharax pusillus, Bryconexodon juruenae, Grundulus cochae, Hasemania crenuchoides, Hasemania melanura, Hemigrammus analis, Hemigrammus arua, Hemigrammus barrigonae, Hemigrammus boesemani, Hemigrammus coeruleus, Hemigrammus cylindricus, Hemigrammus gracilis, Hemigrammus haraldi, Hemigrammus hyanuary, Hemigrammus lunatus, Hemigrammus marginatus, Hemigrammus orthus, Hemigrammus parana, Hemigrammus pulcher, Hemigrammus silimoni, Hemigrammus tridens, Hemigrammus ulreyi, Hemigrammus unilineatus, Hemigrammus vorderwikleri, Hemigrammus yinyang, Hemigrammus sp n., Hollandichthys aff. multifasciatus, Hyphessobrycon bentosi, Hyphessobrycon compressus, Hyphessobrycon eilyos, Hyphessobrycon epicharis, Hyphessobrycon eques, Hyphessobrycon micropterus, Jupiaba polylepis, Lignobrycon myersi, Microschemobrycon sp, Moenkhausia bonita, Moenkhausia hemigrammoides, Oligosarcus pintoi, Paracheirodon axelrodi, Parapristella georgiae, Piabina argentea, Psellogrammus kennedyi, Roeboides bonariensis, Salminus hilarii, Serrapinnus heterodon, Serrapinnus notomelas, Tetragonopterus argenteus, Thayeria obliqua

\section{1- aproximadamente lisa, sem pontas ou reentrâncias (Figura 23 C - D)}

Astyanax altiparanae, Astyanax jacobinae, Astyanax mexicanus, Brycinus longipinnis, Brycon cf pesu, Bryconops melanurus, Chalceus spilogyros, Cheirodon interruptus, Deuterodon iguape, Gymnocorymbus ternetzi, Hasemania sp n., Hemigrammus bellottii, Hemigrammus bleheri, Hemigrammus brevis, Hemigrammus levis, Hemigrammus mimus, Hemigrammus newboldi, Hemigrammus ocellifer, Hemigrammus pretoensis, Hemigrammus rhodostomus, Hemigrammus rodwayi, Hemigrammus schmardae, Hemigrammus skolioplatus, Hemigrammus stictus, Hemigrammus taphorni, Hyphessobrycon coelestinus, Hyphessobrycon diancistrus, Hyphessobrycon erythrostigma, Hyphessobrycon megalopterus, Hyphessobrycon takasei, Moenkhausia intermedia, Moenkhausia sanctaefilomenae, Moenkhausia xinguensis, Petitella georgiae, Phenacogaster franciscoensis, Poptella paraguayensis, Pristella maxillaris, Rhinopetitia myersi, Serrasalmus maculatus, Stethaprion erythrops, Triportheus nematurus 
Serra, J. P. - Análise Filogenética das Espécies de Hemigrammus Gill, 1858 (Characiformes, Characidae)

9- Indeterminado

Bryconamericus exodon, Bryconella pallidifrons, Coptobrycon bilineatus, Iguanodectes spilurus

\section{Arcos Branquiais}

104- Forma do basi-hial (ic - 0,038 )

(Serra, 2003: 76; Benine, 2004: 54; Moreira, 2007: 183; Ferreira, 2007: 82;

Bertaco, 2008: 58; Marinho, 2009: 45)

0- acentuadamente triangular, com a região anterior expandida (duas ou mais vezes mais larga que a posterior) (Figura 24)

Astyanax jacobinae, Brycinus longipinnis, Bryconamericus exodon, Bryconella pallidifrons, Bryconexodon juruenae, Bryconops melanurus, Chalceus spilogyros, Cheirodon interruptus, Grundulus cochae, Hasemania melanura, Hemigrammus analis, Hemigrammus arua, Hemigrammus barrigonae, Hemigrammus bellottii, Hemigrammus bleheri, Hemigrammus boesemani, Hemigrammus brevis, Hemigrammus coeruleus, Hemigrammus haraldi, Hemigrammus levis, Hemigrammus lunatus, Hemigrammus mimus, Hemigrammus newboldi, Hemigrammus ocellifer, Hemigrammus orthus, Hemigrammus pretoensis, Hemigrammus pulcher, Hemigrammus rhodostomus, Hemigrammus schmardae, Hemigrammus silimoni, Hemigrammus skolioplatus, Hemigrammus stictus, Hemigrammus unilineatus, Hemigrammus yinyang, Hyphessobrycon diancistrus, Hyphessobrycon eilyos, Hyphessobrycon eques, Hyphessobrycon megalopterus, Hyphessobrycon takasei, Iguanodectes spilurus, Jupiaba polylepis, Moenkhausia bonita, Moenkhausia intermedia, Moenkhausia xinguensis, Parapristella georgiae, Petitella georgiae, Piabina argentea, Rhinopetitia myersi, Salminus hilarii, Serrapinnus heterodon, Tetragonopterus argenteus, Thayeria obliqua

\section{1- mais retangular, com a região dorsal pouco expandida, igual ou pouco} mais larga que a ventral (menos de 2 vezes)

Aphyocharax anisitsi, Aphyocharax pusillus, Astyanax altiparanae, Astyanax mexicanus, Brycon cf pesu, Coptobrycon bilineatus, Deuterodon iguape, Gymnocorymbus ternetzi, Hasemania crenuchoides, Hasemania sp n., Hemigrammus cylindricus, Hemigrammus gracilis, Hemigrammus hyanuary, Hemigrammus parana, Hemigrammus rodwayi, Hemigrammus sp n., Hemigrammus taphorni, Hemigrammus tridens, Hemigrammus ulreyi, Hemigrammus vorderwikleri, Hollandichthys aff. multifasciatus, Hyphessobrycon bentosi, Hyphessobrycon coelestinus, Hyphessobrycon compressus, Hyphessobrycon epicharis, Hyphessobrycon erythrostigma, Hyphessobrycon micropterus, Lignobrycon myersi, Microschemobrycon sp, Moenkhausia hemigrammoides, Moenkhausia sanctaefilomenae, Oligosarcus pintoi, Paracheirodon axelrodi, Phenacogaster franciscoensis, Poptella paraguayensis, Pristella maxillaris, Psellogrammus kennedyi, Roeboides bonariensis, Serrapinnus notomelas, Serrasalmus maculatus, Stethaprion erythrops, Triportheus nematurus

\section{9- Indeterminado}

Hemigrammus marginatus

105- Número de rastros branquiais no $1^{\circ}$ ceratobranq uial (ic $-0,150$ )

(Serra, 2003: 82; Mirande, 2009: 196)

$\underline{0-\text { mais de trinta rastros }}$ 
Lignobrycon myersi, Moenkhausia intermedia, Triportheus nematurus

\section{1- onze a treze rastros}

Cheirodon interruptus, Gymnocorymbus ternetzi, Hemigrammus brevis, Hemigrammus gracilis, Hemigrammus levis, Hemigrammus schmardae, Hyphessobrycon compressus,

Hyphessobrycon diancistrus, Hyphessobrycon epicharis, Hyphessobrycon erythrostigma, Hyphessobrycon megalopterus, Jupiaba polylepis, Moenkhausia bonita, Parapristella georgiae, Petitella georgiae, Roeboides bonariensis, Tetragonopterus argenteus, Thayeria obliqua

\section{2- sete a dez rastros}

Aphyocharax anisitsi, Aphyocharax pusillus, Astyanax altiparanae, Astyanax jacobinae, Astyanax mexicanus, Brycinus longipinnis, Brycon cf pesu, Bryconamericus exodon, Bryconella pallidifrons, Bryconexodon juruenae, Chalceus spilogyros, Coptobrycon bilineatus, Deuterodon iguape, Grundulus cochae, Hasemania crenuchoides, Hasemania melanura, Hasemania sp n., Hemigrammus analis, Hemigrammus arua, Hemigrammus barrigonae, Hemigrammus bellottii, Hemigrammus bleheri, Hemigrammus boesemani, Hemigrammus coeruleus, Hemigrammus cylindricus, Hemigrammus haraldi, Hemigrammus hyanuary, Hemigrammus lunatus, Hemigrammus marginatus, Hemigrammus mimus, Hemigrammus newboldi, Hemigrammus ocellifer, Hemigrammus orthus, Hemigrammus parana, Hemigrammus pretoensis, Hemigrammus pulcher, Hemigrammus rhodostomus, Hemigrammus rodwayi, Hemigrammus silimoni, Hemigrammus skolioplatus, Hemigrammus stictus, Hemigrammus taphorni, Hemigrammus tridens, Hemigrammus ulreyi, Hemigrammus unilineatus, Hemigrammus vorderwikleri, Hemigrammus yinyang, Hemigrammus sp n., Hollandichthys aff. multifasciatus, Hyphessobrycon coelestinus, Hyphessobrycon eilyos, Hyphessobrycon eques, Hyphessobrycon micropterus, Hyphessobrycon takasei, Microschemobrycon sp, Moenkhausia hemigrammoides, Moenkhausia sanctaefilomenae, Moenkhausia xinguensis, Oligosarcus pintoi, Paracheirodon axelrodi, Poptella paraguayensis, Psellogrammus kennedyi, Pristella maxillaris, Rhinopetitia myersi, Salminus hilarii, Serrapinnus heterodon, Serrapinnus notomelas, Serrasalmus maculatus, Stethaprion erythrops

\section{3- um a seis rastros}

Bryconops melanurus, Iguanodectes spilurus, Phenacogaster franciscoensis, Piabina argentea

\section{1, 2- Polimórfico}

Hyphessobrycon bentosi

\section{6- Ceratobranquiais com duas séries de rastros branquiais (ic - 0,250)}

(Serra, 2003: 83)

\section{0- ceratobranquiais três e quatro (Figura 24)}

Aphyocharax anisitsi, Aphyocharax pusillus, Astyanax altiparanae, Astyanax jacobinae, Astyanax mexicanus, Brycinus longipinnis, Brycon cf pesu, Bryconamericus exodon, Bryconella pallidifrons, Bryconops melanurus, Chalceus spilogyros, Deuterodon iguape, Hasemania crenuchoides, Hasemania sp n., Hemigrammus analis, Hemigrammus barrigonae, Hemigrammus bellottii, Hemigrammus bleheri, Hemigrammus boesemani, Hemigrammus brevis, Hemigrammus coeruleus, Hemigrammus cylindricus, Hemigrammus gracilis, Hemigrammus haraldi, Hemigrammus hyanuary, Hemigrammus levis, Hemigrammus lunatus, 
Hemigrammus marginatus, Hemigrammus mimus, Hemigrammus newboldi, Hemigrammus ocellifer, Hemigrammus orthus, Hemigrammus parana, Hemigrammus pretoensis, Hemigrammus pulcher, Hemigrammus rhodostomus, Hemigrammus rodwayi, Hemigrammus schmardae, Hemigrammus silimoni, Hemigrammus skolioplatus, Hemigrammus stictus, Hemigrammus taphorni, Hemigrammus tridens, Hemigrammus ulreyi, Hemigrammus unilineatus, Hemigrammus vorderwikleri, Hemigrammus yinyang, Hemigrammus sp n., Hollandichthys aff. multifasciatus, Hyphessobrycon bentosi, Hyphessobrycon coelestinus, Hyphessobrycon compressus, Hyphessobrycon diancistrus, Hyphessobrycon epicharis, Hyphessobrycon eques, Hyphessobrycon erythrostigma, Hyphessobrycon megalopterus, Hyphessobrycon micropterus, Hyphessobrycon takasei, Jupiaba polylepis, Lignobrycon myersi, Microschemobrycon sp, Moenkhausia bonita, Moenkhausia hemigrammoides, Moenkhausia intermedia, Moenkhausia sanctaefilomenae, Moenkhausia xinguensis, Oligosarcus pintoi, Paracheirodon axelrodi, Parapristella georgiae, Petitella georgiae, Phenacogaster franciscoensis, Piabina argentea, Poptella paraguayensis, Psellogrammus kennedyi, Pristella maxillaris, Roeboides bonariensis, Salminus hilarii, Serrapinnus heterodon, Serrapinnus notomelas, Stethaprion erythrops, Tetragonopterus argenteus, Thayeria obliqua, Triportheus nematurus

\section{1- ceratobranquiais um, dois, três e quatro}

Cheirodon interruptus, Coptobrycon bilineatus, Grundulus cochae, Iguanodectes spilurus, Rhinopetitia myersi, Serrasalmus maculatus

\section{2- ceratobranquiais dois, três e quatro}

Bryconexodon juruenae, Hasemania melanura, Hemigrammus arua

\section{9- Indeterminado}

Gymnocorymbus ternetzi, Hyphessobrycon eilyos

\section{7- Epibranquiais com duas séries de rastros branquiais (ic - 1,0)}

(Serra, 2003: 84)

\section{0- epibranquiais dois e três}

Microschemobrycon sp

\section{1- epibranquiais um, dois e três (Figura 24)}

Aphyocharax anisitsi, Aphyocharax pusillus, Astyanax altiparanae, Astyanax jacobinae, Astyanax mexicanus, Brycinus longipinnis, Brycon cf pesu, Bryconamericus exodon, Bryconella pallidifrons, Bryconexodon juruenae, Bryconops melanurus, Chalceus spilogyros, Cheirodon interruptus, Coptobrycon bilineatus, Deuterodon iguape, Grundulus cochae, Gymnocorymbus ternetzi, Hasemania crenuchoides, Hasemania melanura, Hasemania sp n., Hemigrammus analis, Hemigrammus arua, Hemigrammus barrigonae, Hemigrammus bellottii, Hemigrammus bleheri, Hemigrammus boesemani, Hemigrammus brevis, Hemigrammus coeruleus, Hemigrammus cylindricus, Hemigrammus gracilis, Hemigrammus haraldi, Hemigrammus hyanuary, Hemigrammus levis, Hemigrammus lunatus, Hemigrammus marginatus, Hemigrammus mimus, Hemigrammus newboldi, Hemigrammus ocellifer, Hemigrammus orthus, Hemigrammus parana, Hemigrammus pretoensis, Hemigrammus pulcher, Hemigrammus rhodostomus, Hemigrammus rodwayi, Hemigrammus schmardae, Hemigrammus silimoni, Hemigrammus skolioplatus, Hemigrammus stictus, Hemigrammus taphorni, Hemigrammus tridens, Hemigrammus ulreyi, Hemigrammus unilineatus, Hemigrammus vorderwikleri, Hemigrammus yinyang, Hemigrammus $\mathrm{sp} \mathrm{n} .$, Hollandichthys aff. multifasciatus, 
Hyphessobrycon bentosi, Hyphessobrycon coelestinus, Hyphessobrycon compressus, Hyphessobrycon diancistrus, Hyphessobrycon eilyos, Hyphessobrycon epicharis, Hyphessobrycon eques, Hyphessobrycon erythrostigma, Hyphessobrycon megalopterus, Hyphessobrycon micropterus, Hyphessobrycon takasei, lguanodectes spilurus, Jupiaba polylepis, Lignobrycon myersi, Moenkhausia bonita, Moenkhausia hemigrammoides, Moenkhausia intermedia, Moenkhausia sanctaefilomenae, Moenkhausia xinguensis, Oligosarcus pintoi, Paracheirodon axelrodi, Parapristella georgiae, Petitella georgiae, Phenacogaster franciscoensis, Piabina argentea, Poptella paraguayensis, Psellogrammus kennedyi, Pristella maxillaris, Rhinopetitia myersi, Roeboides bonariensis, Serrapinnus heterodon, Serrapinnus notomelas, Serrasalmus maculatus, Stethaprion erythrops, Tetragonopterus argenteus, Thayeria obliqua, Triportheus nematurus

\section{2- apenas no epibranquial três}

Salminus hilarii

\section{8- Presença de cteniis sobre os rastros branquiais (ic - 0,118 )}

(Langeani, 1998; Serra, 2003: 80; Mirande, 2009: 201).

\section{0 - com ctenii distribuídos por todo o rastro}

Aphyocharax anisitsi, Aphyocharax pusillus, Astyanax altiparanae, Astyanax mexicanus, Brycinus longipinnis, Brycon cf pesu, Bryconexodon juruenae, Bryconops melanurus, Chalceus spilogyros, Cheirodon interruptus, Coptobrycon bilineatus, Gymnocorymbus ternetzi, Hasemania crenuchoides, Hasemania melanura, Hasemania sp n., Hemigrammus analis, Hemigrammus arua, Hemigrammus barrigonae, Hemigrammus bleheri, Hemigrammus coeruleus, Hemigrammus cylindricus, Hemigrammus haraldi, Hemigrammus hyanuary, Hemigrammus levis, Hemigrammus lunatus, Hemigrammus marginatus, Hemigrammus newboldi, Hemigrammus ocellifer, Hemigrammus parana, Hemigrammus pretoensis, Hemigrammus pulcher, Hemigrammus rhodostomus, Hemigrammus rodwayi, Hemigrammus schmardae, Hemigrammus silimoni, Hemigrammus stictus, Hemigrammus taphorni, Hemigrammus tridens, Hemigrammus ulreyi, Hemigrammus unilineatus, Hemigrammus yinyang, Hemigrammus sp n., Hollandichthys aff. multifasciatus, Hyphessobrycon bentosi, Hyphessobrycon compressus, Hyphessobrycon epicharis, Hyphessobrycon eques, Hyphessobrycon erythrostigma, Hyphessobrycon megalopterus, Hyphessobrycon micropterus, Hyphessobrycon takasei, Jupiaba polylepis, Moenkhausia bonita, Moenkhausia hemigrammoides, Moenkhausia intermedia, Moenkhausia sanctaefilomenae, Moenkhausia xinguensis, Oligosarcus pintoi, Parapristella georgiae, Petitella georgiae, Phenacogaster franciscoensis, Poptella paraguayensis, Psellogrammus kennedyi, Pristella maxillaris, Roeboides bonariensis, Salminus hilarii, Serrapinnus heterodon, Serrapinnus notomelas, Serrasalmus maculatus, Stethaprion erythrops, Tetragonopterus argenteus, Thayeria obliqua

\section{1- com ctenii apenas na base dos rastros}

Astyanax jacobinae, Bryconamericus exodon, Bryconella pallidifrons, Deuterodon iguape, Hemigrammus boesemani, Hemigrammus brevis, Hemigrammus gracilis, Hemigrammus mimus, Hemigrammus orthus, Hemigrammus skolioplatus, Hyphessobrycon coelestinus, Hyphessobrycon diancistrus, Hyphessobrycon eilyos, Rhinopetitia myersi

\section{2- sem ctenii}

Grundulus cochae, Hemigrammus bellottii, Hemigrammus vorderwikleri, Lignobrycon myersi, Microschemobrycon sp, Paracheirodon axelrodi, Piabina argentea, Triportheus nematurus

\section{9- Indeterminado}

Iguanodectes spilurus 
109- Cteniis (ic $-0,111$ )

(Serra, 2003: 81)

\section{$\underline{0 \text { - em grande concentração }}$}

Aphyocharax pusillus, Astyanax altiparanae, Astyanax jacobinae, Astyanax mexicanus, Brycinus longipinnis, Brycon cf pesu, Bryconexodon juruenae, Bryconops melanurus, Chalceus spilogyros, Cheirodon interruptus, Deuterodon iguape, Gymnocorymbus ternetzi, Hasemania crenuchoides, Hasemania sp n., Hemigrammus analis, Hemigrammus barrigonae, Hemigrammus boesemani, Hemigrammus coeruleus, Hemigrammus cylindricus, Hemigrammus gracilis, Hemigrammus haraldi, Hemigrammus lunatus, Hemigrammus marginatus, Hemigrammus mimus, Hemigrammus newboldi, Hemigrammus ocellifer, Hemigrammus orthus, Hemigrammus parana, Hemigrammus pretoensis, Hemigrammus pulcher, Hemigrammus rodwayi, Hemigrammus skolioplatus, Hemigrammus stictus, Hemigrammus taphorni, Hemigrammus ulreyi, Hemigrammus sp n., Hyphessobrycon bentosi, Hyphessobrycon compressus, Hyphessobrycon epicharis, Hyphessobrycon eques, Hyphessobrycon erythrostigma, Hyphessobrycon megalopterus, Hyphessobrycon micropterus, Hyphessobrycon takasei, Jupiaba polylepis, Moenkhausia bonita, Moenkhausia hemigrammoides, Moenkhausia intermedia, Moenkhausia sanctaefilomenae, Moenkhausia xinguensis, Oligosarcus pintoi, Parapristella georgiae, Phenacogaster franciscoensis, Poptella paraguayensis, Psellogrammus kennedyi, Pristella maxillaris, Roeboides bonariensis, Salminus hilarii, Serrapinnus heterodon, Serrapinnus notomelas, Serrasalmus maculatus, Stethaprion erythrops, Tetragonopterus argenteus, Thayeria obliqua

\section{1- poucos e geralmente muito esparsos}

Aphyocharax anisitsi, Bryconamericus exodon, Bryconella pallidifrons, Coptobrycon bilineatus, Hasemania melanura, Hemigrammus arua, Hemigrammus bleheri, Hemigrammus brevis, Hemigrammus hyanuary, Hemigrammus levis, Hemigrammus rhodostomus, Hemigrammus schmardae, Hemigrammus silimoni, Hemigrammus tridens, Hemigrammus unilineatus, Hemigrammus yinyang, Hollandichthys aff. multifasciatus, Hyphessobrycon coelestinus, Hyphessobrycon diancistrus, Petitella georgiae, Rhinopetitia myersi

\section{9- Indeterminado}

Grundulus cochae, Hemigrammus bellottii, Hemigrammus vorderwikleri, Hyphessobrycon eilyos, Iguanodectes spilurus, Lignobrycon myersi, Microschemobrycon sp, Paracheirodon axelrodi, Piabina argentea, Triportheus nematurus

\section{0- Projeção óssea anterior e proximal do ceratobranquial 4 (ic - 0,087)}

(Serra, 2003: 85)

\section{0- presente, larga e retangular (Figura $25 \mathrm{~A}$ )}

Bryconamericus exodon, Hasemania crenuchoides, Hemigrammus barrigonae, Hemigrammus gracilis, Hemigrammus lunatus, Hemigrammus marginatus, Hemigrammus mimus, Hemigrammus ocellifer, Hemigrammus parana, Hemigrammus pulcher, Hemigrammus rhodostomus, Hemigrammus sp n., Hemigrammus taphorni, Hemigrammus ulreyi, Hollandichthys aff. multifasciatus, Moenkhausia bonita, Moenkhausia xinguensis, Rhinopetitia myersi, Salminus hilarii

\section{1- presente e afilada (Figuras 24 e 25 B)}

Aphyocharax anisitsi, Astyanax jacobinae, Bryconella pallidifrons, Bryconops melanurus, Cheirodon interruptus, Coptobrycon bilineatus, Grundulus cochae, Gymnocorymbus ternetzi, 
Hasemania melanura, Hasemania sp n., Hemigrammus analis, Hemigrammus arua, Hemigrammus bleheri, Hemigrammus boesemani, Hemigrammus brevis, Hemigrammus coeruleus, Hemigrammus haraldi, Hemigrammus hyanuary, Hemigrammus levis, Hemigrammus newboldi, Hemigrammus pretoensis, Hemigrammus rodwayi, Hemigrammus schmardae, Hemigrammus silimoni, Hemigrammus tridens, Hemigrammus unilineatus, Hemigrammus vorderwikleri, Hemigrammus yinyang, Hyphessobrycon bentosi, Hyphessobrycon coelestinus, Hyphessobrycon compressus, Hyphessobrycon diancistrus, Hyphessobrycon epicharis, Hyphessobrycon eques, Hyphessobrycon erythrostigma, Hyphessobrycon megalopterus, Hyphessobrycon micropterus, Hyphessobrycon takasei, Iguanodectes spilurus, Jupiaba polylepis, Lignobrycon myersi, Moenkhausia hemigrammoides, Moenkhausia intermedia, Moenkhausia sanctaefilomenae, Oligosarcus pintoi, Paracheirodon axelrodi, Parapristella georgiae, Petitella georgiae, Piabina argentea, Poptella paraguayensis, Psellogrammus kennedyi, Pristella maxillaris, Roeboides bonariensis, Serrapinnus heterodon, Serrapinnus notomelas, Stethaprion erythrops, Thayeria obliqua, Triportheus nematurus

\section{2- ausente}

Aphyocharax pusillus, Astyanax mexicanus, Brycinus longipinnis, Brycon cf pesu, Bryconexodon juruenae, Chalceus spilogyros, Hemigrammus bellottii, Hemigrammus orthus, Hemigrammus cylindricus, Hemigrammus skolioplatus, Microschemobrycon sp, Phenacogaster franciscoensis, Serrasalmus maculatus, Tetragonopterus argenteus

\section{9- Indeterminado}

Astyanax altiparanae, Deuterodon iguape, Hemigrammus stictus, Hyphessobrycon eilyos

111- Placa dentígera do ceratobranquial 5 (ic - 0,143 )

(Serra, 2003: 86)

$\underline{0 \text { - dentes concentrados preferencialmente ao redor (nas margens) da }}$

placa

Aphyocharax pusillus, Brycon cf pesu, Hemigrammus analis, Hemigrammus skolioplatus, Moenkhausia sanctaefilomenae, Moenkhausia xinguensis, Parapristella georgiae, Tetragonopterus argenteus

\section{1- dentes distribuídos por toda a placa (Figura 24)}

Aphyocharax anisitsi, Astyanax mexicanus, Brycinus longipinnis, Bryconamericus exodon, Bryconella pallidifrons, Bryconexodon juruenae, Bryconops melanurus, Chalceus spilogyros, Cheirodon interruptus, Coptobrycon bilineatus, Grundulus cochae, Gymnocorymbus ternetzi, Hasemania crenuchoides, Hasemania melanura, Hasemania sp n., Hemigrammus arua, Hemigrammus bellottii, Hemigrammus bleheri, Hemigrammus boesemani, Hemigrammus coeruleus, Hemigrammus cylindricus, Hemigrammus gracilis, Hemigrammus haraldi, Hemigrammus hyanuary, Hemigrammus levis, Hemigrammus lunatus, Hemigrammus marginatus, Hemigrammus mimus, Hemigrammus newboldi, Hemigrammus ocellifer, Hemigrammus orthus, Hemigrammus parana, Hemigrammus pretoensis, Hemigrammus pulcher, Hemigrammus rhodostomus, Hemigrammus rodwayi, Hemigrammus schmardae, Hemigrammus silimoni, Hemigrammus stictus, Hemigrammus taphorni, Hemigrammus tridens, Hemigrammus ulreyi, Hemigrammus unilineatus, Hemigrammus vorderwikleri, Hemigrammus yinyang, Hemigrammus sp $\mathrm{n}$., Hollandichthys aff. multifasciatus, Hyphessobrycon bentosi, Hyphessobrycon compressus, Hyphessobrycon diancistrus, Hyphessobrycon epicharis, Hyphessobrycon eques, Hyphessobrycon erythrostigma, Hyphessobrycon megalopterus, Hyphessobrycon micropterus, Hyphessobrycon takasei, Iguanodectes spilurus, Jupiaba polylepis, Lignobrycon myersi, Microschemobrycon sp, Moenkhausia bonita, Moenkhausia hemigrammoides, Moenkhausia intermedia, Oligosarcus pintoi, Paracheirodon axelrodi, 
Petitella georgiae, Phenacogaster franciscoensis, Piabina argentea, Poptella paraguayensis, Psellogrammus kennedyi, Pristella maxillaris, Rhinopetitia myersi, Roeboides bonariensis, Salminus hilarii, Serrapinnus heterodon, Serrapinnus notomelas, Serrasalmus maculatus, Stethaprion erythrops, Triportheus nematurus

\section{9- Indeterminado}

Astyanax altiparanae, Astyanax jacobinae, Deuterodon iguape, Hemigrammus barrigonae, Hemigrammus brevis, Hyphessobrycon coelestinus, Hyphessobrycon eilyos, Thayeria obliqua

112- Faringo-branquiais 2 e 3 (ic - 0,083)

(Serra, 2003: 87; Moreira, 2007: 207-208)

\section{0 - sem dentes}

Brycinus longipinnis, Iguanodectes spilurus

\section{1- com dentes}

Aphyocharax pusillus, Brycon cf pesu, Bryconamericus exodon, Bryconexodon juruenae, Bryconops melanurus, Chalceus spilogyros, Cheirodon interruptus, Coptobrycon bilineatus, Gymnocorymbus ternetzi, Hasemania melanura, Hasemania sp n., Hemigrammus arua, Hemigrammus bellottii, Hemigrammus bleheri, Hemigrammus haraldi, Hemigrammus hyanuary, Hemigrammus levis, Hemigrammus marginatus, Hemigrammus mimus, Hemigrammus rhodostomus, Hemigrammus sp n., Hemigrammus stictus, Hemigrammus taphorni, Hyphessobrycon bentosi, Hyphessobrycon diancistrus, Hyphessobrycon eques, Hyphessobrycon erythrostigma, Hyphessobrycon megalopterus, Hyphessobrycon takasei, Lignobrycon myersi, Moenkhausia intermedia, Moenkhausia sanctaefilomenae, Moenkhausia xinguensis, Oligosarcus pintoi, Petitella georgiae, Psellogrammus kennedyi, Rhinopetitia myersi, Roeboides bonariensis, Salminus hilarii, Serrapinnus heterodon, Serrapinnus notomelas, Tetragonopterus argenteus, Triportheus nematurus

\section{2- dentes apenas no faringo-branquial 3 (Figura 24)}

Aphyocharax anisitsi, Astyanax jacobinae, Astyanax mexicanus, Bryconella pallidifrons, Deuterodon iguape, Grundulus cochae, Hasemania crenuchoides, Hemigrammus analis, Hemigrammus barrigonae, Hemigrammus boesemani, Hemigrammus brevis, Hemigrammus coeruleus, Hemigrammus cylindricus, Hemigrammus gracilis, Hemigrammus lunatus, Hemigrammus newboldi, Hemigrammus ocellifer, Hemigrammus orthus, Hemigrammus parana, Hemigrammus pretoensis, Hemigrammus pulcher, Hemigrammus rodwayi, Hemigrammus schmardae, Hemigrammus silimoni, Hemigrammus skolioplatus, Hemigrammus tridens, Hemigrammus ulreyi, Hemigrammus unilineatus, Hemigrammus vorderwikleri, Hemigrammus yinyang, Hollandichthys aff. multifasciatus, Hyphessobrycon coelestinus, Hyphessobrycon compressus, Hyphessobrycon eilyos, Hyphessobrycon epicharis, Hyphessobrycon micropterus, Jupiaba polylepis, Microschemobrycon sp, Moenkhausia bonita, Moenkhausia hemigrammoides, Paracheirodon axelrodi, Parapristella georgiae, Phenacogaster franciscoensis, Piabina argentea, Poptella paraguayensis, Serrasalmus maculatus, Stethaprion erythrops, Thayeria obliqua

\section{9- Indeterminado}

Astyanax altiparanae, Pristella maxillaris

\section{Cintura peitoral}

113- Cleitro (ic - 0,063) 
(Serra, 2003: 69)

$\underline{0 \text { - com a região ventral formando uma aba que encobre parcial (mais de }}$ 70\%) ou totalmente a fenestra formada pelo cleitro e coracóide (Figura 27 e 30)

Aphyocharax anisitsi, Aphyocharax pusillus, Astyanax altiparanae, Astyanax mexicanus, Brycinus longipinnis, Brycon of pesu, Bryconamericus exodon, Bryconella pallidifrons, Bryconexodon juruenae, Bryconops melanurus, Chalceus spilogyros, Cheirodon interruptus, Deuterodon iguape, Gymnocorymbus ternetzi, Hasemania melanura, Hasemania sp n., Hemigrammus arua, Hemigrammus barrigonae, Hemigrammus bellottii, Hemigrammus bleheri, Hemigrammus boesemani, Hemigrammus brevis, Hemigrammus coeruleus, Hemigrammus cylindricus, Hemigrammus hyanuary, Hemigrammus levis, Hemigrammus lunatus, Hemigrammus marginatus, Hemigrammus newboldi, Hemigrammus ocellifer, Hemigrammus parana, Hemigrammus pretoensis, Hemigrammus pulcher, Hemigrammus rhodostomus, Hemigrammus rodwayi, Hemigrammus schmardae, Hemigrammus silimoni, Hemigrammus skolioplatus, Hemigrammus stictus, Hemigrammus taphorni, Hemigrammus tridens, Hemigrammus ulreyi, Hemigrammus unilineatus, Hemigrammus vorderwikleri, Hemigrammus yinyang, Hemigrammus sp n., Hyphessobrycon coelestinus, Hyphessobrycon compressus, Hyphessobrycon epicharis, Hyphessobrycon eques, Hyphessobrycon erythrostigma, Hyphessobrycon takasei, Iguanodectes spilurus, Jupiaba polylepis, Moenkhausia bonita, Moenkhausia hemigrammoides, Moenkhausia intermedia, Moenkhausia sanctaefilomenae, Moenkhausia xinguensis, Oligosarcus pintoi, Paracheirodon axelrodi, Parapristella georgiae, Petitella georgiae, Phenacogaster franciscoensis, Piabina argentea, Poptella paraguayensis, Psellogrammus kennedyi, Pristella maxillaris, Roeboides bonariensis, Salminus hilarii,Serrapinnus heterodon, Serrasalmus maculatus, Tetragonopterus argenteus, Thayeria obliqua, Triportheus nematurus

1- com a região ventral sem aba desenvolvida (ou muito reduzida), deixando a fenestra formada pelo cleitro e coracóide totalmente visível em vista lateral ou com apenas a região mais dorsal encoberta (30\% ou menos) (Figura 28)

Astyanax jacobinae, Coptobrycon bilineatus, Grundulus cochae, Hasemania crenuchoides, Hemigrammus analis, Hemigrammus gracilis, Hemigrammus haraldi, Hemigrammus mimus, Hemigrammus orthus, Hollandichthys aff. multifasciatus, Hyphessobrycon bentosi, Hyphessobrycon diancistrus, Hyphessobrycon eilyos, Hyphessobrycon megalopterus, Hyphessobrycon micropterus, Lignobrycon myersi, Microschemobrycon sp, Rhinopetitia myersi, Serrapinnus notomelas, Stethaprion erythrops

114- Pós-cleitros 2 e 3 (ic - 1,0)

(Moreira, 2007: 225 e 228; Mirande, 2009: 248-249): $\underline{0 \text { - ausentes }}$

Iguanodectes spilurus, Lignobrycon myersi, Triportheus nematurus

\section{1- presentes (Figura 27 e 30)}

Aphyocharax anisitsi, Astyanax altiparanae, Astyanax jacobinae, Astyanax mexicanus, Brycinus longipinnis, Brycon cf pesu, Bryconamericus exodon, Bryconella pallidifrons, Bryconexodon juruenae, Bryconops melanurus, Chalceus spilogyros, Cheirodon interruptus, Coptobrycon bilineatus, Deuterodon iguape, Grundulus cochae, Gymnocorymbus ternetzi, Hasemania crenuchoides, Hasemania melanura, Hasemania sp n., Hemigrammus analis, Hemigrammus arua, Hemigrammus barrigonae, Hemigrammus bellottii, Hemigrammus bleheri, Hemigrammus boesemani, Hemigrammus brevis, Hemigrammus coeruleus, Hemigrammus cylindricus, 
Hemigrammus gracilis, Hemigrammus haraldi, Hemigrammus hyanuary, Hemigrammus levis, Hemigrammus lunatus, Hemigrammus marginatus, Hemigrammus mimus, Hemigrammus newboldi, Hemigrammus ocellifer, Hemigrammus orthus, Hemigrammus parana, Hemigrammus pretoensis, Hemigrammus pulcher, Hemigrammus rhodostomus, Hemigrammus rodwayi, Hemigrammus schmardae, Hemigrammus silimoni, Hemigrammus skolioplatus, Hemigrammus stictus, Hemigrammus taphorni, Hemigrammus tridens, Hemigrammus ulreyi, Hemigrammus unilineatus, Hemigrammus vorderwikleri, Hemigrammus yinyang, Hemigrammus sp n., Hollandichthys aff. multifasciatus, Hyphessobrycon bentosi, Hyphessobrycon coelestinus, Hyphessobrycon compressus, Hyphessobrycon diancistrus, Hyphessobrycon eilyos, Hyphessobrycon epicharis, Hyphessobrycon eques, Hyphessobrycon erythrostigma, Hyphessobrycon megalopterus, Hyphessobrycon micropterus, Hyphessobrycon takasei, Jupiaba polylepis, Microschemobrycon sp, Moenkhausia bonita, Moenkhausia hemigrammoides, Moenkhausia intermedia, Moenkhausia sanctaefilomenae, Moenkhausia xinguensis, Oligosarcus pintoi, Paracheirodon axelrodi, Parapristella georgiae, Petitella georgiae, Phenacogaster franciscoensis, Piabina argentea, Poptella paraguayensis, Psellogrammus kennedyi, Pristella maxillaris, Rhinopetitia myersi, Roeboides bonariensis, Salminus hilarii, Serrapinnus heterodon, Serrapinnus notomelas, Serrasalmus maculatus, Stethaprion erythrops, Tetragonopterus argenteus, Thayeria obliqua

\section{2- apenas pós-cleitro 3 ausente}

Aphyocharax pusillus

A maior parte das espécies de Characiformes apresenta pós-cleitro 2 e 3 associados com a região posterior do cleitro. Dentre as espécies examinadas, entretanto, esses dois ossos estão ausentes em lguanodectes spilurus, Lignobrycon myersi e Triportheus nematurus, condição que aparece como sinapomórfica para o clado 98 que agrupa as três referidas espécies. Aphyocharax pusillus apresenta apenas o pós-cleitro 3 ausente.

\section{5- Altura do pós-cleitro 2 (ic - 0,133)}

\section{0- aproximadamente igual ou pouco maior que o pós-cleitro 1 (Figura 30)}

Astyanax altiparanae, Brycinus longipinnis, Brycon cf pesu, Bryconamericus exodon, Bryconops melanurus, Chalceus spilogyros, Deuterodon iguape, Gymnocorymbus ternetzi, Hasemania crenuchoides, Hasemania sp n., Hemigrammus analis, Hemigrammus arua, Hemigrammus bellottii, Hemigrammus bleheri, Hemigrammus boesemani, Hemigrammus coeruleus, Hemigrammus cylindricus, Hemigrammus gracilis, Hemigrammus haraldi, Hemigrammus hyanuary, Hemigrammus levis, Hemigrammus lunatus, Hemigrammus marginatus, Hemigrammus newboldi, Hemigrammus ocellifer, Hemigrammus parana, Hemigrammus pretoensis, Hemigrammus pulcher, Hemigrammus rhodostomus, Hemigrammus schmardae, Hemigrammus skolioplatus, Hemigrammus stictus, Hemigrammus taphorni, Hemigrammus ulreyi, Hemigrammus yinyang, Hemigrammus sp n., Hyphessobrycon bentosi, Hyphessobrycon coelestinus, Hyphessobrycon compressus, Hyphessobrycon eilyos, Hyphessobrycon epicharis, Hyphessobrycon eques, Hyphessobrycon erythrostigma, Hyphessobrycon megalopterus, Hyphessobrycon micropterus, Hyphessobrycon takasei, Jupiaba polylepis, Microschemobrycon sp, Moenkhausia bonita, Moenkhausia hemigrammoides, Moenkhausia intermedia, Moenkhausia sanctaefilomenae, Moenkhausia xinguensis, Oligosarcus pintoi, Parapristella georgiae, Petitella georgiae, Phenacogaster franciscoensis, Piabina argentea, Poptella paraguayensis, Psellogrammus kennedyi, Pristella maxillaris, Roeboides bonariensis, Salminus hilarii,Serrapinnus heterodon, Serrapinnus notomelas, Serrasalmus maculatus, Stethaprion erythrops, Tetragonopterus argenteus, Thayeria obliqua 


\section{1- aproximadamente o dobro do pós-cleitro 1}

Astyanax mexicanus, Bryconella pallidifrons, Cheirodon interruptus, Coptobrycon bilineatus, Grundulus cochae, Hemigrammus barrigonae, Hemigrammus brevis, Hemigrammus mimus, Hemigrammus orthus, Hemigrammus rodwayi, Hemigrammus silimoni, Hemigrammus tridens, Hemigrammus unilineatus, Hemigrammus vorderwikleri, Hyphessobrycon diancistrus, Paracheirodon axelrodi, Rhinopetitia myersi

\section{2- menor que o pós-cleitro 1}

Aphyocharax anisitsi, Aphyocharax pusillus, Hollandichthys multifasciatus

\section{$\underline{0,1-\text { Polimórfico }}$}

Hasemania melanura

\section{9- Indeterminado}

Astyanax jacobinae, Bryconexodon juruenae, Iguanodectes spilurus, Lignobrycon myersi, Triportheus nematurus

\section{6- Projeção óssea posterior no pós-cleitro 3 (ic - 0,500)}

(Serra, 2003: 70; Benine, 2004; 69; Serra \& Langeani, 2006; Ferreira, 2007: 86;

Moreira, 2007: 229; Bertaco, 2008: 76; Marinho, 2009: 52; Mirande, 2009: 250)

\section{0- ausente (Figura 30)}

Aphyocharax anisitsi, Brycon cf pesu, Bryconamericus exodon, Bryconops melanurus, Piabina argentea, Rhinopetitia myersi, Salminus hilarii

\section{1- presente (Figura 27)}

Astyanax altiparanae, Astyanax jacobinae, Astyanax mexicanus, Brycinus longipinnis, Bryconella pallidifrons, Bryconexodon juruenae, Chalceus spilogyros,Cheirodon interruptus, Coptobrycon bilineatus, Deuterodon iguape, Grundulus cochae, Gymnocorymbus ternetzi, Hasemania crenuchoides, Hasemania melanura, Hasemania sp n., Hemigrammus analis, Hemigrammus arua, Hemigrammus barrigonae, Hemigrammus bellottii, Hemigrammus bleheri, Hemigrammus boesemani, Hemigrammus brevis, Hemigrammus coeruleus, Hemigrammus cylindricus, Hemigrammus gracilis, Hemigrammus haraldi, Hemigrammus hyanuary, Hemigrammus levis, Hemigrammus lunatus, Hemigrammus marginatus, Hemigrammus mimus, Hemigrammus newboldi, Hemigrammus ocellifer, Hemigrammus orthus, Hemigrammus parana, Hemigrammus pretoensis, Hemigrammus pulcher, Hemigrammus rhodostomus, Hemigrammus rodwayi, Hemigrammus schmardae, Hemigrammus silimoni, Hemigrammus skolioplatus, Hemigrammus stictus, Hemigrammus taphorni, Hemigrammus tridens, Hemigrammus ulreyi, Hemigrammus unilineatus, Hemigrammus vorderwikleri, Hemigrammus yinyang, Hemigrammus sp n., Hollandichthys aff. multifasciatus, Hyphessobrycon bentosi, Hyphessobrycon coelestinus, Hyphessobrycon compressus, Hyphessobrycon diancistrus, Hyphessobrycon eilyos, Hyphessobrycon epicharis, Hyphessobrycon eques, Hyphessobrycon erythrostigma, Hyphessobrycon megalopterus, Hyphessobrycon micropterus, Hyphessobrycon takasei, Jupiaba polylepis, Microschemobrycon sp, Moenkhausia bonita, Moenkhausia hemigrammoides, Moenkhausia intermedia, Moenkhausia sanctaefilomenae, Moenkhausia xinguensis, Oligosarcus pintoi, Paracheirodon axelrodi, Parapristella georgiae, Petitella georgiae, Phenacogaster franciscoensis, Poptella paraguayensis, Pristella maxillaris, 
Roeboides bonariensis, Serrapinnus heterodon, Serrapinnus notomelas, Serrasalmus maculatus, Stethaprion erythrops, Tetragonopterus argenteus, Thayeria obliqua

\section{9- Indeterminado}

Aphyocharax pusillus, Iguanodectes spilurus, Lignobrycon myersi, Psellogrammus kennedyi, Triportheus nematurus

Segundo Serra \& Langeani (2006), o pós-cleitro 3 encontrado na maioria dos Characiformes é representado por um osso sinuoso, alongado e com aproximadamente a mesma largura em toda extensão. Os autores colocam, porém, que dentro de Characidae, Serrasalmus, Cheirodontinae, Characinae, Stethaprioninae, Iguanodectinae e Tetragonopterinae (sensu Géry, 1977) apresentam uma projeção convexa e posterior, condição considerada pelos autores como apomórfica em Characiformes. Serra \& Langeani (2006) colocam também, que dentro de Characidae, Aphyocharacinae e os representantes do "clado A" de Malabarba \& Weitzman (2003) apresentam o pós-cleitro 3 em condição semelhante àquela considerada plesiomórfica em Characiformes, ou seja, sem projeção posterior convexa. A condição do pós-cleitro 3 encontrada em Aphyocharacinae e no "clado A" é interpretada pelos autores como reversão dentro de Characidae e uma sinapomorfia adicional para o referido clado.

117- Coracóide (ic - 0,500)

(Moreira, 2007: 235; Mirande, 2009: 238)

\section{0- não expandido em uma quilha ventral (Figuras 27 - 30)}

Aphyocharax anisitsi, Aphyocharax pusillus, Astyanax altiparanae, Astyanax jacobinae, Astyanax mexicanus, Brycinus longipinnis, Brycon cf pesu, Bryconamericus exodon, Bryconella pallidifrons, Bryconexodon juruenae, Bryconops melanurus, Chalceus spilogyros, Cheirodon interruptus, Coptobrycon bilineatus, Deuterodon iguape, Grundulus cochae, Gymnocorymbus ternetzi, Hasemania crenuchoides, Hasemania melanura, Hasemania sp n., Hemigrammus analis, Hemigrammus arua, Hemigrammus barrigonae, Hemigrammus bellottii, Hemigrammus bleheri, Hemigrammus boesemani, Hemigrammus brevis, Hemigrammus coeruleus, Hemigrammus cylindricus, Hemigrammus gracilis, Hemigrammus haraldi, Hemigrammus hyanuary, Hemigrammus levis, Hemigrammus lunatus, Hemigrammus marginatus, Hemigrammus mimus, Hemigrammus newboldi, Hemigrammus ocellifer, Hemigrammus orthus, Hemigrammus parana, Hemigrammus pretoensis, Hemigrammus pulcher, Hemigrammus rhodostomus, Hemigrammus rodwayi, Hemigrammus schmardae, Hemigrammus silimoni, Hemigrammus skolioplatus, Hemigrammus stictus, Hemigrammus taphorni, Hemigrammus tridens, Hemigrammus ulreyi, Hemigrammus unilineatus, Hemigrammus vorderwikleri, Hemigrammus yinyang, Hemigrammus sp n., Hollandichthys aff. multifasciatus, Hyphessobrycon bentosi, Hyphessobrycon coelestinus, Hyphessobrycon compressus, Hyphessobrycon diancistrus, Hyphessobrycon eilyos, Hyphessobrycon epicharis, Hyphessobrycon eques, Hyphessobrycon erythrostigma, Hyphessobrycon megalopterus, Hyphessobrycon micropterus, Hyphessobrycon takasei, Iguanodectes spilurus, Jupiaba 
polylepis, Microschemobrycon sp, Moenkhausia bonita, Moenkhausia hemigrammoides, Moenkhausia intermedia, Moenkhausia sanctaefilomenae, Moenkhausia xinguensis, Oligosarcus pintoi, Paracheirodon axelrodi, Parapristella georgiae, Petitella georgiae, Phenacogaster franciscoensis, Piabina argentea, Poptella paraguayensis, Psellogrammus kennedyi, Pristella maxillaris, Rhinopetitia myersi, Roeboides bonariensis, Salminus hilarii, Serrapinnus heterodon, Serrapinnus notomelas, Serrasalmus maculatus, Stethaprion erythrops, Tetragonopterus argenteus, Thayeria obliqua

\section{1- expandido em uma quilha ventral}

Lignobrycon myersi, Triportheus nematurus

\section{8- Fenestra entre o coracóide e o cleitro (ic - 1,0)}

(Moreira, 2007: 248)

$$
\text { 0- bastante ampla (Figuras 27, } 29 \text { - 30) }
$$

Aphyocharax anisitsi, Aphyocharax pusillus, Astyanax altiparanae, Astyanax jacobinae, Astyanax mexicanus, Brycinus longipinnis, Brycon cf pesu, Bryconamericus exodon, Bryconella pallidifrons, Bryconexodon juruenae, Bryconops melanurus, Chalceus spilogyros, Cheirodon interruptus, Coptobrycon bilineatus, Deuterodon iguape, Grundulus cochae, Gymnocorymbus ternetzi, Hasemania crenuchoides, Hasemania melanura, Hasemania sp n., Hemigrammus analis, Hemigrammus arua, Hemigrammus barrigonae, Hemigrammus bellottii, Hemigrammus bleheri, Hemigrammus boesemani, Hemigrammus brevis, Hemigrammus coeruleus, Hemigrammus cylindricus, Hemigrammus gracilis, Hemigrammus haraldi, Hemigrammus hyanuary, Hemigrammus levis, Hemigrammus lunatus, Hemigrammus marginatus, Hemigrammus newboldi, Hemigrammus ocellifer, Hemigrammus orthus, Hemigrammus parana, Hemigrammus pretoensis, Hemigrammus pulcher, Hemigrammus rhodostomus, Hemigrammus rodwayi, Hemigrammus schmardae, Hemigrammus silimoni, Hemigrammus skolioplatus, Hemigrammus stictus, Hemigrammus taphorni, Hemigrammus tridens, Hemigrammus ulreyi, Hemigrammus unilineatus, Hemigrammus vorderwikleri, Hemigrammus yinyang, Hemigrammus sp n., Hollandichthys aff. multifasciatus, Hyphessobrycon bentosi, Hyphessobrycon coelestinus, Hyphessobrycon compressus, Hyphessobrycon diancistrus, Hyphessobrycon eilyos, Hyphessobrycon epicharis, Hyphessobrycon eques, Hyphessobrycon erythrostigma, Hyphessobrycon megalopterus, Hyphessobrycon micropterus, Hyphessobrycon takasei, Iguanodectes spilurus, Jupiaba polylepis, Lignobrycon myersi, Moenkhausia bonita, Moenkhausia hemigrammoides, Moenkhausia intermedia, Moenkhausia sanctaefilomenae, Moenkhausia xinguensis, Oligosarcus pintoi, Paracheirodon axelrodi, Parapristella georgiae, Petitella georgiae, Phenacogaster franciscoensis, Piabina argentea, Poptella paraguayensis, Psellogrammus kennedyi, Pristella maxillaris, Rhinopetitia myersi, Roeboides bonariensis, Salminus hilarii, Serrapinnus heterodon, Serrapinnus notomelas, Serrasalmus maculatus, Stethaprion erythrops, Tetragonopterus argenteus, Thayeria obliqua, Triportheus nematurus

1- reduzida, quase fechada, ossos quase completamente em contato ao longo da extensão (Figura 28)

Hemigrammus mimus, Microschemobrycon sp.

Em Characidae, a região de junção entre os ossos coracóide (ventral) e cleitro (dorsal) é formada por uma porção anterior e outra posterior de contato entre os dois ossos e por uma fenestra bem desenvolvida e ampla, aproximadamente central, localizada na região onde os dois ossos não se 
contactam. Dentre as espécies examinadas, entretanto, $H$. mimus e Microschemobrycon sp, apresentam uma ampla região de contato entre o coracóide e o cleitro, reduzindo a fenestra formada pelos dois ossos a uma pequena abertura mais anterior ou eliminando completamente a fenestra, condição apomórfica dentro de Characidae para o clado 128.

119- Escápula (ic - 0,500)

0- com 2 projeções latero-dorsais e com forame escapular amplo (Figuras 27 e 30)

Aphyocharax anisitsi, Aphyocharax pusillus, Astyanax altiparanae, Astyanax jacobinae, Astyanax mexicanus, Brycinus longipinnis, Brycon cf pesu, Bryconamericus exodon, Bryconella pallidifrons, Bryconexodon juruenae, Bryconops melanurus, Chalceus spilogyros, Cheirodon interruptus, Deuterodon iguape, Gymnocorymbus ternetzi, Hasemania crenuchoides, Hasemania melanura, Hasemania sp n., Hemigrammus analis, Hemigrammus arua, Hemigrammus barrigonae, Hemigrammus bellottii, Hemigrammus bleheri, Hemigrammus boesemani, Hemigrammus brevis, Hemigrammus coeruleus, Hemigrammus cylindricus, Hemigrammus gracilis, Hemigrammus haraldi, Hemigrammus hyanuary, Hemigrammus levis, Hemigrammus lunatus, Hemigrammus marginatus, Hemigrammus mimus, Hemigrammus newboldi, Hemigrammus ocellifer, Hemigrammus orthus, Hemigrammus parana, Hemigrammus pretoensis, Hemigrammus pulcher, Hemigrammus rhodostomus, Hemigrammus rodwayi, Hemigrammus schmardae, Hemigrammus silimoni, Hemigrammus skolioplatus, Hemigrammus stictus, Hemigrammus taphorni, Hemigrammus tridens, Hemigrammus ulreyi, Hemigrammus unilineatus, Hemigrammus vorderwikleri, Hemigrammus yinyang, Hemigrammus sp n., Hollandichthys aff. multifasciatus, Hyphessobrycon bentosi, Hyphessobrycon coelestinus, Hyphessobrycon compressus, Hyphessobrycon diancistrus, Hyphessobrycon eilyos, Hyphessobrycon epicharis, Hyphessobrycon eques, Hyphessobrycon erythrostigma, Hyphessobrycon megalopterus, Hyphessobrycon micropterus, Hyphessobrycon takasei, Iguanodectes spilurus, Jupiaba polylepis, Lignobrycon myersi, Microschemobrycon sp, Moenkhausia bonita, Moenkhausia hemigrammoides, Moenkhausia intermedia, Moenkhausia sanctaefilomenae, Moenkhausia xinguensis, Oligosarcus pintoi, Paracheirodon axelrodi, Parapristella georgiae, Petitella georgiae, Phenacogaster franciscoensis, Piabina argentea, Poptella paraguayensis, Psellogrammus kennedyi, Pristella maxillaris, Rhinopetitia myersi, Roeboides bonariensis, Salminus hilarii, Serrapinnus heterodon, Serrapinnus notomelas, Serrasalmus maculatus, Stethaprion erythrops, Tetragonopterus argenteus, Thayeria obliqua

1- globosa, sem projeções latero-dorsais e com forame escapular reduzido (Figura 29)

Coptobrycon bilineatus, Grundulus cochae, Triportheus nematurus

A escápula encontrada na maioria dos representantes de Characidae tem um formato aproximado de "T", com duas projeções latero-dorsais e um anel ósseo bastante delgado, delimitando um forame escapular amplo. Em $C$. bilineatus, $G$. cochae e $T$. nematurus, entretanto, a escápula aparece como um osso mais globoso, sem projeções latero-dorsais delimitadas e com anel ósseo bastante espesso, delimitando um forame escapular reduzido. Condição 
semelhante e adquirida independentemente é encontrada também em Markianna, Metyinnis, Lepidarchus (Zanata \& Vari, 2005) e Spintherobolus (Weitzman \& Malabarba, 1999). A presença de uma escápula globosa e com forame escapular reduzido aparece como sinapomórfica para o clado 122, que inclui $C$. bilineatus e $G$. cochae e como uma aquisição independente para $T$. nematurus.

120- Postemporal (ic - 0,043)

(Serra, 2003: 72)

\section{$\underline{0 \text { - região posterior larga afilando bruscamente terminando em ponta }}$}

Aphyocharax anisitsi, Aphyocharax pusillus, Astyanax altiparanae, Bryconops melanurus, Coptobrycon bilineatus, Deuterodon iguape, Hasemania crenuchoides, Hasemania sp n., Hemigrammus analis, Hemigrammus barrigonae, Hemigrammus coeruleus, Hemigrammus gracilis, Hemigrammus haraldi, Hemigrammus levis, Hemigrammus ocellifer, Hemigrammus pretoensis, Hemigrammus pulcher, Hemigrammus schmardae, Hemigrammus taphorni, Hemigrammus unilineatus, Hyphessobrycon bentosi, Hyphessobrycon epicharis, Hyphessobrycon eques, Hyphessobrycon erythrostigma, Hyphessobrycon takasei, Jupiaba polylepis, Microschemobrycon sp, Moenkhausia bonita, Moenkhausia hemigrammoides, Moenkhausia intermedia, Moenkhausia xinguensis, Parapristella georgiae, Rhinopetitia myersi, Serrapinnus heterodon, Tetragonopterus argenteus, Triportheus nematurus

\section{1- região posterior larga, afilando progressivamente terminando em}

ponta

Astyanax jacobinae, Astyanax mexicanus, Brycinus longipinnis, Brycon cf pesu, Bryconamericus exodon, Bryconella pallidifrons, Bryconexodon juruenae, Chalceus spilogyros, Grundulus cochae, Gymnocorymbus ternetzi, Hasemania melanura, Hemigrammus arua, Hemigrammus bellottii, Hemigrammus bleheri, Hemigrammus boesemani, Hemigrammus brevis, Hemigrammus cylindricus, Hemigrammus hyanuary, Hemigrammus lunatus, Hemigrammus marginatus, Hemigrammus mimus, Hemigrammus newboldi, Hemigrammus orthus, Hemigrammus parana, Hemigrammus rhodostomus, Hemigrammus rodwayi, Hemigrammus silimoni, Hemigrammus skolioplatus, Hemigrammus stictus, Hemigrammus tridens, Hemigrammus ulreyi, Hemigrammus vorderwikleri, Hemigrammus yinyang, Hemigrammus sp n., Hollandichthys aff. multifasciatus, Hyphessobrycon coelestinus, Hyphessobrycon compressus, Hyphessobrycon diancistrus, Hyphessobrycon eilyos, Hyphessobrycon megalopterus, Iguanodectes spilurus, Lignobrycon myersi, Moenkhausia sanctaefilomenae, Oligosarcus pintoi, Paracheirodon axelrodi, Petitella georgiae, Phenacogaster franciscoensis, Piabina argentea, Poptella paraguayensis, Psellogrammus kennedyi, Pristella maxillaris, Roeboides bonariensis, Serrapinnus notomelas, Serrasalmus maculatus, Stethaprion erythrops, Thayeria obliqua

\section{9- Indeterminado}

Cheirodon interruptus, Hyphessobrycon micropterus, Salminus hilarii

\section{Nadadeira dorsal}

121- Primeiro radial proximal da nadadeira dorsal (ic - 0,231) 
(Langeani; Serra, 2003: 74).

\section{0- polifurcado}

Bryconops melanurus, Hemigrammus bleheri, Hyphessobrycon epicharis

\section{1- trifurcado}

Hemigrammus boesemani, Hemigrammus cylindricus, Hemigrammus rodwayi, Lignobrycon myersi, Paracheirodon axelrodi

\section{2- bifurcado}

Aphyocharax anisitsi, Aphyocharax pusillus, Astyanax altiparanae, Astyanax mexicanus, Brycinus longipinnis, Bryconella pallidifrons, Bryconexodon juruenae, Chalceus spilogyros, Coptobrycon bilineatus, Deuterodon iguape, Hasemania crenuchoides, Hasemania melanura, Hasemania sp n., Hemigrammus analis, Hemigrammus arua, Hemigrammus barrigonae, Hemigrammus bellottii, Hemigrammus brevis, Hemigrammus coeruleus, Hemigrammus gracilis, Hemigrammus haraldi, Hemigrammus hyanuary, Hemigrammus levis, Hemigrammus lunatus, Hemigrammus marginatus, Hemigrammus mimus, Hemigrammus newboldi, Hemigrammus ocellifer, Hemigrammus orthus, Hemigrammus pretoensis, Hemigrammus pulcher, Hemigrammus rhodostomus, Hemigrammus schmardae, Hemigrammus skolioplatus, Hemigrammus stictus, Hemigrammus taphorni, Hemigrammus tridens, Hemigrammus ulreyi, Hemigrammus unilineatus, Hemigrammus vorderwikleri, Hemigrammus yinyang, Hollandichthys aff. multifasciatus, Hyphessobrycon bentosi, Hyphessobrycon coelestinus, Hyphessobrycon compressus, Hyphessobrycon eilyos, Hyphessobrycon eques, Hyphessobrycon erythrostigma, Hyphessobrycon megalopterus, Hyphessobrycon micropterus, Hyphessobrycon takasei, Jupiaba polylepis, Microschemobrycon sp, Moenkhausia bonita, Moenkhausia hemigrammoides, Moenkhausia intermedia, Moenkhausia sanctaefilomenae, Moenkhausia xinguensis, Parapristella georgiae, Petitella georgiae, Phenacogaster franciscoensis, Poptella paraguayensis, Psellogrammus kennedyi, Pristella maxillaris, Rhinopetitia myersi, Roeboides bonariensis, Salminus hilarii, Serrapinnus heterodon, Serrapinnus notomelas, Tetragonopterus argenteus, Thayeria obliqua, Triportheus nematurus

\section{3- simples, com uma ponta}

Grundulus cochae, Gymnocorymbus ternetzi, Iguanodectes spilurus, Piabina argentea, Serrasalmus maculatus

\section{1, 2- Polimórfico}

Cheirodon interruptus, Hemigrammus parana, Hemigrammus sp n., Oligosarcus pintoi

\section{9- Indeterminado}

Astyanax jacobinae, Brycon cf pesu, Bryconamericus exodon, Hemigrammus silimoni, Hyphessobrycon diancistrus, Stethaprion erythrops

\section{2- Ossificação anterior ao primeiro raio da nadadeira dorsal (ic - 0,273 )}

(Serra, 2003: 75; Moreira, 2007: 276)

\section{0 - ausente}

Aphyocharax anisitsi, Aphyocharax Brycinus longipinnis, pusillus, Brycon cf pesu, Bryconamericus exodon, Bryconexodon juruenae, Bryconops melanurus, Grundulus cochae, Hemigrammus analis, Hemigrammus bellottii, Hemigrammus mimus, Hemigrammus orthus, Hemigrammus yinyang, Hollandichthys aff. multifasciatus, Hyphessobrycon diancistrus, Hyphessobrycon eilyos, Iguanodectes spilurus, Jupiaba polylepis, Lignobrycon myersi, 
Microschemobrycon sp, Oligosarcus pintoi, Paracheirodon axelrodi, Phenacogaster franciscoensis, Piabina argentea, Rhinopetitia myersi, Salminus hilarii, Thayeria obliqua, Triportheus nematurus

\section{1- presente, como um pequeno raio vestigial (Figuras 32, $33 \mathrm{~B}$ e E)}

Astyanax altiparanae, Astyanax mexicanus, Bryconella pallidifrons, Chalceus spilogyros, Cheirodon interruptus, Coptobrycon bilineatus, Deuterodon iguape, Gymnocorymbus ternetzi, Hasemania crenuchoides, Hasemania melanura, Hasemania sp n., Hemigrammus arua,Hemigrammus barrigonae, Hemigrammus bleheri, Hemigrammus boesemani, Hemigrammus brevis, Hemigrammus coeruleus, Hemigrammus cylindricus, Hemigrammus gracilis, Hemigrammus haraldi, Hemigrammus hyanuary, Hemigrammus levis, Hemigrammus lunatus, Hemigrammus marginatus, Hemigrammus newboldi, Hemigrammus ocellifer, Hemigrammus parana, Hemigrammus pretoensis, Hemigrammus pulcher, Hemigrammus rhodostomus, Hemigrammus rodwayi, Hemigrammus schmardae. Hemigrammus skolioplatus, Hemigrammus sp n., Hemigrammus stictus, Hemigrammus taphorni, Hemigrammus tridens, Hemigrammus ulreyi, Hemigrammus unilineatus, Hemigrammus vorderwikleri, Hyphessobrycon bentosi, Hyphessobrycon epicharis, Hyphessobrycon eques, Hyphessobrycon erythrostigma, Hyphessobrycon megalopterus, Hyphessobrycon micropterus, Moenkhausia bonita, Moenkhausia hemigrammoides, Moenkhausia intermedia, Moenkhausia sanctaefilomenae, Moenkhausia xinguensis, Parapristella georgiae, Petitella georgiae, Pristella maxillaris, Psellogrammus kennedyi, Roeboides bonariensis, Serrapinnus heterodon, Serrapinnus notomelas, Tetragonopterus argenteus

2- presente, como um espinho bem desenvolvido e articulado, dirigido anteriormente e associado ao primeiro raio da nadadeira dorsal (Figura 33 D)

Poptella paraguayensis, Stethaprion erythrops

3- presente, como um espinho dirigido anteriormente e não articulado, associado ao primeiro proximal radial (Figura $33 \mathrm{C}$ )

Serrasalmus maculatus

\section{$\underline{0,1-P o l i m o ́ r f i c o ~}$}

Hemigrammus silimoni, Hyphessobrycon compressus, Hyphessobrycon takasei

\section{9- Indeterminado}

Astyanax jacobinae, Hyphessobrycon coelestinus

Segundo Reis (1989) e Zanata \& Vari (2005) em muitos Characiformes neotropicais o primeiro raio da nadadeira dorsal é precedido por uma pequena ossificação semelhante a um raio vestigial e de desenvolvimento variável. Em alguns grupos de Characiformes (Stethaprioninae, Curimatidae e Prochilodontidae) essa ossificação é modificada em um espinho com diferentes graus de mobilidade (Reis, 1989; Zanata \& Vari, 2005). A ausência de uma 
ossificação associada ao primeiro raio da nadadeira dorsal é interpretada por Zanata \& Vari (2005) como uma condição derivada para Alestidae.

Reis (1989) e Zanata \& Vari (2005) colocam também que uma ossificação anterior ao primeiro raio da nadadeira dorsal é encontrada em Serrasalminae, entretanto, essa ossificação é parte do primeiro radial proximal da nadadeira dorsal, e não está associada ao primeiro raio não ramificado da nadadeira (Figura $33 \mathrm{C}$ ).

\section{3- Número de raios ramificados na nadadeira dorsal (ic - 0,250)}

(Benine, 2004: 64; Malabarba \& Weitzmna, 2005; Moreira, 2007: 278; Ferreira, 2007: 91; Bertaco, 2008: 67; Marinho, 2009: 56)

\section{0- 9 ou mais raios}

Aphyocharax anisitsi, Aphyocharax pusillus, Astyanax altiparanae, Astyanax jacobinae, Astyanax mexicanus, Brycon cf pesu, Bryconella pallidifrons, Bryconexodon juruenae, Bryconops melanurus, Chalceus spilogyros, Cheirodon interruptus, Deuterodon iguape, Gymnocorymbus ternetzi, Hasemania melanura, Hemigrammus analis, Hemigrammus arua, Hemigrammus barrigonae, Hemigrammus bellottii, Hemigrammus bleheri, Hemigrammus boesemani, Hemigrammus brevis, Hemigrammus coeruleus, Hemigrammus cylindricus, Hemigrammus gracilis, Hemigrammus haraldi, Hemigrammus hyanuary, Hemigrammus levis, Hemigrammus lunatus, Hemigrammus marginatus, Hemigrammus mimus, Hemigrammus newboldi, Hemigrammus ocellifer, Hemigrammus orthus, Hemigrammus parana, Hemigrammus pretoensis, Hemigrammus pulcher, Hemigrammus rhodostomus, Hemigrammus rodwayi, Hemigrammus schmardae, Hemigrammus silimoni, Hemigrammus skolioplatus, Hemigrammus stictus, Hemigrammus taphorni, Hemigrammus tridens, Hemigrammus ulreyi, Hemigrammus unilineatus, Hemigrammus vorderwikleri, Hemigrammus yinyang, Hollandichthys aff. multifasciatus, Hyphessobrycon bentosi, Hyphessobrycon coelestinus, Hyphessobrycon compressus, Hyphessobrycon diancistrus, Hyphessobrycon eilyos, Hyphessobrycon epicharis, Hyphessobrycon eques, Hyphessobrycon erythrostigma, Hyphessobrycon megalopterus, Hyphessobrycon micropterus, Hyphessobrycon takasei, Iguanodectes spilurus, Jupiaba polylepis, Lignobrycon myersi, Microschemobrycon sp, Moenkhausia bonita, Moenkhausia hemigrammoides, Moenkhausia intermedia, Moenkhausia sanctaefilomenae, Moenkhausia xinguensis, Oligosarcus pintoi, Parapristella georgiae, Petitella georgiae, Phenacogaster franciscoensis, Poptella paraguayensis, Psellogrammus kennedyi, Pristella maxillaris, Roeboides bonariensis, Salminus hilarii, Serrapinnus heterodon, Serrapinnus notomelas, Serrasalmus maculatus, Stethaprion erythrops, Tetragonopterus argenteus, Thayeria obliqua, Triportheus nematurus

\section{$\underline{1-8}$}

Brycinus longipinnis, Bryconamericus exodon, Coptobrycon bilineatus, Hasemania crenuchoides, Hasemania sp n., Piabina argentea, Rhinopetitia myersi

\section{$\underline{0,1-\text { Polimórfico }}$}

Grundulus cochae, Hemigrammus sp n., Paracheirodon axelrodi 
Malabarba \& Weitzman (2003) utilizaram a presença de ii, 8 raios na dorsal, juntamente com a presença de quatro dentes na série interna do prémaxilar para delimitar um subgrupo monofilético em Characidae, denomidado "clado A". Na presente análise, a maioria das espécies apresenta ii, 9 ou mais raios na dadeira dorsal. Presença de ii, 8 raios na nadadeira dorsal aparece, além de em representantes do "clado A", em espécies de Hasemania, Coptobrycon e Brycinus, todas provavelmente adquiridas independentemente. Grundulus cochae, Hemigrammus sp.n. e P. axelrodi apresentam condição polimófica, com exemplares apresentando ii, 8 raios e outros com ii, 9 raios na nadadeira dorsal.

\section{Nadadeira anal}

124- Forma da nadadeira anal (ic - 0,200)

(Benine, 2004: 79; Moreira, 2007: 293; Marinho, 2009: 58)

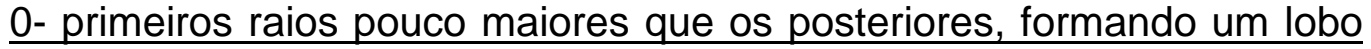
arredondado, raios diminuindo de tamanho suavemente em sentido posterior, nadadeira com concavidade na região mediana (Figura 38 A - B e E)

Aphyocharax anisitsi, Aphyocharax pusillus, Astyanax altiparanae, Astyanax jacobinae, Astyanax mexicanus, Bryconamericus exodon, Bryconella pallidifrons, Bryconexodon juruenae, Bryconops melanurus, Chalceus spilogyros, Cheirodon interruptus, Deuterodon iguape, Hemigrammus analis, Hemigrammus arua, Hemigrammus barrigonae, Hemigrammus bellottii, Hemigrammus bleheri, Hemigrammus boesemani, Hemigrammus brevis, Hemigrammus coeruleus, Hemigrammus cylindricus, Hemigrammus gracilis, Hemigrammus haraldi, Hemigrammus hyanuary, Hemigrammus levis, Hemigrammus marginatus, Hemigrammus mimus, Hemigrammus newboldi, Hemigrammus ocellifer, Hemigrammus orthus, Hemigrammus parana, Hemigrammus pretoensis, Hemigrammus pulcher, Hemigrammus rhodostomus, Hemigrammus rodwayi, Hemigrammus schmardae, Hemigrammus silimoni, Hemigrammus stictus, Hemigrammus tridens, Hemigrammus vorderwikleri, Hemigrammus yinyang, Hemigrammus sp n., Hyphessobrycon bentosi, Hyphessobrycon compressus, Hyphessobrycon diancistrus, Hyphessobrycon eilyos, Hyphessobrycon epicharis, Hyphessobrycon eques, Hyphessobrycon erythrostigma, Hyphessobrycon megalopterus, Iguanodectes spilurus, Jupiaba polylepis, Lignobrycon myersi, Microschemobrycon sp, Moenkhausia bonita, Moenkhausia intermedia, Moenkhausia xinguensis, Oligosarcus pintoi, Paracheirodon axelrodi, Parapristella georgiae, Petitella georgiae, Phenacogaster franciscoensis, Piabina argentea, Pristella maxillaris, Rhinopetitia myersi, Salminus hilarii, Serrapinnus heterodon, Serrapinnus notomelas, Thayeria obliqua, Triportheus nematurus

1 - primeiros raios muito maiores que os seguintes (o dobro dos raios do meio da nadadeira), formando um lobo pontudo, com os raios logo após os mais longos diminuindo de tamanho bruscamente, nadadeira acentuadamente falcada (Figuras 1 e $38 \mathrm{C}-\mathrm{D}$ )

Hemigrammus lunatus, Hemigrammus ulreyi, Hemigrammus unilineatus, Moenkhausia hemigrammoides, Psellogrammus kennedyi 
2- com os raios subiguais, diminuindo de tamanho muito suavemente em sentido posterior, margem distal da nadadeira aproximadamente reta ou convexa (Figura 38F)

Brycon cf pesu, Coptobrycon bilineatus, Grundulus cochae, Gymnocorymbus ternetzi, Hasemania crenuchoides, Hasemania melanura, Hasemania sp n., Hemigrammus skolioplatus, Hemigrammus taphorni, Hollandichthys aff. multifasciatus, Hyphessobrycon micropterus, Moenkhausia sanctaefilomenae, Poptella paraguayensis, Roeboides bonariensis, Serrasalmus maculatus, Tetragonopterus argenteus

\section{9- Indeterminado}

Brycinus longipinnis, Hyphessobrycon coelestinus, Hyphessobrycon takasei, Stethaprion erythrops

Em $H$. unilineatus e $M$. hemigrammoides a nadadeira anal é acentuadamente falcada, sendo que os primeiros raios são muito longos, bem mais longos que os seguintes, formando um lobo pontudo. A maioria das demais espécies analisadas apresenta ou uma nadadeira anal com lobo arredondado e concavidade mediana acentuada na margem distal ou uma nadadeira mais reta ou convexa com os raios diminuindo muito suavemente de tamanho em sentido posterior. A presença de uma nadadeira acentuadamente falcada aparece como sinapomórfica para os clados 152, que inclui $H$. unilineatus e $M$. hemigrammoides e 163, que inclui $H$ lunatus e $H$. ulreyi, condição adquirida independentemente, visto que ambos clados não aparecem mais proximamente relacionados.

\section{5- Número de raios ramificados da nadadeira anal (ic - 0,182)}

(Buckup, 1998: 65; Serra, 2003: 92; Benine, 2004: 78; Moreira, 2007: 292; Bertaco, 2008: 89)

\section{0- acima de trinta e um raios}

Gymnocorymbus ternetzi, Iguanodectes spilurus, Lignobrycon myersi, Poptella paraguayensis, Psellogrammus kennedyi, Roeboides bonariensis, Serrasalmus maculatus, Stethaprion erythrops, Tetragonopterus argenteus, Triportheus nematurus

\section{1- dezesseis a trinta raios}

Aphyocharax anisitsi, Aphyocharax pusillus, Astyanax altiparanae, Astyanax jacobinae, Astyanax mexicanus, Brycinus longipinnis, Brycon cf pesu, Bryconamericus exodon, Bryconexodon juruenae, Bryconops melanurus, Cheirodon interruptus, Deuterodon iguape, Hemigrammus arua, Hemigrammus barrigonae, Hemigrammus bellottii, Hemigrammus boesemani, Hemigrammus brevis, Hemigrammus coeruleus, Hemigrammus cylindricus, 
Hemigrammus gracilis, Hemigrammus haraldi, Hemigrammus levis, Hemigrammus lunatus, Hemigrammus marginatus, Hemigrammus newboldi, Hemigrammus orthus, Hemigrammus parana, Hemigrammus pretoensis, Hemigrammus pulcher, Hemigrammus rodwayi, Hemigrammus silimoni, Hemigrammus stictus, Hemigrammus taphorni, Hemigrammus tridens, Hemigrammus ulreyi, Hemigrammus unilineatus, Hemigrammus yinyang, Hemigrammus sp n., Hyphessobrycon bentosi, Hyphessobrycon coelestinus, Hyphessobrycon compressus, Hyphessobrycon diancistrus, Hyphessobrycon eilyos, Hyphessobrycon epicharis, Hyphessobrycon eques, Hyphessobrycon erythrostigma, Hyphessobrycon megalopterus, Hyphessobrycon micropterus, Hyphessobrycon takasei, Jupiaba polylepis, Moenkhausia bonita, Moenkhausia hemigrammoides, Moenkhausia intermedia, Moenkhausia sanctaefilomenae, Moenkhausia xinguensis, Oligosarcus pintoi, Paracheirodon axelrodi, Piabina argentea, Pristella maxillaris, Rhinopetitia myersi, Salminus hilarii, Serrapinnus heterodon, Serrapinnus notomelas

\section{2- sete a quinze raios}

Bryconella pallidifrons, Chalceus spilogyros, Coptobrycon bilineatus, Grundulus cochae, Hasemania crenuchoides, Hasemania melanura, Hasemania sp n., Hemigrammus analis, Hemigrammus bleheri, Hemigrammus hyanuary, Hemigrammus mimus, Hemigrammus rhodostomus, Hemigrammus vorderwikleri, Microschemobrycon sp, Parapristella georgiae, Petitella georgiae, Thayeria obliqua

\section{1, 0- Polimórfico}

Hollandichthys aff. multifasciatus, Phenacogaster franciscoensis

\section{1, 2- Polimórfico}

Hemigrammus schmardae, Hemigrammus skolioplatus

\section{6- Ganchos na nadadeira anal dos machos (ic - 0,080 )}

(Serra, 2003: 91)

\section{0 - ausentes}

Aphyocharax pusillus, Astyanax jacobinae, Brycinus longipinnis, Brycon cf pesu, Bryconamericus exodon, Chalceus spilogyros, Coptobrycon bilineatus, Deuterodon iguape, Grundulus cochae, Hasemania crenuchoides, Hasemania melanura, Hasemania sp n., Hemigrammus arua, Hemigrammus brevis, Hemigrammus coeruleus, Hemigrammus cylindricus, Hemigrammus gracilis, Hemigrammus marginatus, Hemigrammus orthus, Hemigrammus parana, Hemigrammus rodwayi, Hemigrammus silimoni, Hemigrammus skolioplatus, Hemigrammus sp n., Hyphessobrycon bentosi, Hyphessobrycon coelestinus, Hyphessobrycon eilyos, Hyphessobrycon epicharis, Hyphessobrycon megalopterus, Hyphessobrycon takasei, Jupiaba polylepis, Moenkhausia bonita, Moenkhausia intermedia, Moenkhausia sanctaefilomenae, Pristella maxillaris, Serrasalmus maculatus, Thayeria obliqua, Triportheus nematurus

\section{1- presentes, com número variável, mas sempre mais que um por raio,}

geralmente todos os ganchos pequenos, ou um ou dois ganchos de tamanho

\section{médio a grande por raio, seguidos por ganchos pequenos em cada raio}

Aphyocharax anisitsi, Astyanax altiparanae, Astyanax mexicanus, Bryconella pallidifrons, Bryconexodon juruenae, Bryconops melanurus, Cheirodon interruptus, Gymnocorymbus ternetzi, Hemigrammus analis, Hemigrammus barrigonae, Hemigrammus bellottii, Hemigrammus bleheri, Hemigrammus boesemani, Hemigrammus hyanuary, Hemigrammus levis, Hemigrammus lunatus, Hemigrammus mimus, Hemigrammus newboldi, Hemigrammus 
pretoensis, Hemigrammus rhodostomus, Hemigrammus schmardae, Hemigrammus stictus, Hemigrammus taphorni, Hemigrammus tridens, Hemigrammus ulreyi, Hemigrammus unilineatus, Hemigrammus vorderwikleri, Hollandichthys aff. multifasciatus, Hyphessobrycon compressus, Hyphessobrycon diancistrus, Hyphessobrycon eques, Hyphessobrycon micropterus, Iguanodectes spilurus, Lignobrycon myersi, Moenkhausia hemigrammoides, Oligosarcus pintoi, Paracheirodon axelrodi, Parapristella georgiae, Petitella georgiae, Phenacogaster franciscoensis, Piabina argentea, Poptella paraguayensis, Psellogrammus kennedyi, Salminus hilarii, Serrapinnus heterodon, Serrapinnus notomelas, Stethaprion erythrops, Tetragonopterus argenteus por raio

\section{2- presentes, com apenas um único gancho de tamanho médio a grande}

Hemigrammus haraldi, Hemigrammus ocellifer, Hemigrammus pulcher, Hemigrammus yinyang

\section{9- Indeterminado}

Hyphessobrycon erythrostigma, Microschemobrycon sp, Moenkhausia xinguensis, Rhinopetitia myersi, Roeboides bonariensis

Malabarba \& Weitzman (2003), usam a presença ou não de ganchos na nadadeira anal para discutir relações e propor agrupamentos monofiléticos em Characidae. Lima \& Souza (2009), discutem a presença, forma e distribuição de ganchos na nadadeira anal das espécies de Hemigrammus, dividindo as espécies que apresentam poucos ganchos em cinco diferentes grupos. Os autores propõem que a presença de um único gancho de tamanho médio a grande nos raios da nadadeira anal, somado a presença de duas máculas umerais, mancha negra no pedúnculo caudal e mancha vermelha na porção superior do olho em exemplares vivos, defina um grupo monofilético dentro de Hemigrammus, denominado "Hemigrammus ocellifer species-group", incluindo $H$. pulcher, $H$. haraldi, H. guyanensis, $H$. ocellifer, $H$. neptunus, $H$. luelingi e $H$. yinyang (Lima \& Souza, 2009). Dentre as espécies analisadas, entretanto, $H$. ocellifer aparece mais relacionada a $H$. coeruleus (clado 166), $H$. pulcher a $H$. haraldi (clado 143), e $H$. yinyang com espécies de outros gêneros de Characidae (clado 125).

Avaliar a presença e a morfologia de ganchos nas nadadeiras é bastante difícil, visto que a presença desses ganchos geralmente é sazonal e concomitante com período reprodutivo, levando à necessidade de amostras que contenham exemplares maduros, o que nem sempre é possível. A informação sobre a presença ou não de ganchos também não é fácil de ser obtida em literatura, pois boa parte das descrições originais mais antigas não traz essa informação, além disso, várias descrições originais são feitas com 
base em séries pequenas de exemplares e sem indivíduos maduros. Dessa forma, as diversas morfologias e distribuições dos ganchos da nadadeira anal não puderam ser mais profundamente avaliadas por falta de material suficiente para comparação entre todas as espécies incluídas na análise, porém, uma análise futura mais acurada desse caráter pode trazer mais luz ao relacionamento das espécies de Characidae, bem como daquelas pertencentes ao "Hemigrammus ocellifer species-group" de Lima \& Souza (2009).

\section{Nadadeira adiposa}

127- Nadadeira adiposa (ic - 0,500)

(Serra, 2003: 90; Moreira, 2007: 399; Mirande, 2009: 350)

\section{$\underline{0 \text { - presente }}$}

Aphyocharax anisitsi, Aphyocharax pusillus, Astyanax altiparanae, Astyanax jacobinae, Astyanax mexicanus, Brycinus longipinnis, Brycon cf pesu, Bryconamericus exodon, Bryconella pallidifrons, Bryconexodon juruenae, Bryconops melanurus, Chalceus spilogyros, Cheirodon interruptus, Deuterodon iguape, Gymnocorymbus ternetzi, Hemigrammus analis, Hemigrammus arua, Hemigrammus barrigonae, Hemigrammus bellottii, Hemigrammus bleheri, Hemigrammus boesemani, Hemigrammus brevis, Hemigrammus coeruleus, Hemigrammus cylindricus, Hemigrammus gracilis, Hemigrammus haraldi, Hemigrammus hyanuary, Hemigrammus levis, Hemigrammus lunatus, Hemigrammus marginatus, Hemigrammus mimus, Hemigrammus newboldi, Hemigrammus ocellifer, Hemigrammus orthus, Hemigrammus parana, Hemigrammus pretoensis, Hemigrammus pulcher, Hemigrammus rhodostomus, Hemigrammus rodwayi, Hemigrammus schmardae, Hemigrammus silimoni, Hemigrammus skolioplatus, Hemigrammus stictus, Hemigrammus taphorni, Hemigrammus tridens, Hemigrammus ulreyi, Hemigrammus unilineatus, Hemigrammus vorderwikleri, Hemigrammus yinyang, Hemigrammus sp n., Hollandichthys aff. multifasciatus, Hyphessobrycon bentosi, Hyphessobrycon coelestinus, Hyphessobrycon compressus, Hyphessobrycon diancistrus, Hyphessobrycon eilyos, Hyphessobrycon epicharis, Hyphessobrycon eques, Hyphessobrycon erythrostigma, Hyphessobrycon megalopterus, Hyphessobrycon micropterus, Hyphessobrycon takasei, Iguanodectes spilurus, Jupiaba polylepis, Lignobrycon myersi, Microschemobrycon sp, Moenkhausia bonita, Moenkhausia hemigrammoides, Moenkhausia intermedia, Moenkhausia sanctaefilomenae, Moenkhausia xinguensis, Oligosarcus pintoi, Paracheirodon axelrodi, Parapristella georgiae, Petitella georgiae, Phenacogaster franciscoensis, Piabina argentea, Poptella paraguayensis, Psellogrammus kennedyi, Pristella maxillaris, Rhinopetitia myersi, Roeboides bonariensis, Salminus hilarii, Serrapinnus heterodon, Serrapinnus notomelas, Serrasalmus maculatus, Stethaprion erythrops, Tetragonopterus argenteus, Thayeria obliqua, Triportheus nematurus

\section{1- ausente}

Coptobrycon bilineatus, Grundulus cochae, Hasemania crenuchoides, Hasemania melanura, Hasemania sp $\mathrm{n}$.

Ausência de nadadeira adiposa é um caráter tradicionalmente utilizado para definir o gênero Hasemania. Dentre os Tetragonopterinae (sensu Géry, 1977), além das espécies de Hasemania, nadadeira adiposa está também ausente em Coptobrycon, Grundulus, Nematobrycon, Hyphessobrycon 
taurocephalus e Hyphessobrycon negodagua. Na presente análise, a ausência de nadadeira adiposa aparece como sinapomórfica para os clados 175 , que inclui Hasemania melanura, Hasemania crenuchoides e Hasemania sp.n. e 122 que inclui Coptobrycon bilineatus e Grundulus cochae, condição adquirida independentemente entre as espécies dos dois referidos clados.

\section{Nadadeira caudal}

128- Número de raios na nadadeira caudal (ic - 1,0)

(Moreira, 2007: 309-310)

\section{0-tipicamente 10 no lobo superior +9 no lobo inferior}

Aphyocharax anisitsi, Aphyocharax pusillus, Astyanax altiparanae, Astyanax jacobinae, Astyanax mexicanus, Brycinus longipinnis, Brycon of pesu, Bryconamericus exodon, Bryconella pallidifrons, Bryconexodon juruenae, Bryconops melanurus, Chalceus spilogyros, Cheirodon interruptus, Coptobrycon bilineatus, Deuterodon iguape, Grundulus cochae, Gymnocorymbus ternetzi, Hasemania crenuchoides, Hasemania melanura, Hemigrammus analis, Hemigrammus arua, Hemigrammus barrigonae, Hemigrammus bellottii, Hemigrammus bleheri, Hemigrammus boesemani, Hemigrammus brevis, Hemigrammus coeruleus, Hemigrammus cylindricus, Hemigrammus gracilis, Hemigrammus haraldi. Hemigrammus hyanuary, Hemigrammus levis, Hemigrammus lunatus, Hemigrammus marginatus, Hemigrammus mimus, Hemigrammus newboldi, Hemigrammus ocellifer, Hemigrammus orthus, Hemigrammus parana, Hemigrammus pretoensis, Hemigrammus pulcher, Hemigrammus rhodostomus, Hemigrammus rodwayi, Hemigrammus schmardae, Hemigrammus silimoni, Hemigrammus skolioplatus, Hemigrammus stictus, Hemigrammus taphorni, Hemigrammus tridens, Hemigrammus ulreyi, Hemigrammus unilineatus, Hemigrammus vorderwikleri, Hemigrammus yinyang, Hemigrammus sp n., Hollandichthys aff. multifasciatus, Hyphessobrycon bentosi, Hyphessobrycon coelestinus, Hyphessobrycon compressus, Hyphessobrycon diancistrus, Hyphessobrycon eilyos, Hyphessobrycon epicharis, Hyphessobrycon eques, Hyphessobrycon erythrostigma, Hyphessobrycon megalopterus, Hyphessobrycon micropterus, Hyphessobrycon takasei, Iguanodectes spilurus, Jupiaba polylepis, Lignobrycon myersi, Microschemobrycon sp, Moenkhausia bonita, Moenkhausia hemigrammoides, Moenkhausia intermedia, Moenkhausia sanctaefilomenae, Moenkhausia xinguensis, Oligosarcus pintoi, Paracheirodon axelrodi, Parapristella georgiae, Petitella georgiae, Phenacogaster franciscoensis, Piabina argentea, Poptella paraguayensis, Psellogrammus kennedyi, Pristella maxillaris, Rhinopetitia myersi, Roeboides bonariensis, Salminus hilarii, Serrapinnus heterodon, Serrapinnus notomelas, Serrasalmus maculatus, Stethaprion erythrops, Tetragonopterus argenteus, Thayeria obliqua, Triportheus nematurus

1- tipicamente 9 no lobo superior + 9 no lobo inferior Hasemania sp $\mathrm{n}$.

129- Forma da nadadeira caudal (ic - 0,667)

$$
\underline{0 \text { - nadadeira emarginada }}
$$

Roeboides bonariensis, Serrasalmus maculatus

$\underline{\text { 1- nadadeira bifurcada, lobos afilados e geralmente alongados }}$ 
Aphyocharax anisitsi, Aphyocharax pusillus, Astyanax altiparanae, Astyanax jacobinae, Astyanax mexicanus, Brycinus longipinnis, Bryconamericus exodon, Bryconella pallidifrons, Bryconexodon juruenae, Bryconops melanurus, Chalceus spilogyros, Cheirodon interruptus, Deuterodon iguape, Gymnocorymbus ternetzi, Hasemania melanura, Hemigrammus analis, Hemigrammus arua, Hemigrammus barrigonae, Hemigrammus bellottii, Hemigrammus bleheri, Hemigrammus boesemani, Hemigrammus brevis, Hemigrammus coeruleus, Hemigrammus cylindricus, Hemigrammus gracilis, Hemigrammus haraldi, Hemigrammus hyanuary, Hemigrammus levis, Hemigrammus lunatus, Hemigrammus marginatus, Hemigrammus mimus, Hemigrammus newboldi, Hemigrammus ocellifer, Hemigrammus orthus, Hemigrammus parana, Hemigrammus pretoensis, Hemigrammus pulcher, Hemigrammus rhodostomus, Hemigrammus rodwayi, Hemigrammus schmardae, Hemigrammus silimoni, Hemigrammus skolioplatus, Hemigrammus stictus, Hemigrammus taphorni, Hemigrammus tridens, Hemigrammus ulreyi, Hemigrammus unilineatus, Hemigrammus vorderwikleri, Hemigrammus yinyang, Hemigrammus sp n., Hollandichthys aff. multifasciatus, Hyphessobrycon bentosi, Hyphessobrycon coelestinus, Hyphessobrycon compressus, Hyphessobrycon diancistrus, Hyphessobrycon eilyos, Hyphessobrycon epicharis, Hyphessobrycon eques, Hyphessobrycon erythrostigma, Hyphessobrycon megalopterus, Hyphessobrycon micropterus, Hyphessobrycon takasei, Iguanodectes spilurus, Jupiaba polylepis, Lignobrycon myersi, Microschemobrycon sp, Moenkhausia bonita, Moenkhausia hemigrammoides, Moenkhausia intermedia, Moenkhausia sanctaefilomenae, Moenkhausia xinguensis, Oligosarcus pintoi, Paracheirodon axelrodi, Parapristella georgiae, Petitella georgiae, Phenacogaster franciscoensis, Piabina argentea, Poptella paraguayensis, Psellogrammus kennedyi, Pristella maxillaris, Rhinopetitia myersi, Salminus hilarii, Serrapinnus heterodon, Serrapinnus notomelas, Stethaprion erythrops, Tetragonopterus argenteus, Thayeria obliqua, Triportheus nematurus

\section{2- nadadeira bifurcada, lobos arredondados e geralmente curtos}

Coptobrycon bilineatus, Grundulus cochae, Hasemania crenuchoides, Hasemania sp n.

\section{9- Indeterminado}

Brycon cf pesu

\section{0- Tamanho dos lobos caudais (ic - 1,0)}

\section{$\underline{0 \text { - ambos com o mesmo tamanho }}$}

Aphyocharax anisitsi, Aphyocharax pusillus, Astyanax altiparanae, Astyanax jacobinae, Astyanax mexicanus, Brycinus longipinnis, Brycon cf pesu, Bryconamericus exodon, Bryconella pallidifrons, Bryconexodon juruenae, Bryconops melanurus, Chalceus spilogyros, Cheirodon interruptus, Coptobrycon bilineatus, Deuterodon iguape, Grundulus cochae, Gymnocorymbus ternetzi, Hasemania crenuchoides, Hasemania melanura, Hasemania sp n., Hemigrammus analis, Hemigrammus arua, Hemigrammus barrigonae, Hemigrammus bellottii, Hemigrammus bleheri, Hemigrammus boesemani, Hemigrammus brevis, Hemigrammus coeruleus, Hemigrammus cylindricus, Hemigrammus gracilis, Hemigrammus haraldi, Hemigrammus hyanuary, Hemigrammus levis, Hemigrammus lunatus, Hemigrammus marginatus, Hemigrammus mimus, Hemigrammus newboldi, Hemigrammus ocellifer, Hemigrammus orthus, Hemigrammus parana, Hemigrammus pretoensis, Hemigrammus pulcher, Hemigrammus rhodostomus, Hemigrammus rodwayi, Hemigrammus schmardae, Hemigrammus silimoni, Hemigrammus skolioplatus, Hemigrammus stictus, Hemigrammus taphorni, Hemigrammus tridens, Hemigrammus ulreyi, Hemigrammus unilineatus, Hemigrammus vorderwikleri, Hemigrammus yinyang, Hemigrammus $\mathrm{sp} \mathrm{n}$., Hollandichthys aff. multifasciatus, Hyphessobrycon bentosi, Hyphessobrycon coelestinus, Hyphessobrycon compressus, Hyphessobrycon diancistrus, Hyphessobrycon eilyos, Hyphessobrycon epicharis, Hyphessobrycon eques, Hyphessobrycon erythrostigma, Hyphessobrycon megalopterus, Hyphessobrycon micropterus, Hyphessobrycon takasei, Iguanodectes spilurus, Jupiaba polylepis, Lignobrycon myersi, Microschemobrycon sp, Moenkhausia bonita, Moenkhausia hemigrammoides, Moenkhausia intermedia, Moenkhausia sanctaefilomenae, Moenkhausia 
xinguensis, Oligosarcus pintoi, Paracheirodon axelrodi, Parapristella georgiae, Petitella georgiae, Phenacogaster franciscoensis, Piabina argentea, Poptella paraguayensis, Psellogrammus kennedyi, Pristella maxillaris, Rhinopetitia myersi, Roeboides bonariensis, Salminus hilarii, Serrapinnus heterodon, Serrapinnus notomelas, Serrasalmus maculatus, Stethaprion erythrops, Tetragonopterus argenteus, Triportheus nematurus

\section{1- lobo inferior mais longo que o superior (Figura $41 \mathrm{~F}$ )}

Thayeria obliqua

\section{Vértebras e Supraneurais}

131- Número total de vértebras (incluindo as do Aparelho de Weber) (ic $0,111)$

(Serra, 2003: 99; Benine, 2004: 57; Ferreira, 2007: 100; Bertaco, 2008: 102;

Marinho, 2009: 62; Mirande, 2009: 227)

\section{$\underline{0 \text { - acima de } 35}$}

Aphyocharax pusillus, Astyanax jacobinae, Astyanax mexicanus, Brycinus longipinnis, Brycon cf pesu, Bryconamericus exodon, Bryconops melanurus, Chalceus spilogyros, Cheirodon interruptus, Hollandichthys aff. multifasciatus, Iguanodectes spilurus, Lignobrycon myersi, Oligosarcus pintoi, Phenacogaster franciscoensis, Psellogrammus kennedyi, Roeboides bonariensis, Salminus hilarii, Serrasalmus maculatus, Stethaprion erythrops, Triportheus nematurus

\section{1- 29 a 34}

Aphyocharax anisitsi, Astyanax altiparanae, Bryconella pallidifrons, Bryconexodon juruenae, Coptobrycon bilineatus, Deuterodon iguape, Grundulus cochae, Gymnocorymbus ternetzi, Hasemania crenuchoides, Hasemania melanura, Hasemania sp n., Hemigrammus analis, Hemigrammus arua, Hemigrammus barrigonae, Hemigrammus bellottii, Hemigrammus bleheri, Hemigrammus boesemani, Hemigrammus brevis, Hemigrammus coeruleus, Hemigrammus cylindricus, Hemigrammus gracilis, Hemigrammus haraldi, Hemigrammus hyanuary, Hemigrammus levis, Hemigrammus lunatus, Hemigrammus marginatus, Hemigrammus mimus, Hemigrammus newboldi, Hemigrammus ocellifer, Hemigrammus orthus, Hemigrammus parana, Hemigrammus pretoensis, Hemigrammus pulcher, Hemigrammus rhodostomus, Hemigrammus rodwayi, Hemigrammus schmardae, Hemigrammus silimoni, Hemigrammus skolioplatus, Hemigrammus stictus, Hemigrammus taphorni, Hemigrammus tridens, Hemigrammus ulreyi, Hemigrammus unilineatus, Hemigrammus vorderwikleri, Hemigrammus yinyang, Hemigrammus sp n., Hyphessobrycon bentosi, Hyphessobrycon coelestinus, Hyphessobrycon compressus, Hyphessobrycon diancistrus, Hyphessobrycon eilyos, Hyphessobrycon epicharis, Hyphessobrycon eques, Hyphessobrycon erythrostigma, Hyphessobrycon megalopterus, Hyphessobrycon micropterus, Hyphessobrycon takasei, Jupiaba polylepis, Microschemobrycon sp, Moenkhausia bonita, Moenkhausia hemigrammoides, Moenkhausia intermedia, Moenkhausia sanctaefilomenae, Moenkhausia xinguensis, Paracheirodon axelrodi, Parapristella georgiae, Petitella georgiae, Piabina argentea, Poptella paraguayensis, Pristella maxillaris, Rhinopetitia myersi, Serrapinnus heterodon, Serrapinnus notomelas, Tetragonopterus argenteus, Thayeria obliqua

132- Número de supraneurais (ic - 0,133)

(Serra, 2003: 95; Benine, 2004: 61; Moreira, 2007: 340; Ferreira, 2007: 110;

Bertaco, 2008: 106; Marinho, 2009: 64; Mirande, 2009: 280-281) 


\section{0- sete ou mais}

Brycinus longipinnis, Brycon cf pesu, Chalceus spilogyros, Grundulus cochae, Hollandichthys aff. multifasciatus, Iguanodectes spilurus, Lignobrycon myersi, Salminus hilarii, Triportheus nematurus

\section{1- três a quatro}

Gymnocorymbus ternetzi, Hemigrammus analis, Hemigrammus arua, Hemigrammus barrigonae, Hemigrammus bellottii, Hemigrammus brevis, Hemigrammus coeruleus, Hemigrammus cylindricus, Hemigrammus gracilis, Hemigrammus haraldi, Hemigrammus hyanuary, Hemigrammus levis, Hemigrammus lunatus, Hemigrammus marginatus, Hemigrammus mimus, Hemigrammus ocellifer, Hemigrammus orthus, Hemigrammus parana, Hemigrammus pulcher, Hemigrammus rhodostomus, Hemigrammus rodwayi, Hemigrammus schmardae, Hemigrammus silimoni, Hemigrammus stictus, Hemigrammus taphorni, Hemigrammus tridens, Hemigrammus ulreyi, Hemigrammus unilineatus, Hemigrammus vorderwikleri, Hemigrammus sp n., Hyphessobrycon bentosi, Hyphessobrycon eilyos, Hyphessobrycon eques, Hyphessobrycon erythrostigma, Hyphessobrycon megalopterus, Hyphessobrycon micropterus, Hyphessobrycon takasei, Microschemobrycon sp, Moenkhausia bonita, Moenkhausia hemigrammoides, Moenkhausia sanctaefilomenae, Moenkhausia xinguensis, Paracheirodon axelrodi, Parapristella georgiae, Petitella georgiae, Phenacogaster franciscoensis, Piabina argentea, Poptella paraguayensis, Psellogrammus kennedyi, Pristella maxillaris, Roeboides bonariensis, Serrapinnus notomelas, Stethaprion erythrops, Tetragonopterus argenteus, Thayeria obliqua

\section{2- cinco a seis}

Aphyocharax anisitsi, Aphyocharax pusillus, Astyanax jacobinae, Astyanax mexicanus, Bryconamericus exodon, Bryconella pallidifrons, Bryconexodon juruenae, Bryconops melanurus, Cheirodon interruptus, Coptobrycon bilineatus, Hasemania melanura, Hasemania $\mathrm{sp}$ n., Hemigrammus newboldi, Hemigrammus skolioplatus, Hemigrammus yinyang, Hyphessobrycon coelestinus, Hyphessobrycon compressus, Jupiaba polylepis, Moenkhausia intermedia, Serrapinnus heterodon, Serrasalmus maculatus

\section{$\underline{0,2-P o l i m o ́ r f i c o ~}$}

Hasemania crenuchoides

\section{1, 2- Polimórfico}

Astyanax altiparanae, Deuterodon iguape, Hemigrammus bleheri, Hemigrammus boesemani, Hemigrammus pretoensis, Hyphessobrycon epicharis, Oligosarcus pintoi, Rhinopetitia myersi

\section{9- Indeterminado}

Hyphessobrycon diancistrus

133- Forma dos supraneurais (ic - 0,048)

(Serra, 2003: 96; Benine, 2004: 62; Ferreira, 2007: 111; Moreira, 2007: 338;

Bertaco, 2008: 105; Marinho, 2009: 65)

$\underline{0 \text { - maioria dos supraneurais com a região dorsal mais larga que } 0}$ restante do corpo do osso, estreitando em sentido ventral (Figura 33 A, B e D) 
Astyanax altiparanae, Grundulus cochae, Gymnocorymbus ternetzi, Hasemania melanura, Hasemania sp n., Hemigrammus arua, Hemigrammus barrigonae, Hemigrammus boesemani, Hemigrammus cylindricus, Hemigrammus haraldi, Hemigrammus levis, Hemigrammus lunatus, Hemigrammus parana, Hemigrammus pulcher, Hemigrammus rodwayi, Hemigrammus stictus, Hemigrammus taphorni, Hemigrammus unilineatus, Hollandichthys aff. multifasciatus, Hyphessobrycon compressus, Hyphessobrycon diancistrus, Hyphessobrycon epicharis, Hyphessobrycon eques, Hyphessobrycon erythrostigma, Hyphessobrycon micropterus, Jupiaba polylepis, Moenkhausia hemigrammoides, Moenkhausia sanctaefilomenae, Moenkhausia xinguensis, Phenacogaster franciscoensis, Poptella paraguayensis, Roeboides bonariensis, Stethaprion erythrops, Tetragonopterus argenteus

\section{1- maioria dos supraneurais com aproximadamente a mesma largura em}

\section{toda a extensão do osso (Figura 33, C e E)}

Aphyocharax anisitsi, Aphyocharax pusillus, Astyanax jacobinae, Astyanax mexicanus, Brycinus longipinnis, Brycon of pesu, Bryconamericus exodon, Bryconella pallidifrons, Bryconexodon juruenae, Bryconops melanurus, Chalceus spilogyros, Cheirodon interruptus, Coptobrycon bilineatus, Deuterodon iguape, Hasemania crenuchoides, Hemigrammus analis, Hemigrammus bellottii, Hemigrammus brevis, Hemigrammus coeruleus, Hemigrammus gracilis, Hemigrammus hyanuary, Hemigrammus marginatus, Hemigrammus mimus, Hemigrammus newboldi, Hemigrammus ocellifer, Hemigrammus pretoensis, Hemigrammus schmardae, Hemigrammus silimoni, Hemigrammus skolioplatus, Hemigrammus tridens, Hemigrammus ulreyi, Hemigrammus vorderwikleri, Hemigrammus yinyang, Hyphessobrycon bentosi, Hyphessobrycon coelestinus, Hyphessobrycon takasei, Iguanodectes spilurus, Lignobrycon myersi, Microschemobrycon sp, Moenkhausia bonita, Moenkhausia intermedia, Paracheirodon axelrodi, Parapristella georgiae, Petitella georgiae, Piabina argentea, Psellogrammus kennedyi, Pristella maxillaris, Rhinopetitia myersi, Salminus hilarii, Serrapinnus heterodon, Serrapinnus notomelas, Serrasalmus maculatus, Thayeria obliqua, Triportheus nematurus

\section{$\underline{0,1-\text { Polimórfico }}$}

Hemigrammus orthus, Hemigrammus rhodostomus, Hemigrammus sp n., Hyphessobrycon eilyos, Hyphessobrycon megalopterus

\section{9- Indeterminado}

Hemigrammus bleheri, Oligosarcus pintoi

$$
\text { 134- Supraneurais (ic - 0,077) }
$$

(Serra, 2003: 97)

$\underline{0-}$ um ou mais supraneurais apresentando o eixo principal do osso

\section{bifurcado (Figura $33 \mathrm{~B}$ e D)}

Astyanax altiparanae, Astyanax mexicanus, Gymnocorymbus ternetzi, Hasemania melanura, Hemigrammus brevis, Hemigrammus cylindricus, Hemigrammus parana, Hemigrammus pulcher, Hemigrammus rhodostomus, Hyphessobrycon eilyos, Jupiaba polylepis, Moenkhausia xinguensis, Oligosarcus pintoi, Phenacogaster franciscoensis, Poptella paraguayensis, Psellogrammus kennedyi, Stethaprion erythrops, Tetragonopterus argenteus

\section{1- todos os supraneurais apresentando o eixo principal do osso simples}

(Figura $33 \mathrm{~A}, \mathrm{C}$ e E) 
Aphyocharax anisitsi, Aphyocharax pusillus, Astyanax jacobinae, Brycinus longipinnis, Brycon cf pesu, Bryconamericus exodon, Bryconella pallidifrons, Bryconexodon juruenae, Bryconops melanurus, Chalceus spilogyros, Cheirodon interruptus, Coptobrycon bilineatus, Deuterodon iguape, Grundulus cochae, Hasemania crenuchoides, Hasemania sp n., Hemigrammus analis, Hemigrammus barrigonae, Hemigrammus bellottii, Hemigrammus bleheri, Hemigrammus boesemani, Hemigrammus coeruleus, Hemigrammus gracilis, Hemigrammus haraldi, Hemigrammus hyanuary, Hemigrammus lunatus, Hemigrammus marginatus, Hemigrammus mimus, Hemigrammus ocellifer, Hemigrammus orthus, Hemigrammus pretoensis, Hemigrammus rodwayi, Hemigrammus schmardae, Hemigrammus silimoni, Hemigrammus skolioplatus, Hemigrammus stictus, Hemigrammus taphorni, Hemigrammus tridens, Hemigrammus ulreyi, Hemigrammus unilineatus, Hemigrammus vorderwikleri, Hemigrammus yinyang, Hemigrammus sp n., Hollandichthys aff. multifasciatus, Hyphessobrycon bentosi, Hyphessobrycon coelestinus, Hyphessobrycon compressus, Hyphessobrycon epicharis, Hyphessobrycon eques, Hyphessobrycon erythrostigma, Hyphessobrycon megalopterus, Hyphessobrycon micropterus, Hyphessobrycon takasei, Iguanodectes spilurus, Lignobrycon myersi, Microschemobrycon sp, Moenkhausia bonita, Moenkhausia hemigrammoides, Moenkhausia intermedia, Moenkhausia sanctaefilomenae, Paracheirodon axelrodi, Parapristella georgiae, Piabina argentea, Pristella maxillaris, Rhinopetitia myersi, Roeboides bonariensis, Salminus hilarii, Serrapinnus heterodon, Serrapinnus notomelas, Serrasalmus maculatus, Thayeria obliqua, Triportheus nematurus

\section{0,1- Polimórfico}

Hemigrammus arua, Hemigrammus levis, Hemigrammus newboldi, Hyphessobrycon diancistrus, Petitella georgiae

\section{5- Supraneurais (ic - 0,056)}

(Marinho, 2009: 67)

\section{0- sem expansões ósseas laminares}

Aphyocharax anisitsi, Aphyocharax pusillus, Astyanax jacobinae, Brycon cf pesu, Bryconella pallidifrons, Grundulus cochae, Hemigrammus arua, Hemigrammus coeruleus, Hemigrammus hyanuary, Hemigrammus mimus, Hemigrammus orthus, Hemigrammus schmardae, Hemigrammus silimoni, Hemigrammus skolioplatus, Hemigrammus vorderwikleri, Hyphessobrycon bentosi, Hyphessobrycon coelestinus, Hyphessobrycon eques, Hemigrammus yinyang, Iguanodectes spilurus, Lignobrycon myersi, Microschemobrycon sp, Paracheirodon axelrodi, Parapristella georgiae, Pristella maxillaris, Rhinopetitia myersi, Salminus hilarii, Serrapinnus heterodon, Stethaprion erythrops, Triportheus nematurus

\section{1- com expansões ósseas laminares (Figura 33)}

Astyanax altiparanae, Astyanax mexicanus, Brycinus longipinnis, Bryconamericus exodon, Bryconexodon juruenae, Bryconops melanurus, Chalceus spilogyros, Cheirodon interruptus, Coptobrycon bilineatus, Deuterodon iguape, Gymnocorymbus ternetzi, Hasemania crenuchoides, Hasemania melanura, Hasemania sp n., Hemigrammus analis, Hemigrammus barrigonae, Hemigrammus bleheri, Hemigrammus boesemani, Hemigrammus brevis, Hemigrammus cylindricus, Hemigrammus gracilis, Hemigrammus haraldi, Hemigrammus levis, Hemigrammus lunatus, Hemigrammus marginatus, Hemigrammus newboldi, Hemigrammus ocellifer, Hemigrammus parana, Hemigrammus pretoensis, Hemigrammus pulcher, Hemigrammus rodwayi, Hemigrammus stictus, Hemigrammus taphorni, Hemigrammus tridens, Hemigrammus ulreyi, Hemigrammus unilineatus, Hemigrammus sp n., Hollandichthys aff. multifasciatus, Hyphessobrycon compressus, Hyphessobrycon diancistrus, Hyphessobrycon epicharis, Hyphessobrycon erythrostigma, Hyphessobrycon megalopterus, Hyphessobrycon micropterus, Hyphessobrycon takasei, Jupiaba polylepis, Moenkhausia bonita, Moenkhausia hemigrammoides, Moenkhausia intermedia, Moenkhausia sanctaefilomenae, Moenkhausia xinguensis, Oligosarcus pintoi, Petitella georgiae, Phenacogaster franciscoensis, Piabina 
argentea, Poptella paraguayensis, Psellogrammus kennedyi, Roeboides bonariensis, Serrapinnus notomelas, Serrasalmus maculatus, Tetragonopterus argenteus, Thayeria obliqua

\section{$\underline{0,1-\text { Polimórfico }}$}

Hemigrammus bellottii, Hemigrammus rhodostomus, Hyphessobrycon eilyos

\section{6- Supraneural, anterior ao espinho neural da quarta vértebra (ic - $0,500)$}

(Fink \& Fink, 1996: 58; Serra, 2003: 98; Benine, 2004: 63; Moreira, 2007: 335;

Bertaco, 2008: 104; Mirande, 2009: 279).

\section{0 - presente}

Brycinus longipinnis, Brycon cf pesu, Bryconops melanurus, Chalceus spilogyros, Lignobrycon myersi, Salminus hilarii, Triportheus nematurus

\section{1- ausente}

Aphyocharax anisitsi, Aphyocharax pusillus, Astyanax altiparanae, Astyanax jacobinae, Astyanax mexicanus, Bryconamericus exodon, Bryconella pallidifrons, Bryconexodon juruenae, Cheirodon interruptus, Coptobrycon bilineatus, Deuterodon iguape, Grundulus cochae, Gymnocorymbus ternetzi, Hasemania crenuchoides, Hasemania melanura, Hasemania sp n., Hemigrammus analis, Hemigrammus arua, Hemigrammus barrigonae, Hemigrammus bellottii, Hemigrammus bleheri, Hemigrammus boesemani, Hemigrammus brevis, Hemigrammus coeruleus, Hemigrammus cylindricus, Hemigrammus gracilis, Hemigrammus haraldi, Hemigrammus hyanuary, Hemigrammus levis, Hemigrammus lunatus, Hemigrammus marginatus, Hemigrammus mimus, Hemigrammus newboldi, Hemigrammus ocellifer, Hemigrammus orthus, Hemigrammus parana, Hemigrammus pretoensis, Hemigrammus pulcher, Hemigrammus rhodostomus, Hemigrammus rodwayi, Hemigrammus schmardae, Hemigrammus silimoni, Hemigrammus skolioplatus, Hemigrammus stictus, Hemigrammus taphorni, Hemigrammus tridens, Hemigrammus ulreyi, Hemigrammus unilineatus, Hemigrammus vorderwikleri, Hemigrammus yinyang, Hemigrammus sp n., Hollandichthys aff. multifasciatus, Hyphessobrycon bentosi, Hyphessobrycon coelestinus, Hyphessobrycon compressus, Hyphessobrycon diancistrus, Hyphessobrycon eilyos, Hyphessobrycon epicharis, Hyphessobrycon eques, Hyphessobrycon erythrostigma, Hyphessobrycon megalopterus, Hyphessobrycon micropterus, Hyphessobrycon takasei, Iguanodectes spilurus, Jupiaba polylepis, Microschemobrycon sp, Moenkhausia bonita, Moenkhausia hemigrammoides, Moenkhausia intermedia, Moenkhausia sanctaefilomenae, Moenkhausia xinguensis, Oligosarcus pintoi, Paracheirodon axelrodi, Parapristella georgiae, Petitella georgiae, Phenacogaster franciscoensis, Piabina argentea, Poptella paraguayensis, Psellogrammus kennedyi, Pristella maxillaris, Rhinopetitia myersi, Roeboides bonariensis, Serrapinnus heterodon, Serrapinnus notomelas, Serrasalmus maculatus, Stethaprion erythrops, Tetragonopterus argenteus, Thayeria obliqua

\section{Escamas}

137- Linha lateral (ic - 0,111 )

(Serra, 2003: 88; Benine, 2004: 88; Moreira, 2007: 351; Bertaco, 2008: 109; Marinho, 2009: 86; Mirande, 2009: 91)

\section{0- completa}


Astyanax altiparanae, Astyanax jacobinae, Astyanax mexicanus, Brycinus longipinnis, Brycon cf pesu, Bryconamericus exodon, Bryconexodon juruenae, Bryconops melanurus, Chalceus spilogyros, Deuterodon iguape, Gymnocorymbus ternetzi, Iguanodectes spilurus, Jupiaba polylepis, Lignobrycon myersi, Microschemobrycon sp, Moenkhausia bonita, Moenkhausia hemigrammoides, Moenkhausia intermedia, Moenkhausia xinguensis, Oligosarcus pintoi, Phenacogaster franciscoensis, Piabina argentea, Poptella paraguayensis, Rhinopetitia myersi, Roeboides bonariensis, Salminus hilarii, Serrasalmus maculatus, Serrapinnus heterodon, Stethaprion erythrops, Tetragonopterus argenteus, Triportheus nematurus

\section{1 - incompleta}

Aphyocharax anisitsi, Aphyocharax pusillus, Bryconella pallidifrons, Cheirodon interruptus, Coptobrycon bilineatus, Grundulus cochae, Hasemania crenuchoides, Hasemania melanura, Hasemania sp n., Hemigrammus analis, Hemigrammus arua, Hemigrammus bellottii, Hemigrammus bleheri, Hemigrammus boesemani, Hemigrammus brevis, Hemigrammus coeruleus, Hemigrammus cylindricus, Hemigrammus gracilis, Hemigrammus haraldi, Hemigrammus hyanuary, Hemigrammus levis, Hemigrammus lunatus, Hemigrammus marginatus, Hemigrammus mimus, Hemigrammus newboldi, Hemigrammus ocellifer, Hemigrammus orthus, Hemigrammus parana, Hemigrammus pretoensis, Hemigrammus pulcher, Hemigrammus rhodostomus, Hemigrammus rodwayi, Hemigrammus schmardae, Hemigrammus silimoni, Hemigrammus skolioplatus, Hemigrammus stictus, Hemigrammus taphorni, Hemigrammus tridens, Hemigrammus ulreyi, Hemigrammus unilineatus, Hemigrammus vorderwikleri, Hemigrammus yinyang, Hemigrammus sp n., Hollandichthys aff. multifasciatus, Hyphessobrycon bentosi, Hyphessobrycon coelestinus, Hyphessobrycon compressus, Hyphessobrycon diancistrus, Hyphessobrycon eilyos, Hyphessobrycon epicharis, Hyphessobrycon eques, Hyphessobrycon erythrostigma, Hyphessobrycon megalopterus, Hyphessobrycon micropterus, Hyphessobrycon takasei, Paracheirodon axelrodi, Parapristella georgiae, Petitella georgiae, Pristella maxillaris, Serrapinnus notomelas, Thayeria obliqua

\section{$\underline{0,1-\text { Polimórfico }}$}

Hemigrammus barrigonae, Moenkhausia sanctaefilomenae, Psellogrammus kennedyi

Segundo Zanata \& Vari (2005) redução na linha lateral ocorreu independentemente em vários grupos de Characiformes, especialmente aqueles com tamanho de corpo reduzido. Hemigrammus e Moenkhausia tradicionalmente são diferenciados pela presença de linha lateral incompleta em Hemigrammus e completa em Moenkhausia. Vários autores, entretanto questionam o uso da linha lateral na diagnose desses dois gêneros, levando a que espécies com linha lateral incompleta sejam descritas em Moenkhausia e não em Hemigrammus como é o caso de Moenkhausia pyrophthalma Costa (1994) e Moenkhausia diktyota Lima \& Toledo-Piza (2001). Na descrição de Moenkhausia diktyota, Lima e Toledo-Piza (2001) optam por alocar a espécie em Moenkhausia e não em Hemigrammus devido à semelhança geral do corpo que a espécie apresenta em relação a outras espécies já descritas de Moenkahusia. 
$\mathrm{Na}$ presente análise, linha lateral incompleta aparece como sinapomórfica para o clado mais abrangente 181, sofrendo reversão para linha lateral completa no clado 121 e pontualmente nas espécies de Moenkahusia, Microschemobrycon e em Serrapinnus heterodon.

Zanata \& Vari (2005) colocam que redução no número de escamas com poros na linha lateral também ocorre em alguns representantes da família africana Alestidae. Essa condição entretando foi provavelmente adquirida independentemente em relação às espécies de Characidae.

\section{8- Escamas sobre a nadadeira caudal (ic - 0,067)}

(Serra, 2003: 89; Benine, 2004: 93; Marinho, 2009: 85; Mirande, 2009: 324)

\section{$\underline{0 \text { - ausentes ou com escamas apenas na base }}$}

Aphyocharax anisitsi, Aphyocharax pusillus, Astyanax altiparanae, Astyanax jacobinae, Astyanax mexicanus, Brycon of pesu, Bryconamericus exodon, Bryconexodon juruenae, Bryconops melanurus, Chalceus spilogyros, Cheirodon interruptus, Coptobrycon bilineatus, Deuterodon iguape, Grundulus cochae, Hasemania crenuchoides, Hasemania melanura, Hasemania sp n., Hemigrammus mimus, Hemigrammus orthus, Hemigrammus stictus, Hemigrammus tridens, Hemigrammus ulreyi, Hollandichthys aff. multifasciatus, Hyphessobrycon coelestinus, Hyphessobrycon compressus, Hyphessobrycon diancistrus, Hyphessobrycon eilyos, Hyphessobrycon epicharis, Hyphessobrycon eques, Hyphessobrycon megalopterus, Hyphessobrycon micropterus, Hyphessobrycon takasei, Jupiaba polylepis, Iguanodectes spilurus, Lignobrycon myersi, Oligosarcus pintoi, Paracheirodon axelrodi, Phenacogaster franciscoensis, Piabina argentea, Pristella maxillaris, Psellogrammus kennedyi, Roeboides bonariensis, Salminus hilarii, Serrapinnus heterodon, Serrapinnus notomelas, Serrasalmus maculatus, Triportheus nematurus

\section{1 - base e lobos cobertos com escamas}

Bryconella pallidifrons, Gymnocorymbus ternetzi, Hemigrammus analis, Hemigrammus arua, Hemigrammus barrigonae, Hemigrammus bellottii, Hemigrammus bleheri, Hemigrammus boesemani, Hemigrammus brevis, Hemigrammus coeruleus, Hemigrammus cylindricus, Hemigrammus gracilis, Hemigrammus haraldi, Hemigrammus hyanuary, Hemigrammus levis, Hemigrammus lunatus, Hemigrammus marginatus, Hemigrammus newboldi, Hemigrammus ocellifer, Hemigrammus parana, Hemigrammus pretoensis, Hemigrammus pulcher, Hemigrammus rhodostomus, Hemigrammus rodwayi, Hemigrammus schmardae, Hemigrammus silimoni, Hemigrammus skolioplatus, Hemigrammus taphorni, Hemigrammus unilineatus, Hemigrammus vorderwikleri, Hemigrammus yinyang, Hemigrammus $\mathrm{sp} \mathrm{n}$., Hyphessobrycon bentosi, Microschemobrycon sp, Moenkhausia bonita, Moenkhausia hemigrammoides, Moenkhausia intermedia, Moenkhausia sanctaefilomenae, Moenkhausia xinguensis, Parapristella georgiae, Petitella georgiae, Poptella paraguayensis, Rhinopetitia myersi, Stethaprion erythrops, Tetragonopterus argenteus, Thayeria obliqua

\section{9- Indeterminado}

Brycinus longipinnis, Hyphessobrycon erythrostigma 
Hemigrammus é tradicionalmente separado de Hyphessobycon apenas pela presença de nadadeira caudal coberta de escamas, versus nadadeira caudal nua. Segundo Eigenmann (1918) boa parte das espécies descritas em ambos os gêneros, entretando apresentam condições intermediárias de escamação na nadadeira caudal, variando entre nadadeira com base e lobos cobertos por escamas, nadadeira com escamas apenas na base (podendo ser escamas pequenas ou grandes), até nadadeira completamente nua. Algumas espécies descritas em Hemigrammus apresentam poucas escamas apenas na base da nadadeira, não se prolongando pelos lobos, como $H$. stictus; outras, entretanto apresentam escamas distribuídas característicamente por toda a base e lobos da nadadeira, porém a distribuição ao longo dos lobos também é variável. Na presente análise, a avaliação da presença de escamas sobre a nadadeira caudal, aparece como um caráter altamente homoplástico. Nadadeira caudal com escamas aparece como sinapomórfico para o clado mais inclusivo 173, que inclui todas as espécies de Hemigrammus e Moenkhausia analisadas. Dentro do clado 173, entretanto, o caráter sofre inúmeras reversões para nadadeira caudal nua.

A avaliação da escamação presente na nadadeira caudal é bastante difícil de ser realizada, visto que as espécies podem apresentar escamas de tamanho diferente e diferentes graus de escamação sobre a base e lobos, o que dificulta a delimitação dos estados dos caracteres. Além disso, as escamas presentes na nadadeira caudal são facilmente perdidas durante o processo de fixação, o que torna bastante difícil a simples constatação da presença ou não de escamas sobre a nadadeira caudal de algumas espécies.

139- Número de séries longitudinais de escamas sobre os raios da nadadeira anal (ic - 0,333)

(Serra, 2003: 94; Moreira, 2007: 358)

\section{0 - duas séries ou mais}

Brycon cf pesu, Gymnocorymbus ternetzi, Hemigrammus pretoensis, Moenkhausia sanctaefilomenae, Poptella paraguayensis, Psellogrammus kennedyi, Roeboides bonariensis, Salminus hilarii, Serrasalmus maculatus, Stethaprion erythrops, Tetragonopterus argenteus, Triportheus nematurus

\section{1- uma série}


Aphyocharax anisitsi, Aphyocharax pusillus, Astyanax altiparanae, Astyanax jacobinae, Astyanax mexicanus, Bryconamericus exodon, Bryconella pallidifrons, Bryconexodon juruenae, Bryconops melanurus, Chalceus spilogyros, Cheirodon interruptus, Deuterodon iguape, Hasemania crenuchoides, Hasemania melanura, Hasemania sp n., Hemigrammus analis, Hemigrammus arua, Hemigrammus barrigonae, Hemigrammus bellottii, Hemigrammus bleheri, Hemigrammus boesemani, Hemigrammus brevis, Hemigrammus coeruleus, Hemigrammus cylindricus, Hemigrammus gracilis, Hemigrammus haraldi, Hemigrammus hyanuary, Hemigrammus levis, Hemigrammus lunatus, Hemigrammus marginatus, Hemigrammus mimus, Hemigrammus newboldi, Hemigrammus ocellifer, Hemigrammus orthus, Hemigrammus parana, Hemigrammus pulcher, Hemigrammus rhodostomus, Hemigrammus rodwayi, Hemigrammus schmardae, Hemigrammus silimoni, Hemigrammus skolioplatus, Hemigrammus stictus, Hemigrammus taphorni, Hemigrammus tridens, Hemigrammus ulreyi, Hemigrammus unilineatus, Hemigrammus vorderwikleri, Hemigrammus yinyang, Hemigrammus sp n., Hollandichthys aff. multifasciatus, Hyphessobrycon bentosi, Hyphessobrycon coelestinus, Hyphessobrycon compressus, Hyphessobrycon eilyos, Hyphessobrycon eques, Hyphessobrycon megalopterus, Hyphessobrycon micropterus, Hyphessobrycon takasei, Iguanodectes spilurus, Jupiaba polylepis, Microschemobrycon sp, Moenkhausia bonita, Moenkhausia hemigrammoides, Moenkhausia intermedia, Oligosarcus pintoi, Paracheirodon axelrodi, Parapristella georgiae, Petitella georgiae, Phenacogaster franciscoensis, Piabina argentea, Pristella maxillaris, Rhinopetitia myersi, Serrapinnus heterodon, Serrapinnus notomelas, Thayeria obliqua

\section{2- ausente}

Brycinus longipinnis, Coptobrycon bilineatus, Grundulus cochae, Lignobrycon myersi

\section{$\underline{0,1-\text { Polimórfico }}$}

Moenkhausia xinguensis

\section{9- Indeterminado}

Hyphessobrycon diancistrus, Hyphessobrycon epicharis, Hyphessobrycon erythrostigma

\section{0- Número de escamas sobre os raios da nadadeira anal (na série longitudinal mais próxima à base dos raios) (ic $-0,231$ )}

(Serra, 2003: 93)

\section{0 - mais de 30 escamas}

Brycon cf pesu, Gymnocorymbus ternetzi, Moenkhausia intermedia, Phenacogaster franciscoensis, Roeboides bonariensis, Rhinopetitia myersi, Slaminus hilarii, Serrasalmus maculatus, Stethaprion erythrops, Tetragonopterus argenteus

\section{1- duas a seis escamas}

Aphyocharax anisitsi, Aphyocharax pusillus, Astyanax jacobinae, Astyanax mexicanus, Bryconamericus exodon, Bryconella pallidifrons, Bryconops melanurus, Chalceus spilogyros, Hasemania crenuchoides, Hasemania melanura, Hemigrammus analis, Hemigrammus arua, Hemigrammus barrigonae, Hemigrammus bellottii, Hemigrammus bleheri, Hemigrammus boesemani, Hemigrammus brevis, Hemigrammus coeruleus, Hemigrammus cylindricus, Hemigrammus gracilis, Hemigrammus haraldi, Hemigrammus hyanuary, Hemigrammus levis, Hemigrammus lunatus, Hemigrammus marginatus, Hemigrammus mimus, Hemigrammus newboldi, Hemigrammus ocellifer, Hemigrammus orthus, Hemigrammus parana, Hemigrammus 
pulcher, Hemigrammus rhodostomus, Hemigrammus rodwayi, Hemigrammus schmardae, Hemigrammus silimoni, Hemigrammus skolioplatus, Hemigrammus stictus, Hemigrammus taphorni, Hemigrammus tridens, Hemigrammus ulreyi, Hemigrammus unilineatus, Hemigrammus vorderwikleri, Hemigrammus yinyang, Hemigrammus sp n., Hyphessobrycon bentosi, Hyphessobrycon coelestinus, Hyphessobrycon compressus, Hyphessobrycon eilyos, Hyphessobrycon eques, Hyphessobrycon megalopterus, Hyphessobrycon micropterus, Iguanodectes spilurus, Microschemobrycon sp, Moenkhausia bonita, Moenkhausia hemigrammoides, Oligosarcus pintoi, Paracheirodon axelrodi, Parapristella georgiae, Petitella georgiae, Piabina argentea, Pristella maxillaris, Serrapinnus heterodon, Serrapinnus notomelas, Thayeria obliqua

\section{2- sete a vinte escamas}

Astyanax altiparanae, Bryconexodon juruenae, Deuterodon iguape, Hasemania sp n., Hemigrammus pretoensis, Hyphessobrycon takasei, Hollandichthys multifasciatus, Jupiaba polylepis, Moenkhausia sanctaefilomenae, Moenkhausia xinguensis, Poptella paraguayensis, Psellogrammus kennedyi, Triportheus nematurus

\section{3- ausentes}

Coptobrycon bilineatus, Grundulus cochae

\section{1, 2- Polimórfico}

Cheirodon interruptus

\section{9- Indeterminado}

Brycinus longipinnis, Hyphessobrycon diancistrus, Hyphessobrycon epicharis, Hyphessobrycon erythrostigma, Lignobrycon myersi

\section{1- Distribuição longitudinal das escamas sobre a nadadeira anal (na série longitudinal mais próxima à base dos raios) (ic $-0,200$ )}

\section{0 - ao longo de toda a nadadeira}

Brycon cf pesu, Gymnocorymbus ternetzi, Hemigrammus pretoensis, Roeboides bonariensis, Salminus hilarii, Serrasalmus maculatus, Stethaprion erythrops, Tetragonopterus argenteus, Triportheus nematurus

\section{1- apenas sobre o primeiro terço}

Aphyocharax anisitsi, Aphyocharax pusillus, Astyanax jacobinae, Astyanax mexicanus, Bryconamericus exodon, Bryconella pallidifrons, Bryconexodon juruenae, Bryconops melanurus, Cheirodon interruptus, Deuterodon iguape, Hasemania crenuchoides, Hasemania melanura, Hemigrammus analis, Hemigrammus arua, Hemigrammus barrigonae, Hemigrammus bellottii, Hemigrammus bleheri, Hemigrammus boesemani, Hemigrammus brevis, Hemigrammus coeruleus, Hemigrammus cylindricus, Hemigrammus gracilis, Hemigrammus haraldi, Hemigrammus hyanuary, Hemigrammus levis, Hemigrammus lunatus, Hemigrammus marginatus, Hemigrammus mimus, Hemigrammus newboldi, Hemigrammus ocellifer, Hemigrammus orthus, Hemigrammus parana, Hemigrammus pulcher, Hemigrammus rhodostomus, Hemigrammus rodwayi, Hemigrammus schmardae, Hemigrammus silimoni, Hemigrammus skolioplatus, Hemigrammus stictus, Hemigrammus taphorni, Hemigrammus tridens, Hemigrammus ulreyi, Hemigrammus unilineatus, Hemigrammus vorderwikleri, Hemigrammus yinyang, Hemigrammus sp n., Hollandichthys aff. multifasciatus, 
Hyphessobrycon bentosi, Hyphessobrycon coelestinus, Hyphessobrycon compressus, Hyphessobrycon eilyos, Hyphessobrycon eques, Hyphessobrycon megalopterus, Hyphessobrycon micropterus, Hyphessobrycon takasei, lguanodectes spilurus, Jupiaba polylepis, Microschemobrycon sp, Moenkhausia bonita, Moenkhausia hemigrammoides, Moenkhausia intermedia, Moenkhausia xinguensis, Paracheirodon axelrodi, Parapristella georgiae, Petitella georgiae, Phenacogaster franciscoensis, Piabina argentea, Pristella maxillaris, Rhinopetitia myersi, Serrapinnus heterodon, Serrapinnus notomelas, Thayeria obliqua

\section{2- prolongando-se até $50 \%$ ou mais, mas sem atingir o final da}

\section{nadadeira}

Astyanax altiparanae, Chalceus spilogyros, Hasemania sp n., Moenkhausia sanctaefilomenae, Oligosarcus pintoi, Poptella paraguayensis, Psellogrammus kennedyi

\section{9- Ideterminado}

Brycinus longipinnis, Coptobrycon bilineatus, Grundulus cochae, Hyphessobrycon diancistrus, Hyphessobrycon epicharis, Hyphessobrycon erythrostigma, Lignobrycon myersi

\section{Colorido}

\section{2- Colorido da cabeça em vida (ic - 1,0)}

\section{0 - igual ao do restante do corpo, sem coloração distintamente vermelha}

\section{na cabeca}

Aphyocharax anisitsi, Aphyocharax pusillus, Astyanax altiparanae, Astyanax jacobinae, Astyanax mexicanus, Brycinus longipinnis, Brycon cf pesu, Bryconamericus exodon, Bryconella pallidifrons, Bryconexodon juruenae, Bryconops melanurus, Chalceus spilogyros, Cheirodon interruptus, Coptobrycon bilineatus, Deuterodon iguape, Grundulus cochae, Gymnocorymbus ternetzi, Hasemania crenuchoides, Hasemania melanura, Hasemania sp n., Hemigrammus analis, Hemigrammus arua, Hemigrammus barrigonae, Hemigrammus bellottii, Hemigrammus boesemani, Hemigrammus brevis, Hemigrammus coeruleus, Hemigrammus cylindricus, Hemigrammus gracilis, Hemigrammus haraldi, Hemigrammus hyanuary, Hemigrammus levis, Hemigrammus lunatus, Hemigrammus marginatus, Hemigrammus mimus, Hemigrammus newboldi, Hemigrammus ocellifer, Hemigrammus orthus, Hemigrammus parana, Hemigrammus pretoensis, Hemigrammus pulcher, Hemigrammus rodwayi, Hemigrammus schmardae, Hemigrammus silimoni, Hemigrammus skolioplatus, Hemigrammus stictus, Hemigrammus taphorni, Hemigrammus tridens, Hemigrammus ulreyi, Hemigrammus unilineatus, Hemigrammus vorderwikleri, Hemigrammus yinyang, Hemigrammus sp n., Hollandichthys aff. multifasciatus, Hyphessobrycon bentosi, Hyphessobrycon coelestinus, Hyphessobrycon compressus, Hyphessobrycon diancistrus, Hyphessobrycon eilyos, Hyphessobrycon epicharis, Hyphessobrycon eques, Hyphessobrycon erythrostigma, Hyphessobrycon megalopterus, Hyphessobrycon micropterus, Hyphessobrycon takasei, lguanodectes spilurus, Jupiaba polylepis, Lignobrycon myersi, Microschemobrycon sp, Moenkhausia bonita, Moenkhausia hemigrammoides, Moenkhausia intermedia, Moenkhausia sanctaefilomenae, Moenkhausia xinguensis, Oligosarcus pintoi, Paracheirodon axelrodi, Parapristella georgiae, Phenacogaster franciscoensis, Piabina argentea, Poptella paraguayensis, Psellogrammus kennedyi, Pristella maxillaris, Rhinopetitia myersi, Roeboides bonariensis, Salminus hilarii, Serrapinnus heterodon, Serrapinnus notomelas, Serrasalmus maculatus, Stethaprion erythrops, Tetragonopterus argenteus, Thayeria obliqua, Triportheus nematurus 
Hemigrammus rhodostomus, $H$. bleheri e $P$. georgiae apresentam uma coloração derivada na cabeça em exemplares vivos. Nessas espécies a região da cabeça é característicamente vermelho-vivo, sendo essa coloração distinta do restante do corpo, condição não observada em nenhuma outra espécie de Characidae, sendo assumida, portanto como uma sinapomorfia exlusiva para o clado 138. Dentro de Characidae, algumas espécies do grupo dos "rosy tetra" também apresentam coloração vermelha na cabeça em exemplares vivos, porém essa coloração pode não ser muito conspícua (apresentando tons de rosa claro ou marrom avermelhado), e é distribuída por todo o corpo dos indivíduos, não estando restrita à cabeça. Sendo assim, a condição encontrada nos "rosy tetra" não é considerada homóloga àquela de $H$. rhodostomus, $H$. bleheri e $P$. georgiae.

143- Cromatóforos expandidos concentrados sobre o infra-orbital 3 (ic $0,125)$

\section{$\underline{0 \text { - ausentes }}$}

Aphyocharax anisitsi, Aphyocharax pusillus, Astyanax altiparanae, Astyanax jacobinae, Astyanax mexicanus, Brycon cf pesu, Bryconamericus exodon, Bryconella pallidifrons, Bryconexodon juruenae, Bryconops melanurus, Chalceus spilogyros, Cheirodon interruptus, Deuterodon iguape, Grundulus cochae, Hasemania crenuchoides, Hasemania melanura, Hasemania sp n., Hemigrammus analis, Hemigrammus arua, Hemigrammus barrigonae, Hemigrammus bellottii, Hemigrammus bleheri, Hemigrammus boesemani, Hemigrammus brevis, Hemigrammus coeruleus, Hemigrammus cylindricus, Hemigrammus gracilis, Hemigrammus haraldi, Hemigrammus hyanuary, Hemigrammus levis, Hemigrammus lunatus, Hemigrammus marginatus, Hemigrammus mimus, Hemigrammus newboldi, Hemigrammus ocellifer, Hemigrammus orthus, Hemigrammus parana, Hemigrammus pretoensis, Hemigrammus pulcher, Hemigrammus rhodostomus, Hemigrammus rodwayi, Hemigrammus silimoni, Hemigrammus skolioplatus, Hemigrammus stictus, Hemigrammus taphorni, Hemigrammus tridens, Hemigrammus ulreyi, Hemigrammus vorderwikleri, Hemigrammus sp n., Hollandichthys aff. multifasciatus, Hyphessobrycon bentosi, Hyphessobrycon coelestinus, Hyphessobrycon compressus, Hyphessobrycon diancistrus, Hyphessobrycon eilyos, Hyphessobrycon megalopterus, Hyphessobrycon micropterus, Hyphessobrycon takasei, Iguanodectes spilurus, Jupiaba polylepis, Lignobrycon myersi, Microschemobrycon sp, Moenkhausia bonita, Moenkhausia hemigrammoides, Moenkhausia intermedia, Moenkhausia sanctaefilomenae, Moenkhausia xinguensis, Paracheirodon axelrodi, Parapristella georgiae, Petitella georgiae, Phenacogaster franciscoensis, Piabina argentea, Poptella paraguayensis, Psellogrammus kennedyi, Rhinopetitia myersi, Roeboides bonariensis, Salminus hilarii, Serrapinnus heterodon, Serrapinnus notomelas, Serrasalmus maculatus, Stethaprion erythrops, Tetragonopterus argenteus, Thayeria obliqua, Triportheus nematurus

\section{1 - presentes}


Coptobrycon bilineatus, Gymnocorymbus ternetzi, Hemigrammus schmardae, Hemigrammus unilineatus, Hemigrammus yinyang, Hyphessobrycon eques, Oligosarcus pintoi, Pristella maxillaris

\section{9- Indeterminado}

Brycinus longipinnis, Hyphessobrycon epicharis, Hyphessobrycon erythrostigma

\section{4- Mancha preta no olho (ic - 0,154)}

\section{$\underline{0 \text { - ausente ou presente apenas na porção superior do olho }}$}

Aphyocharax anisitsi, Aphyocharax pusillus, Astyanax altiparanae, Astyanax jacobinae, Astyanax mexicanus, Brycinus longipinnis, Brycon cf pesu, Bryconamericus exodon, Bryconella pallidifrons, Bryconexodon juruenae, Bryconops melanurus, Chalceus spilogyros, Cheirodon interruptus, Coptobrycon bilineatus, Deuterodon iguape, Grundulus cochae, Hasemania crenuchoides, Hasemania melanura, Hasemania sp n., Hemigrammus analis, Hemigrammus bellottii, Hemigrammus bleheri, Hemigrammus boesemani, Hemigrammus brevis, Hemigrammus cylindricus, Hemigrammus gracilis, Hemigrammus haraldi, Hemigrammus hyanuary, Hemigrammus levis, Hemigrammus marginatus, Hemigrammus ocellifer, Hemigrammus orthus, Hemigrammus parana, Hemigrammus pulcher, Hemigrammus rhodostomus, Hemigrammus rodwayi, Hemigrammus silimoni, Hemigrammus skolioplatus, Hemigrammus taphorni, Hemigrammus tridens, Hemigrammus unilineatus, Hemigrammus yinyang, Hemigrammus sp n., Hollandichthys aff. multifasciatus, Hyphessobrycon coelestinus, Hyphessobrycon compressus, Hyphessobrycon diancistrus, Hyphessobrycon eilyos, Hyphessobrycon epicharis, Iguanodectes spilurus, Jupiaba polylepis, Lignobrycon myersi, Microschemobrycon sp, Moenkhausia bonita, Moenkhausia hemigrammoides, Moenkhausia intermedia, Moenkhausia sanctaefilomenae, Moenkhausia xinguensis, Oligosarcus pintoi, Petitella georgiae, Phenacogaster franciscoensis, Piabina argentea, Poptella paraguayensis, Rhinopetitia myersi, Roeboides bonariensis, Salminus hilarii, Serrapinnus heterodon, Serrasalmus maculatus, Stethaprion erythrops, Thayeria obliqua, Triportheus nematurus

\section{1- presente, como uma faixa vertical}

Gymnocorymbus ternetzi, Hemigrammus coeruleus, Hemigrammus schmardae, Hemigrammus stictus, Hemigrammus vorderwikleri, Hyphessobrycon bentosi, Hyphessobrycon eques, Hyphessobrycon erythrostigma, Hyphessobrycon megalopterus, Hyphessobrycon takasei, Pristella maxillaris, Psellogrammus kennedyi, Serrapinnus notomelas, Tetragonopterus argenteus

\section{2- presente, como uma faixa horizontal}

Hemigrammus barrigonae, Hemigrammus ulreyi, Paracheirodon axelrodi

\section{9- Indeterminado}

Hemigrammus arua, Hemigrammus lunatus, Hemigrammus mimus, Hemigrammus newboldi, Hemigrammus pretoensis, Hyphessobrycon micropterus, Parapristella georgiae

\section{5- Mancha vermelha na porção superior do olho (ic - 0,067)}

\section{$\underline{0 \text { - ausente }}$}

Aphyocharax anisitsi, Astyanax altiparanae, Astyanax jacobinae, Bryconamericus exodon, Bryconexodon juruenae, Chalceus spilogyros, Cheirodon interruptus, Coptobrycon bilineatus, Deuterodon iguape, Gymnocorymbus ternetzi, Hasemania crenuchoides, Hasemania sp n., 
Hemigrammus arua, Hemigrammus barrigonae, Hemigrammus boesemani, Hemigrammus brevis, Hemigrammus cylindricus, Hemigrammus hyanuary, Hemigrammus levis, Hemigrammus lunatus, Hemigrammus marginatus, Hemigrammus newboldi, Hemigrammus parana, Hemigrammus rodwayi, Hemigrammus schmardae, Hemigrammus silimoni, Hemigrammus skolioplatus, Hemigrammus tridens, Hemigrammus ulreyi, Hemigrammus unilineatus, Hemigrammus sp n., Hollandichthys aff. multifasciatus, Hyphessobrycon bentosi, Hyphessobrycon eilyos, Hyphessobrycon megalopterus, Hyphessobrycon micropterus, Hyphessobrycon takasei, Iguanodectes spilurus, Moenkhausia hemigrammoides, Moenkhausia intermedia, Moenkhausia xinguensis, Oligosarcus pintoi, Paracheirodon axelrodi, Parapristella georgiae, Phenacogaster franciscoensis, Poptella paraguayensis, Pristella maxillaris, Roeboides bonariensis, Salminus hilarii, Serrapinnus notomelas, Serrasalmus maculatus, Tetragonopterus argenteus, Thayeria obliqua, Triportheus nematurus

\section{1- presente}

Brycinus longipinnis, Bryconella pallidifrons, Bryconops melanurus, Hemigrammus analis, Hemigrammus bellottii, Hemigrammus bleheri, Hemigrammus coeruleus, Hemigrammus haraldi, Hemigrammus ocellifer, Hemigrammus pulcher, Hemigrammus rhodostomus, Hemigrammus stictus, Hemigrammus vorderwikleri, Hemigrammus yinyang, Hyphessobrycon eques, Hyphessobrycon erythrostigma, Moenkhausia sanctaefilomenae, Petitella georgiae, Piabina argentea

\section{9- Indeterminado}

Aphyocharax pusillus, Astyanax mexicanus, Brycon cf pesu, Grundulus cochae, Hemigrammus gracilis, Hasemania melanura, Hemigrammus mimus, Hemigrammus orthus, Hemigrammus pretoensis, Hemigrammus taphorni, Hyphessobrycon coelestinus, Hyphessobrycon compressus, Hyphessobrycon diancistrus, Hyphessobrycon epicharis, Jupiaba polylepis, Lignobrycon myersi, Microschemobrycon sp, Moenkhausia bonita, Psellogrammus kennedyi, Rhinopetitia myersi, Serrapinnus heterodon, Stethaprion erythrops

\section{6- Mancha negra nas escamas dorsais (ic - 0,167 )}

\section{$\underline{0 \text { - ausente }}$}

Aphyocharax anisitsi, Aphyocharax pusillus, Astyanax altiparanae, Astyanax mexicanus, Brycon cf pesu, Bryconamericus exodon, Bryconella pallidifrons, Bryconexodon juruenae, Bryconops melanurus, Chalceus spilogyros, Cheirodon interruptus, Coptobrycon bilineatus, Grundulus cochae, Gymnocorymbus ternetzi, Hasemania crenuchoides, Hasemania melanura, Hasemania sp n., Hemigrammus arua, Hemigrammus barrigonae, Hemigrammus boesemani, Hemigrammus brevis, Hemigrammus gracilis, Hemigrammus hyanuary, Hemigrammus lunatus, Hemigrammus marginatus, Hemigrammus mimus, Hemigrammus newboldi, Hemigrammus parana, Hemigrammus pretoensis, Hemigrammus pulcher, Hemigrammus rhodostomus, Hemigrammus rodwayi, Hemigrammus silimoni, Hemigrammus skolioplatus, Hemigrammus stictus, Hemigrammus taphorni, Hemigrammus tridens, Hemigrammus ulreyi, Hemigrammus vorderwikleri, Hemigrammus yinyang, Hemigrammus sp n., Hollandichthys aff. multifasciatus, Hyphessobrycon bentosi, Hyphessobrycon coelestinus, Hyphessobrycon compressus, Hyphessobrycon eilyos, Hyphessobrycon epicharis, Hyphessobrycon eques, Hyphessobrycon erythrostigma, Hyphessobrycon megalopterus, Hyphessobrycon micropterus, Hyphessobrycon takasei, Iguanodectes spilurus, Jupiaba polylepis, Lignobrycon myersi, Microschemobrycon sp, Moenkhausia bonita, Moenkhausia intermedia, Moenkhausia sanctaefilomenae, Moenkhausia xinguensis, Oligosarcus pintoi, Paracheirodon axelrodi, Parapristella georgiae, Petitella georgiae, Phenacogaster franciscoensis, Piabina argentea, Poptella paraguayensis, Psellogrammus kennedyi, Pristella maxillaris, Rhinopetitia myersi, Roeboides bonariensis, Salminus hilarii, Serrapinnus heterodon, Serrapinnus notomelas, Serrasalmus maculatus, Stethaprion erythrops, Tetragonopterus argenteus, Thayeria obliqua, Triportheus nematurus

\section{$\underline{1-\text { presente }}$}


Hemigrammus analis, Hemigrammus bellottii, Hemigrammus cylindricus, Hyphessobrycon diancistrus, Hemigrammus haraldi, Hemigrammus levis, Hemigrammus orthus, Moenkhausia hemigrammoides

\section{$\underline{0,1-\text { Polimórfico }}$}

Deuterodon iguape, Hemigrammus bleheri, Hemigrammus coeruleus, Hemigrammus ocellifer, Hemigrammus schmardae, Hemigrammus unilineatus

\section{9- Indeterminado}

Astyanax jacobinae, Brycinus longipinnis

147- Mácula umeral (ic - 0,115)

(Bertaco, 2008: 111-112)

\section{$\underline{0-\text { ausente }}$}

Aphyocharax pusillus, Bryconops melanurus, Cheirodon interruptus, Coptobrycon bilineatus, Hasemania melanura, Hemigrammus bleheri, Hemigrammus boesemani, Hemigrammus brevis, Hemigrammus gracilis, Hemigrammus levis, Hemigrammus marginatus, Hemigrammus mimus, Hemigrammus parana, Hemigrammus rhodostomus, Hemigrammus rodwayi, Hemigrammus silimoni, Hemigrammus sp n., Hemigrammus tridens, Hyphessobrycon compressus, Hyphessobrycon diancistrus, Hyphessobrycon eilyos, Iguanodectes spilurus, Lignobrycon myersi, Microschemobrycon sp, Moenkhausia bonita, Moenkhausia intermedia, Paracheirodon axelrodi, Parapristella georgiae, Petitella georgiae, Serrasalmus maculatus, Serrapinnus heterodon, Serrapinnus notomelas, Thayeria obliqua Triportheus nematurus

\section{1- uma, comecando na altura da $1^{\underline{a}}$ a $3^{\text {a }}$ escama da linha lateral}

Aphyocharax anisitsi, Astyanax altiparanae, Astyanax jacobinae, Astyanax mexicanus, Brycon cf pesu, Bryconamericus exodon, Bryconella pallidifrons, Bryconexodon juruenae, Chalceus spilogyros, Deuterodon iguape, Grundulus cochae, Hasemania crenuchoides, Hasemania sp n., Hemigrammus analis, Hemigrammus barrigonae, Hemigrammus bellottii, Hemigrammus coeruleus, Hemigrammus cylindricus, Hemigrammus hyanuary, Hemigrammus lunatus, Hemigrammus newboldi, Hemigrammus orthus, Hemigrammus schmardae, Hemigrammus skolioplatus, Hemigrammus taphorni, Hemigrammus ulreyi, Hemigrammus unilineatus, Hemigrammus vorderwikleri, Hyphessobrycon bentosi, Hyphessobrycon coelestinus, Hyphessobrycon epicharis, Hyphessobrycon eques, Hyphessobrycon erythrostigma, Hyphessobrycon megalopterus, Hyphessobrycon takasei, Moenkhausia hemigrammoides, Moenkhausia sanctaefilomenae, Moenkhausia xinguensis, Oligosarcus pintoi, Piabina argentea, Psellogrammus kennedyi, Pristella maxillaris, Rhinopetitia myersi, Salminus hilarii

\section{2- duas, a primeira começando na altura da $1^{\underline{a}}$ a $3^{\underline{a}}$ escama da linha}

\section{lateral}

Gymnocorymbus ternetzi, Hemigrammus haraldi, Hemigrammus ocellifer, Hemigrammus pretoensis, Hemigrammus pulcher, Hemigrammus yinyang, Hollandichthys aff. multifasciatus, Poptella paraguayensis, Stethaprion erythrops, Tetragonopterus argenteus

3- uma ou duas, a primeira deslocada posteriormente, começando na altura da $5^{\underline{a}-6} 6^{\underline{a}}$ escama da linha lateral 
Hemigrammus arua, Hemigrammus stictus, Jupiaba polylepis, Phenacogaster franciscoensis, Roeboides bonariensis

\section{$\underline{0,1-\text { Polimórfico }}$}

Hyphessobrycon micropterus

\section{9- Indeterminado}

Brycinus longipinnis

A ausência de mácula umeral foi sugerida como uma sinapomorfia para a subfamília Cheirodontinae por Malabarba (1998) e sugerida por Weitzman \& Malabarba (1999) como tendo surgido independentemente nos diversos grupos de Characidae onde essa condição ocorre. Na presente análise, a ausência de uma mácula umeral aparece sinapomórfica para o clado mais inclusivo 186, com diversas reversões para mácula presente dentro do referido clado.

Lima \& Souza (2009), discutem um possível clado monofilético dentro de Hemigrammus denominado "Hemigrammus ocellifer species-group", formado por espécies que apresentam duas máculas umerais, um único gancho de tamanho médio a grande nos raios da nadadeira anal, mancha negra no pedúnculo caudal e mancha vermelha na porção superior do olho em exemplares vivos, incluindo $H$. pulcher, $H$. haraldi, $H$. guyanensis, $H$. ocellifer, H. neptunus, $H$. luelingi e $H$. yinyang (Lima \& Souza, 2009). Dentre as espécies de Hemigrammus com mácula umeral dupla analisadas, entretanto, $H$. ocellifer aparece mais relacionada à $H$. coeruleus, que apresenta mácula umeral simples (clado 166), $H$. pulcher a $H$. haraldi (clado 143), e $H$. yinyang com espécies de outros gêneros de Characidae (clado 125).

Lima et al., (2009) sugerem que uma mácula umeral única e posteriormente deslocada, como a encontrada em $H$. arua e $H$. stictus possa ser homóloga à segunda mácula umeral das espécies de Hemigrammus que apresentam mácula umeral dupla. Essa hipótese, entretanto, é refutada pela presente análise, pois além de $H$. arua e $H$. stictus não aparecerem mais próximas filogenéticamente, nenhuma das duas espécies também aparece mais proximamente relacionada com aquelas de Hemigrammus que apresentam duas máculas umerais, a saber: $H$. ocelliffer, $H$. pulcher, $H$. haraldi e H. yinyang. 
148- Mácula umeral (ic - 0,125)

\section{0- alongada verticalmente}

Aphyocharax anisitsi, Astyanax jacobinae, Astyanax mexicanus, Bryconamericus exodon, Bryconella pallidifrons, Deuterodon iguape, Grundulus cochae, Gymnocorymbus ternetzi, Hasemania crenuchoides, Hasemania sp n., Hemigrammus arua, Hemigrammus bellottii, Hemigrammus coeruleus, Hemigrammus cylindricus, Hemigrammus haraldi, Hemigrammus newboldi, Hemigrammus ocellifer, Hemigrammus pretoensis, Hemigrammus pulcher, Hemigrammus schmardae, Hemigrammus skolioplatus, Hemigrammus taphorni, Hemigrammus unilineatus, Hemigrammus yinyang. Hyphessobrycon bentosi, Hyphessobrycon coelestinus, Hyphessobrycon eques, Hyphessobrycon erythrostigma, Hyphessobrycon megalopterus, Hyphessobrycon micropterus, Hyphessobrycon takasei, Moenkhausia hemigrammoides, Moenkhausia sanctaefilomenae, Oligosarcus pintoi, Piabina argentea, Poptella paraguayensis, Psellogrammus kennedyi, Rhinopetitia myersi, Stethaprion erythrops, Tetragonopterus argenteus

\section{1- alongada horizontalmente}

Astyanax altiparanae, Hemigrammus barrigonae, Hemigrammus ulreyi, Hyphessobrycon epicharis

\section{2- arredondada}

Bryconexodon juruenae, Chalceus spilogyros, Hemigrammus analis, Hemigrammus hyanuary, Hemigrammus lunatus, Hemigrammus orthus, Hemigrammus stictus, Jupiaba polylepis, Moenkhausia xinguensis, Phenacogaster franciscoensis, Pristella maxillaris, Roeboides bonariensis, Salminus hilarii

\section{$\underline{0,2-P o l i m o ́ r f i c o ~}$}

Hemigrammus vorderwikleri

\section{9- Indeterminado}

Aphyocharax pusillus, Brycinus longipinnis, Brycon cf pesu, Bryconops melanurus, Cheirodon interruptus, Coptobrycon bilineatus, Hasemania melanura, Hemigrammus bleheri, Hemigrammus boesemani, Hemigrammus brevis, Hemigrammus gracilis, Hemigrammus levis, Hemigrammus marginatus, Hemigrammus mimus, Hemigrammus parana, Hemigrammus rhodostomus, Hemigrammus rodwayi, Hemigrammus silimoni, Hemigrammus sp n., Hemigrammus tridens, Hollandichthys aff. multifasciatus, Hyphessobrycon compressus, Hyphessobrycon diancistrus, Hyphessobrycon eilyos, Iguanodectes spilurus, Lignobrycon myersi, Microschemobrycon sp, Moenkhausia bonita, Moenkhausia intermedia, Paracheirodon axelrodi, Parapristella georgiae, Petitella georgiae, Serrapinnus heterodon, Serrapinnus notomelas, Serrasalmus maculatus, Thayeria obliqua, Triportheus nematurus

\section{9- Região clara (halo) após a mácula umeral ou entre a primeira e a segunda mácula umeral (ic - 0,125)}

\section{$\underline{0 \text { - ausente }}$}

Astyanax altiparanae, Astyanax jacobinae, Astyanax mexicanus, Brycinus longipinnis, Brycon cf pesu, Bryconamericus exodon, Bryconella pallidifrons, Bryconexodon juruenae, Chalceus spilogyros, Deuterodon iguape, Grundulus cochae, Hasemania crenuchoides, Hasemania sp n., Hemigrammus analis, Hemigrammus arua, Hemigrammus barrigonae, Hemigrammus bellottii, 
Hemigrammus cylindricus, Hemigrammus hyanuary, Hemigrammus lunatus, Hemigrammus newboldi, Hemigrammus orthus, Hemigrammus pretoensis, Hemigrammus schmardae, Hemigrammus skolioplatus, Hemigrammus taphorni, Hemigrammus ulreyi, Hemigrammus unilineatus, Hemigrammus vorderwikleri, Hollandichthys aff. multifasciatus, Hyphessobrycon bentosi, Hyphessobrycon coelestinus, Hyphessobrycon epicharis, Hyphessobrycon eques, Hyphessobrycon micropterus, Hyphessobrycon takasei, Moenkhausia hemigrammoides, Moenkhausia sanctaefilomenae, Moenkhausia xinguensis, Oligosarcus pintoi, Phenacogaster franciscoensis, Piabina argentea, Psellogrammus kennedyi, Pristella maxillaris, Rhinopetitia myersi, Roeboides bonariensis, Salminus hilarii, Serrasalmus maculatus, Tetragonopterus argenteus

\section{1 - presente}

Gymnocorymbus ternetzi, Hemigrammus coeruleus, Hemigrammus haraldi, Hemigrammus ocellifer, Hemigrammus pulcher, Hemigrammus stictus, Hemigrammus yinyang, Hyphessobrycon megalopterus, Jupiaba polylepis, Poptella paraguayensis, Stethaprion erythrops

\section{9- Indeterminado}

Aphyocharax anisitsi, Aphyocharax pusillus, Bryconops melanurus, Cheirodon interruptus, Coptobrycon bilineatus, Hasemania melanura, Hemigrammus bleheri, Hemigrammus boesemani, Hemigrammus brevis, Hemigrammus gracilis, Hemigrammus levis, Hemigrammus marginatus, Hemigrammus mimus, Hemigrammus parana, Hemigrammus rhodostomus, Hemigrammus rodwayi, Hemigrammus silimoni, Hemigrammus sp n., Hemigrammus tridens, Hyphessobrycon compressus, Hyphessobrycon diancistrus, Hyphessobrycon eilyos, Hyphessobrycon erythrostigma, Iguanodectes spilurus, Lignobrycon myersi, Microschemobrycon sp, Moenkhausia bonita, Moenkhausia intermedia, Paracheirodon axelrodi, Parapristella georgiae, Petitella georgiae, Serrapinnus heterodon, Serrapinnus notomelas, Thayeria obliqua, Triportheus nematurus

Algumas espécies apresentam uma região com menor concentração de cromatóforos (ou totalmente desprovida deles) logo após a mácula umeral, ou entre a primeira e a segunda mácula. Essa região é mais clara que o restante do corpo e ajuda a delimitar o entorno da mácula umeral. Região clara após a mácula umeral ou entre a primeira e a segunda mácula, apesar de não ser uma característica amplamente distribuída, pode ser encontrada em alguns representantes de Characidae analisados (notadamente entre aqueles que apresentam mais de uma mácula umeral). $\mathrm{Na}$ presente análise, essa característica aparece como tendo surgido diversas vezes e independentemente.

\section{0- Listra negra longitudinal no corpo (ic - 0,167 )}

\section{0- ausente}

Astyanax jacobinae, Brycon cf pesu, Chalceus spilogyros, Grundulus cochae, Hemigrammus mimus, Hemigrammus pretoensis, Hollandichthys aff. multifasciatus, Hyphessobrycon bentosi, Hyphessobrycon eilyos, Hyphessobrycon eques, Hyphessobrycon erythrostigma, 
Serra, J. P. - Análise Filogenética das Espécies de Hemigrammus Gill, 1858 (Characiformes, Characidae)

Hyphessobrycon megalopterus, Hyphessobrycon micropterus, Hyphessobrycon takasei, Microschemobrycon sp, Roeboides bonariensis, Salminus hilarii, Serrasalmus maculatus

\section{1- presente, começando em contato com a mácula umeral (ou região}

\section{umeral) ou posterior a ela}

Aphyocharax anisitsi, Aphyocharax pusillus, Astyanax altiparanae, Astyanax mexicanus, Brycinus longipinnis, Bryconamericus exodon, Bryconella pallidifrons, Bryconexodon juruenae, Bryconops melanurus, Cheirodon interruptus, Coptobrycon bilineatus, Deuterodon iguape, Gymnocorymbus ternetzi, Hasemania crenuchoides, Hasemania melanura, Hasemania sp n., Hemigrammus analis, Hemigrammus arua, Hemigrammus bellottii, Hemigrammus bleheri, Hemigrammus boesemani, Hemigrammus brevis, Hemigrammus cylindricus, Hemigrammus gracilis, Hemigrammus haraldi, Hemigrammus hyanuary, Hemigrammus levis, Hemigrammus lunatus, Hemigrammus marginatus, Hemigrammus newboldi, Hemigrammus ocellifer, Hemigrammus orthus, Hemigrammus parana, Hemigrammus pulcher, Hemigrammus rhodostomus, Hemigrammus rodwayi, Hemigrammus schmardae, Hemigrammus silimoni, Hemigrammus skolioplatus, Hemigrammus stictus, Hemigrammus taphorni, Hemigrammus tridens, Hemigrammus unilineatus, Hemigrammus vorderwikleri, Hemigrammus yinyang, Hemigrammus sp n., Hyphessobrycon coelestinus, Hyphessobrycon compressus, Hyphessobrycon diancistrus, Iguanodectes spilurus, Jupiaba polylepis, Lignobrycon myersi, Moenkhausia bonita, Moenkhausia hemigrammoides, Moenkhausia intermedia, Moenkhausia sanctaefilomenae, Moenkhausia xinguensis, Oligosarcus pintoi, Paracheirodon axelrodi, Parapristella georgiae, Petitella georgiae, Phenacogaster franciscoensis, Piabina argentea, Poptella paraguayensis, Psellogrammus kennedyi, Pristella maxillaris, Rhinopetitia myersi, Serrapinnus heterodon, Serrapinnus notomelas, Stethaprion erythrops, Thayeria obliqua

\section{2- presente, começando anterior à mácula umeral, e atravessando-a}

\section{horizontalmente}

Hemigrammus barrigonae, Hemigrammus ulreyi

\section{9- Indeterminado}

Hemigrammus coeruleus, Hyphessobrycon epicharis, Tetragonopterus argenteus, Triportheus nematurus

\section{1- Listra negra longitudinal no corpo (ic - 0,063)}

0- fina, apenas uma linha bastante fina, como uma única série de

\section{cromatóforos (às vezes pouco conspícua)}

Aphyocharax anisitsi, Aphyocharax pusillus, Astyanax altiparanae, Brycinus longipinnis, Bryconexodon juruenae, Cheirodon interruptus, Gymnocorymbus ternetzi, Hasemania melanura, Hemigrammus analis, Hemigrammus arua, Hemigrammus bellottii, Hemigrammus bleheri, Hemigrammus boesemani, Hemigrammus brevis, Hemigrammus cylindricus, Hemigrammus haraldi, Hemigrammus hyanuary, Hemigrammus levis, Hemigrammus lunatus, Hemigrammus ocellifer, Hemigrammus orthus, Hemigrammus parana, Hemigrammus pulcher, Hemigrammus rhodostomus, Hemigrammus rodwayi, Hemigrammus schmardae, Hemigrammus silimoni, Hemigrammus stictus, Hemigrammus taphorni, Hemigrammus tridens, Hemigrammus unilineatus, Hemigrammus vorderwikleri, Hemigrammus yinyang, Hyphessobrycon coelestinus, Hyphessobrycon compressus, Hyphessobrycon diancistrus, Jupiaba polylepis, Moenkhausia bonita, Moenkhausia hemigrammoides, Moenkhausia sanctaefilomenae, Oligosarcus pintoi, Parapristella georgiae, Phenacogaster franciscoensis, Poptella paraguayensis, Rhinopetitia myersi, Serrapinnus heterodon, Serrapinnus notomelas, Stethaprion erythrops, Thayeria obliqua 


\section{1- mais larga, formada por várias séries de cromatóforos (geralmente}

\section{bastante conspícua)}

Astyanax mexicanus, Bryconamericus exodon, Bryconella pallidifrons, Bryconops melanurus, Coptobrycon bilineatus, Deuterodon iguape, Hasemania crenuchoides, Hasemania sp n., Hemigrammus barrigonae, Hemigrammus gracilis, Hemigrammus marginatus, Hemigrammus newboldi, Hemigrammus skolioplatus, Hemigrammus sp n., Hemigrammus ulreyi, Iguanodectes spilurus, Lignobrycon myersi, Paracheirodon axelrodi, Petitella georgiae, Piabina argentea, Pristella maxillaris, Psellogrammus kennedyi

\section{9- Indeterminado}

Astyanax jacobinae, Brycon cf pesu, Chalceus spilogyros, Grundulus cochae, Hemigrammus coeruleus, Hemigrammus mimus, Hemigrammus pretoensis, Hollandichthys aff. multifasciatus, Hyphessobrycon bentosi, Hyphessobrycon eilyos, Hyphessobrycon epicharis, Hyphessobrycon eques, Hyphessobrycon erythrostigma, Hyphessobrycon megalopterus, Hyphessobrycon micropterus, Hyphessobrycon takasei, Microschemobrycon sp, Moenkhausia intermedia, Moenkhausia xinguensis, Roeboides bonariensis, Salminus hilarii, Serrasalmus maculatus, Tetragonopterus argenteus, Triportheus nematurus

\section{2- Mancha negra na nadadeira dorsal (ic - 1,0)}

(Benine, 2004: 100; Mirande, 2009: 339)

$\underline{0 \text { - ausente ou presente e inconspícua, difusa e sem delimitação (mais }}$ uma região escurecida que uma mancha definida) ou conspícua porém não muito delimitada e mais concentrada na região proximal dos raios, prolongando-se distalmente em sentido vertical apenas nos raios mais anteriores (Figura $37 \mathrm{E}$ )

Aphyocharax anisitsi, Aphyocharax pusillus, Astyanax altiparanae, Astyanax jacobinae, Astyanax mexicanus, Brycinus longipinnis, Brycon cf pesu, Bryconamericus exodon, Bryconella pallidifrons, Bryconexodon juruenae, Bryconops melanurus, Chalceus spilogyros, Cheirodon interruptus, Coptobrycon bilineatus, Deuterodon iguape, Grundulus cochae, Gymnocorymbus ternetzi, Hasemania crenuchoides, Hasemania melanura, Hasemania sp n., Hemigrammus analis, Hemigrammus arua, Hemigrammus barrigonae, Hemigrammus bellottii, Hemigrammus bleheri, Hemigrammus boesemani, Hemigrammus brevis, Hemigrammus coeruleus, Hemigrammus cylindricus, Hemigrammus gracilis, Hemigrammus haraldi, Hemigrammus hyanuary, Hemigrammus levis, Hemigrammus lunatus, Hemigrammus marginatus, Hemigrammus mimus, Hemigrammus newboldi, Hemigrammus ocellifer, Hemigrammus orthus, Hemigrammus parana, Hemigrammus pretoensis, Hemigrammus pulcher, Hemigrammus rhodostomus, Hemigrammus rodwayi, Hemigrammus schmardae, Hemigrammus silimoni, Hemigrammus skolioplatus, Hemigrammus stictus, Hemigrammus taphorni, Hemigrammus tridens, Hemigrammus ulreyi, Hemigrammus vorderwikleri, Hemigrammus yinyang, Hemigrammus sp n., Hollandichthys aff. multifasciatus, Hyphessobrycon coelestinus, Hyphessobrycon diancistrus, Hyphessobrycon eilyos, Iguanodectes spilurus, Jupiaba polylepis, Lignobrycon myersi, Microschemobrycon sp, Moenkhausia bonita, Moenkhausia intermedia, Moenkhausia sanctaefilomenae, Moenkhausia xinguensis, Oligosarcus pintoi, Paracheirodon axelrodi, Parapristella georgiae, Petitella georgiae, Phenacogaster franciscoensis, Piabina argentea, Poptella paraguayensis, Psellogrammus kennedyi, Rhinopetitia myersi, Roeboides bonariensis, Salminus hilarii, Serrapinnus heterodon, Serrapinnus notomelas, Serrasalmus maculatus, Stethaprion erythrops, Tetragonopterus argenteus, Thayeria obliqua, Triportheus nematurus 
1- mancha conspícua e bem delimitada, ocupando os raios da nadadeira em sentido horizontal, mais restrita a porção mediana dos raios (mais deslocadas próxima à base em apenas algumas espécies), com a região proximal e distal da nadadeira hialina (Figura 37 A - D)

Hemigrammus unilineatus, Hyphessobrycon bentosi, Hyphessobrycon compressus, Hyphessobrycon epicharis, Hyphessobrycon eques, Hyphessobrycon erythrostigma, Hyphessobrycon megalopterus, Hyphessobrycon micropterus, Hyphessobrycon takasei, Moenkhausia hemigrammoides, Pristella maxillaris

Algumas espécies de Hemigrammus são tradicionalmente reconhecidas por apresentar mancha negra na nadadeira dorsal: $H$. ulreyi, $H$. elegans, $H$. coeruleus e $H$. unilineatus. Ao invés de uma mancha negra conspícua e delimitada na nadadeira dorsal, entretanto, $H$. ulreyi, $H$. elegans e $H$. coeruleus apresentam somente os primeiros raios da nadadeira mais escuros, com coloração distribuída verticalmente sobre os primeiros raios e com cromatóforos distribuídos por toda a nadadeira, não definindo claramente uma mancha conspícua. Mancha negra na nadadeira dorsal bastante conspícua, distribuída horizontalmente, delimitada e restrita à região mediana da nadadeira dorsal, como a de $H$. unilineatus, só aparece em representantes de Characidae restritos ao clado 161, que inclui também espécies de Hyphessobrycon, Pristella e Moenkahusia, sendo essa mancha, portanto, não homóloga a condição encontrada em $H$. coeruleus, $H$. ulreyi e $H$. elegans. A presença de uma mancha negra e conspícua na nadadeira dorsal aparece como uma sinapomorfia exclusiva para o clado 161.

153- Mancha negra na nadadeira anal (ic - 0,667)

\section{0- ausente (Figura $38 \mathrm{~F}$ )}

Aphyocharax anisitsi, Aphyocharax pusillus, Astyanax altiparanae, Astyanax jacobinae, Astyanax mexicanus, Brycinus longipinnis, Brycon of pesu, Bryconamericus exodon, Bryconella pallidifrons, Bryconexodon juruenae, Bryconops melanurus, Chalceus spilogyros, Cheirodon interruptus, Coptobrycon bilineatus, Deuterodon iguape, Grundulus cochae, Gymnocorymbus ternetzi, Hasemania crenuchoides, Hasemania melanura, Hasemania sp n., Hemigrammus analis, Hemigrammus arua, Hemigrammus barrigonae, Hemigrammus bellottii, Hemigrammus bleheri, Hemigrammus boesemani, Hemigrammus brevis, Hemigrammus cylindricus, Hemigrammus gracilis, Hemigrammus haraldi, Hemigrammus hyanuary, Hemigrammus levis, Hemigrammus lunatus, Hemigrammus marginatus, Hemigrammus mimus, Hemigrammus newboldi, Hemigrammus orthus, Hemigrammus parana, Hemigrammus pretoensis, Hemigrammus pulcher, Hemigrammus rodwayi, Hemigrammus schmardae, Hemigrammus silimoni, Hemigrammus skolioplatus, Hemigrammus stictus, Hemigrammus taphorni, Hemigrammus tridens, Hemigrammus ulreyi, Hemigrammus vorderwikleri, Hemigrammus yinyang, Hemigrammus sp n., Hollandichthys aff. multifasciatus, Hyphessobrycon bentosi, Hyphessobrycon coelestinus, Hyphessobrycon diancistrus, Hyphessobrycon eilyos, 
Iguanodectes spilurus, Jupiaba polylepis, Lignobrycon myersi, Microschemobrycon sp, Moenkhausia bonita, Moenkhausia intermedia, Moenkhausia sanctaefilomenae, Moenkhausia xinguensis, Oligosarcus pintoi, Paracheirodon axelrodi, Parapristella georgiae, Phenacogaster franciscoensis, Piabina argentea, Poptella paraguayensis, Psellogrammus kennedyi, Rhinopetitia myersi, Roeboides bonariensis, Salminus hilarii, Serrapinnus heterodon, Serrapinnus notomelas, Stethaprion erythrops, Tetragonopterus argenteus, Thayeria obliqua, Triportheus nematurus

1- presente, não obliqua, podendo ser mais ou menos conspícua, formando uma faixa na região mais distal dos raios ao longo de toda a extensão da nadadeira (às vezes estendendo-se dorsalmente sobre os primeiros ou os últimos raios da nadadeira) (Figura $38 \mathrm{~A}$ )

Hemigrammus coeruleus, Hemigrammus ocellifer, Hyphessobrycon compressus, Hyphessobrycon eques, Hyphessobrycon erythrostigma, Hyphessobrycon megalopterus, Hyphessobrycon micropterus, Hyphessobrycon takasei, Serrasalmus maculatus

2- conspícua e obliqua, começando na base dos primeiros raios simples, atravessando a porção mediana dos raios seguintes e terminando na ponta do

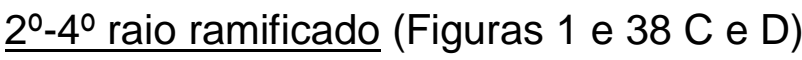

Hemigrammus unilineatus, Moenkhausia hemigrammoides

3- presente, pouco conspícua e não obliqua, restrita aos últimos raios (Figura $38 \mathrm{E}$ )

Hemigrammus rhodostomus, Petitella georgiae

4- presente, conspícua e não obliqua, restrita à porção mediana (ou mais próxima da ponta, mas não atingindo a ponta dos raios) dos primeiros 5 raios da nadadeira (Figura 38 B)

Pristella maxillaris

\section{9- Indeterminado}

Hyphessobrycon epicharis

Apesar de não ser uma condição amplamente distribuída em Characidae, presença de mancha negra na nadadeira anal ocorre em algumas espécies da família, mas quase sempre essa mancha é restrita à região mediana ou distal dos raios da anal e distribuída vertical ou horizontalmente. Hemigrammus unilineatus e $M$. hemigrammoides apresentam uma mancha negra na nadadeira anal, obliquamente disposta, indo da base dos primeiros 
raios simples até a ponta do $2^{\circ}$ a $4^{\circ}$ raio ramificado. Mancha ocupando os primeiros raios anais desde a base até a ponta é relatada por Costa (1994) para Moenkhausia nigromarginata, entretanto essa mancha é distibuída verticalmente sobre os raios, e não obliquamente. Uma mancha distribuída obliquamente como a descrita acima, só ocorre em $H$. unilineatus e $M$. hemigrammoides, e, portanto, é uma sinapomorfia exclusiva para o clado 152 que engloba as duas espécies.

\title{
154- Mancha negra horizontal na região ventral anterior à nadadeira anal e contínua com a mancha negra da nadadeira anal (ic - 1,0)
}

\author{
0- ausente (Figura 38 A, B e E)
}

Aphyocharax anisitsi, Aphyocharax pusillus, Astyanax altiparanae, Astyanax jacobinae, Astyanax mexicanus, Brycinus longipinnis, Brycon cf pesu, Bryconamericus exodon, Bryconella pallidifrons, Bryconexodon juruenae, Bryconops melanurus, Chalceus spilogyros, Cheirodon interruptus, Coptobrycon bilineatus, Deuterodon iguape, Grundulus cochae, Gymnocorymbus ternetzi, Hasemania crenuchoides, Hasemania melanura, Hasemania sp n., Hemigrammus analis, Hemigrammus arua, Hemigrammus barrigonae, Hemigrammus bellottii, Hemigrammus bleheri, Hemigrammus boesemani, Hemigrammus brevis, Hemigrammus coeruleus, Hemigrammus cylindricus, Hemigrammus gracilis, Hemigrammus haraldi, Hemigrammus hyanuary, Hemigrammus levis, Hemigrammus lunatus, Hemigrammus marginatus, Hemigrammus mimus, Hemigrammus newboldi, Hemigrammus ocellifer, Hemigrammus orthus, Hemigrammus parana, Hemigrammus pretoensis, Hemigrammus pulcher, Hemigrammus rhodostomus, Hemigrammus rodwayi, Hemigrammus schmardae, Hemigrammus silimoni, Hemigrammus skolioplatus, Hemigrammus stictus, Hemigrammus taphorni, Hemigrammus tridens, Hemigrammus ulreyi, Hemigrammus vorderwikleri, Hemigrammus yinyang, Hemigrammus sp n., Hollandichthys aff. multifasciatus, Hyphessobrycon bentosi, Hyphessobrycon coelestinus, Hyphessobrycon compressus, Hyphessobrycon diancistrus, Hyphessobrycon eilyos, Hyphessobrycon epicharis, Hyphessobrycon eques, Hyphessobrycon erythrostigma, Hyphessobrycon megalopterus, Hyphessobrycon micropterus, Hyphessobrycon takasei, Iguanodectes spilurus, Jupiaba polylepis, Lignobrycon myersi, Microschemobrycon sp, Moenkhausia bonita, Moenkhausia intermedia, Moenkhausia sanctaefilomenae, Moenkhausia xinguensis, Oligosarcus pintoi, Paracheirodon axelrodi, Parapristella georgiae, Petitella georgiae, Phenacogaster franciscoensis, Piabina argentea, Poptella paraguayensis, Psellogrammus kennedyi, Pristella maxillaris, Rhinopetitia myersi, Roeboides bonariensis, Salminus hilarii, Serrapinnus heterodon, Serrapinnus notomelas, Serrasalmus maculatus, Stethaprion erythrops, Tetragonopterus argenteus, Thayeria obliqua, Triportheus nematurus

$$
\text { 1- presente (Figuras } 1 \text { e } 38 \mathrm{C}-\mathrm{D})
$$

Hemigrammus unilineatus, Moenkhausia hemigrammoides

Hemigrammus unilineatus e $M$. hemigrammoides apresentam uma mancha negra, aproximadamente horizontal, localizada na região ventral do corpo, anterior à nadadeira anal e contínua com a mancha presente sobre os raios da nadadeira anal. Essa condição é exclusiva dentro de Characidae para 
as duas espécies, aparecendo como uma sinapomorfia exclusiva para o clado 152.

\section{5- Linha negra na base ou paralela à base da nadadeira anal (ic - 0,222)}

\section{$\underline{0 \text { - ausente }}$}

Aphyocharax anisitsi, Aphyocharax pusillus, Astyanax altiparanae, Astyanax jacobinae, Astyanax mexicanus, Brycinus longipinnis, Brycon cf pesu, Bryconamericus exodon, Bryconella pallidifrons, Bryconexodon juruenae, Bryconops melanurus, Chalceus spilogyros, Cheirodon interruptus, Deuterodon iguape, Grundulus cochae, Gymnocorymbus ternetzi, Hasemania crenuchoides, Hasemania melanura, Hasemania sp n., Hemigrammus analis, Hemigrammus bleheri, Hemigrammus boesemani, Hemigrammus brevis, Hemigrammus coeruleus, Hemigrammus cylindricus, Hemigrammus gracilis, Hemigrammus haraldi, Hemigrammus hyanuary, Hemigrammus levis, Hemigrammus marginatus, Hemigrammus mimus, Hemigrammus newboldi, Hemigrammus ocellifer, Hemigrammus parana, Hemigrammus pretoensis, Hemigrammus pulcher, Hemigrammus rhodostomus, Hemigrammus rodwayi, Hemigrammus silimoni, Hemigrammus skolioplatus, Hemigrammus stictus, Hemigrammus taphorni, Hemigrammus unilineatus, Hemigrammus yinyang, Hemigrammus sp n., Hollandichthys aff. multifasciatus, Hyphessobrycon bentosi, Hyphessobrycon coelestinus, Hyphessobrycon compressus, Hyphessobrycon diancistrus, Hyphessobrycon eilyos, Hyphessobrycon epicharis, Hyphessobrycon eques, Hyphessobrycon erythrostigma, Hyphessobrycon megalopterus, Hyphessobrycon micropterus, Hyphessobrycon takasei, Iguanodectes spilurus, Jupiaba polylepis, Microschemobrycon sp, Moenkhausia bonita, Moenkhausia hemigrammoides, Moenkhausia intermedia, Moenkhausia sanctaefilomenae, Moenkhausia xinguensis, Oligosarcus pintoi, Paracheirodon axelrodi, Parapristella georgiae, Petitella georgiae, Phenacogaster franciscoensis, Piabina argentea, Poptella paraguayensis, Psellogrammus kennedyi, Rhinopetitia myersi, Roeboides bonariensis, Salminus hilarii, Serrapinnus heterodon, Serrapinnus notomelas, Serrasalmus maculatus, Stethaprion erythrops, Tetragonopterus argenteus, Thayeria obliqua, Triportheus nematurus

\section{1- rente à base da nadadeira e prolongando-se por toda sua extensão}

(Figura 39 C)

Hemigrammus barrigonae, Hemigrammus ulreyi

2- acima, paralela à base da nadadeira, começando na altura dos primeiros raios (ou raios medianos) e se prolongando longitudinalmente por toda a extensão da nadadeira (Figura $39 \mathrm{~A}$ )

Coptobrycon bilineatus, Hemigrammus arua, Hemigrammus bellottii, Hemigrammus lunatus, Hemigrammus orthus, Hemigrammus schmardae, Hemigrammus tridens, Hemigrammus vorderwikleri, Lignobrycon myersi, Pristella maxillaris

\section{6- Listras negras verticais acima da base da nadadeira anal, posicionadas nos espaços correspondentes às membranas inter-radiais (ic - 0,667) \\ $\underline{0 \text { - ausente }}$}

Aphyocharax anisitsi, Aphyocharax pusillus, Astyanax altiparanae, Astyanax jacobinae, Astyanax mexicanus, Brycinus longipinnis, Brycon cf pesu, Bryconamericus exodon, Bryconella 
pallidifrons, Bryconexodon juruenae, Bryconops melanurus, Chalceus spilogyros, Cheirodon interruptus, Coptobrycon bilineatus, Deuterodon iguape, Grundulus cochae, Gymnocorymbus ternetzi, Hasemania crenuchoides, Hasemania melanura, Hasemania sp n., Hemigrammus analis, Hemigrammus arua, Hemigrammus barrigonae, Hemigrammus brevis, Hemigrammus coeruleus, Hemigrammus gracilis, Hemigrammus haraldi, Hemigrammus hyanuary, Hemigrammus levis, Hemigrammus lunatus, Hemigrammus marginatus, Hemigrammus mimus, Hemigrammus newboldi, Hemigrammus ocellifer, Hemigrammus parana, Hemigrammus pretoensis, Hemigrammus pulcher, Hemigrammus rodwayi, Hemigrammus schmardae, Hemigrammus silimoni, Hemigrammus skolioplatus, Hemigrammus stictus, Hemigrammus taphorni, Hemigrammus tridens, Hemigrammus ulreyi, Hemigrammus unilineatus, Hemigrammus vorderwikleri, Hemigrammus yinyang, Hemigrammus sp n., Hollandichthys aff. multifasciatus, Hyphessobrycon bentosi, Hyphessobrycon coelestinus, Hyphessobrycon compressus, Hyphessobrycon eilyos, Hyphessobrycon epicharis, Hyphessobrycon eques, Hyphessobrycon erythrostigma, Hyphessobrycon megalopterus, Hyphessobrycon micropterus, Hyphessobrycon takasei, Iguanodectes spilurus, Jupiaba polylepis, Lignobrycon myersi, Microschemobrycon sp, Moenkhausia bonita, Moenkhausia hemigrammoides, Moenkhausia intermedia, Moenkhausia sanctaefilomenae, Moenkhausia xinguensis, Oligosarcus pintoi, Paracheirodon axelrodi, Parapristella georgiae, Phenacogaster franciscoensis, Piabina argentea, Poptella paraguayensis, Psellogrammus kennedyi, Pristella maxillaris, Rhinopetitia myersi, Roeboides bonariensis, Salminus hilarii, Serrapinnus heterodon, Serrapinnus notomelas, Serrasalmus maculatus, Stethaprion erythrops, Tetragonopterus argenteus, Thayeria obliqua, Triportheus nematurus

\section{1- presente, apenas na altura dos primeiros raios (Figura 39 A)}

Hemigrammus bellottii, Hemigrammus orthus

\section{2- presente, ao longo de toda a base da nadadeira (Figura 39 B)}

Hemigrammus bleheri, Hemigrammus boesemani, Hemigrammus cylindricus, Hemigrammus rhodostomus, Hyphessobrycon diancistrus, Petitella georgiae

\section{7- Mancha negra no pedúnculo caudal (ic- 0,074)}

(Marinho, 2009: 78)

\section{0-presente, diferente do estado 1 (Figura $40 \mathrm{~A}$ )}

Aphyocharax pusillus, Astyanax altiparanae, Astyanax jacobinae, Astyanax mexicanus, Brycinus longipinnis, Bryconexodon juruenae, Cheirodon interruptus, Deuterodon iguape, Hasemania melanura, Hemigrammus pretoensis, Hemigrammus rodwayi, Hemigrammus skolioplatus, Hemigrammus sp n., Hyphessobrycon coelestinus, Hyphessobrycon diancistrus, Lignobrycon myersi, Oligosarcus pintoi, Moenkhausia bonita, Petitella georgiae, Phenacogaster franciscoensis, Psellogrammus kennedyi, Roeboides bonariensis, Salminus hilarii, Serrapinnus heterodon, Serrapinnus notomelas, Tetragonopterus argenteus, Thayeria obliqua Triportheus nematurus

\section{1- presente, atingindo a base da nadadeira caudal e alta, estendendo-se}

dorsal e ventralmente sobre os raios marginais (Figura 40)

Hemigrammus newboldi, Hemigrammus ocellifer, Hemigrammus schmardae, Hemigrammus tridens, Hemigrammus vorderwikleri, Moenkhausia sanctaefilomenae

\section{$\underline{\text { 2- ausente }}$}


Aphyocharax anisitsi, Brycon cf pesu, Bryconamericus exodon, Bryconella pallidifrons, Bryconops melanurus, Chalceus spilogyros, Coptobrycon bilineatus, Grundulus cochae, Gymnocorymbus ternetzi, Hasemania crenuchoides, Hasemania sp n., Hemigrammus analis, Hemigrammus arua, Hemigrammus barrigonae, Hemigrammus bellottii, Hemigrammus bleheri, Hemigrammus boesemani, Hemigrammus brevis, Hemigrammus coeruleus, Hemigrammus cylindricus, Hemigrammus gracilis, Hemigrammus haraldi, Hemigrammus hyanuary, Hemigrammus levis, Hemigrammus lunatus, Hemigrammus marginatus, Hemigrammus mimus, Hemigrammus orthus, Hemigrammus parana, Hemigrammus pulcher, Hemigrammus rhodostomus, Hemigrammus silimoni, Hemigrammus stictus, Hemigrammus taphorni, Hemigrammus ulreyi, Hemigrammus unilineatus, Hemigrammus yinyang, Hollandichthys aff. multifasciatus, Hyphessobrycon bentosi, Hyphessobrycon compressus, Hyphessobrycon eilyos, Hyphessobrycon epicharis, Hyphessobrycon eques, Hyphessobrycon erythrostigma, Hyphessobrycon megalopterus, Hyphessobrycon micropterus, Hyphessobrycon takasei, Iguanodectes spilurus, Jupiaba polylepis, Microschemobrycon sp, Moenkhausia hemigrammoides, Moenkhausia intermedia, Moenkhausia xinguensis, Paracheirodon axelrodi, Parapristella georgiae, Poptella paraguayensis, Pristella maxillaris, Rhinopetitia myersi, Serrasalmus maculatus, Stethaprion erythrops

\section{$\underline{0,2-P o l i m o ́ r f i c o ~}$}

Piabina argentea

Mancha no pedúnculo caudal atingindo a base da nadadeira caudal e alta, ocupando toda a altura dessa região, como a descrita no estado 1 é encontrada, dentre as espécies analisadas em $H$. schmardae, $H$. vorderwinkleri e H. tridens (clado 133), Hemigrammus boesemani (clado 140), Hemigrammus ocellifer (clado 166), Moenkhausia sanctaefilomenae e Hemigrammus newboldi. Mancha semelhante também é relata para outras espécies de Moenkhausia, como M. pyrophthalma e M. oligolepis, indicando que essa condição apareceu mais de uma vez e independentemente em Characidae.

\section{8- Mancha negra sobre a região mediana e/ou distal dos lobos da nadadeira caudal (ic $-0,364$ )}

(Benine, 2004: 103; Bertaco, 2008: 120; Marinho, 2009: 81)

\section{0- ausente (Figura $41 \mathrm{C}$ )}

Aphyocharax anisitsi, Aphyocharax pusillus, Astyanax altiparanae, Astyanax jacobinae, Astyanax mexicanus, Brycinus longipinnis, Bryconella pallidifrons, Bryconexodon juruenae, Chalceus spilogyros, Cheirodon interruptus, Coptobrycon bilineatus, Deuterodon iguape, Grundulus cochae, Gymnocorymbus ternetzi, Hasemania crenuchoides, Hasemania melanura, Hasemania sp n., Hemigrammus analis, Hemigrammus arua, Hemigrammus barrigonae, Hemigrammus bellottii, Hemigrammus boesemani, Hemigrammus brevis, Hemigrammus coeruleus, Hemigrammus cylindricus, Hemigrammus gracilis, Hemigrammus haraldi, Hemigrammus hyanuary, Hemigrammus levis, Hemigrammus lunatus, Hemigrammus mimus, Hemigrammus newboldi, Hemigrammus ocellifer, Hemigrammus orthus, Hemigrammus parana, Hemigrammus pretoensis, Hemigrammus pulcher, Hemigrammus rodwayi, Hemigrammus schmardae, Hemigrammus skolioplatus, Hemigrammus stictus, Hemigrammus taphorni, Hemigrammus tridens, Hemigrammus ulreyi, Hemigrammus unilineatus, Hemigrammus vorderwikleri, Hemigrammus yinyang, Hollandichthys aff. multifasciatus, Hyphessobrycon 
bentosi, Hyphessobrycon compressus, Hyphessobrycon epicharis, Hyphessobrycon eques, Hyphessobrycon erythrostigma, Hyphessobrycon micropterus, Hyphessobrycon takasei, Jupiaba polylepis, Lignobrycon myersi, Microschemobrycon sp, Moenkhausia hemigrammoides, Moenkhausia sanctaefilomenae, Moenkhausia xinguensis, Oligosarcus pintoi, Paracheirodon axelrodi, Parapristella georgiae, Phenacogaster franciscoensis, Piabina argentea, Poptella paraguayensis, Psellogrammus kennedyi, Pristella maxillaris, Rhinopetitia myersi, Salminus hilarii, Serrapinnus heterodon, Serrapinnus notomelas, Stethaprion erythrops, Tetragonopterus argenteus, Triportheus nematurus

\section{1 - somente sobre o lobo superior com disposição variável (Figura $41 \mathrm{E}$ )}

Bryconops melanurus, Iguanodectes spilurus, Hemigrammus silimoni

2- em ambos os lobos, transversal, atingindo ou não a ponta dos raios (Figura $41 \mathrm{~A}-\mathrm{B}$ )

Bryconamericus exodon, Hemigrammus marginatus, Hemigrammus sp n., Hyphessobrycon coelestinus, Hyphessobrycon diancistrus, Hyphessobrycon eilyos, Moenkhausia bonita, Moenkhausia intermedia

3- em ambos lobos, obliqua e mais restrita à região mediana, sem atingir a ponta dos raios (ponta dos lobos hialina) (Figura $41 \mathrm{D}$ )

Hemigrammus bleheri, Hemigrammus rhodostomus, Petitella georgiae

\section{4- somente sobre o lobo inferior, longitudinal (Figura $41 \mathrm{~F}$ )}

Thayeria obliqua

\section{9- Indeterminado}

Brycon cf pesu, Hyphessobrycon megalopterus, Roeboides bonariensis, Serrasalmus maculatus

Diversas espécies dentro de Characidae apresentam manchas negras sobre os lobos caudais, distribuídas de diferentes formas: mancha somente no lobo superior (podendo ser longitudinal ou transversalmente distribuída), como ocorre, por exemplo nas espécies do grupo Moenkahusia lepidura e em Bryconops melanurus; mancha somente sobre o lobo inferior (Iongitudinalmente distribuída), presente nas espécies de Thayeria; mancha em ambos os lobos, obliqua e mais restrita à região mediana dos lobos, que ocorre em $H$. bleheri, $H$. rhodostomus e $P$. georgiae; e por fim mancha em ambos os lobos, transversalmente distribuída, podendo ou não atingir a ponta dos raios, como ocorre dentre outros em $H$. marginatus, $B$. exodon, $M$. bonita e $H$. diancistrus. Mancha como descrita no estado 2, além de em $H$. marginatus, $H$. 
sp. n. e M. bonita, ocorre em algumas outras espécies de Characidae, como por exemplo, Hyphessobrycon diancistrus, Bryconamericus exodon, Moenkhausia dichroura e Moenkhausia intermedia. Dentre essas espécies somente $M$. dichroura não foi aqui analisada, e todas as demais espécies não aparecem proximamente relacionadas ao clado de $M$. bonita, $H$. marginatus e $H$. sp n., sendo a presença de mancha nos lobos caudais como a descrita no estado 2, provavelmente adquirida de forma independente várias vezes dentro de Characide. Mancha nos lobos caudais como a descrita no estado 3, só foi observada para $H$. bleheri, $H$. rhodostomus e $P$. georgiae, sendo, portanto, uma sinapomorfia exclusiva para o clado 138 que une as três espécies.

159- Mancha negra sobre os raios medianos da nadadeira caudal (ic $0,071)$

(Bertaco, 2008: 119)

\section{0 - ausente}

Aphyocharax anisitsi, Aphyocharax pusillus, Astyanax jacobinae, Brycon cf pesu, Bryconexodon juruenae, Chalceus spilogyros, Cheirodon interruptus, Coptobrycon bilineatus, Deuterodon iguape, Grundulus cochae, Gymnocorymbus ternetzi, Hemigrammus analis, Hemigrammus arua, Hemigrammus bellottii, Hemigrammus coeruleus, Hemigrammus cylindricus, Hemigrammus haraldi, Hemigrammus lunatus, Hemigrammus marginatus, Hemigrammus mimus, Hemigrammus orthus, Hemigrammus pulcher, Hemigrammus silimoni, Hemigrammus stictus, Hemigrammus taphorni, Hemigrammus ulreyi, Hemigrammus unilineatus, Hemigrammus yinyang, Hollandichthys aff. multifasciatus, Hyphessobrycon bentosi, Hyphessobrycon compressus, Hyphessobrycon diancistrus, Hyphessobrycon eilyos, Hyphessobrycon epicharis, Hyphessobrycon eques, Hyphessobrycon erythrostigma, Hyphessobrycon megalopterus, Hyphessobrycon micropterus, Hyphessobrycon takasei, Jupiaba polylepis, Lignobrycon myersi, Microschemobrycon sp, Moenkhausia hemigrammoides, Moenkhausia intermedia, Moenkhausia xinguensis, Paracheirodon axelrodi, Piabina argentea, Poptella paraguayensis, Pristella maxillaris, Rhinopetitia myersi, Roeboides bonariensis, Serrapinnus notomelas, Serrasalmus maculatus, Stethaprion erythrops, Tetragonopterus argenteus

\section{1 - presente, não se prolongando até a ponta dos raios}

Hemigrammus barrigonae, Hemigrammus boesemani, Hemigrammus gracilis, Hemigrammus hyanuary, Hemigrammus ocellifer, Hemigrammus schmardae, Hemigrammus tridens, Hemigrammus vorderwikleri, Moenkhausia sanctaefilomenae, Parapristella georgiae, Phenacogaster franciscoensis, Psellogrammus kennedyi, Serrapinnus heterodon

\section{2- presente, se prolongando até a ponta dos raios}

Astyanax altiparanae, Astyanax mexicanus, Bryconamericus exodon, Bryconella pallidifrons, Bryconops melanurus, Hasemania crenuchoides, Hasemania melanura, Hasemania sp n., Hemigrammus bleheri, Hemigrammus levis, Hemigrammus newboldi, Hemigrammus parana, Hemigrammus pretoensis, Hemigrammus rhodostomus, Hemigrammus rodwayi, Hemigrammus skolioplatus, Hemigrammus sp n., Hyphessobrycon coelestinus, Iguanodectes spilurus, 
Moenkhausia bonita, Oligosarcus pintoi, Petitella georgiae, Salminus hilarii, Thayeria obliqua, Triportheus nematurus

\section{1, 2- Polimórfico}

Hemigrammus brevis

\section{9- Indeterminado}

Brycinus longipinnis

\section{0- Raios marginais, dorsais e ventrais da nadadeira caudal (ic - 1,0)}

\section{$\underline{0-\text { hialinos }}$}

Aphyocharax anisitsi, Aphyocharax pusillus, Astyanax altiparanae, Astyanax jacobinae, Astyanax mexicanus, Brycinus longipinnis, Brycon cf pesu, Bryconamericus exodon, Bryconella pallidifrons, Bryconexodon juruenae, Bryconops melanurus, Chalceus spilogyros, Cheirodon interruptus, Coptobrycon bilineatus, Deuterodon iguape, Grundulus cochae, Gymnocorymbus ternetzi, Hasemania crenuchoides, Hasemania melanura, Hasemania sp n., Hemigrammus analis, Hemigrammus arua, Hemigrammus barrigonae, Hemigrammus bellottii, Hemigrammus bleheri, Hemigrammus boesemani, Hemigrammus brevis, Hemigrammus coeruleus, Hemigrammus cylindricus, Hemigrammus gracilis, Hemigrammus haraldi, Hemigrammus hyanuary, Hemigrammus levis, Hemigrammus lunatus, Hemigrammus marginatus, Hemigrammus mimus, Hemigrammus newboldi, Hemigrammus orthus, Hemigrammus parana, Hemigrammus pretoensis, Hemigrammus pulcher, Hemigrammus rhodostomus, Hemigrammus rodwayi, Hemigrammus schmardae, Hemigrammus silimoni, Hemigrammus skolioplatus, Hemigrammus stictus, Hemigrammus taphorni, Hemigrammus tridens, Hemigrammus ulreyi, Hemigrammus unilineatus, Hemigrammus vorderwikleri, Hemigrammus yinyang, Hemigrammus sp n., Hollandichthys aff. multifasciatus, Hyphessobrycon bentosi, Hyphessobrycon coelestinus, Hyphessobrycon compressus, Hyphessobrycon diancistrus, Hyphessobrycon eilyos, Hyphessobrycon epicharis, Hyphessobrycon eques, Hyphessobrycon erythrostigma, Hyphessobrycon megalopterus, Hyphessobrycon micropterus, Hyphessobrycon takasei, Iguanodectes spilurus, Jupiaba polylepis, Lignobrycon myersi, Microschemobrycon sp, Moenkhausia bonita, Moenkhausia hemigrammoides, Moenkhausia intermedia, Moenkhausia sanctaefilomenae, Moenkhausia xinguensis, Oligosarcus pintoi, Paracheirodon axelrodi, Parapristella georgiae, Petitella georgiae, Phenacogaster franciscoensis, Piabina argentea, Poptella paraguayensis, Psellogrammus kennedyi, Pristella maxillaris, Rhinopetitia myersi, Roeboides bonariensis, Salminus hilarii, Serrapinnus heterodon, Serrapinnus notomelas, Serrasalmus maculatus, Stethaprion erythrops, Tetragonopterus argenteus, Thayeria obliqua, Triportheus nematurus

\section{1- escuros (Figura $40 \mathrm{~A}$ )}

Hemigrammus ocellifer

\section{Miscelânea}

161- Poros nasais (ic - 1,0)

(Moreira, 2007: 402)

\section{0-próximos e confluentes (Figura 42 A)}

Aphyocharax anisitsi, Aphyocharax pusillus, Astyanax altiparanae, Astyanax jacobinae, Astyanax mexicanus, Brycinus longipinnis, Brycon cf pesu, Bryconamericus exodon, Bryconella pallidifrons, Bryconexodon juruenae, Bryconops melanurus, Chalceus spilogyros, Cheirodon 
interruptus, Deuterodon iguape, Gymnocorymbus ternetzi, Hasemania crenuchoides, Hasemania melanura, Hasemania sp n., Hemigrammus analis, Hemigrammus arua, Hemigrammus barrigonae, Hemigrammus bellottii, Hemigrammus bleheri, Hemigrammus boesemani, Hemigrammus brevis, Hemigrammus coeruleus, Hemigrammus cylindricus, Hemigrammus gracilis, Hemigrammus haraldi, Hemigrammus hyanuary, Hemigrammus levis, Hemigrammus lunatus, Hemigrammus marginatus, Hemigrammus mimus, Hemigrammus newboldi, Hemigrammus ocellifer, Hemigrammus orthus, Hemigrammus parana, Hemigrammus pretoensis, Hemigrammus pulcher, Hemigrammus rhodostomus, Hemigrammus rodwayi, Hemigrammus schmardae, Hemigrammus silimoni, Hemigrammus skolioplatus, Hemigrammus stictus, Hemigrammus taphorni, Hemigrammus tridens, Hemigrammus ulreyi, Hemigrammus unilineatus, Hemigrammus vorderwikleri, Hemigrammus yinyang, Hemigrammus sp n., Hollandichthys aff. multifasciatus, Hyphessobrycon bentosi, Hyphessobrycon coelestinus, Hyphessobrycon compressus, Hyphessobrycon diancistrus, Hyphessobrycon eilyos, Hyphessobrycon epicharis, Hyphessobrycon eques, Hyphessobrycon erythrostigma, Hyphessobrycon megalopterus, Hyphessobrycon micropterus, Hyphessobrycon takasei, Iguanodectes spilurus, Jupiaba polylepis, Lignobrycon myersi, Microschemobrycon sp, Moenkhausia bonita, Moenkhausia hemigrammoides, Moenkhausia intermedia, Moenkhausia sanctaefilomenae, Moenkhausia xinguensis, Oligosarcus pintoi, Paracheirodon axelrodi, Parapristella georgiae, Petitella georgiae, Phenacogaster franciscoensis, Piabina argentea, Poptella paraguayensis, Psellogrammus kennedyi, Pristella maxillaris, Rhinopetitia myersi, Roeboides bonariensis, Salminus hilarii, Serrapinnus heterodon, Serrapinnus notomelas, Serrasalmus maculatus, Stethaprion erythrops, Tetragonopterus argenteus, Thayeria obliqua, Triportheus nematurus

\section{1- distantes e não confluentes (Figura 42 B)}

Coptobrycon bilineatus, Grundulus cochae

A grande maioria das espécies de Characidae apresenta os orifícios nasais, anterior e posterior, próximos e confluentes, condição interpretada como plesiomófica para a família. Coptobrycon bilinieatus e Grundulus, entretanto, apresentam os orifícios nasais, anterior e posterior, amplamente separados, condição interpretada como sinapomórfica para o clado 122, que une as duas espécies. Buckup (1993) mostra que representantes de Characidiinae também apresentam orifícios nasais amplamente separados, entretanto 0 autor coloca que essa característica foi adquirida independentemente da encontrada em outros Characiformes, e sugere que a presença de narinas distantemente localizadas esteja associada com miniaturização.

162- Forma do corpo (ic - 1,0)

\section{$\underline{0 \text { - relativamente baixo e alongado }}$}

Aphyocharax anisitsi, Aphyocharax pusillus, Astyanax altiparanae, Astyanax jacobinae, Astyanax mexicanus, Brycinus longipinnis, Brycon cf pesu, Bryconamericus exodon, Bryconella pallidifrons, Bryconexodon juruenae, Bryconops melanurus, Chalceus spilogyros, Cheirodon interruptus, Coptobrycon bilineatus, Deuterodon iguape, Grundulus cochae, Hasemania crenuchoides, Hasemania melanura, Hasemania sp n., Hemigrammus analis, Hemigrammus 
arua, Hemigrammus barrigonae, Hemigrammus bellottii, Hemigrammus bleheri, Hemigrammus boesemani, Hemigrammus brevis, Hemigrammus coeruleus, Hemigrammus cylindricus, Hemigrammus gracilis, Hemigrammus haraldi, Hemigrammus hyanuary, Hemigrammus levis, Hemigrammus lunatus, Hemigrammus marginatus, Hemigrammus mimus, Hemigrammus newboldi, Hemigrammus ocellifer, Hemigrammus orthus, Hemigrammus parana, Hemigrammus pretoensis, Hemigrammus pulcher, Hemigrammus rhodostomus, Hemigrammus rodwayi, Hemigrammus schmardae, Hemigrammus silimoni, Hemigrammus skolioplatus, Hemigrammus stictus, Hemigrammus taphorni, Hemigrammus tridens, Hemigrammus ulreyi, Hemigrammus unilineatus, Hemigrammus vorderwikleri, Hemigrammus yinyang, Hemigrammus sp n., Hollandichthys aff. multifasciatus, Hyphessobrycon bentosi, Hyphessobrycon coelestinus, Hyphessobrycon compressus, Hyphessobrycon diancistrus, Hyphessobrycon eilyos, Hyphessobrycon epicharis, Hyphessobrycon eques, Hyphessobrycon erythrostigma, Hyphessobrycon megalopterus, Hyphessobrycon micropterus, Hyphessobrycon takasei, Iguanodectes spilurus, Jupiaba polylepis, Lignobrycon myersi, Microschemobrycon sp, Moenkhausia bonita, Moenkhausia hemigrammoides, Moenkhausia intermedia, Moenkhausia sanctaefilomenae, Moenkhausia xinguensis, Oligosarcus pintoi, Paracheirodon axelrodi, Parapristella georgiae, Petitella georgiae, Phenacogaster franciscoensis, Piabina argentea, Pristella maxillaris, Rhinopetitia myersi, Salminus hilarii, Serrapinnus heterodon, Serrapinnus notomelas, Thayeria obliqua, Triportheus nematurus

\section{1 - alto e geralmente ovalado}

Gymnocorymbus ternetzi, Poptella paraguayensis, Psellogrammus kennedyi, Roeboides bonariensis, Serrasalmus maculatus, Stethaprion erythrops, Tetragonopterus argenteus

\section{3- Altura do corpo em relação ao CP (ic - 0,333)}

\section{0 - menos de $50 \%$ do $\mathrm{CP}$}

Aphyocharax anisitsi, Aphyocharax pusillus, Astyanax altiparanae, Astyanax jacobinae, Astyanax mexicanus, Brycinus longipinnis, Bryconamericus exodon, Bryconella pallidifrons, Bryconexodon juruenae, Chalceus spilogyros, Cheirodon interruptus, Coptobrycon bilineatus, Deuterodon iguape, Grundulus cochae, Hasemania crenuchoides, Hasemania melanura, Hasemania sp n., Hemigrammus analis, Hemigrammus arua, Hemigrammus barrigonae, Hemigrammus bellottii, Hemigrammus bleheri, Hemigrammus boesemani, Hemigrammus brevis, Hemigrammus coeruleus, Hemigrammus cylindricus, Hemigrammus gracilis, Hemigrammus haraldi, Hemigrammus hyanuary, Hemigrammus levis, Hemigrammus lunatus, Hemigrammus marginatus, Hemigrammus mimus, Hemigrammus newboldi, Hemigrammus ocellifer, Hemigrammus orthus, Hemigrammus parana, Hemigrammus pretoensis, Hemigrammus pulcher, Hemigrammus rhodostomus, Hemigrammus rodwayi, Hemigrammus schmardae, Hemigrammus silimoni, Hemigrammus skolioplatus, Hemigrammus stictus, Hemigrammus taphorni, Hemigrammus tridens, Hemigrammus ulreyi, Hemigrammus unilineatus, Hemigrammus vorderwikleri, Hemigrammus yinyang, Hemigrammus sp n., Hollandichthys aff. multifasciatus, Hyphessobrycon bentosi, Hyphessobrycon coelestinus, Hyphessobrycon compressus, Hyphessobrycon diancistrus, Hyphessobrycon eilyos, Hyphessobrycon epicharis, Hyphessobrycon eques, Hyphessobrycon erythrostigma, Hyphessobrycon megalopterus, Hyphessobrycon micropterus, Hyphessobrycon takasei, Iguanodectes spilurus, Jupiaba polylepis, Lignobrycon myersi, Microschemobrycon sp, Moenkhausia bonita, Moenkhausia hemigrammoides, Moenkhausia intermedia, Moenkhausia sanctaefilomenae, Moenkhausia xinguensis, Oligosarcus pintoi, Paracheirodon axelrodi, Parapristella georgiae, Petitella georgiae, Phenacogaster franciscoensis, Piabina argentea, Psellogrammus kennedyi, Pristella maxillaris, Rhinopetitia myersi, Roeboides bonariensis, Salminus hilarii, Serrapinnus heterodon, Serrapinnus notomelas, Stethaprion erythrops, Thayeria obliqua, Triportheus nematurus

\section{1 - mais de $50 \%$ do CP}

Gymnocorymbus ternetzi, Poptella paraguayensis, Serrasalmus maculatus, Tetragonopterus argenteus 


\section{9- Indeterminado}

Brycon cf pesu, Bryconops melanurus

\section{4- Quilha ventral de espinhos após a nadadeira peitoral (ic - 1,0$)$}

\section{$\underline{0 \text { - ausente }}$}

Aphyocharax anisitsi, Aphyocharax pusillus, Astyanax altiparanae, Astyanax jacobinae, Astyanax mexicanus, Brycinus longipinnis, Brycon of pesu, Bryconamericus exodon, Bryconella pallidifrons, Bryconexodon juruenae, Bryconops melanurus, Chalceus spilogyros, Cheirodon interruptus, Coptobrycon bilineatus, Deuterodon iguape, Grundulus cochae, Gymnocorymbus ternetzi, Hasemania crenuchoides, Hasemania melanura, Hasemania sp n., Hemigrammus analis, Hemigrammus arua, Hemigrammus barrigonae, Hemigrammus bellottii, Hemigrammus bleheri, Hemigrammus boesemani, Hemigrammus brevis, Hemigrammus coeruleus, Hemigrammus cylindricus, Hemigrammus gracilis, Hemigrammus haraldi, Hemigrammus hyanuary, Hemigrammus levis, Hemigrammus lunatus, Hemigrammus marginatus, Hemigrammus mimus, Hemigrammus newboldi, Hemigrammus ocellifer, Hemigrammus orthus, Hemigrammus parana, Hemigrammus pretoensis, Hemigrammus pulcher, Hemigrammus rhodostomus, Hemigrammus rodwayi, Hemigrammus schmardae, Hemigrammus silimoni, Hemigrammus skolioplatus, Hemigrammus stictus, Hemigrammus taphorni, Hemigrammus tridens, Hemigrammus ulreyi, Hemigrammus unilineatus, Hemigrammus vorderwikleri, Hemigrammus yinyang, Hemigrammus sp n., Hollandichthys aff. multifasciatus, Hyphessobrycon bentosi, Hyphessobrycon coelestinus, Hyphessobrycon compressus, Hyphessobrycon diancistrus, Hyphessobrycon eilyos, Hyphessobrycon epicharis, Hyphessobrycon eques, Hyphessobrycon erythrostigma, Hyphessobrycon megalopterus, Hyphessobrycon micropterus, Hyphessobrycon takasei, Iguanodectes spilurus, Jupiaba polylepis, Lignobrycon myersi, Microschemobrycon sp, Moenkhausia bonita, Moenkhausia hemigrammoides, Moenkhausia intermedia, Moenkhausia sanctaefilomenae, Moenkhausia xinguensis, Oligosarcus pintoi, Paracheirodon axelrodi, Parapristella georgiae, Petitella georgiae, Phenacogaster franciscoensis, Piabina argentea, Poptella paraguayensis, Psellogrammus kennedyi, Pristella maxillaris, Rhinopetitia myersi, Roeboides bonariensis, Salminus hilarii, Serrapinnus heterodon, Serrapinnus notomelas, Stethaprion erythrops, Tetragonopterus argenteus, Thayeria obliqua, Triportheus nematurus

\section{1- presente}

Serrasalmus maculatus

165- Pseudotímpano entre a 1a e 2⿳a costelas (ic - 0,125)

(Malabarba, 1998: 1; Moreira, 2007: 396; Bertaco, 2008: 122; Mirande, 2009: 335)

\section{$\underline{0 \text { - ausente }}$}

Aphyocharax anisitsi, Aphyocharax pusillus, Astyanax altiparanae, Astyanax mexicanus, Brycinus longipinnis, Brycon of pesu, Bryconamericus exodon, Bryconella pallidifrons, Bryconexodon juruenae, Bryconops melanurus, Chalceus spilogyros, Coptobrycon bilineatus, Deuterodon iguape, Grundulus cochae, Gymnocorymbus ternetzi, Hasemania crenuchoides, Hasemania melanura, Hasemania sp n., Hemigrammus analis, Hemigrammus arua, Hemigrammus barrigonae, Hemigrammus bellottii, Hemigrammus bleheri, Hemigrammus boesemani, Hemigrammus brevis, Hemigrammus coeruleus, Hemigrammus cylindricus, Hemigrammus gracilis, Hemigrammus haraldi, Hemigrammus hyanuary, Hemigrammus levis, Hemigrammus lunatus, Hemigrammus marginatus, Hemigrammus newboldi, Hemigrammus ocellifer, Hemigrammus orthus, Hemigrammus parana, Hemigrammus pretoensis, 
Hemigrammus pulcher, Hemigrammus rhodostomus, Hemigrammus rodwayi, Hemigrammus schmardae, Hemigrammus stictus, Hemigrammus taphorni, Hemigrammus tridens, Hemigrammus ulreyi, Hemigrammus unilineatus, Hemigrammus vorderwikleri, Hemigrammus yinyang, Hemigrammus sp n., Hollandichthys aff. multifasciatus, Hyphessobrycon coelestinus, Hyphessobrycon compressus, Hyphessobrycon diancistrus, Hyphessobrycon eilyos, Iguanodectes spilurus, Jupiaba polylepis, Moenkhausia bonita, Moenkhausia hemigrammoides, Moenkhausia intermedia, Moenkhausia sanctaefilomenae, Moenkhausia xinguensis, Oligosarcus pintoi, Parapristella georgiae, Petitella georgiae, Piabina argentea, Poptella paraguayensis, Pristella maxillaris, Rhinopetitia myersi, Salminus hilarii, Serrasalmus maculatus, Stethaprion erythrops, Tetragonopterus argenteus, Thayeria obliqua, Triportheus nematurus

\section{1- presente}

Cheirodon interruptus, Hemigrammus mimus, Hyphessobrycon bentosi, Hyphessobrycon eques, Hyphessobrycon erythrostigma, Hyphessobrycon megalopterus, Hyphessobrycon micropterus, Hyphessobrycon takasei, Microschemobrycon sp, Paracheirodon axelrodi, Phenacogaster franciscoensis, Psellogrammus kennedyi, Roeboides bonariensis, Serrapinnus heterodon, Serrapinnus notomelas

\section{9- Indeterminado}

Astyanax jacobinae, Hemigrammus silimoni, Hemigrammus skolioplatus, Hyphessobrycon epicharis, Lignobrycon myersi

Malabarba (1998) discute a presença de uma abertura derivada da redução da musculatura entre a $1^{\underline{a}}$ e $2^{\text {a }}$ costelas pleurais; o autor define a forma triangular e ampla dessa abertura como sendo sinapomórfica para Cheirodontinae. Na presente análise, foi considerado como pseudotímpano qualquer hiato derivado da redução da musculatura entre a $1^{\underline{a}}$ e $2^{\underline{a}}$ costelas, independente dessa abertura apresentar a forma triangular ou não. Dentre as espécies analisadas, pseudotímpano foi observado em $H$. mimus e Microschemobrycon sp. (clado 128), representantes de Characinae (Roeboides bonariensis, clado 99 e Phenacogaster franciscoensis, clado 109), Cheirodontinae (Cheirodon interruptus, Serrapinnus hetorodon e S. notomelas, clado 178), "rosy tetra" (Hyphessobrycon bentosi, H. eques, H. megalopterus, H. micropterus, H. takasei e $H$. erythrostigma, clado 160), Paracheirodon axelrodi (clado 123), e em Psellogrammus kennedyi (clado 100). A presença de pseudotímpano, entretanto, como sugerido por Malabarba (1998) parece ter surgido diversas vezes e independentemente em cada um dos agrupamentos citados acima. 


\section{2- Reconstrução Filogenética}

\section{Consideracõos gerais}

A análise filogenética realizada a partir de 95 táxons e 165 caracteres resultou em 6 árvores igualmente parcimoniosas, com 1849 passos, Índice de Consistência 0,13 e Índice de Retenção 0,44; a árvore de consenso estrito é apresentada na figura 43. Nas 6 árvores mais parcimoniosas geradas, alguns agrupamentos de espécies de Hemigrammus já estão presentes, entretando, boa parte das espécies do gênero aparecem em uma grande politomia, incluindo $H$. unilineatus, espécie-tipo. Devido ao grande número de caracteres homoplásticos presentes na análise, repesagens sucessivas foram aplicadas ao resultado inicial obtido no intuito de dar maior peso aos caracteres com maior consistência na sustentação dos clados e consequentemente melhorar a resolução das relações encontradas. Após repesagens sucessivas, foi gerada uma única árvore mais parcimoniosa, com Índice de Consistência de 0,35 e Índice de Retenção de 0,61 (Figuras 44 - 49). Segundo Lucena (1993), a complexidade dos táxons e a história evolutiva dos caracteres analisados podem contribuir para baixos valores de índices de consistência, o que é esperado quando se trabalha com Characidae. Vários dos caractes incluídos na análise são bastante homoplásticos, porém a importância da utilização de caracteres, mesmo que altamente homoplásticos, tem sido discutida recentemente por vários autores e segundo Moreira (2007) esses caracteres apresentam-se importantes para a sustentação da topologia dos cladogramas resultantes.

De acordo com o único cladograma mais parcimonioso obtido após repesagens (Figura 44) o gênero Hemigrammus em seu sensu atual não forma um grupo monofilético. Dentro do clado monofilético que engloba todas as espécies de Hemigrammus analisadas (clado 173, Figuras 44 e 45), estão incluídas também espécies de vários outros gêneros de Characidae, inclusive representantes de diferentes subfamílias. Em um clado menos inclusivo, que engloba a maior parte das espécies de Hemigrammus analisadas (clado 164, Figuras 44 e 46) espécies de outros gêneros de pequenos tetragonopterineos (sensu Géry, 1977) aparecem proximamente relacionadas a espécies de Hemigrammus, são eles: Hyphessobrycon (incluindo $H$. compressus, espécie- 
tipo); Pristella (monotípico); Moenkhausia; Petitella (monotípico); Paracheirodon (incluindo P. axelrodi, espécie típo); Grundulus; Coptobrycon (monotípico) e Bryconella (monotípico). Hemigrammus unilineatus, espécie-tipo do gênero, aparece mais proximamente relacionada com representantes de Hyphessobrycon, Moenkhausia e Pristella e as demais espécies de Hemigrammus estão mais proximamente relacionadas a outras espécies do gênero ou a espécies de outros gêneros de Characidae do que a $H$. unilineatus. Os resultados obtidos sugerem grandes mudanças para o que se conhecia até hoje como Hemigrammus, visto que desmembra o gênero em várias subunidades menores (cada subunidade será discutida ao longo do texto) e faz outras importantes modificações em outros gêneros de Characidae, especialmente em Hyphessobrycon, o que levará a necessidade de várias adequações taxonômicas para mais de 150 espécies conhecidas hoje dentro de Characidae.

O presente trabalho não teve como objetivo avaliar o monofiletismo, nem as interrelações de Characidae, bem como de nenhum de seus subgrupos (subfamílias ou gêneros), exceto Hemigrammus. Uma análise com esse objetivo precisaria englobar um número maior de táxons que representassem todas as subfamílias de Characidae, bem como representantes do maior número possível de seus gêneros incertae sedis (Lima et al., 2003; Mirande 2009), além de um número maior de caracteres mais inclusivos, compatíveis com a análise filogenética de uma família. Sendo assim, a seguir são apresentados e discutidos apenas os principais clados obtidos na presente análise, ou seja, aqueles menos inclusivos (exceto clado 173) e que contenham espécies de Hemigrammus, e dentro desses clados são discutidos apenas os caracteres com maior peso para sua sustentação. A lista completa das sinapomorfias de todos os clados é apresentada no Apêndice 1 e a lista com a mudança dos estados de cada caráter no Apêndice 2.

\section{Clado 173}

(Figuras 44 e 45)

Táxons: Astyanax altiparanae, Astyanax jacobinae, Astyanax mexicanus, Bryconella pallidifrons, Bryconexodon juruenae, Coptobrycon bilineatus, Deuterodon iguape, Grundulus cochae, Gymnocorymbus ternetzi, Hemigrammus analis, Hemigrammus arua, Hemigrammus barrigonae, 
Hemigrammus bellottii, Hemigrammus bleheri, Hemigrammus boesemani, Hemigrammus brevis, Hemigrammus coeruleus, Hemigrammus cylindricus, Hemigrammus gracilis, Hemigrammus haraldi, Hemigrammus hyanuary, Hemigrammus levis, Hemigrammus lunatus, Hemigrammus marginatus, Hemigrammus mimus, Hemigrammus newboldi, Hemigrammus ocellifer, Hemigrammus orthus, Hemigrammus parana, Hemigrammus pretoensis, Hemigrammus pulcher, Hemigrammus rhodostomus, Hemigrammus rodwayi, Hemigrammus schmardae, Hemigrammus silimoni, Hemigrammus skolioplatus, Hemigrammus stictus, Hemigrammus taphorni, Hemigrammus tridens, Hemigrammus ulreyi, Hemigrammus unilineatus, Hemigrammus vorderwikleri, Hemigrammus yinyang, Hemigrammus $\mathrm{sp} \mathrm{n}$., Hollandichthys aff. multifasciatus, Hyphessobrycon bentosi, Hyphessobrycon coelestinus, Hyphessobrycon compressus, Hyphessobrycon diancistrus, Hyphessobrycon eilyos, Hyphessobrycon epicharis, Hyphessobrycon eques, Hyphessobrycon erythrostigma, Hyphessobrycon megalopterus, Hyphessobrycon micropterus, Hyphessobrycon takasei, Jupiaba polylepis, Microschemobrycon sp, Moenkhausia bonita, Moenkhausia hemigrammoides, Moenkhausia intermedia, Moenkhausia sanctaefilomenae, Moenkhausia xinguensis, Oligosarcus pintoi, Paracheirodon axelrodi, Parapristella georgiae, Petitella georgiae, Phenacogaster franciscoensis, Poptella paraguayensis, Psellogrammus kennedyi, Pristella maxillaris, Roeboides bonariensis, Serrasalmus maculatus, Stethaprion erythrops, Tetragonopterus argenteus, Thayeria obliqua

\section{Caracteres:}

-21 (1) - rinoesfenóide com extensão dorsal, contactando o crânio anteriormente e dorsalmente e o pterosfenóide posteriormente, osso com formato mais triangular (3 pontas) (com reversões internas no clado);

Esse estado de caráter está presente na maior parte das espécies de pequeno porte de Tetragonopterinae (sensu Géry, 1977) incluídas no clado 173, além de nos representantes de Stethaprioninae. Dentro do clado 173, reversão para o estado 0 - rinoesfenóide sem extensão dorsal, contactando 0 crânio apenas anteriormente e o pterosfenóide posteriormente, osso aproximadamente retangular ou mais arredondado - ocorre nos clados 102 (com Serrasalminae, Characinae, Psellogrammus, Gymnocorymbus e Tetragonopterus), clado 112 (espécies de Astyanax, Bryconexodon, Hollandichthys, Oligosarcus e Characinae), clado 116 (Astyanax jacobinae) e clado 122 (Coptobrycon e Grundulus).

-73 (1) - infra-orbitais 2 e 3 com sobreposição pequena (com reversões internas no clado); 
-83 (1) - palatino estreito e geralmente mais alongado, com a porção mediana estreita e as porções anterior e posterior mais largas (com reversões internas no clado);

-103 (1) - Margem posterior do eixo médio do uro-hial em vista lateral aproximadamente lisa, sem pontas ou reentrâncias (com reversões internas no clado);

-132 (1) - três a quatro supraneurais (com reversões internas no clado);

-138 (1) - nadadeira caudal com base e lobos cobertos com escamas (com reversões internas clado);

-148 (2) - mácula umeral arredondada (com reversões internas clado);

Nesse clado estão presentes todas as espécies de Hemigrammus analisadas, a maior parte das espécies de Tetragonopterinae (sensu Géry, 1977) incluídas na análise e também os represantes de Serrasalminae, Characinae e Stethaprioninae. Fora do clado 173, ficaram algumas das espécies tradicionalmente consideradas como grupos irmãos ou mais basais em Characidae (Brycinus longipinnis, Chalceus spilogyros, Lignobrycon myersi, Salminus hilarii, Triportheus nematurus e Bryconops melanurus) e também os representantes de Hasemania e do "clado A" de Malabarba \& Weitzman (2003) e das subfamílias Iguanodectinae, Aphyocharacinae e Cheirodontinae.

\section{Hemigrammus levis}

(Figuras 44 e 45)

Caracteres:

-28 (2) - processo do supraocciital não ultrapassando a vertical que passa pela porção final do basioccipital;

-33 (1) - dentes do maxilar predominantemente curvos posteriormente;

-34 (1) - maioria dos dentes do maxilar com três cúspides;

-36 (1) - dentes do maxilar considerávelmente menores que os do prémaxilar, geralmente pequenos e estreitos; 
-39 (1) - maxilar com aproximadamente a mesma largura em toda sua extensão;

-55 (0) - dentário com 11-20 dentes; maiores;

-56 (1) - maioria dos dentes do dentário cônicos, após os anteriores

-67 (1) - infra-orbital 1 com comprimento igual ao infra-orbital 2;

-71 (1) - infra-orbital 1 retangular, quadrado ou mais arredondado;

-92 (1) - projeção óssea ventral do metapterigóide direcionada ao quadrado ausente;

-105 (1) - 11 a 13 rastros branquiais no $1^{\circ}$ ceratobranquial;

-109 (1) - presença de poucos cteniis e geralmente esparsos, sobre os rastros branquais;

-146 (1) - presença de manchas negras na escamas dorsais.

H. levis aparece como a espécie mais basal dentre todas as de Hemigrammus analisadas, e incluídas no clado 173. Géry (1977) distribuiu as espécies de Hemigammus até então descritas, em 5 diferentes grupos de acordo com a presença de manchas negras no corpo (especialmente manchas umeral e caudal). H. levis foi colocada por Géry (1977) no " $H$. tridens-group", que incluia espécies com uma mancha caudal, sem mácula umeral, e geralmente uma linha longitudinal no corpo. Juntamente com $H$. levis, estavam inseridas no grupo $H$. brevis, $H$. hyanuary, $H$. mattei, $H$, tridens, $H$. micropterus, $H$. boesemani, $H$. rodway, $H$. rhodostomus e $H$. marginatus. Na presente análise, entretanto, $H$. levis não aparece proximamente relacionada a nenhuma das espécies incluídas no "H. tridens-group" de Géry (1977).

\section{Hemigrammus stictus}

(Figuras 44 e 45)

\section{Caracteres:}

$$
\text { - 2(1) - fontanela do frontal retangular; }
$$

-10 (0) - asas laterais do mesetmóide com 50\% ou menos de sua superfície encoberta pelo vômer, em vista ventral com uma porção muito grande exposta; 
-35 (0) - todas as cúspides dos dentes do maxilar com aproximadamente o mesmo tamanho;

-53 (0) - seis ou mais dentes anteriores e mais largos no dentário;

-59 (1) - osso corono-meckeliano reduzido;

-93 (0) - fenestra na região posterior do metapterigóide aberta posteriormente;

-120 (1) - postemporal com a região posterior larga, afilando progressivamente e terminando em ponta;

-138 (0) - nadadeira caudal nua ou com escamas apenas na base;

-144 (1) - mancha preta no olho presente como uma faixa vertical;

-145 (1) - mancha vermelha no olho presente;

-147 (3) - mácula umeral deslocada posteriormente, começando na altura da $5^{\underline{a}-6^{\underline{a}}}$ escama da linha lateral;

-149 (1) - região clara após a mácula umeral presente.

H. stictus é a segunda espécie mais basal dentre as de Hemigrammus incluídas na análise, fazendo parte do clado 172. $H$. stictus foi alocada no " $H$. bellottii-group" de Géry (1977) que incluía espécies que apresentavam mácula umeral e ausência de mancha caudual; além de $H$. stictus, estavam incluídas também no grupo: $H$. analis, $H$. bellottii, $H$. microstomus, $H$. lunatus, $H$. aereus, H. cupreus e H. cylindricus. De acordo com os resultados aqui apresentados, todas as espécies analisadas do "He. bellottii-group" de Géry (1977) aparecem mais relacionadas a outras de Hemigrammus ou Characidae do que a $H$. stictus. Géry (1964a) compara $H$. stictus e $H$. lunatus e coloca que as espécies são semelhantes na forma do corpo, entretanto, $H$. stictus e $H$. lunatus não aparecem aqui como proximamente relacionadas.

\section{Clado 170}

(Figuras 44 e 45)

Táxons: Parapristella georgiae, Hemigrammus analis, Thayeria obliqua.

\section{Caracteres:}


-4 (1) - metades do frontal se contatam ou sobrepõem após o mesetmóide;

-28 (2) - processo do supraoccipital não ultrapassando a vertical que passa pela porção final do basioccipital;

-41 (0) - maxilar terminando na vertical que passa posteriormente ao etmóide lateral (com reversão para 1 - terminando aproximadamente na vertical que passa pelo etmóide lateral em $\mathrm{H}$. analis);

-43 (0) - dentes da série externa do pré-maxilar desalinhados;

-58 (0) - articulação entre o quadrado e ângulo-articular na vertical que passa pelo meio da órbita ou posterior a ela (com reversão para 1 - na vertical que passa pelo etmóide lateral ou anterior à porção mediana da órbita em $T$. obliqua);

-72 (1) - infra-orbital 2 com uma projeção antero-dorsal sobre o infraorbital 1 (com reversão para 0 - sem projeção antero-dorsal em $H$. analis);

-111 (0) - placa dentígera do ceratobranquial 5 com dentes concentrados nas margens ( 9 - indeterminado em $T$. obliqua);

-125 (2) - nadadeira anal com sete a quinze raios ramificados.

Adicionalmente, $H$. analis, $P$. georgiae e $T$. obliqua apresentam linha lateral incompleta e padrão geral do corpo bastante semelhante: baixo, alongado e relativamente cilíndrico.

Eigenmann (1908), na descrição de Thayeria, coloca que o gênero é muito próximo de Hemigrammus, sendo que as únicas diferenças entre eles são o lobo inferior da nadadeira caudal mais longo que o superior, e uma mancha negra que se prolonga desde o pedúnculo caudal até o lobo inferior da nadadeira caudal em Thayeria. Géry (1964b) ao descrever Parapristella também coloca que o gênero é muito próximo de Hemigrammus, justificando Parapristella como válido apenas por apresentar o maxilar com muitos dentes (6-8 dentes em $P$. georgiae e cerca de 12 em $P$. aubyinei). Acredito que uma análise que inclua as demais espécies de Thayeria e de Parapristella, possa mostrar Thayeria, Parapristella e $H$. analis como um grupo único monofilético.

\section{Hemigrammus barrigonae}

(Figuras 44 e 45) 
Caracteres:

$-2(1)$ - fontanela do frontal retangular;

-10 (0) - asas laterais do mesetmóide com 50\% ou menos de sua superfície encoberta pelo vômer, em vista ventral com uma porção muito grande exposta;

-16 (0) - ponte óssea que liga o etmóide lateral ao vômer totalmente encoberta pelo frontal, não visível ou com apenas uma pequena porção visível em vista dorsal;

-83 (0) - palatino largo, geralmente curto, com a largura não mudando muito ao longo do comprimento; retangular;

-110 (0) - projeção óssea anterior do ceratobranquial 4 presente, larga e

-115 (1) - pós-cleitro 2 aproximadamente o dobro do tamanho do póscleitro 1;

-133 (0) - maioria dos supraneurais com a região dorsal mais larga que o restante do osso, estreitando em sentido ventral;

-144 (2) - mancha preta no olho presente como uma faixa horizontal;

-148 (1) - mácula umeral alongada horizontalmente;

-150 (2) - listra negra longitudinal no corpo começando anterior à mácula umeral e atravessando-a horizontalmente;

-151 (1) - listra negra longitudinal no corpo, conspícua e mais larga, formada por várias séries de cromatóforos;

-155 (1) - presença de uma linha negra rente à base da nadadeira anal e prolongando-se por toda sua extensão;

-157 (0) - mancha negra no pedúnculo caudal presente e baixa;

-159 (1) - mancha negra sobre os raios medianos da nadadeira caudal presente e não se prolongando até a ponta dos raios.

Hemigrammus barrigonae foi incluída no " $H$. ocellifer-group" de Géry (1977), que incluía as espécies de Hemigrammus que apresentavam uma ou duas máculas umerais, mancha caudal e geralmente uma linha negra longitudinal no corpo, juntamente com $H$. megaceps, $H$. guyanensis, $H$. iota, $H$. vorderwinkleri, $H$. schmardae, $H$. melanochrous, $H$. pretoensis, $H$. luelingi, $H$. ocellifer e $H$. pulcher (Géry, 1977). Entretanto, na presente análise, $H$. 
barrigonae não aparece mais proximamente relacionada a nenhuma das espécies alocadas por Géry (1977) no "H. ocellifer-group".

$\mathrm{Na}$ descrição de $H$. barrigonae, Eignemann \& Henn (1914) colocam que a espécie apresenta número variável de escamas perfuradas na linha lateral. Taphorn (1992 e 2003) também coloca que H. barrigonae apresenta uma linha lateral de comprimento variável, desde com poucas escamas perfuradas até com linha lateral quase completa. Taphorn (2003) coloca ainda que $H$. barrigonae apresenta um padrão de colorido muito semelhante ao de Moenkhausia copei e Moenkahusia collettii, relatando que muitas vezes inclusive, essas espécies são confundidas, especialmente os exemplares de $H$. barrigonae que apresentam linha lateral quase completa com aqueles de $M$. copei. Ainda segundo Taphorn (2003), da mesma forma que $H$. barrigonae, $M$. collettii, também apresenta uma linha lateral de comprimento variável. $M$. collettii e $M$. copei não foram incluídas na presente análise, e a questão sobre uma possível proximidade filogenética decorrente do padrão de colorido semelhanete deve ser avaliada futuramente.

Hemigrammus barrigonae apresenta forma do corpo e padrão de colorido bastante semelhante ao de $H$. ulrey, as duas espécies inclusive compartilham alguns estados de caracteres aqui analisados, como: 83 (0) palatino largo, gerlamente curto, com a largura não mudando muito ao longo do comprimento; 144 (2) - mancha preta no olho presente como uma faixa horizontal; 148 (1) - mácula umeral alongada horizontalmente; 150 (2) - listra negra longitudinal no corpo começando anterior à mácula umeral e atravessando-a horizontalmente; 151 (1) - listra negra longitudinal no corpo, conspícua e mais larga, formada por várias séries de cromatóforos; 155 (1) presença de uma linha negra rente à base da nadadeira anal e prolongando-se por toda sua extensão. As duas espécies, entretanto não aparecem na presente análise como proximamente relacionadas.

\section{Clado 166}

(Figuras 44 e 45)

Táxons: Hemigrammus coeruleus, Hemigrammus ocellifer.

\section{Caracteres:}

-67 (1) - infra-orbital 1 com comprimento menor que o do infra-orbital 2; 
-101 (0) - margem posterior das abas do uro-hial aproximadamente reta, não confluente com o eixo mediano $(0,1$ - polimórfico em $H$. ocellifer);

-145 (1) - mancha vermelha no olho presente;

-149 (1) - região clara presente após a mácula umeral;

A mancha clara após a mácula umeral é bastante conspícua em $H$. ocellifer e $H$. coeruleus, envolvendo como um todo a mácula umeral, (a primeira no caso de $H$. ocellifer que apresenta duas).

-153 (1) - presença de uma mancha negra na nadadeira anal, mais ou menos conspícua, formando uma faixa na região mais distal dos raios ao longo de toda a nadadeira.

A mancha negra presente na nadadeira anal de $H$. ocellifer e $H$. coeruleus é pouco conspícua e relativamente clara. Em $H$. coeruleus, a mancha começa se estendendo verticalmente sobre os raios mais anteriores da nadadeira anal e depois se prolonga em sentido posterior, ao longo de toda a extensão, apenas na margem distal da nadadeira; já em $H$. ocellifer a mancha está presente somente margeando toda a nadadeira distalmente.

Hemigrammus ocellifer foi incluída em " $H$. ocellifer-group" de Géry (1977), que englobava as espécies de Hemigrammus com uma ou duas máculas umerais, mancha caudal e geralmente uma linha negra longitudinal, juntamente com $H$. megaceps, $H$. barrigonae, $H$. guyanensis, $H$. iota, $H$. vorderwinkleri, $H$. schmardae, $H$. melanochrous, $H$. pretoensis, $H$. luelingi e $H$. pulcher (Géry, 1977). Já $H$. coeruleus foi incluída por Géry (1977) no "H. unilineatus-group", que englobava as espécies com uma mancha na nadadeira dorsal, compreendendo também $H$. ulreyi, $H$. elegans e $H$. unilineatus. De acordo com os resultados aqui obtidos, entretanto, $H$. ocellifer e $H$. coeruleus aparecem mais proximamente relacionadas entre si do que a qualquer outra espécie do " $H$. ocellifer-group" e do " $H$. unilineatus-group" de Géry (1977) incluídas na análise.

Hemigrammus neptunus é uma das espécies de Hemigrammus que teve apenas exemplares em álcool examinados. Essa espécie apresenta um padrão de colorido bastante semelhante ao de $H$. ocellifer, consistindo em duas máculas umerais, com uma área clara ao redor da primeira mácula; mancha no 
pedúnculo caudal alta, se prolongando sobre a base da nadadeira caudal, atingindo os raios mais marginais da nadadeira caudal e nesses raios se prolongando em sentido posterior. Além disso, em vida essas espécies também apresentam padrão de colorido semelhante. Devido a esse padrão de colorido bastante característico, essas duas espécies provavelmente são bastante próximas (como já sugerido por Zarske \& Géry, 2002), e em uma análise filogenética devem ficar alocadas como espécies irmãs e o clado formado por elas como grupo irmão de $H$. coeruleus.

\section{Clado 163}

(Figuras 44 e 45)

Táxons: Hemigrammus ulreyi, Hemigrammus lunatus

\section{Caracteres:}

-37 (2) - maxilar com quatro a dez dentes;

-110 (0) - projeção óssea anterior do certatobranquial 4 presente, larga e retangular;

-124 (1) - nadadeira anal acentuadamente falcada, com os primeiros raios muito maiores que os demais, os raios logo após os primeiros maiores diminuem de tamanho bruscamente;

-126 (1) - ganchos na nadadeira anal presentes, com número variável, mas sempre mais que um por raio, geralmente todos pequenos ou com um ou dois médios a grande seguidos de pequenos;

-144 (2) - mancha preta no olho presente como uma faixa horizontal (9 indeterminado para $H$. lunatus);

-148 (1) - mácula umeral alongada horizontalmente (com reversão para 2 - mácula umeral arredondada em $H$. lunatus);

-155 (1) - presença de uma linha negra rente à base da nadadeira anal e se prolongado por toda sua extensão (com reversão para 2 - linha paralela à base da nadadeira em $H$. lunatus).

Esse caráter apresenta o estado 1 como sinapomórfico para o clado 163, porém sofre reversão para o estado 2 em $H$. lunatus. $H$. lunatus não apresenta uma linha negra característica rente à base da nadadeira anal, porém, nessa região pode ser observada uma concentração maior de cromatóforos, como relato por Durbin (in Eigenmman, 1918) e Britskli et al. (2007) 
H. ulreyi foi incluída por Géry (1977) no " $H$. unilineatus-group", que englobava as espécies com uma mancha na nadadeira dorsal, compreendendo também $H$. coeruleus, $H$. elegans e $H$. unilineatus. Já $H$. lunatus foi incluída no "H. bellottii-group" de Géry (1977), que alocava as espécies que apresentavam mácula umeral, ausência de mancha caudal e geralmente uma linha negra longitudinal estreita ao longo do corpo, juntamente com $H$. stictus, $H$. analis, $H$. bellottii, $H$. microstomus, $H$. aereus, $H$. cupreus e $H$. cylindricus. Entretanto, de acordo com os resultados aqui apresentados $H$. ulreyi e $H$. lunatus aparecem mais proximamente relacionadas entre si do que a qualquer outra espécie examinada de " $H$. unilineatus-group" e " $H$. bellottii-group" de Géry (1977).

\section{Clado 161 - gênero "Hemigrammus"}

(Figuras 44 e 46)

Táxons: Hemigrammus unilineatus, Moenkhausia hemigrammoides, Pristella maxillaris, Hyphessobrycon bentosi, Hyphessobrycon compressus, Hyphessobrycon megalopterus, Hyphessobrycon micropterus, Hyphessobrycon epicharis, Hyphessobrycon eques, Hyphessobrycon erythrostigma, Hyphessobrycon takasei.

\section{Caracteres:}

-14 (0) - região posterior do mesetmóide terminando após a região de inserção do etmóide lateral (com reversão para 1 - terminando antes ou aproximadamente na mesma altura da região de inserção do etmóide lateral em $H$. eques, $H$. megalopterus, $H$. micropterus, $H$. takasei e $P$. maxillaris);

-41 (0) - maxilar terminando na vertical que passa posteriormente ao etmóide lateral (com reversão para 1 - terminando aproximadamente na vertical que passa pelo etmóide lateral em $H$. compressus, $H$. eques, $H$. megalopterus, H. micropterus e M. hemigrammoides);

-96 (1) - região póstero-ventral do quadrado distante do metapterigóide (com reversão para 0 - próxima ou em contato com o metapterigóide em $H$. compressus, $H$. eques, $H$. megalopterus, $H$. micropterus e $H$. takasel);

-120 (0) - região posterior do postemporal larga e afilando buscamente (com reversão para 1 - região posterior larga, afilando progressivamente terminando em ponta em $\mathrm{H}$. compressus, $\mathrm{H}$. megalopterus e P. maxillaris);

-152 (1) - presença de uma mancha negra conspícua e delimitada na nadadeira dorsal. 
O estado 1 do caráter 152 - presença de uma mancha negra conspícua na nadadeira dorsal - aparece como uma sinapomorfia exclusiva para o clado 161, incluindo além da espécie-tipo de Hemigrammus ( $H$. unilineatus), a espécie-tipo de Hyphessobrycon ( $H$. compressus) e a espécie-tipo de Pristella ( $P$. maxillaris). De acordo com o Princípio da Prioridade estabelecido pelo Código Internacional de Nomenclatura Zoológica (ICZN, 1999), Hemigrammus, por ser o nome mais antigo proposto torna-se o nome válido para o gênero, passando Hyphessobrycon e Pristella a serem sinônimos júniores e não mais gêneros válidos. A nova conformação de Hemigrammus acarretará grandes mudanças taxonômicas dentro de Characidae, visto que todas as espécies de Hemigrammus e Hyphessobrycon que não se enquadram no sensu atual de Hemigrammus (tratado a partir daqui como "Hemigrammus") ficarão como espécies incertae sedis em Characidae até que estudos futuros possam esclarecer seus relacionamentos.

Além disso, a presença de uma mancha negra na nadadeira dorsal já foi proposta por outros autores (Géry, 1977; Weitzman \& Palmer, 1997a e b; Mirande, 2009) como indicadora de possível relacionamento entre algumas espécies de Characidae. Weitzman \& Palmer (1977a) chamaram esse agrupamento de "rosy tetra" e apresentaram como possivelmente relacionadas (como rosy tetra sensu strictu ou grupos próximos) mais de 30 espécies de cinco gêneros de Characidae: Hyphessobrycon $(H$. epicharis, $H$. eques, $H$. compressus, $H$. milleri, $H$. copelandi, $H$. bentosi, $H$. panamensis, $H$. minor, $H$. rosaceus, $H$. equadoriensis, $H$. megalopterus, $H$. micropterus, $H$. erytrostigma, $H$. uruguayensis, $H$. melasemion, $H$. axelrodi, $H$. haraldschultzi, $H$. simulatus, $H$. roseus, $H$. sweglesi, $H$. georgettae, $H$. takasei, $H$. socolofi, $H$. werneri, $H$. pyrrhonotus, $H$. loweae, $H$. hasemani, $H$. pulchripinnis e $H$. amandae); Cheirodon (C. troemneri); Hemigrammus ( $H$. unilineatus); Pristella ( $P$. maxillaris) e Moenkhausia ( $M$. pittieri e $M$. hemigrammoides). Weitzman \& Palmer (1997a) sugeriram a proximidade entre as espécies acima citadas baseando-se, além da presença de mancha negra conspícua na nadadeira dorsal, também na presença de algumas outras características encontradas em apenas algumas espécies, como: coloração predominantemente rosada ou avermelhada do corpo; colorido das nadadeiras dorsal e anal em vida; corpo alto e relativamente comprimido lateralmente, mudanças ontogenéticas nos 
dentes e nas séries de dentes e caracteres sexuais secundários (machos maduros com nadadeira dorsal e lobo da nadadeira anal alongados). Os autores, apesar de sugerirem a existência daquele grupo monofilético, enfatizam a necessidade de uma análise com enfoque filogenético das várias espécies que formariam o referido grupo, a fim de corroborar sua hipótese, destacando que para muitas espécies possivelmente pertencentes aos "rosy tetra" existe pouco material disponível em coleções, muitas vezes apenas com indivíduos juvenis e imaturos (Weitzman \& Palmer, 1997a). Géry (1977) também reconheceu a semelhança de várias espécies com mancha negra na nadadeira dorsal, porém, agrupou essas espécies separadamente (inclusive em gêneros diferentes) devido à presença de duas séries de dentes no prémaxilar (espécies do "Hy. bentosi-group", Hemigrammus unilineatus e Moenkhausia hemigrammoides) ou uma série irregular de dentes (espécies do "Pristella-group").

No presente trabalho, apenas algumas das espécies sugeridas por Weitzman \& Palmer (1997a) como integrantes ou relacionadas aos "rosy tetra" foram analisadas, visto que o objetivo do presente trabalho não foi o de avaliar a relação entre todos os integrantes do grupo sugerido pelos autores e sim analisar o monofiletismo e a relação das espécies do gênero Hemigrammus. Porém, os resultados aqui encontrados corroboram a proposta de Weitzman \& Palmer (1997a) relativa à existência desse grupo monofilético dentro de Characidae.

Outros trabalhos recentes, apesar de avaliarem poucas espécies de Hemigrammus e Hyphessobrycon, já sugerem uma maior proximidade entre $H$. unilineatus e espécies pertencentes aos "rosy tetra". Benine (2004) apresenta um cladograma onde $H$. unilineatus aparece como grupo irmão de um clado formado por Hyphessobrycon herbertaxelrodii (não pertencete aos "rosy tetra"), Hyphessobrycon eques e Hyphessobrycon compressus (os dois últimos, integrantes dos "rosy tetra"). Dentre os táxons sugeridos por Weitzman \& Palmer (1997a) como pertencentes ou próximos ao grupo dos "rosy tetra", Mirande (2009) utilizou em sua análise $H$. unilineatus, $H$. pulchripinnis, $H$. eques, $H$. socolofi e $P$. maxillaris. Todas essas espécies formaram um clado único e monofilético (Mirande, 2009: pg 6, figura 1) sustentado pela presença de uma mancha negra conspícua na nadadeira dorsal. Javonillo et al. (2010), 
dentre os representates dos "rosy tetra" utilizaram $H$. eques, $H$. erytrostigma, $H$. megalopterus e Pristella; as três primeiras espécies aparecem formando um agrupamento monofilético, já Pristella por sua vez aparece mais proximamente relacionada a $H$. rodwayi. Javonillo et al. (2010), entretanto não incluiram na análise as espécies tipo de Hemigrammus, $H$. unilineatus, e de Hyphessobrycon, H. compressus.

Além da presença de uma mancha negra na nadadeira dorsal, outros caracteres já foram sugeridos em literatura como importantes para o relacionamento dos "rosy tetra", como desenvolvimento das nadadeiras dos machos durante o período reprodutivo, presença de um pseudotímpano, mudanças ontogenéticas nos dentes e padrão de colorido em vida. Com relação ao colorido em vida, é interessante notar que vários dos integrantes do grupo apresentam a ponta das nadadeiras dorsal e anal (e em vários casos também da pélvica) com colorido distintamente branco; em alguns casos podem ocorrer também manchas amarelas, alaranjadas ou avermelhadas, especialmente próximo à mancha negra da nadadeira dorsal. Além disso, algumas espécies incluídas nos "rosy tetras" apresentam o corpo com tons de rosa ou avermelhado. Sendo assim, é importante ressaltar que apenas parte das espécies sugeridas por Weitzman \& Palmer (1997a) e dos caracteres já citados em literatura como importantes para o relacionamento dos "rosy tetras" foram aqui analisados. Dessa forma, fica claro que uma análise mais ampla, que englobe o maior número possível das espécies sugeridas como pertencentes aos "rosy tetra", e também um maior número de caracteres (especialmente aqueles relacionados a dimorfismo sexual secundário, colorido em vida e modificações ontogenéticas nos dentes) é de extrema importância para esclarecer a nova composição real do gênero "Hemigrammus" e o relacionamento de suas espécies entre si.

\section{Dentro do clado 161, o clado menos inclusivo 152 apresenta:}

Táxons: Hemigrammus unilineatus, Moenkhausia hemigrammoides

\section{Caracteres:}

-73 (0) - infra-orbitais 2 e 3 sem sobreposição (0, 1 - polimórfico em $M$. hemigrammoides); 
-92 (1) - projeção óssea ventral do metapterigóide direcionada ao quadrado ausente (0,1 - polimórfico em M. hemigrammoides);

-124 (1) - nadadeira anal com primeiros raios muito maiores que os seguintes (o dobro dos raios do meio da nadadeira), formando um lobo pontudo, com os raios logo após os mais longos diminuindo de tamanho bruscamente, nadadeira acentuadamente falcada;

-126 (1) - ganchos na nadadeira anal presentes, com número variável, mas sempre mais que um por raio, geralmente todos os ganchos pequenos, ou um ou dois ganchos de tamanho médio a grande por raio, seguidos por ganchos pequenos em cada raio;

-133 (0) - maioria dos supraneurais com a região dorsal mais larga que o restante do corpo do osso, estreitando em sentido ventral;

-153 (2) - mancha negra na nadadeira anal conspícua e obliqua, começando na base dos primeiros raios simples, atravessando a porção mediana dos raios seguintes e terminando na ponta do $2^{\circ}-4^{\circ}$ raio ramificado;

Condição observada apenas para as duas espécies que compõem o clado 152, sendo portanto uma sinapomorfia exclusiva para o clado

-154 (1) - mancha negra na região ventral anterior à nadadeira anal e contínua com a mancha negra da nadadeira anal presente;

Condição observada apenas para $H$. unilineatus e $M$. hemigrammoides, sendo portanto mais uma sinapomorfia exclusiva para o clado 152.

Adicionalmente, $H$. unilineatus e $M$. hemigrammoide apresentam em vida, ao redor da mancha negra da nadadeira dorsal (dorsal e ventralmente) e anterior à mancha negra da nadadeira anal, um colorido bastante intenso, geralmente amarelo ou branco (raramente em tons avermelhados) (Planquette et al., 1996). Dessa forma, a avaliação do colorido em vida de $H$. unilineatus e M. hemigrammoides pode revelar caracteres adicionais importantes para a sustenção do clado 152.

Géry (1965a), na descrição de $M$. hemigrammoides coloca que a espécie é muito semelhante a $H$. unilineatus, diferindo basicamente por apresentar uma linha lateral completa (versus incompleta em $H$. unilineatus) e poucos dentes (1 - 3) tricuspidados no maxilar (versus 5 - 6 geralmente cônicos em $H$. unilineatus). Weitzman \& Palmer (1997a) discutem a possível proximidade filogenética de $H$. unilineatus com $M$. hemigrammoides baseados 
no padrão único de colorido da nadadeira anal apresentado por essas duas espécies, hipótese essa corroborada aqui.

Dentro do clado 161, o clado menos inclusivo 160 apresenta:

Táxons: Hyphessobrycon bentosi, Hyphessobrycon takasei, Hyphessobrycon eques, Hyphessobrycon erythrostigma, Hyphessobrycon epicharis, Hyphessobrycon micropterus, Hyphessobrycon megalopterus, Hyphessobrycon compressus, Pristella maxillaris.

\section{Caracteres:}

-6 (3) - ramo superior da linha lateral cefálica do frontal, próximo ao parietal ausente (com reversão para 1 - ramo superior da linha lateral cefálica do frontal presente, não se prolongando pelo parietal em $H$. compressus e 1, 3 - polimórfico em H. takasei);

-34 (0) - maioria dos dentes do maxilar com 4 ou mais cúspides (com reversão para 1 - maioria dos dentes do maxilar com três cúspides em $H$. compressus, $H$. epicharis, $H$. eques, $H$. micropterus e $2-$ maioria cônicos em $H$. erythrostigma, H. megalopterus, P. maxillaris;

-36 (0) - dentes do maxilar semelhantes aos dentes do pré-maxilar, aproximadamente do mesmo tamanho (com reversão para 1 - dentes do maxilar consideravelmente menores que os do pré-maxilar, geralmente pequenos e estreitos em $H$. megalopterus, $P$. maxillaris);

-63 (0) - antorbital alongado, com a largura não mudando muito ao longo do comprimento, osso pode ser estreito ou largo (com reversão para 1 antorbital com porção dorsal afilada e ventral mais larga em $H$. bentosi, $H$. compressus, $H$. eques, $P$. maxillaris e 2 - antorbital bastante largo com a porção ventral pouco mais larga que o restante do osso em $H$. micropterus;

-88 (2) - ectopterigóide sobrepõe-se ao quadrado (com reversão para 0 - ectopterigóide contata o quadrado em $H$. epicharis, $H$. megalopterus, $P$. maxillaris e 0, 2 - polimórfico em $H$. bentosi);

-93 (0) - fenestra na região posterior do metapterigóide aberta posteriormente (com reversão para 1 - totalmente fechada em $H$. epicharis, $H$. eques, $H$. erythrostigma, $P$. maxillaris);

-112 (1) - faringo-branquiais 2 e 3 com dentes (com reversão para 2 dentes apenas no faringo-branquial 3 em $H$. compressus, $H$. epicharis, $H$. micropterus);

-144 (1) - mancha preta no olho presente, como uma faixa vertical (com reversão para 0 - mancha preta no olho ausente ou presente apenas na porção superior em $H$. compressus, $H$. epicharis e 9 - indeterminado em $H$. micropterus); 
-150 (0) - listra negra longitudinal no corpo ausente (com reversão para 1 - presente, começando em contato com a mácula umeral (ou região umeral) ou posterior a ela em $H$. compressus, $P$. maxillaris e 9 - indeterminado em $H$. epicharis);

-165 (1) - pseudotímpano entre a $1^{\underline{a}}$ e $2^{\underline{a}}$ costelas presente (com reversão para 0 - pseudotímpano ausente em $H$. compressus, $P$. maxillaris e 9 - indeterminado em $H$. epicharis);

Várias espécies do grupo dos "rosy tetra" apresentam um pseudotímpano bem desenvolvido entre a $1^{\underline{a}}$ e $2^{\underline{a}}$ costela pleural, semelhante ao obervado para os representantes de Cheirodontinae, como colocado por Malabarba (1998). Dentre as espécies agrupadas em "Hemigrammus", pseudotímpano foi observado em $H$. bentosi, $H$. eques, $H$. megalopterus, $H$. micropterus, $H$. takasei e $H$. erythrostigma; adicionalmente, Malabarba (1998) relata a presença de um pseudotímpano também para $H$. pyrrhonotus, outra espécie de "rosy tetra". Em $H$. unilineatus, M. hemigrammoides, $H$. compressus e P. maxillaris, foi observada apenas uma redução da musculatura na região correspondente ao pseudotímpano, sem a ocorrência de um hiato muscular. Dessa forma, a presença de um pseudotímpano, em uma análise com as demais espécies possívelmente integrantes de "Hemigrammus", pode aparecer como uma sinapomorfia delimitando um agrupamento menor e derivado dentro do gênero.

Além dos caracteres já apresentados acima, algumas das espécies reconhecidas como integrantes do grupo dos "rosy tetra" apresentam em vida o corpo rosado, avermelhado ou em tons de marrom avermelhado (característica que deu nome ao grupo), como por exemplo, $H$. epicharis, $H$. bentosi, $H$. erythrostigma, $H$. socolofi, $H$. pyrrhonotus e $H$. eques, dentre outros. Dentro de "rosy tetra" ainda, existem espécies que possuem uma mancha vermelha na região umeral de exemplares vivos (os chamados "bleeding heart tetras"), como $H$. socolofi e $H$. erythrostigma. Os referidos caracteres de colorido em vida não foram analisados devido à falta de informação disponível para grande parte das espécies incluídas na análise, porém, é muito provável que sejam características derivadas e certamente constituam sinapomorfias adicionais para "Hemigrammus" ou possam delimitar clados menos inclusivos dentro do gênero. Dessa forma, uma melhor avaliação do colorido em vida das espécies 
dará maior suporte para a nova concepção de "Hemigrammus" e ajudará a esclarecer de forma mais precisa a relação entre suas espécies.

Dentro do clado 161, o clado menos inclusivo 159 apresenta:

Táxons: Hyphessobrycon takasei, Hyphessobrycon eques, Hyphessobrycon erythrostigma, Hyphessobrycon epicharis, Hyphessobrycon micropterus, Hyphessobrycon megalopterus, Hyphessobrycon compressus, Pristella maxillaris.

\section{Caracteres:}

-14 (1) - região posterior do mesetmóide terminando antes ou aproximadamente na mesma altura da região de inserção do etmóide lateral (com reversão para 0 - terminando após a região de inserção do etmóide lateral em $H$. bentosi, $H$. compressus, $H$. epicharis, $H$. erythrostigma);

-50 (0) -região anteiror do dentário com declividade acentuada deixando a região anterior bem mais afilada que a posterior (com reversão para 1 região anterior do dentário sem declividade, ou com esta pouco acentuada, deixando a região anterior do dentário aproximadamente da mesma altura que região posterior $H$. epicharis, $H$. erythrostigma, $P$. maxillaris);

-53 (0) - seis ou mais dentes anteriores e mais largos no dentário (com reversão para 2 - quatro a cinco dentes anteriores e mais largos no dentário em $H$. compressus, $P$. maxillaris; 0, 2 - polimórfico em $H$. megalopterus);

-56 (0) - maioria dos dentes do dentário cuspidados após os dentes anteriores maiores (com reversão para 1 - maioria dos dentes do dentário cônicos após os dentes anteriores maiores em $H$. compressus, $H$. megalopterus, $H$. micropterus);

-96 (0) - região póstero-ventral do quadrado próxima ou em contato com o metapterigóide (com reversão para 1 - região póstero-ventral do quadrado distante do metapterigóide em $H$. epicharis, $H$. erythrostigma, $P$. maxillaris);

-138 (0) - nadadeira caudal com escamas ausentes ou apenas na base;

-153 (1) - mancha negra na nadadeira anal presente, não obliqua, podendo ser mais ou menos conspícua, formando uma faixa na região mais distal dos raios ao longo de toda a extensão da nadadeira (às vezes estendendo-se dorsalmente sobre os primeiros ou os últimos raios da nadadeira) (com reversão para 4 - mancha negra presente, conspícua e não obliqua, restrita à porção mediana (ou mais próxima da ponta, mas não atingindo a ponta dos raios) dos primeiros 5 raios da nadadeira em $P$. maxillaris);

Dentro do clado 161, o clado menos inclusivo 158 apresenta: 
Táxons: Hyphessobrycon eques, Hyphessobrycon erythrostigma, Hyphessobrycon epicharis, Hyphessobrycon micropterus, Hyphessobrycon megalopterus, Hyphessobrycon compressus, Pristella maxillaris.

\section{Caracteres:}

-24 (0) - processo lateral do esfenótico sobreposto pelo frotal (com reversão para 1 - processo lateral do esfenótico apenas contatado pelo frotal em H. megalopterus, H. micropterus);

-34 (1) - maioria dos dentes do maxilar com três cúspides (com reversão para 2 - maioria dos dentes do maxilar cônicos em $H$. erythrostigma, $H$. megalopterus, $P$. maxillaris);

-41 (1) - maxilar terminando aproximadamente na vertical que passa pelo etmóide lateral (com reversão para 0- terminando na vertical que passa posteriormente ao etmóide lateral em $H$. epicharis, $H$. erythrostigma);

-45 (0) - série interna do pré-maxilar com seis ou mais dentes;

-70 (1) - infra-orbitais 1 e 2 em contato ou com sobreposição (com reversão para 0 - infra-orbitais 1 e 2 sem contato ou sobreposição em $H$. compressus, $H$. megalopterus, $P$. maxillaris);

-93 (1) - fenestra na região posterior do metapterigóide totalmente fechada (com reversão para 0 - fenestra aberta posteriormente em $H$. compressus, H. megalopterus, H. micropterus);

-98 (1) - canal latero-sensorial do pré-opérculo não atingindo essa margem, geralmente ficando restrito a região $d$ a articulação entre hiomandibular e opérculo (com reversão para 0 - canal lateros-sensorial próximo ou atingindo a margem dorsal formada pelo hiomandibular e o opérculo em $H$. erythrostigma, P. maxillaris);

-133 (0) - maioria dos supraneurais com a região dorsal mais larga que o restante do corpo do osso, estreitando em sentido ventral (com reversão para 1 - maioria dos supraneurais com aproximadamente a mesma largura em toda a extensão do osso em $P$. maxillaris e 0,1-polimórfico em $H$. megalopterus);

-145 (1) - mancha vermelha no olho presente (com reversão para 0 mancha vermelha no olho ausente em $H$. megalopterus, $H$. micropterus, $P$. maxillaris e 9 - indeterminado em $H$. compressus, $H$. epicharis);

\section{Dentro do clado 161, o clado menos inclusivo 157 apresenta:}

Táxons: Hyphessobrycon erythrostigma, Hyphessobrycon epicharis, Hyphessobrycon micropterus, Hyphessobrycon megalopterus, Hyphessobrycon compressus, Pristella maxillaris.

\section{Caracteres:}


-37 (2) - quatro a dez dentes no maxilar (1, 0 -polimórfico em $P$. maxillaris; 1, 2 - polimórfico em $H$. megalopterus; 2, 3 -polimórfico em $H$. compressus e $H$. micropterus);

-46 (3) - dentes da série interna do pré-maxilar com três cúspides (com reversão para 2 - cônicos em $H$. megalopterus e 2, 3 - polimórfico em $H$. erythrostigma);

-50 (1) - região anterior do dentário sem declividade, ou com esta pouco acentuada, deixando a região anterior do dentário aproximadamente da mesma altura que região posterior (com reversão para 0 - com declividade acentuada deixando a região anterior bem mais afilada que a posterior em $H$. megalopterus, $H$. micropterus);

-56 (1) - maioria dos dentes do dentário cônicos após os dentes anteriores maiores (9-indeterminado em $H$. megalopterus, $H$. micropterus);

-75 (0) - infra-orbital 4 com as margens anterior e posterior aproximadamente da mesma altura $(9$ - indeterminado em $H$. compressus e $H$. megalopterus);

-92 (1) - projeção óssea ventral do metapterigóide direcionada ao quadrado ausente (com reversão para 0 - projeção óssea ventral do metapterigóide direcionada ao quadrado presente em H. compressus);

-104 (1) - basi-hial retangular, com a região dorsal pouco expandida, igual ou pouco mais larga que a ventral, menos de 2 vezes (com reversão para 0 - basi-hial acentuadamente triangular, com a região anterior expandida, duas ou mais vezes mais larga que a posterior em $H$. megalopterus);

-105 (1) - 11 a 13 rastros branquiais no $1^{\circ}$ ceratobranquial (com reversão para 2 - sete a 10 rastros branquiais no $1^{\circ}$ ceratobranquial em $H$. micropterus, $P$. maxillaris);

-112 (2) - dentes apenas no faringo-branquial 3 (com reversão para 1 faringo-branquiais 2 e 3 com dentes em $H$. erythrostigma, $H$. megalopterus; 9 indeterminado em P. maxillaris);

\section{Dentro do clado 161, o clado menos inclusivo 156 apresenta:}

Táxons: Hyphessobrycon erythrostigma, Hyphessobrycon epicharis

\section{Caracteres:}

-14 (0) - região posterior do mesetmóide terminando após a região de inserção do etmóide lateral;

-29 (1) - processo do supraoccipital muito inclinado dorsalmente em relação ao eixo longitudinal do crânio; 
-41 (0) - maxilar terminando na vertical que passa posteriormente ao etmóide lateral;

-52 (0) - dentes do dentário diminuindo gradualmente de tamanho em sentido posterior;

-96 (1) - região póstero-ventral do quadrado distante do metapterigóide;

\section{Dentro do clado 161, o clado menos inclusivo 155 apresenta:}

Táxons: Hyphessobrycon micropterus, Hyphessobrycon megalopterus, Hyphessobrycon compressus, Pristella maxillaris.

\section{Caracteres:}

-69 (1) - canal sensorial do infra-orbital 1 ausente (com reversão para 2 canal sensorial do infra-orbital 1 presente e curto, atingindo no máximo $50 \%$ da extensão do osso em $H$. micropterus);

-70 (0) - infra-orbitais 1 e 2 sem contato ou sobreposição (com reversão para 1 - Infra-orbitais 1 e 2 em contato ou com sobreposição em $H$. micropterus);

-93 (0) - fenestra na região posterior do metapterigóide aberta posteriormente (com reversão para 1 - fenestra na região posterior do metapterigóide totalmente fechada em $P$. maxillaris);

-120 (1) - postemporal com região posterior larga, afilando progressivamente terminando em ponta;

-145 (0) - mancha vermelha no olho ausente (com reversão para 1 presente em $H$. erythrostigma e 9 - indeterminado em $H$. compressus, $H$. epicharis);

\section{Dentro do clado 161, o clado menos inclusivo 154 apresenta:}

Táxons: Hyphessobrycon micropterus, Hyphessobrycon megalopterus

\section{Caracteres:} convexas;

-5 (1) - fontanela do parietal abaulada, com as margens laterais

-24 (1) - processo lateral do esfenótico apenas contactado pelo frontal;

-26 (3) - processo posterior do pterótico em vista dorsal ausente;

-50 (0) - região anterior do dentário com declividade acentuada deixando a região anterior bem mais afilada que a posterior;

-54 (0) - dentes anteriores do dentário estreitos, geralmente com três cúspides, semelhantes aos do pré-maxilar; 
-64 (1) - nasal ausente (com reversão para 2 - nasal presente e muito reduzido, restrito à porção mediana da distância entre a margem anterior do frontal e as asas laterais do mesetmóide em $H$. micropterus);

-76 (2) - infra-orbital 4 aproximadamente tão longo quanto alto (9 indeterminado em $H$. megalopterus);

$-80(0)$ - infra-orbital 6 ausente;

-113 (1) - cleitro com a região ventral sem aba desenvolvida (ou muito reduzida), deixando a fenestra formada pelo cleitro e coracóide totalmente visível em vista lateral ou com apenas a região mais dorsal encoberta (30\% ou menos);

Dentro do clado 161, o clado menos inclusivo 153 apresenta:

Táxons: Hyphessobrycon compressus, Pristella maxillaris.

\section{Caracteres:}

-43 (0) - dentes da série externa do pré-maxilar desalinhados (9 indeterminado em $P$. maxillaris);

-53 (2) - quatro a cinco dentes anteriores e mais largos do dentário;

-63 (1) - antorbital com porção dorsal afilada e ventral mais larga;

-148 (2) - mácula umeral arredondada (9 - indeterminado em $H$. compressus);

-150 (1) - listra negra longitudinal no corpo presente, começando em contato com a mácula umeral (ou região umeral) ou posterior a ela;

-165 (0) - pseudotímpano entre a $1^{\underline{a}}$ e $2^{\underline{a}}$ costelas ausente;

P. maxillaris apresenta padrão de colorido em vida das nadadeiras dorsal e anal semelhante ao de $H$. unilineatus e $M$. hemigrammoides, indicando que em uma análise que inclua as referidas características, essas espécies possam aparecer mais proximamente relacionadas.

\section{Clado 150}

(Figuras 44 e 47)

Táxons: Hemigrammus gracilis, Hemigrammus silimoni

\section{Caracteres:}

-33 (1) - dentes do maxilar predominantemente curvos posteriormente; 
-34 (2) - maioria dos dentes do maxilar cônicos;

-49 (0) - dentário retangular, com aproximadamente a mesma altura em toda extensão;

-88 (1) - ectopterigóide não contacta o quadrado.

Hemigrammus gracilis foi colocada por Géry (1977) no " $H$. gracilisgroup", caracterizado por apresentar espécies com corpo alongado e sem manchas negras pelo corpo (ou com essas muito claras), incluindo também $H$. erythrozonus e $H$. mimus. Entretanto, $H$. mimus não aparece na presente análise como proximamente relacionada a $H$. gracilis. $H$. erythrozonus é uma das espécies de Hemigrammus não analisadas e sua posição dentro de Characidae, bem como seu possível relacionamento com $H$. gracilis permanece desconhecido.

Hemigrammus silimoni é uma espécie recentemente descrita (Britki \& Lima, 2008), e caracteizada pela presença de um ocelo na porção inferior do lobo dorsal da nadadeira caudal. Como destacado por Britski \& Lima (2008) a mancha presente no lobo superior de $H$. silimoni não encontra similar dentro de Hemigrammus, nem de Characidae. Dessa forma, a posição filogenética da espécie dentro da família ainda precisa ser melhor avaliada em estudos futuros, principalmente aqueles que incluam outras espécies de Characidae com apenas o lobo superior manchado (especialmente espécies de Moenkhausia) a fim de testar a homologia e a distribuição dessa característica.

\section{Clado 148}

(Figuras 44 e 47)

Táxons: Hemigrammus rodwayi, Hemigrammus parana, Moenkhausia bonita, Hemigrammus marginatus, Hemigrammus sp. $\mathrm{n}$.

\section{Caracteres:}

-24 (0) - processo lateral do esfenótico sobreposto pelo frontal (com reversão para 1 - apenas contatado pelo frontal em $H$. marginatus e $H$. sp.n.);

-39 (0) - maxilar com a região mediana ou posterior mais larga que o restante do osso (com reversão para 1 - maxilar com aproximadamente a mesma largura em todo osso em $H$. marginatus e $H$. sp. n.); 
-104 (1) - basi-hial retangular, com a região dorsal pouco expandida, igual ou pouco mais larga que a ventral (com reversão para 0 - basi-hial acentuadamente triangular, com a região anterior expnadida, duas ou mais vezes mais larga que a posterior e $M$. bonita e estado 9-indeterminado para $H$. marginatus);

-159 (2) - mancha sobre os raios caudais medianos presente e não se prolongando até a ponta dos raios (com reversão para 0 - mancha sobre os raios medianos ausente em $H$. marginatus);

Hemigrammus rodwayi é uma espécie de Hemigrammus pouco discutida em literatura. Géry (1977) alocou H. rodwayi dentro de "H. tridens group" que incluía espécies com mancha caudal e sem mácula umeral, juntamente com $H$. levis, $H$. brevis, $H$. hyanuary, $H$. mattei, $H$. tridens, $H$. micropterus, $H$. boesemani, $H$. rhodostomus e $H$. marginatus. Durbin (1909) na descrição de $H$. rodwayi coloca que os machos dessa espécie, quando vivos, apresentam uma mancha brilhante vermelho-cereja na base de cada lobo caudal e têm essa mesma coloração vermelha distribuída também sobre as nadadeiras anal e dorsal; os machos apresentam ainda uma barra branca na margem anterior da anal; já as fêmeas não apresentam a barra branca na anal e possuem coloração amarelada nos locais correspondentes às manchas vermelhas dos machos. Benine et al. (2004) na descrição de M. bonita, colocam que os exemplares apresentam, em vida, parte de cada um dos raios simples mais longos das nadadeiras dorsal e anal brancos, o restante das nadadeiras com coloração avermelhada ou amarelada; apresentam também pigmentação amarelada na base da nadadeira caudal. Os autores colocam ainda que a pigmentção amarelada ou avermelhada das nadadeiras é muito mais conspícua em machos dominantes (Benine et al., 2004). Na descrição do colorido em vida de $H$. parana, Marinho et al. (2008) relatam que a espécie apresenta as nadadeiras amareladas e a porção média dos lobos caudais vermelha ou alaranjada. $H$. marginatus apresenta em vida coloração muito semelhante à descrita para $M$. bonita. Sendo assim, penso que o padrão de colorido em vida das espécies incluídas no clado 145 deve ser melhor avaliado e incluído em estudos futuros de filogenia, pois pode revelar caracteres adicionais importantes para sustentação do grupo.

Apesar de todas as espécies incluídas no clado 148 apresentarem corpo relativamente baixo, ausência de mácula umeral e colorido em vida 
semelhante, os caracteres que sustentam o referido clado são altamente homoplásticos e a proximidade filogenética de $H$. rodwayi com as demais espécies incluídas no grupo deve ser melhor avaliada futuramente.

Dentro do clado 148, o clado menos inclusivo 147 apresenta:

Táxons: Hemigrammus parana, Moenkhausia bonita, Hemigrammus marginatus, Hemigrammus sp. $\mathrm{n}$.

\section{Caracteres:}

-2 (1) - fontanela do frontal retangular (com reversão para 0 - fontanela do frontal triangular em $H$. marginatus);

-19 (0) - órbito-esfenóide sem projeção dirigida ao pteroesfenóide, ou com apenas uma ponta;

-43 (0) - dentes da série externa do pré-maxilar desalihados (com reversão para 0 - dentes alinhados em $H$. marginatus e $H$. sp. n.);

-89 (1) - concavidade na região dorso-posterior do metapterigóide próxima à união com o hiomandibular presente e acentuada (com reversão para 0 - concavidade ausente ou pouco conspícua em $H$. marginatus e $H$. sp. n.);

-92- (0) - projeção óssea ventral do metapterigóide direcionada ao quadrado;

-110 (0) - projeção óssea anterior do ceratobranquial 4 presente, larga e retangular;

Hemigrammus parana, é uma espécie recentemente descrita para a bacia do Alto rio Paraná (Marinho et al., 2008); da mesma forma que $H$. rodwayi, $H$, parana poderia ser encaixado no " $H$. tridens group" de Géry (1977) devido a ausência de mácula umeral e a presença de mancha caudal.

\section{Dentro do clado 148, o clado menos inclusivo 146 apresenta:}

Táxons: Moenkahusia bonita, Hemigrammus marginatus, Hemigrammus sp. n.

\section{Caracteres:}

-10 (0) - asas laterais do mesetmóide com 50\% ou menos de sua superfície encoberta pelo vômer em vista entral, sendo quase inteiramente visualizadas em vista ventral; 
-67 (1) - infra-orbital 1 com comprimento igual ao do infra-orbital 2 (com reversão para 0 - infra-orbital 1 com comprimento menor que 0 infra-orbital 2 em H. sp. n.);

-133 (1) - maioria dos supraneurais com aproximadamente a mesma largura em toda a extensão do osso (caráter polimórfico 0, 1 em H. sp. n.);

-158 (2) - mancha negra em ambos os lobos da nadadeira caudal, transversal, atingindo ou não a ponta dos raios;

Apesar de provavelmente ter aparecido mais de uma vez e independentemente dentro de Characidae, a presença de mancha distribuída transversalmente sobre os lobos da nadadeira caudal aparece como uma sinapomorfia para o clado 146. H. parana, que faz parte do clado 147 não apresenta mancha negra conspícua sobre os lobos da nadadeira caudal, entretanto essa espécie apresenta uma concentração maior de cromatóforos nessa mesma região, o que pode ser mais um indício de relacionamento entre as espécies que compõem o clado 147.

Benine et al. (2004), na descrição de M. bonita, colocam que a espécie é parecida com espécies de Hemigrammus, especialmente no que se refere à forma e tamanho do corpo e pigmentação. Os autores ainda colocam que dentro de Hemigrammus, $M$. bonita é muito semelhante a $H$. marginatus, pois ambas espécies apresentam uma linha negra médio-lateral e a presença de manchas na nadadeira caudal. Benine (2004) e Marinho (2009) também apresentam em suas análises, um clado monofilético que inclui $M$. bonita e $H$. marginatus, como espécies irmãs.

Hemigrammus sp n. é uma espécie provavelmente nova para a bacia do Alto rio Paraná. A espécie é muito semelhante a $H$. marginatus, porém diferenças de colorido na nadadeira caudal sugerem que a espécie esteja sendo erroneamente identificada para a bacia como $H$. marginatus.

\section{Clado 143}

(Figuras 44 e 47)

Táxons: Hemigrammus pulcher, Hemigrammus haraldi

\section{Caracteres:}


-10 (0) - asas laterais do mesetmóide com $50 \%$ ou menos de sua superfície encoberta pelo vômer em vista entral, sendo quase inteiramente visualizadas em vista ventral;

-33 (1) - dentes do maxilar predominantemente curvos ventralmente;

-43 (0) - dentes da série externa do pré-maxilar desalinhados ;

-67 (1) - infra-orbital 1- com comprimento igual ao do infra-orbital 2;

-89 (1) - concavidade na região dorso-posterior do metapterigóide próxima à união com o hiomandibular presente e acentuada;

-120 (0) - postemporal com a região posterior larga afilando bruscamente terminando em ponta;

-126 (2) - ganchos na nadadeira anal presentes, com apenas um único gancho de tamanho médio a grande por raio;

-145 (1) - mancha vermelha no olho presente;

-147 (2) - duas máculas umerais, a primeira começando na altura da $1^{\underline{a}}$ a 3르 escama da linha lateral;

-149 (1) - presença de uma região clara após a mácula umeral ou entre a primeira e a segunda mácula umeral

Adicionalmente, $H$. pulcher e $H$. haraldi apresentam ainda mancha negra deslocada para a parte inferior do pedúnculo caudal (estendendo-se anteriormente apenas em $H$. pulcher); ausência de mancha negra nas nadadeiras e porção superior do pedúnculo caudal com colorido intenso amarelo ou avermelhado em vida.

Hemigrammus pulcher foi descrito por Ladiges (1938) para a região de Tabatinga e lquitos no Perú. Géry (1961) descreveu H. haraldi (para região de São Paulo de Olivença, AM, alto rio Solimões, Brasil), como sendo uma subespécie de $H$. pulcher $(H$. pulcher haraldi) dada a semelhança entre as duas espécies; e separou-as apenas por observar em $H$. haraldi $(=H$. pulcher haraldi): nadadeira dorsal localizada mais posteriormente; pedúnculo caudal mais curto (quase tão alto quanto longo) e mancha negra no pedúnculo caudal não se estendendo anteriormente. Observando material dessas espécies, porém, encontrei em um mesmo lote (do Brasil), espécies tanto com a mancha no pedúnculo caudal estendendo-se anteriormente (característico de $H$. 
pulcher) quanto com a mancha mais curta, não se estendendo anteriormente (característico de $H$. haraldi). Portanto, acho que $H$. pulcher e $H$. haraldi possam ser sinônimas, sendo assim uma revisão taxonômica dessas espécies é necessária, a fim de confirmar sua validade e definir sua real distribuição.

\section{Clado 141}

(Figuras 44 e 47)

Táxons: Hemigrammus arua, Hemigrammus boesemani, Hyphessobrycon diancistrus, Hemigrammus bleheri, Hemigrammus rhodostomus, Petitella georgiae.

\section{Caracteres:}

-6 (1) - ramo superior da linha lateral cefálica do frontal, próximo ao parietal, presente e não se prolongando pelo parietal (com reversão para 0 ramo superior presente e prolongando-se pelo parietal em $H$. bleheri, $H$. rhodostomus e $P$. georgiae e 0, 1- polimórfico em $H$. arua);

-12 (1) - lâmina dorsal do mesetmóide tão larga quanto a porção ventral do osso (com reversão para 0 - lâmina dorsal mais larga que a porção ventral do osso em $H$. diancistrus);

-35 (0) - todas as cúspides dos dentes do maxilar com aproximadamente o mesmo tamanho (com reversão para 1 - uma das cúpides mais desenvolvida que as demais em $P$. georgiae e 9 - indeterminado em $H$. boesemani, $H$. rhodostomus e $H$. diancistrus);

-75 (0) - infra-orbital 4 com as margens anterior e posterior aproximadamente da mesma altura (com reversão para 1 - margem anterior mais alta que a posterior em $H$. rhodostomus e $H$. diancistrus);

-96 (0) - região póstero-ventral do quadardo próxima ou em contato com o metapterigóide (com reversão para 1 - região póstero-ventral distnte do metapterigóide em $H$. rhodostomus e $H$. diancistrus e $0,1-$ polimórfico em $H$. boesemani);

-112 (1) - faringo-branquais 2 e 3 com dentes (com reversão para 2 dentes apenas no faringo-branquial 3 em $H$. boesemani);

Hemigrammus arua é uma das espécies de Hemigrammus mais recentemente descrita (Lima et al., 2009) e é caracterizada pela presença de uma única mácula umeral larga e deslocada posteriormente (começando na altura da $5^{-}-6^{-}$escma da linha lateral), com uma projeção anterior em forma de vírgula. Na descrição da espécie, Lima et al. (2009) discutem que ela possa ser mais relacionada a $H$. stictus, que também apresenta uma mácula umeral única 
e posteriormente deslocada. Tal hipótese, entretanto, não é corroborada pela presente análise, que mostra $H$. arua distantemente localizada de $H$. stictus.

Dentro do clado 141, o clado menos inclusivo 140 apresenta:

Táxons: Hemigrammus boesemani, Hyphessobrycon diancistrus, Hemigrammus bleheri, Hemigrammus rhodostomus, Petitella georgiae.

\section{Caracteres:}

-34 (2) - maioria dos dentes do maxilar cônicos (com reversão para 0 maioria com quatro ou mais cúspides em $H$. bleheri, 1, 2 - polimórfico em $H$. rhodostomus e 9 - indeterminado em $P$. georgiae);

-73 (1) - infra-orbital 2 e 3 com sobreposição pequena (com reversão para 2 - com sobreposição grande em $H$. rhodostomus e $P$. georgiae; 0,1 polimórfico em $H$. boesemani e $H$. diancistrus);

-88 (1) - ectopterigóide não contata o quadrado (com reversão para 0 contata o quadrado em $H$. bleheri e $P$. georgiae; 0, 1-polimórfico em $H$. rhodostomus e 1, 2- polimórfico em $H$. boesemani);

-92 (0) - projeção óssea ventral do metapterigóide direcionada ao quadrado presente (com reversão para 1 - projeção ausente em $H$. diancistrus);

-108 (1) - com ctenii apenas na base dos rastros branquiais (com reversão para 0 - com cteniis distribuídos por todo o rastro em $H$. bleheri, $H$. rhodostomus e $P$. georgiae);

-126 (1) - ganchos na nadadeira anal presentes, com número variável, mas sempre mais que um por raio, geralmente todos os ganchos pequenos, ou um ou dois ganchos de tamanho médio a grande por raio, seguidos por ganchos pequenos em cada raio;

Lima \& Souza (2009), de acordo com a morfologia e distribuição dos ganchos da nadadeira anal, agrupam $H$. rhodostomus, $H$. bleheri e $P$. georgiae, por apresentarem 1 - 3 ganchos de tamanho médio, distribuídos nos 6 - 8 primeiros raios ramificados da anal. Hemigrammus boesemani é colocado em outro grupo, por apresentar 2 ganchos grandes, localizados no último raio simples e no primeiro ramificado da nadadeira anal, seguidos por ganchos menores (Lima \& Souza, 2009). Nas minhas observações, entretanto, os ganchos chamados de grandes por Lima \& Souza (2009) encontrados em $H$. boesemani não são tão claramente maiores que aqueles observados em $H$. rhodostomus, $H$. bleheri e $P$. georgiae; além disso, os ganchos de $H$. bleheri, $H$. rhodostomus e P. georgiae, apesar de serem todos considerados como de 
tamanho médio, em alguns exemplares tendem a diminuir de tamanho quanto mais próximos da margem distal da nadadeira. Weitzman (1977), na descrição de $H$. diancistrus, coloca que os machos maduros apresentam dois grandes ganchos, localizados no último raio simples e primeiro ramificado da nadadeira anal, em observação pessoal, entretanto, pude constatar que alguns exemplares de $H$. diancistrus apresentam pequenas projeções espiniformes, que considerei homólogas a ganchos menores, localizadas posteriormente aos ganchos grandes. Entendo que a condição do tamanho e distribuição dos ganchos encontrada em $H$. boesemani, $H$. diancistrus, $H$. bleheri, $H$. rhodostomus e $P$. georgiae possa ser homóloga, em razão dessas espécies apresentarem mais de um gancho por raio, incluindo ganchos de tamanho médio a grande, seguidos de ganchos menores. A ausência de ganchos no último raio não ramificado de $H$. bleheri, $H$. rhodostomus e $P$. georgia pode ser interpretada como perda secundária para as três espécies (clado 138) e a presença de ganchos em um número maior de raios (6 - 8 primeiros ramificados em $H$. bleheri, $H$. rhodostomus e $P$. georgiae, vs. somente no primeiro ramificado de $H$. boesemani e $H$. diancistrus) pode ser entendida como uma aquisição para aquelas três espécies que juntamente formam um clado monofilético (138, abaixo).

156 (2) - listras negras verticais na base da nadadeira anal, posicionadas nos espaços correspondentes às membranas inter-radiais presentes ao longo de toda a base

Essas listras são bastante conspícuas em $H$. boesemani e pouco conspícuas em $H$. rhodostomus, e a presença delas é uma condição sinapomórfica para o clado 140.

Hemigrammus boesemani e $H$. rhodostomus foram incluídas no " $H$. tridens group" de Géy (1977), que incluía espécies com mancha caudal e sem mácula umeral. Adicionalmente, $H$. bleheri, $H$. diancistrus e $P$. georgiae também apresentam mancha no pedúnculo caudal e não possuem mácula umeral.

Visto que $H$. boesemani, $H$. diancistrus, $H$. rhodostomus e $H$. bleheri não estão proximamente relacionadas à espécie-tipo de Hemigrammus e formam um clado monofilético com $P$. georgiae, única espécie do gênero, proponho que 
as referidas espécies sejam transferidas para Petitella, passando a ser denominadas Petitella boesemani, Petitella diancistrus, Petitella rhodostomus e Petitella bleheri, com $P$. boesemani e $P$. diancistrus formando as espécies mais basais dentro do grupo monofilético.

\section{Dentro do clado 141, o clado menos inclusivo 139 apresenta:}

Táxons: Hyphessobrycon diancistrus, Hemigrammus bleheri, Hemigrammus rhodostomus, Petitella georgiae.

Caracteres:

-19 (0) - órbito-esfenóide sem projeção posterior dirigida ao pteroesfenóide, ou com apenas uma ponta (com reversão para 1 - com uma projeção posterior alongada, dirigida ao pteroesfenóide em $H$. rhodostomus e P. georgiae);

-28 (2) - processo do supraoccipital não ultrapassando a vertical que passa pela porção final do basioccipital;

-39 (0) - maxilar com a região mediana ou posterior mais larga;

-55 (1) - dentário com um a 10 dentes no total $(0,1$ - polimórfico para $H$. rhodostomus);

-71 (1) - infra-orbital 2 retangular, quadrado ou mais arredondado, margens anterior e posterior aproximadamente da mesma altura (com reversão para 0 - infra-orbital 2 triangular em $H$. rhodostomus);

-93 (0) - fenestra na região posterior do metapterigóide aberta posteriormente;

-103 (1) - margem posterior do eixo médio do uro-hial aproximadamente lisa, sem pontas ou reentrâncias;

-145 (1) - mancha vermelha no olho presente (9 - indeterminado em $H$. diancistrus);

-158 (2) - mancha negra em ambos os lobos da nadadeira caudal, transversal, atingindo ou não a ponta dos raios (com reversão para 3 - mancha negra presente em ambos, obliqua e mais restrita à região mediana, sem atingir a ponta dos raios (ponta dos lobos hialina) no clado 138);

Dentro do clado 141, o clado menos inclusivo 138 apresenta:

Táxons: Hemigrammus bleheri, Hemigrammus rhodostomus, Petitella georgiae

\section{Caracteres:}


-4 (1) - metades do frontal se contatam ou sobrepõem após o mesetmóide;

-6 (0) - ramo superior da linha lateral cefálica do frontal, próximo ao parietal presente, prolongando-se totalmente pelo parietal;

-11 (1) - lâmina dorsal do frontal com margens retas ou às vezes levemente côncavas, osso sempre com aspecto retangular, bastante estreito, tão largo ou mais estreito que a fontanela do frontal, deixando uma grande porção do vômer exposta em vista dorsal;

-69 (0) - canal sensorial do infra-orbital 1 presente ao longo de todo 0 osso ou em mais de $50 \%$ de sua extensão; rhodostomus);

-88 (0) - ectopterigóide contata o quadrado (0, 1 - polimórfico em $H$.

-108 (0) - rastros branquiais com ctenii distribuídos por todo o rastro;

-125 (2) - sete a 15 raios ramificados na nadadeira anal;

-133 (1) - maioria dos supraneurais com aproximadamente a mesma largura em toda a extensão do osso $(0,1$ - polimórfico em $H$. rhodostomus e $9-$ indeterminado em $\mathrm{H}$. bleheri);

-142 (1) - colorido da cabeça em vida vermelho intenso, coloração distinta da encontrada no restante do corpo;

O estado 1 do caráter 142 só foi observado para as espécies do clado 138, sendo assumida, portanto como uma sinapomorfia exlusiva para o grupo.

-158 (3) - mancha negra presente em ambos os lobos da nadadeira caudal, obliqua e mais restrita à região mediana, sem atingir a ponta dos raios (ponta dos lobos hialina);

Dentre todas as espécies analisadas o estado 3 do caráter 158 só foi observado para $H$. rhodostomus, $H$. bleheri e $P$. georgiae, aparecendo, portanto, como uma sinapomorfia exclusiva para o grupo.

-159 (2) - mancha negra sobre os raios medianos da nadadeira caudal presente e se prolongando até a ponta dos raios;

Como apresentado acima, alguns dos principais caracteres que sustentam o clado 138 estão relacionados ao padrão de colorido das espécies. Essa hipótese de relacionamento já foi sugerida por Lima \& Souza (2009), que se basearam também na morfologia e distribuição dos ganchos da nadadeira anal. 
A coloração apresentada em vida pelos indivíduos de $H$. bleheri, $H$. rhodostomus e $P$. georgiae, apesar de ainda pouco explorada, revelou caracteres importantes para o esclarecimento do relacionamento filogenético entre as espécies, e certamente uma melhor avaliação desse conjunto potencial de caracteres (como distribuição de manchas brancas e amarelas ao redor das manchas negras da nadadeira caudal) poderão revelar sinapomorfias adicionais para o grupo.

Dentro do clado 141, o clado menos inclusivo 137 apresenta:

Táxons: H. rhodostomus, P. georgiae

Caracteres:

-13 (1) - porção ventral do mesetmóide, abaixo da lâmina dorsal com estreitamento na região médio-anterior;

-19 (1) - órbito-esfenóide com uma projeção posterior alongada, dirigida ao pteroesfenóide;

-35 (1) - dentes do maxilar com uma das cúspides mais desenvolvida que as demais (geralmente a mediana);

-70 (1) - infra-orbitais 1 e 2 em contato ou com sobreposição;

-134 (0) - um ou mais supraneurais apresentando o eixo principal do osso bifurcado (0,1 - polimórfico em $P$. georgiae);

-153 (3) - mancha negra na nadadeira anal presente, pouco conspícua e não oblíqua, restrita aos últimos raios;

Esse estado de caráter é único dentre as espécies analisadas, sendo uma sinapomorfia exclusiva para $H$. rhodostomus e $P$. georgiae

Géry \& Boutiere (1964) na descrição de $P$. georgiae destacam a grande semelhança entre a espécie nova e $H$. rhodostomus, porém optam por descrever um gênero novo para alocar a nova espécie devido às diferenças observadas na morfologia dos dentes do pré-maxilar e dentário, especialmente na presença de uma única série de dentes no pré-maxilar. Entretanto, como poposto por Lima \& Souza (2009) as diferenças observadas nas maxilas e nos dentes de $P$. georgiae (caracteres 42, 1; 50, 0 e 54, 0) aparecem como autapomorfias para a espécie. 


\section{Hemigrammus brevis}

(Figuras 44 e 48)

\section{Caracteres:}

$-2(1)$ - fontanela do frontal retangular;

-5 (1) - fontanela do parietal abaulada e larga;

-24 (0) - processo lateral do esfenóticosobreposto pelo frontal;

-41 (0) - maxilar terminando na vertical que passa posteriormente ao etmóide lateral;

-88 (0) - ectopterigóide contata o quadrado;

-103 (1) - margem posterior do eixo médio do uro-hial aproximadamente lisa, sem pontas ou reentrâncias;

-105 (1) - onze a treze rastros no primeiro ceratobranquial;

-108 (1) - rastros branquais com ctenii apenas na base;

-115 (1) - pós-cleitro 2 aproximadamente o dobro do pós-cleitro 1;

$-134(0)$ - um ou mais supraneurais apresentando o eixo principal do osso bifurcado;

Hemigrammus brevis foi incluída por Géry (1977) no "H. tridens-group", por apresentar uma mancha caudal e uma faixa ou linha longitudinal no corpo e não ter mácula umeral, juntamente com $H$. levis, $H$. hyanuary, $H$. mattei, $H$. tridens, $H$. micropterus, $H$. boesemani, $H$. rodwayi, $H$. rhodostomus e $H$. marginatus. Nos resultados obtidos, $H$. brevis não aparece formando um agrupamento monofilético único com nenhuma das espécies analisadas pertencentes ao "H. tridens-group" de Géry (1977), estando bem distantemente alocada de $H$. levis, $H$. rodwayi e $H$. marginatus. Entretanto, $H$. brevis aparece como a espécie mais basal do clado 136, que inclui também $H$. hyanuary e $H$. tidens, porém nenhuma dessas espécies apresenta maior proximidade filogenética com $H$. brevis do que com outras espécies incluídas no clado. $H$. mattei e $H$. micropterus não foram incluídas na análise e seu relacionamento com $H$. brevis e com as demais espécies de Hemigrammus permanece incerto. 


\section{Hemigrammus hyanuary}

(Figuras 44 e 48)

\section{Caracteres:}

-10 (0) - asas laterais do mesetmóide com 50\% ou menos de sua superfície encoberta pelo vômer; em vista ventral as asas tem uma porção muito grande exposta, sendo quase inteiramente visualizadas;

-16 (0) - ponte óssea que liga o etmóide lateral ao vômer totalmente encoberta pelo frontal, não visível ou com apenas uma pequena porção visível em vista dorsal;

-60 (0) - osso corono-meckeliano exposto em vista medial, com $50 \%$ ou menos de sua superfície encoberta pela cartilagem de Meckel;

-63 (2) - antorbital bastante largo com a porção ventral pouco mais larga que o restante do osso;

-75 (0) - infra-orbital 4 com as margens anterior e posterior aproximadamente da mesma altura;

-99 (0) - abas laterais do uro-hial reduzidas (fundidas com o eixo médio) ou ausentes;

-104 (1) - basi-hial mais retangular, com a região dorsal pouco expandida, igual ou pouco mais larga que a ventral (menos de 2 vezes);

-112 (1) - faringo-branquiais 2 e 3 com dentes;

-125 (2) - nadadeira anal com sete a quinze raios ramificados;

Hemigrammus hyanuary apresenta um padrão de colorido bastante característico, com uma mancha negra sobre os raios medianos da nadadeira caudal (levemente mais deslocada sobre o lobo inferior), que se prolonga anteriormente sobre o pedúnculo caudal (pode ser uma mancha mais curta ou mais projetada anteriormente), ocupando a porção inferior do pedúnculo. Em vida, apresenta uma mancha iridescente acima e a frente da mancha negra do pedúnculo caudal. H. hyanuary foi alocada por Géry (1977) no "H. tridensgroup" (espécies com mancha caudal e sem mácula umeral), juntamente com: $H$. levis, $H$. mattei, $H$. tridens, $H$. micropterus, $H$. boesemani, $H$. rodwayi, $H$. rhodostomus, $H$. marginatus e $H$. brevis. De acordo com os resultados da presente análise $H$. hanuary não aparece proximamente relacionada a nenhuma das espécies analisadas integrantes do " $H$. tridens-group" de Géry (1977). Entretanto, H. hyanuary aparece como a espécie mais basal do clado 
135, que inclui também, $H$. tidens, porém $H$. tridens aparece mais relacionada a outras espécies de Hemigrammus do que a $H$. hyanuary. $H$. mattei e $H$. micropterus não foram incluídas na análise e seu relacionamento com $H$. hyanuary e com as demais espécies de Hemigrammus permanece incerto

\section{Clado 133}

(Figuras 44 e 48)

Táxons: Hemigrammus schmardae, Hemigrammus tridens, Hemigrammus vorderwinkleri.

Caracteres:

-55 (1) - um a dez dentes ao todo no dentário (0, 1 -polimórfico em $H$. schamardae);

-56 (0) - maioria dos dentes do dentário cuspidados após os dentes anteriores maiores;

-89 (1) - concavidade na região dorso-posterior do metapterigóide próxima à união com o hiomandibular presente e acentuada;

-92 (0) - projeção óssea ventral do metapterigóide direcionada ao quadrado presente;

-144 (1) - mancha preta no olho presente como uma faixa vertical (com reversão para 0 - mancha preta ausente ou presente apenas na porção superior do olho em $H$. tridens);

-155 (2) - linha negra paralela à base da nadadeira anal, começando na altura dos primeiros raios (ou raios medianos) e se prolongando longitudinalmente por toda a extensão da nadadeira;

Adicionalmente aos caracteres apresentados acima, as espécies do clado 133 também compartilham o caráter 157, 1 - presença de uma mancha negra no pedúnculo caudal que se prolonga sobre a base da nadadeira caudal, onde ela é alta, estendendo-se dorsal e ventralmente sobre os raios marginais de cada lobo. O caráter 157 (1), entretanto, aparece como sinapomórfico em um nível mais geral, no clado 134, com reversão para 157 (2 - mancha negra no pedúnculo caudal ausente) no clado 131, irmão do clado 133.

Hemigrammus vorderwinkleri e $H$. schmardae não aparecem aqui como espécies irmãs, entretanto, ambas tem diferenciação taxonômica bastante confusa, visto que mostram um padrão de colorido muito semelhante, e 
apresentam caracteres diagnósticos com sobreposição entre elas. Os principais caracteres problemáticos para a diferenciação de $H$. schmardae e $H$. vorderwinkleri são: presença ou ausência e forma da mácula umeral; número de dentes e de cúspides dos dentes do maxilar e número de raios na nadadeira anal. Ellis (in Eigenmann 1918), por exemplo, apresenta para H. schmardae: vinte a vinte e três raios na nadadeira anal; 2 ou 3 pequenos dentes cônicos ou tricuspidados no maxilar; ausência de mancha caudal, e mácula umeral ausente ou pequena e arredondada. Géry (1963), por sua vez, apresenta para H. vorderwinkleri: dezoito raios na nadadeira anal; 2 - 3 dentes tricúspidados no maxilar; uma linha negra na base da nadadeira anal; mácula umeral verticalmente alongada; uma grande mancha negra no pedúnculo caudal e um lobo ventral desenvolvido em ambos os sexos, na região da nadadeira anal. Material de $H$. schmardae (incluindo síntipos) analisado por mim apresenta dezoito a vinte e um raios na nadadeira anal; 2 a 3 dentes com 5 - 6 cúspides no maxilar (dentes grandes, alguns aparentando mais de 6 cúspides); uma mácula umeral (mais ou menos conspícua) verticalmente alongada; mancha negra alta e bastante conspícua no pedúnculo caudal e base da nadadeira caudal; uma listra negra fina paralela à base da anal, e uma mancha negra e vertical no olho. Material examinado por mim de $H$. vorderwinkleri apresenta dezessete a dezoito raios na anal; 1 - 2 dentes tri a pentacuspidados no maxilar; mácula umeral (mais ou menos conspícua) verticalmente alongada ou arredondada; mancha negra vertical no olho; linha negra paralela à base da nadadeira anal pouco conspícua e presente em alguns exemplares e um lobo característico na região da nadadeira anal (só obervado em exemplares que apresentavam ganchos na nadadeira).

Pelo que pude analisar, ambas espécies são muito semelhantes e de difícil diferenciação morfológica, visto que não apresentam caracteres que as separem completamente, dessa forma, fica claro que $H$. schmardae e $H$. vorderwinkleri precisam ser revisadas taxonomicamente a fim de confirmar a validade dos caracteres diagnósticos de ambas espécies e de melhor diferenciá-las.

Dentro do clado 133, o clado menos inclusivo 132 apresenta: Táxons: Hemigrammus tridens, Hemigrammus vorderwinkleri 


\section{Caracteres:}

-81 (2) - infra-orbital 6 sem canal sensorial (ou muito teduzido), restando apenas uma pequena porção óssea (geralmente osso pequeno);

-104 (1) - basi-hial mais retangular, com a região dorsal pouco expandida, igual ou pouco mais larga que a ventral (menos de 2 vezes);

-115 (1) - pós-cleitro 2 aproximadamente o dobro do pós-cleitro 1;

\section{Clado 130}

(Figuras 44 e 48)

Táxons: Hemigrammus bellottii, Hemigrammus orthus, Hyphessobrycon eilyos, Hemigrammus mimus, Microschemobrycon sp.

\section{Caracteres:}

-10 (0) - asas laterais do mesetmóide com $50 \%$ ou menos de sua superfície encoberta pelo vômer; em vista ventral as asas tem uma porção muito grande exposta, sendo quase inteiramente visualizadas (com reversão para 1 - com mais de $50 \%$ de sua superfície encoberta pelo vômer; em vista ventral as asas são quase ou totalmente encobertas em $H$. orthus);

-41 (0) - maxilar terminando na vertical que passa posteriormente ao etmóide lateral (com reversão para 2 - terminando antes da vertical que passa pelo etmóide lateral em $H$. mimus e Microschemobrycon sp);

-81 (2) - infra-orbital 6 sem canal sensorial (ou muito teduzido), restando apenas uma pequena porção óssea, geralmente osso pequeno (com reversão para 1 - com a porção lamelar reduzida, restrito quase que totalmente à porção tubular do canal sensorial cercado apenas por uma lâmina óssea delgada ou com regiões do canal totalmente expostas em $H$. bellottii e $H$. mimus);

-88 (1) - ectopterigóide não contata o quadrado (com reversão par 2 sobrepõe-se ao quadrado em $H$. eilyos);

-108 (2) - rastros branquais sem ctenii (com reversão para 1 - com ctenii apenas na base dos rastros em $H$. mimus, $H$. orthus e $H$. eilyos);

-109 (0) - ctenii em grande concentração (9 - indeterminado em $H$. bellottii e Microschemobrycon sp);

-110 (2) - projeção óssea anterior do ceratobranquial 4 ausente (com reversão para 0 - presente, larga e retangular em $H$. mimus e 9 indeterminado para $H$. eilyos);

-113 (1) - cleitro com a região ventral sem aba desenvolvida (ou muito reduzida), deixando a fenestra formada pelo cleitro e coracóide totalmente visível em vista lateral ou com apenas a região mais dorsal encoberta, 30\% ou menos (com reversão para 0 - com a região ventral formando uma aba que 
encobre parcial (mais de $70 \%$ ) ou totalmente a fenestra formada pelo cleitro e coracóide em H.bellottii);

Dentro do clado 130, o clado menos inclusivo 127 apresenta:

Táxons: Hemigrammus bellotti, Hemigrammus orthus.

\section{Caracteres:}

-24 (0) - processo lateral do esfenótico sobreposto pelo frontal;

-26 (2) - processo posterior do pterótico em vista dorsal presente, porém pouco desenvolvida, não formando uma projeção característica apenas uma protuberância arredondada ou pontiaguda;

-36 (1) - dentes do maxilar consideravelmente menores que os do prémaxilar, geralmente pequenos e estreitos;

-146 (1) - mancha negra nas escamas dorsais presente;

-155 (2) - linha negra paralela à base da nadadeira, começando na altura dos primeiros raios (ou raios medianos) e se prolongando longitudinalmente por toda a extensão da nadadeira;

-156 (1) - listras negras verticais na base da nadadeira anal presentes apenas na altura dos primeiros raios da nadadeira anal

Hemigrammus bellottii é descrita para Tabatinga, Rio Solimões, AM, Brasil, com distribuição nas bacias dos rios Solimões, Negro e Maroni (Lima et al., 2003). H. orthus é descrita para Tukeit, alto rio Essequibo, Guyana, com distribuição registrada para as bacias dos rios Essequibo e baixo Tapajós (Lima et al., 2003). Comparando a redescrição de $H$. bellottii apresentada por Géry (1963) com a descrição original de $H$. orthus de Durbin (1909), pude constatar que a grande maioria das contagens e medidas sugeridas pelos autores são correspondentes entre as referidas espécies. Além disso, a figura de $H$. orthus apresentada em Eigenmann (1918, prancha 21, Figura 1) apresenta o mesmo padrão de colorido daquela apresentada por Géry (1963, figuras 3 e 4; e 1977, pág. 494) para H. bellottii. Géry (1977), Planquette et al. (1996) e Lima et al. (2003) já colocam que $H$. orthus provavelmente seja sinônimo de $H$. bellottii. As duas espécies apresentam exatamente o mesmo padrão de colorido (não só observado como também colocado nas descrições originais e redescrições): mácula umeral verticalmente alongada (às vezes não muito conspícua); linha negra lateral no corpo, começando aproximadamente na altura do início da 
nadadeira dorsal, ou posterior e se prolongando até o pedúnculo caudal; manchas negras aproximadamente arredondadas sobre as escamas da região dorsal; e o que é mais característico, uma linha negra paralela à base da nadadeira anal, começando na altura dos primeiros raios $\left(^{\circ}{ }^{\circ} 4^{\circ}\right.$ aproximadamente) e se prolongando por toda a extensão da base da nadadeira; e presença de listras negras verticais na base da nadadeira anal, apenas sobre os primeiros raios.

Para o presente trabalho, eu examinei lotes de $H$. bellottii (síntipos e não tipos) da bacia do rio Negro e um lote de H. orthus da bacia do Tapajós. O material de $H$. orthus, entretanto, é composto por exemplares bastante pequenos (em torno de $20 \mathrm{~mm}$ de comprimento padrão, ou menos), porém apresentam o padrão de colorido característico da espécie, que é exatamente igual ao de $H$. bellottii. Sendo assim, acredito que o material de $H$. orhtus, proveniente da bacia do rio Tapajós, examinado por mim corresponda na verdade a exemplares (talvez juvenis) de $H$. bellottii e acredito que um exame dos tipos de ambas espécies confirmará a hipótese de Géry (1977), Planquette et al. (1996) e Lima et al. (2003) de que $H$. bellottii e $H$. orthus são na verdade uma única espécie.

\section{Dentro do clado 130, o clado menos inclusivo 129 apresenta:}

Táxons: Hyphessobrycon eilyos, Hemigrammus mimus, Microschemobrycon sp.

\section{Caracteres:}

-12 (1) - lâmina dorsal do mesetmóide tão larga quanto a porção ventral do osso;

-19 (0) - orbito-esfenóide sem projeção posterior dirigida ao pteroesfenóide, ou com apenas uma ponta;

-39 (0) - maxilar com a região mediana ou posterior mais larga;

-45 (0) - seis ou mais dentes na série interna do pré-maxilar;

-46 (2) - dentes da série interna do pré-maxilar cônicos;

-53 (0) - seis ou mais dentes anteriores e mais largos do dentário (9 inderterminado para $H$. mimus e Microschemobrycon); 
-54 (0) - dentes anteriores do dentário estreitos, geralmente com três cúspides, semelhantes aos do pré-maxilar ( 9 - inderterminado para $H$. mimus e Microschemobrycon);

-77 (1) - infra-orbital 4 mais baixo que a metade do infra-orbital 5 (com reversão para 0 - infraorbital 4 tão alto quanto o infra-orbital 5 ou maior que a metade dele em Microschemobrycon sp);

-145 (0) - mancha vermelha no olho ausente $(9$ - indeterminado em H. mimus e Microschemobrycon sp);

-147 (0) - mácula umeral ausente;

-150 (0) - listra negra longitudinal no corpo ausente;

Dentro do clado 130, o clado menos inclusivo 128 apresenta:

Táxons: Hemigrammus mimus, Microschemobrycon sp.

\section{Caracteres:}

$-2(0)$ - fontanela do frontal triangular;

-4 (1) - metades do frontal se contatam ou sobrepõem após o mesetmóide (com reversão para 0 - metades não se contatam ou sobrepõem após o mesetmóide em Microschemobrycon sp);

-6 (0) - ramo superior da linha lateral cefálica do frontal, próximo ao parietal presente e prolongando-se totalmente pelo parietal; estreitas;

-8 (0) - asas laterais do mesetmóide em vista dorsal retangulares e

-14 (1) - região posterior do mesetmóide terminando antes ou aproximadamente na mesma altura da região de inserção do etmóide lateral (com reversão para 0 - região posterior do mesetmóide terminando apóa a região de inserção do etmóide lateral em Microschemobrycon sp);

-16 (0) - ponte óssea que liga o etmóide lateral ao vômer totalmente encoberta pelo frontal, não visível ou com apenas uma pequena porção visível em vista dorsal;

-22 (0) - paresfenóide retilíneo em toda a sua extensão; lateral;

-41 (2) - maxilar terminando antes da vertical que passa pelo etmóide

-50 (0) - região anterior do dentário com declividade acentuada deixando a região anterior bem mais afilada que a posterior; 
-52 (0) - dentes do dentário diminuindo gradualmente de tamanho em sentido posterior;

-63 (0) - antorbital alongado, com a largura não mudando muito ao longo do comprimento (osso pode ser estreito ou largo);

-76 (2) - infra-orbital 4 aproximadamente tão longo quanto alto (com reversão para 0 - mais alto que longo em $H$. mimus);

-83 (2) - palatino largo, com a porção anterior bem mais larga que o restante do osso;

-84 (0) - palatino com ambas as margens laterais aproximadamente retas ou convexas;

-98 (0) - canal latero-sensorial do pré-opérculo próximo ou atingindo a margem dorsal formada pelo hiomandibular e o opérculo;

-118 (1) - fenestra entre o coracóide e o cleitro reduzida, quase fechada, ossos quase completamente em contato ao longo da extensão;

Essa condição aparece como uma sinapomorfia exclusiva para as duas espécies do clado 128.

-125 (2) - sete a quinze raios ramificados na nadadeira anal;

-165 (1) - pseudotímpano entre a $1^{\underline{a}}$ e $2^{\underline{a}}$ costelas presente;

Adicionalmente aos caracteres apresentados acima, $H$. mimus apresenta um padrão de colorido muito semelhante ao de espécies de Microschemobrycon, especialmente em relação à presença de uma pequena macha negra na base de cada lobo da nadadeira caudal. A forma geral do corpo de $H$. mimus (bastante baixo e alongado) também é muito semelhante ao de espécies de Microschemobrycon. Böhlke (1955), na descrição de H. mimus reconhece a gande semelhança entre a espécie e espécies de Microschemobrycon do rio Negro, no que diz respeito a forma geral do corpo e padrão de colorido, notadamente em relação a $M$. callops e $M$. casiquiare. Böhlke (1955), entretanto, opta por descrever a nova espécie em Hemigrammus unicamente por ela apresentar duas séries de dentes no prémaxilar, ao contrário das espécies de Microschemobrycon que apresentam apenas uma. Géry (1963 e 1977) inclusive, coloca H. mimus como uma espécie mimética de Microschemobrycon (o nome $H$. mimus foi dado por 
Böhlke (1955) em alusão ao seu mimetismo/semelhança com Microschemobrycon).

Frente a todo o exposto, aos caracteres sinapomórficos compatilhados por $H$. mimus e Microschemobrycon sp e a indiscutível semelhança entre as duas espécies, é provável que $H$. mimus, na realidade pertença a Microschemobrycon, fato que deve ser comprovado com a inserção futura da espécie-tipo de Microschemobrycon na análise.

\section{Hemigrammus yinyang}

(Figuras 44 e 48)

\section{Caracteres:}

-36 (1) - dentes do maxilar consideravelmente menores que os do prémaxilar, geralmente pequenos e estreitos;

-41 (0) - maxilar terminando na vertical que passa posteriormente ao etmóide lateral;

-46 (3) - dentes da série interna do pré-maxilar tricuspidados;

-96 (1) - região póstero-ventral do quadrado distante do metapterigóide;

-108 (0) - rastros branquiais com ctenii distribuídos por todo o rastro;

-126 (2) - ganchos na nadadadeira anal presentes, com apenas um único gancho de tamanho médio a grande por raio; presentes;

-143 (1) - cromatóforos expandidos concentrados sobre o infra-orbital 3

-147 (2) - duas máculas umerais, a primeira começando na altura da $1^{\text {a }}$ a 3ํㅡㄹ escama da linha lateral; umeral;

-149 (1) - região clara presente entre a primeira e a segunda mácula

Hemigrammus yinyang é uma das espécies de Hemigrammus descritas recentemente (Lima \& Souza, 2009), e é caracterizada pela presença de uma mancha alaranjada (em exemplares vivos), localizada entre a primeira e a segunda máculas umerais negras.

Lima \& Souza (2009), sugerem a inclusão de $H$. yinyang em um grupo denominado "Hemigrammus ocellifer species-group", juntamente com $H$. pulcher, $H$. haraldi, $H$. guyanensis, $H$. ocellifer, $H$. neptunus e $H$. luelingi devido 
a presença de apenas um gancho de tamanho médio a grande nos raios da nadadeira anal, duas máculas umerais, mancha negra no pedúnculo caudal (exceto em $H$. yinyang) e mancha vermelha na porção superior do olho em exemplares vivos. Hemigrammus yinyang, entretanto não aparece mais proximamente relacionada a nenhumas das espécies sugeridas pelos autores como integrantes do "Hemigrammus ocellifer species-group" analisadas, e os caracteres sugeridos por Lima \& Souza (2009) como sustentando o monofiletismo do grupo aparecem como tendo surgido independetemente mais de uma vez dentro de Characidae.

\section{Clado 120}

(Figuras 44 e 49)

Táxons: Hemigrammus newboldi, Hemigrammus cylindricus, Hemigrammus skolioplatus.

\section{Caracteres:}

-18 (1) - orbito-esfenóide com concavidade pouco característica, formando uma região levemente côncava;

-24 (0) - processo lateral do esfenótico sobreposto pelo frontal (com reversão para 1 - apenas sobreposto pelo frontal em $H$. skolioplatus);

-28 (2) - processo do supraoccipital não ultrapassando a vertical que passa pela porção final do basioccipital;

-43 (0) - dentes da série externa do pré-maxilar desalinhados (com reversão para 1 - alinhados em $H$. cylindircus);

-75 (0) - infra-orbital 4 com as margens anterior e posterior aproximadamente da mesma altura ( 9 - indeterminado para $H$. skolioplatus);

-151 (1) - listra negra longitudinal no corpo mais larga, formada por várias séries de cromatóforos, geralmente bastante conspícua (com reversão para 0 - fina, apenas uma linha bastante fina, como uma única série de cromatóforos às vezes pouco conspícua em $\mathrm{H}$. cylindricus);

-159 (2) - Mancha negra sobre os raios medianos da nadadeira caudal presente, se prolongando até a ponta dos raios (com reversão para 0 - mancha sobre os raios medianos da nadadeira caudal ausente em $H$. cylindricus);

Hemigrammus newboldi, segundo Taphorn (1992) é uma espécie muito parecida com outras de Moenkhausia, notadamente M. cotinho, especialmente por ambas apresentarem uma mancha bastante alta e conspícua no pedúnculo 
caudal (envolvendo-o por completo). Eigenmann (1918) e Géry (1977) colocam que $M$. cotinho apresenta uma série irregular de escamas perfuradas na linha lateral, indo desde uma série incompleta de escamas até uma série completa. Frente a isso e à semelhança da mancha negra caudal, Taphorn (1992 e 2003) sugere que $H$. newboldi e $M$. cotinho são espécies muito próximas, podendo ser sinônimas. Moenkhausia cotinho não foi avaliada no presente trabalho e seu relacionamento com $H$. newboldi permanece incerto.

Costa (1994) descreve Moenkhausia pyrophthalma, uma espécie que apresenta linha lateral incompleta e discute outras espécies dentro do gênero como a mesma condição. O autor justifica a alocação de M. pyrophthalma em Moenkhausia por hipotetizar que ela forme um grupo monofilético com outras espécies do gênero, como $M$. sanctaefilomenae e M. oligolepis (que também apresentam populações com linha lateral incompleta), devido à presença de um padrão de colorido reticulado no corpo, de uma mancha (barra) negra conspícua no pedúnculo caudal, precedida por uma área clara e pela presença de pigmentação vermelha na porção superior do olho (Costa, 1994). Moenkahusia cotinho não é incluída por Costa (1994) no grupo acima descrito, por não apresentar padrão reticulado no corpo e por diferenças encontradas por ele na forma da mancha do pedúnculo caudal, entretanto, $M$. cotinho apresenta a mancha negra conspícua no pedúnculo caudal e uma região mais clara no pedúnculo que precede a mancha negra. Hemigrammus newboldi não apresenta padrão reticulado no corpo, porém, apresenta uma mancha negra conspícua e bastante alta no pedúnculo caudal e base da nadadeira, e também apresenta uma área mais clara, anterior à mancha do pedúnculo caudal. Dentre as espécies sugeridas por Costa (1994) como formando um grupo monofilético em Moenkhausia, M. sanctaefilomenae foi a única aqui analisada, não aparecendo, entretanto mais proximamente relacionada a $H$. newboldi.

Penso que uma análise filogenética que inclua os táxons e caracteres sugeridos por Taphorn (1992) e Costa (1994) possa trazer luz ao relacionamento sugerido pelos referidos autores e esclarecer a distribuição, dentro de Characidae, dos estados de caracteres sugeridos por eles.

Dentro do clado 120, o clado menos inclusivo 119 apresenta:

Táxons: Hemigrammus cylindricus, Hemigrammus skolioplatus. 


\section{Caracteres:}

-4 (1) - metades do frontal se contatam ou sobrepõem após o mesetmóide;

-10 (0) - asas laterais do mesetmóide com 50\% ou menos de sua superfície encoberta pelo vômer; em vista ventral as asas tem uma porção muito grande exposta, sendo quase inteiramente visualizadas;

-16 (0) - ponte óssea que liga o etmóide lateral ao vômer totalmente encoberta pelo frontal, não visível ou com apenas uma pequena porção visível em vista dorsal;

-37 (2) - quarto a dez dentes no maxilar;

-45 (0) - seis ou mais dentes na série interna do pré-maxilar $(0,1$ polimórfico em H. skolioplatus);

-58 (0) - articulação entre o quadrado e o ângulo articular na vertical que passa pelo meio da órbita ou posteriormente a ela;

-76 (2) - infra-orbital 4 aproximadamente tão longo quanto alto (9 indeterminado);

-88 (2) - ectopterigóide sobrepõe-se ao quadrado (9 - indeterminado em H. skolioplatus);

-89 (0) - concavidade na região dorso-posterior do metapterigóide próxima à união com o hiomandibular ausente ou pouco conspícua;

-90 (1) - projeção ântero-dorsal do metapterigóide presente e pouco desenvolvida;

-93 (0) - fenestra na região posterior do metapterigóide aberta posteriormente;

-110 (2) - projeção óssea anterior do ceratobranquial 4 ausente;

Hemigrammus cylindricus foi incluída por Géry (1977) no " $H$. bellottiigroup", juntamente com $H$. stictus, $H$. analis, $H$. bellottii, $H$. microstomus, $H$. lunatus, $H$. aereus e $H$. cupreus, por apresentar mácula umeral e ausência de mancha caudal. Dentre as espécies analisadas, entretanto $H$. cylindricus não aparece mais proximamente relacionada a nenhuma outra integrante do " $H$. bellottii-group" de Géry (1977).

Hemigrammus skolioplatus é distinta de todas as demais espécies incluídas em Hemigrammus por apresentar um padrão único de colorido, 
formado por uma faixa longitudinal negra e curva, estendendo-se desde a mácula umeral verticalmente alongada, até porção inferior da base da nadadeira caudal (Bertaco \& Carvalho, 2005).

\section{Hemigrammus taphorni}

(Figuras 44 e 49)

\section{Caracteres:}

-10 (0) - asas laterais do mesetmóide em vista ventral com $50 \%$ ou menos de sua superfície encoberta pelo vômer; em vista ventral as asas tem uma porção muito grande exposta, sendo quase inteiramente visualizadas;

-18 (2) - orbito-esfenóide com concavidade próximo à união com o pteroesfenóide bastante acentuada, formando uma região com uma forte invaginação;

-29 (1) - processo do supraoccipital muito inclinado dorsalmente em relação ao eixo longitudinal do crânio;

-43 (0) - dentes da série externa do pré-maxilar desalinhados;

-58 (0) - articulação entre o quadrado e o dentário na vertical que passa pelo meio da órbita ou posteriormente a ela;

-68 (2) - margem posterior do infra-orbital 1 maior que a margem anterior do infra-orbital 2;

-70 (0) - infra-orbitais 1 e 2 sem contato ou sobreposição;

-73 (0) - infra-orbitais 2 e 3 sem sobreposição;

-77 (1) - infra-orbital 4 mais baixo que a metade do infra-orbital 5;

-89 (0) - concavidade na região dorso-posterior do metapterigóide próxima à união com o hiomandibular ausente ou pouco conspícua;

-90 (0) - projeção ântero-dorsal do metapterigóide ausente;

-120 (0) - postemporal região posterior larga afilando bruscamente terminando em ponta;

-140 (1) - duas a seis escamas sore os raios da nadadeira anal;

-157 (2) - mancha negra no pedúnculo caudal ausente;

Hemigrammus taphorni foi recentemente descrita (Benine \& Lopes, 2007), e seus autores propõem que a espécie esteja mais relacionada, dentro 
de Hemigrammus a $H$. elegans devido ao seu padrão de colorido, sugerindo também que $H$. taphorni e $H$. elegans estejam mais proximamente relacionadas com o grupo dos "rosy tetra". Da mesma forma que $H$. elegans, $H$. taphorni apresenta cromatóforos mais concentrados na nadadeira dorsal, formando uma área mais escurecida, porém não é uma mancha negra conspícua e delimitada como aquela apresentada pelas espécies incluídas no clado 161 ("Hemigrammus"). Hemigrammus taphorni, de acordo com os resultados aqui apresentados não está proximamente relacionado ao grupo dos "rosy tetra", que agora é parte integrante de "Hemigrammus". Hemigrammus elegans não foi aqui avaliada, porém, apesar de ser tradicionalmente reconhecida como tendo uma mancha negra na nadadeira dorsal, não apresenta mancha homóloga a das espécies de "Hemigrammus". A posição filogenética de $H$. elegans, espécie não analisada, entretanto, permanece incerta e seu possível relacionamento com $H$. taphorni precisa ser melhor investigado.

\section{Hemigrammus pretoensis}

(Figuras 44 e 49)

\section{Caracteres:}

-10 (0) - asas laterais do mesetmóide em vista ventral com 50\% ou menos de sua superfície encoberta pelo vômer; em vista ventral as asas tem uma porção muito grande exposta, sendo quase inteiramente visualizadas;

-26 (2) - processo posterior do pterótico em vista dorsal presente, porém pouco desenvolvida, não formando uma projeção característica apenas uma protuberância arredondada ou pontiaguda;

-37 (2) - quatro a dez dentes no maxilar;

-104 (0) - basi-hial acentuadamente triangular, com a região anterior expandida, duas ou mais vezes mais larga que a posterior;

-120 (0) - postemporal com a região posterior larga afilando bruscamente terminando em ponta;

-124 (0) - nadadeira anal com primeiros raios pouco maiores que os posteriores, formando um lobo arredondado, raios diminuindo de tamanho suavemente em sentido posterior, nadadeira com concavidade na região mediana;

-133 (1) - maioria dos supraneurais com aproximadamente a mesma largura em toda a extensão do osso; 
$-150(0)$ - listra negra longitudinal no corpo ausente;

-159 (2) - mancha negra sobre os raios medianos da nadadeira caudal presente, se prolongando até a ponta dos raios;

Hemigrammus pretoensis é uma das espécies de Hemigrammus com indivíduos que atingem o maior porte (se não o maior) dentro do gênero, tanto em relação ao comprimento quanto a altura do corpo. $H$. pretoensis foi sugerida por Géry (1977) como integrante do "H. ocellifer group", que incluía espécies com uma ou duas máculas umerais e uma mancha caudal; e dentro desse grupo ainda, $H$. pretoensis foi colocada junto com as espécies que apresentavam duas máculas umerais: $H$. luelingi, $H$. ocellifer e $H$. pulcher. Géry (1965b), na descrição de $H$. pretoensis coloca que a espécie é muito semelhante a $H$. ocellifer; na presente análise, entretanto, $H$. pretoensis aparece mais proximamente relacionada a espécies de outros gêneros de Characidae que também apresentam corpo mais alto do que à espécies de Hemigrammus.

Lima \& Toledo-Piza (2001) descrevem Moenkhausia diktyota, uma espécie que apresenta linha lateral incompleta, padrão reticulado de colorido do corpo, duas máculas umerais, e mancha negra no pedúnculo caudal longitudinalmene alongada e se prolongando sobre os raios medianos da nadadeira caudal. Os autores sugerem que a espécie possa estar proximamente relacionada a $M$. oligolepis, $M$. sanctaefilomenae e $M$. pyrophthalma, devido à semelhança no padrão de colorido do corpo, grupo sugerido por Costa (1994) como provavelmente monofilético. H. pretoensis é muito semelhante a $H$. diktyota no que se refere ao corpo alto, presença de duas máculas umerais, e de mancha negra no pedúnculo caudal alongada horizontalmente e prolongando-se sobre os raios medianos da nadadeira caudal. Adicionalmente as duas espécies apresentam linha lateral incompleta, entretanto, $H$. pretoensis não apresenta o padrão de colorido reticulado do corpo como o de $H$. diktyota e das outras espécies sugeridas por Costa (1994) como proximamente relacionadas. Moenkhausia diktyota não foi incluída na presente análise e sua relação com $H$. pretoensis permanece incerta, entretando, penso, que $H$. pretoensis possa estar realmente mais relacionada com outras espécies de Characidae que apresentem porte maior e corpo alto 
do que às demais espécies de Hemigrammus e acho que para uma análise mais adequada do relacionamento de $H$. pretoensis é importante que na análise sejam incluídas $M$. diktyota, $M$. oligolepis, $M$. sanctaefilomenae e $M$. pyrophthalma. 


\section{Consideracõos Finais}

A análise filogenética aqui apresentada é bem sucedia em mostrar filogeneticamente que o gênero Hemigrammus, como vinha sendo tradicionalmente reconhecido não constitui uma unidade monofilética. De acordo com os resultados obtidos, Hemigrammus hoje ficaria restrito à $H$. unilineatus, espécie-tipo do gênero, mais Moenkhausia hemigrammoides, Pristella maxillaris, Hyphessobrycon bentosi, H. compressus, H. megalopterus, $H$. micropterus, $H$. epicharis, $H$. eques, $H$. erythrostigma e $H$. takasei. O gênero Hemigrammus, de acordo com a análise filogenética apresentada, tem como sinapomorfia exclusiva a presença de uma mancha negra conspícua e delimitada na região mediana da nadadeira dorsal, com distribuição horizontal na nadadeira. De acordo com a nova conformação do gênero, tanto Hyphessobrycon quanto Pristella por serem nomes mais recentemente disponibilizados em literatura, são propostos como sinônimos sênior de Hemigrammus.

Os resultados aqui apresentados também mostram que agrupamentos tradicionais, baseados especialmente na combinação de presença ou ausência de manchas negras pelo corpo (região umeral, pedúnculo caudal, nadadeiras dorsal e caudal) como o proposto por Géry (1977) são meras conveniências, e não encontram sustentação filogenética. Entretanto, alguns dos caracteres mais importantes para a sustentação dos agrupamentos formados na análise trantam do padrão de colorido das espécies (tanto colorido em álcool quanto em vida), o que mostra a importância da utilização de caracteres de padrão de colorido em um contesto filogenético, juntamente com outros conjuntos de caracteres (no caso osteológicos e morfológicos).

Embora a presente análise possa não ter esclarecido de forma satisfatória todos os aspectos relacionados ao relacionamento filogenético de algumas das espécies que tradicionalmente compunham o gênero, para alguns agrupamentos de espécies que agora não se encaixam na nova concepção de Hemigrammus, informações importantes são disponibilizadas para a condução de novas avaliações. Estudos futuros mais abrangentes, que incluam um universo maior de caracteres, de táxons de Characidae, além das demais espécies válidas de Hemigrammus são necessários para solucionar os 
problemas de realocação e relacionamento filogenético das espécies de Hemigrammus e Hyphessobrycon que não se enquadram na definição de "Hemigrammus" no sensu atual. 


\section{REFERÊNCIAS BIBLIOGRÁFICAS}

Amorim, D.S. 1997. Elementos Básicos de Sistemática Filogenética. São Paulo, Holos e Sociedade Brasileira de Entomologia, 276p.

Benine, R.C. 2004. Análise Filogenética do gênero Moenkhausia Eigenmann, 1903 (Characiformes: Characidae) com a revisão dos táxons do alto Rio Paraná. Tese de Doutorado, Universidade Estadual Paulista/UNESP, Botucatu, 317p.

Benine, R.C.; R.M.C. Castro \& J. Sabino. 2004. Moenkhausia bonita: A New Small Characin Fish from the Rio Paraguay Basin, Southwestern Brazil (Characiformes: Charcidae). Copeia, 2004(1): 68-73.

Benine, R.C. \& G.A.M. Lopes. 2007. A new species of Hemigrammus Gill, 1858 (Characiformes: Characidae) from Rio Caura, Venezuela. Zootaxa, 1610: 53-59.

Bertaco, 2008. Taxonomy and Phylogeny of the Neotropical fish genus Hemibrycon Günther, 1864 (Ostariophysi: Characiformes: Characidae). Tese de Doutorado não publicada, PUC-RS, Porto Alegre, 298p.

Bertaco, V.A. \& T.P. Carvalho. 2005. New Characid fish, Hemigrammus skolioplatus (Characiformes: Characidae) from upper rio Tapajós drainage, Central Brazil. Comunicações do Mususeu de Ciência e Tecnologia da PUCRS, Série Zoologia, Porto Alegre, 18 (2): 141-150.

Bertaco, V.A. \& L.R. Malabarba. 2005. A new species of Hyphessobrycon (Teleostei: Characidae) from the upper rio Tocantins drainage, with bony hooks on fins. Neotropical Ichthyology, 3: 83-88.

Böhlke, J. 1955. Studies on Fishes of the Family Characidae $-\mathrm{N}^{\circ} 8$. The Description of a New Hemigrammus from the Rio Negro of Brazil. Transaction Kansas Academy of Science, 58(1): 229-236. 
Britski, H.A. \& F.C.T. Lima. 2008. A new species of Hemigrammus form the Upper rio Tapajós basin in Brazil (Teleostei: Characiformes: Characidae). Copeia, 2008(3): 565-569.

Britski, H. A.; K. Z. S. Silimon \& B. S. Lopes. 2007. Peixes do Pantanal: Manual de identificação. Brasília, Embrapa, 2ª ed., 227p.

Buckup, P.A. 1993. The monophyly of the Characidiinae, a Neotropical group pf characiforms fishes (Teleostei: Ostariophysi). Zoological Journal of the Linnean Society, 108: 225-245.

Buckup, P.A. 1998. Relationships of the Characidiinae and Phylogeny of Characiform Fishes (Teleostei: Ostariophysi). In: Malabarba, L.R.; Reis, R.E.; Vari, R.P., Lucena, Z.M. \& Lucena, C.A.S. (eds.). Phylogeny and Classification of Neotropical Fishes. Porto Alegre, Edipucrs, 603p.

Calcagnotto, D.; S.A. Schaffer \& R. Desalle. 2005. Relationships among characiform fishes inferred from analysis of nuclear and mitochoncdrial gene sequences. Molecular Phylogenetics and Evolution, 36: 135-153.

Castro, R.M.C. \& M.C. Castro, 1987. Proposta de uma nomenclatura osteológica para Characiformes (Pisces, Ostariophysi). Boletim do Museu Paraense Emilio Goeldi, Série Zoologia, 3(1): 25 - 32.

Costa, W.J.E.M. 1994. Description of two new species of the genus Moenkhausia (Characiformes: Characidae) from the central Brazil. Zoologischer Anzeiger, 232(1-2): 21-29.

Durbin, M.L. 1909. Reports on the expedition to British Guiana of the Indiana University and the Carnegie Museum, 1908. Report $n \div 2$. A new genus and twelve new species of tetragonopterid characins. Annals of the Carnegie Museum, 6(1): 55-72.

Eigenmann, C.H. 1908. Preliminary description of new genera and species of tetragonopterinae characins. (Zoölogical Results of the Thayer Brazilian Expedition). Bulletin: Museum of Comparative Zoölogy, 52(6): 91-106. 
Eigenmann, C.H. 1917. The American Characidae. Memoirs of the Museum of Comparative Zoölogy at Harvard College, 43(Part. 1): 102.

Eigenmann, C.H. 1918. The American Characidae. Memoirs of the Museum of Comparative Zoölogy at Harvard College, 43(Part. 1): 103-208.

Eigenmann, C.H. \& R.S. Eigenmann. 1891. A catalogue of the fresh-water fishes of South America. Proceedings of the United States National Museum, 14(842): 1-81.

Eigenmann, C.H. \& A.W. Henn. 1914. On new species of fishes from Colombia, Ecuador, and Brazil. Contributions from the Zoölogical Laboratory of Indiana University, 24: 231-234.

Eigenmann, C.H. \& F. Ogle. 1907. An annoted list of characin fishes in the United States National Museum and the Museum of Indiana university, with descriptions of new species. Proceedings of the United States National Mususeum, 33(1556): 1-36.

Ellis, M.D. 1911. On the species of Hasemania, Hyphessobrycon, and Hemigrammus collected by J. D. Haseman for the Carnegie Museum. Annals of the Carnegie Museum, 8(2): 148-163

Ferreira, K.M. 2007. Análise Filogenética e revisão taxonômica do gênero Knodus Eigenmann, 1911 (Characiformes: Characidae). Tese de Doutorado não publicada, Universidade de São Paulo/USP, Ribeirão Preto, $549 p$.

Fink, S.V. \& W.L. Fink. 1981. Interrelationships of the Ostariophysan fishes (Teleostei). Zoological Journal of the Linnean Society, London, 72: 297-353.

Fink, S.V. \& W.L. Fink. 1996. Interrelationships of Ostariophysan fishes (Teleostei). In: Interrelationships of Fishes, San Diego, California, 496: 209249. 
Fink, P.L. \& S.H. Weitzman. 1974. The so-called cheirodontin fishes of Central America with descriptions of two new species (Pisces: Characidae). Smithsonian Contributions to Zoology, 172:46.

Géry, J. 1959. Contributions a L'Etude des Poissons Characoides (Ostariophysi). (IV). Nouvelles espèces de Guyane Française du genre Hemigrammus (Tetragonopterinae) avec une liste critique des formes recensees. Bulletin Mensuel de la Société Linnéenne de Lyon, 28(8): 248260.

Géry, J. 1961. Hemigrammus pulcher haraldi a new subspecies of a well-known aquarium Tetra. Tropical Fish Hobbyist, 10: 42-51.

Géry, J. 1963. Three new Tetras from the upper Rio Negro near Tapurucuara. Tropical Fish Hobbyist, 12(3): 9-15, 57-63.

Géry, J. 1964a. Poissons characoïdes de l'Amazonie péruvienne. Beiträge zur Neotropischen Fauna, 4(1): 1-44.

Géry, J. 1964b. Preliminary description of seven new species and two new genera of characoid fishes from the Upper Rio Meta in Colombia. Tropical Fish Hobbyist, 13(4): 25-32, 41-48.

Géry, J. 1965a. Notes on characoid fishes collected in Surinam by Mr. H. P. Pijpers, with description of new forms. Bijdragen tot de Dierkunde, 35: 101126.

Géry, J. 1965b. Poissons characoïdes sud-américains du Senckenberg Muséum, II. Characidae et Crenuchidae de l'Igarapé Preto (Haute Amazonie). Senckenbergiana Biologica, 46(1): 11-45.

Géry, J., 1977. Characoids of the World. T. F. H. Publications, Neptune City, New Jersey, 672p.

Géry, J. \& H. Boutière. 1964. Petitella georgiae gen. et sp. Nov. (Pisces, Cypriniformes, Characoidei). Vie et Milieu Supplement, 17: 473-484. 
Géry, J. \& V. Mahnert. 1986. A new rummy-nose tetr from the Rio Negro, Brazil: Hemigrammus bleheri n. sp (Characidae, Tetragonopterinae). With comments on Paracheirodon. Tropical Fish Hobbyist, 34(11): 37, 40-41, 44-45, 48-49, 52.

Gill, T.N. 1858. Syinopsis of the fresh water fishes of the western portion of the island of Trinidad, W.I. Annas of the Lycium of Natural History of New York, 6(10-13): 363-430.

Gilbert, C. H. 1900. Results of the Branner-Agassiz Expedition to Brazil. Proceedings of the Washington Academy of Sciences, 161-184.

Günther, A. 1864. Catalogue of the fishes in the British Museum. Vol. 5. Catalogue of the Physostomi, containing the families Siluridae, Characinidae, Haplochitonidae, Sternoptychidae, Scopelidae, Stomiatidae in the collection of the British Museum. London, xxii + 455p.

Hennig, W. 1966. Phylogenetic systematic. University of Illinois Press, Urbana, III, 263p.

Hennig, W. 1968. Elementos de una sistemática filogenética. Eudeba Editorial Universitaria de Buenos Aires, 353p.

ICZN (International Commission on Zoological Nomenclature). 1999. International code of zoological nomenclature adopted by the International Union of Biological Sciences. International Trust for Zoological Nomenclature, London, XXIX + 306p.

Javonillo, R.; L.R. Malabarba; S.H. Weitzman \& J.R. Burns. 2010. Relationships among major lineages pf characid fishes (Teleostei: Ostariophysi: Characiformes), based on molecular sequence data. Molecular Phylogenetics and Evolution, 54: 498-511.

Kitching, I.J.; P.L. Forey; C.J. Humphries \& D.M. Williams. 2000. Cladistics. The theory and practice of parsimony analysis. The Systematics Association Publication, $n^{\circ}$ 11, Oxford University Press, 228p. 
Ladiges, W. 1938. Drei neue Fische der Gattungen Hyphessobrycon und Hemigrammus aus dem peruanischen Teil des Amazonas. Zoologischer Anzeiger, 124(3-4): 49-52.

Langeani, F. 1998. Phylogenetic study of the Hemiodontidae (Ostariophysi: Characiformes). Pp. 145-160. In: Malabarba, L.R.; Reis, R.E.; Vari, R.P., Lucena, Z.M. \& Lucena, C.A.S. (eds.). Phylogeny and Classification of Neotropical Fishes. Porto Alegre, Edipucrs 603pp.

Lima, F.C.T.; P.A.,Buckup; N.A. Menezes; C.A.S. Lucena; Z.M.S. Lucena; M. Toledo-Pizza \& A. Zanata. 2007. Família Characidae: Gêneros incertae sedis. Pp. 44-62. In: Buckup, P.A.; N.A., Menezes \& M.S., Ghazzi (Eds), Catálogo das Espécies de Peixes de Água Doce do Brasil, Série Livros (23), Museu Nacional, Universidade Federal do Rio de Janeiro.

Lima, F.C.T.; L.R. Malabarba; P.A. Buckup; J.F.P. Silva; R.P. Vari; A. Harold; R.C. Benine; O. Oyakawa; C.S. Pavanelli; N.A. Menezes; C.A.S. Lucena; M.C.S.L. Malabarba; Z.M.S. Lucena; R.E. Reis; F. Langeani; L. Casatti; V.A. Bertaco; C. Moreira \& P.H.F. Lucinda 2003. Genera Incertae Sedis in Characidae. Pp. 106-169. In: Reis, R.E.; S.O. Kullander \& C.J. Ferraris-Jr (Orgs.), Check List of Freshwater Fishes of South and Central America, Edipucrs, Porto Alegre, 729p.

Lima, F.C.T. \& C.R., Moreira. 2003. Three new species of Hyphessobrycon (Characiformes: Characidae) from the upper rio Araguaia basin in Brazil. Neotropical Ichthyology, 1:21-33.

Lima, F.C.T.; L., Ramos; T., Barreto; A., Cabalzar; G., Tenório; A., Barbosa; F., Tenório \& A.S., Resende. 2005. Peixes do Alto rio Tiquié - Ictiologia e conhecimento dos tuyuka e tukano. Pp. 111-304. In Cabalzar, A. (Org.), Peixes e Gente no Alto rio Tiquié - conhecimentos tukano e tuyuka, ictiologia, etnologia, Instituto Socioambiental, 339p.

Lima, F.C.T. \& L.M., Souza. 2009. A new species of Hemigrammus from the upper rio Negro basin, Brazil, with comments on the presence and arrangement of anal-fin hooks in Hemigrammus and related genera 
(Ostariophysi: Characiformes: Characidae). Aqua, International Journal of Ichthyology, 15(3): 153-168.

Lima, F.C.T. \& M., Toledo-Piza. 2001. New species of Moenkhausia (Characiformes: Characidae) from the Rio Negro of Brazil. Copeia, 2001 (2): 1058-1063.

Lima, F.C.T.; W.B., Wosiacki \& C.S., Ramos. 2009. Hemigrammus arua, a new species of characid (Characiformes, Characidae) from the lower Amazon, Brazil. Neotropical Ichthyology, 7(2): 153-160.

Lucena, C.A.S. 1993. Estudo Filogenético da família Characidae com uma discussão dos grupos naturais propostos (Teleostei, Ostariophysi, Characiformes). Tese de Doutorado não publicada, Universidade de São Paulo/USP, São Paulo, 158p.

Lucena, C.A.S. \& N.A., Menezes. 1998. A phylogenetic analysis of the Roestes Günther and Gilbertolus Eigenmann, with a hypothesis on the relationships of the Cyinodontidae and Acestrorhyinchidae (Teleostei: Ostariophysi: Characiformes). Pp. 261-278. In: Malabarba, L.R.; Reis, R.E.; Vari, R.P., Lucena, Z.M. \& Lucena, C.A.S. (eds.). Phylogeny and Classification of Neotropical Fishes. Porto Alegre, Edipucrs 603p.

Malabarba, L.R. 1998. Monophyly of the Cheirodontinae, characters and major clades (Ostariophysi: Characidae). Pp. 193-233. In: Malabarba, L.R.; Reis, R.E.; Vari, R.P., Lucena, Z.M. \& Lucena, C.A.S. (eds.). Phylogeny and Classification of Neotropical Fishes. Porto Alegre, Edipucrs 603pp.

Malabarba, L.R. \& S.H., Weitzman. 2003. Description of a new genus with six new species from Southern Brazil, Uruguay And Argentina, With A Discussion of a Putative Characid Clade (Teleostei: Characiformes: Characidae). Comunicações do Museu de Ciências e Tecnologia da PUCRS, Série Zoologia, 16: 67-151.

Marinho, M.M.F. 2009. Análise Filogenética e Revisão Taxonômica das espécies de Moenkhausia Eigenmann, 1903 do grupo M. lepidura 
(Ostariophysi: Characiformes: Characidae). Dissertação de Mestrado não publicada, Universiade Estadual Paulista/UNESP, São José do Rio Preto, 242p.

Marinho, M.M.F; F.R0, .Carvalho; F., Langeani \& F., Tatsumi. 2008. A new Hemigrammus Gill from upper rio Paraná system, Southeastern Brazil (Characiformes: Charcidae). Zootaxa, 1724: 52-60.

Menezes, N.A.; S.H., Weitzman; O.Y., Oyakawa; F.C.T., Lima; R.M.C., Castro \& M.J., Weitzman. 2007. Peixes de água doce da Mata Atlântica - Lista Preliminar das espécies e comentários sobre conservação de peixes de água doce Neotropicais (Freshwater fishes of Mata Atlântica Prelimanary listo $f$ species and comments on conservation of Neotropical freshwater fishes). Museu de Zoologia - Universidade de São Paulo, SP, $408 p$.

Mirande, J.M. 2009. Weighted parsimony phylogeny of the family Characidae (Teleostei: Characiformes). Cladistics, 25(2009): 1-40.

Monod, T. 1967. Le complexe urophore des téléostéens: typologie et évolution (note preliminaire). Colloques Internationaux du Centre National de la Recherche Scientifique., 163: 11-31.

Monod, T. 1968. Le complexe urophore des poissons téléostéens. Memoires de I'Institution Fondamental d'Afrique Noire, 81:1-34.

Moreira, C.R. 2007. Relações Filogenéticas na ordem Characiformes (Teleostei: Ostariophysi). Tese de Doutorado não publicada, Universidade de São Paulo, São Pualo, 468p.

Nelson, G. \& N., Platnick. 1981. Systematics and biogeography: Cladistics and vicariance. Columbia University Press, New York, 567p.

Nixon, K. C. \& J. M. Carpenter. 1993. On outgroups. Cladistics, 9: 413-426. 
Planquette, P.; P., Keith \& P. Y., Le Bail. 1996. Atlas des Poissons d'eau douce de Guyane (Tome 1). Museum d'Histoire Naturelle, Ministère de l'Environnement, 431p.

Reis, R.E. 1989. Systematic Revision of the Neotropical characid subfamily Stethaprioninae (Pisces, Characiformes). Comunicações do Museu de Ciência da PUCRS, Série Zoologia, 2(6): 3-86.

Reis, R.E.; S.O., Kullander \& C.J., Ferraris Jr. 2003. Check List of the Freshwater Fishes of South and Central America (CLOFFSCA). 1a Ed. Edipucrs, 729p.

Roberts, T.R. 1969. Osteology and relationships of characoid fishes, particulary the genera Hepsetus, Salminus, Hoplias, Ctenolucius, and Acestrorhyinchus. Proceedings of the California Academy of Sciences, 36(15): 391-500.

Serra, J.P. 2003. Análise Filogenética e Revisão Taxonômica de Hasemania Ellis, 1911 (Characiformes, Characidae). Dissertação de Mestrado não publicada, Universidade Estadual Paulista/UNESP, São José do Rio Preto, $148 p$.

Serra, J.P. \& F., Langeani. 2006. Redescrição e osteCologia de Bryconamericus exodon Eigenmann, 1907 (Ostariophysi, Characiformes, Characidae). Biota Neotropica, 6(3): 1-14.

Taphorn, D.C. 1992. The characiform fishes of the Apure River drainage, Venezuela. BiLlania Edición Especial - № 4, Monografias Cientificas del Museo de Ciencias Naturales, UNELLEZ - Guanara, estado Portuguesa, Venezuela, 537p.

Taphorn, D.C. 2003. Manual de Identificación y biologia de los peces Characiformes de la Cuenca del Rio Apure en Venezuela. Universidade Nacional Experimental de los Llanos Occidentales "Ezequiel Zamora" UNELLEZ, Vice-Rectorado de Producción Agrícola, BioCentro, estado Portuguesa, Venezuela, 393p. 
Taylor, W.R. \& G.C., Van Dyke. 1985. Revised procedures for staining and clearing small fishes and other vertebrates for bone and cartilage. Cybium, 9: 107-119.

Toledo-Pizza, M. 2000. The neotropical fish subfamily Cyinodontinae (Teleostei: Ostariophysi: Characiformes): A Phylogenetic study and a revision of Cyinodon and Raphiodon. American Museum Novitates, 3286: 1-88.

Uj, A. \& J., Géry. 1989. Deux nouvelles espèces de tetras (poissons characoïdes, Characidae auct., Tetragonopterinae) du Paraguay: Hyphessorycon arianae $\mathrm{n}$. sp. et Hemigrammus mahnerti n. sp. Revue Suisse de Zoologie, 96(1): 147-159.

Ulreyi, A.B. 1895. The South American Characinidae collected by Charles Frederick Hartt. Annals of the New York Academy of Sciences, 8: 257-300.

Vari, R.P. 1983. Phylogenetic relationships of the families Curimatidae, Prochilodontidae, Anostomidae, and Chilodontidae (Pisces: Characiformes). Smith. Smithsonian Contributions to Zoology, Washington, 378:1-60.

Vari, R.P. 1995. The neotropical fish family Ctenoluciidae (Teleostei: Ostariophysi: Characiformes): supra and intrafamilial phylogenetic relationships, with a revisionary study. Smithsonian Contributions to Zoology, 564: 1-97.

Vari, R.P. \& A.S., Harold. 1998. The Genus Creagrutus (Teleostei: Characiformes: Characidae): monophyly, relationships, and undetected diversity. In: Malabarba, L.R.; Reis, R.E.; Vari, R.P., Lucena, Z.M. \& Lucena, C.A.S. (eds.). Phylogeny and Classification of Neotropical Fishes. Porto Alegre, Edipucrs, 603pp.

Vari, R.P. \& A.S., Harold. 2001. Phylogenetic Study of the Neotropical fish genera Creagrutus Günther and Piabina Reinhardt (Teleostei: Ostariophysi: Characiformes), with a revision of the Cis-Andean species. Smithsonian Contributions to Zoology, 613: 1-239. 
Weitzman, S.H. 1962. The osteology of Brycon meeki, a generalized Characidae fish, with an osteological definition of the family. Stanford Ichthyol. Bull., 8: 177.

Weitzman, S.H., 1974. Osteology and Evolutionary Relationships of the Sternoptychidae, with a new Classification of Stomiatoid Families. Bulletin of the American Museum of Natural History, New York, 153(3): 327-478.

Weitzman, S.H. 1997. Problem in Systematic Studies of Rosy Tetras and their Significance for Aquarists. Tropical Fish Hobbyist, 150-159.

Weitzman, S.H. \& W., Fink. 1983. Relationships of the Neon Tetras, a Group of South American Freshwater Fishes (Teleostei, Characidae), with Comments on the Phylogeny of new World Characiformes. Bulletin: Museum of Comparative Zoölogy, 150(6): 339-395.

Weitzman, S.H. \& L.R., Malabarba. 1998. Perspectives about the Phylogeny and Classification of the Characidae (Teleostei: Characiformes). Pp. 161170. In: Malabarba, L.R., Reis, R.E.; Vari, R.P.; Lucena, Z.M. \& Lucena, C.A.S (Eds), Phylogeny and Classification of Neotropical Fishes, Edipucrs, Porto Alegre, 729p.

Weitzman, S.H. \& L.R., Malabarba. 1999. Systematics of Spintherobolus (Teleostei: Characidae: Cheirodontinae) from Eastern Brazil. Ichthyological Exploration of Freshwaters, 10(1): 1-43.

Weitzman, S.H. \& L., Palmer. 1997a. A New Species of Hyphessobrycon (Teleostei: Characidae) from the Neblina Region of Venezuela and Brazil, with Comments on the Putative "Rosy Tetra Clade". Ichthyological Exploration of Freshwaters, 7(3): 209-242.

Weitzman, S.H. \& L., Palmer. 1997b. The Rosy Tetra Hyphessobrycon rosaceus, its Identification and Brief History as an Aquarium Fish. Tropical Fish Hobbyist, 45(11): 158-166. 
Wiley, E.O. 1981. Phylogenetics - The theory and practice of phylogenetic systematics. New York, John Wiley \& Sons. 439p.

Wiley, E.O.; D., Siegels-Causey; D.R., Brooks \& V.A., Funk. 1991. The complete cladist: A primer of phylogenetic procedures. Special Publication, no 19, The University of Kansas, Museum of Natural History, Lawrence, 159p.

Zanata, A.M. 2000. Estudo das relações filogenéticas do gênero Brycon Muller \& Troschel, 1844 (Characidae; Characiformes). Tese de Doutorado não publicada, Universidade de São Paulo/USP, São Paulo, 358p.

Zanata, A.M. \& R.P., Vari. 2005. The family Alestidae (Ostariophysi, Characiformes): a phylogenetic analysis of a trans-Atlantic clade. Zoological Journal of the Linnean Society, 145(1): 1-144.

Zarske, A. \& J., Géry. 2002. Hemigrammus neptunus sp. n. - eine neue Salmer-Art (Teleostei, Characiformes, Characidae) aus dem Einzugsebiet dês Rio Manuripi in Bolivien (Departamento Pando). Zoologische Abhandlungen, 52: 23-34.

Zarske, A.; P.Y., Le-Bail \& J., Géry. 2006. New and poorly known Characiform fishes from French Guiana. 1. Two new Tetras of the genera Hemigrammus and Hyphessobrycon (Teleostei: Characiformes: Characidae). Zoologische Abbandlungen (Dresden), 55: 17-30.

Zarske, A. \& J., Géry. 2007. Hemigrammus geisleri sp. n. - ein neuer Glassalmler aus dem zentralen Amazonasgebiet, mit einer ergänzenden Beschreibung von Hemigrammus mimus Böhlke, 1955 (Teleostei: Characiformes: Characidae). Vertebrate Zoology, 57(1): 5-14. 


\section{TABELAS E FIGURAS}


Tabela 1- Espécies válidas de Hemigrammus, seguidas de sua respectiva localidade-tipo e distribuição geográfica.

\section{Espécie}

Hemigrammus aereus Géry, 1959

Hemigrammus analis Durbin, 1909

Hemigrammus arua Lima, Wosiacki \& Ramos, 2009

Hemigrammus barrigonae Eigenmann Colômbia, Barrigona, rio Meta. \& Henn, 1914

Hemigrammus belottii (Steindachner, 1882)

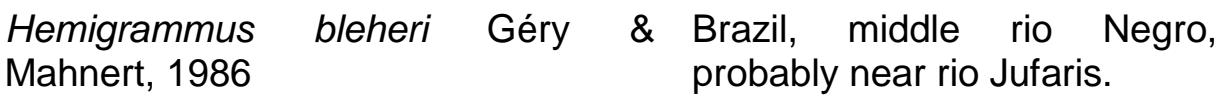

Hemigrammus boesemani Géry, 1959

Hemigrammus brevis Ellis, 1911

Hemigrammus coeruleus Durbin, Brazil, Manacapuru, Solimões 1908

Hemigrammus cupreus Durbin, 1918

Hemigrammus cylindricus Durbin, Guyana, Tumatumari. 1909:

Hemigrammus

(Steindachner, 1882) river, Amazonas. Brazil, Jatuarana, probably near Parintins, Amazonas.

French Guiana, crique Conhecido apenas da Nancibo, basin de la Conté, localidade-tipo um dês formaterus du Mahury.

Guyana, Essequibo river

Rockstone

Rios Essequibo e Demerara; bacias dos rios Apure, Orinoco, baixo Tapajós e Negro; Brasil, Guiana e Venezuela.

Cabeceiras do rio Arapiuns (rio Branco e rio Aruã), bacia do Tapajós e cabeceiras do igarapé Juruti Grande, bacia do rio Amazonas, Pará; Brasil.

Bacia do rio Orinoco Colômbia e Venezuela. Bacia do rio Tiquié; Brasil.

Bacia dos rios Solimões, Negro e Maroni; Brasil e Guiana Francesa

Bacia dos rios Negro e Meta; Brasil e Colômbia.

French Guiana, crique vers Sinnamary, Guyane fse.

Brazil, Barreiras, Lagoas do rio Grande, São Francisco river basin, Bahia.

Rios Maroni, Mana, Iracoubo, Sinnamary, Kouro, Comté, Kaw e alto rio Amazonas no Peru; Guiana Francesa, Peru e Suriname.

Bacia do rio São Francisco; Brasil.

Bacia dos rios Solimões e baixo rio Negro; Brasil.

Bacia do rio Solimões; Brasil.

Rio Essequibo; Guiana.

Bacia dos rios Amazonas e Tapajós; Brasil. Bacia dos rio Apure e Aguaro; 
Venezuela.

Hemigrammus erythrozonus Dubin, Guyana, Erukin, Essequibo Rio Essequibo; Guiana. 1909 river.

Hemigrammus geileri Zarske \& Géry, Brasilien, Para, Igarapé 35 km 2007 nordwestlich Obidos

Conhecido apenas da localidade-tipo

Hemigrammus gracilis (Lütken, 1875) Brazil, Minas Gerais, Lacu Lagoa Santa, São Francisco river basin.

Bbacia dos rios São Francisco e Amazonas; Brasil

Hemigrammus guyanensis Géry, French Guiana, crique Sable 1959 (Hte Mana)

Rios Maroni, Mana, Approuague e Oyapock, Guiana Francesa.

Hemigrammus haraldi Géry, 1961

Brazil, Upper Solimões,

Bacia do alto rio environs of São Paulo de Amazonas; Brasil.

Oliença, in an inland-lake midst the thick forest between Paraná-para and the ParanáCamatia

Hemigrammus hyanuary Durbin, 1918 Brazil, Lake Hyanuary (= Lago Januari), Amazonas.

Bacia do rio Amazonas; Brasil e Peru

Hemigrammus iota Durbin, 1909

Guyana, Glunk Island, Essequibo river

Bacia do rio Essequibo; Guiana

Hemigrammus levis Durbin, 1908

Brazil, Lago do Maximo, Obidos, Villa Bella, lake Jose Assu, Amazon river basin.

Hemigrammus luelingi Géry, 1964

Peru, petit ruisseau faisnt communiquer lê Zapote Cocha avec la Cano Yarina, em bordure du rio Pacaya, afluent du canal de Puinahuna (Brás du bas rio Ucayali.

Hemigrammus lunatus Durbin, 1918 Amazon

Bacia dos rios Amazonas, Paraguai e Suriname; Brasil, Peru e Suriname

Hemigrammus mahnerti Uj \& Géry, Paraguay, dépt. Missiones, 1989 marais pres de Panchito Lopez à 4 km de Yabebyry

Bacia dos rios Paraná e Paraguai; Argentina e Paraguai.

Hemigrammus marginatus Ellis, 1911 Bazil, Bahia, Queimadas, rio Itapicurú.

Bacia dos rios São Francisco, Itapicuru, Paraná, Paraguai, Guaporé, Amazonas e Orinoco; Brasil, Colômbia, Peru e Venezuela. 


\begin{tabular}{|c|c|c|c|}
\hline $\begin{array}{l}\text { Hemigrammus } \\
1918\end{array}$ & matei Eig & enmann, & Argentina \\
\hline Hemigrammus & maxillaris Fowl & er, 1932 & $\begin{array}{l}\text { Brazil, Descalvados, Mato } \\
\text { Grosso }\end{array}$ \\
\hline $\begin{array}{l}\text { Hemigrammus } \\
1945\end{array}$ & megaceps & Fowler, & $\begin{array}{l}\text { Peru, Ninabamba near } \\
\text { Ayacucho, at } 1900 \text { meters } \\
\text { elevation, rio Ucayali basin }\end{array}$ \\
\hline $\begin{array}{l}\text { Hemigrammus } \\
1913\end{array}$ & melanochrous & Fowler, & $\begin{array}{l}\text { Brazil, tributary of Madeira } \\
\text { river near Porto Velho }\end{array}$ \\
\hline $\begin{array}{l}\text { Hemigrammus } \\
1907\end{array}$ & micropterus & Meek, & Venezuela, Los Cstillos \\
\hline $\begin{array}{l}\text { Hemigrammus } \\
1918\end{array}$ & microstomus & Durbin, & $\begin{array}{l}\text { Brazil, Pará, } \\
\text { Amazon basin }\end{array}$ \\
\hline \multicolumn{3}{|c|}{ Hemigrammus mimus Böhlke, 1955} & $\begin{array}{l}\text { Brazil, rapids at Camanáos on } \\
\text { the rio Negro }\end{array}$ \\
\hline
\end{tabular}

Hemigrammus neptunus Zarske \& Bolivien, Dep. Pando, rio Géry, 2002

Hemigrammus newboldi (FernándezYépez, 1949

Hemigrammus ocellifer (Steindachner, Brazil, Amazon, Villa Bella, 1882)

Hemigrammus ora Zarske, Le Bail \& French Guiana, Pripri Yiyi Géry, 2006

Hemigrammus orthus Durbin, 1909

Hemigrammus parana Marinho, Brazil, São Paulo, Santa Clara Carvalho, Langeani \& Tatsumi, 2008

Hemigrammus pretoensis Géry, 1965 Brazil, Igarapé Preto, collatéral de l'Amazone supérieure pres
Manuripi, uneit dês Zusammenflusses mit dem rio Tahumanu, mehrere Fundorte Zwischen Alméndrillo und Bolima

D'Oeste, rio Grande near the old sand port, Known as Velho Adão

Argentina, localidade exata desconhecida

Bacia do rio Paraguai; Brazil

Bacia do alto rio Ucayali; Peru

Bacia do médio rio Madeira; Brazil

Bacia do rio Orinoco; Venezuela

Amazônia Central e bacia do rio Orinoco; Brasil e Venezuela.

Bacia dos rios Amazonas, médio Negro e alto Orinoco; Brazil, Colômbia e Venezuela

Bacia do rio Amazonas; Bolívia

Bacia do rio Orinoco; Venezuela

Rios da Guiana, Suriname, Guiana Francesa, e bacia do rio Amazonas no Brasil e Peru, introduzido em Trinidad e Tobago.

Conhecida apenas para a região costeira da Guiana Francesa.

Guyana, Tukeit, alto rio Essequibo

Rios Essequibo e baixo Tapajós; Brasil e Guiana

Bacia do Alto rio Paraná; Brasil

Bacia do alto rio Solimões; 


\begin{tabular}{|c|c|c|}
\hline & $\begin{array}{l}\text { de Belém, à environ } 60 \mathrm{~km} \text { en } \\
\text { aval de Letícia }\end{array}$ & Brasil \\
\hline Hemigrammus pulcher Ladiges, 1938 & $\begin{array}{l}\text { Peru, dem Stromgebiet } \\
\text { zwischen Tabatinga um lquitos }\end{array}$ & $\begin{array}{l}\text { Bacia do alto rio } \\
\text { Amazonas; Peru }\end{array}$ \\
\hline $\begin{array}{l}\text { Hemigrammus rhodostomus Ahl, } \\
1924\end{array}$ & Brazil, Pará & $\begin{array}{l}\text { Bacia do baixo Amazonas } \\
\text { no Estado do Pará e bacia } \\
\text { do rio Orinoco; Brasil e } \\
\text { Venezuela. }\end{array}$ \\
\hline Hemigrammus rodwayi Durbin, 1909 & Guyana, Georgetown trenches & 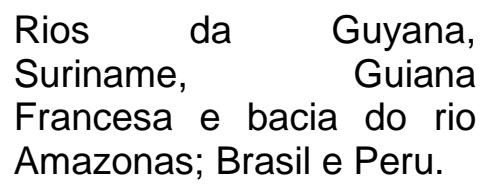 \\
\hline $\begin{array}{l}\text { Hemigrammus } \\
\text { (Steindachner, 1882) }\end{array}$ & $\begin{array}{l}\text { Brazil, Tabatinga, upper } \\
\text { Amazon basin }\end{array}$ & $\begin{array}{l}\text { Bacia dos rios Amazonas, } \\
\text { Negro e Orinoco; Brasil e } \\
\text { Venezuela }\end{array}$ \\
\hline $\begin{array}{l}\text { Hemigrammus silimoni Britski \& Lima, } \\
\text { 2008: }\end{array}$ & $\begin{array}{l}\text { Brazil, Mato Grosso, Sapezal, } \\
\text { rio Juruena, downtream PCH } \\
\text { Telegráfica }\end{array}$ & $\begin{array}{l}\text { Alto rio Juruena, bacia do } \\
\text { rio Tapajós; Brasil }\end{array}$ \\
\hline $\begin{array}{l}\text { Hemigrammus skolioplatus Bertaco \& } \\
\text { Carvalho } 2005\end{array}$ & \begin{tabular}{lrr} 
Brazil, & Mato & \multicolumn{2}{c}{ Grosso, } \\
Comodoro, rio Doze & de \\
Outubro on road BR & 364 \\
between & Comodoro & and \\
Vilhena & &
\end{tabular} & $\begin{array}{l}\text { Bacia do Alto rio Tapajós; } \\
\text { Brasil }\end{array}$ \\
\hline Hemigrammus stictus (Durbin, 1909) & Guyana, Lama Stop-Off & $\begin{array}{l}\text { Bacia dos rios Amazonas, } \\
\text { Negro e Orinoco e rios } \\
\text { costeiros da Guyana; } \\
\text { Brasil, Guyana e } \\
\text { Venezuela. }\end{array}$ \\
\hline $\begin{array}{l}\text { Hemigrammus taphorni Benine \& } \\
\text { Lopes, } 2007\end{array}$ & $\begin{array}{l}\text { Venezuela, State of } \\
\text { rio Caura, Cano } \\
\text { around Salto Para }\end{array}$ & $\begin{array}{l}\text { Conhecido apenas da } \\
\text { localidade-tipo }\end{array}$ \\
\hline $\begin{array}{l}\text { Hemigrammus tridens Eigenmann, } \\
1907\end{array}$ & $\begin{array}{l}\text { Paraguay, Arroyo Pypucu, } \\
\text { Paraguay basin }\end{array}$ & $\begin{array}{l}\text { Bacia do rio Paraguai; } \\
\text { Paraguai }\end{array}$ \\
\hline $\begin{array}{l}\text { Hemigrammus ulreyi (Boulenger, } \\
\text { 1895): }\end{array}$ & $\begin{array}{l}\text { Brazil, } \quad \text { Mato } \quad \text { Grosso, } \\
\text { Descalvados, Paraguay basin }\end{array}$ & $\begin{array}{l}\text { Bacia do rio Paraguai; } \\
\text { Brasil }\end{array}$ \\
\hline Hemigrammus unilineatus (Gill, 1858) & $\begin{array}{l}\text { Trinida and Tobago, Western } \\
\text { Portion of the Island of } \\
\text { Trinidad, W.I. }\end{array}$ & $\begin{array}{l}\text { Bacia dos rios de Trinidad, } \\
\text { rios costeiros da } \\
\text { Venezuela, rios da } \\
\text { Guyana, } \\
\text { Guiana Francesa, bacia } \\
\text { dos rios Guaporé, } \\
\text { Amazonas e rios costeiros } \\
\text { do Nordeste Brasileiro; } \\
\text { Brasil, Guiana Francesa, }\end{array}$ \\
\hline
\end{tabular}




\begin{tabular}{|c|c|c|}
\hline & & $\begin{array}{l}\text { Guyana, Peru, Suriname, } \\
\text { Trinidad e Tobago e } \\
\text { Venezuela }\end{array}$ \\
\hline $\begin{array}{l}\text { Hemigrammus vorderwinkleri Géry, } \\
1963\end{array}$ & $\begin{array}{l}\text { Brazil, Amazonas, near } \\
\text { Tapurucuara, upper rio Negro }\end{array}$ & $\begin{array}{l}\text { Bacia do médio rio Negro; } \\
\text { Brasil }\end{array}$ \\
\hline $\begin{array}{l}\text { Hemigrammus yinyang Lima \& Souza, } \\
2009\end{array}$ & $\begin{array}{l}\text { Brazil, Amazonas, igarapé } \\
\text { Castanha, near Sítio São } \\
\text { Pedro }\end{array}$ & $\begin{array}{l}\text { Tributários do médio rio } \\
\text { Tiquié, um tributário do rio } \\
\text { Uauapés, bacia do alto rio } \\
\text { Negro, estado do } \\
\text { Amazonas; Brazil }\end{array}$ \\
\hline
\end{tabular}


Tabela 2- Dados morfométricos e merísticos de Hemigrammus unilineatus.

$\begin{array}{llll}\text { Caráter N Variação Média Desvio Padrão } & \end{array}$

Comprimento Padrão (mm)

$30 \quad 22,7-26,6$

24,1

2,1

Porcentagens do Comprimento Padrão

\begin{tabular}{lcccc}
\hline Altura do Corpo & 30 & $33,1-42,8$ & 37,3 & 2,4 \\
Comprimento da Cabeça & 30 & $25,3-30,7$ & 27,8 & 1,3 \\
Distância Pré-Dorsal & 29 & $44,7-53,4$ & 50,1 & 2,1 \\
Distância Pré-Ventral & 28 & $44-60,5$ & 47,2 & 3,7 \\
Distância Pélvica-Anal & 28 & $13,8-21,6$ & 17,2 & 1,7 \\
Comprimento da Base da Nadadeira Dorsal & 29 & $12,2-16,1$ & 14,3 & 1,0 \\
Comprimento da Base da Nadadeira Anal & 29 & $33-38,1$ & 35,6 & 1,4 \\
Comprimento da Nadadeira Dorsal & 29 & $26,9-35$ & 31,3 & 2,1 \\
Comprimento da Nadadeira Anal & 28 & $20,1-30,7$ & 26,1 & 2,2 \\
Comprimento da Nadadeira Peitoral & 27 & $18,1-26,3$ & 22,1 & 1,7 \\
Comprimento da Nadadeira Pélvica & 28 & $15,6-20,6$ & 18,2 & 1,2 \\
Comprimento da Nadadeira Caudal & 21 & $25,3-37$ & 32,1 & 2,9 \\
Comprimento do Pedúnculo Caudal & 29 & $8,9-14,6$ & 11,5 & 1,4 \\
Altura do Pedúnculo Caudal & 29 & $8,3-11,6$ & 10,4 & 1,0 \\
\hline
\end{tabular}

Porcentagens do Comprimento da Cabeça

\begin{tabular}{lcccc}
\hline Altura da Cabeça & 30 & $82,2-103,5$ & 93,6 & 5,8 \\
Diâmetro da Orbita & 30 & $39,7-51,4$ & 45,2 & 2,6 \\
Comprimento do Focinho & 30 & $17,9-28,2$ & 22,8 & 2,7 \\
Distância Interorbital & 30 & $28,4-35,6$ & 32,1 & 2,0 \\
\hline
\end{tabular}

\section{Contagens}

\begin{tabular}{lccc}
\hline & N & Variação & Moda \\
\hline Raios da Nadadeira Dorsal & 29 & $9-9$ & 9 \\
Raios da Nadadeira Anais & 28 & $22-27$ & 24 \\
Raios da Nadadeira Caudal & 23 & $8-9+9-10$ & $9-10$ \\
Raios da Nadadeira Pectoral & 29 & $10-12$ & 11 \\
Raios da Nadadeira Pélvica & 28 & 7 & 7 \\
Escamas Perfuradas na Linha Lateral & 25 & $8-15$ & 11 \\
Escamas não Perfuradas na Linha Lateral & 24 & $18-23$ & 21 \\
Escamas ao Redor do Pedúnculo Caudal & 19 & $12-14$ & 12 \\
Séries Longitudinais de Escamas Acima da & 25 & $5-6$ & 5 \\
Linha Lateral & & & \\
Séries Longitudinais de Escamas Abaixo da & 25 & $4-5$ & 4 \\
Linha Lateral & & & \\
Escamas na Base da Anal & 17 & $3-6$ & 6 \\
Dentes no Maxilar & 29 & $3-7$ & 5 \\
Dentes na Série Externa do Pré-maxilar & 30 & $3-5$ & 4 \\
Dentes na Série Interna do Pré-maxilar & 30 & $5-7$ & 5 \\
Dentes no Dentário & 30 & $4-5$ & 4 \\
\hline
\end{tabular}




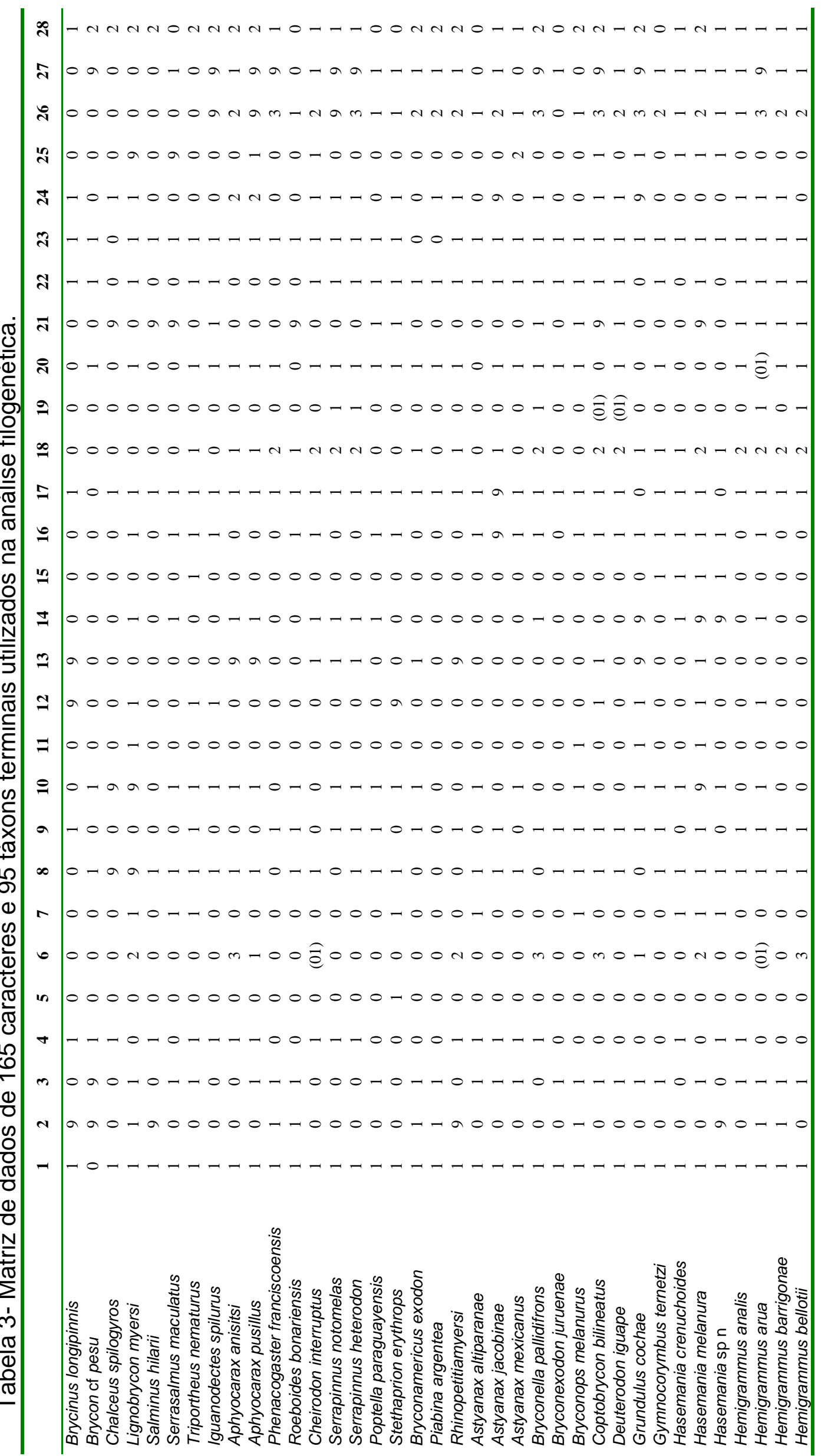




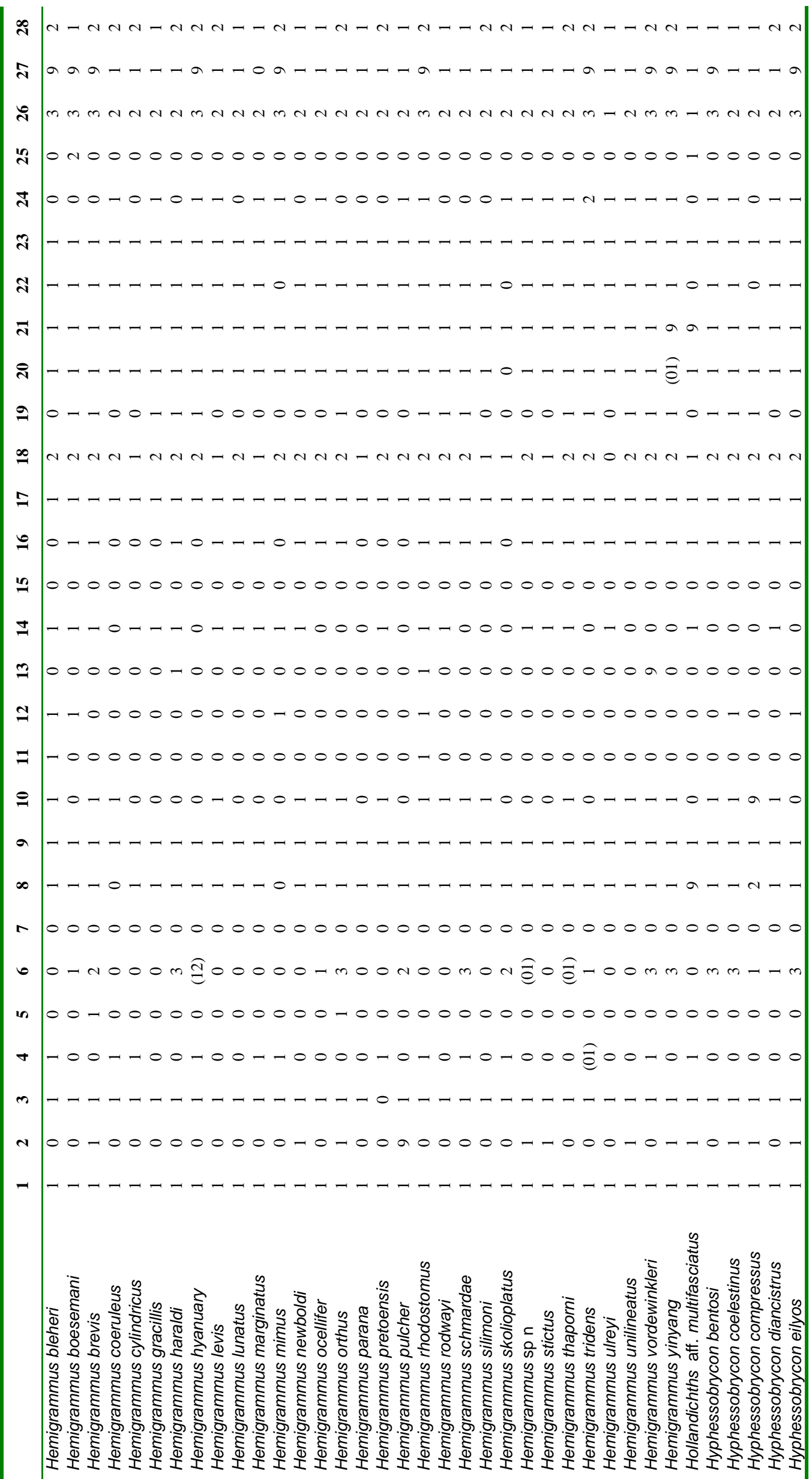




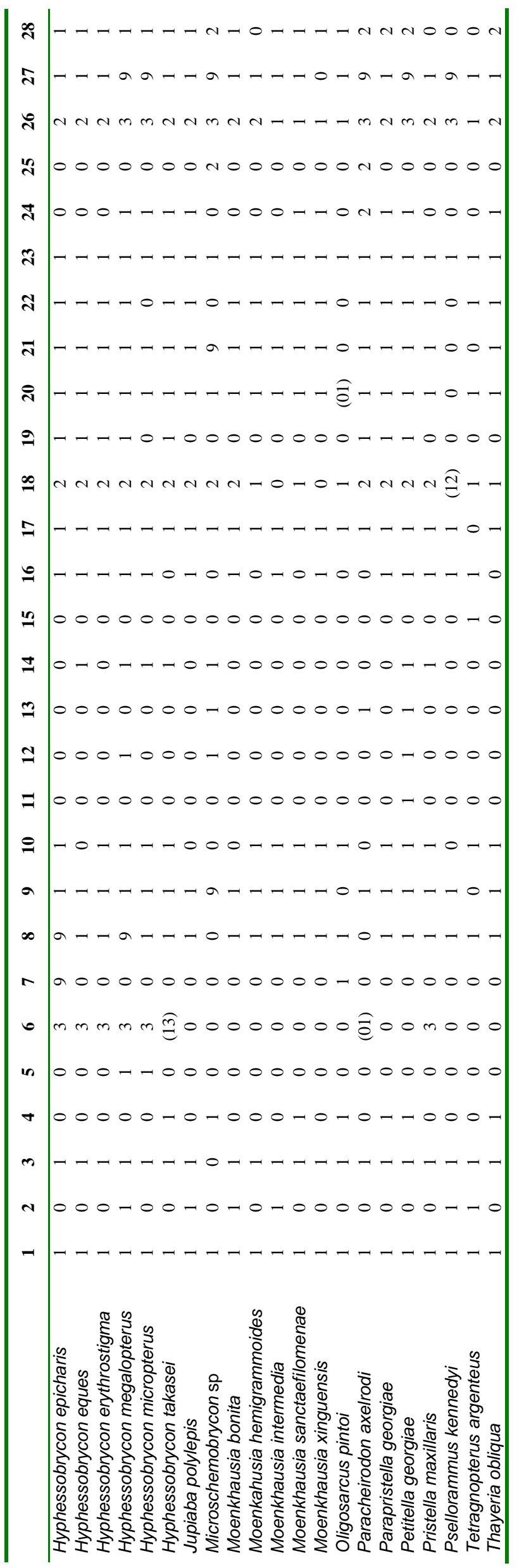




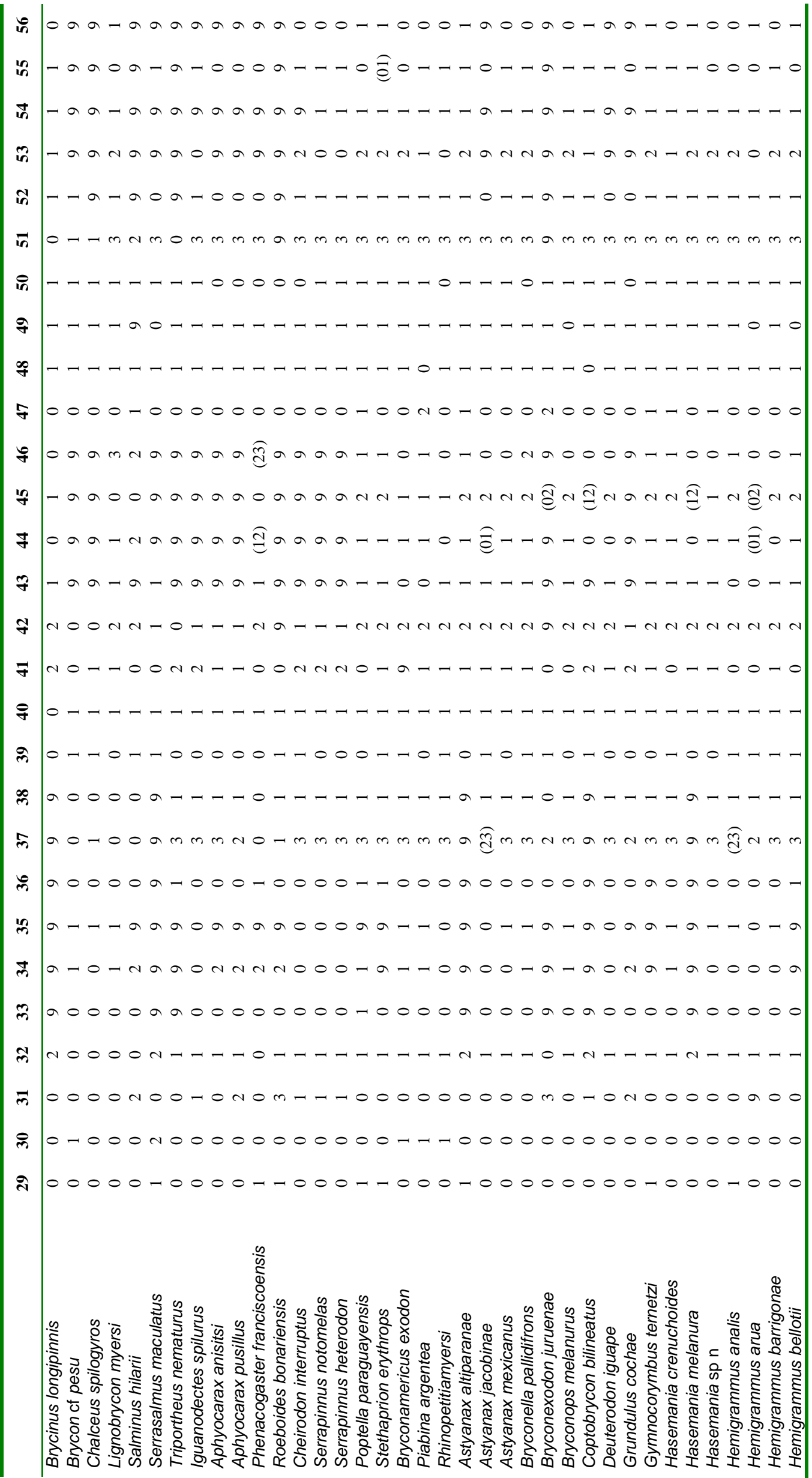




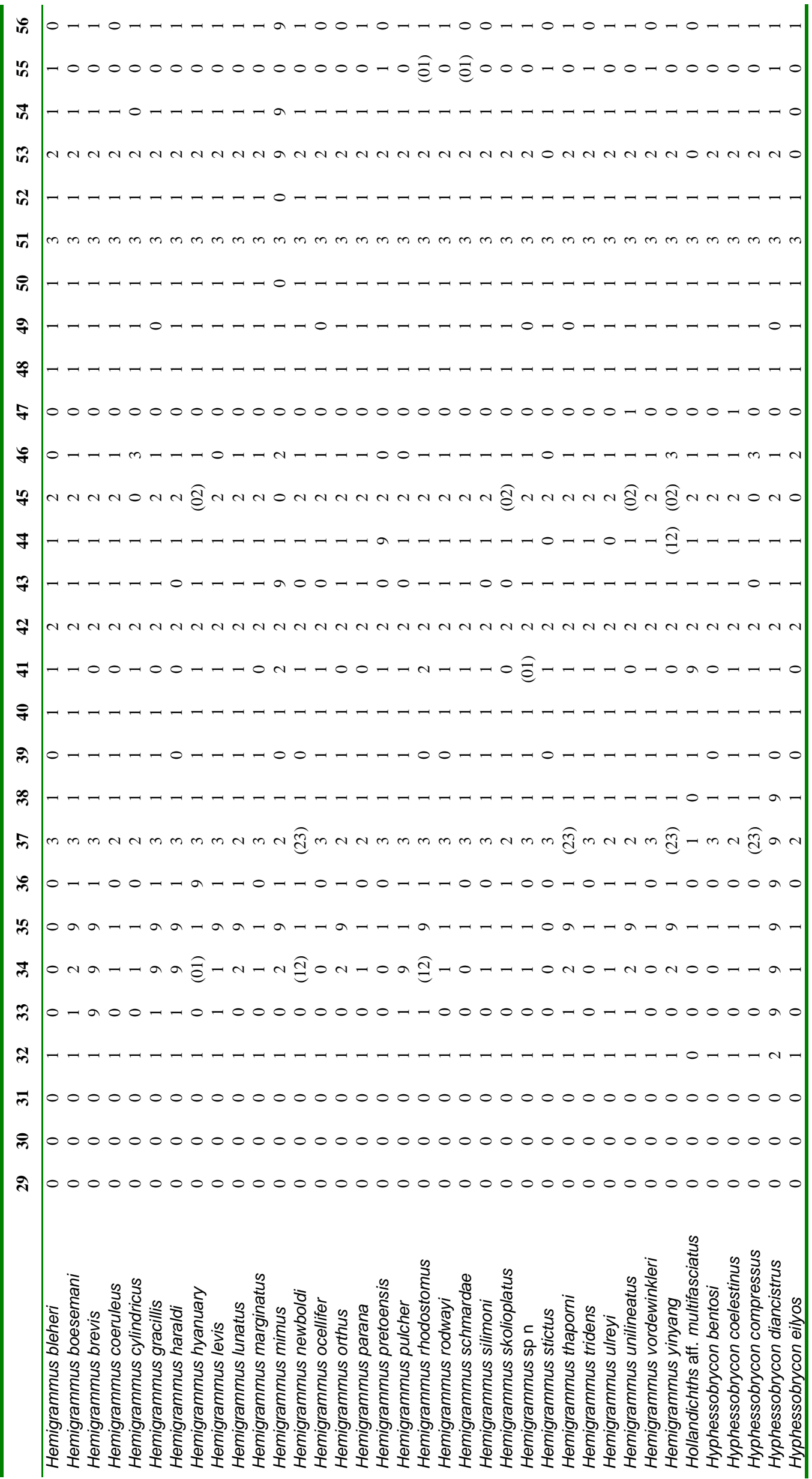




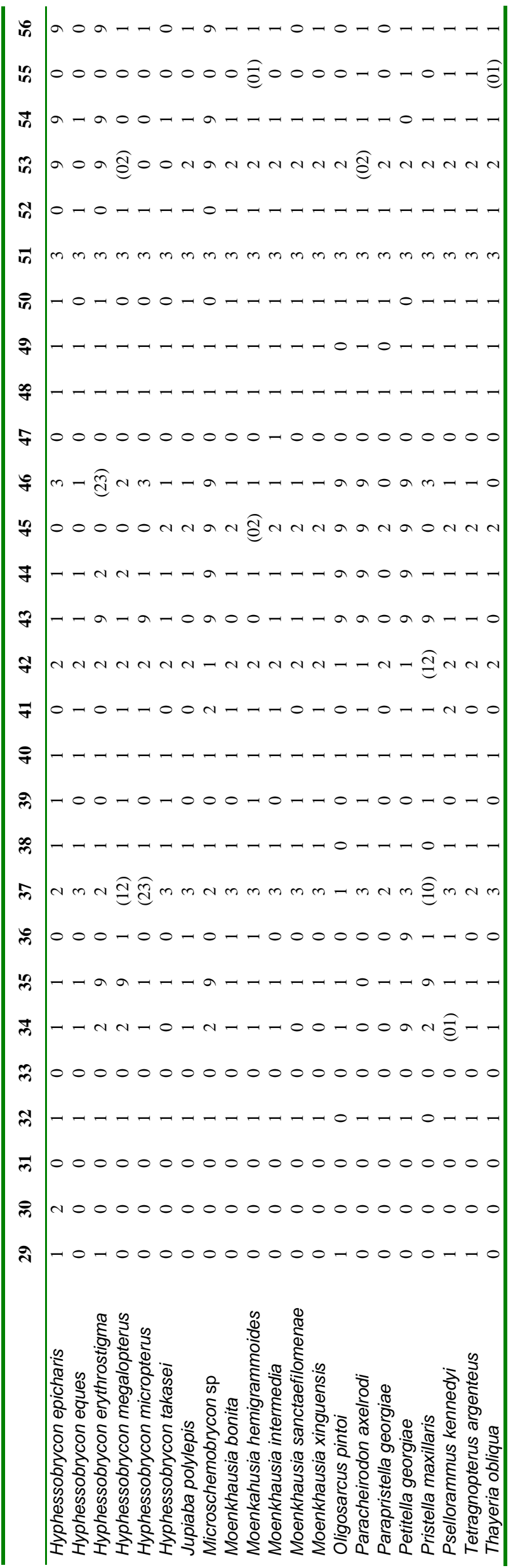




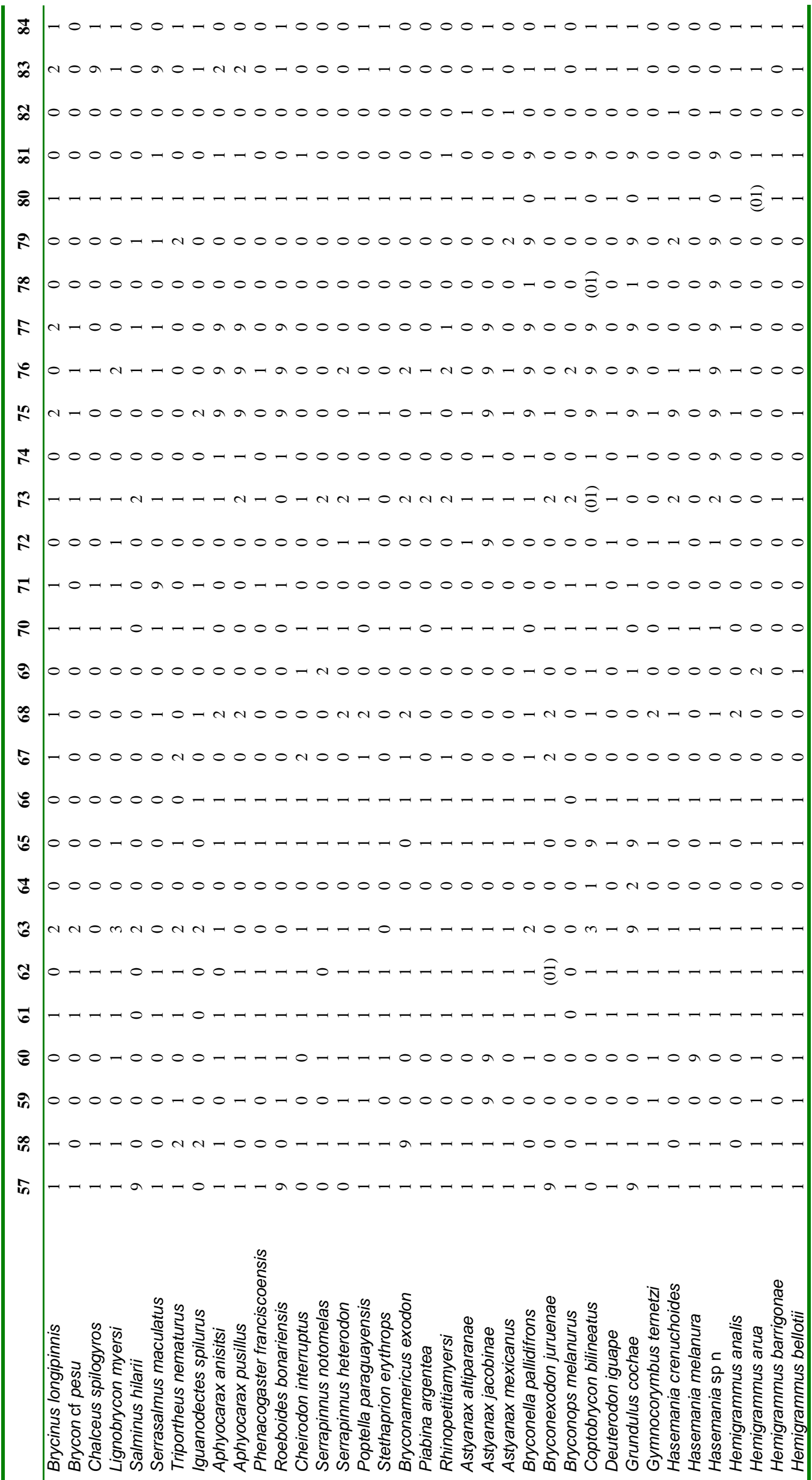




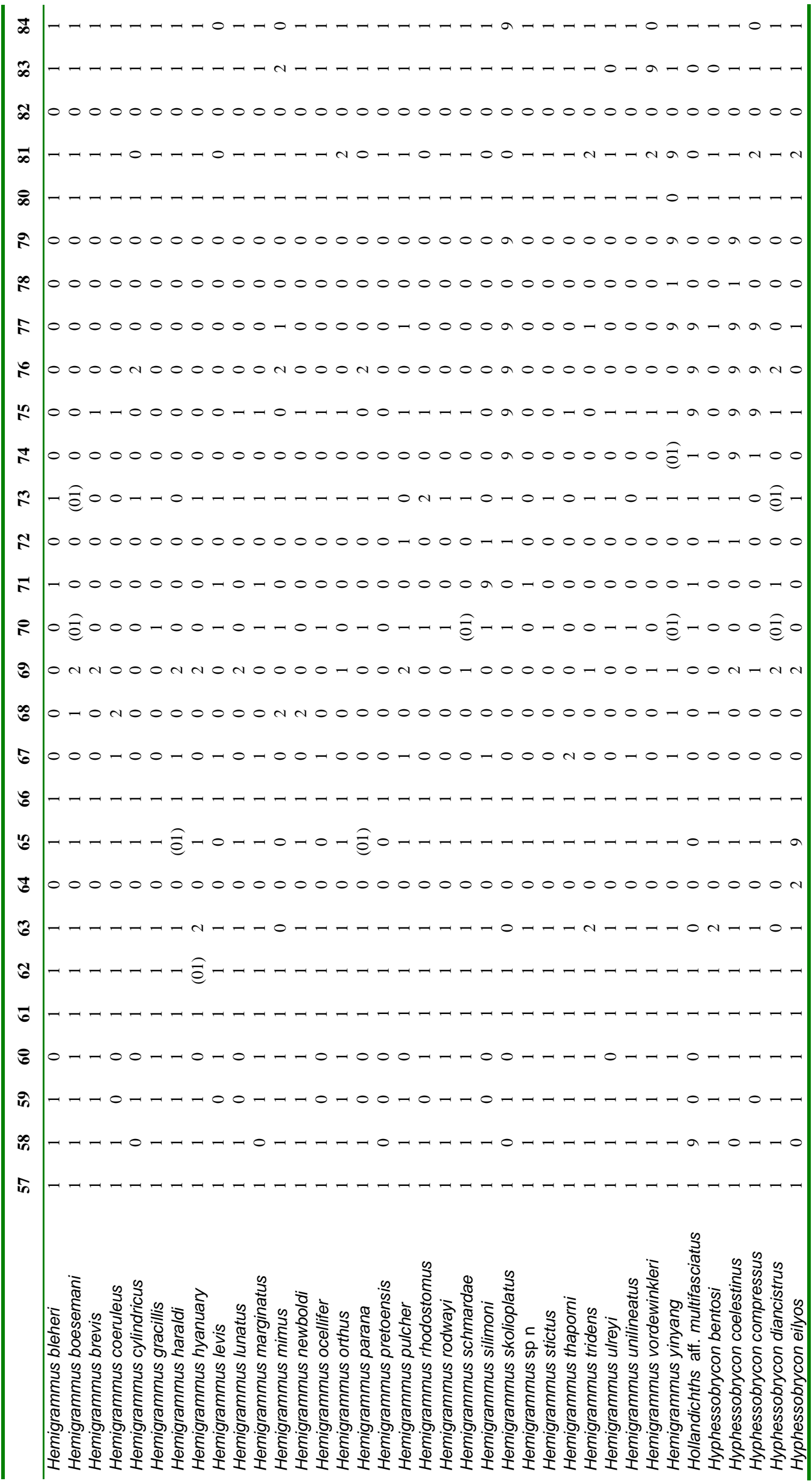




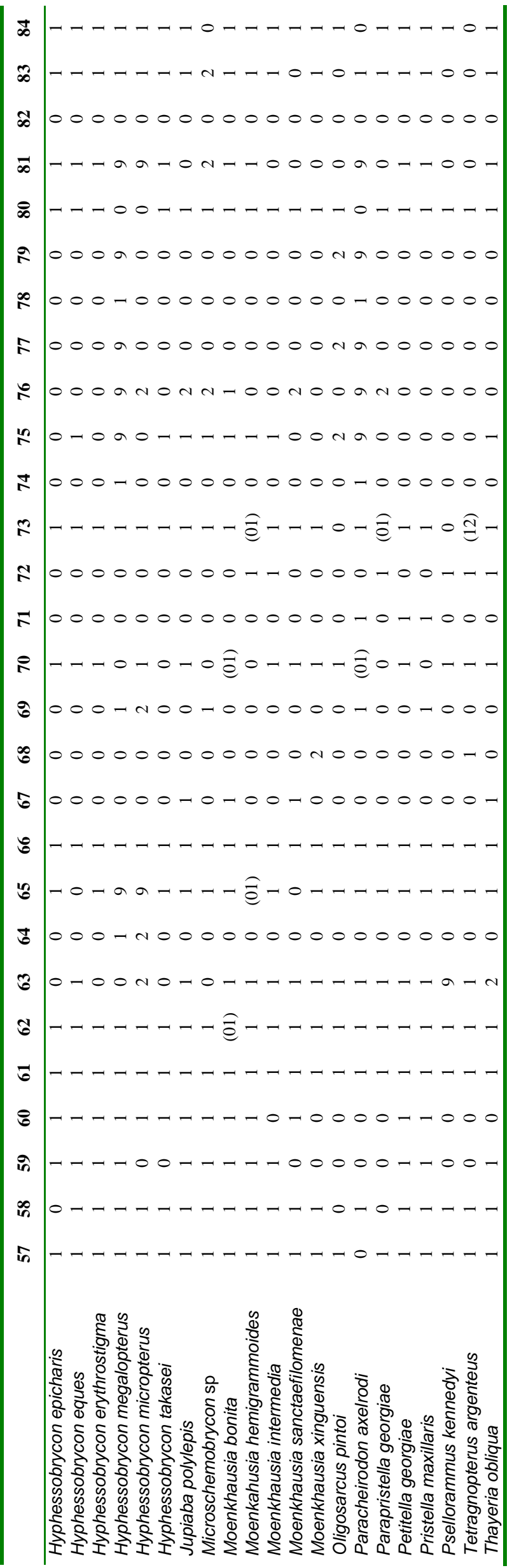




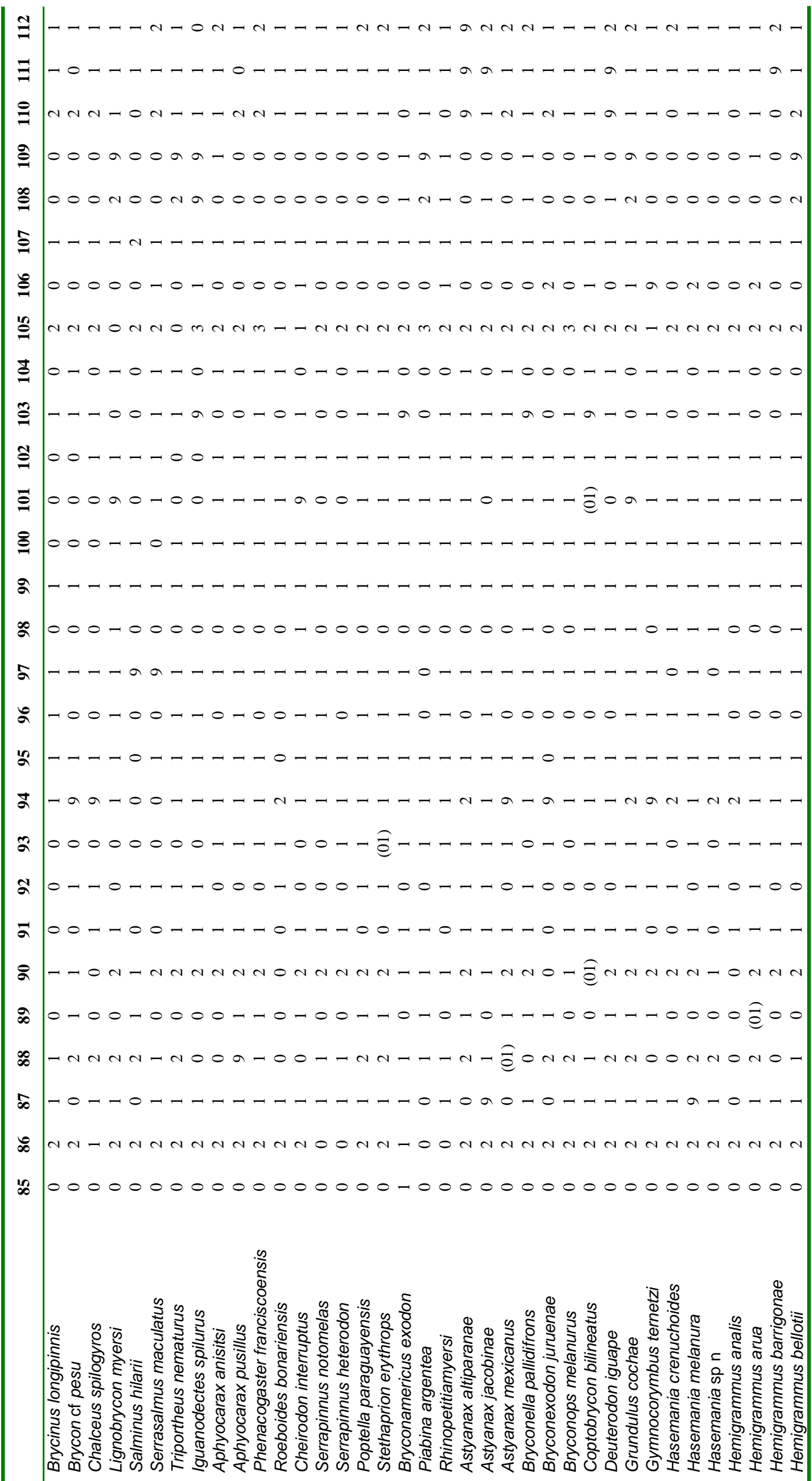




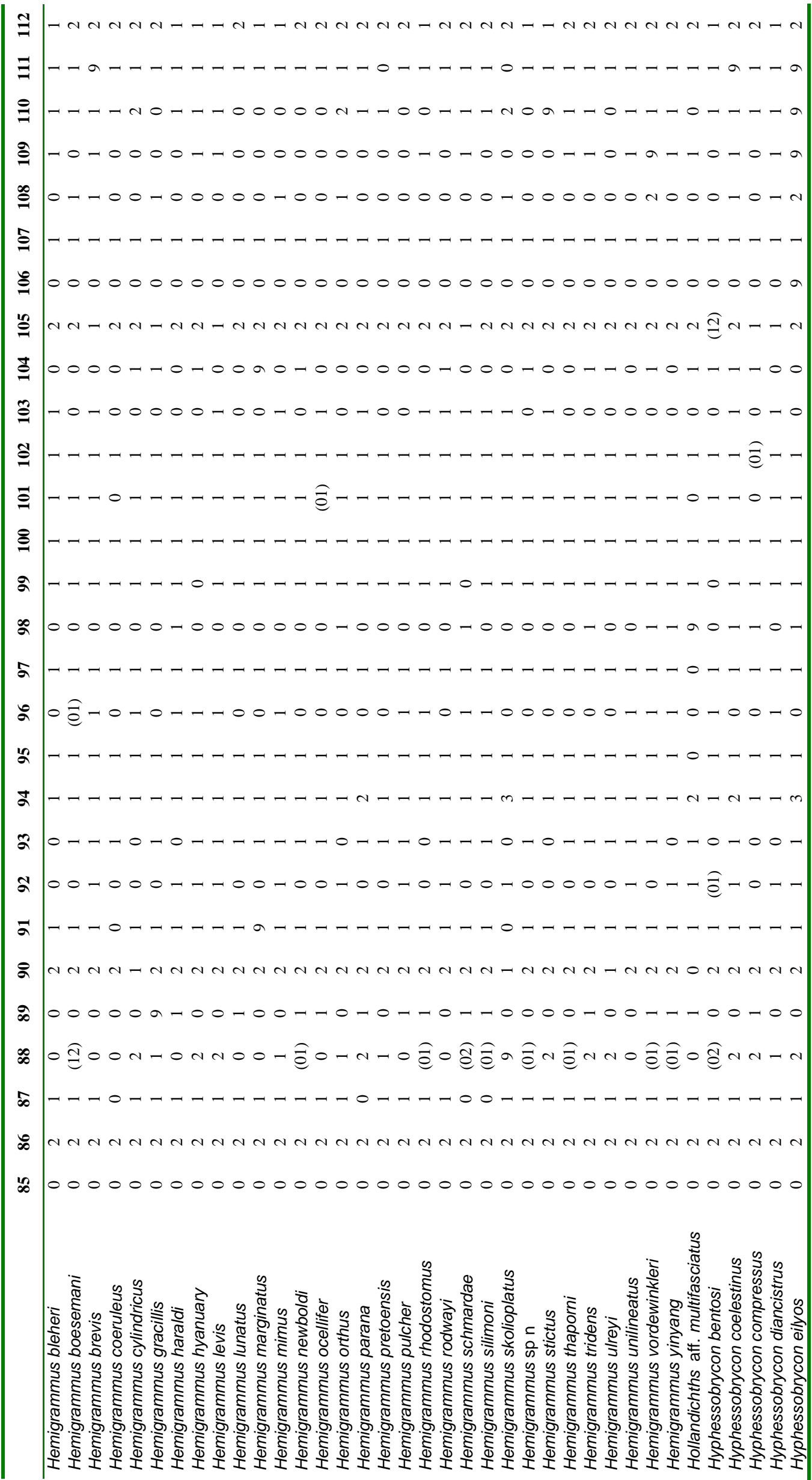




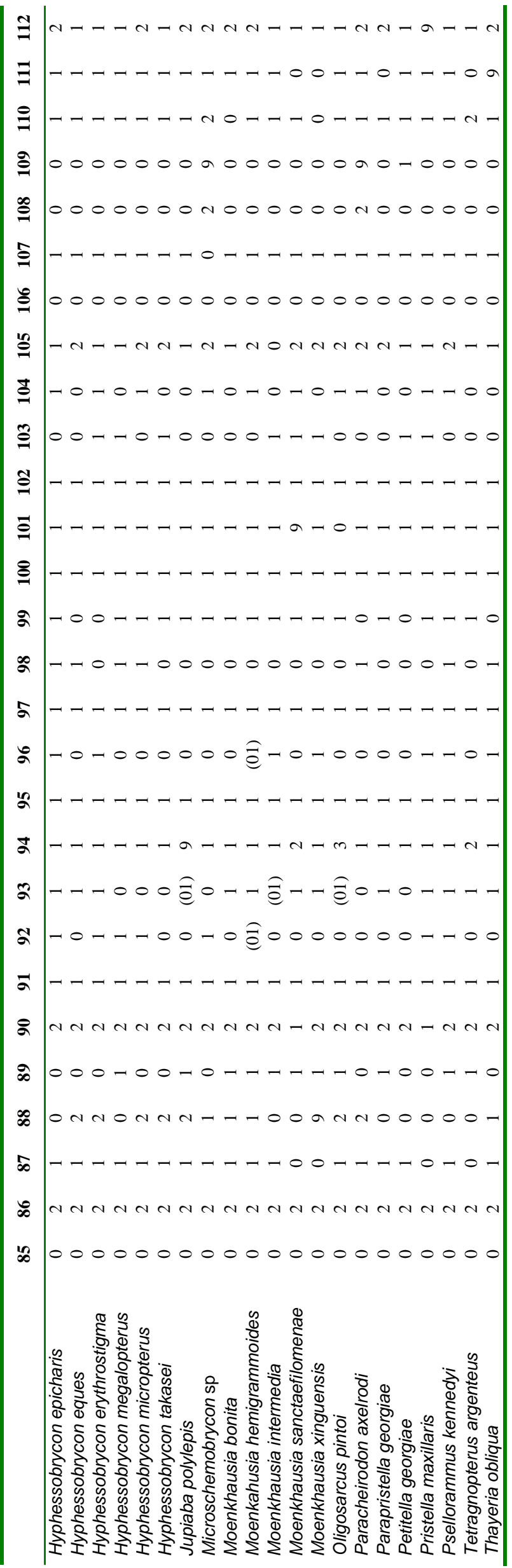




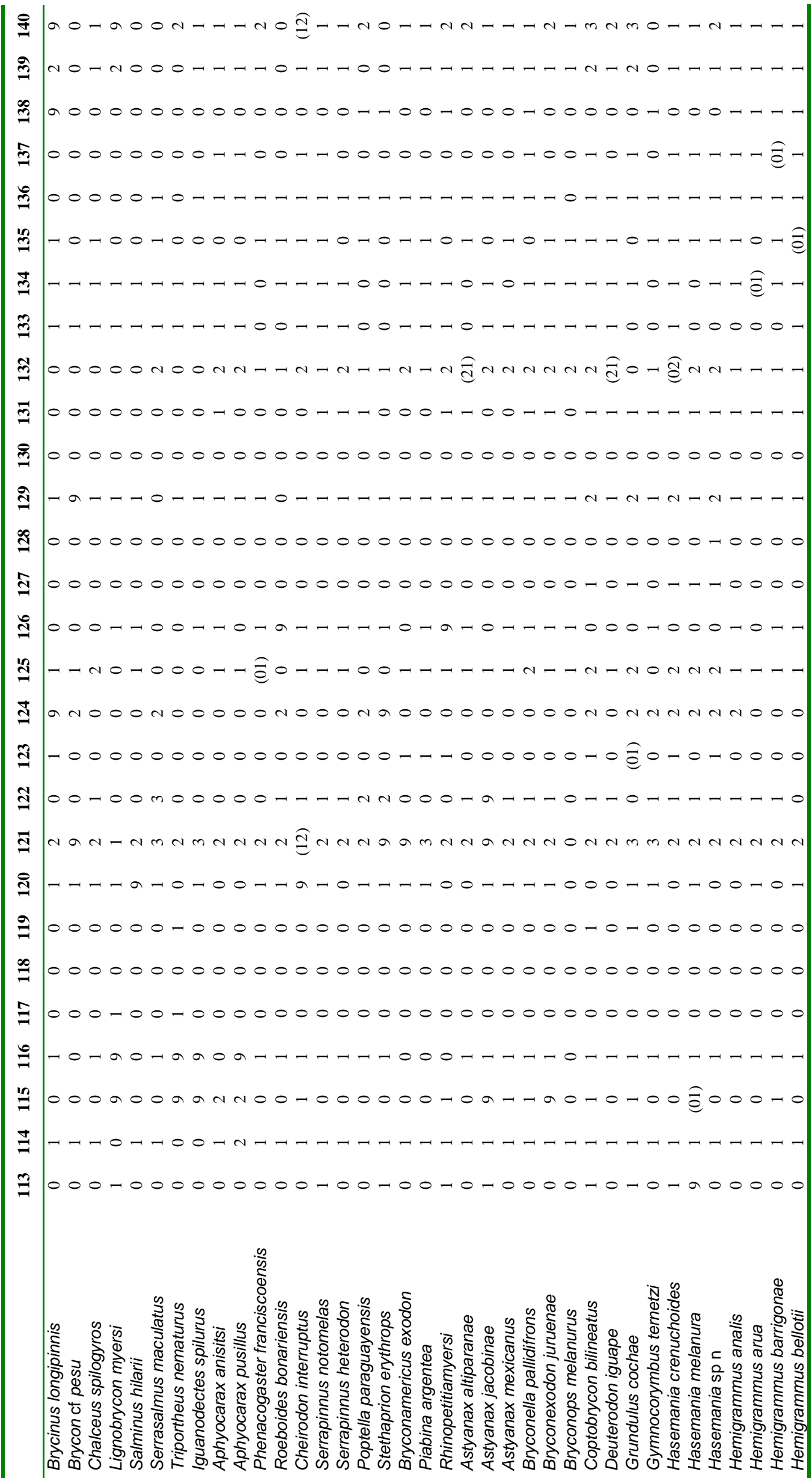




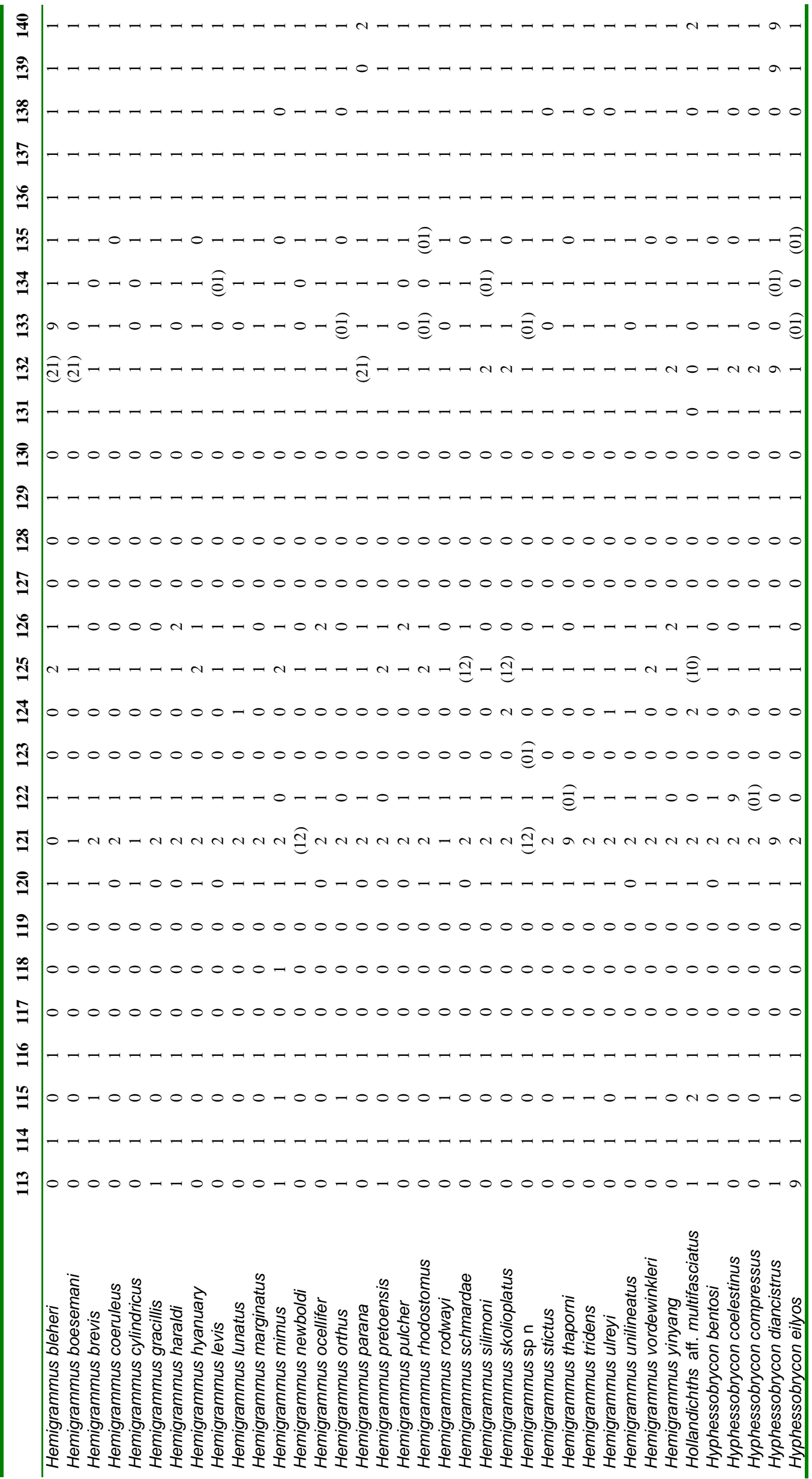




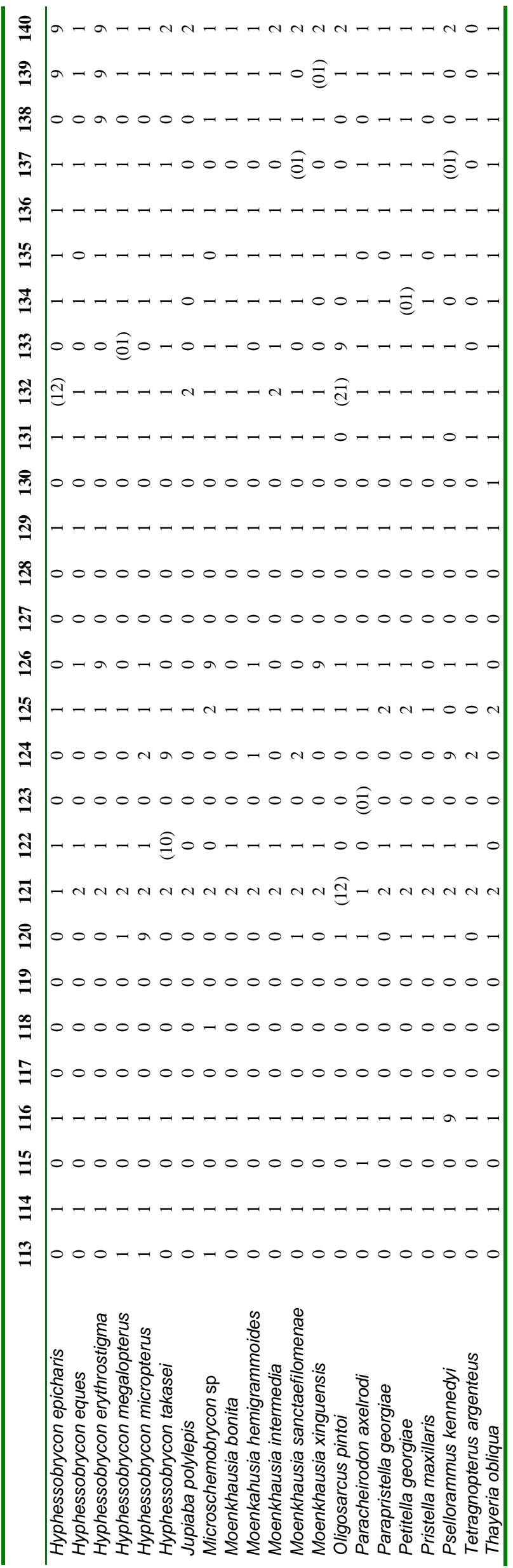




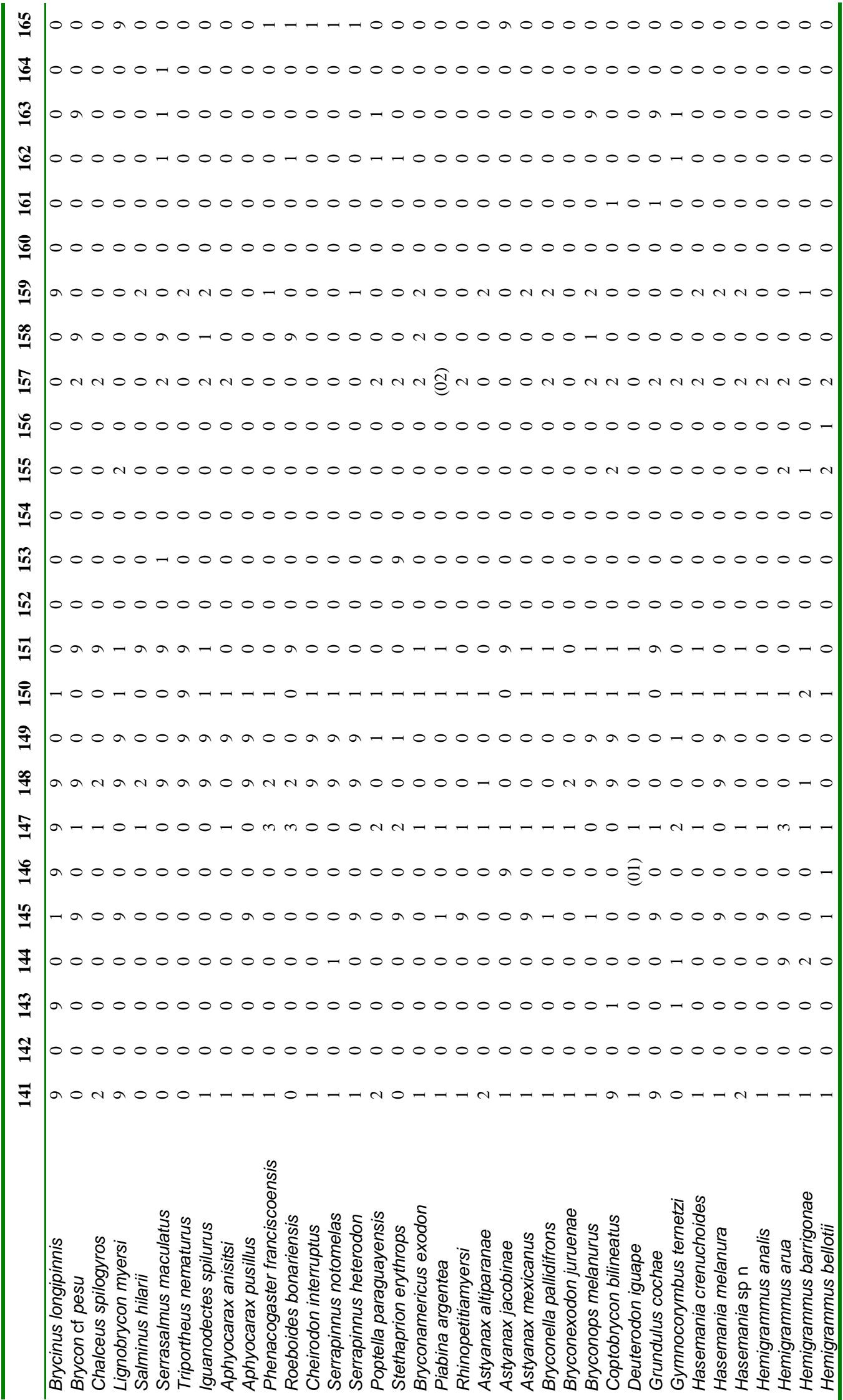




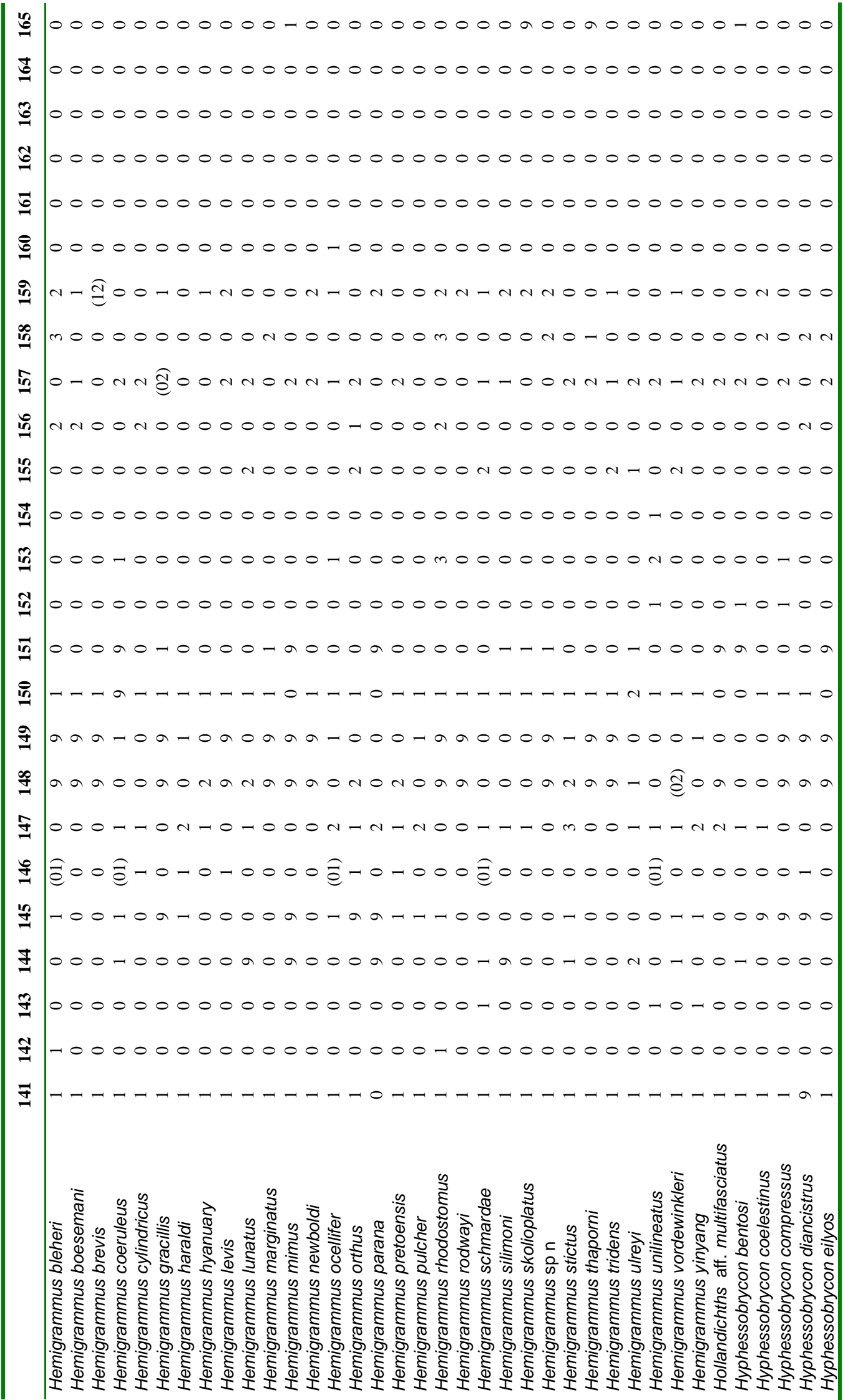




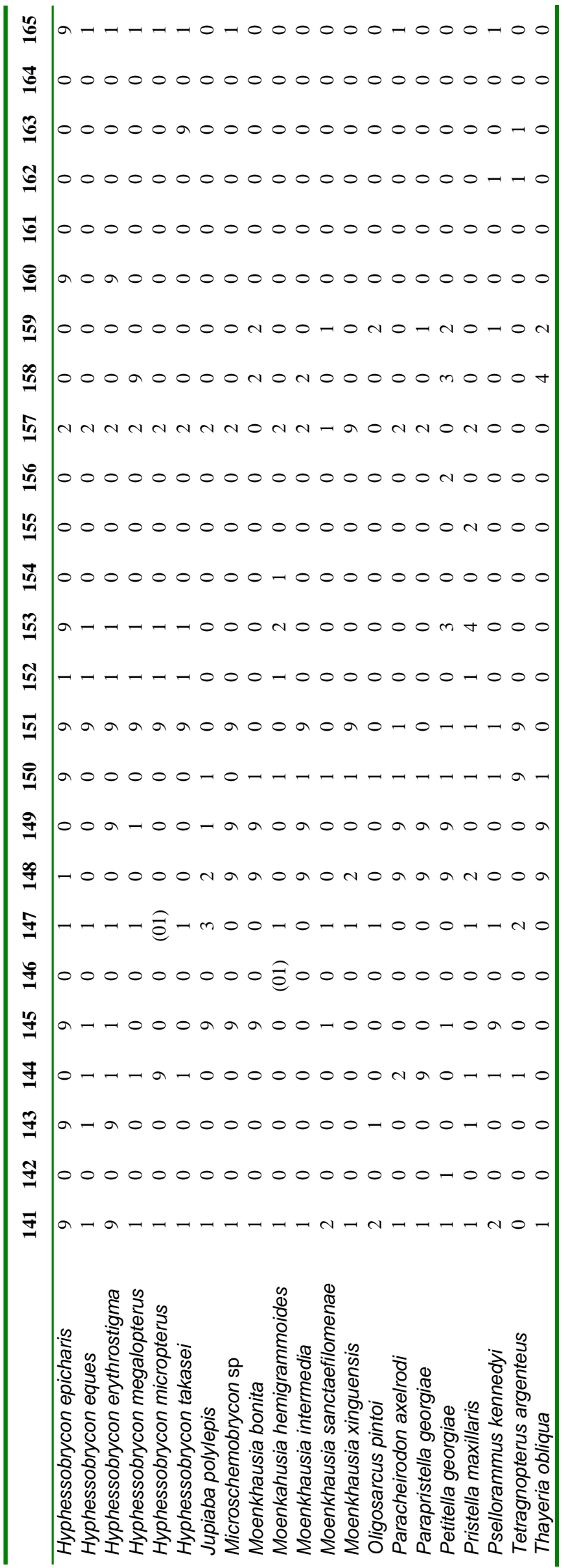




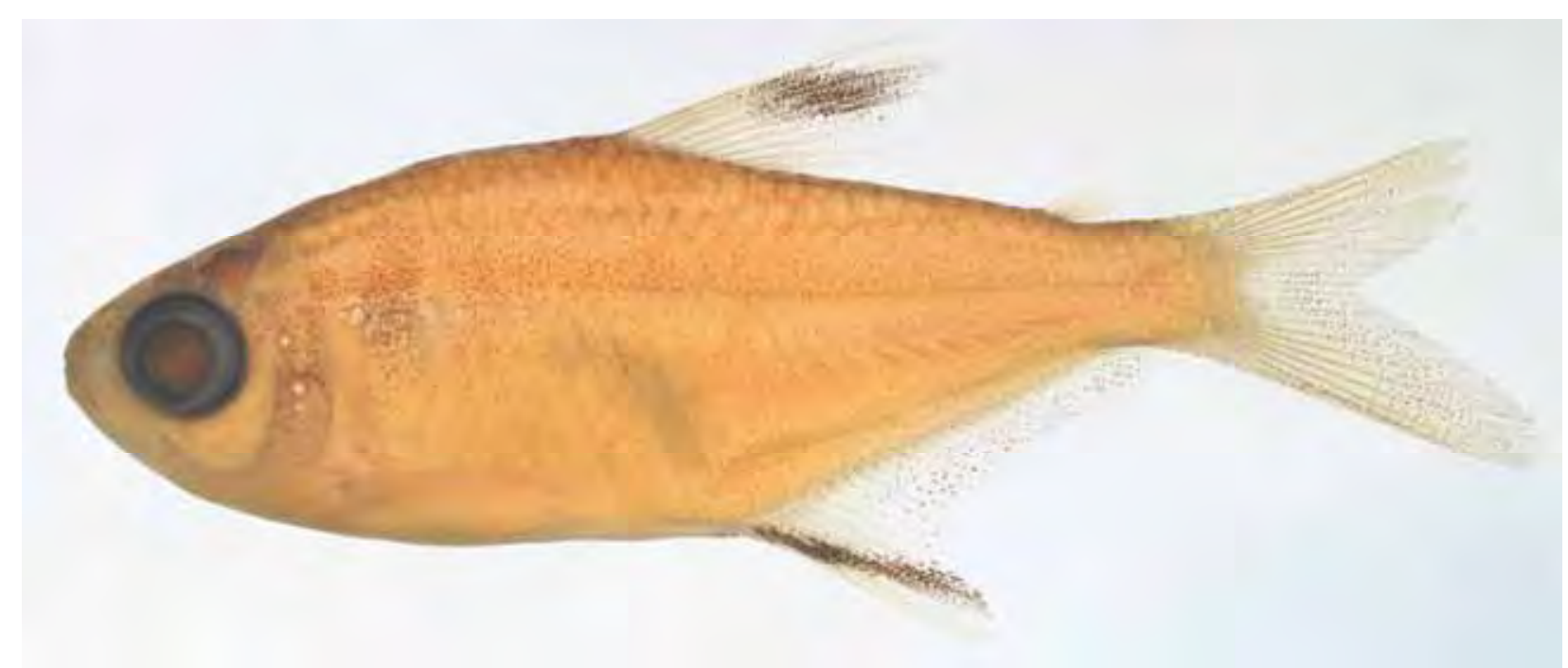

Figura 1- Hemigrammus unilineatus ANSP 147082, 27,7 mm CP, West Indies, Trinidad and Tobago, La Selva river. 


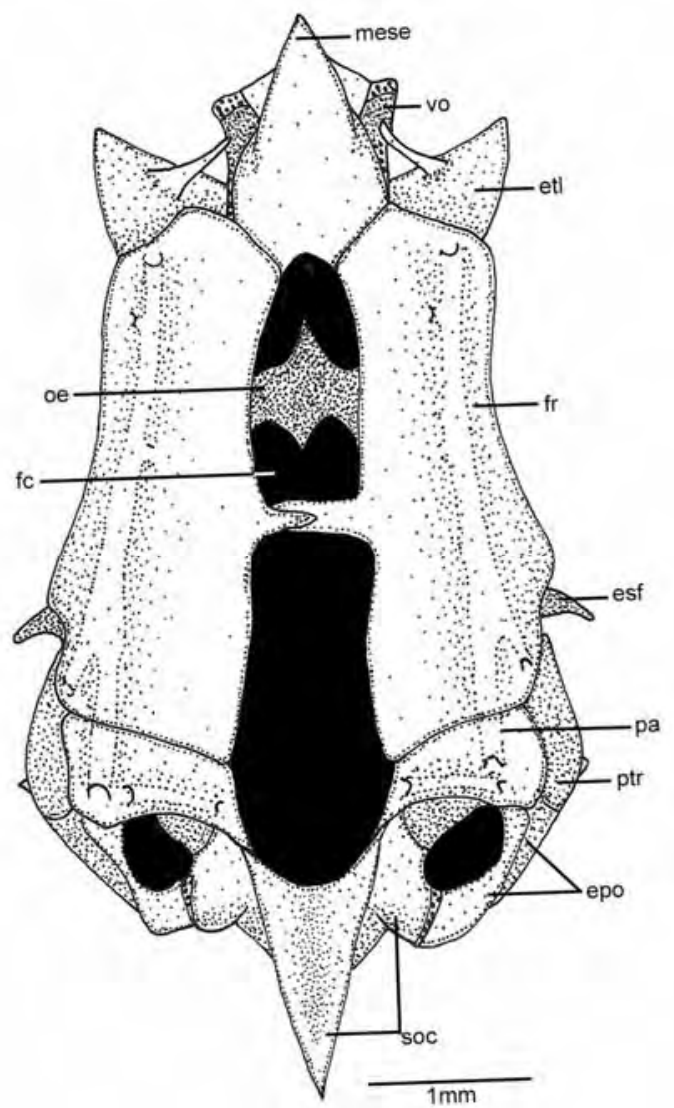

Figura 2- Hemigrammus unilineatus MZUSP 65409, 25,8 mm CP. Crânio, vista dorsal.

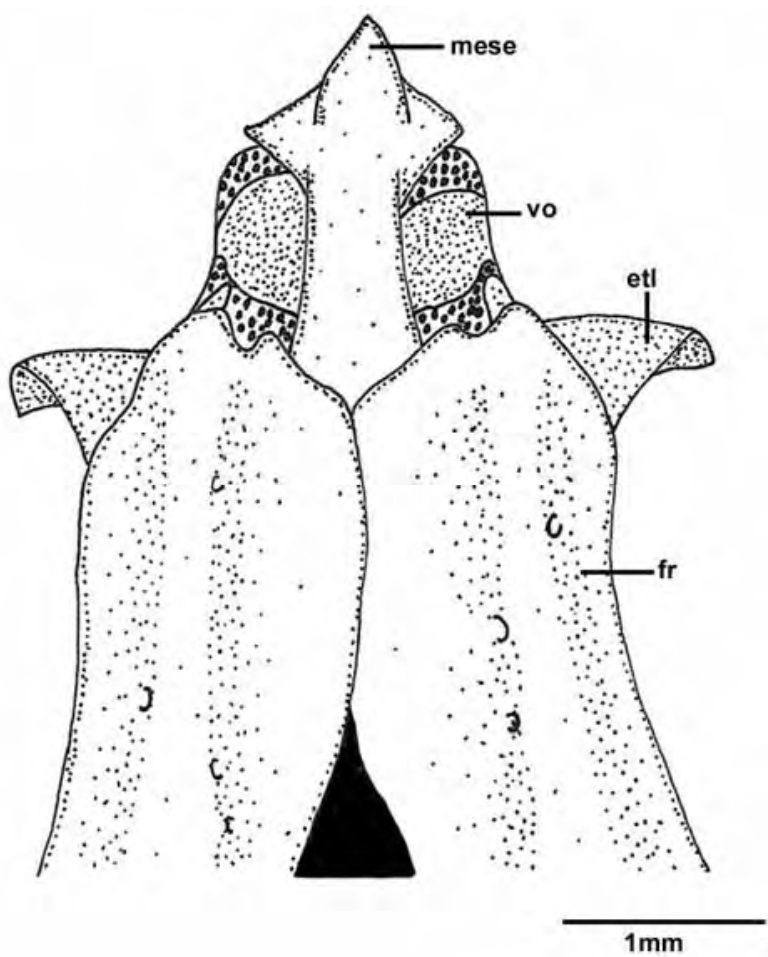

Figura 3- Hemigrammus bleheri MCP 14921, 28,4 mm CP. Região anterior do crânio, vista dorsal. 


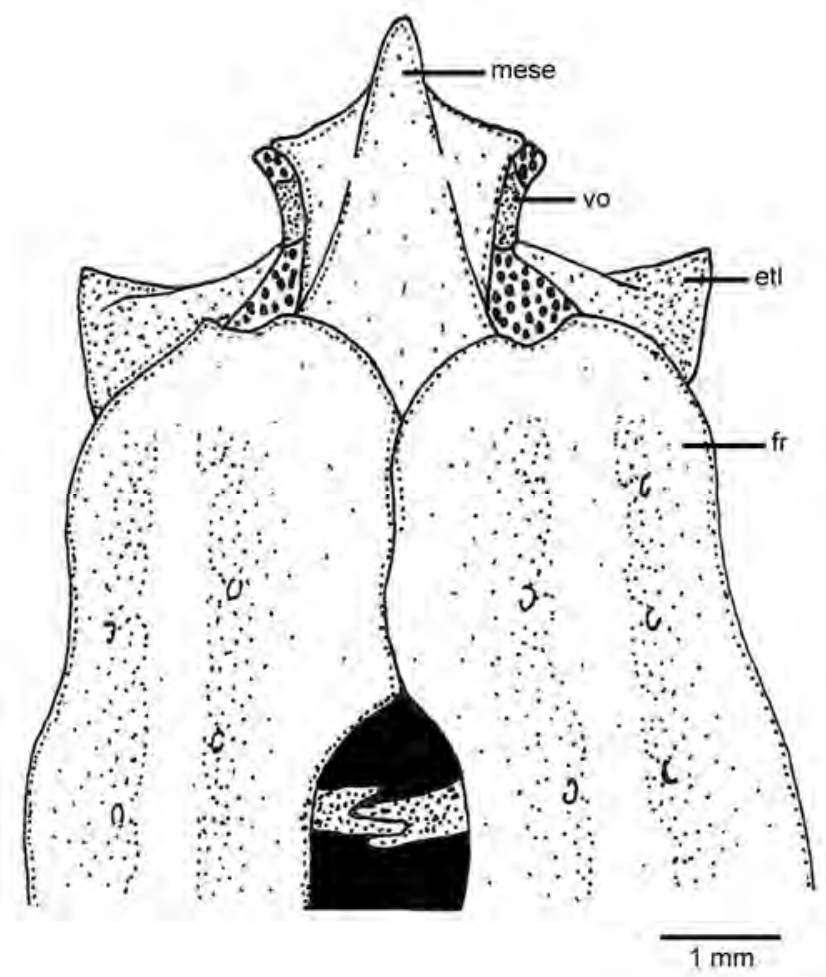

Figura 4- Hasemania crenuchoides DZSJRP 11039, 50,7 mm CP. Região anterior do crânio, vista dorsal.

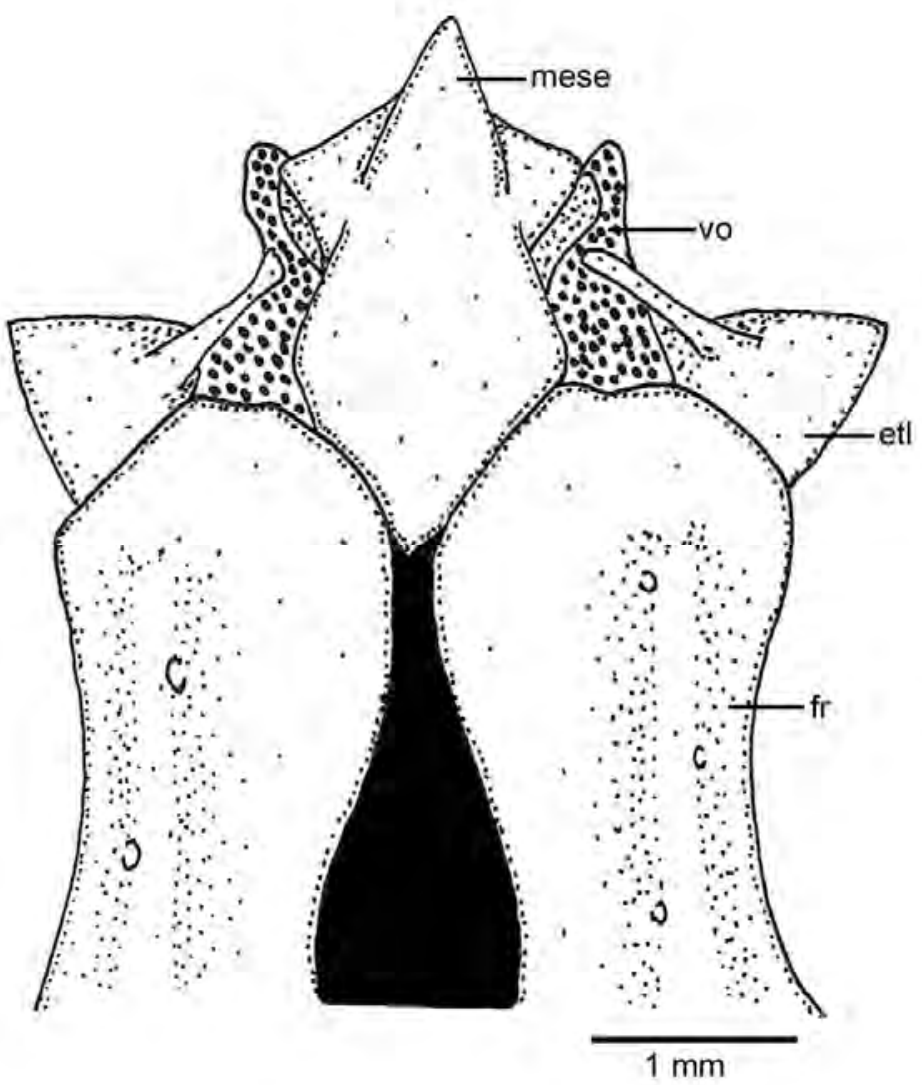

Figura 5- Hemigrammus lunatus MZUSP 90274, 27,8 mm CP. Região antertior do crânio, vista dorrsal. 


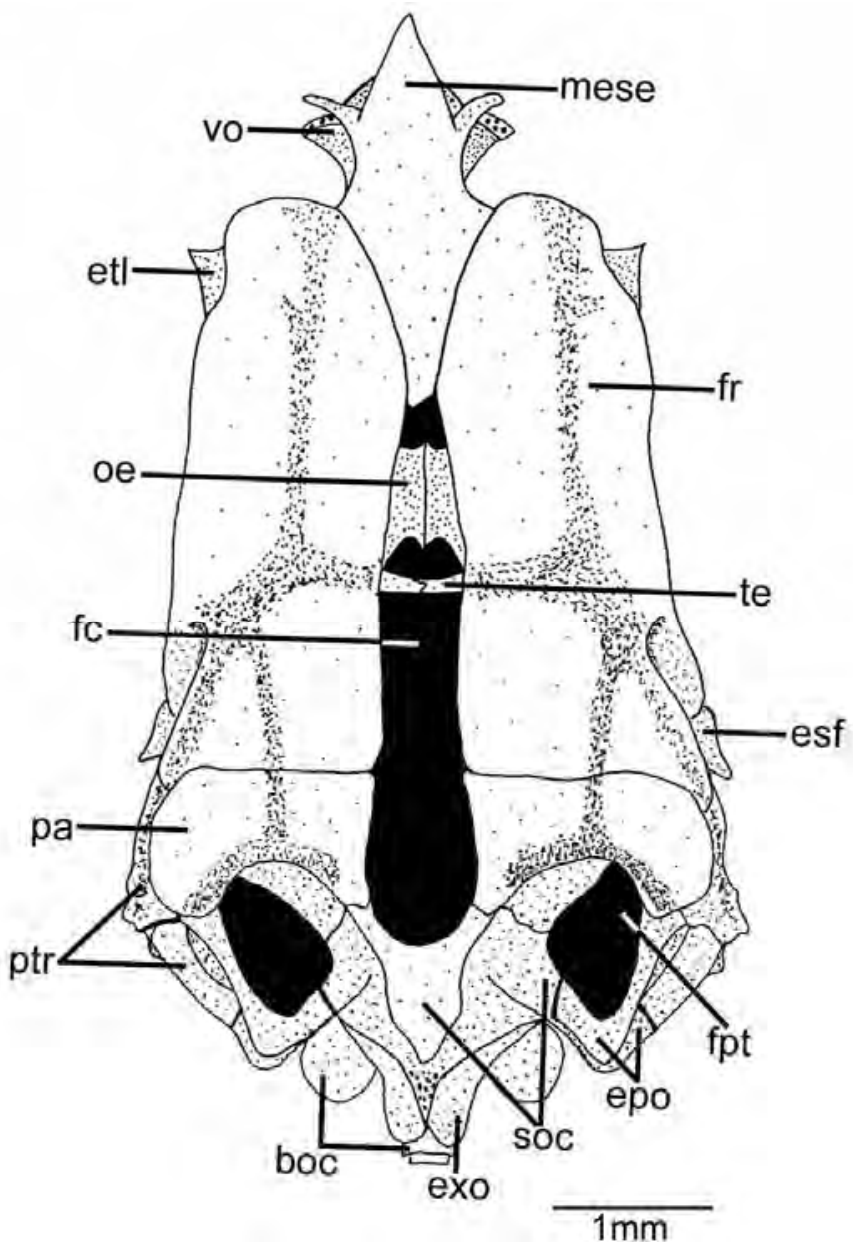

Figura 6- Bryconamericus exodon MZUSP 28026, 43,1 mm CP. Crânio, vista dorsal.

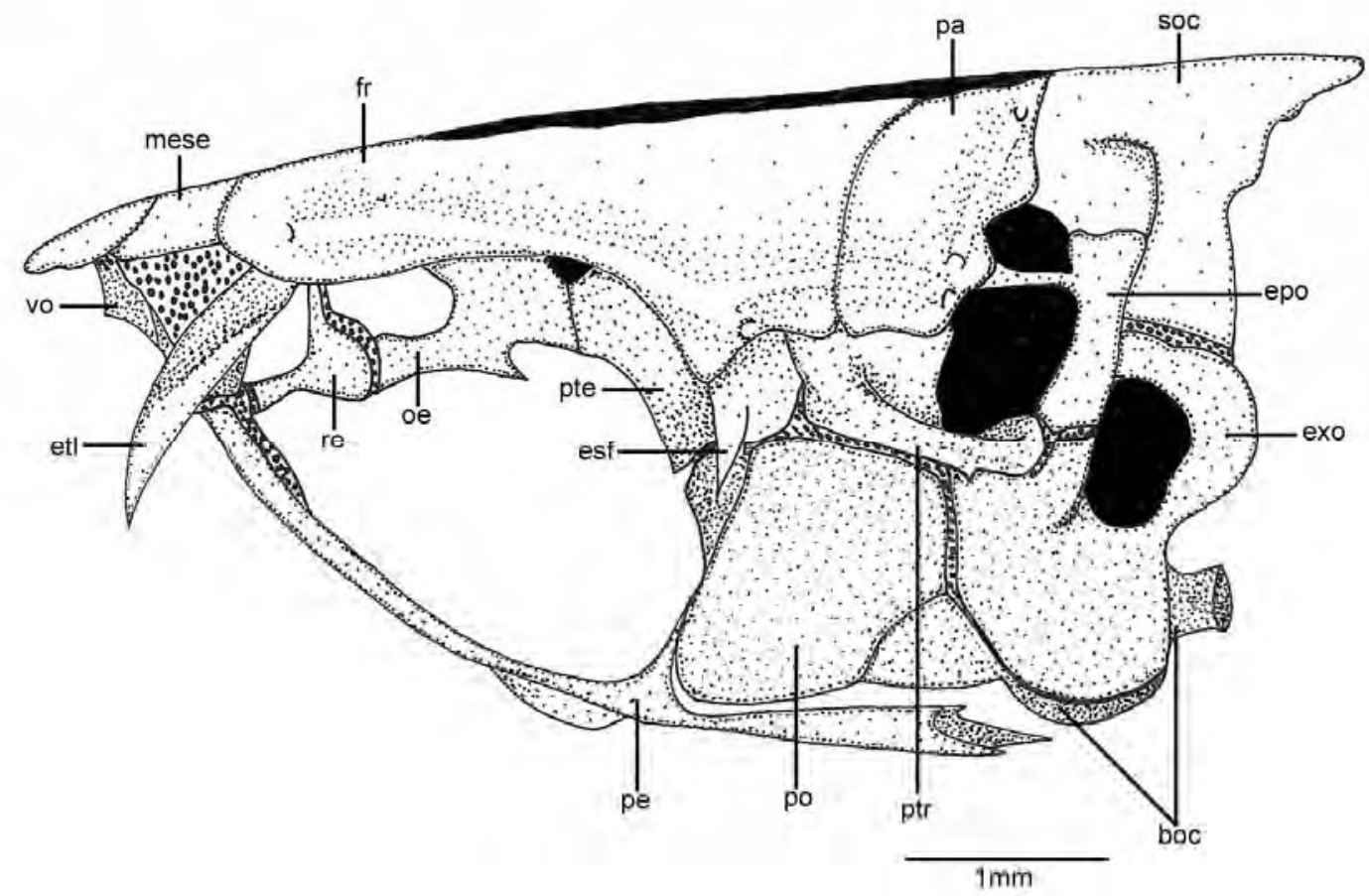

Figura 7- Hemigrammus unilineatus MZUSP 65409, 25,8 mm CP. Crânio, vista lateral. 


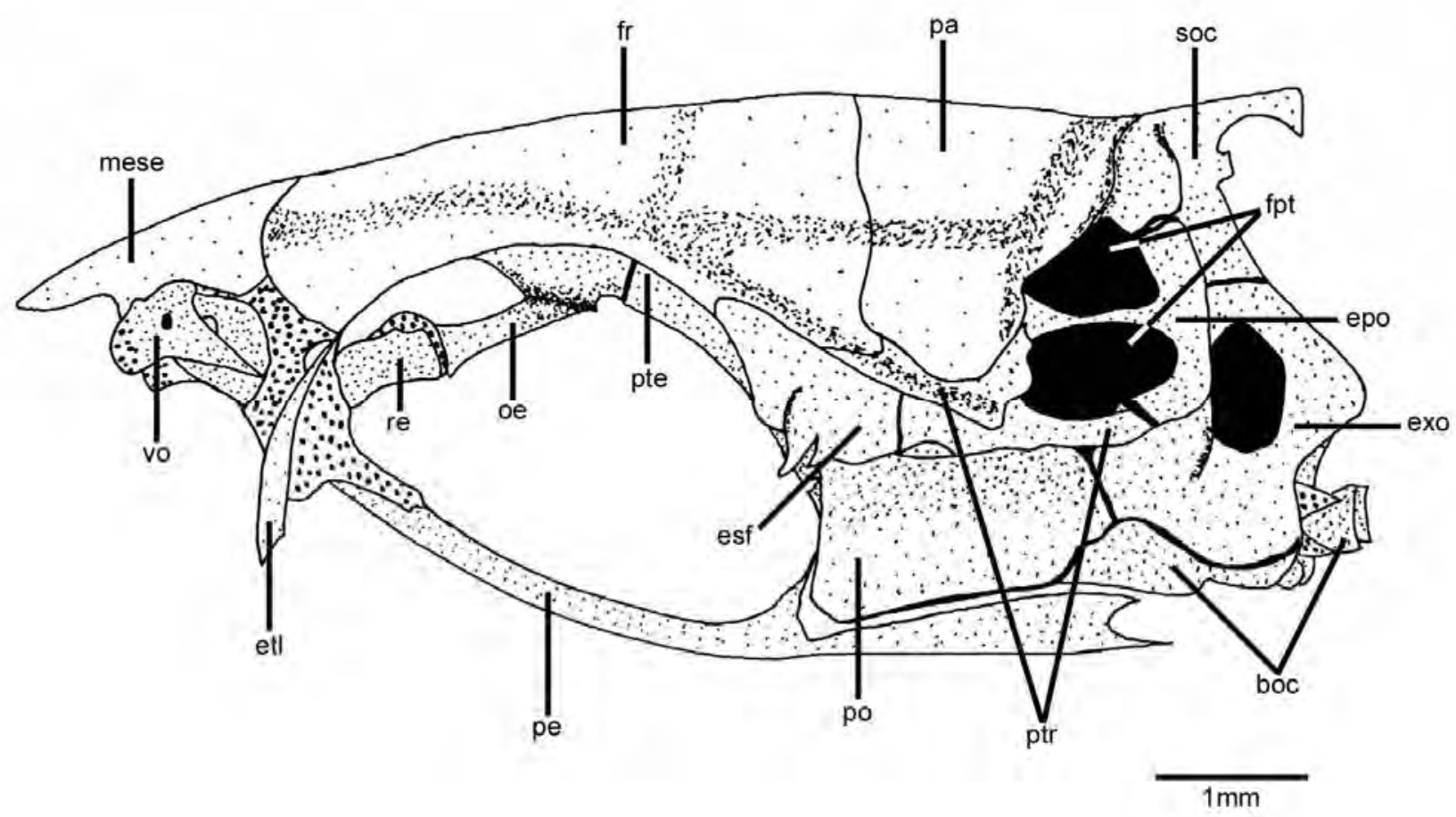

Figura 8- Bryconamericus exodon MZUSP 28026, 43,1 mm CP. Crânio, vista lateral.

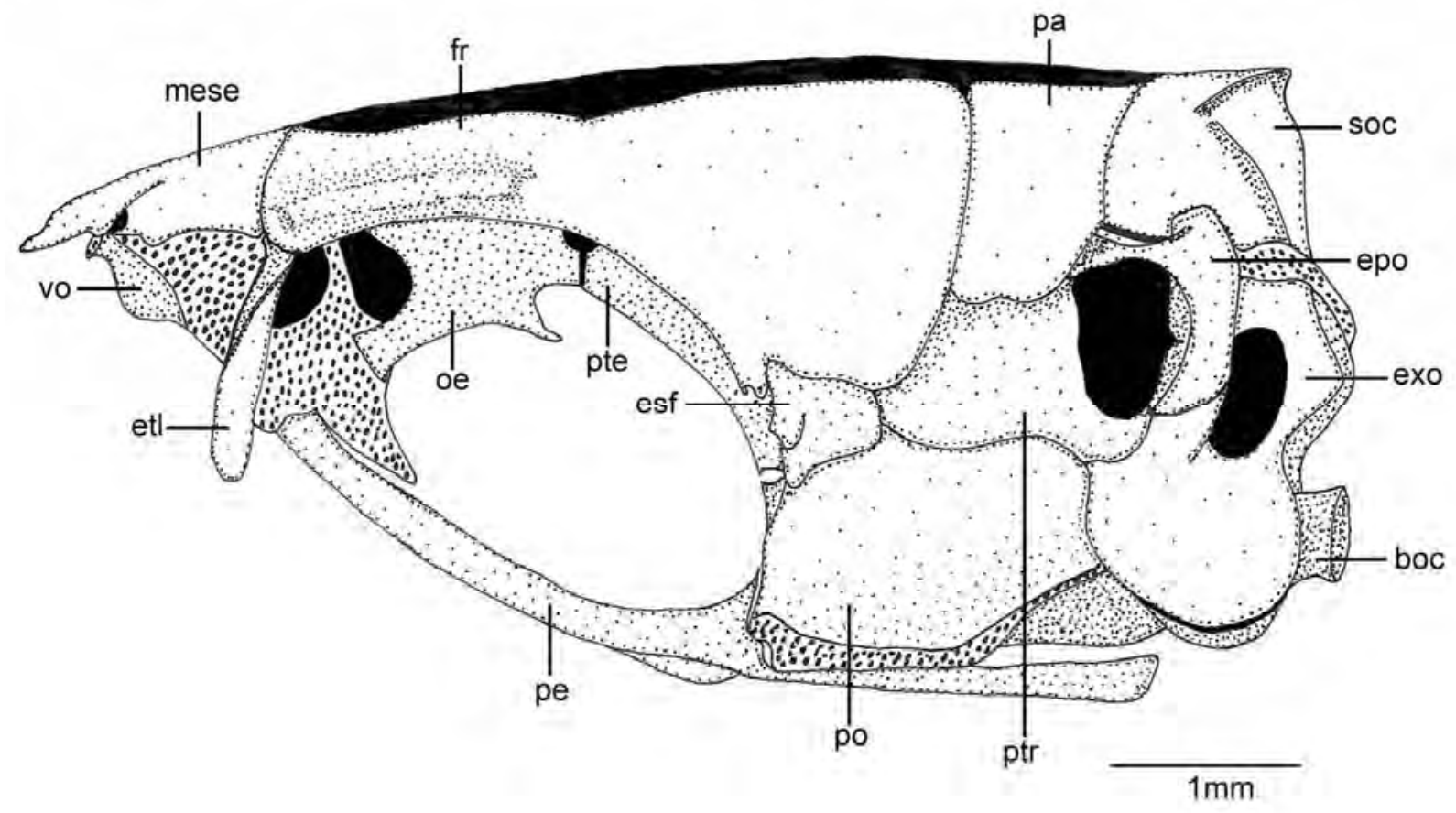

Figura 9- Coptobrycon bilineatus DZSJRP 6890, 36,3 mm CP. Crânio, vista lateral. 


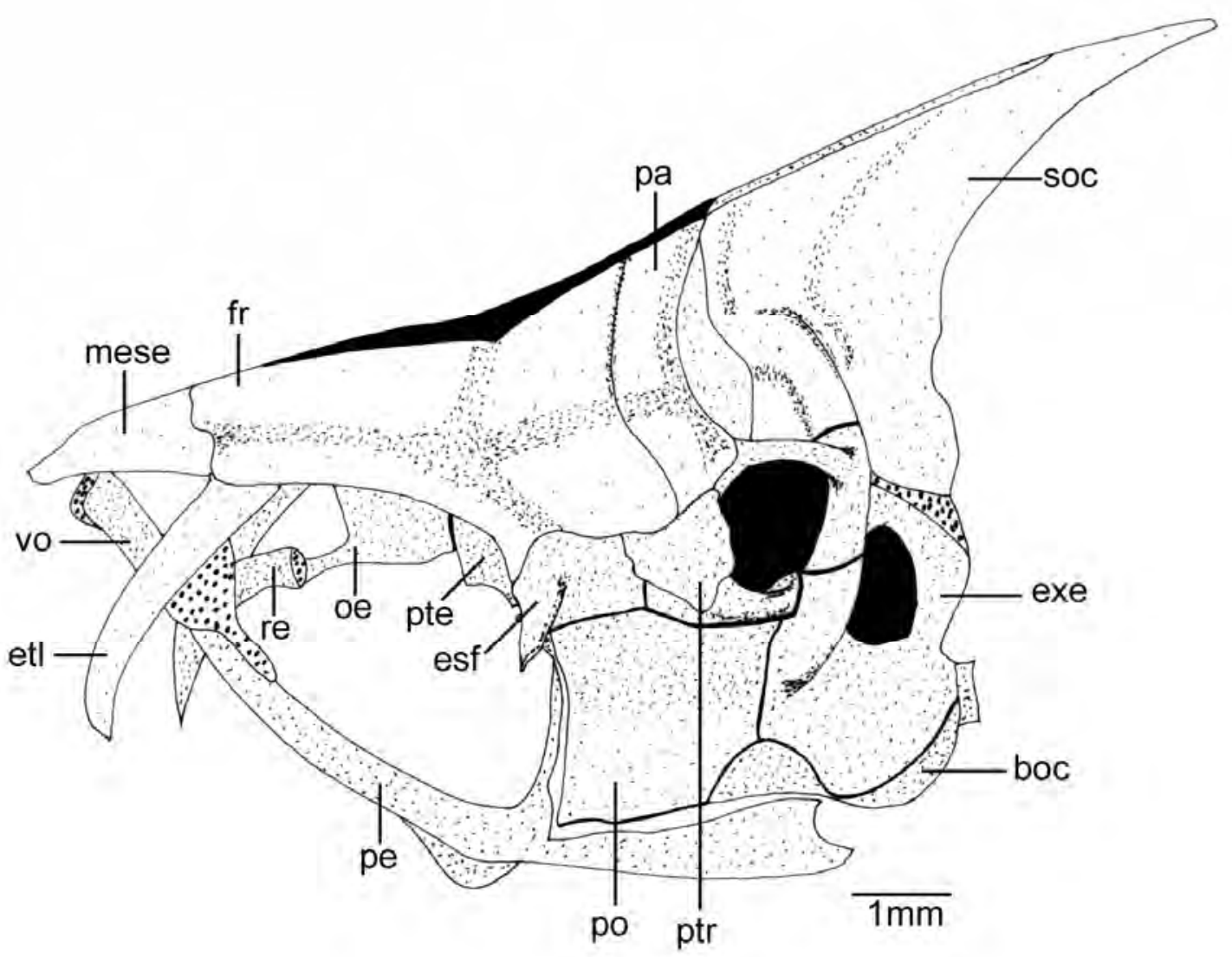

Figura 10- Gymnocorymbus ternetzi DZSJRP 2808, 30,4 mm CP. Crânio, vista lateral.

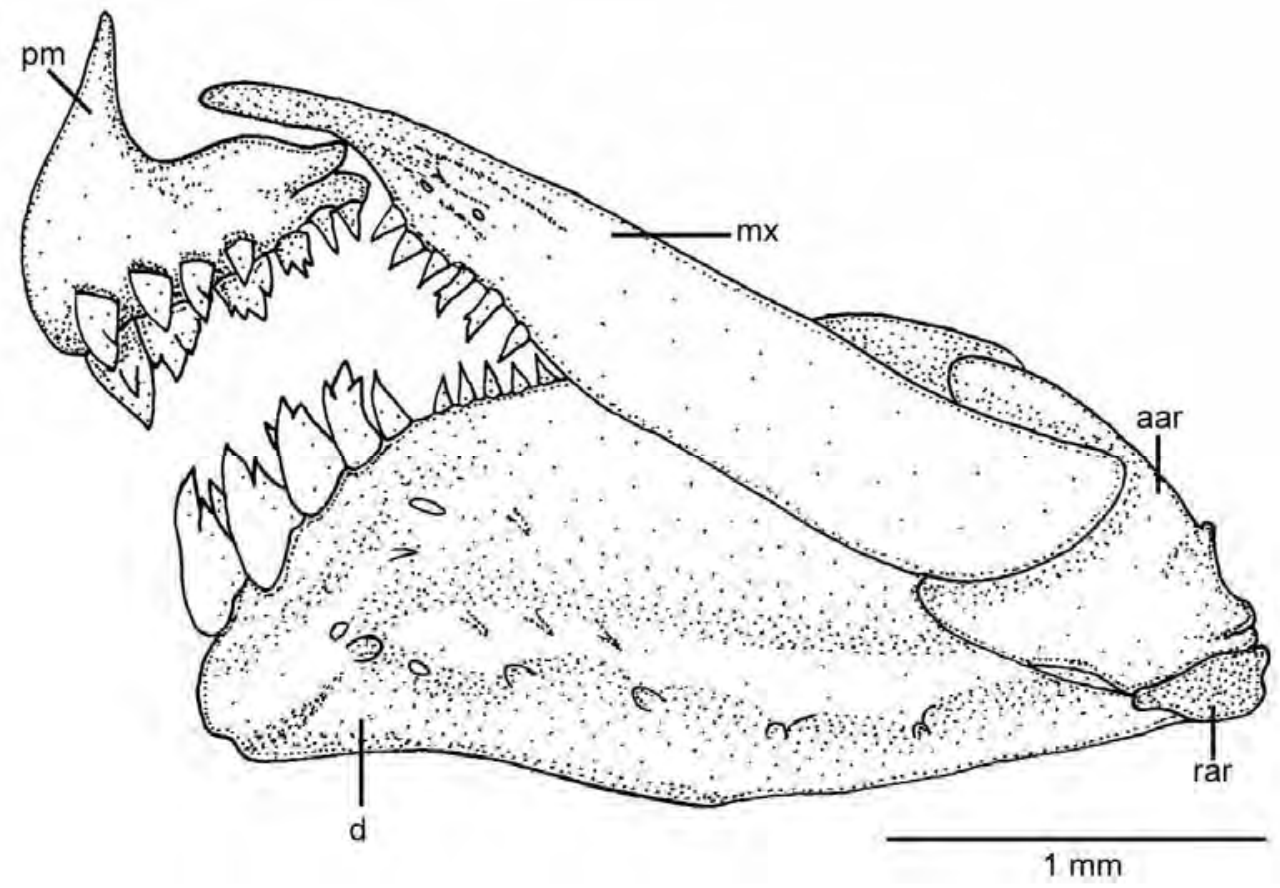

Figura 11- Hemigrammus unilineatus, MZUSP 65409, 25,8 mm CP. Maxilas, vista lateral do lado esquerdo. 


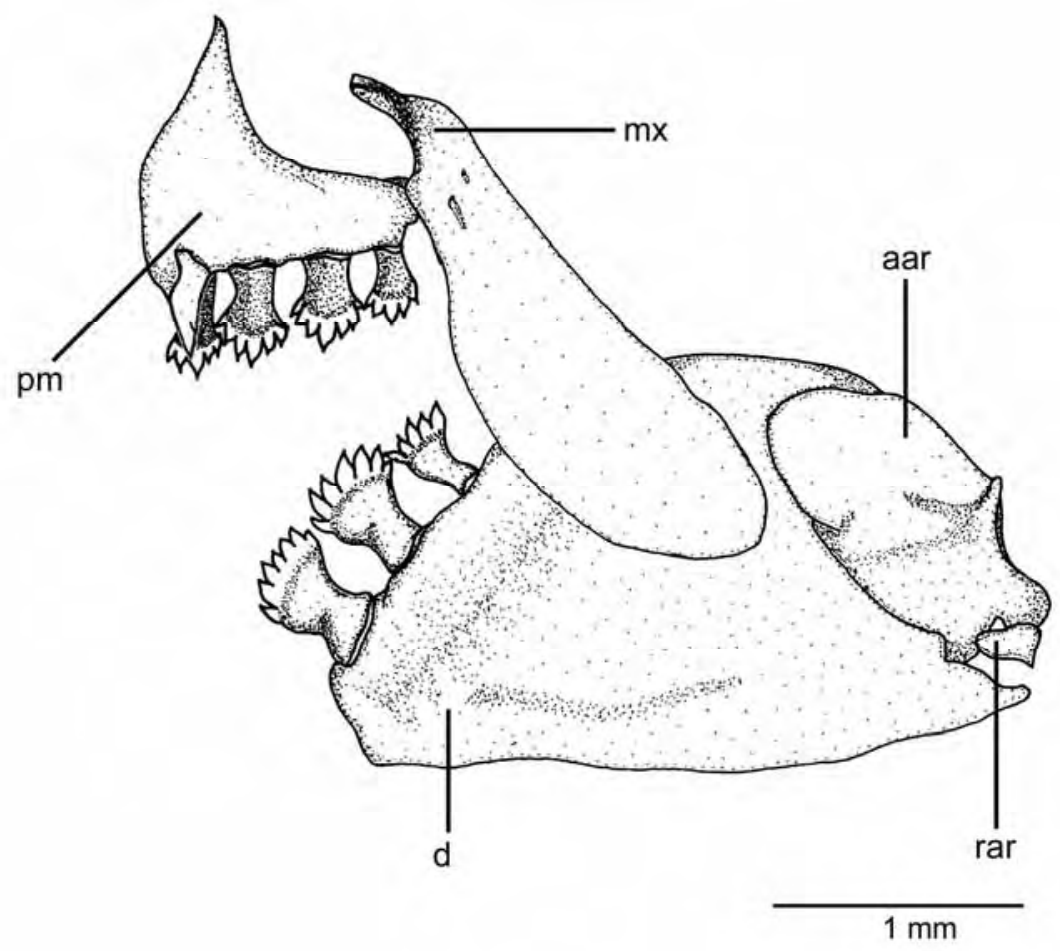

Figura 12- Coptobrycon bilineatus DZSJRP 6890, 36,3 mm CP. Maxilas, vista lateral do lado esquerdo.

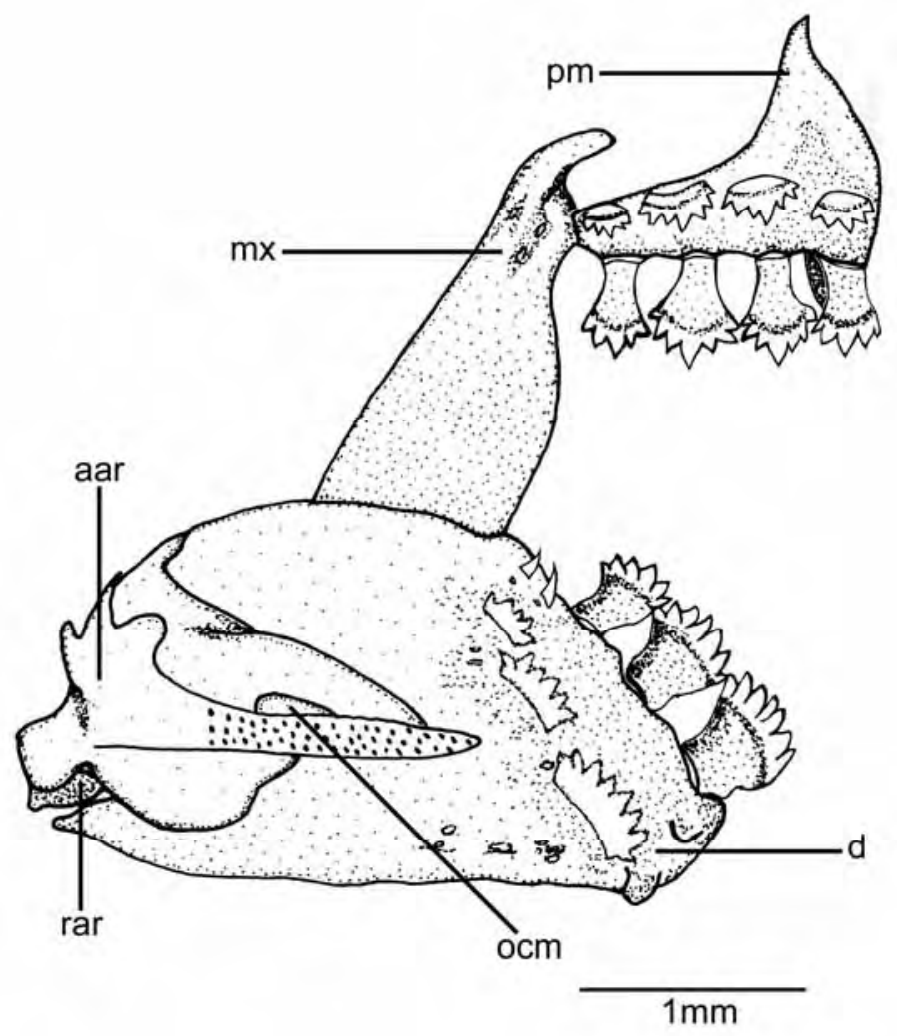

Figura 13- Coptobrycon bilineatus DZSJRP 6890, 36,3 mm CP. Maxilas, vista medial do lado esquerdo. 


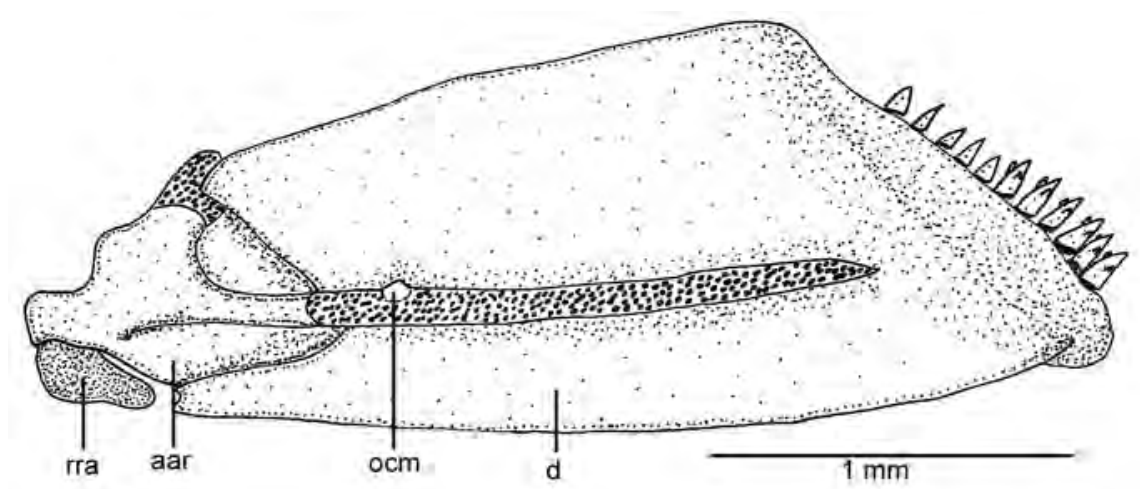

Figura 14- Hemigrammus mimus MZUSP 28018, 22,2 mm CP. Dentário, vista medial do lado esquerdo.

A

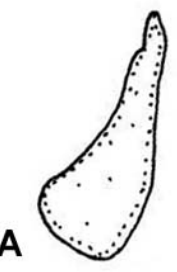

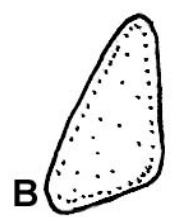
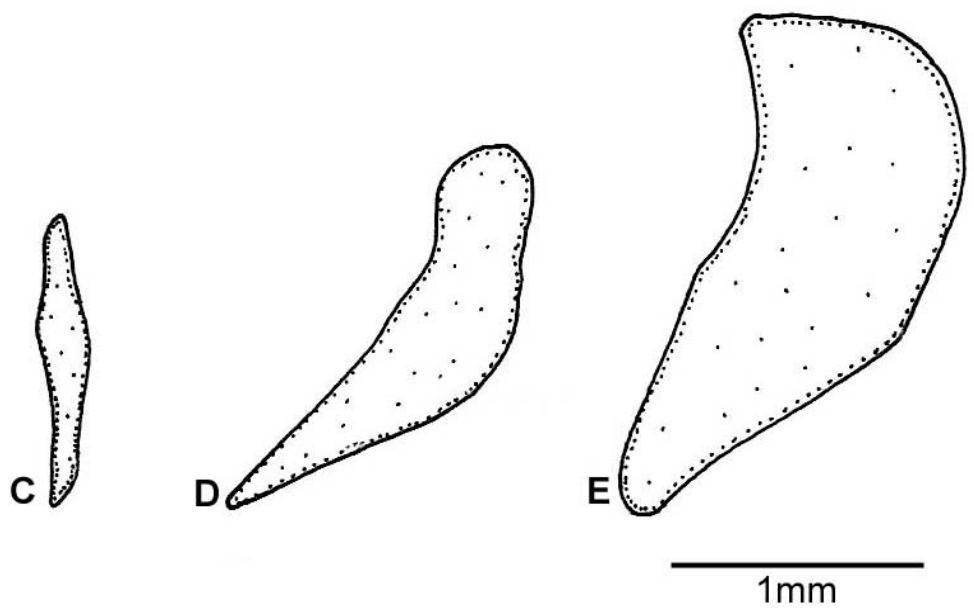

Figura 15- Antorbital. A) Hemigrammus unilineatus MZUSP 65409, 28,5 mm $\mathrm{CP}$; B) Hemigrammus hyanuary MZUSP 42348, 22,8 $\mathrm{mm}$ CP; C) Hemigrammus mimus MZUSP 28018, 22,2 mm CP; D) Coptobrycon bilineatus DZSJRP 6889, 36,3 mm CP e E) Lignobrycon myersi MCP 39737, 51,7 mm CP. 


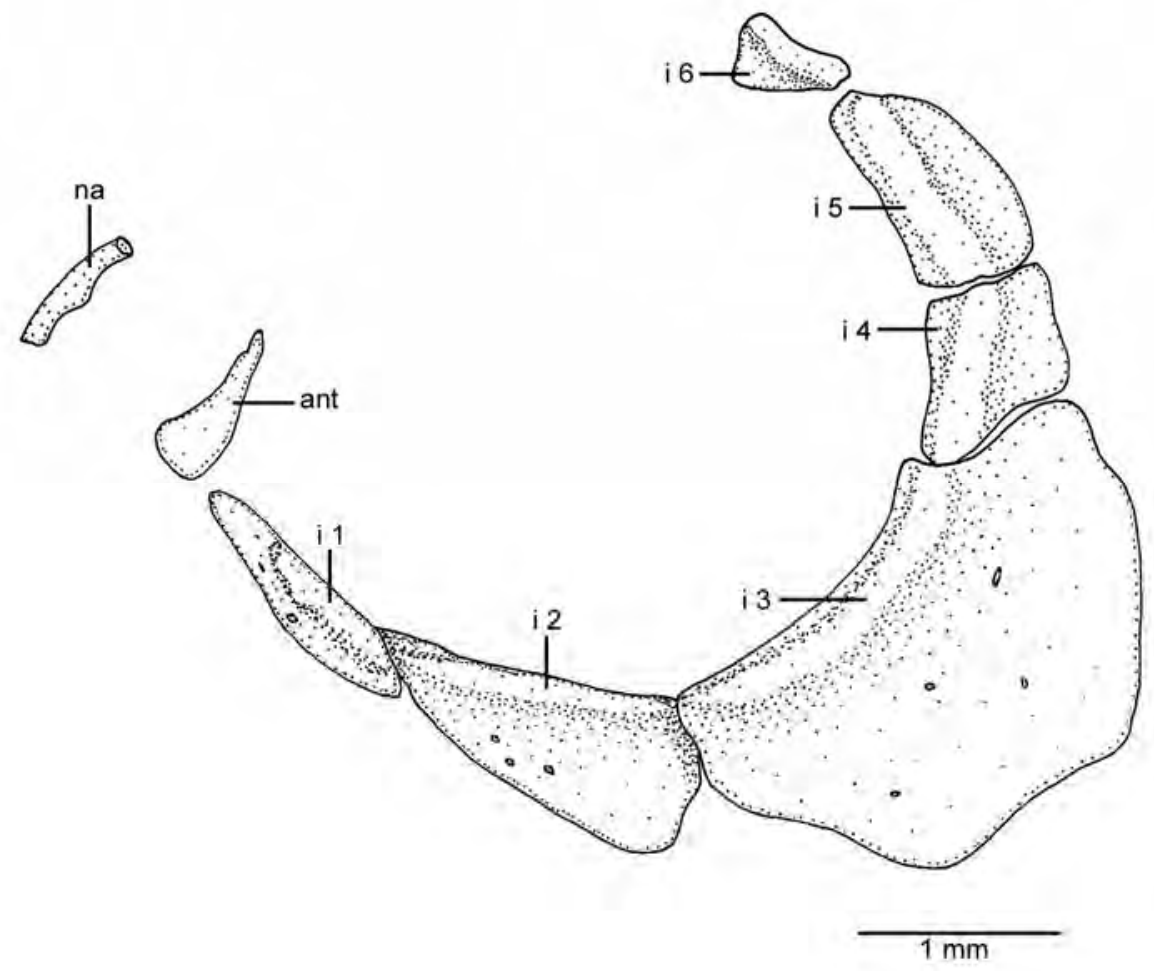

Figura 16- Hemigrammus unilineatus, MZUSP 65409, 25,8 mm CP. Infraorbitais, antorbital e nasal, vista lateral do lado direito (refletido).

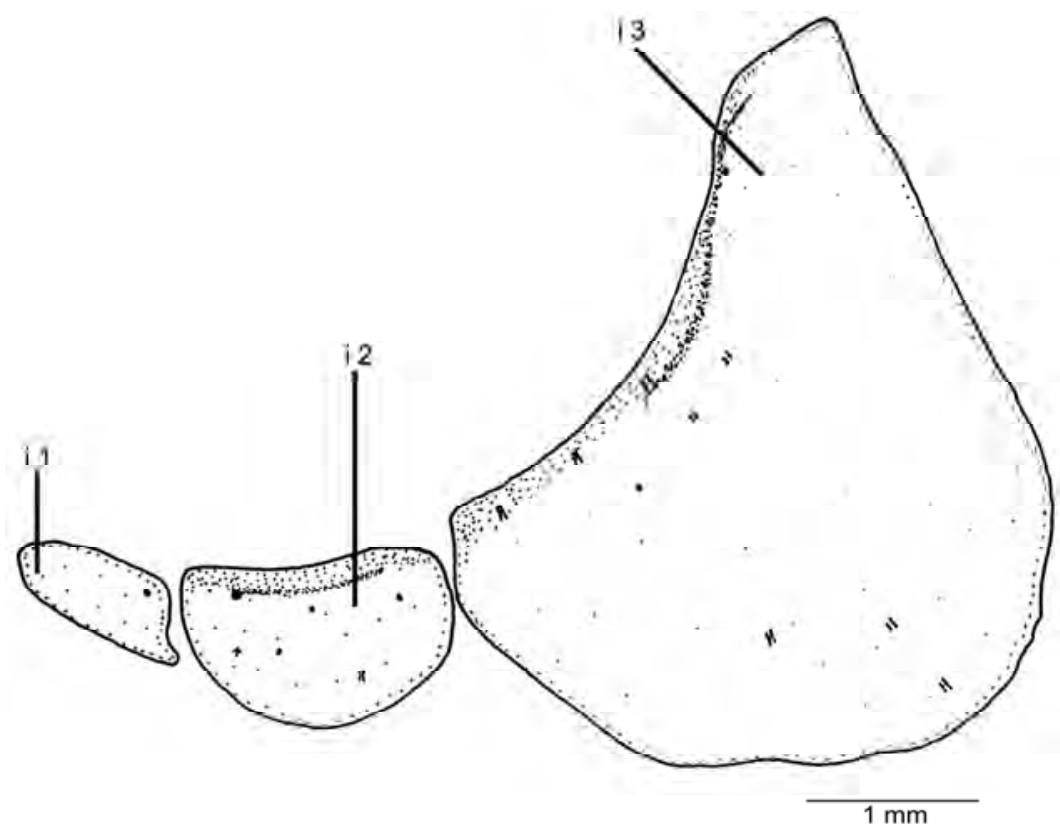

Figura 17- Coptobrycon bilineatus DZSJRP 6890, 36,3 mm CP. Infra-orbitais, vista lateral do lado esquerdo. 


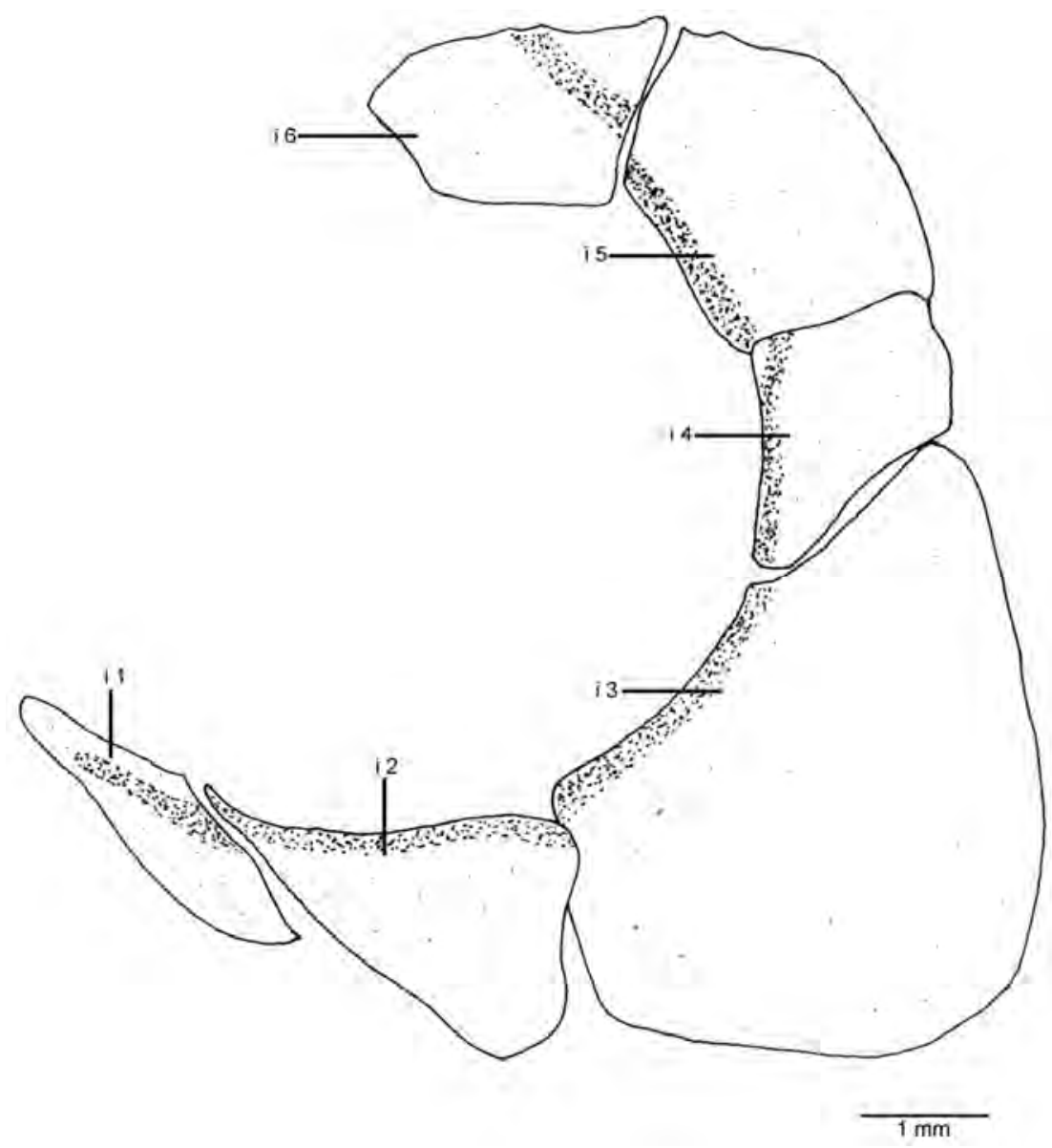

Figura 18- Astyanax altiparanae DZSJRP 3297, 48,8 mm CP. Infra-orbitais, vista lateral do lado esquerdo.
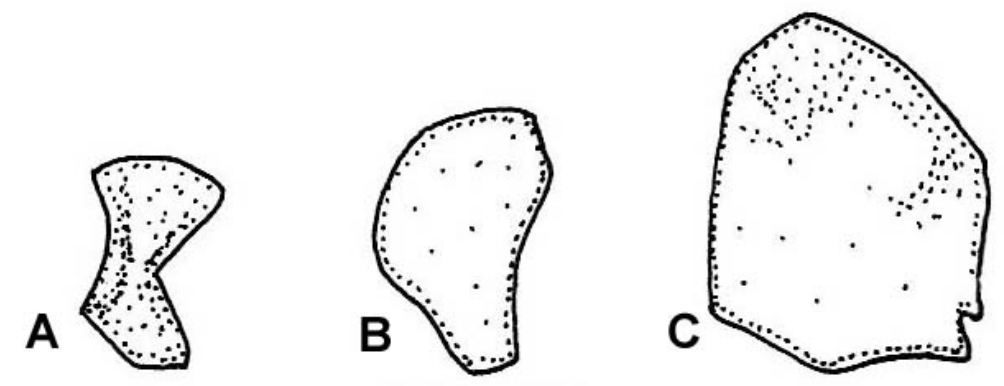

\section{$1 \mathrm{~mm}$}

Figura 19- Palatino. A) Hemigrammus unilineatus MZUSP 65409, 28,5 mm CP; B) Aphyocharax anisitsi DZSJRP 7573, $29 \mathrm{~mm}$ CP e C) Hasemania crenuchoides DZSJRP 11039, 50,7 mm CP. 


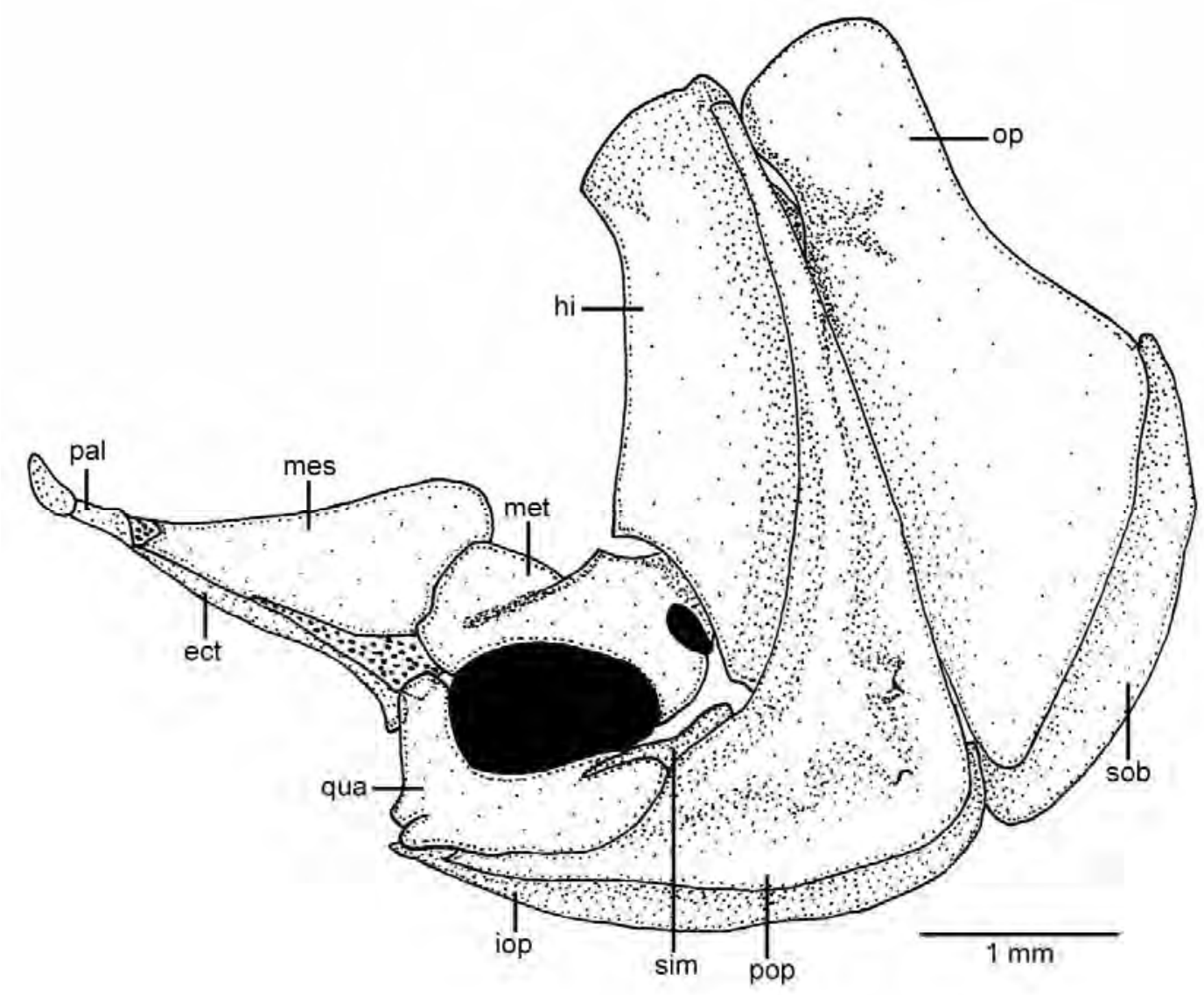

Figura 20- Hemigrammus unilineatus MZUSP 65409, 25,8 mm CP. Arco palatino, suspensório e ossos operculares, vista lateral do lado esquerdo.
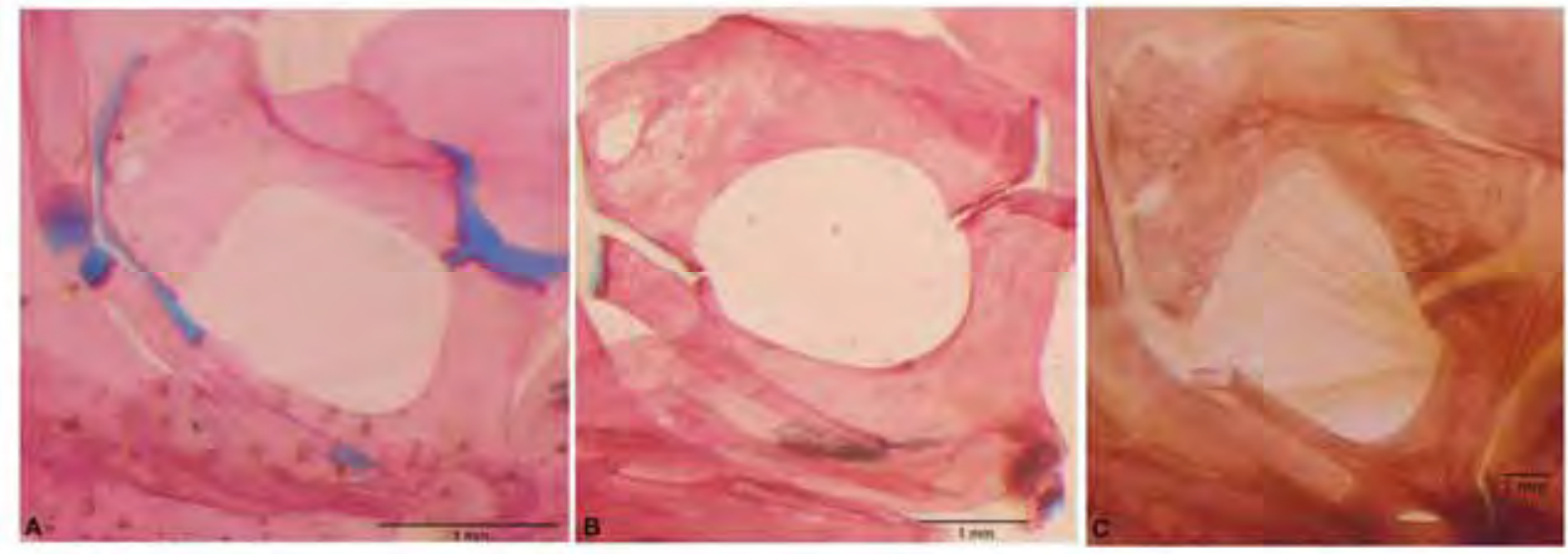

Figura 21- Parte do suspensório esquerdo em vista medial, detalhe da fenestra entre metapterigóide e quadrado. A) Hemigrammus eilyos DZSJRP 3092, 22,9 mm CP; B) Hemigrammus taphorni MCNG 55843, 41,3 mm CP e C) Salminus hilarii DZSJRP 3833, 74,8 mm CP. 


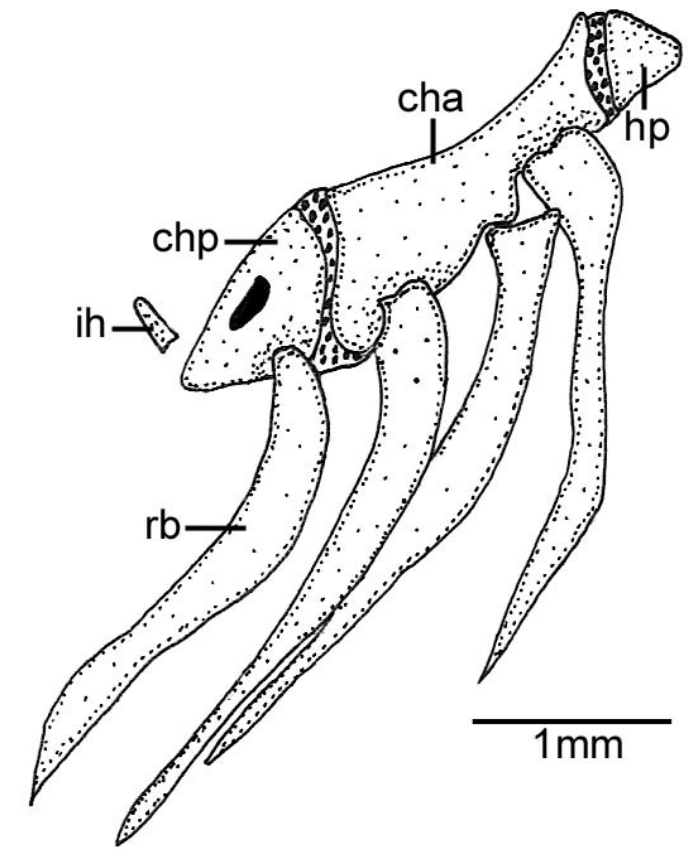

Figura 22- Hemigrammus unilineatus MZUSP 65409, 25,8 mm CP. Parte do arco hióideo, vista ventral do lado esquerdo.
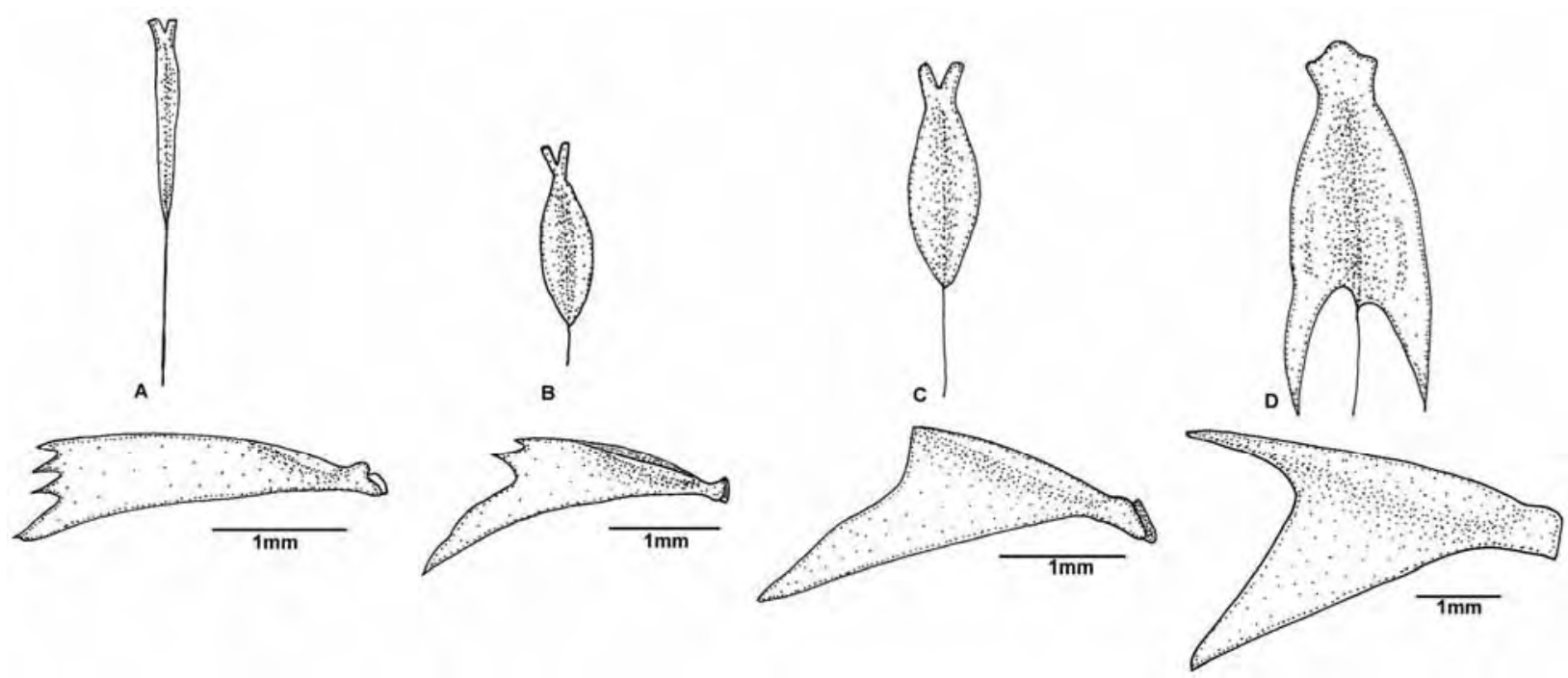

Figura 23- Uro-hial, vista ventral (superior) e vista lateral (inferior). A) Hyphessobrycon bentosi MZUSP 77528, 23,6 mm CP; B) Hemigrammus unilineatus MZUSP 65409, 25,8 mm CP; C) Hemigrammus newboldi MZUSP 103066, 33,6 mm CP e D) Brycon cf pesu DZSJRP 3803, 88,8 mm CP. 


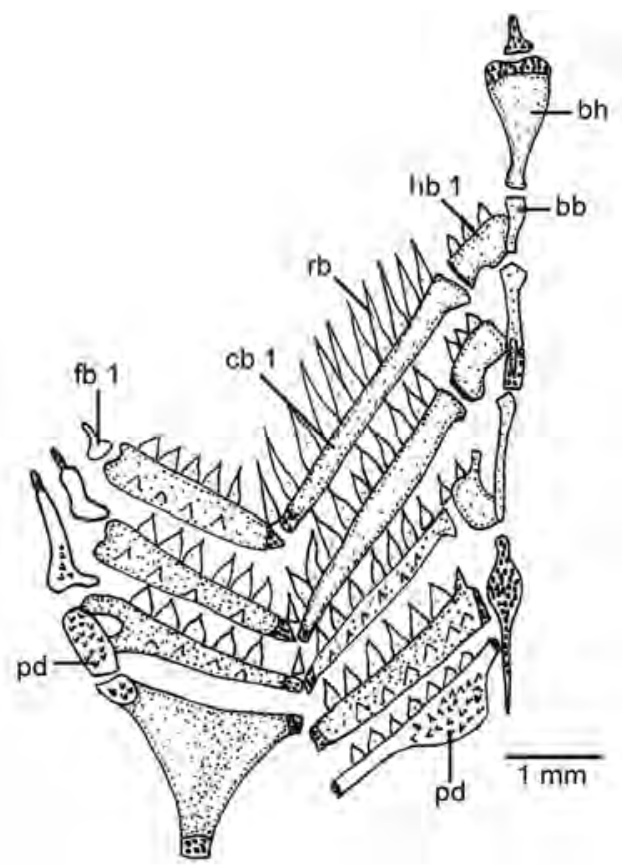

Figura 24- Hemigrammus unilineatus MZUSP 65409, 25,8 mm CP. Parte do arco branquial, vista dorsal.

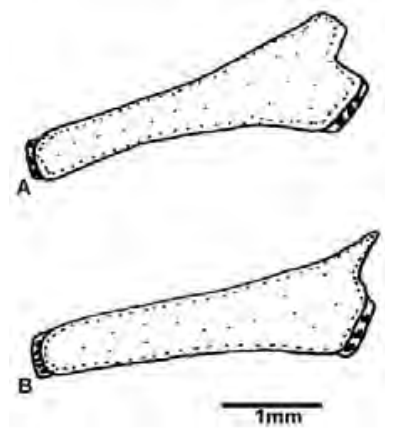

Figura 25- Ceratobranquial 4. A) Hemigrammus marginatus MZUSP 17088, 36,3 mm CP e B) Hemigrammus analis MZUSP 85667, 33,8 mm CP.

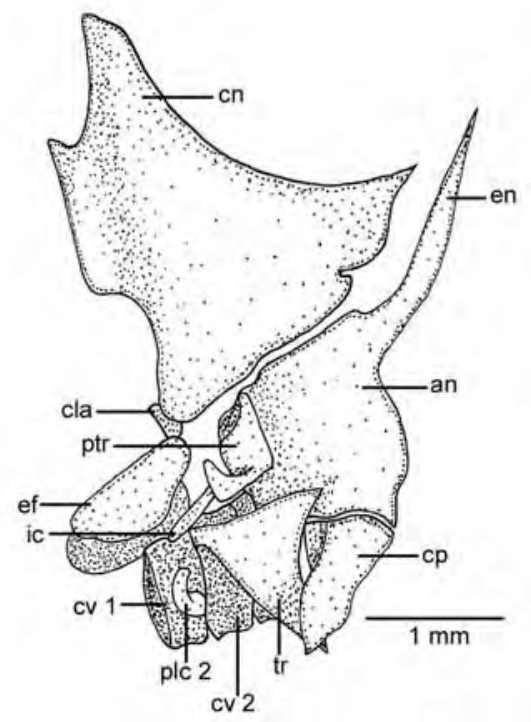

Figura 26- Hemigrammus unilineatus MZUSP 65409, 25,8 mm CP. Aparelho de Weber. 


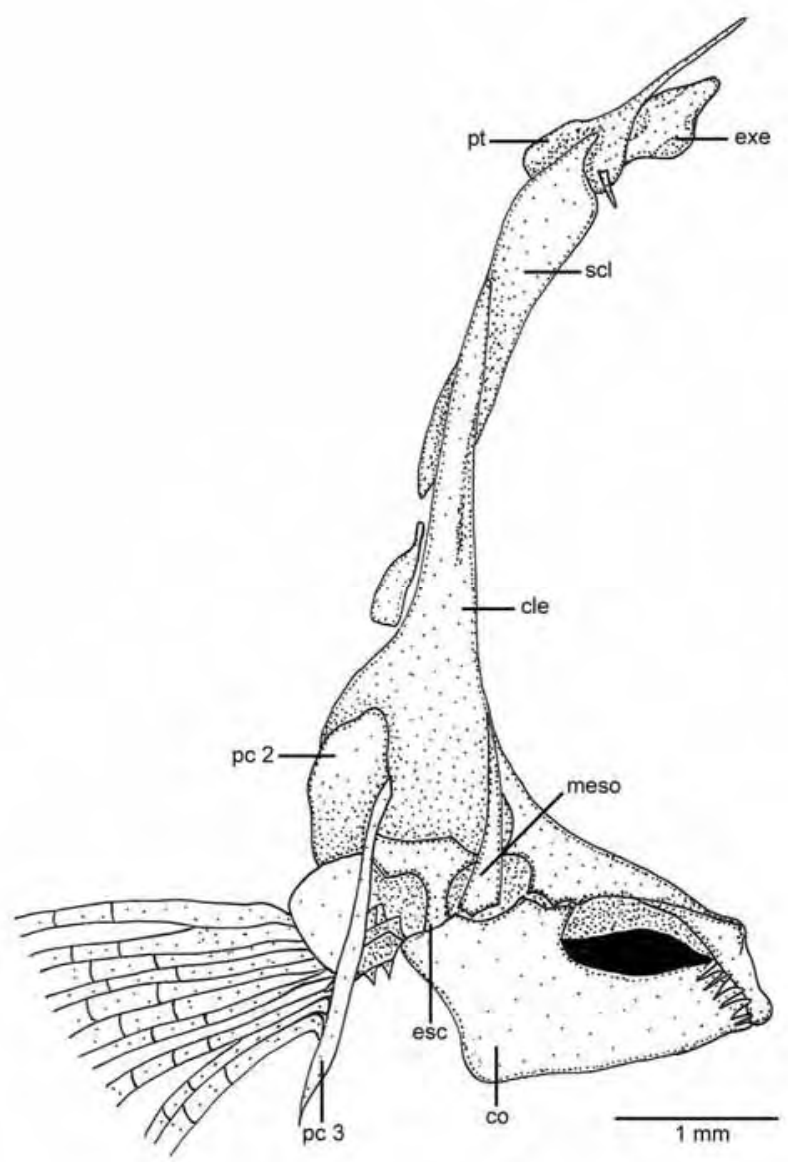

Figura 27- Hemigrammus unilineatus MZUSP 65409, 25,8 mm CP. Cintura e nadadeira peitoral, vista medial do lado esquerdo.

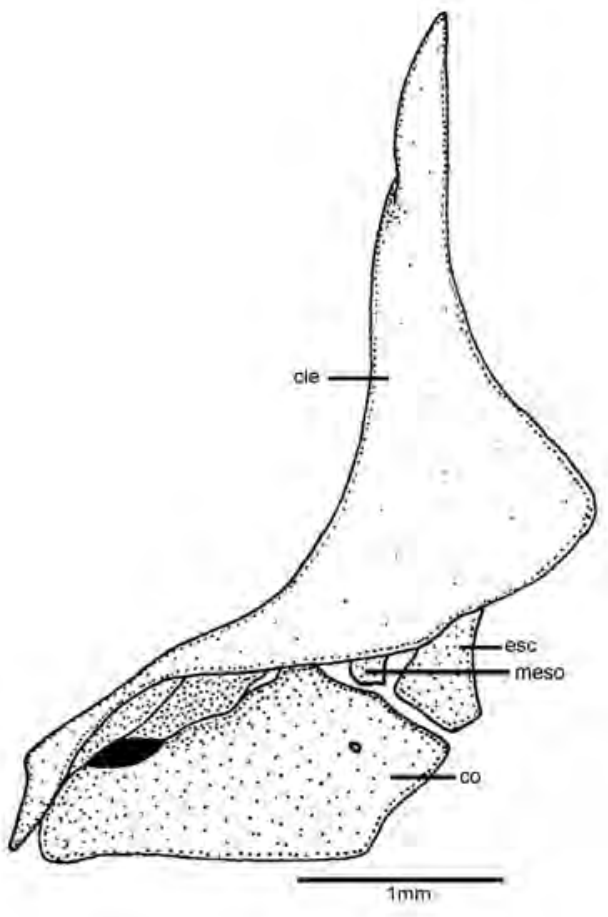

Figura 28- Hemigrammus mimus MZUSP 28018, 22,2 mm CP. Parte da cintura peitoral, vista lateral do lado esquerdo. 


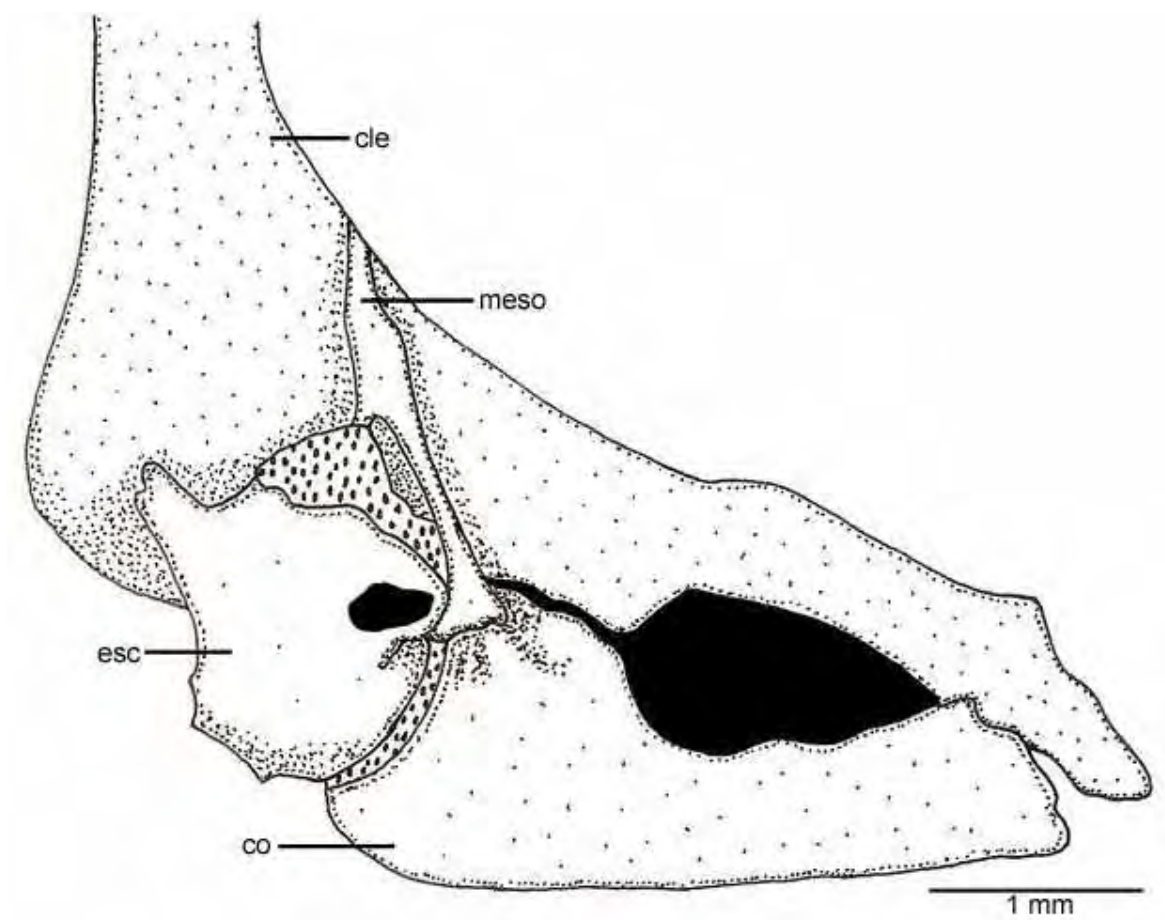

Figura 29- Coptobrycon bilineatus DZSJRP 6890, 36,3 mm CP. Parte da cintura peitoral, vista medial do lado esquerdo.
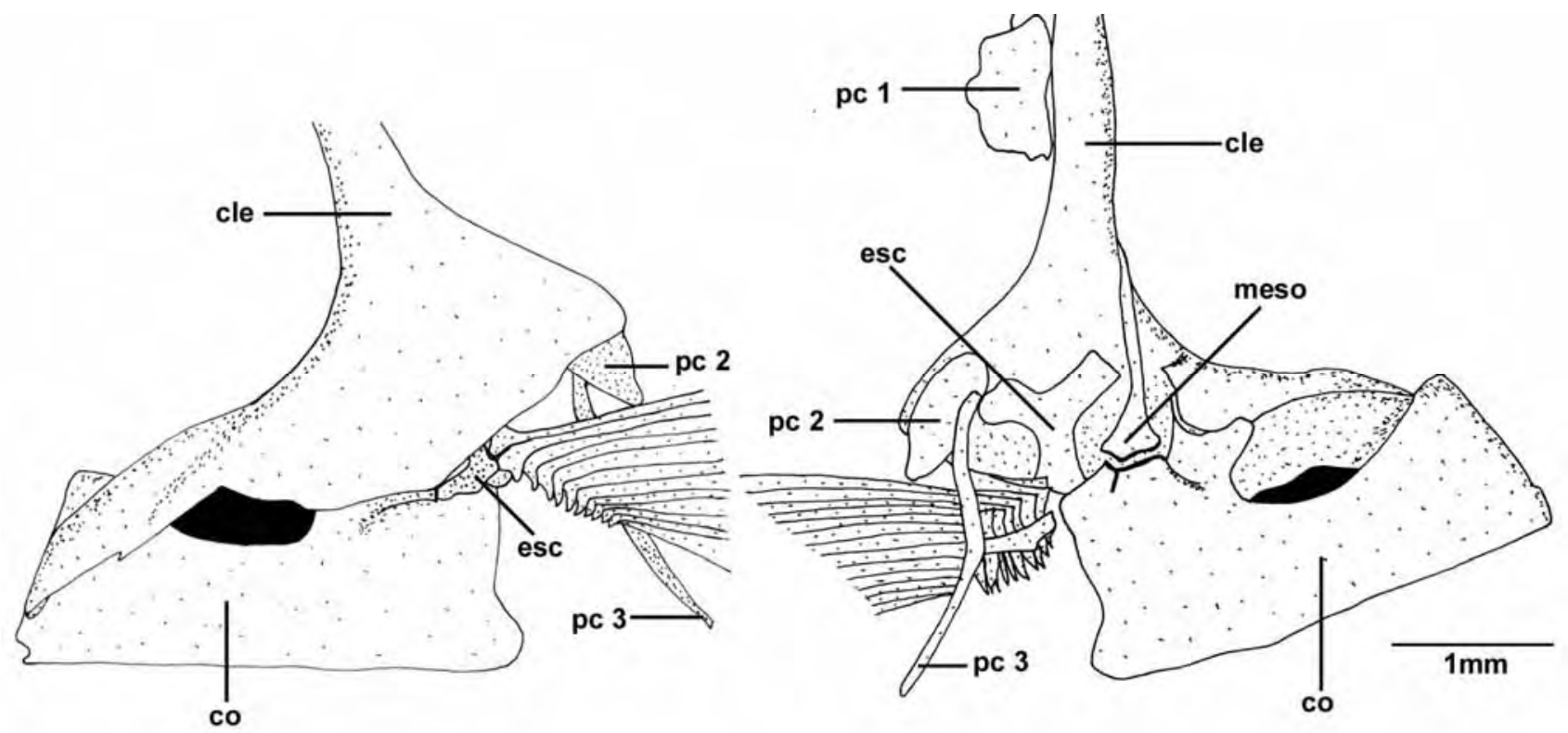

Figura 30- Bryconamericus exodon MZUSP 28026, 43,1 mm CP. Parte da cintura e nadadeira peitoral, respectivamente vista lateral e vista medial do lado esquerdo. 


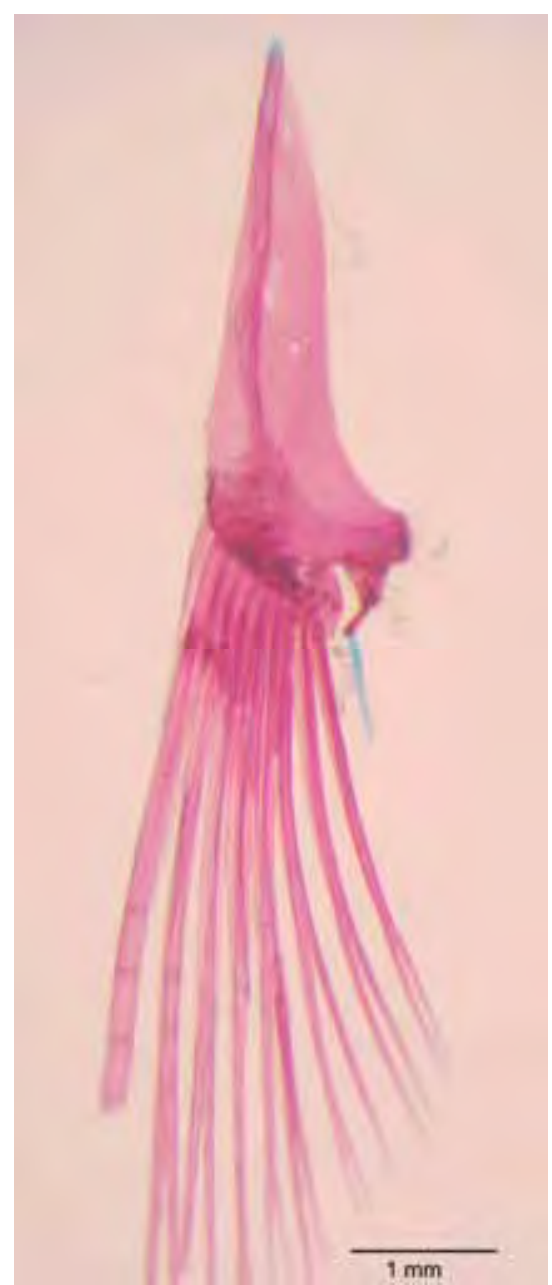

Figura 31- Hemigrammus unilineatus MZUSP 65409, 25,8 mm CP. Cintura e nadadeira pélvica, vista lateral do lado esquerdo.

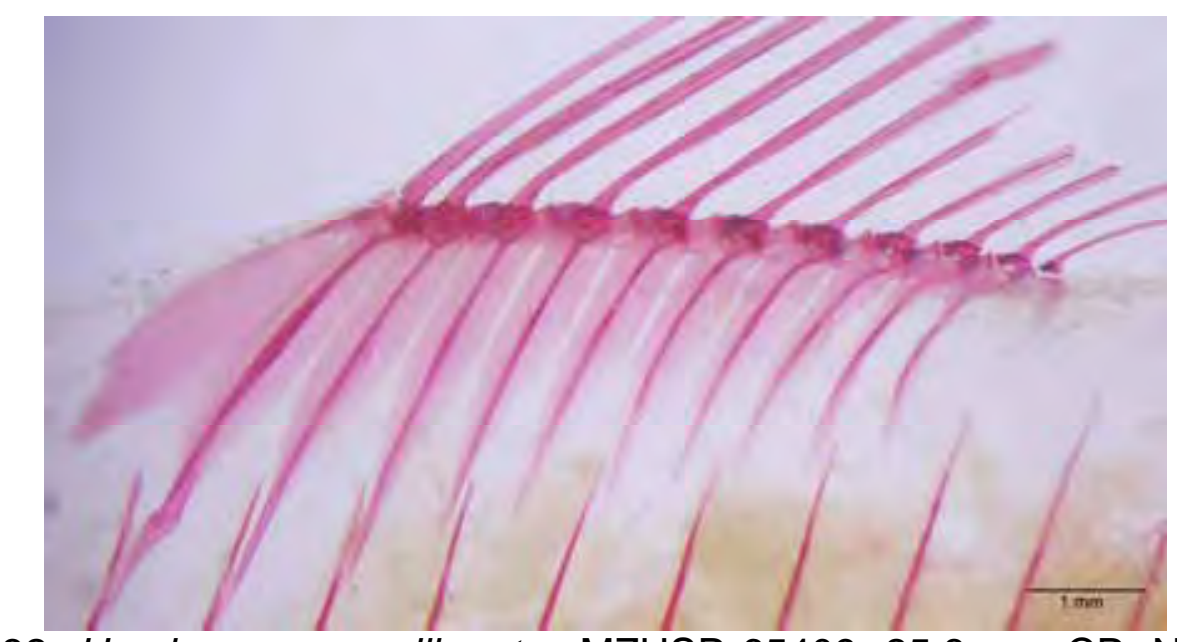

Figura 32- Hemigrammus unilineatus MZUSP 65409, 25,8 mm CP. Nadadeira dorsal. 

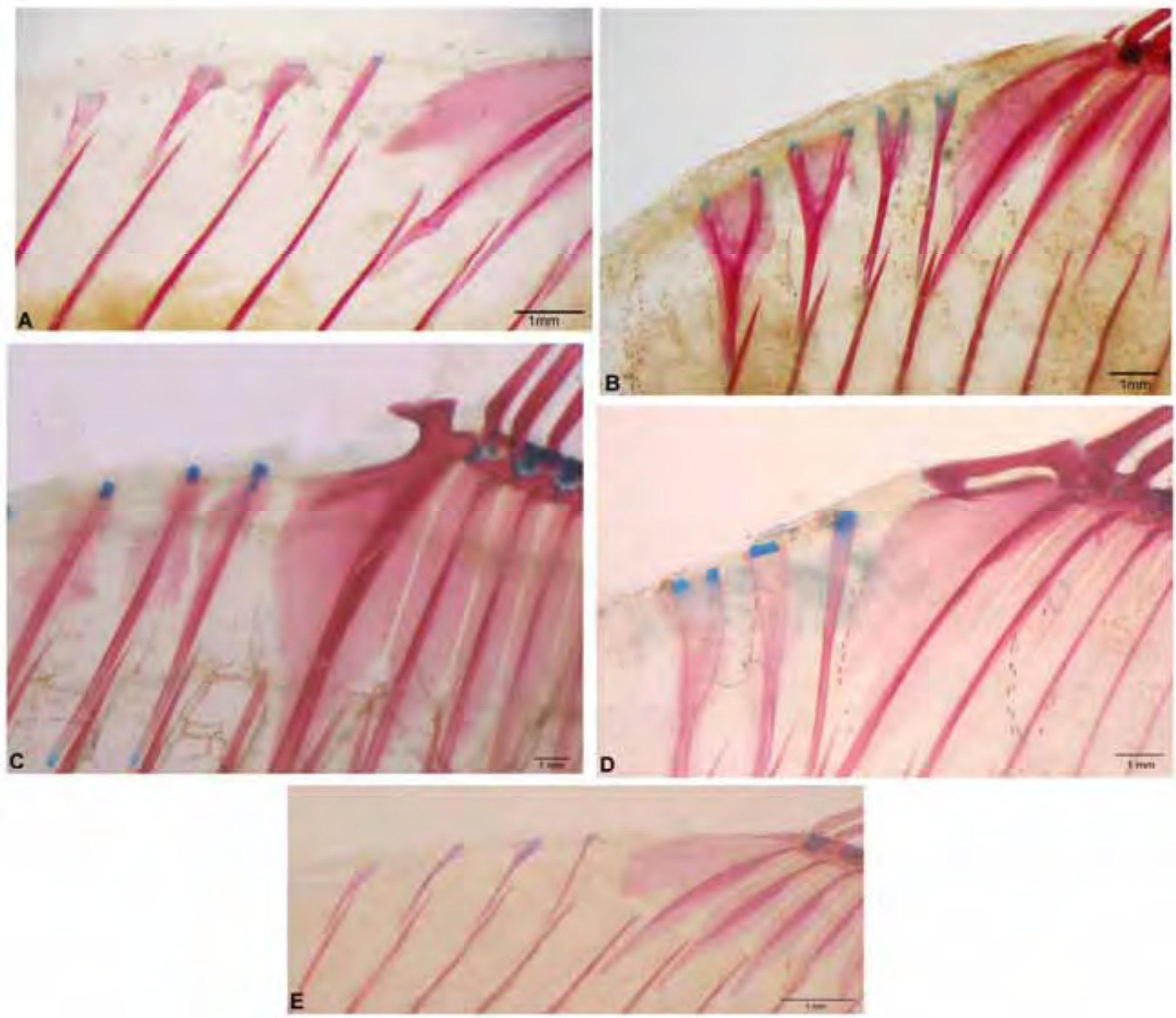

Figura 33- Supraneurais e parte da nadadeira dorsal. A) Hemigrammus unilineatus MZUSP 65409, 25,8 mm CP; B) Gymnocorymbus ternetzi DZSJRP 2808, 37,4 mm CP; C) Serrasalmus maculatus DZSJRP 1903, 47,4 mm CP; D) Poptella paraguayensis DZSJRP 627, 43,2 mm CP e E) Hemigrammus brevis MZUSP 17068, 20,7 mm CP. 


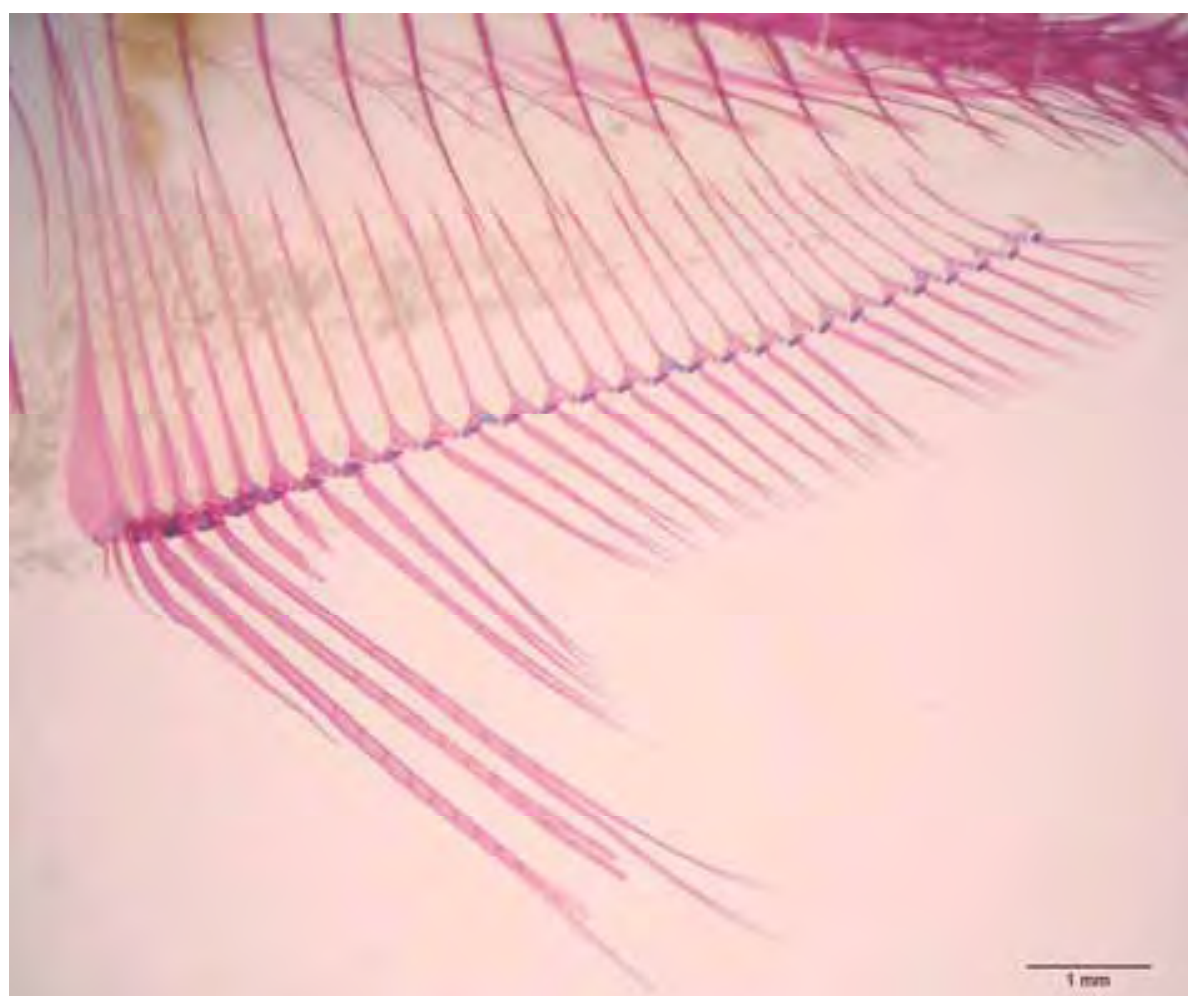

Figura 34- Hemigrammus unilineatus MZUSP 65409, 25,8 mm CP. Nadadeira anal.

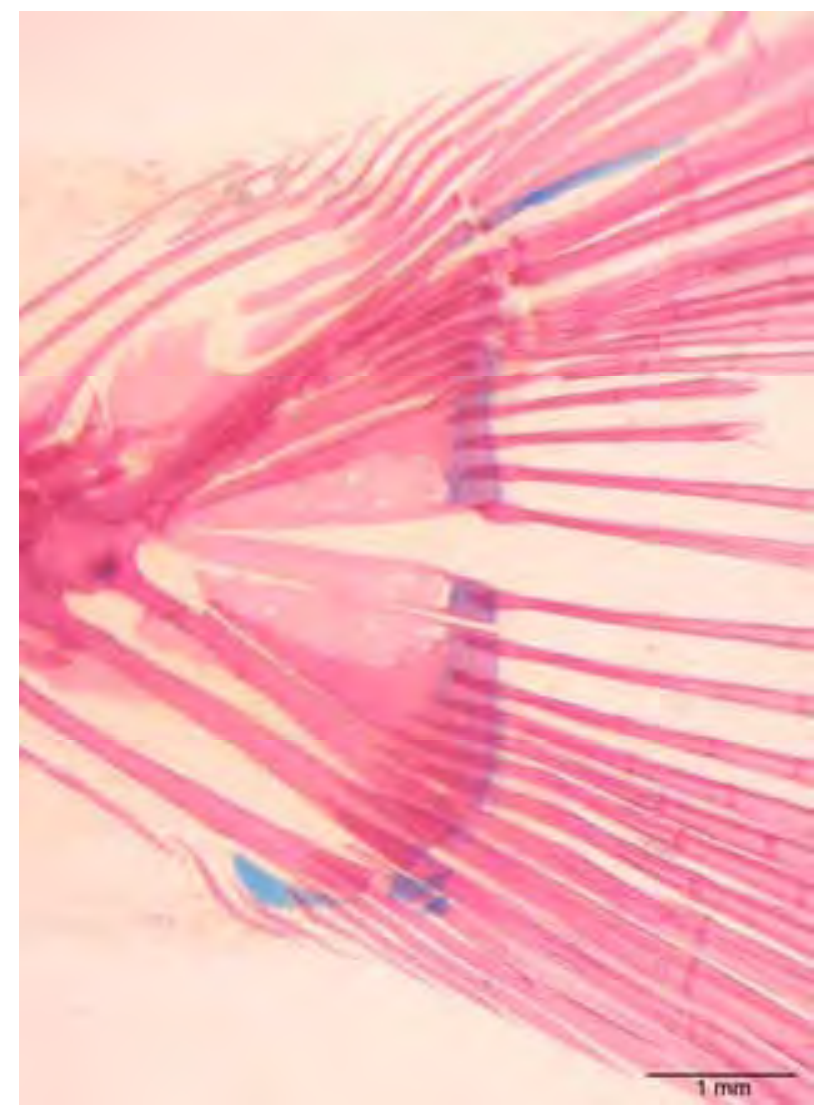

Figura 35- Hemigrammus unilineatus MZUSP 65409, 25,8 mm CP. Nadadeira caudal. 

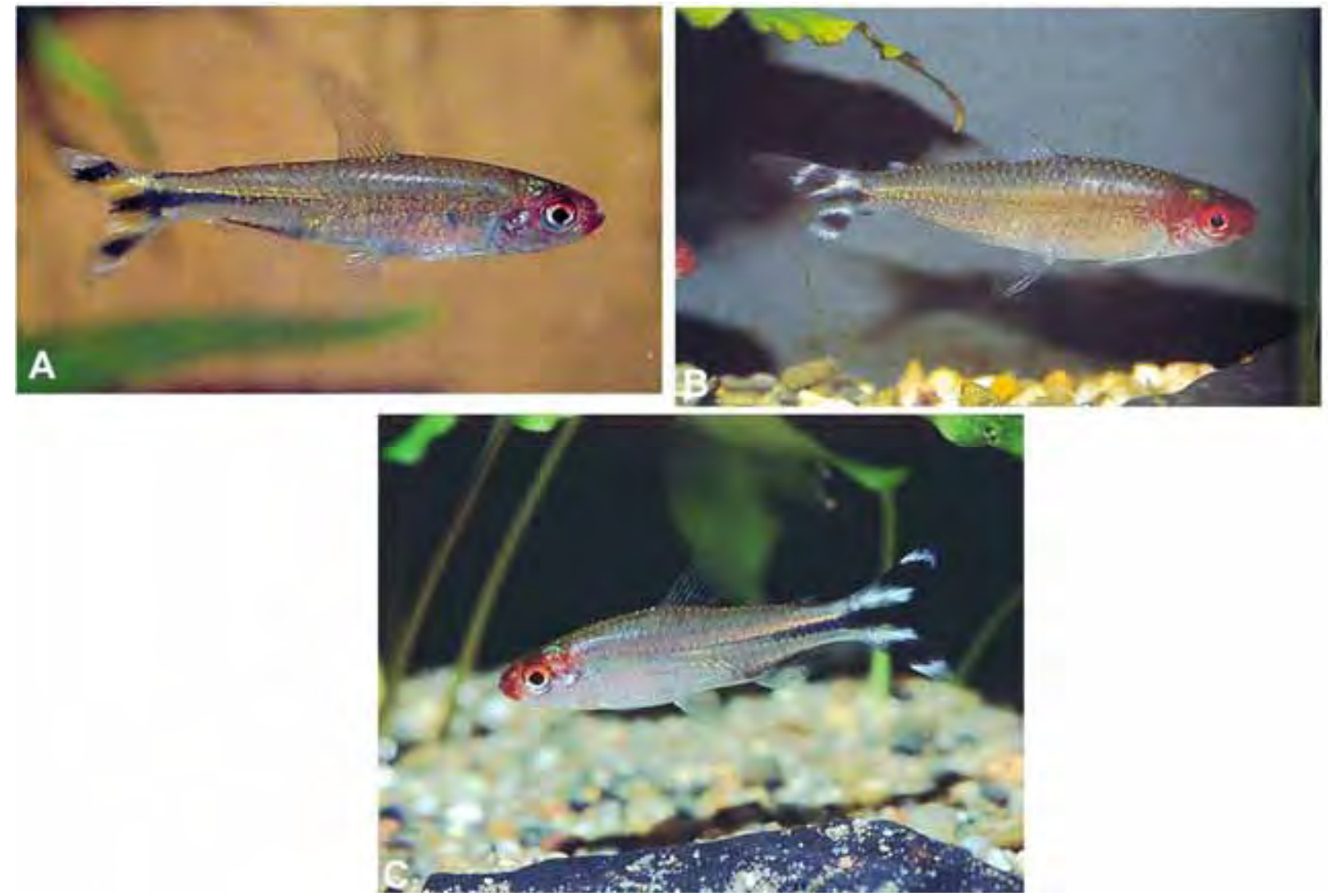

Figura 36- Colorido em vida. A) Hemigrammus rhodostomus; B) Hemigrammus bleheri e C- Petitella georgiae. Figuras extraídas de www.fishbase.org.br. 

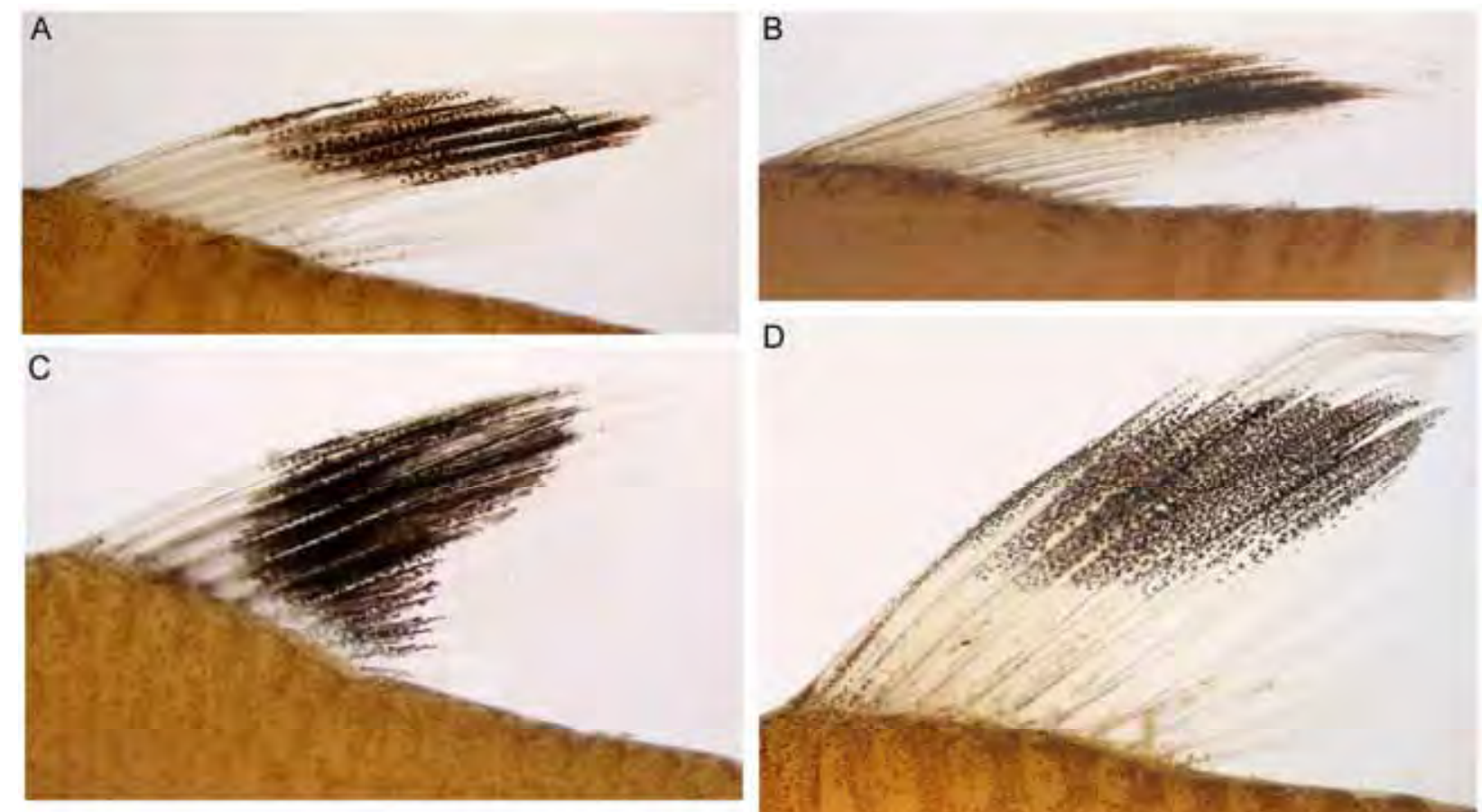

D

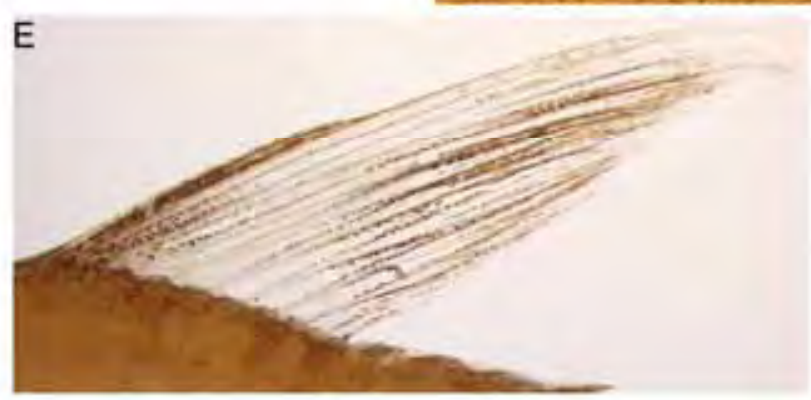

Figura 37- Nadadeira anal: presença de mancha negra. A) Hemigrammus unilineatus MZUSP 65409, 29 mm CP; B) Pristella maxillaris MZUSP 66678, 29,8 mm CP; C) Hyphessobrycon eques DZSJRP 10863, 19,3 mm CP; D) Moenkhausia hemigrammoides MZUSP 92036, 37 mm CP e E) Hemigrammus ulreyi MZUSP 59538, $30 \mathrm{~mm}$ CP. 

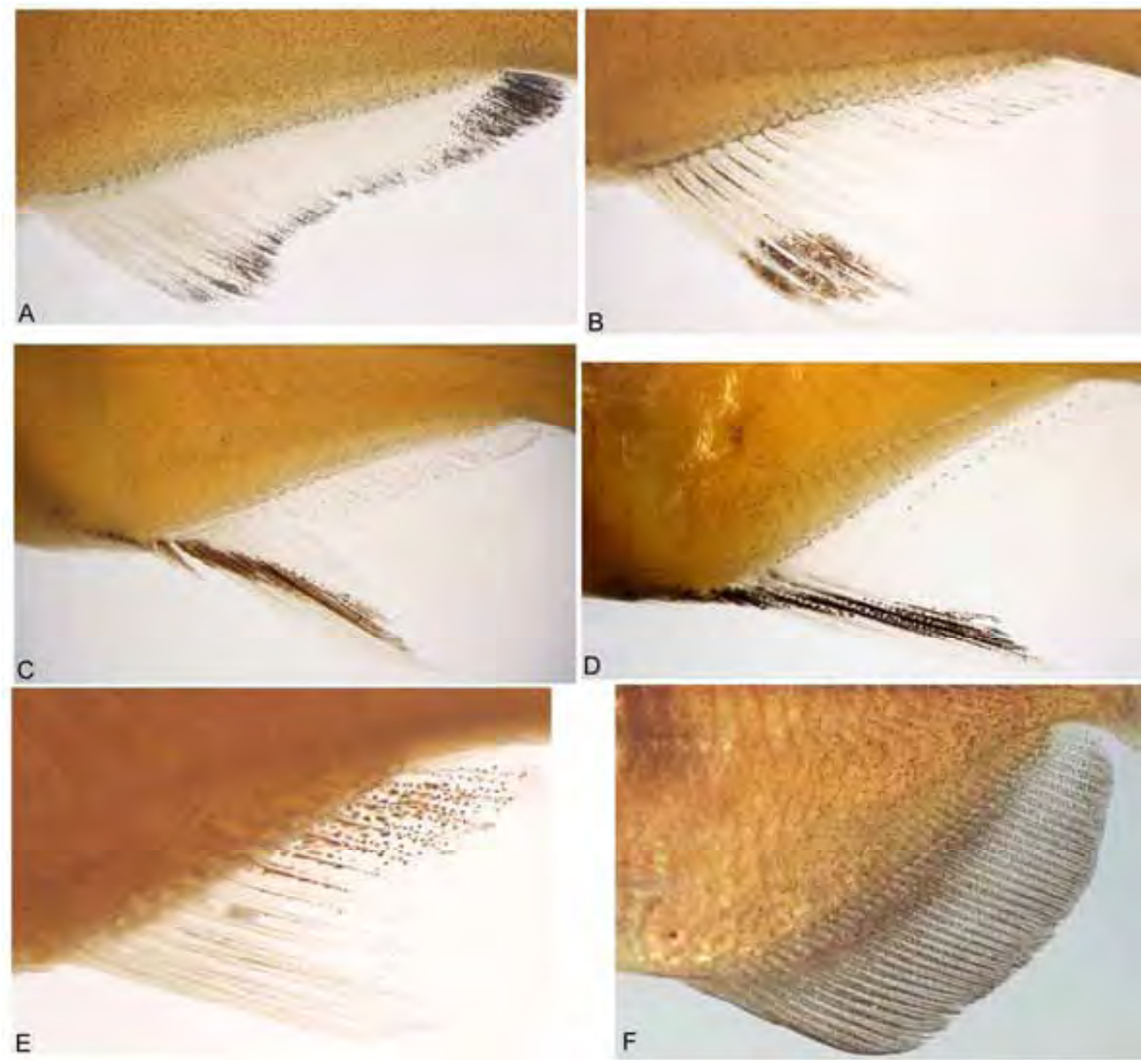

Figura 38- Nadadeira anal: presença de mancha negra. A) Hyphessobrycon eques DZSJRP 10863, 26 mm CP; B) Pristella maxillaris MZUSP 66678, 29,8 $\mathrm{mm} \mathrm{CP}$; C) Hemigrammus unilineatus MZUSP 65409, $29 \mathrm{~mm} \mathrm{CP;} \mathrm{D)}$ Moenkhausia hemigrammoides MZUSP 92936, 37 mm CP; E) Hemigrammus rhodostomus MZUSP 17997, 27,1 mm CP e F) Gymnocorymbus ternetzi DZSJRP 10858, 36,5 mm CP. 

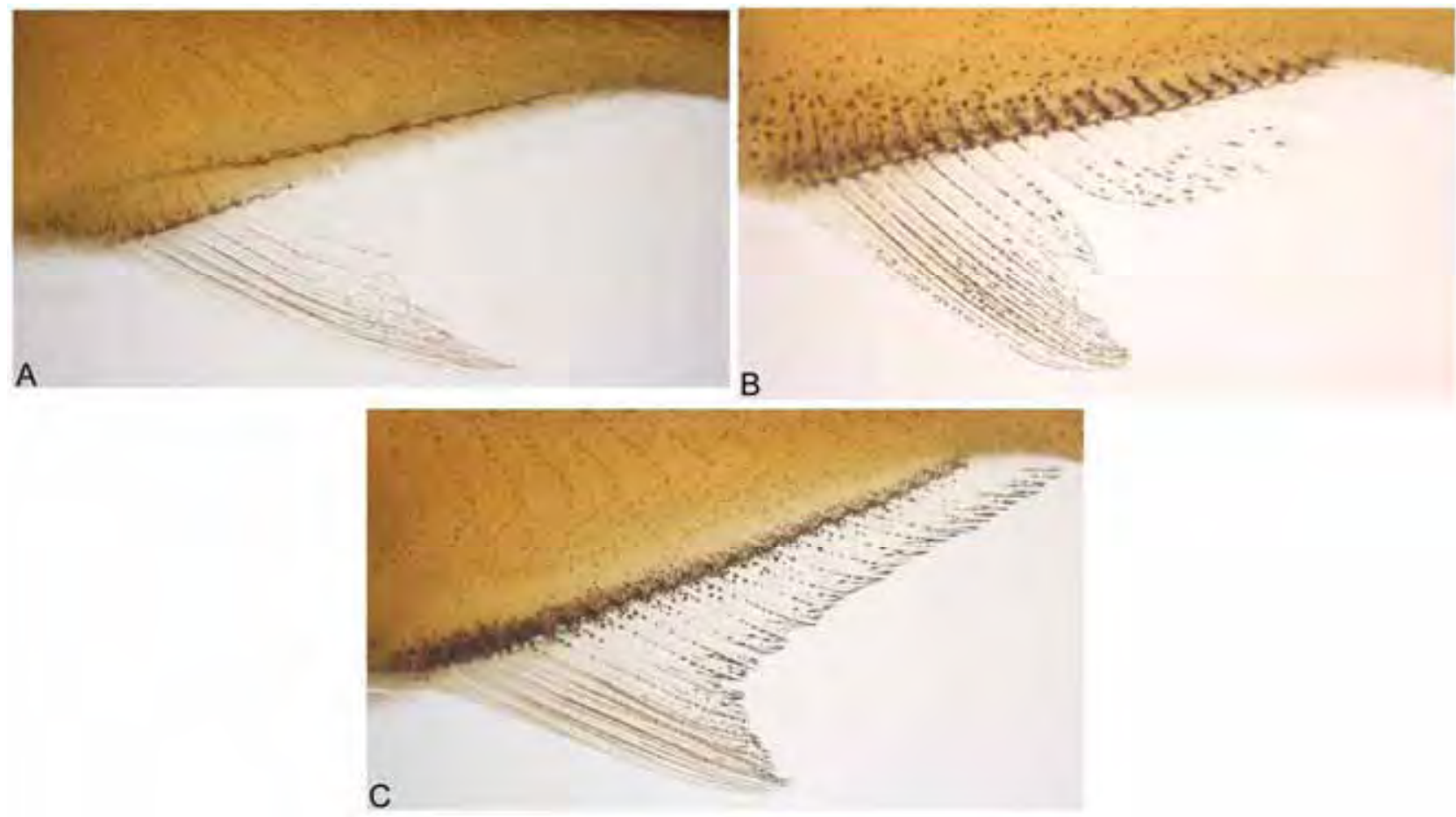

Figura 39- Nadadeira anal: presença de mancha negra na base ou paralela à base da nadadeira. A) Hemigrammus bellottii MZUSP 85729, 28 mm CP; B) Hemigrammus boesemani MZUSP 65445, 26,9 mm CP e C) Hemigrammus barrigonae MZUSP 85006, $35 \mathrm{~mm}$ CP. 


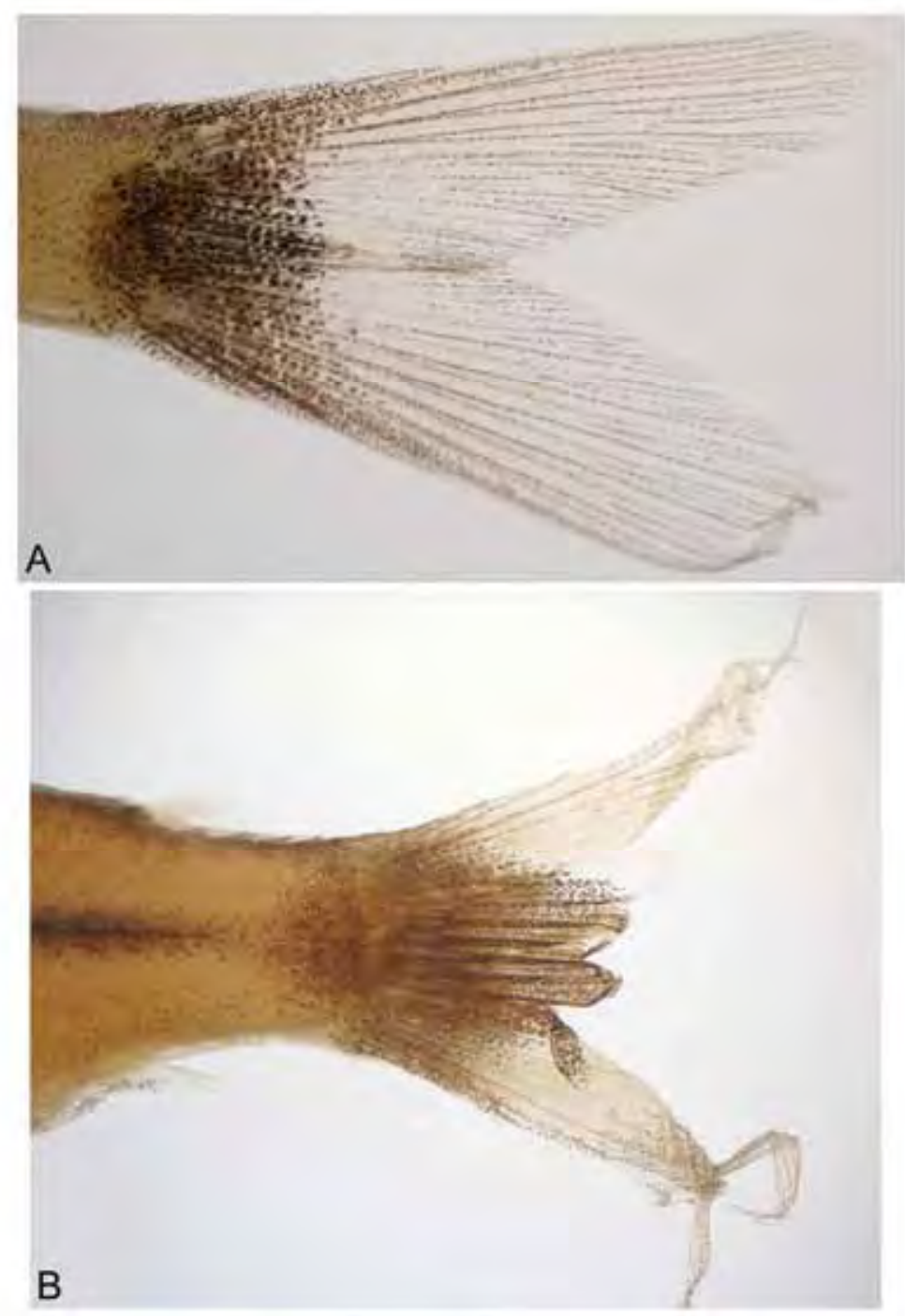

Figura 40- Pedúnculo caudal: presença de mancha negra. A) Hemigrammus ocellifer DZSJRP 11208, 20,9 mm CP e B) Hemigrammus newboldi MZUSP 77793, 31,6 mm CP. 

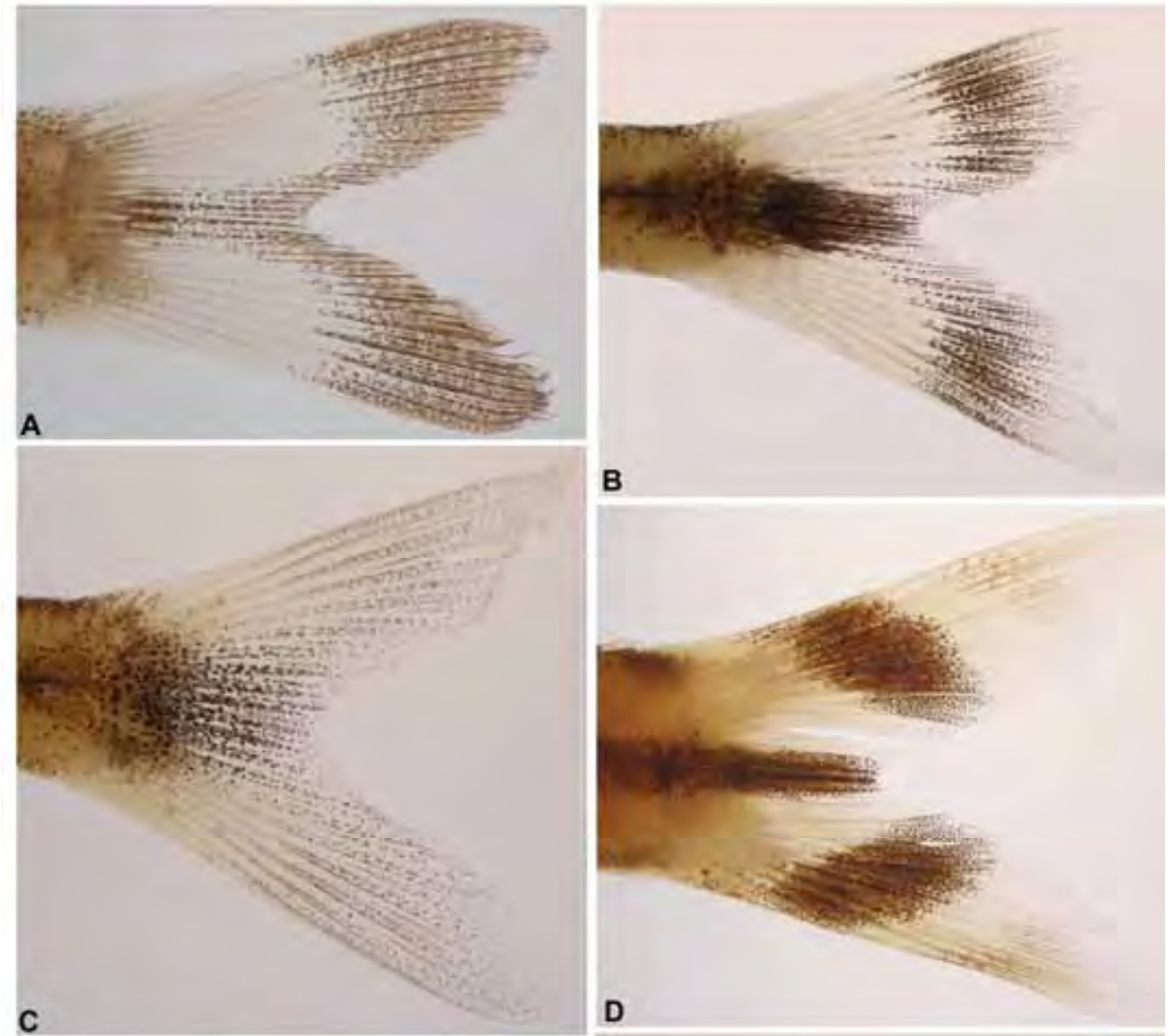

B
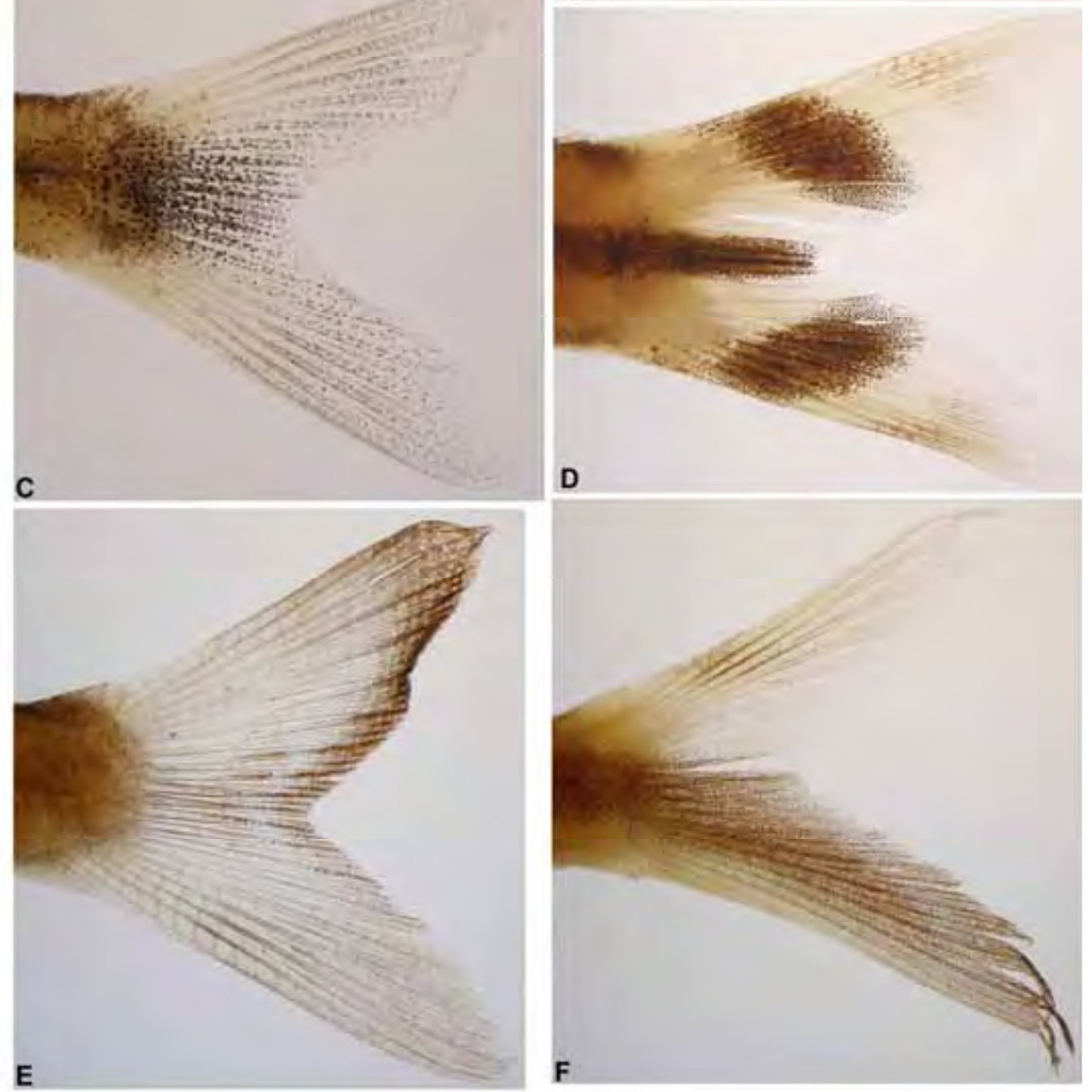

D

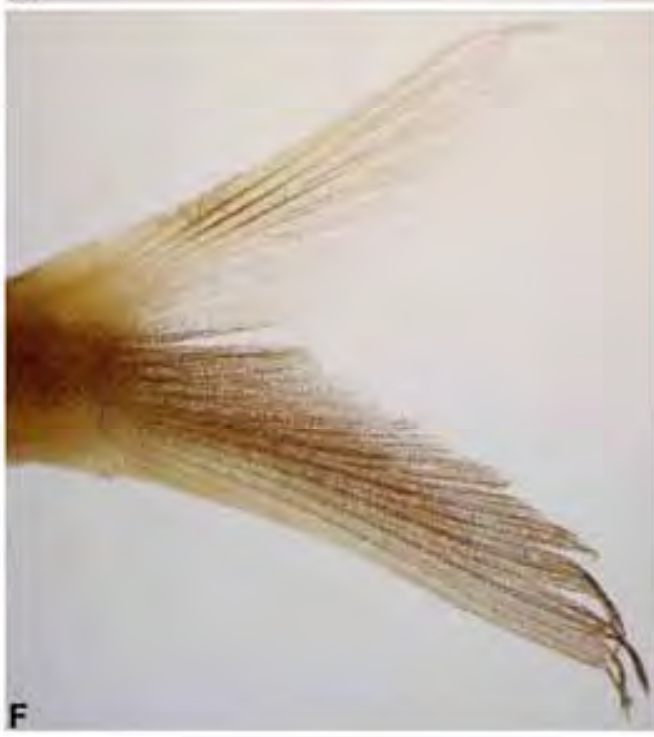

Figura 41- Nadadeira caudal: presença de manhcas negras. A) Bryconamericus exodon MZUSP 54010, 27,5 mm CP; B) Moenkhausia bonita DZSJRP 11372 , 24,8 mm CP; C) Hemigrammus parana DZSJRP 10796, 24,3 mm CP; D) Hemigrammus rhodostomus MZUSP 17997, 27,1 mm CP; E) Bryconops melanurus DZSJRP 6285, 46 mm CP e F) Thayeria obliqua MZUSP 29391, $32,8 \mathrm{~mm} \mathrm{CP}$. 


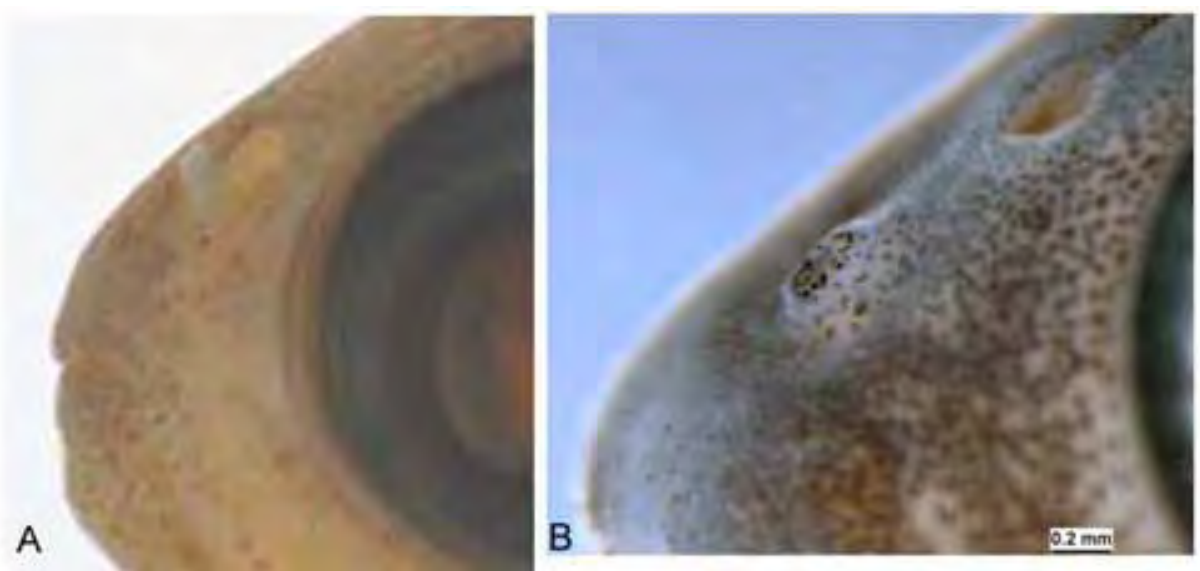

Figura 42- Narinas. A) Hemigrammus unilineatus ANSP 147082, 27,7 mm CP e B) Coptobrycon bilineatus DZSJRP 29,1 mm CP. 


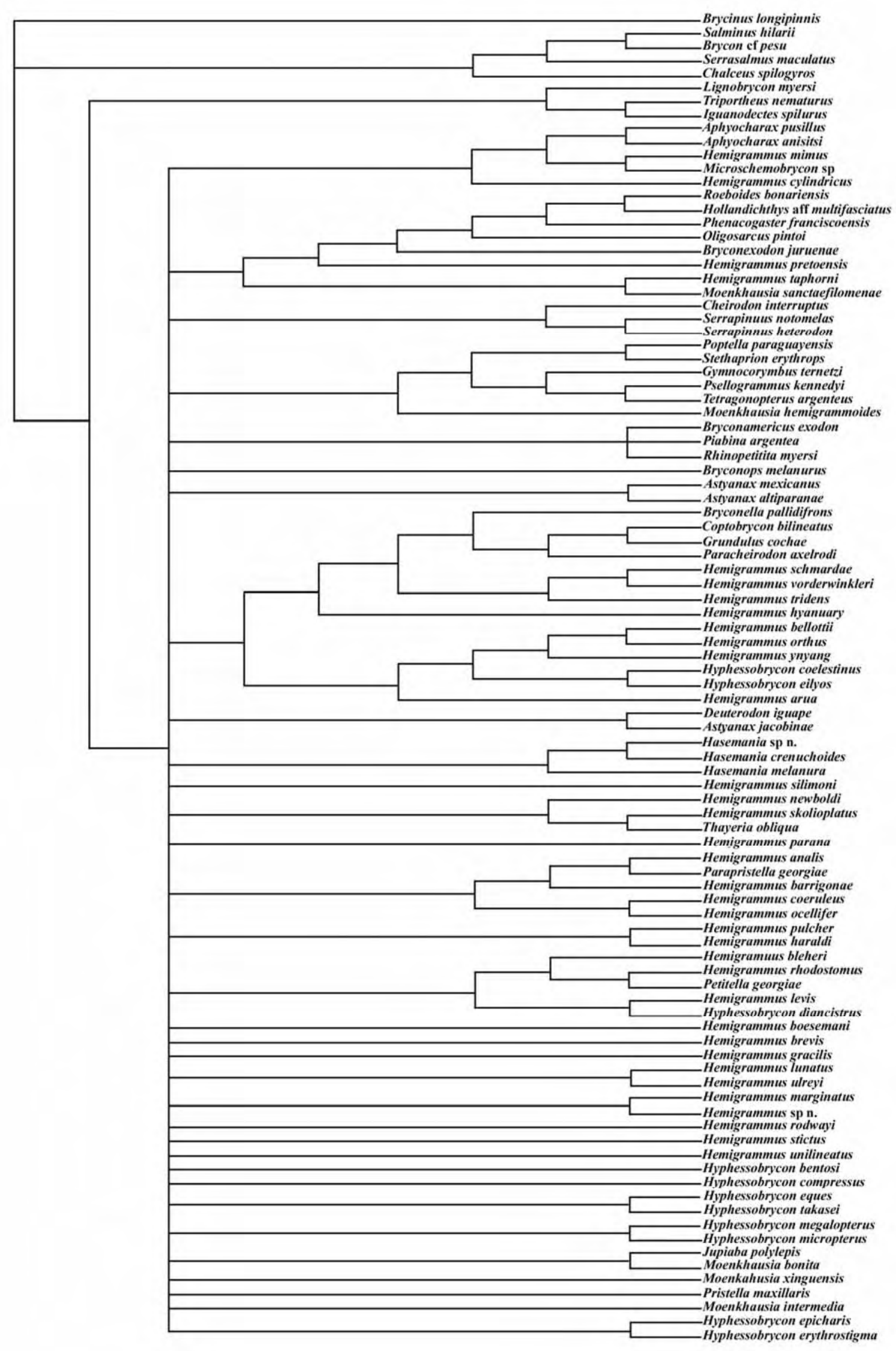

Figura 43- Cladograma de consenso estrito das seis árvores mais parcimoniosas geradas pela análise. IC -0,13; IR -0,44; Passos - 1849 . 


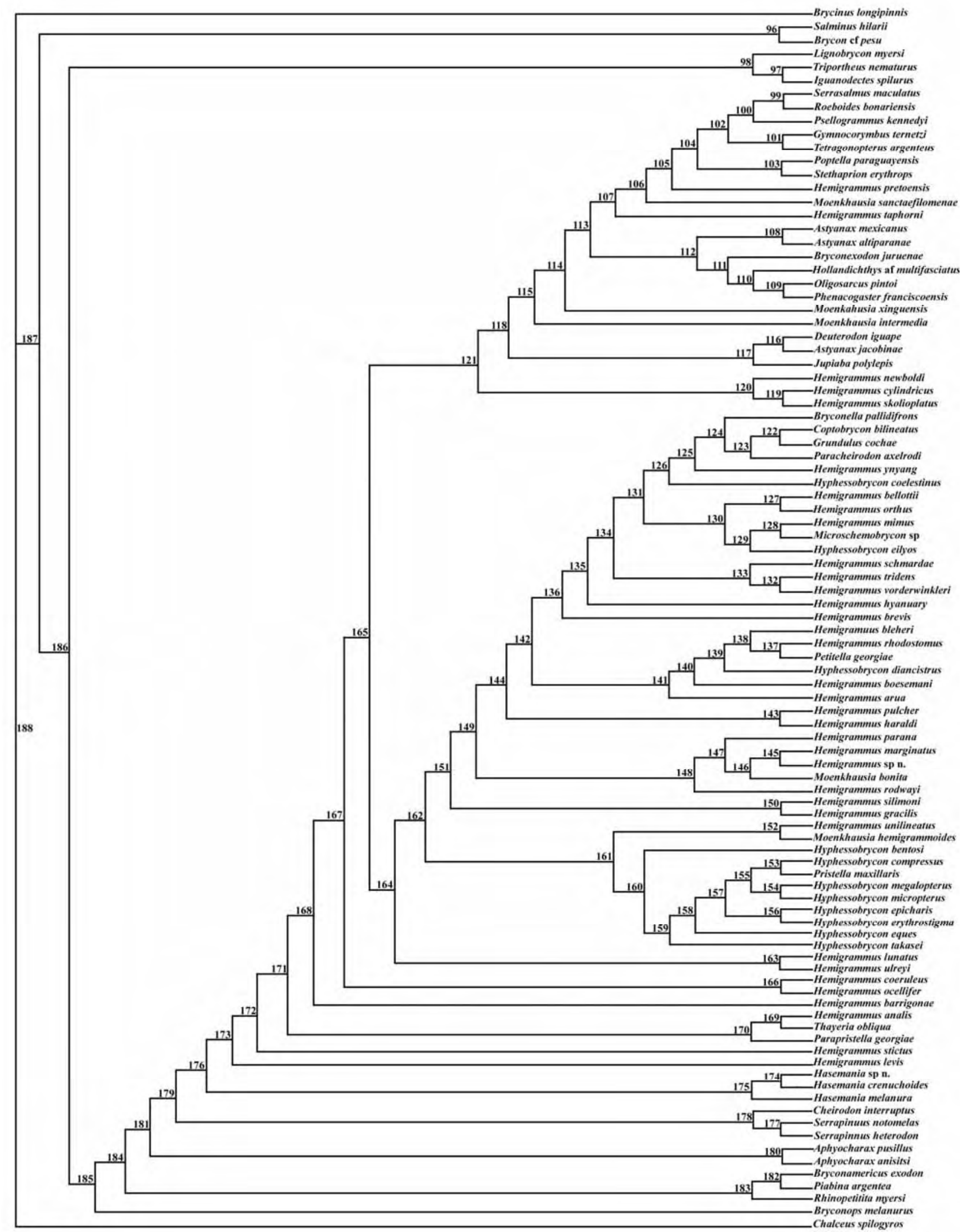

Figura 44 - Única árvore mais parcimoniosa gerada após repesagens. IC 0,$35 ; \mathrm{IR}-0,61$. 


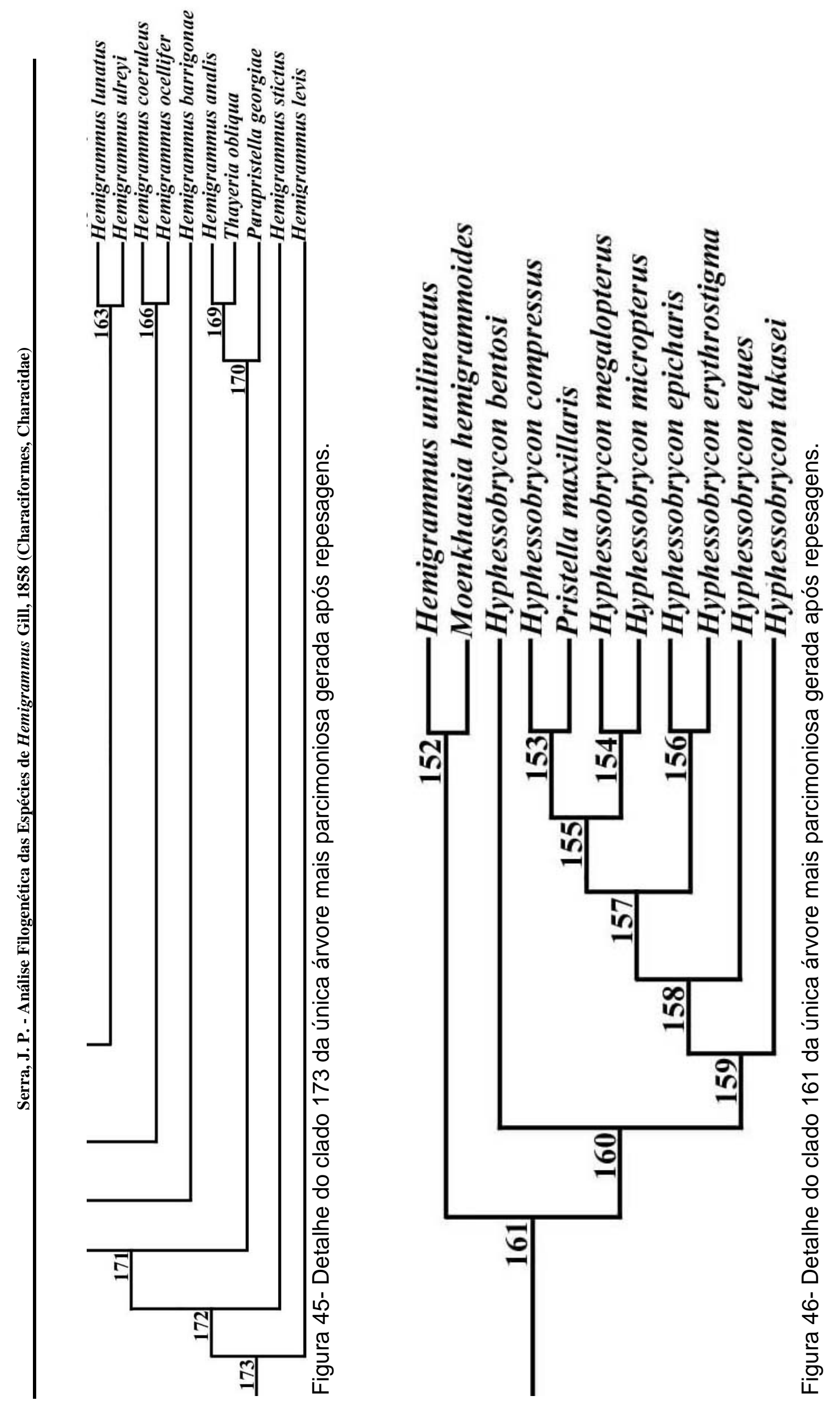

๖̊ 


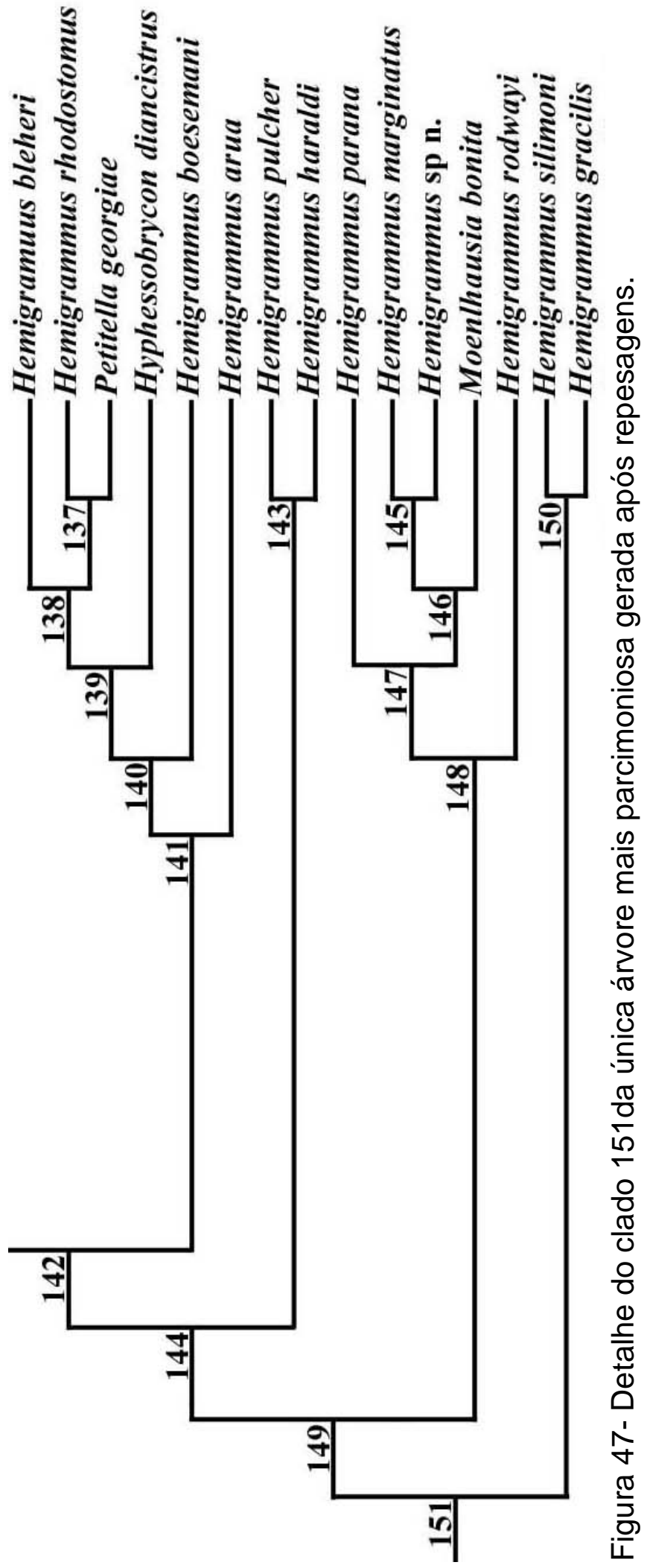




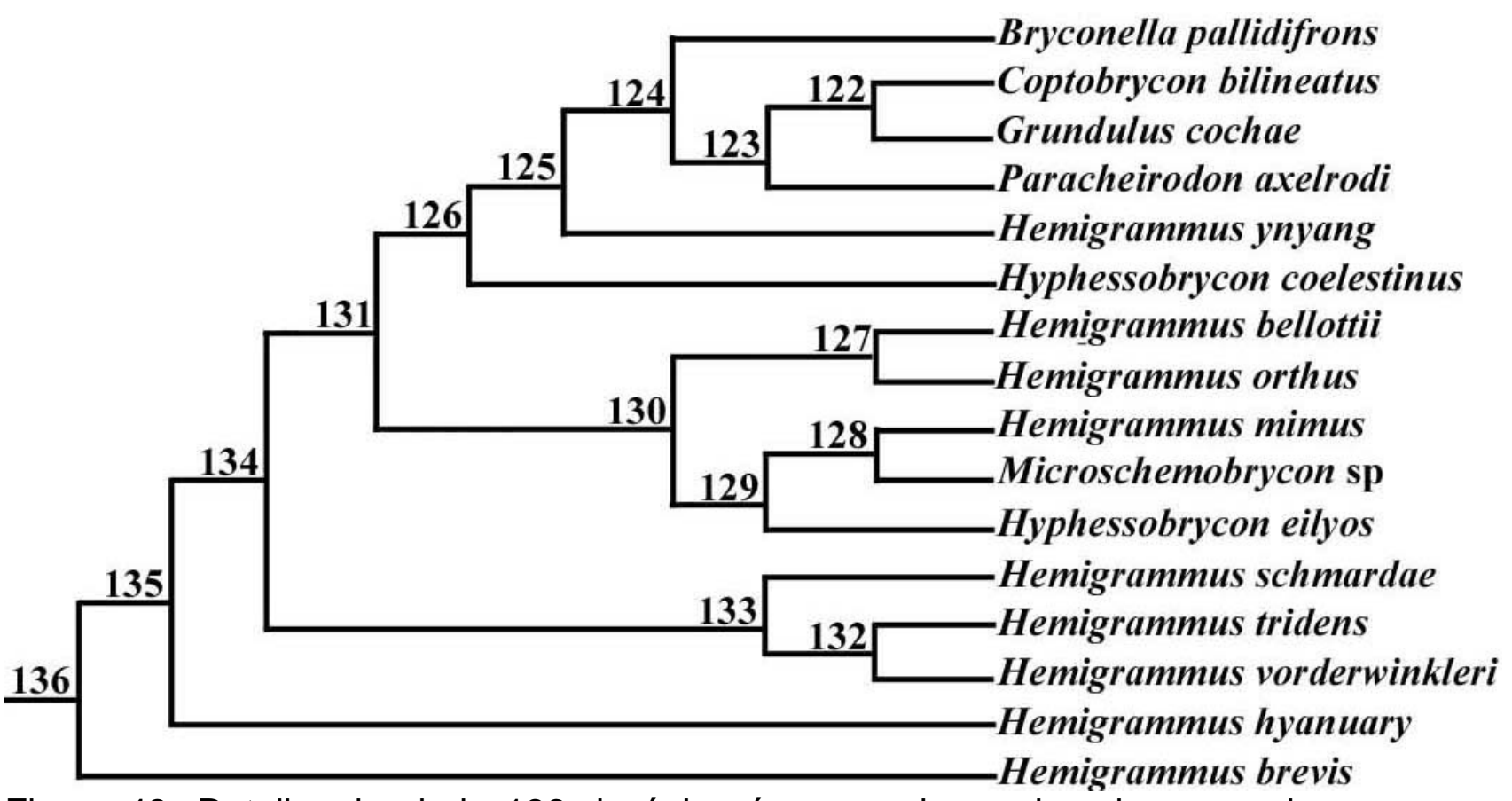

Figura 48- Detalhe do clado 136 da única árvore mais parcimoniosa gerada após repesagens.

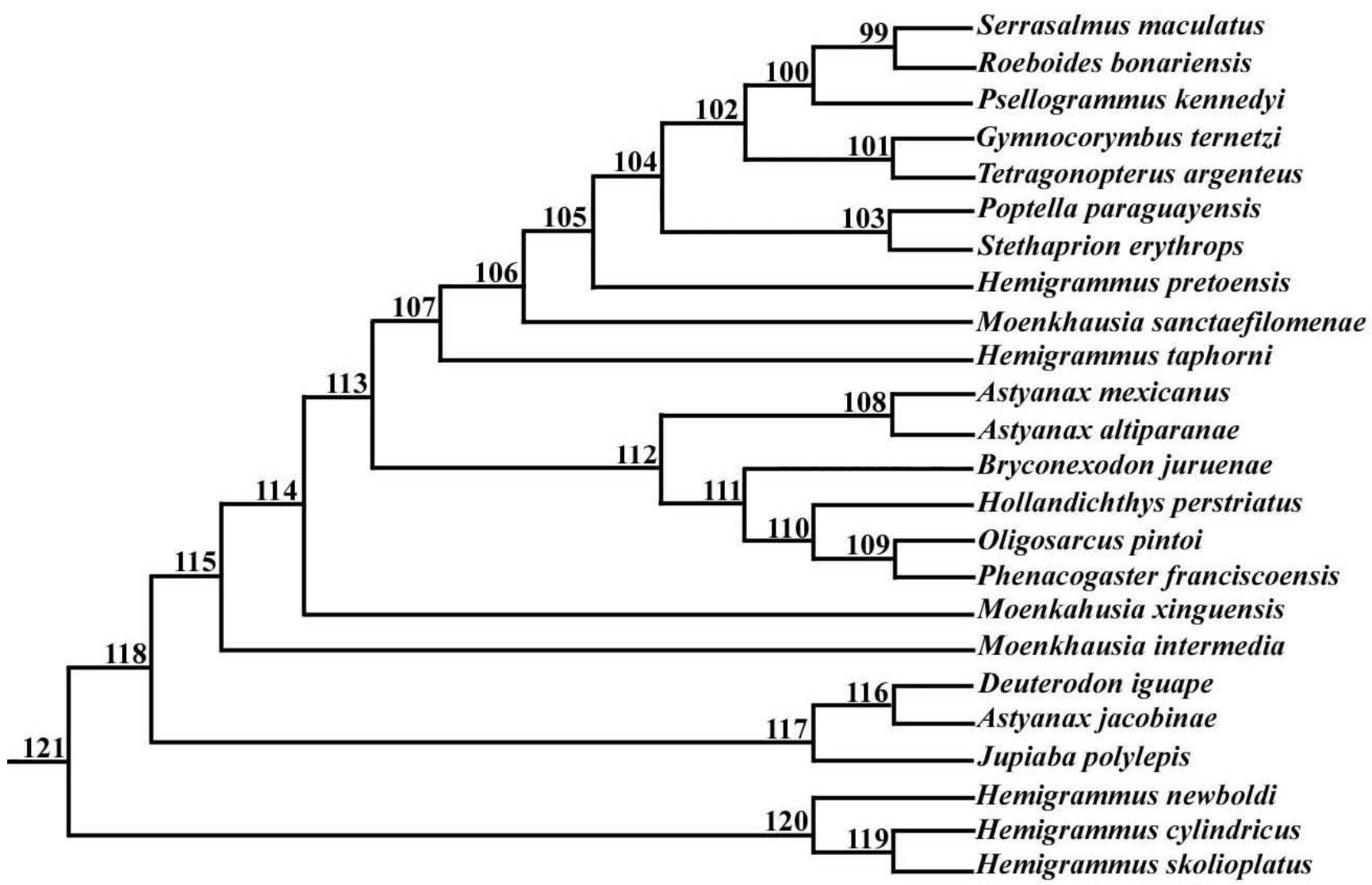

Figura 49- Detalhe do clado 121 da única árvore mais parcimoniosa gerada após repesagens. 


\section{APÊNDICE 1}




\section{Apêndice 1- Lista das sinapomorfias para cada clado e táxon.}

\begin{tabular}{|c|c|c|c|c|}
\hline \multicolumn{2}{|c|}{ node 188 --> Brycinuslongipin 9} & $10.0830==>1$ & 51 & $10.750 \quad 1==>3$ \\
\hline & 28 & $10.1052==>1$ & 65 & 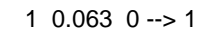 \\
\hline & 32 & $10.2000==>2$ & 76 & $10.100 \quad 1==>2$ \\
\hline & 40 & $11.0001==>0$ & 91 & $\begin{array}{lll}10.100 & 0 & -->1\end{array}$ \\
\hline & 41 & $10.0671==>2$ & 96 & $10.034 \quad 0->1$ \\
\hline & 45 & $10.250 \quad 0-->1$ & 110 & $10.0872==>1$ \\
\hline & 51 & $10.750 \quad 1==>0$ & 141 & $\begin{array}{lll}1 & 0.200 & 0\end{array}->1$ \\
\hline & 62 & $10.167 \quad 1==>0$ & 147 & $10.115 \quad 1==>0$ \\
\hline & 67 & $10.100 \quad 0==>1$ & 148 & $\begin{array}{llll}1 & 0.125 & 2-->0\end{array}$ \\
\hline & 68 & $10.0950==>1$ & 150 & $\begin{array}{llll}1 & 0.167 & 0 & ->\end{array}$ \\
\hline & 75 & $10.0830==>2$ & node_186 --> node_98 & $10.1250==>1$ \\
\hline & 76 & $10.100 \quad 1==>0$ & - & $\begin{array}{llll} & 0.167 & 0 & -->3\end{array}$ \\
\hline & 77 & $10.1820==>2$ & 56 & $10.067 \quad 0->1$ \\
\hline & 83 & $10.143 \quad 0->2$ & 83 & $10.143 \quad 0->1$ \\
\hline & 88 & $10.0542==>1$ & 84 & $\begin{array}{llll}1 & 0.083 & 0 & ->\end{array}$ \\
\hline & 96 & $\begin{array}{llll}1 & 0.034 & 0 & -->1\end{array}$ & 90 & $10.1431==>2$ \\
\hline & 102 & $10.3331==>0$ & 104 & $\begin{array}{llll} & 0.038 & 0 & -->1\end{array}$ \\
\hline & 123 & $10.2500==>1$ & 105 & $10.1502==>0$ \\
\hline & 139 & $10.333 \quad 1==>2$ & 108 & $10.1180==>2$ \\
\hline & 145 & $10.0670==>1$ & 114 & $11.0001==>0$ \\
\hline & 150 & $\begin{array}{lll}1 & 0.167 & 0-->1\end{array}$ & 117 & $\begin{array}{lll}10.500 & 0-->1\end{array}$ \\
\hline node_188 --> node_187 & 2 & $10.0430-->1$ & 125 & $10.1821==>0$ \\
\hline & 8 & $10.2220-->1$ & node_98 --> Lignobryconmyers 4 & $10.0501==>0$ \\
\hline & 10 & 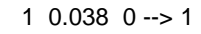 & - & $10.1670==>2$ \\
\hline & 20 & $\begin{array}{llll}1 & 0.143 & 0 & -->\end{array}$ & 11 & $10.200 \quad 0==>1$ \\
\hline & 24 & $\begin{array}{lll}1 & 0.071 & 1-->0\end{array}$ & 14 & $10.0560==>1$ \\
\hline & 34 & $10.0610-->1$ & 24 & $\begin{array}{lll}1 & 0.071 & 0-->1\end{array}$ \\
\hline & 44 & 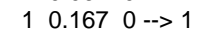 & 32 & $10.2001-->0$ \\
\hline & 53 & $10.154 \quad 1->2$ & 37 & $10.136 \quad 3->0$ \\
\hline & 84 & $10.0831->0$ & 38 & $\begin{array}{lll}1 & 0.250 & 1->0\end{array}$ \\
\hline & 100 & $10.3330-->1$ & 55 & $10.071 \quad 1==>0$ \\
\hline & 116 & $10.500 \quad 1==>0$ & 60 & $10.0630==>1$ \\
\hline & 126 & $10.080 \quad 0-->1$ & 63 & $10.1362==>3$ \\
\hline & 135 & $10.056 \quad 1->0$ & 72 & $10.0590==>1$ \\
\hline & 151 & $10.0630-->1$ & 98 & $10.0830==>1$ \\
\hline node_187 --> node_96 & 39 & $10.037 \quad 0-->1$ & 103 & $10.036 \quad 1==>0$ \\
\hline & 41 & $10.0671==>0$ & 113 & $10.0630==>1$ \\
\hline & 44 & $\begin{array}{lll}1 & 0.167 & 1->\end{array}$ & 121 & $10.2312==>1$ \\
\hline & 46 & 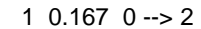 & 139 & $10.3331==>2$ \\
\hline & 58 & $10.125 \quad 1==>0$ & 155 & $10.2220==>2$ \\
\hline & 71 & $10.0831==>0$ & node_98 --> node_97 & $\begin{array}{llll}1 & 0.043 & 1->\end{array}$ \\
\hline & 77 & $10.1820==>1$ & 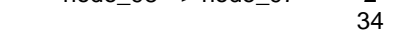 & $\begin{array}{lll}1 & 0.061 & 1->0\end{array}$ \\
\hline & 87 & $10.100 \quad 1==>0$ & 35 & $10.125 \quad 1->0$ \\
\hline & 89 & $10.0430==>1$ & 41 & $10.067 \quad 1==>2$ \\
\hline & 92 & $10.043 \quad 0->1$ & 42 & $\begin{array}{lll}10.167 & 2-->0\end{array}$ \\
\hline & 94 & $\begin{array}{lll}1 & 0.250 & 1-->0\end{array}$ & 53 & $10.1542->0$ \\
\hline & 139 & $10.333 \quad 1==>0$ & 58 & $10.125 \quad 1==>2$ \\
\hline & 140 & $10.231 \quad 1==>0$ & 76 & $10.1002==>0$ \\
\hline node_96 --> Salminushi & ii 10 & $10.038 \quad 1->0$ & 92 & $\begin{array}{llll} & 0.043 & 0 & -->\end{array}$ \\
\hline & 20 & $10.143 \quad 1->0$ & 102 & $10.333 \quad 1==>0$ \\
\hline & 22 & $10.091 \quad 1==>0$ & 159 & $10.0710==>2$ \\
\hline & 31 & $10.3750==>2$ & node_97 --> Triportheusnemat 9 & $10.0830==>1$ \\
\hline & 34 & $\begin{array}{lll}1 & 0.061 & 1->2\end{array}$ & 15 & $10.3330==>1$ \\
\hline & 47 & $10.2000==>1$ & 18 & $10.1000==>1$ \\
\hline & 51 & $10.750 \quad 1=\Rightarrow 2$ & 36 & $10.0560==>1$ \\
\hline & 61 & $10.333 \quad 1==>0$ & 51 & $10.7503==>0$ \\
\hline & 62 & $10.167 \quad 1==>0$ & 59 & $10.0560==>1$ \\
\hline & 70 & $10.0401==>0$ & 67 & $10.1000==>2$ \\
\hline & 73 & $10.083 \quad 1==>2$ & 71 & $10.0831==>0$ \\
\hline & 79 & $10.3330==>1$ & 79 & $10.3330==>2$ \\
\hline & 95 & $10.250 \quad 1=\Rightarrow 0$ & 83 & $\begin{array}{llll} & 0.143 \quad 1->0\end{array}$ \\
\hline & 103 & $10.0361==>0$ & 119 & $10.5000==>1$ \\
\hline & 107 & $11.0001==>2$ & 120 & $10.0431==>0$ \\
\hline & 110 & $10.0872==>0$ & 126 & $10.0801==>0$ \\
\hline & 159 & $10.0710==>2$ & 139 & $10.333 \quad 1==>0$ \\
\hline node_96 --> Bryconsp & 1 & $11.0001==>0$ & 140 & $10.231 \quad 1==>2$ \\
\hline & 17 & $10.250 \quad 1==>0$ & 141 & $\begin{array}{llll} & 0.200 & 1->0\end{array}$ \\
\hline & 30 & $10.5000==>1$ & node_97 --> Iguanodectesspil 3 & $10.100 \quad 1==>0$ \\
\hline & 42 & $10.1672==>0$ & 7 & $\begin{array}{llll}1 & 0.125 & 1-->0\end{array}$ \\
\hline & 75 & $10.0830==>1$ & 21 & $10.1430==>1$ \\
\hline & 100 & $\begin{array}{llll}1 & 0.333 & 1-->0\end{array}$ & 31 & $10.3750==>1$ \\
\hline & 102 & $10.3331==>0$ & 42 & $\begin{array}{llll} & 0.167 & 0 & -->\end{array}$ \\
\hline & 104 & $10.0380==>1$ & 57 & $10.333 \quad 1==>0$ \\
\hline & 111 & $10.143 \quad 1==>0$ & 61 & $10.3331==>0$ \\
\hline & 124 & $10.2000==>2$ & 62 & $10.167 \quad 1==>0$ \\
\hline & 126 & $10.080 \quad 1->0$ & 65 & $\begin{array}{llll} & 0.063 \quad 1->0\end{array}$ \\
\hline & 157 & $10.074 \quad 0-->2$ & 66 & $10.3330==>1$ \\
\hline node_187 --> node_186 & 3 & $10.1000==>1$ & 68 & $10.0950==>1$ \\
\hline & 7 & 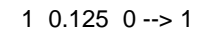 & 75 & $10.0830==>2$ \\
\hline & 16 & $\begin{array}{llll}1 & 0.048 & 0 & -->1\end{array}$ & 88 & $10.0542==>0$ \\
\hline & 32 & 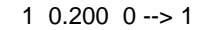 & 104 & $\begin{array}{lll}1 & 0.038 & 1->0\end{array}$ \\
\hline & 37 & $10.136 \quad 0->3$ & 105 & $10.1500==>3$ \\
\hline & 38 & $10.250 \quad 0 \quad->1$ & 106 & $10.250 \quad 0==>1$ \\
\hline
\end{tabular}




\begin{tabular}{|c|c|c|c|c|c|}
\hline & 112 & $10.0831==>0$ & & 96 & $10.034 \quad 0->1$ \\
\hline & 117 & $10.500 \quad 1->0$ & & 103 & $10.0360==>1$ \\
\hline & 121 & $10.2312==>3$ & & 132 & $10.1331==>2$ \\
\hline & 136 & $10.5000==>1$ & node_121 --> node_118 & 39 & $\begin{array}{lll}10.037 \quad 1->0 & 0\end{array}$ \\
\hline & 157 & $10.074 \quad 0-->2$ & & 120 & $10.0431->0$ \\
\hline & 158 & $10.3640==>1$ & & 137 & $10.111 \quad 1==>0$ \\
\hline \multirow{10}{*}{ node_186 --> node_185 } & 9 & $10.0830==>1$ & & 140 & $10.2311==>2$ \\
\hline & 26 & $10.158 \quad 0 \quad->1$ & node_118 --> node_115 & 18 & $10.1002==>0$ \\
\hline & 45 & $10.250 \quad 0==>2$ & & 26 & $10.1582==>1$ \\
\hline & 63 & $10.136 \quad 2->0$ & & 112 & $10.0832==>1$ \\
\hline & 73 & $10.0831==>2$ & node_115 --> node_114 & 34 & $10.0611==>0$ \\
\hline & 101 & $10.1430==>1$ & & 39 & $\begin{array}{llll}1 & 0.037 & 0 & ->1\end{array}$ \\
\hline & 120 & $10.0431==>0$ & & 59 & $10.0561->0$ \\
\hline & 132 & $10.1330==>2$ & & 72 & $10.0591->0$ \\
\hline & 135 & $10.056 \quad 0-->1$ & & 87 & $10.100 \quad 1==>0$ \\
\hline & 157 & $10.0740->2$ & & 110 & $\begin{array}{llll}1 & 0.087 & 1-->0\end{array}$ \\
\hline \multirow[t]{16}{*}{ node_185 --> node_184 } & 7 & $10.1251->0$ & & 126 & $10.0800->1$ \\
\hline & 16 & $10.048 \quad 1->0$ & & 132 & $\begin{array}{lll}1 & 0.133 & 2->1\end{array}$ \\
\hline & 18 & $10.1000==>1$ & & 133 & $10.048 \quad 1==>0$ \\
\hline & 24 & $\begin{array}{llll} & 0.071 & 0 & ->1\end{array}$ & & 157 & $10.0742->0$ \\
\hline & 26 & $10.158 \quad 1-->2$ & node_114 --> node_113 & 4 & $10.0500==>1$ \\
\hline & 27 & $10.2000==>1$ & & 16 & $\begin{array}{llll}1 & 0.048 & 1-->0\end{array}$ \\
\hline & 34 & $10.061 \quad 1->0$ & & 41 & $\begin{array}{lll}10.067 & 1-->0\end{array}$ \\
\hline & 63 & $10.1360->1$ & & 56 & $10.0671==>0$ \\
\hline & 66 & $10.3330==>1$ & & 65 & $\begin{array}{llll} & 0.063 \quad 1->0\end{array}$ \\
\hline & 71 & $10.0831==>0$ & & 94 & $10.2501==>2$ \\
\hline & 88 & $10.0542->0$ & & 96 & $\begin{array}{lll}10.034 & 1->0\end{array}$ \\
\hline & 93 & $10.0590==>1$ & & 104 & $10.0380==>1$ \\
\hline & 103 & $10.036 \quad 1->0$ & & 120 & $\begin{array}{llll} & 0.043 & 0 & -->1\end{array}$ \\
\hline & 131 & 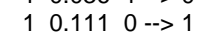 & node_113 --> node_107 & 18 & $10.100 \quad 0->1$ \\
\hline & 136 & $10.5000==>1$ & & 76 & $10.100 \quad 0->1$ \\
\hline & 151 & $10.063 \quad 1->0$ & & 124 & $10.2000==>2$ \\
\hline \multirow{7}{*}{ node_184 --> node_181 } & 2 & $10.043 \quad 1->0$ & & 137 & 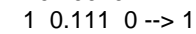 \\
\hline & 14 & $10.0560==>1$ & node_107 --> node_106 & 75 & $10.0831==>0$ \\
\hline & 42 & $10.167 \quad 2->1$ & & 76 & $\begin{array}{llll} & 0.100 & 1-->2\end{array}$ \\
\hline & 60 & $10.0630->1$ & & 110 & $10.0870->1$ \\
\hline & 76 & $10.1002-->0$ & & 139 & $10.3331==>0$ \\
\hline & 90 & $10.143 \quad 1==>2$ & & 141 & $\begin{array}{llll}1 & 0.200 & 1->0\end{array}$ \\
\hline & 137 & $10.1110==>1$ & node_106 --> node_105 & 4 & $10.0501==>0$ \\
\hline node_181 --> node_179 & 16 & $10.048 \quad 0->1$ & & 24 & $10.0711==>0$ \\
\hline & 28 & $10.105 \quad 2-->1$ & & 34 & $10.0610==>1$ \\
\hline & 116 & $10.5000==>1$ & & 56 & $10.0670==>1$ \\
\hline & 122 & $10.2730==>1$ & & 65 & $\begin{array}{llll} & 0.063 & 0 & -->1\end{array}$ \\
\hline node_179 --> node_176 & 4 & $10.0501->0$ & & 88 & $10.054 \quad 0->2$ \\
\hline & 42 & $10.167 \quad 1->2$ & & 112 & $10.0831->2$ \\
\hline & 65 & $10.0631->0$ & & 147 & $10.1151==>2$ \\
\hline & 88 & $10.0540->2$ & node_105 --> node_104 & 16 & $10.0480==>1$ \\
\hline & 133 & $10.048 \quad 1==>0$ & & 28 & $10.105 \quad 1==>0$ \\
\hline & 159 & $\begin{array}{llll} & 0.071 & 0 & -->2\end{array}$ & & 29 & $10.2000==>1$ \\
\hline node_176 --> node_173 & 21 & $10.1430==>1$ & & 72 & $\begin{array}{llll} & 0.059 & 0 & ->1\end{array}$ \\
\hline & 73 & $10.0832==>1$ & & 73 & $10.0831->0$ \\
\hline & 83 & $10.1430==>1$ & & 76 & $10.1002->0$ \\
\hline & 103 & $10.036 \quad 0-->1$ & & 87 & $10.100 \quad 0==>1$ \\
\hline & 132 & $10.1332==>1$ & & 91 & $\begin{array}{llll}1 & 0.100 & 1->0\end{array}$ \\
\hline & 138 & $10.067 \quad 0-->1$ & & 92 & $10.0430==>1$ \\
\hline & 148 & $10.125 \quad 0-->2$ & & 96 & $\begin{array}{llll} & 0.034 & 0 & -->1\end{array}$ \\
\hline node_173 --> node_172 & 44 & $10.167 \quad 1->0$ & & 125 & $10.1821==>0$ \\
\hline & 65 & $10.0630->1$ & & 134 & $10.077 \quad 1==>0$ \\
\hline & 81 & $10.1540==>1$ & & 137 & $\begin{array}{lll}1 & 0.111 & 1->0\end{array}$ \\
\hline & 84 & $10.0830==>1$ & & 140 & $10.2312->0$ \\
\hline & 96 & $10.034 \quad 1==>0$ & & 162 & $11.0000==>1$ \\
\hline & 159 & $\begin{array}{lll}1 & 0.071 & 2->0\end{array}$ & node_104 --> node_102 & 2 & 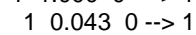 \\
\hline node_172 --> node_171 & 14 & $10.056 \quad 1==>0$ & & 21 & $10.143 \quad 1==>0$ \\
\hline & 18 & $10.100 \quad 1==>2$ & & 55 & $10.0710==>1$ \\
\hline & 60 & $10.063 \quad 1->0$ & & 83 & $\begin{array}{llll} & 0.143 & 1->0\end{array}$ \\
\hline & 70 & $10.040 \quad 1==>0$ & & 88 & $10.0542->0$ \\
\hline & 88 & $10.0542==>0$ & & 103 & $10.0361->0$ \\
\hline & 103 & $10.036 \quad 1->0$ & & 112 & $\begin{array}{lll}1 & 0.083 \quad 2->1\end{array}$ \\
\hline & 112 & $10.083 \quad 1==>2$ & & 144 & $10.154 \quad 0->1$ \\
\hline & 133 & $10.048 \quad 0-->1$ & node_102 --> node_100 & 20 & $10.143 \quad 1==>0$ \\
\hline node_171 --> node_168 & 39 & $10.0370==>1$ & & 22 & $10.0911==>0$ \\
\hline & 147 & $10.1150==>1$ & & 63 & $10.136 \quad 1->0$ \\
\hline & 148 & $10.125 \quad 2->0$ & & 131 & $10.111 \quad 1==>0$ \\
\hline node_168 --> node_167 & 34 & $10.0610 \rightarrow 1$ & & 133 & 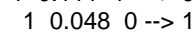 \\
\hline & 44 & $\begin{array}{llll}1 & 0.167 & 0 & -->1\end{array}$ & & 138 & $10.067 \quad 1==>0$ \\
\hline & 46 & $10.1670==>1$ & & 147 & $10.1152->0$ \\
\hline & 55 & $10.071 \quad 1==>0$ & & 151 & $10.0630->1$ \\
\hline & 75 & $10.0830==>1$ & & 165 & 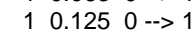 \\
\hline & 126 & $10.080 \quad 1==>0$ & node_100 --> node_99 & 34 & $10.061 \quad 1->2$ \\
\hline node_167 --> node_165 & 56 & $10.0670==>1$ & & 37 & $10.136 \quad 3->1$ \\
\hline & 59 & $10.056 \quad 0 \quad->1$ & & 42 & $10.167 \quad 2->1$ \\
\hline & 120 & $10.043 \quad 0->1$ & & 52 & $10.1431->0$ \\
\hline node_165 --> node_121 & 70 & $10.040 \quad 0==>1$ & & 58 & $10.125 \quad 1==>0$ \\
\hline & 72 & $10.059 \quad 0->1$ & & 71 & 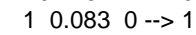 \\
\hline & 81 & $10.154 \quad 1==>0$ & & 72 & $10.0591->0$ \\
\hline & 89 & $10.043 \quad 0->1$ & & 76 & $10.100 \quad 0->1$ \\
\hline
\end{tabular}




\begin{tabular}{|c|c|c|c|}
\hline 77 & $\begin{array}{llll} & 0.182 & 0 & -->1\end{array}$ & node_101 --> Tetragnopterusar 9 & $10.083 \quad 1==>0$ \\
\hline 83 & $10.1430-->1$ & 17 & $10.2501==>0$ \\
\hline 89 & $10.0431==>0$ & 37 & $10.1363==>2$ \\
\hline 96 & $\begin{array}{lll}1 & 0.034 & 1-->0\end{array}$ & 73 & $\begin{array}{lll}1 & 0.0830-->\{12\}\end{array}$ \\
\hline 126 & $10.0801-->0$ & 87 & $10.1001==>0$ \\
\hline 129 & $10.6671==>0$ & 91 & 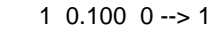 \\
\hline 134 & $10.0770==>1$ & 92 & $10.0431==>0$ \\
\hline 144 & $\begin{array}{llll} & 0.154 & 1-->0\end{array}$ & 96 & $\begin{array}{lll}1 & 0.034 & 1->0\end{array}$ \\
\hline 148 & $10.1250-->2$ & 104 & $10.0381==>0$ \\
\hline 150 & $10.167 \quad 1==>0$ & 110 & $10.0871==>2$ \\
\hline node_99 --> Serrasalmusmacul 2 & $\begin{array}{llll} & 0.043 & 1-->0\end{array}$ & 111 & $10.143 \quad 1==>0$ \\
\hline $\begin{array}{ll}- & 7\end{array}$ & $10.1250==>1$ & 120 & $10.0431==>0$ \\
\hline 9 & $10.0831==>0$ & node_104 --> node_103 & $10.100 \quad 1==>0$ \\
\hline 14 & $10.0560==>1$ & - & $10.0560==>1$ \\
\hline 18 & $10.100 \quad 1==>0$ & 60 & $10.0630==>1$ \\
\hline 26 & $10.158 \quad 1==>0$ & 75 & $10.0830==>1$ \\
\hline 30 & $10.5000==>2$ & 94 & $10.2502==>1$ \\
\hline 32 & $10.200 \quad 1==>2$ & 122 & $10.273 \quad 1==>2$ \\
\hline 49 & $10.100 \quad 1==>0$ & 149 & $10.1250==>1$ \\
\hline 65 & $10.0631==>0$ & 157 & 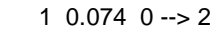 \\
\hline 66 & $10.3331==>0$ & node_103 --> Poptellaparaguay 14 & $10.0560==>1$ \\
\hline 68 & $10.0950==>1$ & - 33 & $10.1250==>1$ \\
\hline 73 & 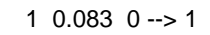 & 39 & $10.0371==>0$ \\
\hline 79 & $10.3330==>1$ & 47 & $10.2000==>1$ \\
\hline 81 & $10.1540==>1$ & 59 & $10.0560==>1$ \\
\hline 84 & $10.0831==>0$ & 67 & $10.1000==>1$ \\
\hline 88 & $10.0540==>1$ & 68 & $10.0950==>2$ \\
\hline 93 & $10.0591==>0$ & 70 & $10.0401==>0$ \\
\hline 94 & $10.2502==>0$ & 73 & $\begin{array}{llll}1 & 0.083 & 0-->1\end{array}$ \\
\hline 100 & $10.3331==>0$ & 140 & 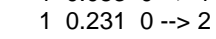 \\
\hline 103 & $10.036 \quad 0-->1$ & 141 & $10.200 \quad 0==>2$ \\
\hline 106 & $10.2500==>1$ & 163 & $10.3330==>1$ \\
\hline 110 & $10.0871==>2$ & node_103 --> Stethaprioneryth 3 & $10.100 \quad 1==>0$ \\
\hline 112 & $10.0831==>2$ & - 5 & $10.250 \quad 0==>1$ \\
\hline 121 & $10.2312==>3$ & 7 & $10.1250==>1$ \\
\hline 122 & $10.273 \quad 1==>3$ & 9 & $10.0831==>0$ \\
\hline 132 & $10.1331==>2$ & 24 & $10.0710==>1$ \\
\hline 153 & $10.6670==>1$ & 41 & $10.0670==>1$ \\
\hline 157 & $\begin{array}{llll}1 & 0.074 & 0 & -->\end{array}$ & 63 & $10.136 \quad 1==>0$ \\
\hline 163 & $10.3330==>1$ & 72 & $\begin{array}{llll}1 & 0.059 & 1-->0\end{array}$ \\
\hline 164 & $11.0000==>1$ & 113 & $10.0630==>1$ \\
\hline 165 & $\begin{array}{lll}1 & 0.125 & 1-->0\end{array}$ & 131 & $10.1111==>0$ \\
\hline node_99 --> Roeboidesbonarie 27 & $10.2001==>0$ & 135 & $10.0561==>0$ \\
\hline 31 & $10.3750==>3$ & node_105 --> Hemigrammuspreto 10 & $10.038 \quad 1==>0$ \\
\hline 50 & $10.0831==>0$ & 26 & $10.158 \quad 1==>2$ \\
\hline 59 & $10.0560==>1$ & 37 & $10.1363==>2$ \\
\hline 60 & $10.0630==>1$ & 104 & $10.0381==>0$ \\
\hline 70 & $10.0401==>0$ & 120 & $\begin{array}{llll}1 & 0.043 & 1->0\end{array}$ \\
\hline 74 & $10.1430==>1$ & 124 & $10.2002==>0$ \\
\hline 90 & $10.1432==>0$ & 133 & $10.0480==>1$ \\
\hline 95 & $10.2501==>0$ & 150 & $10.1671==>0$ \\
\hline 105 & $10.1502==>1$ & 159 & $10.0710==>2$ \\
\hline 133 & $\begin{array}{llll}1 & 0.048 & 1-->0\end{array}$ & node_106 --> Moenkhausiasanct 60 & $10.0630==>1$ \\
\hline 147 & $10.115 \quad 0-->3$ & 67 & $10.100 \quad 0==>1$ \\
\hline node_100 --> Psellorammuskenn 10 & $10.038 \quad 1==>0$ & 83 & $10.143 \quad 1==>0$ \\
\hline 26 & $10.158 \quad 1==>3$ & 90 & $10.1432==>1$ \\
\hline 36 & $10.0560==>1$ & 111 & $10.143 \quad 1==>0$ \\
\hline 39 & $10.0371==>0$ & 126 & $10.0801==>0$ \\
\hline 41 & $10.0670==>2$ & 141 & $10.200 \quad 0-->2$ \\
\hline 91 & $\begin{array}{lll}10.100 & 0-->1\end{array}$ & 145 & $10.0670==>1$ \\
\hline 94 & $10.2502==>1$ & 157 & 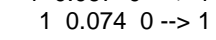 \\
\hline 98 & $10.0830==>1$ & 159 & $10.0710==>1$ \\
\hline 140 & 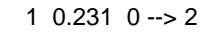 & node_107 --> Hemigrammusthapo 10 & $10.0381==>0$ \\
\hline 141 & $10.200 \quad 0==>2$ & 18 & $\begin{array}{lll}1 & 0.100 & 1->2\end{array}$ \\
\hline 147 & $10.115 \quad 0-->1$ & 29 & $10.2000==>1$ \\
\hline 159 & $10.0710==>1$ & 43 & $10.0771==>0$ \\
\hline node_102 --> node_101 & $10.3330==>1$ & 58 & $10.1251==>0$ \\
\hline- & $10.095 \quad 0 \quad->1$ & 68 & $10.0950==>2$ \\
\hline 84 & $10.0831==>0$ & 70 & $10.0401==>0$ \\
\hline 105 & $10.150 \quad 2==>1$ & 73 & $10.0831==>0$ \\
\hline 163 & $10.3330==>1$ & 77 & $10.1820==>1$ \\
\hline node_101 --> Gymnocorymbuster 2 & $10.0431-->0$ & 89 & $10.0431==>0$ \\
\hline 26 & $10.158 \quad 1==>2$ & 90 & $10.1432==>0$ \\
\hline 39 & $10.0371==>0$ & 120 & $10.0431->0$ \\
\hline 41 & $10.0670==>1$ & 140 & $10.2312==>1$ \\
\hline 47 & $10.200 \quad 0==>1$ & 157 & 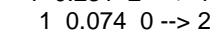 \\
\hline 59 & $10.0560==>1$ & node_113 --> node_112 & $10.1431==>0$ \\
\hline 60 & $10.0630==>1$ & 24 & $10.0711==>0$ \\
\hline 68 & $\begin{array}{lll}1 & 0.095 & 1-->2\end{array}$ & 83 & $\begin{array}{llll}1 & 0.143 & 1->0\end{array}$ \\
\hline 70 & $10.040 \quad 1==>0$ & 88 & $10.054 \quad 0-->2$ \\
\hline 75 & $10.0830==>1$ & 110 & $10.0870==>2$ \\
\hline 103 & 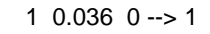 & 112 & $\begin{array}{lll}1 & 0.083 & 1-->2\end{array}$ \\
\hline 121 & $10.2312==>3$ & 132 & $\begin{array}{lll}1 & 0.133 \quad 1->\end{array}$ \\
\hline 143 & $10.1250==>1$ & 138 & $10.0671==>0$ \\
\hline 149 & $10.1250==>1$ & node_112 --> node_108 & $10.0831==>0$ \\
\hline 157 & $\begin{array}{llll}1 & 0.074 & 0 & -->2 \\
\end{array}$ & 16 & $\begin{array}{llll}1 & 0.048 & 0 & ->\end{array}$ \\
\hline
\end{tabular}




\begin{tabular}{|c|c|c|c|c|}
\hline & 27 & $10.200 \quad 1==>0$ & 45 & $10.2502-->0$ \\
\hline & 39 & $10.037 \quad 1==>0$ & 46 & $\begin{array}{llll}1 & 0.167 & 1-> & 2\end{array}$ \\
\hline & 41 & $10.067 \quad 0->1$ & 65 & $10.0630-->1$ \\
\hline & 55 & $10.0710==>1$ & 90 & $\begin{array}{llll}1 & 0.143 & 0 & ->\end{array}$ \\
\hline & 65 & $10.063 \quad 0-->1$ & 94 & $\begin{array}{lll}1 & 0.250 & 2-->1\end{array}$ \\
\hline & 82 & $10.500 \quad 0==>1$ & 95 & $\begin{array}{llll}1 & 0.250 & 0 & -->1\end{array}$ \\
\hline & 84 & $10.083 \quad 1==>0$ & 132 & $\begin{array}{llll}1 & 0.133 & 0 & -->\end{array}$ \\
\hline & 134 & $10.077 \quad 1==>0$ & 134 & $10.077 \quad 1==>0$ \\
\hline & 159 & $10.0710==>2$ & 159 & $\begin{array}{lll}1 & 0.071 & 0-->1\end{array}$ \\
\hline \multicolumn{2}{|c|}{ node_108 --> Astyanaxmexicanu 25} & $10.2220==>2$ & node_109 --> Oligosarcuspinto 2 & $10.043 \quad 1->0$ \\
\hline & 46 & $10.167 \quad 1==>0$ & 7 & $10.1250==>1$ \\
\hline & 76 & $10.1000==>1$ & 9 & $10.0831==>0$ \\
\hline & 79 & $10.3330==>2$ & 10 & $10.0380==>1$ \\
\hline & 88 & $10.0542->\{01\}$ & 42 & $10.1672==>1$ \\
\hline & 115 & $10.1330==>1$ & 49 & $10.1001==>0$ \\
\hline & 131 & $10.111 \quad 1==>0$ & 63 & $10.1360==>1$ \\
\hline & 133 & $10.0480==>1$ & 71 & $\begin{array}{lll} & 0.083 \quad 1->0\end{array}$ \\
\hline & 140 & $10.2312==>1$ & 73 & $10.0831==>0$ \\
\hline & 151 & $10.0630==>1$ & 75 & $\begin{array}{llll}1 & 0.083 & 0 & -->2\end{array}$ \\
\hline \multicolumn{2}{|c|}{ node_108 --> Astyanaxaltipara 7} & $10.1250==>1$ & 77 & $10.1820==>2$ \\
\hline & 20 & $10.143 \quad 1==>0$ & 79 & $10.3330==>2$ \\
\hline & 28 & $10.105 \quad 1==>0$ & 94 & $\begin{array}{llll} & 0.250 & 1-->3\end{array}$ \\
\hline & 29 & $10.2000==>1$ & 110 & $10.0872==>1$ \\
\hline & 32 & $10.200 \quad 1==>2$ & 112 & $10.0832-->1$ \\
\hline & 47 & $10.2000==>1$ & 141 & $10.2001==>2$ \\
\hline & 56 & $10.0670==>1$ & 143 & $10.1250==>1$ \\
\hline & 72 & $10.0590==>1$ & 148 & $10.125 \quad 2->0$ \\
\hline & 92 & $10.0430==>1$ & 159 & $\begin{array}{lll}1 & 0.071 & 1-->2\end{array}$ \\
\hline & 120 & $10.0431->0$ & node_109 --> Phenacogasterfra 4 & $10.0501==>0$ \\
\hline & 141 & $10.200 \quad 1==>2$ & 8 & $10.2221==>0$ \\
\hline & 148 & $10.1250==>1$ & 18 & $10.100 \quad 1==>2$ \\
\hline \multirow{10}{*}{ node_112 --> node_111 } & 10 & $10.038 \quad 1==>0$ & 26 & $10.158 \quad 1==>3$ \\
\hline & 32 & $10.2001==>0$ & 34 & $\begin{array}{llll}1 & 0.061 & 1-->2\end{array}$ \\
\hline & 37 & $10.136 \quad 3-->1$ & 36 & $10.0560==>1$ \\
\hline & 38 & $10.250 \quad 1==>0$ & 37 & $10.1361==>0$ \\
\hline & 58 & $10.125 \quad 1==>0$ & 50 & $10.0831==>0$ \\
\hline & 63 & $10.136 \quad 1==>0$ & 52 & $10.143 \quad 1==>0$ \\
\hline & 90 & $10.1432->0$ & 60 & $10.0630==>1$ \\
\hline & 95 & $10.2501-->0$ & 70 & $10.0401==>0$ \\
\hline & 103 & $10.036 \quad 1==>0$ & 76 & $10.1000==>1$ \\
\hline & 148 & $10.125 \quad 0-->2$ & 84 & $10.0831==>0$ \\
\hline \multicolumn{2}{|c|}{ node_111 --> Bryconexodonjuru 4} & $10.050 \quad 1==>0$ & 88 & $10.0542->1$ \\
\hline & 26 & $10.158 \quad 1==>0$ & 101 & $\begin{array}{llll}1 & 0.143 \quad & 0 & -->1\end{array}$ \\
\hline & 28 & $10.105 \quad 1==>0$ & 103 & $10.0360==>1$ \\
\hline & 31 & $10.3750==>3$ & 105 & $10.1502==>3$ \\
\hline & 37 & $10.136 \quad 1->2$ & 147 & $10.1151==>3$ \\
\hline & 47 & $10.2000==>2$ & 165 & $10.1250==>1$ \\
\hline & 67 & $10.1000==>2$ & node_114 --> Moenkhausiaxingu 27 & $10.2001==>$ \\
\hline & 68 & $10.0950==>2$ & & 0 \\
\hline & 73 & $10.083 \quad 1==>2$ & 68 & $10.0950==>2$ \\
\hline & 83 & $10.143 \quad 0-->1$ & 75 & $10.0831==>0$ \\
\hline & 91 & $10.100 \quad 1==>0$ & 111 & $10.143 \quad 1==>0$ \\
\hline & 104 & $10.038 \quad 1==>0$ & 134 & $10.0771==>0$ \\
\hline & 106 & $10.2500==>2$ & 148 & $10.1250==>2$ \\
\hline & 112 & $10.0832->1$ & node_115 --> Moenkhausiainter 2 & $10.0430==>1$ \\
\hline & 133 & $10.0480==>1$ & 24 & $10.071 \quad 1==>0$ \\
\hline node_111 --> node_110 & 2 & $10.0430-->1$ & 47 & $10.2000==>1$ \\
\hline & 18 & $10.1000==>1$ & 105 & $10.1502==>0$ \\
\hline & 22 & $10.091 \quad 1==>0$ & 147 & $10.115 \quad 1==>0$ \\
\hline & 71 & $10.0830-->1$ & 158 & $10.3640==>2$ \\
\hline & 75 & $10.0831-->0$ & node_118 --> node_117 & $10.0381==>0$ \\
\hline & 87 & $10.1000==>1$ & 88 & $10.0540==>2$ \\
\hline & 101 & $\begin{array}{llll}1 & 0.143 & 1->0\end{array}$ & 96 & $\begin{array}{llll}1 & 0.034 & 1-->0\end{array}$ \\
\hline & 122 & $10.273 \quad 1==>0$ & 138 & $10.0671==>0$ \\
\hline & 131 & $10.111 \quad 1==>0$ & node_117 --> node_116 & $10.0611==>0$ \\
\hline & 132 & $10.1332-->0$ & 35 & $10.1251==>0$ \\
\hline node_110 --> Hollandichths| & er 14 & $10.0560==>1$ & 44 & $\begin{array}{llll} & 0.167 & 1-->0\end{array}$ \\
\hline & 16 & $10.048 \quad 0->1$ & 46 & $10.167 \quad 1==>0$ \\
\hline & 25 & $10.2220==>1$ & 52 & $10.143 \quad 1==>0$ \\
\hline & 53 & $10.1542==>0$ & 59 & $\begin{array}{lll}10.056 \quad 1->0 & 0\end{array}$ \\
\hline & 74 & $10.1430==>1$ & 101 & $10.143 \quad 1==>0$ \\
\hline & 88 & $10.0542-->0$ & 108 & $10.1180==>1$ \\
\hline & 92 & $10.0430==>1$ & 151 & $\begin{array}{lll} & 0.063 & 0-->1\end{array}$ \\
\hline & 97 & $10.3331==>0$ & 157 & $10.0742==>0$ \\
\hline & 109 & $10.1110==>1$ & node_116 --> Deuterodoniguape 55 & $10.0710==>1$ \\
\hline & 110 & $10.0872==>0$ & 98 & $10.0830==>1$ \\
\hline & 113 & $10.0630==>1$ & 104 & $10.0380==>1$ \\
\hline & 115 & $10.1330==>2$ & node 116 --> Astyanaxjacobina 4 & $10.050 \quad 0==>1$ \\
\hline & 124 & $10.200 \quad 0==>2$ & 18 & $10.1002==>1$ \\
\hline & 137 & $10.1110==>1$ & 21 & $10.143 \quad 1==>0$ \\
\hline & 147 & $10.115 \quad 1==>2$ & 39 & $\begin{array}{llll} & 0.037 & 0 & -->1\end{array}$ \\
\hline & 150 & $10.167 \quad 1==>0$ & 74 & $10.1430==>1$ \\
\hline & 157 & $10.0740==>2$ & 88 & $10.0542==>1$ \\
\hline node_110 --> node_109 & 29 & $10.2000==>1$ & 89 & $10.043 \quad 1==>0$ \\
\hline & 34 & $\begin{array}{lll} & 0.061 \quad 0 \rightarrow 1\end{array}$ & 90 & $10.1432=>1$ \\
\hline & 39 & $10.037 \quad 1==>0$ & 92 & $10.0430==>1$ \\
\hline
\end{tabular}




\begin{tabular}{|c|c|c|c|c|c|}
\hline & 96 & $\begin{array}{llll} & 0.034 & 0 & ->\end{array}$ & & 133 & $\begin{array}{lll}1 & 0.048 & 1==>0\end{array}$ \\
\hline & 113 & $10.0630==>1$ & & 157 & $10.0742==>0$ \\
\hline & 120 & $10.0430==>1$ & node_149 --> node_144 & 6 & $10.1670==>2$ \\
\hline & 131 & $10.111 \quad 1==>0$ & & 34 & $\begin{array}{llll}1 & 0.061 & 1-->0\end{array}$ \\
\hline & 135 & $10.056 \quad 1==>0$ & & 69 & $10.1820==>2$ \\
\hline & 140 & $10.2312==>1$ & & 73 & $\begin{array}{llll}1 & 0.083 & 1-->0\end{array}$ \\
\hline & 150 & $10.167 \quad 1==>0$ & & 96 & $\begin{array}{llll}1 & 0.034 & 0 & ->\end{array}$ \\
\hline \multicolumn{2}{|c|}{ node_117 --> Jupiabapolylepis 2} & $10.0430==>1$ & node_144 --> node_142 & 26 & $10.1582==>3$ \\
\hline & 36 & $10.0560==>1$ & & 88 & $\begin{array}{llll}1 & 0.054 & 0 & -->2\end{array}$ \\
\hline & 41 & $10.067 \quad 1==>0$ & & 109 & $10.1110==>1$ \\
\hline & 43 & $10.0771==>0$ & node_142 --> node_136 & 28 & $10.105 \quad 1==>2$ \\
\hline & 60 & $10.0630==>1$ & & 133 & $10.0480==>1$ \\
\hline & 67 & $10.1000==>1$ & & 159 & $10.0710==>1$ \\
\hline & 72 & $\begin{array}{lllll} & 0.059 & 1->>0\end{array}$ & node_136 --> node_135 & 4 & $\begin{array}{llll}1 & 0.050 & 0 & -->1\end{array}$ \\
\hline & 76 & $10.1000==>2$ & & 14 & $10.0561==>0$ \\
\hline & 103 & $10.036 \quad 1==>0$ & & 36 & $\begin{array}{llll}1 & 0.056 & 1-->0\end{array}$ \\
\hline & 105 & $10.1502==>1$ & & 73 & 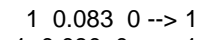 \\
\hline & 122 & $10.273 \quad 1==>0$ & & 126 & $10.080 \quad 0==>1$ \\
\hline & 133 & $10.048 \quad 1==>0$ & & 135 & $10.0561==>0$ \\
\hline & 134 & $10.077 \quad 1==>0$ & & 147 & $10.1150==>1$ \\
\hline & 147 & $10.1151==>3$ & node_135 --> node_134 & 6 & $10.1672==>3$ \\
\hline & 148 & $10.1250==>2$ & & 69 & $10.1822==>1$ \\
\hline & 149 & $10.125 \quad 0==>1$ & & 98 & $10.0830==>1$ \\
\hline \multirow{7}{*}{ node_121 --> node_120 } & 18 & $10.1002==>1$ & & 157 & $\begin{array}{llll}1 & 0.074 & 0 & ->\end{array}$ \\
\hline & 24 & $\begin{array}{llll}1 & 0.071 & 1-->0\end{array}$ & node_134 --> node_131 & 2 & $\begin{array}{llll}1 & 0.043 & 0 & ->\end{array}$ \\
\hline & 28 & $10.1051==>2$ & & 4 & $\begin{array}{llll}1 & 0.050 & 1->0\end{array}$ \\
\hline & 43 & $\begin{array}{llll}1 & 0.077 & 1-->0\end{array}$ & & 34 & $10.0610==>2$ \\
\hline & 75 & $10.0831==>0$ & & 37 & $\begin{array}{llll}1 & 0.136 & 3-->2\end{array}$ \\
\hline & 151 & $\begin{array}{llll}1 & 0.063 & 0 & -->\end{array}$ & & 96 & $10.0341==>0$ \\
\hline \multirow{2}{*}{\multicolumn{2}{|c|}{ node_120 --> Hemigrammusnewbo 56}} & $10.071 \quad 0-->2$ & & 108 & $\begin{array}{llll}1 & 0.118 & 0-->1\end{array}$ \\
\hline & & $10.067 \quad 1==>0$ & & 122 & $10.273 \quad 1==>0$ \\
\hline & 59 & $10.056 \quad 1-->0$ & & 138 & $\begin{array}{llll}1 & 0.067 & 1 & -->0\end{array}$ \\
\hline & 67 & $10.100 \quad 0==>1$ & & 145 & 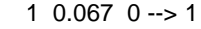 \\
\hline & 73 & $10.083 \quad 1==>0$ & & 157 & $\begin{array}{llll}1 & 0.074 \quad 1->2\end{array}$ \\
\hline & 87 & $10.100 \quad 1==>0$ & & 159 & $10.0711==>0$ \\
\hline & 157 & $10.0742==>1$ & node_131 --> node_126 & 74 & $\begin{array}{llll}1 & 0.143 & 0 & -->\end{array}$ \\
\hline node_120 --> node_119 & 4 & $10.0500==>1$ & & 78 & $10.500 \quad 0==>1$ \\
\hline & 10 & $10.038 \quad 1==>0$ & & 132 & $10.1331==>2$ \\
\hline & 16 & $10.048 \quad 1==>0$ & node_126 --> node_125 & 37 & $10.136 \quad 2->3$ \\
\hline & 37 & $10.136 \quad 3==>2$ & & 46 & $\begin{array}{llll}1 & 0.167 & 1-->0\end{array}$ \\
\hline & 45 & $\begin{array}{llll}1 & 0.250 & 2-->0\end{array}$ & & 67 & $\begin{array}{llll}1 & 0.100 & 0 & -->1\end{array}$ \\
\hline & 58 & $10.125 \quad 1==>0$ & & 68 & $10.095 \quad 0-->1$ \\
\hline & 76 & $10.100 \quad 0 \quad->2$ & & 80 & $10.3331==>0$ \\
\hline & 88 & $10.054 \quad 0-->2$ & & 88 & $\begin{array}{lll}1 & 0.054 & 2-->0\end{array}$ \\
\hline & 89 & $\begin{array}{llll}1 & 0.043 & 1->0\end{array}$ & & 89 & $\begin{array}{llll}1 & 0.043 & 0 & ->\end{array}$ \\
\hline & 90 & $10.1432==>1$ & & 93 & $10.0591==>0$ \\
\hline & 93 & $10.0591==>0$ & & 138 & $\begin{array}{llll}1 & 0.067 & 0 & -->1\end{array}$ \\
\hline & 110 & $10.087 \quad 1==>2$ & node_125 --> node_124 & 2 & $\begin{array}{lll}1 & 0.043 & 1->0\end{array}$ \\
\hline node_119 --> Hemigrammuscy & n 14 & $10.056 \quad 0==>1$ & & 8 & $\begin{array}{lll}1 & 0.2221==>0\end{array}$ \\
\hline & 43 & $\begin{array}{llll}1 & 0.077 & 0 & -->1\end{array}$ & & 10 & $10.0381==>0$ \\
\hline & 46 & $10.167 \quad 1==>3$ & & 55 & $10.0710==>1$ \\
\hline & 54 & $10.250 \quad 1==>0$ & & 59 & $10.0561==>0$ \\
\hline & 70 & $10.040 \quad 1==>0$ & & 84 & $\begin{array}{lll}1 & 0.083 & 1->0\end{array}$ \\
\hline & 72 & $10.0591->0$ & & 115 & $10.1330==>1$ \\
\hline & 103 & $10.036 \quad 1==>0$ & & 125 & $\begin{array}{lll}1 & 0.182 & 1->\end{array}$ \\
\hline & 104 & $10.038 \quad 0==>1$ & & 151 & $10.0630==>1$ \\
\hline & 121 & $10.2312==>1$ & node_124 --> Bryconellapalli & id 3 & $10.100 \quad 1==>0$ \\
\hline & 132 & $10.1332==>1$ & & 4 & $10.0500==>1$ \\
\hline & 133 & $10.048 \quad 1==>0$ & & 14 & $10.0560==>1$ \\
\hline & 134 & $10.077 \quad 1==>0$ & & 34 & $10.0612==>1$ \\
\hline & 146 & $10.1670==>1$ & & 46 & $\begin{array}{llll}1 & 0.167 & 0 & ->\end{array}$ \\
\hline & 151 & $10.063 \quad 1->0$ & & 50 & $10.0831==>0$ \\
\hline & 156 & $10.6670==>2$ & & 56 & $10.067 \quad 1==>0$ \\
\hline & 159 & $\begin{array}{llll}1 & 0.071 & 2-->0\end{array}$ & & 58 & $10.1251==>0$ \\
\hline node_119 --> Hemigrammussk & oli 6 & $10.1670==>2$ & & 63 & $10.136 \quad 1==>2$ \\
\hline & 20 & $10.143 \quad 1==>0$ & & 122 & $10.2730==>1$ \\
\hline & 22 & $10.0911==>0$ & & 159 & $10.0710==>2$ \\
\hline & 24 & $\begin{array}{llll}1 & 0.071 & 0 & -->1\end{array}$ & node_124 --> node_123 & 6 & $\begin{array}{llll}1 & 0.167 & 3->1\end{array}$ \\
\hline & 36 & $10.0560==>1$ & & 13 & $10.1430==>1$ \\
\hline & 41 & $10.067 \quad 1==>0$ & & 25 & 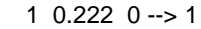 \\
\hline & 63 & $10.136 \quad 1==>0$ & & 35 & $\begin{array}{llll}1 & 0.125 & 1->0\end{array}$ \\
\hline & 91 & $10.100 \quad 1==>0$ & & 42 & $\begin{array}{lll}1 & 0.167 & 2-->1\end{array}$ \\
\hline & 92 & $10.0430==>1$ & & 44 & $\begin{array}{llll}1 & 0.167 & 1->0\end{array}$ \\
\hline & 94 & $10.250 \quad 1==>3$ & & 57 & $10.3331==>0$ \\
\hline & 96 & $10.034 \quad 1-->0$ & & 60 & $10.0631==>0$ \\
\hline & 108 & $10.1180==>1$ & & 67 & $\begin{array}{llll}1 & 0.100 & 1 & -->0\end{array}$ \\
\hline & 111 & $10.143 \quad 1==>0$ & & 68 & $10.0951->0$ \\
\hline & 124 & $10.2000==>2$ & & 71 & $10.0830==>1$ \\
\hline & 135 & $10.056 \quad 1==>0$ & & 88 & $\begin{array}{llll}1 & 0.054 & 0 & ->\end{array}$ \\
\hline & 157 & $10.0742==>0$ & & 89 & $10.0431-->0$ \\
\hline node_165 --> node_164 & 14 & $10.0560-->1$ & & 92 & $\begin{array}{lll}10.043 & 1->0\end{array}$ \\
\hline & 36 & $10.0560==>1$ & & 104 & $\begin{array}{llll}1 & 0.038 & 0 & ->\end{array}$ \\
\hline node_164 --> node_162 & 19 & $10.0830==>1$ & & 108 & $\begin{array}{llll}1 & 0.118 & 1-->2\end{array}$ \\
\hline & 60 & $10.0630==>1$ & & 123 & $\begin{array}{llll}1 & 0.250 & 0 & -->1\end{array}$ \\
\hline node_162 --> node_151 & 147 & $10.115 \quad 1==>0$ & & 138 & $\begin{array}{llll}1 & 0.067 & 1-->0\end{array}$ \\
\hline node_151 --> node_149 & 92 & $\begin{array}{llll}1 & 0.043 & 0 & -->1\end{array}$ & & 145 & $\begin{array}{llll}1 & 0.067 & 1 & ->0 \\
\end{array}$ \\
\hline
\end{tabular}




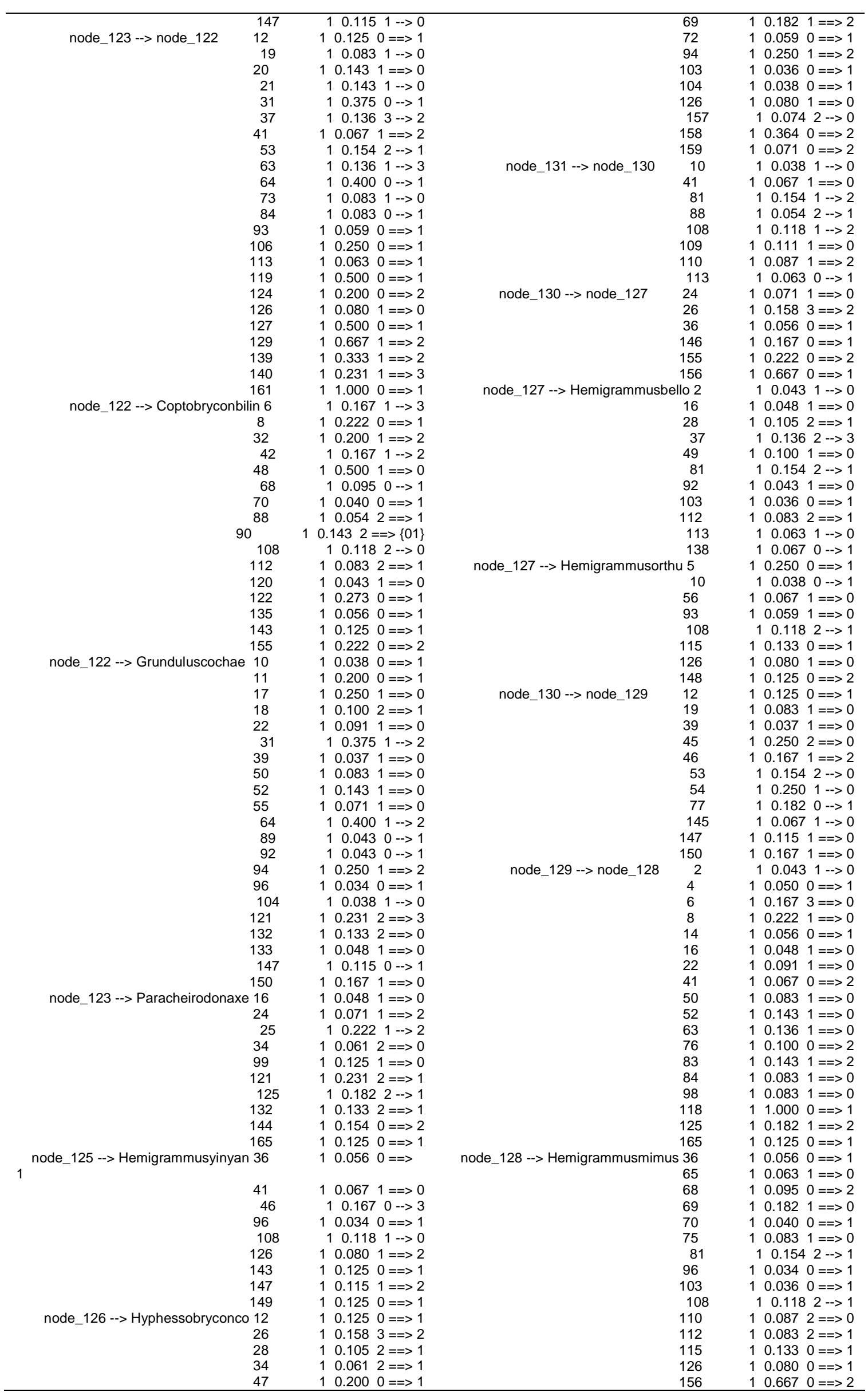




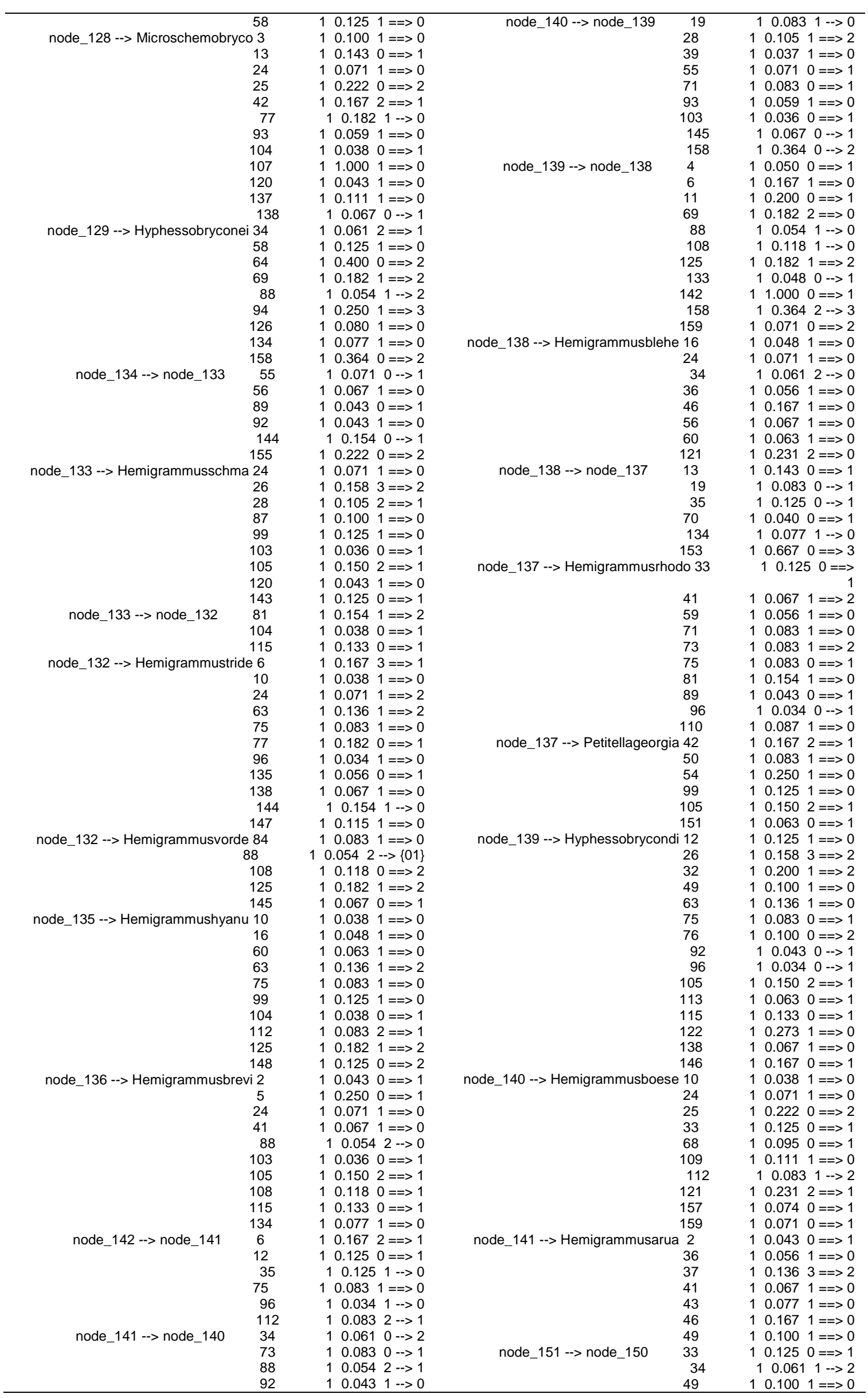




\begin{tabular}{|c|c|c|c|c|c|}
\hline & 108 & 10.118 & $80-->1$ & 88 & $\begin{array}{llll}1 & 0.054 & 0 & -->1\end{array}$ \\
\hline & 53 & 10.154 & $2==>0$ & node_150 --> Hemigrammussilim 28 & $10.1051==>2$ \\
\hline & 106 & 10.250 & $0==>2$ & 67 & $10.1000==>2$ \\
\hline & 135 & 10.056 & $1==>0$ & 73 & $10.0831==>0$ \\
\hline & 147 & 10.115 & $0==>3$ & 109 & $10.1110==>1$ \\
\hline & 155 & 10.222 & $0==>2$ & 115 & $10.1330==>1$ \\
\hline & 157 & 10.074 & $0==>2$ & 135 & $10.0561==>0$ \\
\hline \multirow{10}{*}{ node_144 --> node_143 } & 10 & 10.038 & $1==>0$ & 158 & $10.3640==>1$ \\
\hline & 33 & 10.125 & $0==>1$ & node_150 --> Hemigrammusgraci 10 & $10.0381==>0$ \\
\hline & 43 & 10.077 & $1==>0$ & 16 & $10.0481==>0$ \\
\hline & 67 & 10.100 & $0==>1$ & 41 & $10.0671==>0$ \\
\hline & 89 & 10.043 & $0==>1$ & 70 & $10.0400==>1$ \\
\hline & 120 & 10.043 & $31->0$ & 75 & $10.0831==>0$ \\
\hline & 126 & 10.080 & $0==>2$ & 104 & $10.0380==>1$ \\
\hline & 145 & 10.067 & $0==>1$ & 105 & $10.1502==>1$ \\
\hline & 147 & 10.115 & $0==>2$ & 108 & $10.1180==>1$ \\
\hline & 149 & 10.125 & $0==>1$ & 110 & $10.087 \quad 1==>0$ \\
\hline \multicolumn{2}{|c|}{ node_143 --> Hemigrammuspulch 14} & 10.056 & $1==>0$ & 113 & $10.0630==>1$ \\
\hline & 16 & 10.048 & $1==>0$ & 120 & $\begin{array}{llll} & 0.043 & 1-->0\end{array}$ \\
\hline & 19 & 10.083 & $1==>0$ & 151 & $10.0630==>1$ \\
\hline & 46 & 10.167 & $1==>0$ & 159 & $10.0710==>1$ \\
\hline & 60 & 10.063 & $1==>0$ & node_162 --> node_161 & $\begin{array}{llll}1 & 0.056 & 1->0\end{array}$ \\
\hline & 70 & 10.040 & $0==>1$ & -5 & $\begin{array}{llll}1 & 0.067 & 1->0\end{array}$ \\
\hline & 72 & 10.059 & $0==>1$ & 96 & 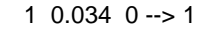 \\
\hline & 77 & 10.182 & $0==>1$ & 120 & $\begin{array}{llll}1 & 0.043 & 1-->0\end{array}$ \\
\hline & 110 & 10.087 & $1==>0$ & 152 & $11.0000==>1$ \\
\hline & 134 & 10.077 & $1==>0$ & node_161 --> node_152 & $10.0831-->0$ \\
\hline \multicolumn{2}{|c|}{ node_143 --> Hemigrammusharal 6} & 10.167 & $2==>3$ & -- & $\begin{array}{llll}1 & 0.043 & 0 & ->\end{array}$ \\
\hline & 13 & 10.143 & $0==>1$ & 124 & $10.2000==>1$ \\
\hline & 24 & 10.071 & $1==>0$ & 126 & $10.0800==>1$ \\
\hline & 28 & 10.105 & $1==>2$ & 133 & $10.048 \quad 1==>0$ \\
\hline & 39 & 10.037 & $1==>0$ & 153 & $10.6670==>2$ \\
\hline & 41 & 10.067 & $1==>0$ & 154 & $11.0000==>1$ \\
\hline & 75 & 10.083 & $1==>0$ & node_152 --> Hemigrammusunili 2 & $10.0430==>1$ \\
\hline & 93 & 10.059 & $1==>0$ & 33 & $10.1250==>1$ \\
\hline & 98 & 10.083 & $0==>1$ & 34 & $10.0611==>2$ \\
\hline & 112 & 10.083 & $2==>1$ & 37 & $10.1363==>2$ \\
\hline & 113 & 10.063 & $0==>1$ & 47 & $10.200 \quad 0==>1$ \\
\hline & 146 & 10.167 & $0==>1$ & 67 & $10.100 \quad 0==>1$ \\
\hline node_149 --> node_148 & 24 & 10.071 & $1==>0$ & 70 & $10.040 \quad 0==>1$ \\
\hline & 39 & 10.037 & $1==>0$ & 109 & $10.1110==>1$ \\
\hline & 104 & 10.038 & $0==>1$ & 115 & $10.1330==>1$ \\
\hline & 159 & 10.071 & $0==>2$ & 143 & $10.1250==>1$ \\
\hline node_148 --> node_147 & 2 & 10.043 & $0==>1$ & node_152 --> Moenkahusiahemig 16 & $10.0481==>0$ \\
\hline & 19 & 10.083 & $1==>0$ & 18 & $10.1002==>1$ \\
\hline & 43 & 10.077 & $71-->0$ & 19 & $10.0831==>0$ \\
\hline & 89 & 10.043 & $30-->1$ & 24 & $10.0711==>0$ \\
\hline & 92 & 10.043 & $31-->0$ & 28 & $10.105 \quad 1==>0$ \\
\hline & 110 & 10.087 & $1==>0$ & 41 & $\begin{array}{llll}1 & 0.067 & 0 & -->\end{array}$ \\
\hline node_147 --> Hemigrammuspar & n 68 & 10.095 & $0==>2$ & 43 & $10.0771==>0$ \\
\hline & 134 & 10.077 & $1==>0$ & 72 & $10.0590==>1$ \\
\hline & 157 & 10.074 & $0==>2$ & 88 & $10.0540==>1$ \\
\hline node_147 --> node_146 & 10 & 10.038 & $1==>0$ & 89 & $10.0430==>1$ \\
\hline & 67 & 10.100 & $00-->1$ & 104 & $10.0380==>1$ \\
\hline & 133 & 10.048 & $0==>1$ & 137 & $\begin{array}{lll}1 & 0.111 & 1==>0\end{array}$ \\
\hline & 158 & 10.364 & $0==>2$ & node_161 --> node_160 & $10.1670==>3$ \\
\hline node_146 --> node_145 & 24 & 10.071 & $0==>1$ & $--2+2-1$ & $\begin{array}{lll}1 & 0.061 & 1-->0\end{array}$ \\
\hline & 36 & 10.056 & $1==>0$ & 36 & $10.0561==>0$ \\
\hline & 39 & 10.037 & $0==>1$ & 63 & $\begin{array}{llll}1 & 0.136 & 1-->0\end{array}$ \\
\hline & 41 & 10.067 & $71-->0$ & 88 & $\begin{array}{llll}1 & 0.054 & 0 & ->\end{array}$ \\
\hline & 43 & 10.077 & $7 \quad 0-->1$ & 93 & $\begin{array}{llll}1 & 0.059 & 1-->0\end{array}$ \\
\hline & 71 & 10.083 & $0==>1$ & 112 & $10.0832==>1$ \\
\hline & 89 & 10.043 & $31-->0$ & 144 & $10.1540==>1$ \\
\hline & 112 & 10.083 & $2==>1$ & 150 & $10.167 \quad 1==>0$ \\
\hline & 151 & 10.063 & $0==>1$ & 165 & $10.1250==>1$ \\
\hline node_145 --> Hemigrammusma & gi 2 & 10.043 & $1==>0$ & node_160 --> Hyphessobryconbe 26 & $10.1582==>3$ \\
\hline & 4 & 10.050 & $0==>1$ & 39 & $10.0371==>0$ \\
\hline & 18 & 10.100 & $2==>1$ & 63 & 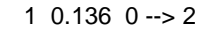 \\
\hline & 27 & 10.200 & $1==>0$ & 68 & $10.0950==>1$ \\
\hline & 58 & 10.125 & $1==>0$ & 72 & $10.0590==>1$ \\
\hline & 70 & 10.040 & $0==>1$ & 75 & $10.0831==>0$ \\
\hline & 159 & 10.071 & $2==>0$ & 77 & $10.1820==>1$ \\
\hline node_145 --> Hemigrammussp & It 49 & 10.100 & $1==>0$ & 83 & $10.1431==>0$ \\
\hline & 67 & 10.100 & $01-->0$ & 99 & $10.125 \quad 1==>0$ \\
\hline & 73 & 10.083 & $1==>0$ & 104 & $10.0380==>1$ \\
\hline & 75 & 10.083 & $1==>0$ & 113 & $10.0630==>1$ \\
\hline node_146 --> Moenkhausiabo & it 14 & 10.056 & $1==>0$ & 135 & $10.0561==>0$ \\
\hline & 76 & 10.100 & $0==>1$ & node_160 --> node_159 & $10.0560-->1$ \\
\hline & 88 & 10.054 & $0==>1$ & - & $\begin{array}{lll}10.083 & 1->0\end{array}$ \\
\hline & 104 & 10.038 & $1==>0$ & 53 & $10.1542==>0$ \\
\hline & 105 & 10.150 & $2==>1$ & 56 & $\begin{array}{llll} & 0.067 & 1-->0\end{array}$ \\
\hline & 120 & 10.043 & $1==>0$ & 96 & $\begin{array}{llll}1 & 0.034 & 1-->0\end{array}$ \\
\hline & 137 & 10.111 & $1==>0$ & 138 & $10.0671==>0$ \\
\hline node_148 --> Hemigrammusrod & a 70 & 10.040 & $0==>1$ & 89 & $10.0430==>1$ \\
\hline & 103 & 10.036 & $0==>1$ & 103 & $10.0360==>1$ \\
\hline & 115 & 10.133 & $0==>1$ & 104 & $10.038 \quad 1==>0$ \\
\hline
\end{tabular}




\begin{tabular}{|c|c|c|c|c|}
\hline & 121 & $10.2312==>1$ & 112 & $\begin{array}{lll}1 & 0.083 & 2-->1\end{array}$ \\
\hline & 153 & $10.6670==>1$ & 149 & $10.1250==>1$ \\
\hline \multirow{9}{*}{ node_159 --> node_158 } & 24 & $10.071 \quad 1==>0$ & node_154 --> Hyphessobryconmi 19 & $10.0831==>0$ \\
\hline & 34 & $\begin{array}{llll}1 & 0.061 & 0 & -->1\end{array}$ & 22 & $10.091 \quad 1==>0$ \\
\hline & 41 & $10.0670-->1$ & 39 & $10.0371==>0$ \\
\hline & 45 & $10.2502==>0$ & 59 & $10.0561==>0$ \\
\hline & 70 & $10.040 \quad 0 \quad->1$ & 63 & $\begin{array}{llll}1 & 0.136 & 0 & -->2\end{array}$ \\
\hline & 93 & $10.0590-->1$ & 64 & $\begin{array}{lll}1 & 0.400 & 1-->2\end{array}$ \\
\hline & 98 & $10.0830==>1$ & 69 & $10.1821==>2$ \\
\hline & 133 & $10.0481==>0$ & 70 & 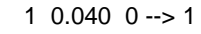 \\
\hline & 145 & $10.067 \quad 0-->1$ & 105 & $10.150 \quad 1==>2$ \\
\hline \multirow[t]{9}{*}{ node_158 --> node_157 } & 37 & $10.1363==>2$ & 124 & $10.2000==>2$ \\
\hline & 46 & $10.1671==>3$ & 126 & $10.0800==>1$ \\
\hline & 50 & $\begin{array}{llll}1 & 0.083 & 0 & -->\end{array}$ & node_157 --> node_156 & $10.0561==>0$ \\
\hline & 56 & $\begin{array}{llll} & 0.067 & 0 & ->\end{array}$ & - & $10.2000==>1$ \\
\hline & 75 & $10.0831==>0$ & 41 & $\begin{array}{llll}1 & 0.067 & 1-->0\end{array}$ \\
\hline & 92 & $10.0430==>1$ & 52 & $10.143 \quad 1==>0$ \\
\hline & 104 & $10.038 \quad 0==>1$ & 96 & $10.0340==>1$ \\
\hline & 105 & $10.1502==>1$ & node_156 --> Hyphessobryconep 30 & $10.5000==>2$ \\
\hline & 112 & $\begin{array}{llll}1 & 0.083 & 1-->2\end{array}$ & 58 & $10.1251==>0$ \\
\hline \multirow[t]{5}{*}{ node_157 --> node_155 } & 69 & $10.1820==>1$ & 88 & $10.0542==>0$ \\
\hline & 70 & $\begin{array}{llll}1 & 0.040 & 1-->0\end{array}$ & 121 & $10.2312==>1$ \\
\hline & 93 & $10.0591-->0$ & 144 & $10.1541==>0$ \\
\hline & 120 & $10.0430==>1$ & 148 & $10.1250==>1$ \\
\hline & 145 & $\begin{array}{llll}1 & 0.067 & 1-->0\end{array}$ & node_156 --> Hyphessobryconer 34 & $10.0611==>2$ \\
\hline \multirow[t]{6}{*}{ node_155 --> node_153 } & 43 & $10.077 \quad 1-->0$ & 39 & $10.0371==>0$ \\
\hline & 53 & $10.1540==>2$ & 44 & $10.1671==>2$ \\
\hline & 63 & $\begin{array}{llll}1 & 0.136 & 0 & -->\end{array}$ & 98 & $10.0831==>0$ \\
\hline & 148 & $10.1250-->2$ & 99 & $10.1251==>0$ \\
\hline & 150 & $10.1670==>1$ & 103 & $10.0360==>1$ \\
\hline & 165 & $10.125 \quad 1==>0$ & 112 & $10.0832-->1$ \\
\hline node_153 --> Hyphessobryco & co 2 & $10.0430==>1$ & node_158 --> Hyphessobryconeq 10 & $10.0381==>0$ \\
\hline & 6 & $10.1673==>1$ & - 39 & $10.0371==>0$ \\
\hline & 8 & $10.222 \quad 1==>2$ & 63 & $\begin{array}{llll} & 0.136 & 0 & -->1\end{array}$ \\
\hline & 14 & $10.0561==>0$ & 65 & $10.0631==>0$ \\
\hline & 22 & $10.0911==>0$ & 99 & $10.1251==>0$ \\
\hline & 59 & $10.0561==>0$ & 126 & $10.0800==>1$ \\
\hline & 73 & $10.083 \quad 1==>0$ & 135 & $10.0561==>0$ \\
\hline & 74 & $10.143 \quad 0==>1$ & 143 & $10.1250==>1$ \\
\hline & 81 & $10.154 \quad 1==>2$ & node_159 --> Hyphessobryconta 4 & $10.050 \quad 0==>1$ \\
\hline & 84 & $10.0831==>0$ & 16 & $10.0481==>0$ \\
\hline & 89 & $10.0430==>1$ & 59 & $10.0561==>0$ \\
\hline & 92 & $10.0431==>0$ & 103 & $10.0360==>1$ \\
\hline & 101 & $10.143 \quad 1==>0$ & 140 & $10.2311==>2$ \\
\hline & 126 & $10.0800==>1$ & node_164 --> node_163 & $10.1363==>2$ \\
\hline & 132 & $10.1331==>2$ & - & $10.0871==>0$ \\
\hline & 144 & $10.1541==>0$ & 124 & $10.2000==>1$ \\
\hline & 147 & $10.1151==>0$ & 126 & $10.0800==>1$ \\
\hline node_153 --> Pristellamax & lla 19 & $10.0831==>0$ & 144 & $\begin{array}{llll} & 0.154 & 0-->2\end{array}$ \\
\hline & 28 & $10.1051==>0$ & 148 & 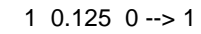 \\
\hline & 32 & $10.2001==>0$ & 155 & 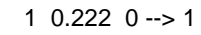 \\
\hline & 34 & $10.0611==>2$ & node_163 --> Hemigrammuslunat 10 & $10.0381==>0$ \\
\hline & 36 & $10.0560==>1$ & 24 & $10.0711==>0$ \\
\hline & 37 & $10.1362==>\{01\}$ & 34 & $10.0611==>2$ \\
\hline & 38 & $10.250 \quad 1==>0$ & 59 & $\begin{array}{llll}1 & 0.056 & 1-->0\end{array}$ \\
\hline & 71 & $10.0830==>1$ & 69 & $10.1820==>2$ \\
\hline & 87 & $10.1001==>0$ & 89 & $10.0430==>1$ \\
\hline & 88 & $10.0542==>0$ & 133 & $10.0481==>0$ \\
\hline & 90 & $10.1432==>1$ & 148 & $\begin{array}{llll}1 & 0.125 & 1-->2\end{array}$ \\
\hline & 93 & $10.0590-->1$ & 155 & $\begin{array}{llll}1 & 0.222 & 1-->2\end{array}$ \\
\hline & 96 & $10.0340==>1$ & node_163 --> Hemigrammusulrey 18 & $10.1002==>0$ \\
\hline & 98 & $10.0831==>0$ & 26 & $10.1582==>1$ \\
\hline & 103 & $10.0360==>1$ & 33 & $10.1250==>1$ \\
\hline & 133 & $10.0480==>1$ & 44 & $10.167 \quad 1==>0$ \\
\hline & 135 & $10.056 \quad 1==>0$ & 70 & $10.0400==>1$ \\
\hline & 143 & $10.1250==>1$ & 83 & $10.143 \quad 1==>0$ \\
\hline & 151 & $10.0630==>1$ & 88 & $10.0540==>2$ \\
\hline & 153 & $10.667 \quad 1==>4$ & 90 & $10.1432==>1$ \\
\hline & 155 & $10.2220==>2$ & 104 & $10.0380==>1$ \\
\hline node_155 --> node_154 & 5 & $10.250 \quad 0==>1$ & 138 & $\begin{array}{lll}1 & 0.067 \quad 1==>0\end{array}$ \\
\hline & 24 & $10.0710==>1$ & 150 & $10.1671==>2$ \\
\hline & 26 & $10.1582==>3$ & 151 & $10.0630==>1$ \\
\hline & 50 & $\begin{array}{lllll}1 & 0.083 & 1->0\end{array}$ & node_167 --> node_166 & $10.100 \quad 0==>1$ \\
\hline & 54 & $10.250 \quad 1==>0$ & - & $\begin{array}{lll} & 0.143 \quad 1->0\end{array}$ \\
\hline & 64 & $10.400 \quad 0-->1$ & 145 & $10.0670==>1$ \\
\hline & 76 & $10.100 \quad 0-->2$ & 149 & $10.1250==>1$ \\
\hline & 80 & $10.3331==>0$ & 153 & $10.6670==>1$ \\
\hline & 113 & $10.0630==>1$ & node_166 --> Hemigrammuscoeru 4 & $10.0500==>1$ \\
\hline node_154 --> Hyphessobrycon & ne 2 & $10.0430==>1$ & 8 & $10.2221==>0$ \\
\hline & 12 & $10.1250==>1$ & 16 & $10.0481==>0$ \\
\hline & 34 & $10.0611==>2$ & 28 & $10.1051==>2$ \\
\hline & 36 & $10.0560==>1$ & 37 & $10.1363==>2$ \\
\hline & 44 & $10.167 \quad 1==>2$ & 41 & $10.0671==>0$ \\
\hline & 46 & $10.1673==>2$ & 10 & $10.0381==>0$ \\
\hline & 74 & $10.143 \quad 0==>1$ & 35 & $10.1251==>0$ \\
\hline & 78 & $10.500 \quad 0==>1$ & 53 & $10.1542==>0$ \\
\hline
\end{tabular}




\begin{tabular}{|c|c|c|c|c|}
\hline & 88 & $10.0542==>0$ & 59 & $10.0560==>1$ \\
\hline & 68 & $10.0950==>2$ & 93 & $10.0591==>0$ \\
\hline & 73 & $10.083 \quad 1==>0$ & 120 & $10.0430==>1$ \\
\hline & 87 & $10.100 \quad 1==>0$ & 138 & $\begin{array}{llll}1 & 0.067 & 1->0\end{array}$ \\
\hline & 91 & $10.100 \quad 1==>0$ & 144 & $10.1540==>1$ \\
\hline & 135 & $10.056 \quad 1==>0$ & 145 & $10.0670==>1$ \\
\hline & 144 & $10.1540==>1$ & 147 & $10.1150==>3$ \\
\hline \multicolumn{2}{|c|}{ node 166 --> Hemigrammusocell 6} & $10.1670==>1$ & 149 & $10.1250==>1$ \\
\hline & 34 & $\begin{array}{llll}1 & 0.061 \quad 1->0\end{array}$ & node_173 --> Hemigrammuslevis 28 & $\begin{array}{llll} & 0.105 & 1-->2\end{array}$ \\
\hline & 43 & $10.077 \quad 1==>0$ & 33 & $10.1250==>1$ \\
\hline & 49 & $10.100 \quad 1==>0$ & 34 & $10.0610-->1$ \\
\hline & 65 & $10.0631==>0$ & 36 & $10.0560==>1$ \\
\hline & 70 & $10.0400==>1$ & 39 & $10.0370==>1$ \\
\hline & 89 & $10.0430==>1$ & 55 & $10.0711==>0$ \\
\hline & 103 & $10.0360==>1$ & 56 & $10.0670==>1$ \\
\hline & 110 & $10.0871==>0$ & 67 & $10.1000==>1$ \\
\hline & 126 & $10.0800==>2$ & 71 & $10.0830==>1$ \\
\hline & 147 & $10.115 \quad 1==>2$ & 92 & $10.0430==>1$ \\
\hline & 157 & $10.0742==>1$ & 105 & $10.1502==>1$ \\
\hline & 159 & $10.0710==>1$ & 109 & $10.1110==>1$ \\
\hline & 160 & $11.000 \quad 0==>1$ & 146 & $10.1670==>1$ \\
\hline \multicolumn{2}{|c|}{ node_168 --> Hemigrammusbarri 2} & $10.0430==>1$ & node_176 --> node_175 & $10.1250==>1$ \\
\hline & 10 & $10.038 \quad 1==>0$ & -5 & $10.3330==>1$ \\
\hline & 16 & $10.048 \quad 1==>0$ & 20 & $10.1431==>0$ \\
\hline & 83 & $10.143 \quad 1==>0$ & 24 & $10.071 \quad 1==>0$ \\
\hline & 110 & $10.0871==>0$ & 25 & $10.2220==>1$ \\
\hline & 115 & $10.1330==>1$ & 60 & $\begin{array}{llll} & 0.063 & 1-->0\end{array}$ \\
\hline & 133 & $10.048 \quad 1-->0$ & 76 & $10.1000==>1$ \\
\hline & 144 & $10.1540==>2$ & 98 & $10.0830==>1$ \\
\hline & 148 & $\begin{array}{lllll}1 & 0.125 & 0 & -->1\end{array}$ & 124 & $10.2000==>2$ \\
\hline & 150 & $10.167 \quad 1==>2$ & 125 & $10.1821==>2$ \\
\hline & 151 & $10.0630==>1$ & 126 & $10.0801==>0$ \\
\hline & 155 & $10.2220==>1$ & 127 & $10.500 \quad 0==>1$ \\
\hline & 157 & $10.0742==>0$ & node_175 --> node_174 & $10.1001==>0$ \\
\hline & 159 & $10.0710==>1$ & $--2+2+2=-$ & 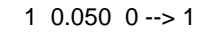 \\
\hline \multirow{8}{*}{ node_171 --> node_170 } & 4 & $10.0500==>1$ & 9 & $10.0831==>0$ \\
\hline & 28 & $10.105 \quad 1-->2$ & 22 & $10.0911==>0$ \\
\hline & 41 & $\begin{array}{llll} & 0.067 & 1->0\end{array}$ & 26 & $10.1582==>1$ \\
\hline & 43 & $10.077 \quad 1==>0$ & 47 & $10.200 \quad 0==>1$ \\
\hline & 58 & $10.125 \quad 1->0$ & 68 & $10.0950==>1$ \\
\hline & 72 & $10.0590-->1$ & 72 & $10.0590==>1$ \\
\hline & 111 & $10.143 \quad 1==>0$ & 79 & $\begin{array}{llll}1 & 0.333 & 0 & -->\end{array}$ \\
\hline & 125 & $10.1821==>2$ & 82 & $10.5000==>1$ \\
\hline \multirow[t]{4}{*}{ node_170 --> node_169 } & 16 & $10.0481==>0$ & 91 & $10.1001==>0$ \\
\hline & 44 & $\begin{array}{llll} & 0.167 & 0 & -->\end{array}$ & 92 & $10.0430==>1$ \\
\hline & 88 & $10.0540==>1$ & 93 & $10.0591==>0$ \\
\hline & 122 & $10.273 \quad 1==>0$ & 94 & $10.2501==>2$ \\
\hline node_169 --> Hemigrammusan & ali 3 & $10.100 \quad 1==>0$ & 97 & $10.3331==>0$ \\
\hline & 14 & $10.0560==>1$ & 104 & $10.0380==>1$ \\
\hline & 24 & $10.071 \quad 1==>0$ & 123 & $10.2500==>1$ \\
\hline & 39 & $10.0370==>1$ & 129 & $10.6671==>2$ \\
\hline & 41 & $\begin{array}{llll}1 & 0.067 & 0 & --> \\
1\end{array}$ & 147 & $10.1150==>1$ \\
\hline & 65 & $10.063 \quad 1==>0$ & 151 & $10.0630==>1$ \\
\hline & 72 & $10.0591-->0$ & node_174 --> HasemaniaspCrenu 17 & $10.2501==>0$ \\
\hline & 113 & $10.0630==>1$ & $\begin{array}{ll}- & 45\end{array}$ & $10.2502==>1$ \\
\hline & 145 & $10.0670==>1$ & 55 & $10.0711==>0$ \\
\hline & 146 & $10.1670==>1$ & 65 & $\begin{array}{llll}1 & 0.063 & 0 & -->\end{array}$ \\
\hline & 147 & $10.1150==>1$ & 80 & $10.3331==>0$ \\
\hline node_169 --> Thayeriaobliqu & 18 & $10.1002==>1$ & 90 & $10.1432==>1$ \\
\hline & 34 & $10.0610==>1$ & 103 & $\begin{array}{llll} & 0.036 & 0 & ->\end{array}$ \\
\hline & 56 & $10.0670==>1$ & 128 & $11.0000==>1$ \\
\hline & 58 & $\begin{array}{llll} & 0.125 & 0 & -->1\end{array}$ & 140 & $10.231 \quad 1==>2$ \\
\hline & 59 & $10.0560==>1$ & 141 & $10.2001==>2$ \\
\hline & 63 & $10.136 \quad 1==>2$ & node_174 --> Hasemaniacrenuch 34 & $\begin{array}{lll}10.061 & 0-->1\end{array}$ \\
\hline & 67 & $10.1000==>1$ & - & $10.0370==>1$ \\
\hline & 70 & $10.0400==>1$ & 41 & $10.0671==>0$ \\
\hline & 75 & $10.0830==>1$ & 46 & $10.1670==>1$ \\
\hline & 96 & $10.0340==>1$ & 53 & $10.1542==>1$ \\
\hline & 98 & $10.0830==>1$ & 58 & $10.1251==>0$ \\
\hline & 99 & $10.125 \quad 1==>0$ & 88 & $10.0542==>0$ \\
\hline & 105 & $10.1502==>1$ & 110 & $10.0871==>0$ \\
\hline & 120 & $10.0430==>1$ & 112 & $10.0831==>2$ \\
\hline & 126 & $10.0801==>0$ & 113 & $10.0630==>1$ \\
\hline & 130 & $11.0000==>1$ & 133 & $10.0480==>1$ \\
\hline & 157 & $10.0742==>0$ & node_175 --> Hasemaniamelanur 6 & $10.167 \quad 0==>2$ \\
\hline & 158 & $10.3640==>4$ & $\begin{array}{ll}- & 11\end{array}$ & $10.2000==>1$ \\
\hline & 159 & $10.0710==>2$ & 12 & $10.1250==>1$ \\
\hline node_170 --> Parapristellage & o 19 & $10.0830==>1$ & 13 & $10.1430==>1$ \\
\hline & 37 & $10.1363==>2$ & 18 & $10.1001==>2$ \\
\hline & 49 & $10.100 \quad 1==>0$ & 28 & $10.105 \quad 1-->2$ \\
\hline & 55 & $10.071 \quad 1==>0$ & 32 & $10.2001==>2$ \\
\hline & 76 & $10.1000==>2$ & 44 & $10.1671==>0$ \\
\hline & 81 & $10.154 \quad 1==>0$ & 56 & $10.0670==>1$ \\
\hline & 89 & $10.0430==>1$ & 6 & $\begin{array}{llll} & 0.167 & 1-->3\end{array}$ \\
\hline & 135 & $10.056 \quad 1==>0$ & 62 & $10.1671==>0$ \\
\hline & 159 & $10.0710==>1$ & 73 & $10.0832==>1$ \\
\hline
\end{tabular}




\begin{tabular}{|c|c|c|c|c|c|}
\hline \multicolumn{2}{|l|}{ node_172 --> Hemigrammusstict 2} & 10.043 & $0==>1$ & 96 & $10.034 \quad 1==>0$ \\
\hline & 73 & 10.083 & $2==>0$ & 109 & $10.1110==>1$ \\
\hline & 106 & 10.250 & $0==>2$ & 112 & $10.0831==>2$ \\
\hline & 120 & 10.043 & $0==>1$ & 147 & $10.1150==>1$ \\
\hline & 134 & 10.077 & $1==>0$ & node_184 --> node_183 & $10.222 \quad 1==>0$ \\
\hline & 157 & 10.074 & $2==>0$ & 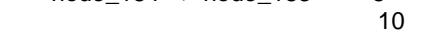 & $\begin{array}{llll} & 0.038 & 1->0\end{array}$ \\
\hline node_179 --> node_178 & 3 & 10.100 & $1==>0$ & 30 & $10.5000==>1$ \\
\hline & 13 & 10.143 & $0==>1$ & 39 & $\begin{array}{llll} & 0.037 & 0 & ->\end{array}$ \\
\hline & 18 & 10.100 & $1==>2$ & 45 & $10.2502==>1$ \\
\hline & 31 & 10.375 & $0==>1$ & 67 & $\begin{array}{llll}1 & 0.100 & 0 & -->1\end{array}$ \\
\hline & 35 & 10.125 & $1==>0$ & 86 & $10.5002==>0$ \\
\hline & 41 & 10.067 & $1==>2$ & 88 & $\begin{array}{lll} & 0.054 \quad 0->1\end{array}$ \\
\hline & 57 & 10.333 & $1==>0$ & 108 & $10.1180==>1$ \\
\hline & 93 & 10.059 & $91->0$ & 109 & $10.1110==>1$ \\
\hline & 101 & 10.143 & $31->0$ & 110 & $\begin{array}{llll} & 0.087 & 1->0\end{array}$ \\
\hline & 157 & 10.074 & $2==>0$ & 123 & $10.250 \quad 0==>1$ \\
\hline & 165 & 10.125 & $0==>1$ & 147 & $10.1150==>1$ \\
\hline node_178 --> Cheirodoninter & ru 9 & 10.083 & $1==>0$ & node_183 --> node_182 & $10.050 \quad 1==>0$ \\
\hline & 10 & 10.038 & $1==>0$ & 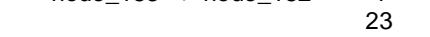 & $10.5001==>0$ \\
\hline & 25 & 10.222 & $0==>1$ & 34 & $\begin{array}{lll} & 0.061 & 0-->1\end{array}$ \\
\hline & 39 & 10.037 & $0==>1$ & 43 & $10.077 \quad 1==>0$ \\
\hline & 50 & 10.083 & $1==>0$ & 120 & $10.0430==>1$ \\
\hline & 60 & 10.063 & $31->0$ & 121 & $\begin{array}{lll} & 0.231 & 2-->3\end{array}$ \\
\hline & 67 & 10.100 & $0==>2$ & 131 & $\begin{array}{llll}1 & 0.111 & 1->0\end{array}$ \\
\hline & 69 & 10.182 & $0==>1$ & 151 & $\begin{array}{lll}10.063 & 0->1\end{array}$ \\
\hline & 73 & 10.083 & $2==>1$ & node_182 --> Bryconamericusex 10 & $\begin{array}{llll}1 & 0.038 & 0 & -->1\end{array}$ \\
\hline & 81 & 10.154 & $0==>1$ & 13 & $10.1430==>1$ \\
\hline & 89 & 10.043 & $0==1$ & 24 & $10.071 \quad 1==>0$ \\
\hline & 98 & 10.083 & $0==>1$ & 55 & $10.071 \quad 1==>0$ \\
\hline & 103 & 10.036 & $60->1$ & 65 & $10.0631==>0$ \\
\hline & 105 & 10.150 & $2==>1$ & 68 & $10.0950==>2$ \\
\hline & 106 & 10.250 & $0==1$ & 85 & $11.0000==>1$ \\
\hline & 115 & 10.133 & $0==>1$ & 86 & $10.500 \quad 0==>1$ \\
\hline & 131 & 10.111 & $11->0$ & 126 & $10.0801==>0$ \\
\hline node_178 --> node_177 & 16 & 10.048 & $81->0$ & 158 & $10.3640==>2$ \\
\hline & 19 & 10.083 & $0==>1$ & 159 & $10.0710==>2$ \\
\hline & 26 & 10.158 & $82-->3$ & node_182 --> Piabinaargentea 9 & $10.083 \quad 1==>0$ \\
\hline & 53 & 10.154 & $2==>0$ & $\begin{array}{ll}- & 18\end{array}$ & $10.100 \quad 1==>0$ \\
\hline & 86 & 10.500 & $2==>0$ & 39 & $\begin{array}{llll} & 0.037 & 1->0\end{array}$ \\
\hline & 88 & 10.054 & $40->1$ & 46 & $10.1670==>1$ \\
\hline node_177 --> Serrapinnusnoto & $\mathrm{m} 8$ & 10.222 & $1==>0$ & 47 & $10.200 \quad 0==>2$ \\
\hline & 62 & 10.167 & $1==>0$ & 48 & $10.5001==>0$ \\
\hline & 69 & 10.182 & $0==>2$ & 53 & $10.1542==>1$ \\
\hline & 104 & 10.038 & $0==>1$ & 67 & $10.1001->0$ \\
\hline & 113 & 10.063 & $0==>1$ & 70 & $10.040 \quad 1==>0$ \\
\hline & 120 & 10.043 & $0==>1$ & 75 & $10.0830==>1$ \\
\hline & 132 & 10.133 & $2==>1$ & 76 & $10.1002==>1$ \\
\hline & 144 & 10.154 & $0==>1$ & 87 & $10.1001==>0$ \\
\hline node_177 --> Serrapinnushete & 59 & 10.056 & $0==>1$ & 89 & $10.0430==>1$ \\
\hline & 68 & 10.095 & $0==>2$ & 96 & $10.0341==>0$ \\
\hline & 72 & 10.059 & $0==>1$ & 97 & $10.3331==>0$ \\
\hline & 76 & 10.100 & $0==>2$ & 105 & $10.1502==>3$ \\
\hline & 93 & 10.059 & $90-->1$ & 108 & $10.1181==>2$ \\
\hline & 96 & 10.034 & $1==>0$ & 110 & $\begin{array}{llll} & 0.087 & 0 & ->1\end{array}$ \\
\hline & 135 & 10.056 & $1==>0$ & 112 & $10.0831==>2$ \\
\hline & 137 & 10.111 & $1==>0$ & 132 & $10.1332==>1$ \\
\hline & 159 & 10.071 & $0==>1$ & 145 & $10.0670==>1$ \\
\hline node_181 --> node_180 & 6 & 10.167 & $70->1$ & node_183 --> Rhinopetitiamyer 3 & $10.100 \quad 1==>0$ \\
\hline & 9 & 10.083 & $1==>0$ & 6 & $10.1670==>2$ \\
\hline & 22 & 10.091 & $1==>0$ & 35 & $10.125 \quad 1==>0$ \\
\hline & 24 & 10.071 & $1==>2$ & 44 & $10.167 \quad 1==>0$ \\
\hline & 34 & 10.061 & $10-->2$ & 50 & $10.0831==>0$ \\
\hline & 50 & 10.083 & $1==>0$ & 53 & $10.1542==>0$ \\
\hline & 52 & 10.143 & $1==>0$ & 77 & $10.1820==>1$ \\
\hline & 55 & 10.071 & $1==>0$ & 81 & $10.1540==>1$ \\
\hline & 68 & 10.095 & $0==>2$ & 91 & $10.100 \quad 1==>0$ \\
\hline & 70 & 10.040 & $1==>0$ & 92 & $10.0430==>1$ \\
\hline & 74 & 10.143 & $0==>1$ & 103 & $\begin{array}{llll} & 0.036 & 0 & ->1\end{array}$ \\
\hline & 81 & 10.154 & $0==>1$ & 106 & $10.250 \quad 0==>1$ \\
\hline & 83 & 10.143 & $0==>2$ & 113 & $10.0630==>1$ \\
\hline & 104 & 10.038 & $0==>1$ & 115 & $10.1330==>1$ \\
\hline & 115 & 10.133 & $0==>2$ & 135 & $10.056 \quad 1->0$ \\
\hline & 135 & 10.056 & $1->0$ & 138 & $10.0670==>1$ \\
\hline node_180 --> Aphyocaraxpusi & II 25 & 10.222 & $0==>1$ & 140 & $10.231 \quad 1==>2$ \\
\hline & 31 & 10.375 & $0==>2$ & node_185 --> Bryconopsmelanur 4 & $10.0501==>0$ \\
\hline & 37 & 10.136 & $3==>2$ & 11 & $10.2000==>1$ \\
\hline & 58 & 10.125 & $1==>0$ & 21 & $10.1430==>1$ \\
\hline & 59 & 10.056 & $0==>1$ & 41 & $10.0671==>0$ \\
\hline & 63 & 10.136 & $1==>0$ & 49 & $10.100 \quad 1==>0$ \\
\hline & 89 & 10.043 & $0==>1$ & 58 & $10.125 \quad 1==>0$ \\
\hline & 110 & 10.087 & $1==>2$ & 61 & $10.3331==>0$ \\
\hline & 111 & 10.143 & $1==>0$ & 62 & $10.167 \quad 1==>0$ \\
\hline & 114 & 11.000 & $1==>2$ & 65 & $\begin{array}{llll} & 0.063 & 1->0\end{array}$ \\
\hline & 126 & 10.080 & $1==>0$ & & \\
\hline & 131 & 10.111 & $11->0$ & & \\
\hline & 157 & 10.074 & $2==>0$ & & \\
\hline
\end{tabular}




\begin{tabular}{|c|c|}
\hline $\begin{array}{r}\text { node_180 --> Aphyocaraxanisit } 3 \\
96 \\
105 \\
121 \\
145 \\
158 \\
159 \\
\text { node_188 --> Chalceusspilogyr } 22 \\
23 \\
37 \\
39 \\
42 \\
63 \\
86 \\
90 \\
91 \\
92 \\
122 \\
125 \\
141 \\
157\end{array}$ & $\begin{array}{rrrr}1 & 0.100 & 1==>0 \\
1 & 0.034 & 1=>0 \\
1 & 0.150 & 2==>3 \\
1 & 0.231 & 2==>0 \\
1 & 0.067 & 0==>1 \\
1 & 0.364 & 0==>1 \\
1 & 0.071 & 0==>2 \\
1 & 0.091 & 1==>0 \\
1 & 0.500 & 1==>0 \\
1 & 0.136 & 0-->1 \\
1 & 0.037 & 0-->1 \\
1 & 0.167 & 2==>0 \\
1 & 0.136 & 2==>0 \\
1 & 0.500 & 2==>1 \\
1 & 0.143 & 1==>0 \\
1 & 0.100 & 0-->1 \\
1 & 0.043 & 0-->1 \\
1 & 0.273 & 0==>1 \\
1 & 0.182 & 1==>2 \\
1 & 0.200 & 0=>2 \\
1 & 0.074 & 0-->2\end{array}$ \\
\hline
\end{tabular}




\section{APÊNDICE 2}


Apêndice 2- Lista da mudança dos estados dos caracteres. Número do caráter, seguido pelo índice de consistência, número de passos e mudança de estado.

\begin{tabular}{|c|c|c|c|c|c|c|c|}
\hline 1 & 1.000 & 1 & node_96 1 ==> 0 Bryconsp & & 1 & & node_108 $0==>1$ Astyanaxaltipara \\
\hline \multirow[t]{23}{*}{2} & 0.043 & 1 & node_188 $0-->1$ node_187 & & 1 & & node_109 $0==>1$ Oligosarcuspinto \\
\hline & 1 & & node_98 1 -->0 node_97 & & 1 & & node_176 $0==>1$ node_ 175 \\
\hline & 1 & & node_184 1 -->0 node_181 & \multirow[t]{9}{*}{8} & 0.222 & 1 & node_188 $0-->1$ node_187 \\
\hline & 1 & & node_104 $0->1$ node_102 & & 1 & & node_109 $1==>0$ Phenacogasterfra \\
\hline & 1 & & node_99 1 -->0 Serrasalmusmacul & & 1 & & node_125 $1==>0$ node $\_124$ \\
\hline & 1 & & node_1011 -->0 Gymnocorymbuster & & 1 & & node_122 $0==>1$ Coptobryconbilin \\
\hline & 1 & & node $1110-->1$ node 110 & & 1 & & node $1291==>0$ node 128 \\
\hline & 1 & & node 1091 -->0 Oligosarcuspinto & & 1 & & node_153 1 ==> 2 Hyphessobryconco \\
\hline & 1 & & node_115 $0==>1$ Moenkhausiainter & & 1 & & node_166 $1==>0$ Hemigrammuscoeru \\
\hline & 1 & & node_117 $0==>1$ Jupiabapolylepis & & 1 & & node_177 $1==>0$ Serrapinnusnotom \\
\hline & 1 & & node_134 0 --> 1 node_131 & & 1 & & node_184 $1==>0$ node_183 \\
\hline & 1 & & node_1251 -->0 node_124 & \multirow[t]{12}{*}{9} & 0.083 & 1 & node_188 $0==>1$ Brycinuslongipin \\
\hline & 1 & & node_127 1 -->0 Hemigrammusbello & & 1 & & node_97 $0==>1$ Triportheusnemat \\
\hline & 1 & & node_129 $1-->0$ node_128 & & 1 & & node_186 $0==>1$ node_185 \\
\hline & 1 & & node $1360==>1$ Hemigrammusbrevi & & 1 & & node 991 ==> 0 Serrasalmusmacul \\
\hline & 1 & & node $1410==>1$ Hemigrammusarua & & 1 & & node_ $1011==>0$ Tetragnopterusar \\
\hline & 1 & & node_148 $0==>1$ node_147 & & 1 & & node_103 $1==>0$ Stethaprioneryth \\
\hline & 1 & & node_145 1 ==> 0 Hemigrammusmargi & & 1 & & node_112 $1==>0$ node_108 \\
\hline & 1 & & node_152 $0==>1$ Hemigrammusunili & & 1 & & node_109 1 ==> 0 Oligosarcuspinto \\
\hline & 1 & & node_153 $0==>1$ Hyphessobryconco & & 1 & & node $1751==>0$ node 174 \\
\hline & 1 & & node_154 $0==>1$ Hyphessobryconme & & 1 & & node $1781==>0$ Cheirodoninterru \\
\hline & 1 & & node_168 $0==>1$ Hemigrammusbarri & & 1 & & node_181 $1==>0$ node_180 \\
\hline & 1 & & node_$-1720==>1$ Hemigrammusstict & & 1 & & node_182 $1==>0$ Piabinaargentea \\
\hline \multirow[t]{10}{*}{3} & 0.100 & 1 & node_187 $0==>1$ node_186 & \multirow[t]{26}{*}{10} & 0.038 & 1 & nōde_188 0 --> 1 node_187 \\
\hline & 1 & & node_97 1 ==> 0 Iguanodectesspil & & 1 & & node_96 1 --> 0 Salminushilarii \\
\hline & 1 & & node_103 $1==>0$ Stethaprioneryth & & 1 & & node_100 1 ==> 0 Psellorammuskenn \\
\hline & 1 & & node_124 1==> 0 Bryconellapallid & & 1 & & node_105 $1==>0$ Hemigrammuspreto \\
\hline & 1 & & node_128 1 ==> 0 Microschemobryco & & 1 & & node_107 $1==>0$ Hemigrammusthapo \\
\hline & 1 & & node_169 $1==>0$ Hemigrammusanali & & 1 & & node_112 $1==>0$ node_111 \\
\hline & 1 & & node $\_1751==>0$ node 174 & & 1 & & node_109 $0==>1$ Oligosarcuspinto \\
\hline & 1 & & node_$\_-1791==>0$ node_178 & & 1 & & node_-118 $1==>0$ node_117 \\
\hline & 1 & & node_180 1 ==> 0 Aphyōcaraxanisit & & 1 & & node_-120 $1==>0$ node $\_119$ \\
\hline & 1 & & node_183 $1==>0$ Rhinopetitiamyer & & 1 & & node_125 $1==>0$ node_124 \\
\hline 4 & 0.050 & 1 & nōde_98 $1==>0$ Lignobryconmyers & & 1 & & node_122 $0==>1$ Grund̄uluscochae \\
\hline & 1 & & node_179 $1-->0$ node_176 & & 1 & & node_131 1 -->0 node_130 \\
\hline & 1 & & node_114 $0==>1$ node_ 113 & & 1 & & node_127 0 --> 1 Hemigrammusorthu \\
\hline & 1 & & node_106 $1==>0$ node_105 & & 1 & & node_132 $1==>0$ Hemigrammustride \\
\hline & 1 & & node_-111 $1==>0$ Bryconexodonjuru & & 1 & & node_135 $1==>0$ Hemigrammushyanu \\
\hline & 1 & & node $1091==>0$ Phenacogasterfra & & 1 & & node $1401==>0$ Hemigrammusboese \\
\hline & 1 & & node_116 $0==>1$ Astyanaxjacobina & & 1 & & node_144 $1==>0$ node_143 \\
\hline & 1 & & node_120 $0==>1$ node_ 119 & & 1 & & node_147 $1==>0$ node_146 \\
\hline & 1 & & node_136 0 --> 1 node_1 135 & & 1 & & node_150 $1==>0$ Hemigrammusgraci \\
\hline & 1 & & node_134 1 -->0 node_131 & & 1 & & node_158 1 ==>0 Hyphessobryconeq \\
\hline & 1 & & node_124 $0==>1$ Bryconellapallid & & 1 & & node_163 $1==>0$ Hemigrammuslunat \\
\hline & 1 & & node $1290==>1$ node 128 & & 1 & & node $1681=\Rightarrow 0$ Hemigrammusbarri \\
\hline & 1 & & node_139 $0==>1$ node_138 & & 1 & & node_172 $1==>0$ Hemigrammusstict \\
\hline & 1 & & node_145 $0==>1$ Hemigrammusmargi & & 1 & & node_178 $1==>0$ Cheirodoninterru \\
\hline & 1 & & node_159 $0==>1$ Hyphessobryconta & & 1 & & node_184 1 -->0 node_183 \\
\hline & 1 & & node_166 $0==>1$ Hemigrammuscoeru & & 1 & & node_182 0 --> 1 Bryconamericusex \\
\hline & 1 & & node $-1710==>1$ node_ 170 & 11 & 0.200 & 1 & node_98 $0==>1$ Lignobryconmyers \\
\hline & 1 & & node_175 $0->1$ node_174 & & 1 & & node_122 $0==>1$ Grunduluscochae \\
\hline & 1 & & node_183 $1==>0$ node 182 & & 1 & & node_139 $0==>1$ node_138 \\
\hline & 1 & & node_185 $1==>0$ Bryconopsmelanur & & 1 & & node_175 $0==>1$ Hasemaniamelanur \\
\hline 5 & 0.250 & 1 & node_103 $0==>1$ Stethaprioneryth & & 1 & & node_185 $0==>1$ Bryconopsmelanur \\
\hline & 1 & & node_127 $0==>1$ Hemigrammusorthu & 12 & 0.125 & 1 & nöde_186 $0==>1$ node_98 \\
\hline & 1 & & node_136 $0==>1$ Hemigrammusbrevi & & 1 & & node_123 $0==>1$ node_122 \\
\hline & 1 & & node_155 $0==>1$ node_154 & & 1 & & node_126 $0==>1$ Hyphessobryconco \\
\hline 6 & 0.167 & 1 & node_98 $0==>2$ Lignobryconmyers & & 1 & & node_130 $0==>1$ node_129 \\
\hline & 1 & & node_119 $0==>2$ Hemigrammusskoli & & 1 & & node_142 $0==>1$ node_141 \\
\hline & 1 & & node $1490==>2$ node_144 & & 1 & & node_139 1 ==> 0 Hyphessobrycondi \\
\hline & 1 & & node_135 $2==>3$ node_134 & & 1 & & node $1540=\Rightarrow 1$ Hyphessobryconme \\
\hline & 1 & & node_1243 --> 1 node_ 123 & & 1 & & node_175 $0==>1$ Hasemaniamelanur \\
\hline & 1 & & node_122 1 --> 3 Coptobryconbilin & 13 & 0.143 & 1 & node_124 $0==>1$ node_123 \\
\hline & 1 & & node_129 $3==>0$ node_128 & & 1 & & node_128 $0==>1$ Microschemobryco \\
\hline & 1 & & node_132 $3==>1$ Hemigrammustride & & 1 & & node_138 $0==>1$ node_137 \\
\hline & 1 & & node_142 $2==>1$ node_ 141 & & 1 & & node_143 $0==>1$ Hemigrammusharal \\
\hline & 1 & & node $1391==>0$ node 138 & & 1 & & node $1750=\Rightarrow 1$ Hasemaniamelanur \\
\hline & 1 & & node $1432==>3$ Hemigrammusharal & & 1 & & node $1790=\Rightarrow 1$ node 178 \\
\hline & 1 & & node $1610==>3$ node 160 & & 1 & & node $1820==>1$ Bryconamericusex \\
\hline & 1 & & node $1533==>1$ Hyphessobryconco & 14 & 0.056 & 1 & node $980==>1$ Lignobryconmyers \\
\hline & 1 & & node_166 $0==>1$ Hemigrammusocell & & 1 & & node $\_18 \overline{0} 0==>1$ node $\_181$ \\
\hline & 1 & & node $1750==>2$ Hasemaniamelanur & & 1 & & node_172 $1==>0$ node_171 \\
\hline & 1 & & node_1810 -->1 node_180 & & 1 & & node_99 $0==>1$ Serrasalmusmacul \\
\hline & 1 & & node_180 1 --> 3 Aphyocaraxanisit & & 1 & & node_103 $0==>1$ Poptellaparaguay \\
\hline & 1 & & node_183 $0==>2$ Rhinopetitiamyer & & 1 & & node_110 $0==>1$ Hollandichthsper \\
\hline 7 & 0.125 & 1 & node $1870-->1$ node 186 & & 1 & & node $1190==>1$ Hemigrammuscylin \\
\hline & 1 & & node_97 $1-->0$ Iguanodectesspil & & 1 & & node_165 0 -->1 node_164 \\
\hline & 1 & & node_1851 -->0 node_184 & & 1 & & node_136 $1==>0$ node $\_135$ \\
\hline & 1 & & node_99 $0==>1$ Serrasalmusmacul & & 1 & & node_124 $0==>1$ Bryconellapallid \\
\hline & 1 & & node_103 $0==>1$ Stethaprioneryth & & 1 & & node_ $1290==>1$ node_128 \\
\hline
\end{tabular}




\begin{tabular}{|c|c|c|c|c|c|c|c|}
\hline & 1 & & node_143 1 ==> 0 Hemigrammuspulch & & 1 & & node_129 1 ==> 0 node_128 \\
\hline & 1 & & node_146 1 ==> 0 Moenkhausiabonit & & 1 & & node_153 $1==>0$ Hyphessobryconco \\
\hline & 1 & & node_162 $1->0$ node_161 & & 1 & & node_154 $1==>0$ Hyphessobryconmi \\
\hline & 1 & & node_-160 0 -->1 node_159 & & 1 & & node_$\_1751==>0$ node_174 \\
\hline & 1 & & node_153 1 ==> 0 Hyphessobryconco & & 1 & & node_-181 $1==>0$ node_180 \\
\hline & 1 & & node_157 $1==>0$ node_156 & & 1 & & node_188 $1==>0$ Chalceusspilogyr \\
\hline & 1 & & node_169 $0==>1$ Hemigrammusanali & 23 & 0.500 & 1 & node_183 $1==>0$ node_182 \\
\hline \multirow[t]{3}{*}{15} & 0.333 & 1 & node_97 $0==>1$ Triportheusnemat & & 1 & & node_18 $1==>0$ Chalceusspilogyr \\
\hline & 1 & & node_102 $0==>1$ node_101 & 24 & 0.071 & 1 & nōde_188 1 -->0 node_187 \\
\hline & 1 & & node $\_1760==>1$ node_175 & & 1 & & node_98 0 --> 1 Lignobryconmyers \\
\hline \multirow[t]{21}{*}{16} & 0.048 & 1 & node_187 0 --> 1 node_186 & & 1 & & node_185 0 --> 1 node_184 \\
\hline & 1 & & 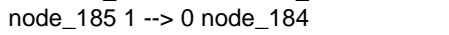 & & 1 & & node_-106 $1==>0$ node__105 \\
\hline & 1 & & node_-1810 --> 1 node_179 & & 1 & & node_103 $0==>1$ Stethaprioneryth \\
\hline & 1 & & node_114 $1-->0$ node_113 & & 1 & & node_113 $1==>0$ node_112 \\
\hline & 1 & & node_105 $0==>1$ node__104 & & 1 & & node_115 $1==>0$ Moenkhausiainter \\
\hline & 1 & & node_112 0 --> 1 node_108 & & 1 & & node_1211 -->0 node_120 \\
\hline & 1 & & node_110 0 --> 1 Hollandichthsper & & 1 & & node_119 0 --> 1 Hemigrammusskoli \\
\hline & 1 & & node_-120 $1==>0$ node_119 & & 1 & & node_123 $1==>2$ Paracheirodonaxe \\
\hline & 1 & & node_123 1 ==> 0 Paracheirodonaxe & & 1 & & node_-130 $1==>0$ node_127 \\
\hline & 1 & & node_127 $1==>0$ Hemigrammusbello & & 1 & & node_128 $1==>0$ Microschemobryco \\
\hline & 1 & & node_129 $1==>0$ node_128 & & 1 & & node_133 $1==>0$ Hemigrammusschma \\
\hline & 1 & & node_135 1 ==> 0 Hemigrammushyanu & & 1 & & node_132 $1==>2$ Hemigrammustride \\
\hline & 1 & & node_138 $1==>0$ Hemigrammusblehe & & 1 & & node_136 1 ==>0 Hemigrammusbrevi \\
\hline & 1 & & node_1431 $1==>0$ Hemigrammuspulch & & 1 & & node_138 $1==>0$ Hemigrammusblehe \\
\hline & 1 & & node_150 1 ==>0 Hemigrammusgraci & & 1 & & node_140 $1==>0$ Hemigrammusboese \\
\hline & 1 & & node_152 $1==>0$ Moenkahusiahemig & & 1 & & node_143 $1==>0$ Hemigrammusharal \\
\hline & 1 & & node_159 1 ==> 0 Hyphessobryconta & & 1 & & node_149 $1==>0$ node_148 \\
\hline & 1 & & node_166 $1==>0$ Hemigrammuscoeru & & 1 & & node_146 $0==>1$ node_145 \\
\hline & 1 & & node_168 $1==>0$ Hemigrammusbarri & & 1 & & node_152 1 ==> 0 Moenkahusiahemig \\
\hline & 1 & & node_170 1 ==> 0 node_169 & & 1 & & node_159 $1==>0$ node_158 \\
\hline & 1 & & node_178 1 -->0 node_177 & & 1 & & node_155 $0==>1$ node_154 \\
\hline 17 & 0.250 & 1 & node_96 $1==>0$ Bryconsp & & 1 & & node_163 $1==>0$ Hemigrammuslunat \\
\hline & 1 & & node_101- $1==>0$ Tetragnopterusar & & 1 & & node_169 $1==>0$ Hemigrammusanali \\
\hline & 1 & & node_122 1 ==> 0 Grunduluscochae & & 1 & & node_176 $1==>0$ node_175 \\
\hline & 1 & & node_174 $1==>0$ HasemaniaspCrenu & & 1 & & node_181 $1==>2$ node_180 \\
\hline 18 & 0.100 & 1 & node_97 $0==>1$ Triportheusnemat & & 1 & & node_182 $1==>0$ Bryconamericusex \\
\hline & 1 & & node_185 $0==>1$ node_184 & 25 & 0.222 & 1 & nōde_108 $0==>2$ Astyanaxmexicanu \\
\hline & 1 & & node_172 $1==>2$ node_171 & & 1 & & node_110 $0==>1$ Hollandichthsper \\
\hline & 1 & & node_118 $2==>0$ node_115 & & 1 & & node_124 0 --> 1 node_123 \\
\hline & 1 & & node_1130 -->1 node_107 & & 1 & & node_123 1 --> 2 Paracheirodonaxe \\
\hline & 1 & & node_99 $1==>0$ Serrāalmusmacul & & 1 & & node_128 $0==>2$ Microschemobryco \\
\hline & 1 & & node_104 $1==>0$ node_103 & & 1 & & node_140 $0==>2$ Hemigrammusboese \\
\hline & 1 & & node_107 1 --> 2 Hemigrammusthapo & & 1 & & node_$\_1760==>1$ node_175 \\
\hline & 1 & & node_111 $0==>1$ node_110 & & 1 & & node_178 $0==>1$ Cheirodoninterru \\
\hline & 1 & & node_109 1 ==> 2 Phenacogasterfra & & 1 & & node_180 $0==>1$ Aphyocaraxpusill \\
\hline & 1 & & node_116 $2==>1$ Astyanaxjacobina & 26 & 0.158 & 1 & nōde_186 0 --> 1 node_185 \\
\hline & 1 & & node_-1212 ==> 1 node_120 & & 1 & & 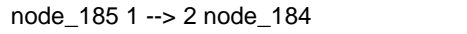 \\
\hline & 1 & & node_122 2 ==> 1 Grund̄uluscochae & & 1 & & node_118 $2==>1$ node__115 \\
\hline & 1 & & node_145 $2==>1$ Hemigrammusmargi & & 1 & & node_99 $1==>0$ Serrasalmusmacul \\
\hline & 1 & & node_152 2 ==> 1 Moenkahusiahemig & & 1 & & node_100 $1==>3$ Psellorammuskenn \\
\hline & 1 & & node_163 $2==>0$ Hemigrammusulrey & & 1 & & node_101 $1==>2$ Gymnocorymbuster \\
\hline & 1 & & node_169 $2==>1$ Thayeriaobliqua & & 1 & & node_105 $1==>2$ Hemigrammuspreto \\
\hline & 1 & & node_175 $1==>2$ Hasemaniamelanur & & 1 & & node_111 $1==>0$ Bryconexodonjuru \\
\hline & 1 & & node_179 $1==>2$ node_178 & & 1 & & node_109 1 ==> 3 Phenacogasterfra \\
\hline & 1 & & node_182 $1==>0$ Piabinaargentea & & 1 & & node_144 2 ==> 3 node_142 \\
\hline 19 & 0.083 & 1 & nōde_164 $0==>1$ node_162 & & 1 & & node_126 $3==>2$ Hyphessobryconco \\
\hline & 1 & & node_123 $1-->0$ node_122 & & 1 & & node $\_1303==>2$ node 127 \\
\hline & 1 & & node_$\_1301==>0$ node_ 129 & & 1 & & node_133 $3==>2$ Hemigrammusschma \\
\hline & 1 & & node_140 1 -->0 node_-̄139 & & 1 & & node_139 3 ==> 2 Hyphessobrycondi \\
\hline & 1 & & node_138 $0-->1$ node_137 & & 1 & & node_160 $2==>3$ Hyphessobryconbe \\
\hline & 1 & & node_143 $1==>0$ Hemigrammuspulch & & 1 & & node_155 $2==>3$ node_154 \\
\hline & 1 & & node_148 $1==>0$ node_147 & & 1 & & node_163 $2==>1$ Hemigrammusulrey \\
\hline & 1 & & node_-152 $1==>0$ Moenkahusiahemig & & 1 & & node_175 $2==>1$ node_174 \\
\hline & 1 & & node_153 $1==>0$ Pristellamaxilla & & 1 & & node_178 2 --> 3 node_177 \\
\hline & 1 & & node_154 $1==>0$ Hyphessobryconmi & 27 & 0.200 & 1 & nōde_185 $0==>1$ node_184 \\
\hline & 1 & & node_170 $0==>1$ Parapristellageo & & 1 & & node_99 $1==>0$ Roeboidesbonarie \\
\hline & 1 & & node_178 $0==>1$ node_177 & & 1 & & node_112 $1==>0$ node_108 \\
\hline 20 & 0.143 & 1 & nōde_188 0 --> 1 node_e_187 & & 1 & & node_114 $1==>0$ Moenkhausiaxingu \\
\hline & 1 & & node_96 1 -->0 Salminushilarii & & 1 & & node_145 $1==>0$ Hemigrammusmargi \\
\hline & 1 & & node_102 $1==>0$ node_100 & 28 & 0.105 & 1 & node_188 2 ==> 1 Brycinuslongipin \\
\hline & 1 & & node_108 $1==>0$ Astyanaxaltipara & & 1 & & node_1812 --> 1 node_179 \\
\hline & 1 & & node_119 1 ==>0 Hemigrammusskoli & & 1 & & node_105 1 ==>0 node_104 \\
\hline & 1 & & node_123 $1==>0$ node_122 & & 1 & & node_108 $1==>0$ Astyanaxaltipara \\
\hline & 1 & & node_$\_1761==>0$ node_175 & & 1 & & node_111 $1==>0$ Bryconexodonjuru \\
\hline 21 & 0.143 & 1 & node_97 $0==>1$ Iguanodectesspil & & 1 & & node_121 $1==>2$ node_120 \\
\hline & 1 & & node_ $1760==>1$ node_173 & & 1 & & node_142 $1==>2$ node_136 \\
\hline & 1 & & node_104 $1==>0$ node_102 & & 1 & & node_126 2 ==> 1 Hyphessobryconco \\
\hline & 1 & & node_$\_1131==>0$ node_-112 & & 1 & & node_127 $2==>1$ Hemigrammusbello \\
\hline & 1 & & node_116 $1==>0$ Astyanaxjacobina & & 1 & & node_133 $2==>1$ Hemigrammusschma \\
\hline & 1 & & node_123 1 --> 0 node_122 & & 1 & & node_140 $1==>2$ node $\_139$ \\
\hline & 1 & & node_185 $0==>1$ Bryconopsmelanur & & 1 & & node_143 $1==>2$ Hemigrammusharal \\
\hline 22 & 0.091 & 1 & node_96 1 ==> 0 Salminushilarii & & 1 & & node_150 $1==>2$ Hemigrammussilim \\
\hline & 1 & & node_102 $1==>0$ node_100 & & 1 & & node_152 1 ==>0 Moenkahusiahemig \\
\hline & 1 & & node $-1111==>0$ node_ 110 & & 1 & & node_153 $1==>0$ Pristellamaxilla \\
\hline & 1 & & node_$\_1191==>0$ Hemigrammusskoli & & 1 & & node_166 $1==>2$ Hemigrammuscoeru \\
\hline & 1 & & node_122 $1==>0$ Grunduluscochae & & 1 & & node_1711 -->2 node_170 \\
\hline
\end{tabular}


Serra, J. P. - Análise Filogenética das Espécies de Hemigrammus Gill, 1858 (Characiformes, Characidae)

\begin{tabular}{|c|c|c|c|c|c|c|c|}
\hline & 1 & & node_173 1 --> 2 Hemigrammuslevis & & 1 & & node_125 $0==>1$ Hemigrammusyinyan \\
\hline & 1 & & node_175 1 --> 2 Hasemaniamelanur & & 1 & & node_130 $0==>1$ node_127 \\
\hline \multirow[t]{5}{*}{29} & 0.200 & 1 & node_105 $0==>1$ node_104 & & 1 & & node_128 $0==>1$ Hemigrammusmimus \\
\hline & 1 & & node_10 $\overline{7} 0==>1$ Hemigrammusthapo & & 1 & & node_138 $1==>0$ Hemigrammusblehe \\
\hline & 1 & & node_108 $0==>1$ Astyanaxaltipara & & 1 & & node_141 $1==>0$ Hemigrammusarua \\
\hline & 1 & & node_110 $0==>1$ node_109 & & 1 & & node_146 $1==>0$ node_145 \\
\hline & 1 & & node_157 $0==>1$ node_156 & & 1 & & node_161 $1==>0$ node_160 \\
\hline \multirow[t]{4}{*}{30} & 0.500 & 1 & node_96 $0==>1$ Bryconsp & & 1 & & node_153 $0==>1$ Pristellamaxilla \\
\hline & 1 & & node_99 $0==>2$ Serrasalmusmacul & & 1 & & node_154 $0==>1$ Hyphessobryconme \\
\hline & 1 & & node_156 $0==>2$ Hyphessobryconep & & 1 & & node_173 $0==>1$ Hemigrammuslevis \\
\hline & 1 & & node_184 $0==>1$ node_183 & 37 & 0.136 & 1 & node_187 0 --> 3 node_186 \\
\hline 31 & 0.375 & 1 & node_96 $0==>2$ Sāminushilarii & & 1 & & node_98 3 --> 0 Lignobryconmyers \\
\hline & 1 & & node_97 $0==>1$ Iguanodectesspil & & 1 & & node_100 3 -->1 node_99 \\
\hline & 1 & & node_99 $0==>3$ Roeboidesbonarie & & 1 & & node_101 $3==>2$ Tetragnopterusar \\
\hline & 1 & & node_111 $0==>3$ Bryconexodonjuru & & 1 & & node_105 $3==>2$ Hemigrammuspreto \\
\hline & 1 & & node_123 0 --> 1 node_122 & & 1 & & node_112 3 --> 1 node_111 \\
\hline & 1 & & node_-122 1 --> 2 Grunduluscochae & & 1 & & node_-111 1 --> 2 Bryconexodonjuru \\
\hline & 1 & & node_-179 $0==>1$ node_178 & & 1 & & node_109 1 ==> 0 Phenacogasterfra \\
\hline & 1 & & node_180 $0==>2$ Aphyocaraxpusill & & 1 & & node_120 $3==>2$ node_119 \\
\hline 32 & 0.200 & 1 & node_188 $0==>2$ Brycinuslongipin & & 1 & & node_134 3 --> 2 node_131 \\
\hline & 1 & & node_187̄ 0 --> 1 node_186 & & 1 & & node_126 2 --> 3 node_125 \\
\hline & 1 & & node_98 1 -->0 Lignobryconmyers & & 1 & & node_123 3 --> 2 node_122 \\
\hline & 1 & & node_99 $1==>2$ Serrasalmusmacul & & 1 & & node_127 2 --> 3 Hemigrammusbello \\
\hline & 1 & & node_108 1 ==> 2 Astyanaxaltipara & & 1 & & node_141 $3==>2$ Hemigrammusarua \\
\hline & 1 & & node_112 1 ==> 0 node_111 & & 1 & & node_152 $3==>2$ Hemigrammusunili \\
\hline & 1 & & node_122 $1==>2$ Coptobryconbilin & & 1 & & node_158 $3==>2$ node_157 \\
\hline & 1 & & node_139 $1==>2$ Hyphessobrycondi & & 1 & & node_153 $2==>\{01\}$ Pristellamaxilla \\
\hline & 1 & & node_153 $1==>0$ Pristellamaxilla & & 1 & & node_164 $3==>2$ node_163 \\
\hline & 1 & & node_175 $1==>2$ Hasemaniamelanur & & 1 & & node_-166 $3==>2$ Hemigrammuscoeru \\
\hline 33 & 0.125 & 1 & nōde_103 $0==>1$ Poptellaparaguay & & 1 & & node_170 $3==>2$ Parapristellageo \\
\hline & 1 & & node_137 $0==>1$ Hemigrammusrhodo & & 1 & & node_180 $3==>2$ Aphyocaraxpusill \\
\hline & 1 & & node_140 $0==>1$ Hemigrammusboese & & 1 & & node_188 0 --> 1 Chalceusspilogyr \\
\hline & 1 & & node_-144 $0==>1$ node_143 & 38 & 0.250 & 1 & nóde_187 0 --> 1 node_186 \\
\hline & 1 & & node $\_1510==>1$ node_150 & & 1 & & node_98 1 -->0 0 Lignobryconmyers \\
\hline & 1 & & node_152 $0==>1$ Hemigrammusunili & & 1 & & node_112 $1==>0$ node_111 \\
\hline & 1 & & node_163 $0==>1$ Hemigrammusulrey & & 1 & & node_153 $1==>0$ Pristellamaxilla \\
\hline & 1 & & node_173 $0==>1$ Hemigrammuslevis & 39 & 0.037 & 1 & nōde_187 0 --> 1 node_96 \\
\hline 34 & 0.061 & 1 & node_188 0 --> 1 node_187 & & 1 & & node_171 $0==>1$ node_168 \\
\hline & 1 & & node_96 1 --> 2 Salminushilarii & & 1 & & node_121 1 -->0 node_118 \\
\hline & 1 & & node_98 1 -->0 node_97 & & 1 & & node_115 0 --> 1 node_114 \\
\hline & 1 & & node_185 1 -->0 node_184 & & 1 & & node_100 $1==>0$ Psellorammuskenn \\
\hline & 1 & & node_-168 $0-->1$ node_167 & & 1 & & node_101 $1==>0$ Gymnocorymbuster \\
\hline & 1 & & node_$\_1151==>0$ node_ 114 & & 1 & & node_103 1 ==> 0 Poptellaparaguay \\
\hline & 1 & & node_106 $0==>1$ node_105 & & 1 & & node_-112 $1==>0$ node_108 \\
\hline & 1 & & node_100 1 --> 2 node_ 99 & & 1 & & node_110 $1==>0$ node_109 \\
\hline & 1 & & node_-110 0 -->1 node_109 & & 1 & & node_116 0 --> 1 Astyanaxjacobina \\
\hline & 1 & & node_109 1 --> 2 Phenāacogasterfra & & 1 & & node_122 $1==>0$ Grunduluscochae \\
\hline & 1 & & node_117 $1==>0$ node_116 & & 1 & & node_130 1 ==> 0 node_129 \\
\hline & 1 & & node_149 1 -->0 node_- 144 & & 1 & & node_140 $1==>0$ node_139 \\
\hline & 1 & & node_$\_1340==>2$ node $\_131$ & & 1 & & node_143 $1==>0$ Hemigrammusharal \\
\hline & 1 & & node_124 $2==>1$ Bryconellapallid & & 1 & & node_149 $1==>0$ node_148 \\
\hline & 1 & & node_123 $2==>0$ Paracheirodonaxe & & 1 & & node_146 $0==>1$ node_145 \\
\hline & 1 & & node_126 2 ==> 1 Hyphessobryconco & & 1 & & node_160 1 ==> 0 Hyphessobryconbe \\
\hline & 1 & & node_129 2 ==> 1 Hyphessobryconei & & 1 & & node_154 1 ==>0 Hyphessobryconmi \\
\hline & 1 & & node_141 0 --> 2 node_140 & & 1 & & node_156 1 ==>0 Hyphessobryconer \\
\hline & 1 & & node_1382 -->0 Hemigrammusblehe & & 1 & & node_158 1 ==> 0 Hyphessobryconeq \\
\hline & 1 & & node_1511 --> 2 node_150 & & 1 & & node_169 $0==>1$ Hemigrammusanali \\
\hline & 1 & & node_152 $1==>2$ Hemigrammusunili & & 1 & & node_173 $0==>1$ Hemigrammuslevis \\
\hline & 1 & & node_1611 -> 0 node_160 & & 1 & & node_174 $0==>1$ Hasemaniacrenuch \\
\hline & 1 & & node_159 $0-->1$ node_158 & & 1 & & node_178 $0==>1$ Cheirodoninterru \\
\hline & 1 & & node_153 $1==>2$ Pristellamaxilla & & 1 & & node_184 0 --> 1 node_183 \\
\hline & 1 & & node_154 $1==>2$ Hyphessobryconme & & 1 & & node_182 1 -->0 0 Piabinaargentea \\
\hline & 1 & & node_156 $1==>2$ Hyphessobryconer & & 1 & & node_188 $0-->1$ Chalceusspilogyr \\
\hline & 1 & & node_163 $1==>2$ Hemigrammuslunat & 40 & 1.000 & 1 & node_188 $1==>0$ Brycinuslongipin \\
\hline & 1 & & node_166 1 -->0 0 Hemigrammusocell & 41 & 0.067 & 1 & node_188 $1==>2$ Brycinuslongipin \\
\hline & 1 & & node_169 $0==>1$ Thayeriaobliqua & & 1 & & node_18̄̄ $1==>0$ node_96 \\
\hline & 1 & & node_173 0 --> 1 Hemigrammuslevis & & 1 & & node_-98 $1==>2$ node_- 97 \\
\hline & 1 & & node_174 0 --> 1 Hasemaniacrenuch & & 1 & & node_114 1 -->0 node_113 \\
\hline & 1 & & node_-1810 --> 2 node_180 & & 1 & & node_100 $0==>2$ Psellorammuskenn \\
\hline & 1 & & node_183 $0-->1$ node_182 & & 1 & & node_101 $0==>1$ Gymnocorymbuster \\
\hline 35 & 0.125 & 1 & node_98 1 -->0 node_97 & & 1 & & node_103 $0==>1$ Stethaprioneryth \\
\hline & 1 & & node $\_11 \overline{7} 1==>0$ node_$\_\overline{11} 6$ & & 1 & & node_1120 -->1 node_108 \\
\hline & 1 & & node_124 $1-->0$ node_123 & & 1 & & node_117 $1==>0$ Jupiabapolylepis \\
\hline & 1 & & node_142 $1->0$ node_141 & & 1 & & node_119 $1==>0$ Hemigrammusskoli \\
\hline & 1 & & node_138 $0->1$ node_137 & & 1 & & node_123 $1==>2$ node_122 \\
\hline & 1 & & node_172 $1==>0$ Hemigrammusstict & & 1 & & node_125 $1==>0$ Hemigrammusyinyan \\
\hline & 1 & & node_179 $1==>0$ node_178 & & 1 & & node_131 $1==>0$ node_130 \\
\hline & 1 & & node_183 $1==>0$ Rhinopetitiamyer & & 1 & & node_-129 $0==>2$ node_128 \\
\hline 36 & 0.056 & 1 & node_97 $0==>1$ Triportheusnemat & & 1 & & node_136 $1==>0$ Hemigrammusbrevi \\
\hline & 1 & & node_100 $0==>1$ Psellorammuskenn & & 1 & & node_137 1==> 2 Hemigrammusrhodo \\
\hline & 1 & & node_104 $0==>1$ node_103 & & 1 & & node_141 $1==>0$ Hemigrammusarua \\
\hline & 1 & & node_109 $0==>1$ Phenacogasterfra & & 1 & & node_143 $1==>0$ Hemigrammusharal \\
\hline & 1 & & node_117 $0==>1$ Jupiabapolylepis & & 1 & & node_1461 -->0 node_145 \\
\hline & 1 & & node_119 $0==>1$ Hemigrammusskoli & & 1 & & node_150 $1==>0$ Hemigrammusgraci \\
\hline & 1 & & node_165 $0==>1$ node_164 & & 1 & & node_162 $1-->0$ node_161 \\
\hline & 1 & & node_1361 $->0$ node_135 & & 1 & & node_152 0 --> 1 Moenkahusiahemig \\
\hline
\end{tabular}




\begin{tabular}{|c|c|c|c|c|c|c|c|}
\hline & 1 & & node_159 0 -->1 node_158 & & 1 & & node_139 1 ==> 0 Hyphessobrycondi \\
\hline & 1 & & node_1571 -->0 node_156 & & 1 & & node_141 $1==>0$ Hemigrammusarua \\
\hline & 1 & & node_166 $1==>0$ Hemigrammuscoeru & & 1 & & node_145 $1==>0$ HemigrammusspAlt \\
\hline & 1 & & node_171 1 -->0 node_170 & & 1 & & node_151 $1==>0$ node_150 \\
\hline & 1 & & node_169 $0->1$ Hemigrammusanali & & 1 & & node_166 $1==>0$ Hemigrammusocell \\
\hline & 1 & & node_174 $1==>0$ Hasemaniacrenuch & & 1 & & node_170 $1==>0$ Parapristellageo \\
\hline & 1 & & node_179 $1==>2$ node_178 & & 1 & & node_185 $1==>0$ Bryconopsmelanur \\
\hline & 1 & & node_185 1 ==> 0 Bryconopsmelanur & 50 & 0.083 & 1 & node_99 1 ==> 0 Roeboidesbonarie \\
\hline \multirow[t]{12}{*}{42} & 0.167 & 1 & node_96 $2==>0$ Bryconsp & & 1 & & node_109 $1==>0$ Phenacogasterfra \\
\hline & 1 & & node_98 $2-->0$ node_97 & & 1 & & node_124 $1==>0$ Bryconellapallid \\
\hline & 1 & & node_97 0 --> 1 Iguanodectesspil & & 1 & & node_122 $1==>0$ Grunduluscochae \\
\hline & 1 & & node_184 $2->1$ node_181 & & 1 & & node_129 $1==>0$ node_128 \\
\hline & 1 & & node_179 $1->2$ node_176 & & 1 & & node_137 $1==>0$ Petitellageorgia \\
\hline & 1 & & node_100 2 -->1 node_99 & & 1 & & node_160 $1-->0$ node_159 \\
\hline & 1 & & node 1092 ==> 1 Oligosarcuspinto & & 1 & & node $1580-->1$ node 157 \\
\hline & 1 & & node $1242-->1$ node 123 & & 1 & & node $1551-->0$ node 154 \\
\hline & 1 & & node_1221 --> 2 Coptobryconbilin & & 1 & & node_178 $1==>0$ Cheirodoninterru \\
\hline & 1 & & node_128 $2==>1$ Microschemobryco & & 1 & & node_181 $1==>0$ node_180 \\
\hline & 1 & & node_137 $2==>1$ Petitellageorgia & & 1 & & node_183 $1==>0$ Rhinopetitiamyer \\
\hline & 1 & & node_188 $2==>0$ Chalceusspilogyr & 51 & 0.750 & 1 & node_188 $1==>0$ Brycinuslongipin \\
\hline \multirow[t]{13}{*}{43} & 0.077 & 1 & node_107 $1==>0$ Hemigrammusthapo & & 1 & & node_96 $1==>2$ Salminushilarii \\
\hline & 1 & & node_11 $\overline{7} 1==>0$ Jupiabapolylepis & & 1 & & node_187 $1==>3$ node_186 \\
\hline & 1 & & node_1211 -->0 node_120 & & 1 & & node_97 $3==>0$ Triportheusnemat \\
\hline & 1 & & node_1190 0 -> 1 Hemigrammuscylin & 52 & 0.143 & 1 & node_1001 -->0 node_99 \\
\hline & 1 & & node_141 $1==>0$ Hemigrammusarua & & 1 & & node_10 $\overline{9} 1==>0$ Phenacogasterfra \\
\hline & 1 & & node_144 $1==>0$ node_143 & & 1 & & node_117 $1==>0$ node_116 \\
\hline & 1 & & node_148 1 -> 0 node_147 & & 1 & & node_122 $1==>0$ Grunduluscochae \\
\hline & 1 & & node_146 0 --> 1 node_145 & & 1 & & node_129 $1==>0$ node_128 \\
\hline & 1 & & node_152 $1==>0$ Moenkahusiahemig & & 1 & & node_157 $1==>0$ node_156 \\
\hline & 1 & & node $1551-->0$ node 153 & & 1 & & node $-1811==>0$ node -180 \\
\hline & 1 & & node 1661 ==> 0 Hemigrammusocell & 53 & 0.154 & 1 & nōde 1881 --> 2 nōe 187 \\
\hline & 1 & & node $\_1711==>0$ node $\_170$ & & 1 & & node_98 $2-->0$ node_97 \\
\hline & 1 & & node $\_1831==>0$ node_182 & & 1 & & node_110 $2==>0$ Hollandichthsper \\
\hline 44 & 0.167 & 1 & node_188 $0->1$ node_187 & & 1 & & node_123 2 --> 1 node_122 \\
\hline & 1 & & node_187 1 --> 2 node_96 & & 1 & & node_1302 -->0 node_129 \\
\hline & 1 & & node_1731 -->0 node_172 & & 1 & & node_141 $2==>0$ Hemigrammusarua \\
\hline & 1 & & node_168 $0-->1$ node_167 & & 1 & & node_160 $2==>0$ node_159 \\
\hline & 1 & & node_-117 1 -->0 node_116 & & 1 & & node_155 $0==>2$ node_153 \\
\hline & 1 & & node_124 1 -->0 node_123 & & 1 & & node_172 $2==>0$ Hemigrammusstict \\
\hline & 1 & & node_154 $1==>2$ Hyphessobryconme & & 1 & & node_174 $2==>1$ Hasemaniacrenuch \\
\hline & 1 & & node_156 1 ==> 2 Hyphessobryconer & & 1 & & node_178 $2==>0$ node_177 \\
\hline & 1 & & node $1631==>0$ Hemigrammusulrey & & 1 & & node_182 $2==>1$ Piabinaargentea \\
\hline & 1 & & node_170 $0-->1$ node_169 & & 1 & & node_1832 ==> 0 Rhinopetitiamyer \\
\hline & 1 & & node $\_1751==>0$ Hasemaniamelanur & 54 & 0.250 & 1 & node_119 $1==>0$ Hemigrammuscylin \\
\hline & 1 & & node_183 $1==>0$ Rhinopetitiamyer & & 1 & & node_130 1 --> 0 node_129 \\
\hline 45 & 0.250 & 1 & nōde_188 0 --> 1 Brycinuslongipin & & 1 & & node_137 $1==>0$ Petitellageorgia \\
\hline & 1 & & 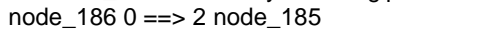 & & 1 & & node_155 1 ==> 0 node_154 \\
\hline & 1 & & node_1102 -->0 node_109 & 55 & 0.071 & 1 & node_98 1 ==> 0 Lignobryconmyers \\
\hline & 1 & & node_120 $2-->0$ node_119 & & 1 & & node_168 $1==>0$ node_167 \\
\hline & 1 & & node_130 $2==>0$ node_129 & & 1 & & node_104 $0==>1$ node_102 \\
\hline & 1 & & node_159 $2==>0$ node_158 & & 1 & & node_112 $0==>1$ node_108 \\
\hline & 1 & & node_174 $2==>1$ HasemaniaspCrenu & & 1 & & node_116 $0==>1$ Deuterodoniguape \\
\hline & 1 & & node $1842==>1$ node 183 & & 1 & & node $1250==>1$ node 124 \\
\hline 46 & 0.167 & 1 & node_187 0 --> 2 node_96 & & 1 & & node_122 $1==>0$ Grunduluscochae \\
\hline & 1 & & node_186 $0-->3$ node_98 & & 1 & & node_134 $0-->1$ node_133 \\
\hline & 1 & & node_168 $0==>1$ nodē_167 & & 1 & & node_140 $0==>1$ node_ 139 \\
\hline & 1 & & node_108 $1==>0$ Astyanaxmexicanu & & 1 & & node_170 $1==>0$ Parapristellageo \\
\hline & 1 & & node_1101 --> 2 node_109 & & 1 & & node_173 $1==>0$ Hemigrammuslevis \\
\hline & 1 & & node $\_1171==>0$ node $\_116$ & & 1 & & node_174 $1==>0$ HasemaniaspCrenu \\
\hline & 1 & & node_119 $1==>3$ Hemigrammuscylin & & 1 & & node_ $1811==>0$ node_180 \\
\hline & 1 & & node $1261-->0$ node 125 & & 1 & & node $1821==>0$ Bryconamericusex \\
\hline & 1 & & node 1240 --> 2 Bryconellapallid & 56 & 0.067 & 1 & nöde $1860->1$ node 98 \\
\hline & 1 & & node_125 0 --> 3 Hemigrammusyinyan & & 1 & & node_167 $0==>1$ node_165 \\
\hline & 1 & & node_130 $1==>2$ node_129 & & 1 & & node_114 $1==>0$ node_113 \\
\hline & 1 & & node_138 $1==>0$ Hemigrammusblehe & & 1 & & node_106 $0==>1$ node_105 \\
\hline & 1 & & node $1411==>0$ Hemigrammusarua & & 1 & & node_108 $0==>1$ Astyanaxaltipara \\
\hline & 1 & & node_143 $1==>0$ Hemigrammuspulch & & 1 & & node_120 $1==>0$ Hemigrammusnewbo \\
\hline & 1 & & node $1581==>3$ node_ 157 & & 1 & & node_124 $1==>0$ Bryconellapallid \\
\hline & 1 & & node_154 $3==>2$ Hyphessobryconme & & 1 & & node_127 $1==>0$ Hemigrammusorthu \\
\hline & 1 & & node $1740==>1$ Hasemaniacrenuch & & 1 & & node_134 $1==>0$ node_133 \\
\hline & 1 & & node_182 $0==>1$ Piabinaargentea & & 1 & & node_138 $1==>0$ Hemigrammusblehe \\
\hline 47 & 0.200 & 1 & node_96 $0==>1$ Salminushilarii & & 1 & & node_160 1 -->0 node_159 \\
\hline & 1 & & node_ $1010==>1$ Gymnocorymbuster & & 1 & & node_158 $0-->1$ node_157 \\
\hline & 1 & & node_103 $0==>1$ Poptellaparaguay & & 1 & & node_169 $0==>1$ Thayeriaobliqua \\
\hline & 1 & & node_108 $0==>1$ Astyanaxaltipara & & 1 & & node_173 $0==>1$ Hemigrammuslevis \\
\hline & 1 & & node $1110==>2$ Bryconexodonjuru & & 1 & & node $1750=\Rightarrow 1$ Hasemaniamelanur \\
\hline & 1 & & node_115 $0==>1$ Moenkhausiainter & 57 & 0.333 & 1 & node_97 $1==>0$ Iguanodectesspil \\
\hline & 1 & & node_126 $0==>1$ Hyphessobryconco & & 1 & & node_124 $1==>0$ node_123 \\
\hline & 1 & & node_152 $0==>1$ Hemigrammusunili & & 1 & & node_179 $1==>0$ node_178 \\
\hline & 1 & & node $1750==>1$ node_ 174 & 58 & 0.125 & 1 & node_187 $1==>0$ node_96 \\
\hline & 1 & & node_182 $0==>2$ Piabinaargentea & & 1 & & node_98 $1==>2$ node_97 \\
\hline 48 & 0.500 & 1 & node_122 $1==>0$ Coptobryconbilin & & 1 & & node_100 $1==>0$ node_99 \\
\hline & 1 & & node_182 $1==>0$ Piabinaargentea & & 1 & & node_107 $1==>0$ Hemigrammusthapo \\
\hline 49 & 0.100 & 1 & node_99 $1==>0$ Serrasalmusmacul & & 1 & & node $-1121==>0$ node_ 111 \\
\hline & 1 & & node_109 $1==>0$ Oligosarcuspinto & & 1 & & node_120 $1==>0$ node_119 \\
\hline & 1 & & node $1271==>0$ Hemigrammusbello & & 1 & & node_124 $1==>0$ Bryconellapallid \\
\hline
\end{tabular}




\begin{tabular}{|c|c|c|c|c|c|c|c|}
\hline & 1 & & node_126 1 ==> 0 Hyphessobryconco & & 1 & & node_112 0 -->1 node_108 \\
\hline & 1 & & node_129 $1==>0$ Hyphessobryconei & & 1 & & node_110 0 --> 1 node_109 \\
\hline & 1 & & node $1451==>0$ Hemigrammusmargi & & 1 & & node_128 $1==>0$ Hemigrammusmimus \\
\hline & 1 & & node_156 $1==>0$ Hyphessobryconep & & 1 & & node_158 $1==>0$ Hyphessobryconeq \\
\hline & 1 & & node $1711->0$ node 170 & & 1 & & node $1661=\Rightarrow 0$ Hemigrammusocell \\
\hline & 1 & & node_169 $0-->1$ Thayeriaobliqua & & 1 & & node_169 $1==>0$ Hemigrammusanali \\
\hline & 1 & & node $1741==>0$ Hasemaniacrenuch & & 1 & & node_174 0 --> 1 HasemaniaspCrenu \\
\hline & 1 & & node_180 1 ==> 0 Aphyocaraxpusill & & 1 & & node_182 1 ==>0 Bryconamericusex \\
\hline & 1 & & node_185 $1==>0$ Bryconopsmelanur & & 1 & & node_185 1 -->0 Bryconopsmelanur \\
\hline \multirow[t]{17}{*}{59} & 0.056 & 1 & $\begin{array}{l}\text { node_97 } 0==>1 \text { Triportheusnemat } \\
\text { node_167 } 0-->1 \text { node_165 }\end{array}$ & 66 & 0.333 & 1 & $\begin{array}{l}\text { node_97 } 0==>1 \text { lguanodectesspil } \\
\text { node_185 } 0==>1 \text { node_184 }\end{array}$ \\
\hline & 1 & & node_115 1 -->0 node_114 & & 1 & & node_99 $1==>0$ Serrasalmusmacul \\
\hline & 1 & & node_99 $0==>1$ Roeboidesbonarie & 67 & 0.100 & 1 & node_188 $0==>1$ Brycinuslongipin \\
\hline & 1 & & node_101 $0==>1$ Gymnocorymbuster & & 1 & & node_97 $0==>2$ Triportheusnemat \\
\hline & 1 & & node_103 $0==>1$ Poptellaparaguay & & 1 & & node_103 $0==>1$ Poptellaparaguay \\
\hline & 1 & & node_117 1 -->0 node_116 & & 1 & & node_106 $0==>1$ Moenkhausiasanct \\
\hline & 1 & & node_1201 -->0 Hemigrammusnewbo & & 1 & & node_ $1110==>2$ Bryconexodonjuru \\
\hline & 1 & & node $1251==>0$ node 124 & & 1 & & node_117 $0==>1$ Jupiabapolylepis \\
\hline & 1 & & node $1371==>0$ Hemigrammusrhodo & & 1 & & node $1200==>1$ Hemigrammusnewbo \\
\hline & 1 & & node $1531==>0$ Hyphessobryconco & & 1 & & node 1260 --> 1 node 125 \\
\hline & 1 & & node_154 $1==>0$ Hyphessobryconmi & & 1 & & node_124 1 -->0 node_123 \\
\hline & 1 & & node_159 $1==>0$ Hyphessobryconta & & 1 & & node_144 $0==>1$ node_ 143 \\
\hline & 1 & & node_1631 $->0$ Hemigrammuslunat & & 1 & & node_147 $0->1$ node_146 \\
\hline & 1 & & node_169 $0==>1$ Thayeriaobliqua & & 1 & & node_145 1 -->0 HemigrammusspAlt \\
\hline & 1 & & node_172 $0==>1$ Hemigrammusstict & & 1 & & node $1500==>2$ Hemigrammussilim \\
\hline & 1 & & node_177 $0==>1$ Serrapinnusheter & & 1 & & node_152 $0==>1$ Hemigrammusunili \\
\hline & 1 & & node_180 $0==>1$ Aphyocaraxpusill & & 1 & & node_167 $0==>1$ node_166 \\
\hline \multirow[t]{16}{*}{60} & 0.063 & 1 & node_98 $0==>1$ Lignobryconmyers & & 1 & & node_169 $0==>1$ Thayeriaobliqua \\
\hline & 1 & & node_1840 -->1 node_181 & & 1 & & node_173 $0==>1$ Hemigrammuslevis \\
\hline & 1 & & node_172 1 -->0 node_171 & & 1 & & node_178 $0==>2$ Cheirodoninterru \\
\hline & 1 & & node_99 $0==>1$ Roeboidesbonarie & & 1 & & node_184 0 --> 1 node_183 \\
\hline & 1 & & node_101 $0==>1$ Gymnocorymbuster & & 1 & & node_182 1 -->0 Piabinaargentea \\
\hline & 1 & & node $1040==>1$ node_103 & 68 & 0.095 & 1 & nóde_188 $0==>1$ Brycinuslongipin \\
\hline & 1 & & node $1060==>1$ Moenkhausiasanct & & 1 & & node $9 \overline{7} 0==>1$ Iguanodectesspil \\
\hline & 1 & & node $1090==>1$ Phenacogasterfra & & 1 & & node $990==>1$ Serrasalmusmacul \\
\hline & 1 & & node $1170==>1$ Jupiabapolylepis & & 1 & & node 1020 --> 1 node 101 \\
\hline & 1 & & node_164 $0==>1$ node_162 & & 1 & & node_1011 --> 2 Gymnocorymbuster \\
\hline & 1 & & node_124 $1==>0$ node_123 & & 1 & & node_103 $0==>2$ Poptellaparaguay \\
\hline & 1 & & node $1351==>0$ Hemigrammushyanu & & 1 & & node $1070==>2$ Hemigrammusthapo \\
\hline & 1 & & node $1381==>0$ Hemigrammusblehe & & 1 & & node_111 $0==>2$ Bryconexodonjuru \\
\hline & 1 & & node $1431==>0$ Hemigrammuspulch & & 1 & & node_114 $0==>2$ Moenkhausiaxingu \\
\hline & 1 & & node_176 $1->0$ node_175 & & 1 & & node_126 $0->1$ node_125 \\
\hline & 1 & & node_1781 -->0 Cheirodoninterru & & 1 & & node_124 1 -->0 node_123 \\
\hline \multirow[t]{3}{*}{61} & 0.333 & 1 & node_96 $1==>0$ Salminushilarii & & 1 & & node_122 $0->1$ Coptobryconbilin \\
\hline & 1 & & node_97 $1==>0$ Iguanodectesspil & & 1 & & node_128 $0==>2$ Hemigrammusmimus \\
\hline & 1 & & node_185 $1==>0$ Bryconopsmelanur & & 1 & & node $1400==>1$ Hemigrammusboese \\
\hline 62 & 0.167 & 1 & node_188 $1==>0$ Brycinuslongipin & & 1 & & node $1470==>2$ Hemigrammusparan \\
\hline & 1 & & node_96 $1==>0$ Salminushilarii & & 1 & & node_160 $0==>1$ Hyphessobryconbe \\
\hline & 1 & & node $971==>0$ Iguanodectesspil & & 1 & & node $1660==>2$ Hemigrammuscoeru \\
\hline & 1 & & node $1771==>0$ Serrapinnusnotom & & 1 & & node $1750=\Rightarrow 1$ node 174 \\
\hline & 1 & & node_180 1 ==> 0 Aphyocaraxanisit & & 1 & & node_177 $0==>2$ Serrapinnusheter \\
\hline & 1 & & node_185 $1==>0$ Bryconopsmelanur & & 1 & & node $\_1810==>2$ node 180 \\
\hline 63 & 0.136 & 1 & node_98 $2==>3$ Lignobryconmyers & & 1 & & node_182 $0==>2$ Bryconamericusex \\
\hline & 1 & & node_186 $2->0$ node_185 & 69 & 0.182 & 1 & node_149 $0==>2$ node 144 \\
\hline & 1 & & node $1850 \rightarrow 1$ node 184 & & 1 & & node_135 $2=>1$ node $13 \overline{4}$ \\
\hline & 1 & & node_102 1 -->0 node_100 & & 1 & & node $1261==>2$ Hyphessobryconco \\
\hline & 1 & & node_103 $1==>0$ Stethaprioneryth & & 1 & & node $1281==>0$ Hemigrammusmimus \\
\hline & 1 & & node_112 $1==>0$ node_ 111 & & 1 & & node_129 $1==>2$ Hyphessobryconei \\
\hline & 1 & & node_109 $0==>1$ Oligosarcuspinto & & 1 & & node_139 $2==>0$ node_138 \\
\hline & 1 & & node_119 $1==>0$ Hemigrammusskoli & & 1 & & node_157 $0==>1$ node_155 \\
\hline & 1 & & node_124 $1==>2$ Bryconellapallid & & 1 & & node_154 $1==>2$ Hyphessobryconmi \\
\hline & 1 & & node_1231 --> 3 node_122 & & 1 & & node_163 $0==>2$ Hemigrammuslunat \\
\hline & 1 & & node $1291==>0$ node 128 & & 1 & & node $1780=\Rightarrow 1$ Cheirodoninterru \\
\hline & 1 & & node $1321==>2$ Hemigrammustride & & 1 & & node $1770==>2$ Serrapinnusnotom \\
\hline & 1 & & node_135 $1==>2$ Hemigrammushyanu & 70 & 0.040 & 1 & node_96 $1==>0$ Salminushilarii \\
\hline & 1 & & node_139 $1==>0$ Hyphessobrycondi & & 1 & & node_172 $1==>0$ node_ 171 \\
\hline & 1 & & node_1611 -->0 node_160 & & 1 & & node_165 $0==>1$ node_- 121 \\
\hline & 1 & & node_160 0 --> 2 Hyphessobryconbe & & 1 & & node_99 $1==>0$ Roeboidesbonarie \\
\hline & 1 & & node_155 $0->1$ node_153 & & 1 & & node_1011 ==>0 Gymnocorymbuster \\
\hline & 1 & & node 1540 --> 2 Hyphessobryconmi & & 1 & & node $1031==>0$ Poptellaparaguay \\
\hline & 1 & & node_158 $0->1$ Hyphessobryconeq & & 1 & & node $1071==>0$ Hemigrammusthapo \\
\hline & 1 & & node $1691==>2$ Thayeriaobliqua & & 1 & & node_109 $1==>0$ Phenacogasterfra \\
\hline & 1 & & node_180 $1==>0$ Aphyocaraxpusill & & 1 & & node $1191==>0$ Hemigrammuscylin \\
\hline & 1 & & node_188 $2==>0$ Chalceusspilogyr & & 1 & & node_122 $0==>1$ Coptobryconbilin \\
\hline 64 & 0.400 & 1 & node_1230 $0->1$ node_122 & & 1 & & node_128 $0==>1$ Hemigrammusmimus \\
\hline & 1 & & node_122 $1-->2$ Grunduluscochae & & 1 & & node_138 $0==>1$ node_137 \\
\hline & 1 & & node_129 $0==>2$ Hyphessobryconei & & 1 & & node_143 $0==>1$ Hemigrammuspulch \\
\hline & 1 & & node $1550-->1$ node 154 & & 1 & & node $1450==>1$ Hemigrammusmargi \\
\hline & 1 & & node 1541 --> 2 Hyphèssobryconmi & & 1 & & node $1480==>1$ Hemigrammusrodwa \\
\hline 65 & 0.063 & 1 & node_187 $0-->1$ node_186 & & 1 & & node $1500==>1$ Hemigrammusgraci \\
\hline & 1 & & node_971--> 0 Iguanodectesspil & & 1 & & node_152 $0==>1$ Hemigrammusunili \\
\hline & 1 & & node_179 $1->0$ node_176 & & 1 & & node_159 0 --> 1 node_158 \\
\hline & 1 & & node_173 $0->1$ node_172 & & 1 & & node_157 1 -->0 node_155 \\
\hline & 1 & & node_114 1 -->0 node_113 & & 1 & & node_154 0 --> 1 Hyphessobryconmi \\
\hline & 1 & & node_106 0 --> 1 node_105 & & 1 & & node_163 $0==>1$ Hemigrammusulrey \\
\hline & 1 & & node $991==>0$ Serrasalmusmacul & & 1 & & node_166 $0==>1$ Hemigrammusocell \\
\hline
\end{tabular}


Serra, J. P. - Análise Filogenética das Espécies de Hemigrammus Gill, 1858 (Characiformes, Characidae)

\begin{tabular}{|c|c|c|c|c|c|c|c|}
\hline & 1 & & node_169 $0==>1$ Thayeriaobliqua & & 1 & & node_182 $0==>1$ Piabinaargentea \\
\hline & 1 & & node_181 $1==>0$ node_180 & 76 & 0.100 & 1 & node_188 1 ==>0 Brycinuslongipin \\
\hline & 1 & & node $1821==>0$ Piabinaargentea & & 1 & & node $18 \overline{7} 1==>2$ node 186 \\
\hline \multirow[t]{12}{*}{71} & 0.083 & 1 & node_187 $1==>0$ node_96 & & 1 & & node_98 2 ==> 0 node_97 \\
\hline & 1 & & node_97 $1==>0$ Triportheusnemat & & 1 & & node_1842 -->0 node_181 \\
\hline & 1 & & node $1851==>0$ node 184 & & 1 & & node $1130-->1$ node 107 \\
\hline & 1 & & node_100 0 --> 1 node_ 99 & & 1 & & node_107 1 --> 2 node_106 \\
\hline & 1 & & node_1110 --> 1 node_110 & & 1 & & node_1052 -->0 node_104 \\
\hline & 1 & & node_1091 -->0 Oligosarcuspinto & & 1 & & node_100 0 --> 1 node_99 \\
\hline & 1 & & node_124 $0==>1$ node_123 & & 1 & & node_108 $0==>1$ Astyanaxmexicanu \\
\hline & 1 & & node $1400==>1$ node -139 & & 1 & & node_109 $0==>1$ Phenacogasterfra \\
\hline & 1 & & node_137 $1==>0$ Hemigrammusrhodo & & 1 & & node_117 $0==>2$ Jupiabapolylepis \\
\hline & 1 & & node_146 $0==>1$ node 145 & & 1 & & node_120 0 --> 2 node_119 \\
\hline & 1 & & node $1530==>1$ Pristellamaxilla & & 1 & & node $1290==>2$ node 128 \\
\hline & 1 & & node $1730==>1$ Hemigrammuslevis & & 1 & & node 1390 ==> 2 Hyphessobrycondi \\
\hline \multirow[t]{17}{*}{72} & 0.059 & 1 & node_98 $0==>1$ Lignobryconmyers & & 1 & & node_146 $0==>1$ Moenkhausiabonit \\
\hline & 1 & & node_1650 0 -> 1 node_121 & & 1 & & node_155 0 --> 2 node_154 \\
\hline & 1 & & node_1151 -->0 node_114 & & 1 & & node_170 $0==>2$ Parapristellageo \\
\hline & 1 & & node_1050 0 -> 1 node_104 & & 1 & & node_176 $0==>1$ node_175 \\
\hline & 1 & & node_1001 -->0 node_99 & & 1 & & node_177 $0==>2$ Serrapinnusheter \\
\hline & 1 & & node_1031 -->0 Stethaprioneryth & & 1 & & node_182 2 ==> 1 Piabinaargentea \\
\hline & 1 & & node_108 $0==>1$ Astyanaxaltipara & 77 & 0.182 & 1 & node_188 $0==>2$ Brycinuslongipin \\
\hline & 1 & & node_1171 -->0 Jupiabapolylepis & & 1 & & node_187 $0==>1$ node_96 \\
\hline & 1 & & node_1191 -->0 Hemigrammuscylin & & 1 & & node_100 0 --> 1 node_ 99 \\
\hline & 1 & & node_126 $0==>1$ Hyphessobryconco & & 1 & & node_107 $0==>1$ Hemigrammusthapo \\
\hline & 1 & & node_143 $0==>1$ Hemigrammuspulch & & 1 & & node_109 $0==>2$ Oligosarcuspinto \\
\hline & 1 & & node_152 $0==>1$ Moenkahusiahemig & & 1 & & node_130 0 --> 1 node_129 \\
\hline & 1 & & node_160 $0==>1$ Hyphessobryconbe & & 1 & & node_1281 -->0 Microschemobryco \\
\hline & 1 & & node $1710-->1$ node 170 & & 1 & & node $1320=\Rightarrow 1$ Hemigrammustride \\
\hline & 1 & & node 1691 --> 0 Hemigrammusanali & & 1 & & node $1430==>1$ Hemigrammuspulch \\
\hline & 1 & & node $1750==>1$ node 174 & & 1 & & node $1600==>1$ Hyphessobryconbe \\
\hline & 1 & & node_177 $0==>1$ Serrapinnusheter & & 1 & & node_183 $0==>1$ Rhinopetitiamyer \\
\hline 73 & 0.083 & 1 & node_96 $1==>2$ Salminushilarii & 78 & 0.500 & 1 & node $\_1310==>1$ node 126 \\
\hline & 1 & & node_186 $1==>2$ node_185 & & 1 & & node_154 $0==>1$ Hyphessobryconme \\
\hline & 1 & & node $1762==>1$ node -173 & 79 & 0.333 & 1 & node_96 $0==>1$ Salminushilarii \\
\hline & 1 & & node_1051 -->0 node_104 & & 1 & & node_97 $0==>2$ Triportheusnemat \\
\hline & 1 & & node_99 0 --> 1 Serrasalmusmacul & & 1 & & node_99 $0==>1$ Serrasalmusmacul \\
\hline & 1 & & node_101 $0-->\{12\}$ Tetragnopterusar & & 1 & & node_108 $0==>2$ Astyanaxmexicanu \\
\hline & 1 & & node_103 0 --> 1 Poptellaparaguay & & 1 & & node_109 $0==>2$ Oligosarcuspinto \\
\hline & 1 & & node $1071==>0$ Hemigrammusthapo & & 1 & & node_175 $0-->2$ node_174 \\
\hline & 1 & & node_111 $1==>2$ Bryconexodonjuru & 80 & 0.333 & 1 & node_126 $1==>0$ node_125 \\
\hline & 1 & & node_109 $1==>0$ Oligosarcuspinto & & 1 & & node_ $15 \overline{5} 1==>0$ node_ $15 \overline{4}$ \\
\hline & 1 & & node_120 $1==>0$ Hemigrammusnewbo & & 1 & & node_174 $1==>0$ HasemaniaspCrenu \\
\hline & 1 & & node_1491 -->0 node_144 & 81 & 0.154 & 1 & node_173 $0==>1$ node_172 \\
\hline & 1 & & node $1360-->1$ node 135 & & 1 & & node $16 \overline{5} 1=\Rightarrow 0$ node $12 \overline{1}$ \\
\hline & 1 & & node $1231-->0$ node 122 & & 1 & & node $990==>1$ Serrasalmusmacul \\
\hline & 1 & & node_1410 --> 1 node_140 & & 1 & & node_1311 --> 2 node_130 \\
\hline & 1 & & node_137 $1==>2$ Hemigrammusrhodo & & 1 & & node_127 2 --> 1 Hemigrammusbello \\
\hline & 1 & & node_145 $1==>0$ HemigrammusspAlt & & 1 & & node_128 $2->1$ Hemigrammusmimus \\
\hline & 1 & & node_150 $1==>0$ Hemigrammussilim & & 1 & & node_133 $1==>2$ node 132 \\
\hline & 1 & & node_1611 -->0 node_152 & & 1 & & node_137 $1==>0$ Hemigrammusrhodo \\
\hline & 1 & & node_153 $1==>0$ Hyphessobryconco & & 1 & & node_153 $1==>2$ Hyphessobryconco \\
\hline & 1 & & node_166 $1==>0$ Hemigrammuscoeru & & 1 & & node_170 $1==>0$ Parapristellageo \\
\hline & 1 & & node_175 $2==>0$ Hasemaniamelanur & & 1 & & node_178 $0==>1$ Cheirodoninterru \\
\hline & 1 & & node $1782==>1$ Cheirodoninterru & & 1 & & node $1810==>1$ node 180 \\
\hline & 1 & & node_180 $2==>1$ Aphyocaraxanisit & & 1 & & node_183 $0==>1$ Rhinopetitiamyer \\
\hline 74 & 0.143 & 1 & node_99 $0==>1$ Roeboidesbonarie & 82 & 0.500 & 1 & node_112 $0==>1$ node_108 \\
\hline & 1 & & node_ $1100==>1$ Hollandichthsper & & 1 & & node $\_17 \overline{5} 0==>1$ node $\_17 \overline{4}$ \\
\hline & 1 & & node_116 $0==>1$ Astyanaxjacobina & 83 & 0.143 & 1 & node_188 0 --> 2 Brycinuslongipin \\
\hline & 1 & & node $1310->1$ node 126 & & 1 & & node $18 \overline{6} 0-->1$ node 98 \\
\hline & 1 & & node 1530 ==> 1 Hyphessobryconco & & 1 & & node 971 -->0 Triportheusnemat \\
\hline & 1 & & node_154 $0==>1$ Hyphessobryconme & & 1 & & node_ $1760==>1$ node 173 \\
\hline & 1 & & node_181 $0==>1$ node 180 & & 1 & & node_104 1 -->0 node_102 \\
\hline 75 & 0.083 & 1 & node $1880==>2$ Brycinuslongipin & & 1 & & node_100 $0->1$ node_99 \\
\hline & 1 & & node_96 $0==>1$ Bryconsp & & 1 & & node_106 $1==>0$ Moenkhausiasanct \\
\hline & 1 & & node $970==>2$ Iguanodectesspil & & 1 & & node $1131-->0$ node 112 \\
\hline & 1 & & node_ $1680==>1$ node 167 & & 1 & & node_111 $0-->1$ Bryconexodonjuru \\
\hline & 1 & & node $1071==>0$ node $\_106$ & & 1 & & node_129 $1==>2$ node_128 \\
\hline & 1 & & node_101 $0==>1$ Gymnocorymbuster & & 1 & & node_160 $1==>0$ Hyphessobryconbe \\
\hline & 1 & & node $1040==>1$ node 103 & & 1 & & node_163 $1==>0$ Hemigrammusulrey \\
\hline & 1 & & node_1111 -->0 node_ 110 & & 1 & & node_168 $1==>0$ Hemigrammusbarri \\
\hline & 1 & & node_109 $0-->2$ Oligosarcuspinto & & 1 & & node $1810==>2$ node 180 \\
\hline & 1 & & node_114 $1==>0$ Moenkhausiaxingu & 84 & 0.083 & 1 & node_188 $1->0$ node_187 \\
\hline & 1 & & node_121 $1==>0$ node 120 & & 1 & & node_186 $0-->1$ node_98 \\
\hline & 1 & & node_128 $1==>0$ Hemigrammusmimus & & 1 & & node_173 $0==>1$ node_172 \\
\hline & 1 & & node $1321==>0$ Hemigrammustride & & 1 & & node 991 ==> 0 Serrasalmusmacul \\
\hline & 1 & & node_135 $1==>0$ Hemigrammushyanu & & 1 & & node_102 $1==>0$ node_101 \\
\hline & 1 & & node_142 $1==>0$ node_141 & & 1 & & node_112 $1==>0$ node_108 \\
\hline & 1 & & node_137 $0==>1$ Hemigrammusrhodo & & 1 & & node $1091==>0$ Phenacogasterfra \\
\hline & 1 & & node_139 $0==>1$ Hyphessobrycondi & & 1 & & node_1251 -->0 node_124 \\
\hline & 1 & & node $1431==>0$ Hemigrammusharal & & 1 & & node $1230-->1$ node 122 \\
\hline & 1 & & node_145 $1==>0$ HemigrammusspAlt & & 1 & & node_129 $1==>0$ node 128 \\
\hline & 1 & & node_150 $1==>0$ Hemigrammusgraci & & 1 & & node_1321 $==>0$ Hemigrammusvorde \\
\hline & 1 & & node $1601==>0$ Hyphessobryconbe & & 1 & & node $1531==>0$ Hyphessobryconco \\
\hline & 1 & & node $1581==>0$ node 157 & 85 & 1.000 & 1 & node_182 $0==>1$ Bryconamericusex \\
\hline & 1 & & node_169 $0==>1$ Thayeriaobliqua & 86 & 0.500 & 1 & node_178 $2==>0$ node_177 \\
\hline
\end{tabular}


Serra, J. P. - Análise Filogenética das Espécies de Hemigrammus Gill, 1858 (Characiformes, Characidae)

\begin{tabular}{|c|c|c|c|c|c|c|c|}
\hline & 1 & & node_184 $2==>0$ node_183 & & 1 & & node_188 1 ==>0 Chalceusspilogyr \\
\hline & 1 & & node_182 $0==>1$ Bryconamericusex & 91 & 0.100 & 1 & node_187 0 --> 1 node_186 \\
\hline & 1 & & node $1882==>1$ Chalceusspilogyr & & 1 & & node $10 \overline{5} 1 \rightarrow>0$ node $10 \overline{4}$ \\
\hline \multirow[t]{10}{*}{87} & 0.100 & 1 & node_187 $1==>0$ node_96 & & 1 & & node_100 0 --> 1 Psellorammuskenn \\
\hline & 1 & & node_ $11 \overline{5} 1==>0$ node_ $11 \overline{4}$ & & 1 & & node_101 0 --> 1 Tetragnopterusar \\
\hline & 1 & & node_105 $0==>1$ node_104 & & 1 & & node_111 $1==>0$ Bryconexodonjuru \\
\hline & 1 & & node_101 $1==>0$ Tetragnopterusar & & 1 & & node_119 $1==>0$ Hemigrammusskoli \\
\hline & 1 & & node $-1110==>1$ node 110 & & 1 & & node_166 $1==>0$ Hemigrammuscoeru \\
\hline & 1 & & node_120 $1==>0$ Hemigrammusnewbo & & 1 & & node_175 $1==>0$ node $\_174$ \\
\hline & 1 & & node_133 $1==>0$ Hemigrammusschma & & 1 & & node_183 $1==>0$ Rhinopetitiamyer \\
\hline & 1 & & node_153 $1==>0$ Pristellamaxilla & & 1 & & node_188 $0-->1$ Chalceusspilogyr \\
\hline & 1 & & node_166 $1==>0$ Hemigrammuscoeru & 92 & 0.043 & 1 & node_187 0 --> 1 node_96 \\
\hline & 1 & & node_182 $1==>0$ Piabinaargentea & & 1 & & node_980-->1 node_97 \\
\hline \multirow[t]{37}{*}{88} & 0.054 & 1 & nóde_188 $2==>1$ Brycinuslongipin & & 1 & & node_105 $0==>1$ node_104 \\
\hline & 1 & & node_97 $2==>0$ Iguanodectesspil & & 1 & & node_101 $1==>0$ Tetragnopterusar \\
\hline & 1 & & node $1852->0$ node 184 & & 1 & & node $1080==>1$ Astyanaxaltipara \\
\hline & 1 & & node_179 $0-->2$ node_176 & & 1 & & node_110 $0==>1$ Hollandichthsper \\
\hline & 1 & & node $\_1722==>0$ node $\_171$ & & 1 & & node_116 $0==>1$ Astyanaxjacobina \\
\hline & 1 & & node_106 $0-->2$ node_105 & & 1 & & node_119 $0==>1$ Hemigrammusskoli \\
\hline & 1 & & node_104 $2->0$ node_102 & & 1 & & node_151 $0-->1$ node_149 \\
\hline & 1 & & node_99 $0==>1$ Serrasalmusmacul & & 1 & & node_124 1 -->0 node_123 \\
\hline & 1 & & node_113 $0->2$ node_112 & & 1 & & node_122 $0-->1$ Grunduluscochae \\
\hline & 1 & & node_108 $2->\{01\}$ Astyanaxmexicanu & & 1 & & node_127 $1==>0$ Hemigrammusbello \\
\hline & 1 & & node_1102 -->0 Hollandichthsper & & 1 & & node $1341==>0$ node_133 \\
\hline & 1 & & node_109 2 --> 1 Phenacogasterfra & & 1 & & node_1411 -->0 node_ 140 \\
\hline & 1 & & node_ $1180==>2$ node_117 & & 1 & & node_139 0 --> 1 Hyphessobrycondi \\
\hline & 1 & & node_116 $2==>1$ Astyanaxjacobina & & 1 & & node_148 1 -->0 node_147 \\
\hline & 1 & & node_120 $0-->2$ node_119 & & 1 & & node_1610 --> 1 node_152 \\
\hline & 1 & & node $1440-->2$ node 142 & & 1 & & node $1580==>1$ node 157 \\
\hline & 1 & & node $1262-->0$ node 125 & & 1 & & node $1531==>0$ Hyphèssobryconco \\
\hline & 1 & & node $1240-->2$ node 123 & & 1 & & node $1730==>1$ Hemigrammuslevis \\
\hline & 1 & & node_122 2 ==> 1 Coptobryconbilin & & 1 & & node_175 $0==>1$ node 174 \\
\hline & 1 & & node_1312 --> 1 node_130 & & 1 & & node_183 $0==>1$ Rhinopetitiamyer \\
\hline & 1 & & node_129 1 --> 2 Hyphessobryconei & & 1 & & node_188 $0-->1$ Chalceusspilogyr \\
\hline & 1 & & node_132 $2->\{01\}$ Hemigrammusvorde & 93 & 0.059 & 1 & node_185 $0==>1$ node_184 \\
\hline & 1 & & node_1362 $2->0$ Hemigrammusbrevi & & 1 & & node_99 $1=\Rightarrow 0$ Serrasalmusmacul \\
\hline & 1 & & node_1412 -> 1 node_140 & & 1 & & node_120 $1==>0$ node_119 \\
\hline & 1 & & node_139 1 -->0 node_138 & & 1 & & node_126 $1==>0$ node_125 \\
\hline & 1 & & node_146 $0==>1$ Moenkhausiabonit & & 1 & & node_123 $0==>1$ node_122 \\
\hline & 1 & & node $1510->1$ node 150 & & 1 & & node $1271==>0$ Hemigrammusorthu \\
\hline & 1 & & node_152 $0==>1$ Moenkahusiahemig & & 1 & & node_128 $1==>0$ Microschemobryco \\
\hline & 1 & & node_161 $0->2$ node_160 & & 1 & & node $1401==>0$ node_139 \\
\hline & 1 & & node_153 $2==>0$ Pristellamaxilla & & 1 & & node_143 $1==>0$ Hemigrammusharal \\
\hline & 1 & & node $1542==>0$ Hyphessobryconme & & 1 & & node $1611->0$ node 160 \\
\hline & 1 & & node $1562==>0$ Hyphessobryconep & & 1 & & node $1590-->1$ node 158 \\
\hline & 1 & & node $1630==>2$ Hemigrammusulrey & & 1 & & node $1571-->0$ node 155 \\
\hline & 1 & & node_170 $0==>1$ node_169 & & 1 & & node_1530 --> 1 Pristellamaxilla \\
\hline & 1 & & node_174 $2==>0$ Hasemaniacrenuch & & 1 & & node_172 $1==>0$ Hemigrammusstict \\
\hline & 1 & & node_178 $0->1$ node_177 & & 1 & & node $1751==>0$ node_174 \\
\hline & 1 & & node_184 $0->1$ node_183 & & 1 & & node_179 $1-->0$ node_ 178 \\
\hline 89 & 0.043 & 1 & node_1870 ==> 1 node_96 & & 1 & & node_177 0 --> 1 Serrapinnusheter \\
\hline & 1 & & node_165 $0->1$ node_121 & 94 & 0.250 & 1 & node_1871 -->0 node_96 \\
\hline & 1 & & node_100 $1==>0$ node_99 & & 1 & & node_ $1141==>2$ node_ 113 \\
\hline & 1 & & node_107 $1==>0$ Hemigrammusthapo & & 1 & & node_99 $2==>0$ Serrasalmusmacul \\
\hline & 1 & & node_116 $1==>0$ Astyanaxjacobina & & 1 & & node_100 $2==>1$ Psellorammuskenn \\
\hline & 1 & & node_1201 $->0$ node_119 & & 1 & & node_104 $2==>1$ node_103 \\
\hline & 1 & & node_126 $0->1$ node_125 & & 1 & & node_1102 $->1$ node_109 \\
\hline & 1 & & node_124 $1->0$ node_123 & & 1 & & node_109 1 --> 3 Oligosarcuspinto \\
\hline & 1 & & node $1220-->1$ Grunduluscochae & & 1 & & node $1191=\Rightarrow 3$ Hemigrammusskoli \\
\hline & 1 & & node $1340==>1$ node 133 & & 1 & & node 1221 ==> 2 Grunduluscochae \\
\hline & 1 & & node $1370==>1$ Hemigrammusrhodo & & 1 & & node $1261==>2$ Hyphessobryconco \\
\hline & 1 & & node_144 $0==>1$ node_143 & & 1 & & node_129 $1==>3$ Hyphessobryconei \\
\hline & 1 & & node_148 $0-->1$ node_147 & & 1 & & node_175 $1==>2$ node_ 174 \\
\hline & 1 & & node_146 1 -->0 node_145 & 95 & 0.250 & 1 & node_96 $1==>0$ Salminushilarii \\
\hline & 1 & & node_152 $0==>1$ Moenkahusiahemig & & 1 & & node_99 $1==>0$ Roeboidesbonarie \\
\hline & 1 & & node $1530==>1$ Hyphessobryconco & & 1 & & node $1121-->0$ node 111 \\
\hline & 1 & & node_154 $0==>1$ Hyphessobryconme & & 1 & & node_110 0 --> 1 node_109 \\
\hline & 1 & & node_163 $0==>1$ Hemigrammuslunat & 96 & 0.034 & 1 & node_188 $0->1$ Brycinuslongipin \\
\hline & 1 & & node_166 $0==>1$ Hemigrammusocell & & 1 & & node_187 $0-->1$ node_186 \\
\hline & 1 & & node $1700=\Rightarrow 1$ Parapristellageo & & 1 & & node_173 $1==>0$ node_ 172 \\
\hline & 1 & & node_178 $0==>1$ Cheirodoninterru & & 1 & & node_165 $0-->1$ node_121 \\
\hline & 1 & & node_180 $0==>1$ Aphyocaraxpusill & & 1 & & node_114 $1->0$ node_113 \\
\hline & 1 & & node_182 $0==>1$ Piabinaargentea & & 1 & & node_105 0 --> 1 node_104 \\
\hline 90 & 0.143 & 1 & node $1861==>2$ node 98 & & 1 & & node $1001->0$ node 99 \\
\hline & 1 & & node $18 \overline{4} 1==>2$ node $18 \overline{1}$ & & 1 & & node 1011 -->0 Tetragnopterusar \\
\hline & 1 & & node $992==>0$ Roeboidesbonarie & & 1 & & node $1181-->0$ node 117 \\
\hline & 1 & & node_106 $2==>1$ Moenkhausiasanct & & 1 & & node_116 0 --> 1 Astyanaxjacobina \\
\hline & 1 & & node_107 $2==>0$ Hemigrammusthapo & & 1 & & node_119 1 -->0 Hemigrammusskoli \\
\hline & 1 & & node_112 $2->0$ node_111 & & 1 & & node_149 $0-->1$ node_144 \\
\hline & 1 & & node_110 $0->2$ node_109 & & 1 & & node_134 $1==>0$ node_ 131 \\
\hline & 1 & & node $1162==>1$ Astyanaxjacobina & & 1 & & node $1220==>1$ Grunduluscochae \\
\hline & 1 & & node_120 $2==>1$ node 119 & & 1 & & node $1250==>1$ Hemigrammusyinyan \\
\hline & 1 & & node_122 $2==>01\}$ Coptobryconbilin & & 1 & & node $1280==>1$ Hemigrammusmimus \\
\hline & 1 & & node $1532==>1$ Pristellamaxilla & & 1 & & node $1321==>0$ Hemigrammustride \\
\hline & 1 & & node $1632=\Rightarrow 1$ Hemigrammusulrey & & 1 & & node_142 1 -->0 node_141 \\
\hline & 1 & & node_1742 ==> 1 HasemaniaspCrenu & & 1 & & node_137 $0-->1$ Hemigrammusrhodo \\
\hline
\end{tabular}




\begin{tabular}{|c|c|c|c|c|c|c|}
\hline & 1 & & node_1390 --> 1 Hyphessobrycondi & & 1 & node_128 $0==>1$ Microschemobryco \\
\hline & 1 & & node_162 0 --> 1 node_161 & & 1 & node_133 $0==>1$ node_132 \\
\hline & 1 & & node_160 1 -->0 node_159 & & 1 & node_135 $0==>1$ Hemigrammushyanu \\
\hline & 1 & & node_153 $0==>1$ Pristellamaxilla & & 1 & node_149 $0==>1$ node_148 \\
\hline & 1 & & node $1570==>1$ node_156 & & 1 & node_146 $1==>0$ Moenkhausiabonit \\
\hline & 1 & & node_169 $0==>1$ Thayeriaobliqua & & 1 & node_150 $0==>1$ Hemigrammusgraci \\
\hline & 1 & & node $1771==>0$ Serrapinnusheter & & 1 & node $1520==>1$ Moenkahusiahemig \\
\hline & 1 & & node_180 1 ==> 0 Aphyocaraxanisit & & 1 & node_160 $0==>1$ Hyphessobryconbe \\
\hline & 1 & & node_182 $1==>0$ Piabinaargentea & & 1 & node_158 $0==>1$ node_157 \\
\hline & 1 & & node_1851 -->0 Bryconopsmelanur & & 1 & node_154 $1==>0$ Hyphessobryconme \\
\hline \multirow[t]{3}{*}{97} & 0.333 & 1 & node_110 $1==>0$ Hollandichthsper & & 1 & node_163 $0==>1$ Hemigrammusulrey \\
\hline & 1 & & node_ $17 \overline{5} 1==>0$ node_174 & & 1 & node_175 $0==>1$ node_ 174 \\
\hline & 1 & & node_182 $1==>0$ Piabinaargentea & & 1 & node_177 $0==>1$ Serrapinnusnotom \\
\hline \multirow[t]{12}{*}{98} & 0.083 & 1 & node_98 $0==>1$ Lignobryconmyers & & 1 & node $\_1810==>1$ node 180 \\
\hline & 1 & & node_100 $0==>1$ Psellorammuskenn & 105 & 0.150 & 1 node_186 $2==>0$ node_98 \\
\hline & 1 & & node $1160==>1$ Deuterodoniguape & & 1 & node $97 \overline{0}==>3$ Iguanodectesspil \\
\hline & 1 & & node_135 $0==>1$ node_134 & & 1 & node_99 2 ==> 1 Roeboidesbonarie \\
\hline & 1 & & node_$\_1291==>0$ node_128 & & 1 & node_-102 2 ==> 1 node_101 \\
\hline & 1 & & node_143 $0==>1$ Hemigrammusharal & & 1 & node_109 2 ==> 3 Phenacogasterfra \\
\hline & 1 & & node_159 $0==>1$ node_158 & & 1 & node_115 $2==>0$ Moenkhausiainter \\
\hline & 1 & & node_153 $1==>0$ Pristellamaxilla & & 1 & node_117 $2==>1$ Jupiabapolylepis \\
\hline & 1 & & node_156 $1==>0$ Hyphessobryconer & & 1 & node_133 $2==>1$ Hemigrammusschma \\
\hline & 1 & & node_169 $0==>1$ Thayeriaobliqua & & 1 & node_136 2 ==> 1 Hemigrammusbrevi \\
\hline & 1 & & node $1760==>1$ node 175 & & 1 & node_1372 ==> 1 Petitellageorgia \\
\hline & 1 & & node_178 $0==>1$ Cheirodoninterru & & 1 & node_139 2 ==> 1 Hyphessobrycondi \\
\hline \multirow[t]{8}{*}{99} & 0.125 & 1 & node_123 $1==>0$ Paracheirodonaxe & & 1 & node_146 $2==>1$ Moenkhausiabonit \\
\hline & 1 & & node_133 $1==>0$ Hemigrammusschma & & 1 & node_150 $2==>1$ Hemigrammusgraci \\
\hline & 1 & & node_135 1 ==> 0 Hemigrammushyanu & & 1 & node_158 $2==>1$ node_157 \\
\hline & 1 & & node_137 $1==>0$ Petitellageorgia & & 1 & node_154 $1==>2$ Hyphēessobryconmi \\
\hline & 1 & & node_160 $1==>0$ Hyphessobryconbe & & 1 & node_169 $2==>1$ Thayeriaobliqua \\
\hline & 1 & & node 1561 ==> 0 Hyphessobryconer & & 1 & node $1732==>1$ Hemigrammuslevis \\
\hline & 1 & & node_158 $1==>0$ Hyphessobryconeq & & 1 & node_178 $2==>1$ Cheirodoninterru \\
\hline & 1 & & node_169 $1==>0$ Thayeriaobliqua & & 1 & node_182 $2==>3$ Piabinaargentea \\
\hline 100 & 0.333 & 1 & node_188 0 --> 1 node_187 & & 1 & node_185 $2==>3$ Bryconopsmelanur \\
\hline & 1 & & node_96 $1->0$ Bryconsp & 106 & 0.250 & 1 node_97 $0==>1$ lguanodectesspil \\
\hline & 1 & & node_99 $1==>0$ Serrasalmusmacul & & 1 & node_99 $\overline{0}==>1$ Serrasalmusmacul \\
\hline 101 & 0.143 & 1 & node_186 $0==>1$ node_185 & & 1 & node_1110 $0=>2$ Bryconexodonjuru \\
\hline & 1 & & node_111 $1-->0$ node_110 & & 1 & node_123 $0==>1$ node_122 \\
\hline & 1 & & node_109 0 --> 1 Phenāacogasterfra & & 1 & node_141 $0==>2$ Hemigrammusarua \\
\hline & 1 & & node_117 $1==>0$ node_ 116 & & 1 & node_175 $0==>2$ Hasemaniamelanur \\
\hline & 1 & & node_153 $1==>0$ Hyphessobryconco & & 1 & node_178 $0==>1$ Cheirodoninterru \\
\hline & 1 & & node_167 $1->0$ node_166 & & 1 & node_183 $0==>1$ Rhinopetitiamyer \\
\hline & 1 & & node_1791 -->0 node_178 & 107 & 1.000 & 1 node_96 $1==>2$ Salminushilarii \\
\hline 102 & 0.333 & 1 & node_188 $1==>0$ Brycinuslongipin & & 1 & node_128 $1==>0$ Microschemobryco \\
\hline & 1 & & node_96 $\overline{1}==>0$ Bryconsp & 108 & 0.118 & node_186 $0==>2$ node_98 \\
\hline & 1 & & node $981==>0$ node 97 & & 1 & node $11 \overline{\overline{7}} 0==>1$ node $11 \overline{6}$ \\
\hline 103 & 0.036 & 1 & node_96 $1==>0$ S̄alminushilarii & & 1 & node_119 $0==>1$ Hemigrammusskoli \\
\hline & 1 & & node_98 $\overline{1}==>0$ Lignobryconmyers & & 1 & node_134 0 --> 1 node_131 \\
\hline & 1 & & node_185 1 -->0 node_184 & & 1 & node_124 $1-->2$ node_123 \\
\hline & 1 & & node_176 0 -->1 node_173 & & 1 & node_122 2 --> 0 Coptōbryconbilin \\
\hline & 1 & & node_172 $1->0$ node_171 & & 1 & node_125 1 -->0 Hemigrammusyinyan \\
\hline & 1 & & node $\_1650==>1$ node $\_121$ & & 1 & node_1311 --> 2 node_130 \\
\hline & 1 & & node_-104 $1-->0$ node_102 & & 1 & node_127 2 --> 1 Hemigrammusorthu \\
\hline & 1 & & node 990 --> 1 Serrasalmusmacul & & 1 & node_128 2 --> 1 Hemigrammusmimus \\
\hline & 1 & & node_101 0 --> 1 Gymnocorymbuster & & 1 & node_132 $0==>2$ Hemigrammusvorde \\
\hline & 1 & & node_$-1121==>0$ node_111 & & 1 & node_136 $0==>1$ Hemigrammusbrevi \\
\hline & 1 & & node_109 $0==>1$ Phenacogasterfra & & 1 & node_141 0 --> 1 node_140 \\
\hline & 1 & & node_117 $1==>0$ Jupiabapolylepis & & 1 & node_139 1 -->0 node_138 \\
\hline & 1 & & node_119 $1==>0$ Hemigrammuscylin & & 1 & node_150 $0==>1$ Hemigrammusgraci \\
\hline & 1 & & node_126 $0==>1$ Hyphessobryconco & & 1 & node_184 $0==>1$ node_183 \\
\hline & 1 & & node_127 $0==>1$ Hemigrammusbello & & 1 & node_182 $1==>2$ Piabinaargentea \\
\hline & 1 & & node $1280==>1$ Hemigrammusmimus & 109 & 0.111 & node $1100==>1$ Hollandichthsper \\
\hline & 1 & & node_133 $0==>1$ Hemigrammusschma & & 1 & node $-14 \overline{4} 0==>1$ node $\_142$ \\
\hline & 1 & & node_136 $0==>1$ Hemigrammusbrevi & & 1 & node_$-1311==>0$ node_130 \\
\hline & 1 & & node_140 $0==>1$ node_139 & & 1 & node_140 $1==>0$ Hemigrammusboese \\
\hline & 1 & & node_148 $0==>1$ Hemigrammusrodwa & & 1 & node_150 $0==>1$ Hemigrammussilim \\
\hline & 1 & & node_153 $0==>1$ Pristellamaxilla & & 1 & node_152 $0==>1$ Hemigrammusunili \\
\hline & 1 & & node_154 $0==>1$ Hyphessobryconme & & 1 & node_173 $0==>1$ Hemigrammuslevis \\
\hline & 1 & & node_156 $0==>1$ Hyphessobryconer & & 1 & node_180 $0==>1$ Aphyocaraxanisit \\
\hline & 1 & & node_159 $0==>1$ Hyphessobryconta & & 1 & node_184 $0==>1$ node_183 \\
\hline & 1 & & node_166 $0==>1$ Hemigrammusocell & 110 & 0.087 & node_96 $2==>0$ Salminushilarii \\
\hline & 1 & & node_174 0 --> 1 HasemaniaspCrenu & & 1 & node_1872 $==>1$ node_186 \\
\hline & 1 & & node_178 $0->1$ Cheirodoninterru & & 1 & node_115 $1->0$ node_ 114 \\
\hline & 1 & & node_183 0 --> 1 Rhinopetitiamyer & & 1 & node_107 $0-->1$ node_106 \\
\hline 104 & 0.038 & 1 & node_96 $0==>1$ Bryconsp & & 1 & node_99 $1==>2$ Serrasalmusmacul \\
\hline & 1 & & node $1860->1$ node 98 & & 1 & node $1011==>2$ Tetragnopterusar \\
\hline & 1 & & node 971 --> 0 Iguanōdectesspil & & 1 & node $1130==>2$ node 112 \\
\hline & 1 & & node $-1140==>1$ node_113 & & 1 & node_110 $2==>0$ Hollandichthsper \\
\hline & 1 & & node_1011 ==> 0 Tetragnopterusar & & 1 & node_109 $2==>1$ Oligosarcuspinto \\
\hline & 1 & & node_105 $1==>0$ Hemigrammuspreto & & 1 & node_120 $1==>2$ node_119 \\
\hline & 1 & & node_111 $1==>0$ Bryconexodonjuru & & 1 & node_131 $1==>2$ node_130 \\
\hline & 1 & & node_116 $0==>1$ Deuterodoniguape & & 1 & node_128 $2==>0$ Hemigrammusmimus \\
\hline & 1 & & node_119 $0==>1$ Hemigrammuscylin & & 1 & node_137 $1==>0$ Hemigrammusrhodo \\
\hline & 1 & & node $1240-->1$ node 123 & & 1 & node $1431==>0$ Hemigrammuspulch \\
\hline & 1 & & node_1221 -->0 Grunduluscochae & & 1 & node_148 $1==>0$ node $\_147$ \\
\hline & 1 & & node $1260==>1$ Hyphessobryconco & & 1 & node_150 $1==>0$ Hemigrammusgraci \\
\hline
\end{tabular}




\begin{tabular}{|c|c|c|c|c|c|c|}
\hline & 1 & & node_164 $1==>0$ node_163 & & 1 & node_108 1 --> 0 Astyanaxaltipara \\
\hline & 1 & & node_166 $1==>0$ Hemigrammusocell & & 1 & node_116 $0==>1$ Astyanaxjacobina \\
\hline & 1 & & node $1681==>0$ Hemigrammusbarri & & 1 & node_122 $1==>0$ Coptobryconbilin \\
\hline & 1 & & node $1741==>0$ Hasemaniacrenuch & & 1 & node $1281=\Rightarrow 0$ Microschemobryco \\
\hline & 1 & & node $1801==>2$ Aphyocaraxpusill & & 1 & node $1331==>0$ Hemigrammusschma \\
\hline & 1 & & node_184 $1->0$ node_183 & & 1 & node_1441 -->0 node_143 \\
\hline & 1 & & node_182 0 --> 1 Piabinaargentea & & 1 & node_146 $1==>0$ Moenkhausiabonit \\
\hline \multirow[t]{7}{*}{111} & 0.143 & 1 & node_96 $1==>0$ Bryconsp & & 1 & node_1501 -->0 Hemigrammusgraci \\
\hline & 1 & & node_ $101 \overline{1}==>0$ Tetragnopterusar & & 1 & node_162 $1->0$ node_161 \\
\hline & 1 & & node $1061==>0$ Moenkhausiasanct & & 1 & node $1570=\Rightarrow 1$ node 155 \\
\hline & 1 & & node $1141==>0$ Moenkhausiaxingu & & 1 & node $1690==>1$ Thayeriaobliqua \\
\hline & 1 & & node_119 $1==>0$ Hemigrammusskoli & & 1 & node_172 $0==>1$ Hemigrammusstict \\
\hline & 1 & & node $1711==>0$ node $\_170$ & & 1 & node_175 $0==>1$ Hasemaniamelanur \\
\hline & 1 & & node_180 $1==>0$ Aphyocaraxpusill & & 1 & node_177 $0==>1$ Serrapinnusnotom \\
\hline \multirow[t]{24}{*}{112} & 0.083 & 1 & node_97 $1==>0$ Iguanodectesspil & & 1 & node_183 $0==>1$ node_182 \\
\hline & 1 & & node_172 $1==>2$ node_ 171 & 121 & 0.231 & 1 node_98 $2==>1$ Lignobryconmyers \\
\hline & 1 & & node $1182==>1$ node 115 & & 1 & node_97 $==>3$ Iguanodectesspil \\
\hline & 1 & & node_106 1 --> 2 node_105 & & 1 & node_99 $2==>3$ Serrasalmusmacul \\
\hline & 1 & & node_104 $2->1$ node_102 & & 1 & node_101 $2==>3$ Gymnocorymbuster \\
\hline & 1 & & node 991 ==> 2 Serrasalmusmacul & & 1 & node $1192==>1$ Hemigrammuscylin \\
\hline & 1 & & node_1131 --> 2 node_112 & & 1 & node_122 2 ==> 3 Grunduluscochae \\
\hline & 1 & & node_1112 --> 1 Bryconexodonjuru & & 1 & node_123 $2==>1$ Paracheirodonaxe \\
\hline & 1 & & node_109 2 --> 1 Oligosarcuspinto & & 1 & node_138 $2==>0$ Hemigrammusblehe \\
\hline & 1 & & node_122 $2==>1$ Coptobryconbilin & & 1 & node_140 $2==>1$ Hemigrammusboese \\
\hline & 1 & & node_127 $2==>1$ Hemigrammusbello & & 1 & node_148 $2==>1$ Hemigrammusrodwa \\
\hline & 1 & & node $1282=\Rightarrow 1$ Hemigrammusmimus & & 1 & node $1562==>1$ Hyphessobryconep \\
\hline & 1 & & node_135 $2==>1$ Hemigrammushyanu & & 1 & node_1832 --> 3 node_182 \\
\hline & 1 & & node_142 $2->1$ node_141 & & 1 & node_185 $2==>0$ Bryconopsmelanur \\
\hline & 1 & & node_1401--> 2 Hemigrammusboese & 122 & 0.273 & 1 node_181 $0==>1$ node 179 \\
\hline & 1 & & node $1432==>1$ Hemigrammusharal & & 1 & node_99 $1==>3$ Serrasalmusmacul \\
\hline & 1 & & node_146 $2==>1$ node_145 & & 1 & node_104 $1==>2$ node_103 \\
\hline & 1 & & node $1612==>1$ node_160 & & 1 & node $-1111==>0$ node_ 110 \\
\hline & 1 & & node_158 1 --> 2 node_157 & & 1 & node_117 $1==>0$ Jupiabapolylepis \\
\hline & 1 & & node $1542->1$ Hyphessobryconme & & 1 & node $1341==>0$ node 131 \\
\hline & 1 & & node 1562 --> 1 Hyphessobryconer & & 1 & node $1240==>1$ Bryconellapallid \\
\hline & 1 & & node $1741==>2$ Hasemaniacrenuch & & 1 & node $1220==>1$ Coptobryconbilin \\
\hline & 1 & & node_180 $1==>2$ Aphyocaraxanisit & & 1 & node_139 $1==>0$ Hyphessobrycondi \\
\hline & 1 & & node_182 $1==>2$ Piabinaargentea & & 1 & node_ $1701==>0$ node_169 \\
\hline \multirow[t]{16}{*}{113} & 0.063 & 1 & node_98 $0==>1$ Lignobryconmyers & & 1 & node_188 $0==>1$ Chalceusspilogyr \\
\hline & 1 & & node_103 $0==>1$ Stethaprioneryth & 123 & 0.250 & node_188 $0==>1$ Brycinuslongipin \\
\hline & 1 & & node_110 $0==>1$ Hollandichthsper & & 1 & node_124 $0-->1$ node_123 \\
\hline & 1 & & node_116 $0==>1$ Astyanaxjacobina & & 1 & node $-1750==>1$ node $\_174$ \\
\hline & 1 & & node_123 $0==>1$ node_ 122 & & 1 & node_184 $0==>1$ node_183 \\
\hline & 1 & & node_1310 --> 1 node_$\overline{1} 30$ & 124 & 0.200 & node_96 $0==>2$ Bryconsp \\
\hline & 1 & & node_127 1 -> 0 Hemigrammusbello & & 1 & node $1130==>2$ node 107 \\
\hline & 1 & & node_139 $0==>1$ Hyphessobrycondi & & 1 & node_105 $2==>0$ Hemigrammuspreto \\
\hline & 1 & & node $1430==>1$ Hemigrammusharal & & 1 & node $1100==>2$ Hollandichthsper \\
\hline & 1 & & node_150 $0==>1$ Hemigrammusgraci & & 1 & node_119 $0==>2$ Hemigrammusskoli \\
\hline & 1 & & node_160 $0==>1$ Hyphessobryconbe & & 1 & node_$\_1230==>2$ node_122 \\
\hline & 1 & & node $1550=\Rightarrow 1$ node 154 & & 1 & node $1610=\Rightarrow 1$ node 152 \\
\hline & 1 & & node $1690==>1$ Hemigrammusanali & & 1 & node $1540==>2$ Hyphèssobryconmi \\
\hline & 1 & & node $1740==>1$ Hasemaniacrenuch & & 1 & node_164 $0==>1$ node_163 \\
\hline & 1 & & node_177 $0==>1$ Serrapinnusnotom & & 1 & node_176 $0==>2$ node_175 \\
\hline & 1 & & node_183 $0==>1$ Rhinopetitiamyer & 125 & 0.182 & 1 node_186 $1==>0$ node_98 \\
\hline 114 & 1.000 & 1 & node $1861==>0$ node 98 & & 1 & node $1051==>0$ node 104 \\
\hline & 1 & & node_180 $1==>2$ Aphyocaraxpusill & & 1 & node_1251 --> 2 node_124 \\
\hline 115 & 0.133 & 1 & node_108 $0==>1$ Astyanaxmexicanu & & 1 & node_123 $2->1$ Paracheirodonaxe \\
\hline & 1 & & node_ $1100==>2$ Hollandichthsper & & 1 & node_129 $1==>2$ node_128 \\
\hline & 1 & & node_125 $0==>1$ node_124 & & 1 & node_132 1 ==> 2 Hemigrammusvorde \\
\hline & 1 & & node_127 $0==>1$ Hemigrammusorthu & & 1 & node_135 $1==>2$ Hemigrammushyanu \\
\hline & 1 & & node_128 $0==>1$ Hemigrammusmimus & & 1 & node_139 $1==>2$ node_138 \\
\hline & 1 & & node $1330==>1$ node_132 & & 1 & node_ $1711==>2$ node_ 170 \\
\hline & 1 & & node $1360=\Rightarrow 1$ Hemigrammusbrevi & & 1 & node $1761==>2$ node 175 \\
\hline & 1 & & node $1390==>1$ Hyphessobrycondi & & 1 & node $1881==>2$ Chalceusspilogyr \\
\hline & 1 & & node_148 $0==>1$ Hemigrammusrodwa & 126 & 0.080 & 1 node_188 $0->1$ node_187 \\
\hline & 1 & & node_150 $0==>1$ Hemigrammussilim & & 1 & node_96 $1-->0$ Bryconsp \\
\hline & 1 & & node_152 $0==>1$ Hemigrammusunili & & 1 & node_97 $1==>0$ Triportheusnemat \\
\hline & 1 & & node_168 $0==>1$ Hemigrammusbarri & & 1 & node_168 $1==>0$ node_ 167 \\
\hline & 1 & & node $1780==>1$ Cheirodoninterru & & 1 & node $1150-->1$ node $\overline{1} 14$ \\
\hline & 1 & & node $1810==>2$ node 180 & & 1 & node_1001 -->0 node_99 \\
\hline & 1 & & node_183 $0==>1$ Rhinopetitiamyer & & 1 & node_106 $1==>0$ Moenkhausiasanct \\
\hline 116 & 0.500 & 1 & node_188 $1==>0$ node_187 & & 1 & node_136 $0==>1$ node_135 \\
\hline & 1 & & node $1810==>1$ node 179 & & 1 & node_123 $1==>0$ node_122 \\
\hline 117 & 0.500 & 1 & node_186 $0->1$ node_98 & & 1 & node_125 $1==>2$ Hemigrammusyinyan \\
\hline & 1 & & node_97 1 --> 0 Iguanodectesspil & & 1 & node_126 $1==>0$ Hyphessobryconco \\
\hline 118 & 1.000 & 1 & node_129 $0==>1$ node_128 & & 1 & node_127 $1==>0$ Hemigrammusorthu \\
\hline 119 & 0.500 & 1 & node_97 $0==>1$ Triportheusnemat & & 1 & node_129 $1==>0$ Hyphessobryconei \\
\hline & 1 & & node $1230==>1$ node 122 & & 1 & node $1410=\Rightarrow 1$ node 140 \\
\hline 120 & 0.043 & 1 & node_97 $1==>0$ Triportheusnemat & & 1 & node_144 $0==>2$ node $\_143$ \\
\hline & 1 & & node $1861==>0$ node 185 & & 1 & node_161 $0==>1$ node $\_152$ \\
\hline & 1 & & node_167 $0-->1$ node_165 & & 1 & node_153 $0==>1$ Hyphessobryconco \\
\hline & 1 & & node_121 1 -->0 node_118 & & 1 & node_154 $0==>1$ Hyphessobryconmi \\
\hline & 1 & & node_114 $0 \rightarrow 1$ node_113 & & 1 & node_158 $0==>1$ Hyphessobryconeq \\
\hline & 1 & & node $1011==>0$ Tetragnopterusar & & 1 & node $1640=\Rightarrow 1$ node 163 \\
\hline & 1 & & node_105 1 -->0 Hemigrammuspreto & & 1 & node_166 $0==>2$ Hemigrammusocell \\
\hline & 1 & & node 1071 --> 0 Hemigrammusthapo & & 1 & node_169 $1==>0$ Thayeriaobliqua \\
\hline
\end{tabular}


Serra, J. P. - Análise Filogenética das Espécies de Hemigrammus Gill, 1858 (Characiformes, Characidae)

\begin{tabular}{|c|c|c|c|c|c|c|}
\hline & 1 & & node_176 1 ==> 0 node_175 & 136 & 0.500 & node_97 $0==>1$ Iguanodectesspil \\
\hline & 1 & & node_180 $1==>0$ Aphyocaraxpusill & & 1 & node_185 $0==>1$ node_184 \\
\hline & 1 & & node_182 $1==>0$ Bryconamericusex & 137 & 0.111 & node_184 $0==>1$ node_181 \\
\hline \multirow[t]{2}{*}{127} & 0.500 & 1 & node_123 $0==>1$ node_122 & & 1 & node $\_1211==>0$ node 118 \\
\hline & 1 & & node_ $1760==>1$ node 175 & & 1 & node_1130 -->1 node_107 \\
\hline 128 & 1.000 & 1 & node $1740==>1$ HasemaniaspCrenu & & 1 & node_1051 -->0 node_104 \\
\hline \multirow[t]{3}{*}{129} & 0.667 & 1 & node_100 $1==>0$ node_99 & & 1 & node_$\_1100==>1$ Hollandichthsper \\
\hline & 1 & & node $123 \overline{1}==>2$ node $12 \overline{-}$ & & 1 & node $1281==>0$ Microschemobryco \\
\hline & 1 & & node $1751==>2$ node 174 & & 1 & node $1461==>0$ Moenkhausiabonit \\
\hline 130 & 1.000 & 1 & node_169 $0==>1$ Thayeriaobliqua & & 1 & node_152 $1==>0$ Moenkahusiahemig \\
\hline \multirow[t]{9}{*}{131} & 0.111 & 1 & node_185 $0-->1$ node_184 & & 1 & node_177 $1==>0$ Serrapinnusheter \\
\hline & 1 & & node_102 $1==>0$ node_ $10 \overline{0}$ & 138 & 0.067 & 1 node_176 0 --> 1 node_173 \\
\hline & 1 & & node_103 $1==>0$ Stethaprioneryth & & 1 & node $1021==>0$ node 100 \\
\hline & 1 & & node_108 $1==>0$ Astyanaxmexicanu & & 1 & node $\_1131==>0$ node $\_112$ \\
\hline & 1 & & node $1111==>0$ node 110 & & 1 & node $1181==>0$ node 117 \\
\hline & 1 & & node_116 $1==>0$ Astyanaxjacobina & & 1 & node_134 1 -->0 node_ 131 \\
\hline & 1 & & node_1781 -->0 Cheirodoninterru & & 1 & node_1260 --> 1 node_125 \\
\hline & 1 & & node_1801-->0 Aphyocaraxpusill & & 1 & node_124 $1-->0$ node_123 \\
\hline & 1 & & node_183 $1->0$ node_182 & & 1 & node_127 0 --> 1 Hemigrammusbello \\
\hline 132 & 0.133 & 1 & node_186 $0==>2$ node_185 & & 1 & node_128 0 --> 1 Microschemobryco \\
\hline & 1 & & node_ $1762==>1$ node_ 173 & & 1 & node_132 $1==>0$ Hemigrammustride \\
\hline & 1 & & node_$-1651==>2$ node_ 121 & & 1 & node_139 1 ==> 0 Hyphessobrycondi \\
\hline & 1 & & node_115 2 --> 1 node_$\_-114$ & & 1 & node_160 $1==>0$ node_159 \\
\hline & 1 & & node 991 ==> 2 Serrasalmusmacul & & 1 & node $1631==>0$ Hemigrammusulrey \\
\hline & 1 & & node_1131 -->2 node_112 & & 1 & node_172 $1-->0$ Hemigrammusstict \\
\hline & 1 & & node_1112 -->0 node_110 & & 1 & node_183 $0==>1$ Rhinopetitiamyer \\
\hline & 1 & & node_110 0 -->1 node_109 & 139 & 0.333 & node_188 $1==>2$ Brycinuslongipin \\
\hline & 1 & & node_119 $2==>1$ Hemigrammuscylin & & 1 & node_187 $1==>0$ node_96 \\
\hline & 1 & & node $\_1311==>2$ node $\_126$ & & 1 & node_98 $1==>2$ Lignobryconmyers \\
\hline & 1 & & node_-122 $2==>0$ Grunduluscochae & & 1 & node_97 $1==>0$ Triportheusnemat \\
\hline & 1 & & node_123 $2==>1$ Paracheirodonaxe & & 1 & node_107 $1==>0$ node_106 \\
\hline & 1 & & node_153 $1==>2$ Hyphessobryconco & & 1 & node_123 $1==>2$ node_122 \\
\hline & 1 & & node_177 $2==>1$ Serrapinnusnotom & 140 & 0.231 & 1 node_187 $1==>0$ node_96 \\
\hline & 1 & & node_182 $2==>1$ Piabinaargentea & & 1 & node_97 $==>2$ Triportheusnemat \\
\hline 133 & 0.048 & 1 & node_179 $1==>0$ node_176 & & 1 & node_121 $1==>2$ node_118 \\
\hline & 1 & & node_172 $0->1$ node_171 & & 1 & node_1052 -->0 node_104 \\
\hline & 1 & & node_115 $1==>0$ node $\_114$ & & 1 & node_100 0 --> 2 Psellorammuskenn \\
\hline & 1 & & node_102 0 --> 1 node_- 100 & & 1 & node_103 0 --> 2 Poptellaparaguay \\
\hline & 1 & & node 991 -->0 Roeboidesbonarie & & 1 & node_1072 ==> 1 Hemigrammusthapo \\
\hline & 1 & & node $1050==>1$ Hemigrammuspreto & & 1 & node_108 $2==>1$ Astyanaxmexicanu \\
\hline & 1 & & node_108 $0==>1$ Astyanaxmexicanu & & 1 & node_116 2 ==> 1 Astyanaxjacobina \\
\hline & 1 & & node_ $1110==>1$ Bryconexodonjuru & & 1 & node_123 $1==>3$ node_ 122 \\
\hline & 1 & & node_117 $1==>0$ Jupiabapolylepis & & 1 & node_159 $1==>2$ Hyphessobryconta \\
\hline & 1 & & node_119 $1==>0$ Hemigrammuscylin & & 1 & node_174 $1==>2$ HasemaniaspCrenu \\
\hline & 1 & & node_151 $1==>0$ node_149 & & 1 & node_183 $1==>2$ Rhinopetitiamyer \\
\hline & 1 & & node_142 $0==>1$ node_136 & 141 & 0.200 & 1 node_187 $0->1$ node_186 \\
\hline & 1 & & node_122 $1==>0$ Grunduluscochae & & 1 & node_97 $1-->0$ Triportheusnemat \\
\hline & 1 & & node_1390 $0->1$ node_138 & & 1 & node_1071 -->0 node_106 \\
\hline & 1 & & node_ $1470==>1$ node $\_146$ & & 1 & node_100 $0==>2$ Psellorammuskenn \\
\hline & 1 & & node_161 $1==>0$ node_152 & & 1 & node_103 $0==>2$ Poptellaparaguay \\
\hline & 1 & & node_159 $1==>0$ node_158 & & 1 & node_106 $0-->2$ Moenkhausiasanct \\
\hline & 1 & & node_153 $0==>1$ Pristellamaxilla & & 1 & node_108 $1==>2$ Astyanaxaltipara \\
\hline & 1 & & node $1631==>0$ Hemigrammuslunat & & 1 & node 1091 ==> 2 Oligosarcuspinto \\
\hline & 1 & & node 1681 -->0 Hemigrammusbarri & & 1 & node_174 $1==>2$ HasemaniaspCrenu \\
\hline & 1 & & node $1740==>1$ Hasemaniacrenuch & & 1 & node_188 0 --> 2 Chalceusspilogyr \\
\hline 134 & 0.077 & 1 & node_105 $1==>0$ node_104 & 142 & 1.000 & 1 node_139 $0==>1$ node 138 \\
\hline & 1 & & node_ $1000==>1$ node_99 & 143 & 0.125 & node_101 $0==>1$ Gymnocorymbuster \\
\hline & 1 & & node_112 $1==>0$ node_108 & & 1 & node_109 $0==>1$ Oligosarcuspinto \\
\hline & 1 & & node $1101==>0$ node_109 & & 1 & node_122 $0=\Rightarrow 1$ Coptobryconbilin \\
\hline & 1 & & node_114 $1==>0$ Moenkhausiaxingu & & 1 & node_125 $0==>1$ Hemigrammusyinyan \\
\hline & 1 & & node_117 $1==>0$ Jupiabapolylepis & & 1 & node_133 $0==>1$ Hemigrammusschma \\
\hline & 1 & & node_119 $1==>0$ Hemigrammuscylin & & 1 & node_152 $0==>1$ Hemigrammusunili \\
\hline & 1 & & node_129 $1==>0$ Hyphessobryconei & & 1 & node_153 $0==>1$ Pristellamaxilla \\
\hline & 1 & & node_136 $1==>0$ Hemigrammusbrevi & & 1 & node_158 $0==>1$ Hyphessobryconeq \\
\hline & 1 & & node_1381 -->0 node_137 & 144 & 0.154 & node_104 $0->1$ node_102 \\
\hline & 1 & & node_143 $1==>0$ Hemigrammuspulch & & 1 & node_1001 -->0 node_99 \\
\hline & 1 & & node $1471==>0$ Hemigrammusparan & & 1 & node $1230==>2$ Paracheirodonaxe \\
\hline & 1 & & node $1751==>0$ Hasemaniamelanur & & 1 & node_134 0 --> 1 node_133 \\
\hline 135 & 0.056 & 1 & node_188 1 --> 0 node_187 & & 1 & node_132 1 -->0 Hemigrammustride \\
\hline & 1 & & node_1860 $0->1$ node_185 & & 1 & node $1610==>1$ node_160 \\
\hline & 1 & & node_1031 ==> 0 Stethaprioneryth & & 1 & node_153 1 ==> 0 Hyphessobryconco \\
\hline & 1 & & node_116 $1==>0$ Astyanaxjacobina & & 1 & node_156 $1==>0$ Hyphessobryconep \\
\hline & 1 & & node_119 $1==>0$ Hemigrammusskoli & & 1 & node_164 0 --> 2 node_163 \\
\hline & 1 & & node_136 $1==>0$ node_135 & & 1 & node_166 $0==>1$ Hemigrammuscoeru \\
\hline & 1 & & node_122 $0==>1$ Coptobryconbilin & & 1 & node_168 $0==>2$ Hemigrammusbarri \\
\hline & 1 & & node_132 $0==>1$ Hemigrammustride & & 1 & node_172 $0==>1$ Hemigrammusstict \\
\hline & 1 & & node_141 $1==>0$ Hemigrammusarua & & 1 & node_177 $0==>1$ Serrapinnusnotom \\
\hline & 1 & & node_150 $1==>0$ Hemigrammussilim & 145 & 0.067 & 1 node_188 $0==>1$ Brycinuslongipin \\
\hline & 1 & & node_160 $1==>0$ Hyphessobryconbe & & 1 & node_106 $0==>1$ Moenkhausiasanct \\
\hline & 1 & & node_153 $1==>0$ Pristellamaxilla & & 1 & node_134 $0-->1$ node_131 \\
\hline & 1 & & node_158 $1==>0$ Hyphessobryconeq & & 1 & node_1241 -->0 node_123 \\
\hline & 1 & & node $1661==>0$ Hemigrammuscoeru & & 1 & node $1301->0$ node 129 \\
\hline & 1 & & node $1701==>0$ Parapristellageo & & 1 & node_132 $0==>1$ Hemigrammusvorde \\
\hline & 1 & & node_177 $1==>0$ Serrapinnusheter & & 1 & node_140 $0-->1$ node_139 \\
\hline & 1 & & node_1811 -->0 node_180 & & 1 & node_144 $0==>1$ node_ 143 \\
\hline & 1 & & node $1831->0$ Rhinopetitiamyer & & 1 & node_159 $0->1$ node 158 \\
\hline
\end{tabular}


Serra, J. P. - Análise Filogenética das Espécies de Hemigrammus Gill, 1858 (Characiformes, Characidae)

\begin{tabular}{|c|c|c|c|c|c|c|c|}
\hline & 1 & & node_157 1 -->0 node_155 & & 1 & & node_163 $0==>1$ Hemigrammusulrey \\
\hline & 1 & & node_167 $0==>1$ node $\_166$ & & 1 & & node_168 $0==>1$ Hemigrammusbarri \\
\hline & 1 & & node_169 $0==>1$ Hemigrammusanali & & 1 & & node_175 $0==>1$ node_174 \\
\hline & 1 & & node_172 $0==>1$ Hemigrammusstict & & 1 & & node_183 $0-->1$ node_182 \\
\hline & 1 & & node_182 $0==>1$ Piabinaargentea & 152 & 1.000 & 1 & node_162 $0==>1$ node_161 \\
\hline & 1 & & node_185 $0==>1$ Bryconopsmelanur & 153 & 0.667 & 1 & node_99 $0==>1$ Serrasalmusmacul \\
\hline \multirow[t]{6}{*}{146} & 0.167 & 1 & node_119 $0==>1$ Hemigrammuscylin & & 1 & & node_138 $0==>3$ node_137 \\
\hline & 1 & & node_ $1300==>1$ node_ 127 & & 1 & & node_ $1610==>2$ node_152 \\
\hline & 1 & & node_139 $0==>1$ Hyphessobrycondi & & 1 & & node $1600==>1$ node_159 \\
\hline & 1 & & node_143 $0==>1$ Hemigrammusharal & & 1 & & node_153 $1==>4$ Pristellamaxilla \\
\hline & 1 & & node_169 $0==>1$ Hemigrammusanali & & 1 & & node_167 $0==>1$ node_166 \\
\hline & 1 & & node_173 $0==>1$ Hemigrammuslevis & 154 & 1.000 & 1 & node_161 $0==>1$ node_152 \\
\hline \multirow[t]{26}{*}{147} & 0.115 & 1 & node_187 $1==>0$ node_186 & 155 & 0.222 & 1 & node_98 $0==>2$ Lignobryconmyers \\
\hline & 1 & & node_ $1710==>1$ node_168 & & 1 & & node_122 $0==>2$ Coptobryconbilin \\
\hline & 1 & & node $1061==>2$ node 105 & & 1 & & node $1300==>2$ node 127 \\
\hline & 1 & & node $1022-->0$ node $\overline{1} 00$ & & 1 & & node $1340==>2$ node 133 \\
\hline & 1 & & node_99 0 --> 3 Roeboidesbonarie & & 1 & & node_141 $0==>2$ Hemigrammusarua \\
\hline & 1 & & node_100 0 --> 1 Psellorammuskenn & & 1 & & node_153 $0==>2$ Pristellamaxilla \\
\hline & 1 & & node_110 $1==>2$ Hollandichthsper & & 1 & & node_1640 --> 1 node_163 \\
\hline & 1 & & node_109 $1==>3$ Phenacogasterfra & & 1 & & node_1631 --> 2 Hemigrammuslunat \\
\hline & 1 & & node_115 $1==>0$ Moenkhausiainter & & 1 & & node_168 $0==>1$ Hemigrammusbarri \\
\hline & 1 & & node_117 $1==>3$ Jupiabapolylepis & 156 & 0.667 & 1 & node_119 $0==>2$ Hemigrammuscylin \\
\hline & 1 & & node_162 $1==>0$ node 151 & & 1 & & node_130 $0==>1$ node_127 \\
\hline & 1 & & node $\_1360==>1$ node_135 & & 1 & & node $1410==>2$ node 140 \\
\hline & 1 & & node_124 1 -->0 node_-123 & 157 & 0.074 & 1 & node_96 0 --> 2 Bryconsp \\
\hline & 1 & & node_122 0 --> 1 Grunduluscochae & & 1 & & node_97 0 --> 2 lguanodectesspil \\
\hline & 1 & & node_125 $1==>2$ Hemigrammusyinyan & & 1 & & node_186 0 --> 2 node_185 \\
\hline & 1 & & node_130 $1==>0$ node_129 & & 1 & & node_1152-->0 node_114 \\
\hline & 1 & & node_132 $1==>0$ Hemigrammustride & & 1 & & node_99 0 --> 2 Serrasalmusmacul \\
\hline & 1 & & node $1410==>3$ Hemigrammusarua & & 1 & & node 1010 --> 2 Gymnocorymbuster \\
\hline & 1 & & node $1440==>2$ node 143 & & 1 & & node $1040-->2$ node 103 \\
\hline & 1 & & node_153 1 ==> 0 Hyphēssobryconco & & 1 & & node_106 0 --> 1 Moenkhausiasanct \\
\hline & 1 & & node_166 $1==>2$ Hemigrammusocell & & 1 & & node_107 0 --> 2 Hemigrammusthapo \\
\hline & 1 & & node_169 $0==>1$ Hemigrammusanali & & 1 & & node_110 $0==>2$ Hollandichthsper \\
\hline & 1 & & node $1720==>3$ Hemigrammusstict & & 1 & & node_117 $2==>0$ node_116 \\
\hline & 1 & & node_175 $0==>1$ node_ 174 & & 1 & & node_120 2 ==> 1 Hemigrammusnewbo \\
\hline & 1 & & node_180 $0==>1$ Aphyocaraxanisit & & 1 & & node_1192 ==> 0 Hemigrammusskoli \\
\hline & 1 & & node_184 $0=\Rightarrow 1$ node_183 & & 1 & & node $1512==>0$ node_149 \\
\hline 148 & 0.125 & 1 & node_187 2 -->0 node_186 & & 1 & & node_1350 --> 1 node_134 \\
\hline & 1 & & node_176 $0-->2$ node_173 & & 1 & & node_134 $1->2$ node_131 \\
\hline & 1 & & node_1712 -->0 node_168 & & 1 & & node_126 2 -->0 Hyphessobryconco \\
\hline & 1 & & node_100 0 --> 2 node_99 & & 1 & & node $1400==>1$ Hemigrammusboese \\
\hline & 1 & & node_108 $0==>1$ Astyanaxaltipara & & 1 & & node $1410==>2$ Hemigrammusarua \\
\hline & 1 & & node_112 $0-->2$ node_111 & & 1 & & node $1470==>2$ Hemigrammusparan \\
\hline & 1 & & node 1092 -->0 Oligos̄arcuspinto & & 1 & & node $1662==>1$ Hemigrammusocell \\
\hline & 1 & & node $1140==>2$ Moenkhausiaxingu & & 1 & & node $1682==>0$ Hemigrammusbarri \\
\hline & 1 & & node_117 $0==>2$ Jupiabapolylepis & & 1 & & node_169 2 ==> 0 Thayeriaobliqua \\
\hline & 1 & & node_127 $0==>2$ Hemigrammusorthu & & 1 & & node_175 $2==>0$ Hasemaniamelanur \\
\hline & 1 & & node_135 $0==>2$ Hemigrammushyanu & & 1 & & node $1792==>0$ node_178 \\
\hline & 1 & & node_155 0 --> 2 node_153 & & 1 & & node $1802==>0$ Aphyocaraxpusill \\
\hline & 1 & & node_156 $0==>1$ Hyphessobryconep & & 1 & & node_188 0 --> 2 Chalceusspilogyr \\
\hline & 1 & & node_164 $0-->1$ node_163 & 158 & 0.364 & 1 & node_97 $0==>1$ Iguanodectesspil \\
\hline & 1 & & node_163 1 --> 2 Hemigrammuslunat & & 1 & & node_1150 $0=>2$ Moenkhausiainter \\
\hline & 1 & & node_168 $0->1$ Hemigrammusbarri & & 1 & & node_126 $0==>2$ Hyphessobryconco \\
\hline 149 & 0.125 & 1 & node_101 $0==>1$ Gymnocorymbuster & & 1 & & node_129 $0==>2$ Hyphessobryconei \\
\hline & 1 & & node_104 $0==>1$ node_103 & & 1 & & node_140 0 --> 2 node_139 \\
\hline & 1 & & node_117 $0==>1$ Jupiabapolylepis & & 1 & & node_139 $2->3$ node_138 \\
\hline & 1 & & node_125 $0==>1$ Hemigrammusyinyan & & 1 & & node $1470==>2$ node $\_146$ \\
\hline & 1 & & node $1440=\Rightarrow 1$ node 143 & & 1 & & node $1500=\Rightarrow 1$ Hemigrammussilim \\
\hline & 1 & & node $1540==>1$ Hyphèssobryconme & & 1 & & node $1690==>4$ Thayeriaobliqua \\
\hline & 1 & & node $1670==>1$ node 166 & & 1 & & node $1820==>2$ Bryconamericusex \\
\hline & 1 & & node $1720==>1$ Hemigrammusstict & & 1 & & node $1850==>1$ Bryconopsmelanur \\
\hline 150 & 0.167 & 1 & node_188 0 --> 1 Brycinuslongipin & 159 & 0.071 & 1 & node_96 $0==>2$ Salminushilarii \\
\hline & 1 & & node_1870 0 -> 1 node_186 & & 1 & & node_98 $0==>2$ node_97 \\
\hline & 1 & & node_100 $1==>0$ node_99 & & 1 & & node_179 $0-->2$ node_176 \\
\hline & 1 & & node_105 $1==>0$ Hemigrammuspreto & & 1 & & node_173 $2->0$ node_172 \\
\hline & 1 & & node $1101==>0$ Hollandichthsper & & 1 & & node $1000=\Rightarrow 1$ Psellorammuskenn \\
\hline & 1 & & node_116 1 ==> 0 Astyanaxjacobina & & 1 & & node_105 $0==>2$ Hemigrammuspreto \\
\hline & 1 & & node_122 $1==>0$ Grunduluscochae & & 1 & & node_106 $0==>1$ Moenkhausiasanct \\
\hline & 1 & & node $1301==>0$ node 129 & & 1 & & node_ $1120==>2$ node_108 \\
\hline & 1 & & node $\_1611==>0$ node $\_160$ & & 1 & & node_110 0 --> 1 node_109 \\
\hline & 1 & & node $-1550==>1$ node_ 153 & & 1 & & node_109 1 --> 2 Oligosarcuspinto \\
\hline & 1 & & node_163 $1==>2$ Hemigrammusulrey & & 1 & & node_121 $0-->2$ node_120 \\
\hline & 1 & & node_168 $1==>2$ Hemigrammusbarri & & 1 & & node_1192 -->0 Hemigrammuscylin \\
\hline 151 & 0.063 & 1 & node_188 $0-->1$ node_187 & & 1 & & node_142 $0==>1$ node_136 \\
\hline & 1 & & node $1851->0$ node 184 & & 1 & & node $1341==>0$ node 131 \\
\hline & 1 & & node_102 $0-->1$ node_100 & & 1 & & node_124 $0==>2$ Bryconellapallid \\
\hline & 1 & & node_108 $0==>1$ Astyanaxmexicanu & & 1 & & node_126 $0==>2$ Hyphessobryconco \\
\hline & 1 & & node_117 $0->1$ node_116 & & 1 & & node_139 $0==>2$ node_138 \\
\hline & 1 & & node_121 $0->1$ node_120 & & 1 & & node $1400==>1$ Hemigrammusboese \\
\hline & 1 & & node_119 1 -->0 Hemigrammuscylin & & 1 & & node_149 $0==>2$ node_148 \\
\hline & 1 & & node $1250==>1$ node 124 & & 1 & & node_145 $2==>0$ Hemigrammusmargi \\
\hline & 1 & & node_137 $0==>1$ Petitellageorgia & & 1 & & node_150 $0==>1$ Hemigrammusgraci \\
\hline & 1 & & node $1460==>1$ node 145 & & 1 & & node $1660==>1$ Hemigrammusocell \\
\hline & 1 & & node $1500=\Rightarrow 1$ Hemigrammusgraci & & 1 & & node $1680==>1$ Hemigrammusbarri \\
\hline & 1 & & node_153 $0==>1$ Pristellamaxilla & & 1 & & node_169 $0==>2$ Thayeriaobliqua \\
\hline
\end{tabular}




\begin{tabular}{|c|c|c|}
\hline & 1 & node_170 $0==>1$ Parapristellageo \\
\hline & 1 & node_177 $0==>1$ Serrapinnusheter \\
\hline & 1 & node_182 $0==>2$ Bryconamericusex \\
\hline & 1 & node_185 $0==>2$ Bryconopsmelanur \\
\hline 160 & 1.000 & 1 node_166 $0==>1$ Hemigrammusocell \\
\hline 161 & 1.000 & node_-123 $0==>1$ node_122 \\
\hline 162 & 1.000 & node_105 $0==>1$ node_104 \\
\hline 163 & 0.333 & node_99 $0==>1$ Serrasalmusmacul \\
\hline & 1 & node_1020 ==> 1 node_101 \\
\hline & 1 & node_103 $0==>1$ Poptellaparaguay \\
\hline 164 & 1.000 & node_99 $0==>1$ Serrasalmusmacul \\
\hline 165 & 0.125 & node_102 0 --> 1 node_100 \\
\hline & 1 & node_99 1 --> 0 Serrasalmūsmacul \\
\hline & 1 & node_109 $0==>1$ Phenacogasterfra \\
\hline & 1 & node_123 $0==>1$ Paracheirodonaxe \\
\hline & 1 & node_129 $0==>1$ node_128 \\
\hline & 1 & node_161 $0==>1$ node_160 \\
\hline & 1 & node_155 $1==>0$ node_153 \\
\hline & 1 & node_179 $0==>1$ node_178 \\
\hline
\end{tabular}


Autorizo a reprodução xerográfica para fins de pesquisa.

São José do Rio Preto, 14/04/2010

Jane Piton Serra 